Portland State University

PDXScholar

$7-29-2020$

\title{
Synthesizing Expressive Behaviors for Humanoid Robots
}

Mathias Irwan Sunardi

Portland State University

Follow this and additional works at: https://pdxscholar.library.pdx.edu/open_access_etds

Part of the Robotics Commons

Let us know how access to this document benefits you.

\section{Recommended Citation}

Sunardi, Mathias Irwan, "Synthesizing Expressive Behaviors for Humanoid Robots" (2020). Dissertations and Theses. Paper 5566.

https://doi.org/10.15760/etd.7440

This Dissertation is brought to you for free and open access. It has been accepted for inclusion in Dissertations and Theses by an authorized administrator of PDXScholar. Please contact us if we can make this document more accessible: pdxscholar@pdx.edu. 
Synthesizing Expressive Behaviors for Humanoid Robots

by

Mathias Irwan Sunardi

A dissertation submitted in partial fulfillment of the requirements for the degree of

Doctor of Philosophy

in

Electrical and Computer Engineering

Dissertation Committee:

Marek Perkowski, Chair

Christof Teuscher

Xiaoyu Song

Gerardo Lafferriere

Portland State University

2020 
(c) 2020 Mathias Irwan Sunardi 


\begin{abstract}
Humanoid robots are expected to be able to communicate with expressive gestures at the same level of proficiency as humans. However, creating expressive gestures for humanoid robots is difficult and time consuming due to the high number of degrees of freedom (DOF) and the iterations needed to get the desired expressiveness. Current robot motion editing software has varying levels of sophistication of motion editing tools ranging from basic ones that are text-only, to ones that provide graphical user interfaces (GUIs) which incorporate advanced features, such as curve editors and inverse kinematics. These tools enable users to create simple motions; but creating expressive motions is laborious and demands a lot of patience, as well as technical and artistic skills from the user. Therefore, most humanoid robots have a limited range of expressive motions with little variety and executed the same way each time. Future robots should be able to generate expressive gestures on the fly during interaction with humans.

This work presents several new methods for creating expressive motions in humanoid robots that we have not seen in other robot motion editors. The first is a new method of composing robot gestures and behaviors that uses algebraic expressions by utilizing probabilistic operators that are extensions of simple algebraic operators such as concatenation, union, repetition, and subtraction. This method also allows hierarchical composition by reusing previously-defined behaviors in another expression, enabling generation of highly complex behaviors. I implemented this method as a tool called Robot Expressive Behavior Language (REBeL). The utility of this method is demonstrated by creating various behaviors for the HROS-1 mini humanoid robot and the adult-human-sized robot Mr. Jeeves. The second method analyzes MIDI music to extract timing information. This method allows motions to be executed with
\end{abstract}


more rhythmic and dynamic varieties without requiring the user to manually specify and edit the motion data. The third method uses Kochanek-Bartels interpolation parameters of tension, bias, and continuity to produce follow-through, overlapping, and anticipation effects from traditional animation principles. Additionally, I employ multiresolution analysis using wavelets to filter motion data in two tasks: 1) The first task was to reduce jerk when concatenating two or more motion data where the joins are discontinuous. We found that jerk was reduced not only at the joins, but everywhere else in the motion data without deviating much from the original data. 2) The second task was to create various motion expressions. By reconstructing the motion data, using only a subset of the filterbanks obtained from the multiresolution analysis, we found that different wavelets produced different effects such as tremors, stuttering, and the 'lazy' version of the motion. In addition, the Haar wavelet was used to discretize values from our motion dataset for machine learning. This discretization method reduces the number of unique values in the dataset from over two million to just above two hundred values/bins, which makes it suitable for using one-hot encoding to train machine learning models.

In this work, I also present my results of using multiple machine learning models in two tasks. The first task was to evaluate the quality of robot motions by training classifiers that discriminate between motion capture data and robot motion data. For this task, I used binary decision tree, naïve Bayes, support vector machine (SVM), and a long short-term memory (LSTM) network as classifier models. A robot motion data sample is considered 'good' if the classifier misclassifies the sample as motion capture data. The second task is to produce new motion samples using generative models. I built three generative models: a LSTM network, a variational auto encoder (VAE) network, and a generative adversarial network (GAN) that was based on the deep convolutional GAN (DCGAN) model. My results show that all three generative 
models can produce data that are better than random noise and that have similar characteristics to motion capture data. The LSTM model especially benefits from the one-hot encoding of the training data. However, most of the data produced by these models do not always show meaningful communicative gestures. I also present my building a motion dataset to train these machine learning models using samples from motion capture data and existing robot motion data. 


\section{DEDICATION}

This dissertation is dedicated to my parents, who have been the backbone of support during my time working on this dissertation. Without their constant and enduring moral, spiritual, and financial supports, I would not have reached the end of this journey. 


\section{ACKNOWLEDGEMENTS}

Over the course of working on my dissertation, I have had the privilege of meeting and working with some truly remarkable people to whom I owe my deepest respect and gratitude.

First of all, it has been my great privilege to work on this dissertation under the supervision and guidance of Dr. Marek Perkowski. As my academic adviser, Dr Perkowiski's contribution to this work has been immeasurable. I am very grateful for his support on this project and for his mentorship over many years that has greatly expanded my horizons.

I would also like to thank Dr. Christof Teuscher, Dr. Xiaoyu Song, and Dr. Gerardo Lafferriere for the honor of agreeing to be on my dissertation committee and whose kind and helpful advice greatly improved my dissertation.

Thanks also to the many friends and colleagues who have helped me in my research, notably: Rick Armstrong, for playing a pivotal role in the development of the "Mr. Jeeves" robot and for inspiring me by his example to be a better engineer. And also thanks to Husnu Melih Erdogan and Mohamed Abidalrekab, for always being a dependable source of engaging discussions about robotics, machine learning, and cultural dynamics. Thanks also to Cassie Winter for the fun discussions on traditional animation concepts. Additional thanks go out to my colleagues Ellen Weeks, Dennis Gilbert, Will Garrick, Mike Gostomski, and Brennen Florey at the Office of Information Technology for giving me the opportunity to contribute to interesting projects and learn new technologies in a high-performing workplace and for their personal support for me over many years while I was completing this research.

Finally, I want to give thanks to my family for providing me with constant moral and financial support all these years, and to my wonderful girlfriend (and occasional 
copy-editor), April, who has been on my side throughout the highs and lows of this journey. Throughout this time, they have been a constant source of love, encouragement, and prayers. I will forever be grateful. 


\section{TABLE OF CONTENTS}

Abstract ..................... i

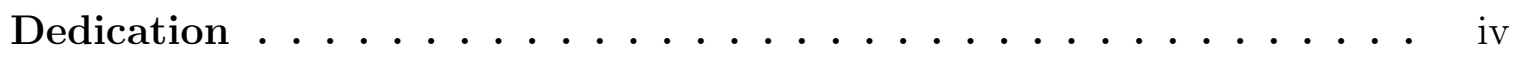

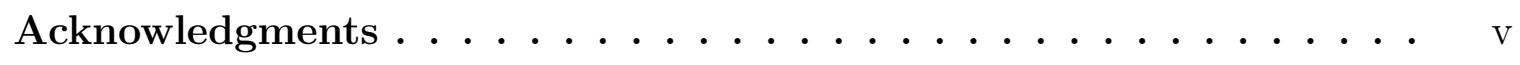

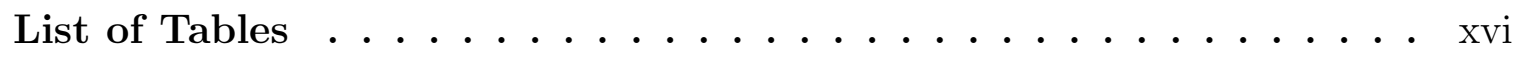

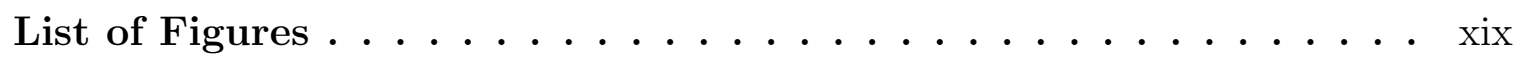

Chapter 1 Introduction ........................ 1

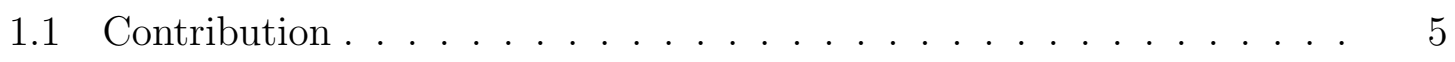

1.1.1 Primary contributions ................. 5

1.1.2 Auxiliary contributions . . . . . . . . . . . . . 7

1.2 Overview of Contributions ................... 8

1.3 List of Publications . . . . . . . . . . . . . . . . . 12

1.4 Scope and Limitations . . . . . . . . . . . . . . . 13

1.5 Overview of Dissertation . . . . . . . . . . . . . 14

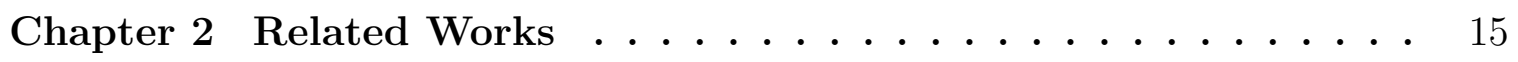


2.1 Expressive Behaviors of Social Robots _... . . . . . . . 15

2.2 Motion Vocabulary from Performance Arts and Traditional Animation 19

2.2 .1 Laban Movement Analysis . . . . . . . . . . . . . . . . . 20

2.2 .2 Principles of Animation _ . . . . . . . . . . . . . . 21

2.3 Humanoid Robot Motion Editing Tools . . . . . . . . . . . . . . 28

2.3.1 Animation Process using Motion Editors . . . . . . . . . . 30

2.3 .2 Pose Editor . . . . . . . . . . . . . . . . . . . . . . . 32

2.3 .3 Sequence Editor . . . . . . . . . . . . . . . . . . . . . 35

2.3.3.1 Tabular Sequence Editor . . . . . . . . . . . . . 37

2.3.3.2 Timeline Editor . . . . . . . . . . . . . . . 40

2.3.3.3 Curve Editor . . . . . . . . . . . . . . . . . . 42

2.3.4 Simulated/Virtual Robot . . . . . . . . . . . . . . . . 43

2.3.5 Dynamics Simulator and Inverse Kinematics . . . . . . . . 46

2.3.6 Flow Diagram Editor . . . . . . . . . . . . . . . . . 46

2.3 .7 Scripting . . . . . . . . . . . . . . . . . . . 49

2.4 Behavior Markup Language and its Related Frameworks . . . . . . 52

2.5 Synthesis Using Motion Capture Data _. . . . . . . . . . . 55

2.6 Dynamic Models and Biologically-inspired Methods . . . . . . . . 57

2.7 Synthesis Using Machine Learning Methods . . . . . . . . . . . . . . 60

2.8 Computational Creativity $\ldots \ldots \ldots \ldots$

2.8 .1 Single-domain Blending . . . . . . . . . . . . . 66 
2.8.2 Cross-Domain Blending . . . . . . . . . . . . . 66

2.8 .3 Other models of cognition $\ldots \ldots \ldots$. . . . . . . 67

2.8.4 Intersections Between My Work and Computational Creativity: Similarities and Differences . . . . . . . . . . . . . 68

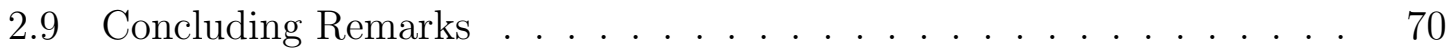

Chapter 3 Hardware and Software Platforms . . . . . . . . . 71

3.1 Robot Operating System (ROS) _ . . . . . . . . . . . . 71

3.2 The Robot Jeeves . . . . . . . . . . . . . . . . . . . . . . . . . . . 73

3.2 .1 Contributors . . . . . . . . . . . . . . . . . . 73

3.2 .2 Mobile Base . . . . . . . . . . . . . . . . . . . 77

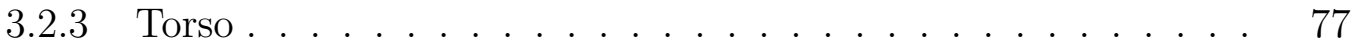

3.2 .4 Neck and Head . . . . . . . . . . . . . . . . . . . 79

3.2.5 Speech and Dialogue System . . . . . . . . . . . . 81

3.2 .6 User Interface . . . . . . . . . . . . . . . . . . . . . . . 83

3.2 .7 Source Code . . . . . . . . . . . . . . . . . . . . . . . . 83

$3.3 \quad$ HROS $-1 \ldots \ldots \ldots \ldots \ldots$

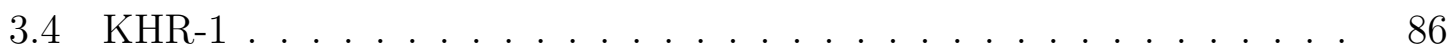

3.5 Lynxmotion Arm . . . . . . . . . . . . . . . . . . 87

3.6 Representation of Motion Data . . . . . . . . . . . . . . . . 89

3.7 Summary . . . . . . . . . . . . . . . . . . . . . . . . . . 93

Chapter 4 Algebraic Expressions for Expressive Behaviors . . . . . . 94 
4.1 Introduction . . . . . . . . . . . . . . . . . . . . 94

4.2 Composing Expressive Behaviors _. . . . . . . . . . . 95

4.3 Robot Expressive Behavior Language (REBeL) _ . . . . . . . . . 101

4.3 .1 Library of Basic Actions . . . . . . . . . . . . . . . 101

$4.3 .2 \quad$ Behavior Expressions . . . . . . . . . . . . . . . . . . . 102

4.3.2.1 Notation . . . . . . . . . . . . . . . . . . . 104

4.3.2.2 Generating Behaviors _. . . . . . . . . . . . 105

4.3.2.3 Similarity and Differences with Regular Expression . 106

4.3.2.4 Behavior Expression Operators _. . . . . . . . 107

4.3.2.4.1 Concatenation . . . . . . . . . . . 107

4.3.2.4.2 Union . . . . . . . . . . . . . . . 111

4.3.2.4.3 Repetition . . . . . . . . . . . . 115

4.3.2.4.4 Subtraction ............. . 118

4.3.2.5 Other/Future Behavior Expression Operations . . . . 122

4.3.2.5.1 Concurrency . . . . . . . . . . . . 122

4.3 .3 The REBeL Pipeline . . . . . . . . . . . . . . . . . . . 127

4.4 Results . . . . . . . . . . . . . . . . . . . . . . . . 128

4.4 .1 Probabilistic Behaviors . . . . . . . . . . . . . . . . . . . 129

4.4.1.1 Concatenation . . . . . . . . . . . . . 129

4.4.1.2 Union . . . . . . . . . . . . . . . . . . . . 131

4.4.1.3 Repetition . . . . . . . . . . . . . . . 131

4.4 .2 On HROS-1 Robot . . . . . . . . . . . . . . . . . . . 134

4.4 .3 Subtraction . . . . . . . . . . . . . . . 137

4.5 Multimodality . . . . . . . . . . . . . . . . . . . . 139 
4.6 My Contributions . . . . . . . . . . . . . . . . . . . . 141

Chapter 5 Music to Motion ................. 145

5.1 Introduction . . . . . . . . . . . . . . . . . 145

5.2 Related Works . . . . . . . . . . . . . . . . . . . . 149

5.2.1 Music and Motion . . . . . . . . . . . . . . . . 149

5.2 .2 Motion as Signals . . . . . . . . . . . . . . . . . . . 150

5.2.3 Synchronized Speech and Gestures . . . . . . . . . . . . . . 152

5.3 The Melodic Motion System . . . . . . . . . . . . . . . . 153

5.3.1 Parsing and Information Extraction of Music Data . . . . . 154

5.3.1.1 First Stage - Parsing/Collecting Note Events per Beat 155

5.3.1.2 Second Stage - Information Extraction . . . . . . . 156

5.3.2 Free Mode and Scenario Mode . . . . . . . . . . . . 161

5.3.2.1 Free/Dance Mode . . . . . . . . . . . . . . 161

5.3.2.2 Scenario Mode ... . . . . . . . . . . . . . . 165

5.4 Experiment . . . . . . . . . . . . . . . . 168

5.5 Results . . . . . . . . . . . . . . . . . . . . 171

5.5.1 Free/Dance Mode . . . . . . . . . . . . . . . . . . 172

5.5.2 Scenario Mode . . . . . . . . . . . . . . . . 174

5.6 Results from Non-parametric Analysis . . . . . . . . . . . . . . 176

5.6 .1 Results from First Survey . . . . . . . . . . . . . . . 179

5.6.1.1 Free/Dance Mode . . . . . . . . . . . . . 179 
5.6.1.2 Scenario Mode . . . . . . . . . . . . . . . . . . . . 182

5.6 .2 Summary ........................ 183

5.6.3 Results from Second Survey . . . . . . . . . . . . . . . . . . 183

5.6.3.1 Music Survey . . . . . . . . . . . . . . . 183

5.6.3.2 Free/Dance Mode . . . . . . . . . . . . . . . 184

5.6.3.3 Scenario Mode . . . . . . . . . . . . . . . 187

5.6.3.4 Summary ................... . . 189

5.6.4 Comparison of Results from Repeated-measure ANOVA versus Friedman Test . . . . . . . . . . . . . . . . . . . . . . . . . . 189

5.7 Discussion . . . . . . . . . . . . . . . . . . . . . . 192

5.8 Conclusions and Future Works . . . . . . . . . . . . . . . . . 195

5.9 Additional Videos . . . . . . . . . . . . . . . . . . . . . . . . . 198

5.10 Contributions . . . . . . . . . . . . . . . . . . 198

5.11 Future Works . . . . . . . . . . . . . . . . . . . 199

Chapter 6 Parameters for Motion Texturing . . . . . . . . . 200

6.1 Motion Texture .................... 203

6.2 Approaches of Executing Motion Data . . . . . . . . . . . . 205

6.3 Interpolating Gesture Data . . . . . . . . . . . . . . . . . . 208

6.3.1 Kochanek-Bartels Interpolation . . . . . . . . . . . 208

6.3.2 Applying KB Interpolation to Motion Data . . . . . . . . . . 211

6.4 Multiresolution Analysis . . . . . . . . . . . . . . . . . . 216 
6.4.1 Changing Motion Expressions . . . . . . . . . . . . . . 220

6.4.2 Merging of Motion Data . . . . . . . . . . . . . 221

6.5 Using Wavelets . . . . . . . . . . . . . . . . . . . 225

6.5.1 Transitions in Concatenation of Motion Data . . . . . . . . . 233

6.5.2 Discretized Steps . . . . . . . . . . . . . . . . . 244

6.5.3 Reduction .................... 246

6.5.4 'Lazy' or 'Bounded' Effect . . . . . . . . . . . . . . . . . . . . 250

6.5.5 Noise/jitter . . . . . . . . . . . . . . . . . 251

6.5.6 Linearize . . . . . . . . . . . . . . . . . . 254

6.5.7 Miscellaneous Effects . . . . . . . . . . . . . . . 254

6.6 Legibility of Wavelet Motion Effects on Actual Robot . . . . . . . . . 257

6.7 Contributions of This Work . . . . . . . . . . . . . . . 259

\section{Chapter 7 Machine Learning Models for Evaluating Synthesized Robot}

Motion .................... 262

7.1 Related Works . . . . . . . . . . . . . . . . 266

7.2 The Dataset . . . . . . . . . . . . . . . . . 267

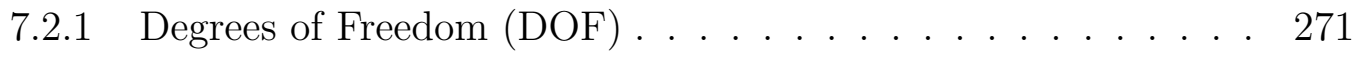

7.2 .2 Joint Angle Units . . . . . . . . . . . . . . . . . . . . . . . . 272

7.2.3 Frame Rate . . . . . . . . . . . . . . . . . . . . . 273

7.2.4 Dataset Size . . . . . . . . . . . . . . . . 274

7.2.5 Dataset Cleanup . . . . . . . . . . . . . . . 275 
7.3 The Discriminator/Classifier Models . . . . . . . . . . . 278

7.4 Naïve Bayes Classifier _ . . . . . . . . . . . . . . . . . . 279

7.4.1 Naïve Bayes Classification Results . . . . . . . . . . . . . . . . 281

7.5 Binary Decision Tree . . . . . . . . . . . . . . . . . . 281

7.5.1 Decision Tree Classification Results . . . . . . . . . . . . 283

7.5.2 Support Vector Machine . . . . . . . . . . . . . . . . . 284

7.5.3 SVM Classification Results . . . . . . . . . . . . . . 287

7.6 Long Short-Term Memory Network . . . . . . . . . . . . . . . 287

7.6 .1 The Model . . . . . . . . . . . . . . . . . . . . . . . 290

7.6.2 Classification Results . . . . . . . . . . . . . . . . . . 292

7.7 Contributions . . . . . . . . . . . . . . . . . . . . . . . 293

Chapter 8 Generative Models . . . . . . . . . . . . . . . 295

8.1 The LSTM Model . . . . . . . . . . . . . . . . . . . . . . . . . . . . 299

8.1.1 Dataset Preprocessing . . . . . . . . . . . . . . . . . 299

8.1.2 The LSTM Network Model _. . . . . . . . . . . . . . . 303

8.1.3 LSTM Generation Results . . . . . . . . . . . . . . . . . 303

8.2 Variational Auto Encoder . . . . . . . . . . . . . . 305

8.2 .1 Loss Function . . . . . . . . . . . . . . . . . . . . . . . . . . . 309

8.2.2 Motion Generation using VAE . . . . . . . . . . . . . . . 314

8.2 .3 Results . . . . . . . . . . . . . . . . . . . . 315

8.3 Generative Adversarial Network . . . . . . . . . . . . . . . . . . 321 
8.3.1 The Transposed Convolution Layer . . . . . . . . . . . . . . . 326

8.3.2 The Motion Generator GAN Model . . . . . . . . . . . . . . . 326

8.3.3 The Discriminator Model . . . . . . . . . . . . . . . . . . 327

8.3.4 Generator Input and Output Modes . . . . . . . . . . . . . . . 329

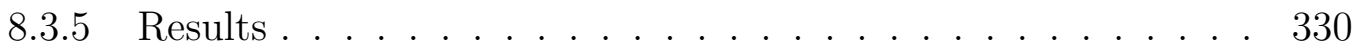

8.4 Evaluation of Motion Quality . . . . . . . . . . . . . . . 333

8.5 Contributions . . . . . . . . . . . . . . . . . . . . 338

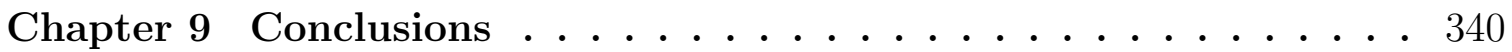

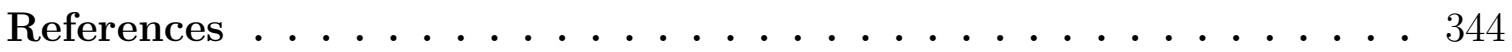

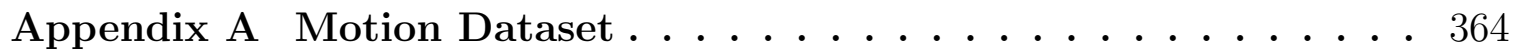




\section{LIST OF TABLES}

2.1 Pose editors and their widgets (note: Spinners always include editable textbox $\ldots \ldots \ldots \ldots \ldots \ldots \ldots \ldots$

2.2 Scripting command code from $[10] \ldots \ldots \ldots \ldots \ldots$

2.3 Example of actual script from $[10] . \ldots \ldots \ldots \ldots \ldots$

3.1 Example motion data representation for a "wave left hand" gesture. Each row is the robot's pose at one time step and each column is a DOF. 93

4.1 Similarities and differences between behavior expressions of REBeL and regular expressions. ${ }^{\dagger}$ Read: subtract $b$ from $a$. See section 4.4.4 for more details. ${ }^{\ddagger}$ Assumed groupings of operands unless explicitly stated using parentheses. . . . . . . . . . . . . . . . . 108

4.2 Probabilistic Concatenation . . . . . . . . . . . . . . . 110

4.3 Calculating concatenation Probabilities for Behavior Expressions from Table $4.2 \ldots \ldots \ldots \ldots \ldots$

4.4 Output set of the second variant probabilistic Concatenation . . . . 111

4.5 Union probability usage examples . . . . . . . . . . . . . . . . 114

4.6 Repetition usage example . . . . . . . . . . . . . . . 116

4.7 Repetition with Probability usage examplesge . . . . . . . . . 117

4.8 Additional Subtraction Example Usage . . . . . . . . . . . . . . . 122

4.9 Example usage of concurrency operation . . . . . . . . . . . 123 
4.10 Concurrency Delay Argument Usage and Examples . . . . . . . . . . 124

4.11 Concurrency Priority Argument Usage and Examples . . . . . . . . . 125

4.12 Supported Tasks comparison between the HROS Robot Motion Editor (RME) [15], Choreographe [16], Improv [11], BML Sequencer [75], and REBeL . . . . . . . . . . . . . . . . . . . 144

5.1 One of the note events in beat $3 \ldots \ldots \ldots 156$

5.2 Choosing Note Events Information and Production of $D i s_{i, j}$ and $A c c_{i, j}$ for a joint $j$ with maxrange $_{j}=1400$ and maxacc $_{j}=30 \ldots 160$

5.3 Allowable Movements for the Lynxmotion Robot DOFs . . . . . . . . 164

5.4 A few examples of generated motion data for the Lynxmotion robot . 164

5.5 KHR-1 Pose and Gesture Library . . . . . . . . . . . . . . . . 167

5.6 Compilation of hypothesis testing results of the first and second survey 190

5.7 Null hypothesis acceptance/rejection using ANOVA versus Friedman test for data in the first and second survey (see Table 5.6) . . . . . . 191

5.8 Post-hoc analysis using Scheffe versus Wilcoxon Signed-rank (WSR) tests for data in the first and second survey to find pairs of categories with significantly different ratings $(A=\operatorname{Robot} A, B=\operatorname{Robot} B$, $\mathrm{C}=$ RobotC, $\mathrm{D}=$ RobotD, $\mathrm{E}=$ RobotE) . . . . . . . . . . . 191

6.1 Differences between traditional keyframe animation and robot animation.202

6.2 Parameters to tune for executing expressive motions using our method 205

6.3 Jerk range in concatenation results $\left(\frac{d^{3} s}{d t^{3}}\right)$ (lower is better). . . . . . . . 241

6.4 Mean squared error of filtering with respect to original data (lower is better . . . . . . . . . . . . . . . . . . 241

6.5 Wavelets, and the filterbanks to use to create transition effects. $N$ is the number of resulting filterbanks from the discrete wavelet transform (DWT). ( $N / 2$ is usually rounded down to the nearest integer) . . . . 245 
8.1 LSTM motion generator parameters . . . . . . . . . . . . . . . 303

8.2 Encoder parameters . . . . . . . . . . . . . . . . 315

8.3 Decoder parameters . . . . . . . . . . . . . . . . 316

8.4 GAN generator parameters . . . . . . . . . . . . . 328

8.5 GAN discriminator parameters . . . . . . . . . . . . . . . 329

8.6 Evaluation of motion data generated by REBeL with additional texturing (TP: True Positive, TN: True Negative, FP: False Positive, FN: False Negative). Dataset: 0 motion capture samples, 1811 REBeL samples. . . . . . . . . . . . . . . . . . . . . 337

8.7 Evaluation of motion data generated by GAN motion generator (TP: True Positive, TN: True Negative, FP: False Positive, FN: False Negative). Dataset consists of 50 motion capture samples and 48 GANgenerated samples. . . . . . . . . . . . . . . . 337

8.8 Evaluation of motion data generated by VAE motion generator (TP: True Positive, TN: True Negative, FP: False Positive, FN: False Negative). Dataset consists of 320 motion capture samples and 319 VAEgenerated samples. . . . . . . . . . . . . . . . . . . . 337 


\section{LIST OF FIGURES}

1.1 Some examples of expressive robots: (a) KISMET [7], (b) LEONARDO [9], (c) KASPAR [3] . . . . . . . . . . . . . . . . . . . . 2

1.2 Robots used in this dissertation: (a) Mr. Jeeves robot, (b) HROS-1 mini humanoid robot. . . . . . . . . . . . . . . . .

2.1 Example of Anticipation principle in a jumping action [47]. Anticipation happens at the second frame when the character crouches downward, indicating he is about to leap upward. . . . . . . . . . . .

2.2 Example of Overlapping Action. In creating the animation of 'raising an arm', instead of only rotating at the shoulder, adding successive bending to the wrist and elbow (a technique called 'breaking of joints') makes the raising arm motion appear more organic rather than mechanical $[47] \ldots \ldots \ldots \ldots \ldots \ldots$. . . . . . . . . . .

2.3 The general animation process using motion editor tools for humanoid robots. . . . . . . . . . . . . . . . . . . .

2.4 Various pose editors (From top left, clockwise): WinRME, Choreographe (for Nao robot), RoboPlus Motion 2.0, Pololu Maestro, Choreonoid $[59] \ldots \ldots \ldots \ldots \ldots \ldots \ldots$

2.5 Puppeteering on the HROS-1 robot. The servos with ID 1-6 have values "????" because those servos are turned off so the author can move them into the desired pose by moving the robot (right). . . . .

2.6 Interface of the RME tool (left) and WinRME tool (right) for creating motions on HROS-1 humanoid robot [55] . . . . . . . . . . . . 38

2.7 Pololu Maestro Control Center sequence editor[57]. . . . . . . . . . 
2.8 Timeline editors: (a) Choreographe, (b) RoboPlus Motion 2.0, and (c) Lynxmotion Flowbotics . . . . . . . . . . . . . . . . . . . 41

2.9 Timeline editor in Blender . . . . . . . . . . . . . . . . . . . . 41

2.10 Choreographe detailed timeline editor $[16] \ldots \ldots$. . . . . . . . . . 42

2.11 Choreographe curve editor interface $[16] \ldots \ldots$. . . . . . . . . . 43

2.12 Examples of Bezier curves [63]. The nodes that do not lie on the curve are the control points. Top row: one control point creates quadratic Bezier curves. Bottom row: two control points create cubic Bezier curves. Notice that the end points in the quadratic and cubic curves remain in the same coordinates, and the shapes of the curves are determined by the positions of the control points. . . . . . . . . . . . . 44

2.13 Virtual robots in (a) Choreographe, (b) WinRME, (c) RoboPlus Motion $2.0 \ldots \ldots \ldots \ldots \ldots \ldots \ldots \ldots \ldots \ldots \ldots \ldots \ldots \ldots$

2.14 Flow diagram editor from (a) HeartToHeart4 [51], (b) Choreographe [16], and (c) Lego Mindstorms EV3 [56] . . . . . . . . . . . . . .

2.15 The Improv ACTION script defines low-level motion of the DOF of the agent (example from [11]). Each row in the "Talk Gesture1" ACTION block refers to a DOF (R_UP_ARM, R_LO_ARM, R_HAND). The three columns of numbers refer to the rotation in Pitch, Roll, and Yaw rotations in degrees, respectively. The number 25:55 refers to range of motion for the Pitch, 0 for Roll, and -35:65 for Yaw rotations of R_UP_ARM. The numbers in braces refer to interpolation using Perlin noise [64]. N0, N1, N2 refers to different configurations of Perlin

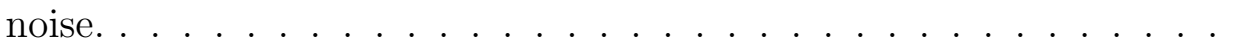

2.16 The Improv SCRIPT "greeting" defines a complete sequence of highlevel actions for an agent's greeting behavior (example from [11]). .

2.17 An example of a BML script (example from [70]). The speech "Yes, that was great" is synchronized with a head nod which starts at the beginning of the speech and ends after saying "Yes" at the "sync1" mark. 53

2.18 The BML Sequencer interface (Image from [75]). . . . . . . . . . . 54 
2.19 Visualization of motion capture data in Mokka tool (left) [77] and

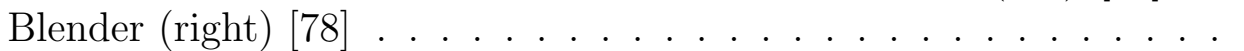

2.20 Interpolation and extrapolation using Fourier transform on two different styles of walking animation (image from [85]). The two reference animations are: (a) "Normal" walk, and (b) "Tired" walk. Adjusting the interpolation coefficient $\mathrm{s}$ in the Fourier domain, (c) shows a "slightly tired" walk $(\mathrm{s}=0.5),(\mathrm{d})$ "very tired" walk $(\mathrm{s}=2.0)$, (e) "brisk" walk $(\mathrm{s}=-0.5) \ldots \ldots \ldots \ldots \ldots \ldots$

2.21 Examples of applying genetic algorithms for gait generation without prior knowledge. On the left, examples of "creatures" created by Sims' algorithm for different kinds of locomotion. From top left, clockwise: for swimming, walking, and jumping [93]. (right) Some of the different bipedal models used by Geijtenbeek to develop walking, jumping, trotting gaits using genetic algorithm without being given gait examples $[29] \ldots \ldots \ldots \ldots \ldots \ldots \ldots$

2.22 Braitenberg vehicles light-approaching behavior (left) and light-avoiding behavior (right) are created by the hard-wiring the light sensors (circles) to the motors (grey cylinders) to turn the wheels (black cylinders). Filled circles indicate high intensity at the sensor. Solid and dashed lines are just to differentiate how the left and right motors are connected to the sensors. . . . . . . . . . . . . . . . . . .

2.23 Walking gait on dynamic terrains and obstacles learned using deep reinforcement learning [98]. . . . . . . . . . . . . . . . . .

2.24 Handwriting generation from an LSTM (recurrent) network of a given text "from his travels it might have been". The first line is from real handwriting data, the remaining lines were generated using recurrent neural network [100]. . . . . . . . . . . . . . . . . . .

2.25 A Conceptual Blending Network for 'houseboat' [115] . . . . . . . . .

3.1 Two types of interaction between two nodes in ROS: by service (top path) and by publish-subscribe mechanism via Topics (bottom path).

3.2 The Mr. Jeeves robot. . . . . . . . . . . . . . . . 
3.3 Various facial expressions of Jeeves with our modifications from the original code by Burgess . . . . . . . . . . . . . . . . . . . 74

3.4 3D model of Jeeves' mobile base core components _ . . . . . . . 78

3.5 Jeeves' head and neck mechanism. . . . . . . . . . . . . . . . 82

3.6 Jeeves' head/neck and face system. The animation of the eyes and mouth is controlled by an Arduino program from AdaFruit [127] that has been modified to be a ROS node, and the neck servo movements are controlled by a ROS node in the RaspberryPi. The head/neck and face system is connected to the Jeeves Main PC by connecting both in the same network via ethernet to a router. . . . . . . . . . . 82

3.7 Jeeves expressing the number 8 using hands. . . . . . . . . . . . 83

3.8 Jeeves' navigation user interface running on an Apple iPad. . . . . . . 84

3.9 HROS-1 robot $\ldots \ldots \ldots \ldots \ldots$

3.10 KHR-1 robot. . . . . . . . . . . . . . . . . . . . . . . . . . 87

3.11 Sample poses of the modified Lynxmotion AL5A Arm generated by music data $[130] . \ldots \ldots \ldots$. . . . . . . . . . . . . . 88

3.12 DOFs of the Lynxmotion robot. DOF 1 is the yaw rotation, DOF 2 and 3 perform pitch rotation and together creates the 'chicken neck' motion, DOF 4 is used to perform a roll to tilt the 'face' sideways. . . 88

3.13 Motion data in WinRME PoseAnimator (sequence editor) [55]. . . . 90

4.1 Five basic poses of the HROS-1 robot for hand-waving gestures: HOME, R_ARM_RAISE, R_ARM_OUT, L_ARM_RAISE, L_ARM_OUT. . . . 97

4.2 The sequence of poses (motion) of the WAVE_RIGHT_ARM gesture. 97

4.3 Concatenation with probability for the two expressions in Table 4.2. . 112

4.4 Concatenation with probability alternative version for the expressions in Table 4.4. . . . . . . . . . . . . . . . . . . . . . . . . . 112 
4.5 Graph for the first and third expressions in Table 4.5: (a) $(+a b),(\mathrm{b})$ $(+a b c d[0.1,0.3,0.4,0.2]) \ldots \ldots \ldots \ldots \ldots \ldots$

4.6 (a) Graph for expression 1 from table 4.6, (b) graph for expression 1 from Table 4.7, (c) graph for expression 3 from Table 4.7. The symbols $\_a$ and $\_b$ denote the symbols $\mathrm{a}$ and $\mathrm{b}$ are the second instances in the sequence.

4.7 Two types of conflicts: compatible (top) and incompatible (bottom).

4.8 The REBeL pipeline. A behavior expression is given as input to the behavior expression parser. The behavior expression parser will evaluate the expression and produce an instance of behavior sequence. Each symbol in the behavior sequence is converted into their corresponding motion data from the Database of Poses. The output of the behavior expression parser is a sequence of numbers which correspond to joint angle positions (i.e. sequence of poses) and some timing information (i.e. Time, PauseTime). The timing information is used to interpolate the poses to create the desired motion dynamics. Additionally, expressions can be added by applying filtering methods [13]. The interpolated and filtered motion data is then converted into motor commands. .

4.9 Occurrences of the output set from probabilistic concatenation operation for the expression: ( $\& a b c \mathrm{p})$ with $p$ is the probability value, where: (top) $p=0.5$, (middle) $p=0.25$, (bottom) $p=0.7$.

4.10 Occurrences of the output set from the probabilistic union operation for the expression: $(+A B C \mathrm{p})$ with $p$ as the probability value, where: (top) $p=0.5$, (middle) $p=0.2$, (bottom) $p=[0.3,0.15,0.55] . \ldots$

4.11 Occurrences of output set of a repetition expression $(* G)$ with different probability values $p$. (a) With default probability 0.5 , (b) $p=0.2$; $80 \%$ of occurrences is the empty string, (c) $p=2.6$ repeats at least twice, (d) with Gaussian probability distribution by specifying mean and standard deviations $\mu=10, \sigma=2.5$ produces sequences of symbol $G$ with length around $10 \ldots \ldots \ldots \ldots \ldots \ldots$

4.12 The sequence of poses of the "Yes" gesture on HROS-1 robot. Order: left to right, top to bottom. . . . . . . . . . . . . .

4.13 The sequence of poses of the "Wow" gesture on HROS-1 robot. Order: left to right, top to bottom. . . . . . . . . . . . 
4.14 HROS-1 model in the Gazebo simulation environment. (Snapshots at various moments in simulation) . . . . . . . . . . . . . 135

4.15 Motion data of the expression 4.25: (a) the original sequence (Eq. 4.26) with the stand poses at around frames 110, 290, 430, and 600 onward., (b) after the subtraction of stand pose (Eq. 4.28). . . . . . . . . 140

4.16 Video of animating Mr. Jeeves' head using REBeL. . . . . . . . . . . 141

5.1 High-level view of the Melodic Motion System. Input to the system is a MIDI information of a song represented in .XML format. Music information such as beat, pitch, note-on velocity, etc. are extracted as Time markers, Dis and Acc. This information is used to generate a sequence of actions by the Action Generator block, or regulate the execution of a Scenario by the Scenario Executor block. A scenario is a sequence of motions or gestures. The Gesture Library stores a set of gestures or poses that the user can use to create Scenarios. . . . . . . 154

5.2 MIDI music data in XML format from .med file . . . . . . . . 157

5.3 Output of the first stage of parsing. Each bin (row) is a list with the entries: tick/time mark, number of note events in this beat, the list of note events in this beat. Beat 3 and 4 each have multiple note events, while Beat 1-3 and 5 have none. Each note event is a list with entries: tick/time mark, duration (in ticks), note code, note-on velocity, noteoff velocity. . . . . . . . . . . . . . . . . .

5.4 Configuration of the Lynxmotion robot. The degrees of freedom (DOFs) on the robot are: base (1), lower arm (2), upper arm (3), and 'face' (4). The two-headed arrows near each DOF indicate the directions for the motions of the DOF. . . . . . . . . . . . . .

5.5 Some sample poses of the Lynxmotion robot which are generated by the Action Generator in Free/Dance mode using the displacement information extracted from music data. . . . . . . . . . . . . . .

5.6 Timed Events (Displacement and Acceleration) are applied to gestures by first associating each stroke of the gesture to a time marker. The Timed Events on each time marker are then applied to each stroke. Application of displacement value to stroke is scaled by a factor $\alpha \ldots 168$ 
5.7 Means and standard error for Free/Dance Mode from the first survey.

5.8 Survey result for the question: "Which robot performance did you like best?" . . . . . . . . . . . . . . . . . .

5.9 Means and standard error for Scenario Mode from the first survey. .

5.10 Survey result for the question: "Which robot performance did you like best?" . . . . . . . . . . . . . . . . . .

5.11 Means and standard error for Free/Dance Mode for the second survey. 181

5.12 Survey result for the question: "Which robot performance did you like

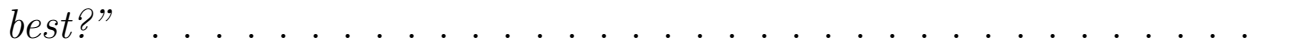

5.13 Ratings of music versus Free/Dance Mode video clips of three subjects (A, B, and $\mathrm{C}$ ). The four bars over 'Music' represent the ratings for four music pieces used: M1, M2, M3, M4. The five bars over 'DQ1', 'DQ2', and 'DQ3' represent the five video clips in each Free/Dance Mode survey question and labeled as 'music piece/video clip': 'M1/RobotA', 'M2/RobotC', 'M3/RobotB', 'M4/RobotD', 'M1/RobotE' . . . . . . .

5.14 Ratings of music versus Scenario Mode video clips of three subjects (A, B, and $\mathrm{C})$. These responses correspond to the same subjects in Figure 5.13. The three bars over 'Music' represent the ratings for three music pieces used: M1, M2, M4. The three bars over 'SQ1', 'SQ2', and 'SQ3' represent only the three video clips in each Scenario Mode survey question which uses music and labeled as 'music piece/video clip': 'M1/RobotB', 'M2/RobotD', 'M4/RobotC' . . . . . . . . . . . 185

5.15 Means and standard error for Scenario Mode from the second survey

5.16 Result for Q4: "Which robot performance did you like best?" from the second survey . . . . . . . . . . . . . . . . . . . . . . . . . . 188

5.17 Video demonstration of motions timed by music information from Lullaby of Birdland (https://youtu.be/JuDXhHRo2Kw) . . . . . . . . . 198

6.1 Four Hermite basis functions $h_{1}(s), h_{2}(s), h_{3}(s), h_{4}(s)$ (image from: [141]), where $s$ is on the x-axis, $h_{i}(s)$ is on the y-axis, and $s \in[0,1] . \quad 210$ 
6.2 The effects of different Bias values at a single point (image from: [141]). $($ Tension $=-0.2$, Continuity $=0.0$ ) (left) Bias=-1.0, (middle) Bias=0.0, (right) $\mathrm{Bias}=1.0 \ldots \ldots \ldots \ldots \ldots$

6.3 The effects of different Continuity values at a single point (image from: $[141])$. (Tension=0.0, Bias=0.0) (left) Continuity=-1.0, (middle) Continuity $=0.0$, (right) Continuity $=1.0 \ldots \ldots \ldots$

6.4 The effects of different Tension values at a point on a curve (image from: [141]). (Bias=0.0 and Continuity =0.0). (left) Tension=-1.0,

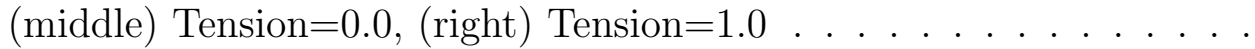

6.5 Comparison of results between setting Tension to 1.0 while keeping the other parameters to 0.0 and setting Continuity to -1.0 and other parameters to 0.0 (image from: [141]). The shape of the curve looks the same, but the spreads of the interpolated points are different. With Tension 1.0, interpolated points near the data points are spaced closer together which creates an acceleration and deceleration effects. With Continuity -1.0, the interpolated points are evenly spaced thus will not exhibit acceleration and deceleration. . . . . . . . . . . .

6.6 Comparison of (left) Kochanek-Bartels interpolation with tension, bias, and continuity parameters set to 0 , and (right) cubic interpolation. The dots represent the original data points and the dashed line is linear

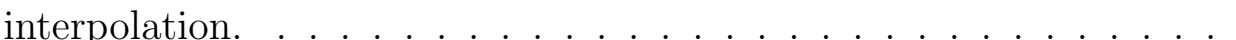

6.7 Results of KB interpolation results with tension and bias set to 0.0 , and continuity (from left to right, top to bottom): -2.0, -1.0, 1.0, 2.0 (solid line). Dashed line is the result of linear interpolation. Dash-dot line is the result of $\mathrm{KB}$ interpolation with tension, bias, and continuity set to 0.0 (equivalent to Catmull-Rom 'cardinal' interpolation). . . . .

6.8 Results of KB interpolation with continuity and bias set to 0.0 , and tension (left to right, top to bottom): $-2.0,-1.0,1.0,2.0$. . . . . . . 216

6.9 Results of KB interpolation with tension and continuity set to 0.0, and bias (left to right, top to bottom): $-2.0,-1.0,1.0,2.0$. . . . . . . . 217

6.10 Cascades of multiresolution filtering. The symbol $2 \downarrow$ indicates downsampling to half the resolution (the width of the blocks illustrates the size of the data). 
6.11 Comparison of filtering motion data using Fourier transform (left column) and multiresolution filtering (right column). From top to bottom: more high frequency components (least filtered) to fewer high frequency components (most filtered). The cutoff value indicates the threshold where frequency components with magnitude higher than this value are removed (set to zero). . . . . . . .

6.12 Top left: two motion data to merge: red-dotted line (motion 1) and yellow-dashed line (motion 2). Top right: solid blue line shows the combined motion with magnitudes of 1.0 on all filter bands for both motion 1 and motion 2. Bottom left: the combined motion (solid blue line) obtained with magnitudes of 0.5 for all filter bands for both motion 1 and motion 2 creates an 'average' motion. Bottom right: the combined motion obtained by adding only the last Laplacian of motion $2 L_{K, 2}$ with magnitude 1.0 to the last Laplacian of motion $1 L_{K, 1}$. . .

6.13 Examples of wavelet usage on time series data ( $x=$ time, $y=f(x))$ : (a) detecting anomalies/change (spike of orange line indicates where frequency change happens), (b) denoising data (green line is the denoised data). The wavelet called Debauchies5 was used in both cases. . . .

6.14 Time vs. frequency resolutions for Fourier transform (FT) (left) and wavelet transform (WT) (right). In FT, there is only frequency resolution, while in WT the time-frequency resolution changes with frequency. Note that the area of the inner rectangles for WT are the same but at different widths and heights, showing the trade-offs between time and frequency resolutions at different frequency levels. . .

6.15 Plots of wavelets: Debauchies6, Coiflet3, Haar, and Symlet6 (image

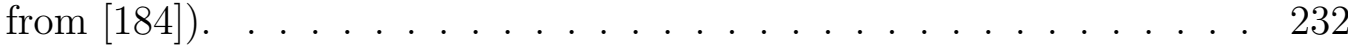

6.16 Transition filter (image from [187]). . . . . . . . . . . . . . 234

6.17 Concatenation of two different motion data using transition filter[187] (left column) vs. multiresolution filtering (right column). The pairs of figures in each row show the same concatenation of two motion data. The kernel Daubechies5 was used for all multiresolution filtering results. The lines labeled "concatenated" show the two motion data concatenated without any modifications. . . . . . . . . . . . 236 
6.18 Three examples of jerk plot from concatenated motions of two motion data from Fig. 6.17. The first plot (top) is for Concatenated 1, the second plot (middle) is for Concatenated 2, and the third (bottom) is for Concatenated 3. The joins of the two motion data in each plot can be seen by the highest jerk amplitude near the middle of each plot. The solid yellow plot is the jerk of the concatenated motion without filtering, the red dotted plot with triangle markers is the jerk from transition filter, and the blue dashed plot with circle marker is the jerk from multiresolution filtering. . . . . . . . . . . . . . . . . .

6.19 Comparison plots of applying transition filter (TF), multiresolution filter (MRF), and multiresolution filter after transition filter ( $\mathrm{TF}+\mathrm{MRF})$. Each stacked pair of plots shows the concatenated motion data as in Fig. 6.17: (a) Concatenated 1, (b) Concatenated 2, and (c) Concatenated 3 . The plots on the top are joint angles over time, while the plots on the bottom show the jerk profiles. Unlike Fig. 6.18 where the whole length of the motion data is shown, here the plots are zoomed in around the joins/transition points where the differences are significant. 240

6.20 Three arbitrary motion data to concatenate . . . . . . . . . . . . 241

6.21 Concatenation of 3 motion data from Fig. 6.20: (a) Using van Breemen's transition filter ('transition filtered' line) to concatenate three motion data, (b) using multiresolution filtering ('filtered' line) to concatenate the same set of motion data. Joins between motion data are at Timesteps 140 and 330. . . . . . . . . . . . . . . . 242

6.22 Stuttering effect using the bior1.5 wavelet. The dashed line is the original motion data, the solid line is the reconstructed data. (a) shows reconstruction using two lowest filterbanks, (b) uses three filterbanks and captures more details but higher number of 'stutters'. . . . . . . 247

6.23 The top plot is a signal reconstructed using the bior1.5 wavelet. The bottom plot is the reduced signal: the same signal but only consisting of values when they change. . . . . . . . . . . . . . . . . . . 248

6.24 Comparison of 'reduction' versus 'downsampling'. The 'reduction' method captures details such as the peak near frame 100 in the original signal in frame 4 of the reconstructed signal, whereas the dowsampled signal misses the peak in frame 3 . The reduction signal is the same as in Figure 6.23. . . . . . . . . . . . . . . . . . . . . . . . . . . . . . . . 249 
6.25 'Lazy' effect. The three motion data were randomly picked from our concatenated motion dataset. All motion data were filtered using the symlet5 wavelet and reconstructed with only the lowest filterbank and other filterbanks set to zero. Many detailed parts are missing in the reconstructed data, but retain the overall 'shape' of the original data.

6.26 Energy level comparison of the original signal ('original') versus the reconstructed signal ('sym5') for the 'lazy' effect using sym5 wavelet. Each plot corresponds to each of the figures in Figure 6.25 in the same order top to bottom. . . . . . . . . . . . . . . . . . . 252

6.27 Concatenated motion data (top), with added random noise $(\mu=0.0, \sigma=$ 0.1 ), and using rbio3.3 wavelet reconstructed with lowest half of the filterbanks. . . . . . . . . . . . . . . . . . 253

6.28 Effects of applying various rbio wavelets on a motion data. In the title of each plot, 'coeffs' indicate the number of bandpass as the result of the multiresolution analysis using wavelet transform. $\operatorname{coeffs}_{i}=1.0$ indicates the bandpass being used for reconstruction, and coeffs $\mathrm{s}_{i}=0.0$ indicates the bandpass are not being used (the bandpass coefficients are set to zero). . . . . . . . . . . . . . . . . . . 255

6.29 Using the bior wavelets to 'linearize' motion data. The dashed red line is the original data, the solid blue line is the reconstructed data. 'levels' indicate the number of bandpass banks used in the inverse transform to reconstruct the data, starting from the lowest bandpass. . . . . . . 256

6.30 Video demonstration on the effects of using different wavelets (https: //youtu.be/9LoDtXDUNC8). . . . . . . . . . . . . 259

7.1 Training the classifier of some model $C$. Given a dataset of size $M, m \in$ $M, \mathbf{X}_{m}$ is the vector of motion sequence, $Y_{m}$ is the true label, and $\hat{Y}_{m}$ is the output (prediction) of the classifier. . . . . . . . . . . . 265

7.2 Using a classifier model as a way to measure the performance of the motion generator $S \ldots \ldots \ldots \ldots$. . . . . . . . . . . 265

7.3 The skeleton model from the motion capture dataset in BVH format.

7.4 Interface of the WinRME tool for creating motions on HROS-1 humanoid robot. . . . . . . . . . . . . . . . 270 
7.5 A sample with only constant values in all timesteps indicates a static pose. X-axis: time/frames, y-axis: joint angle in radians. . . . . . . . 276

7.6 Confusion matrix for Naïve Bayes. The class 0 is represented by label "<0.5" and class 1 is labelled " $\geq 0.5 "$. . . . . . . . . . . . . . . . . 281

7.7 Trained decision tree model (shown only 4 out of 10 levels) . . . . . 284

7.8 Confusion matrix for a trained decision tree model. The class 0 is represented by label " $<0.5 "$ and class 1 is labelled " $\geq 0.5 " . \ldots .285$

7.9 Confusion matrix for a trained SVM classifier. The class 0 is represented by label " $<0.5 "$ and class 1 is labelled " $\geq 0.5 " . \ldots \ldots . .287$

7.10 An LSTM node. $\mathbf{c}_{t-1}$ is the contents of memory cell from the previous timestep. $\mathbf{h}_{t-1}$ is the output of the node at the previous timestep. $\mathbf{x}_{t}$ is the current input to the node. $f_{t}, o_{t}, i_{t}$ are forget gate, output gate, and input gate, respectively. $r_{t}$ is an intermediate network for regularizing the new values for the memory cell. tanh indicates the tanh activation function. $c_{t}$ and $h_{t}$ are the state of the memory cell and output of the node at the current timestep. $\otimes$ indicates element-wise multiplication, $\oplus$ indicates element-wise addition. . . . . . . . . . . . .

7.11 The basic LSTM motion classifier network. The left image is the compact representation of the recurrent network, and the loop at the LSTM layer indicates recurrent process. $\mathrm{FC}=$ fully connected output layer. The image on the right is the 'unrolled' depiction of the same classifier network. $\mathbf{h}_{t}$ and $\mathbf{c}_{t}$ indicates the output and cell state at time $t$ of the LSTM layer, respectively. The index $i$ indicates the index of samples, e.g. the labeled sample $\left(x_{i}, y_{i}\right)$. Notice that only the last output of the sequence of the LSTM layer $\mathbf{h}_{t}$ is used by FC to make the class prediction $\hat{y}^{i}$

7.12 Four randomly-selected samples from the test set and their classification results from the trained LSTM motion classifier. Class: $0=$ robot motion, $1=$ motion capture. . . . . . . . . . . . .

7.13 False positives predictions (top), and false negative prediction (bottom). Class: $0=$ robot motion, $1=$ motion capture. . . . . . . . 
8.1 Plots of the range of unique values in our motion dataset. The x-axis shows the number of unique values in the dataset. The y-axis shows the range of values. The values are sorted from low to high. (a) There are over 2 million unique values in the motion capture dataset which ranges from -6 to 6 . (b) There are over twenty-six thousand unique values in the robot motion dataset which ranges from -2.5 to 2.5. Left: unique values in the original dataset, right: the unique values after discretization. . . . . . . . . . . . . . . . . 301

8.2 Reproducing sample data using discretized values. Solid red line is the real data, ' $\mathrm{X}$ ' marks represents the discretized values. (a) is a sample from the motion capture dataset, (b) is a sample from the robot motion dataset.

8.3 (a) Input to the trained LSTM generator, (b) the output produced by the LSTM generator. $\ldots \ldots \ldots$

8.4 Two samples of gestural motions produced by the LSTM motion generator model (Top and bottom). Each sample consists of 100 frames; each pose shown here are taken one for every 10 poses. . . . . . . .

8.5 Video demonstrations of the motion samples produced by the LSTM network (https://youtu.be/pIFBIe4HxyU) . . . . . . . . . 306

8.6 The basic architecture of an auto encoder model. . . . . . . . . . . . 307

8.7 Variational Auto Encoder model. The latent variables z are produced by sampling a set of Gaussian distributions parameterized by mean $\mu$

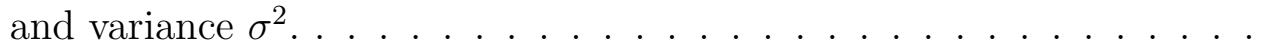

8.8 Training of variational autoencoder without the reparameterization trick (left), and with the reparameterization (right). Image from [212].

8.9 The motion generator VAE that uses only fully-connected, feed-forward network layers. The parameters are given in Tables 8.2 and 8.3. $(\mu=$ z_mean, $\Sigma=z_{-}$var $) \ldots \ldots \ldots \ldots$

8.10 Unfiltered VAE output for various values of with latent variables $z$ of size 2. The columns are the variations for the latent variable $z_{1}$ (left to right), and the rows are the variations for $z_{2}$ (top to bottom). . . . 318

8.11 Output of VAE from Fig. 8.10 filtered using the symlet4 wavelet. . . 319 
8.12 Output of VAE model with convolutional decoder model based on our GAN generator (Table 8.4) with latent variables $z$ of size 2 . The columns are the variations for the latent variable $z_{1}$ (left to right), and the rows are the variations for $z_{2}$ (top to bottom). . . . . . .

8.13 Two samples of gestural motions produced by the VAE motion generator model (Top and bottom). Each sample consists of 100 frames; each pose shown here are taken one for every 10 poses. . . . . . . . . 320

8.14 Video demonstrations of the motion samples produced by the VAE model (https://youtu .be/PQ0d5k_9_dc). . . . . . . . . . . . . . 321

8.15 Basic architecture of GAN. $G_{\theta}$ is the generator network with parameters (weights) $\theta, D$ is the discriminator network with parameters (i.e. weights) $\psi$. "Synth. Data" $G_{\theta}(z)$ is synthetic data produced by the generator. $\mathbf{x}$ is feature vector of the "real data" which is sampled from the training data. . . . . . . . . . . . . . . . . . . 322

8.16 Discriminator loss . . . . . . . . . . . . . . . . . 325

8.17 Generator loss . . . . . . . . . . . . . . . . . 325

8.18 Three steps of the operation of the transposed convolution layer (im-

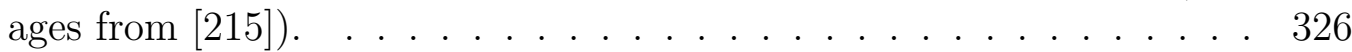

8.19 Our GAN generator model based on the generator of the DCGAN

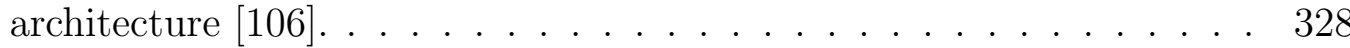

8.20 Samples of generator output with $|\mathbf{z}|=2$ and random values of $z_{i}$ during training at epoch 100. . . . . . . . . . . . . . . 331

8.21 Samples of generator output with $|\mathbf{z}|=4$ and random values of $z_{i}$

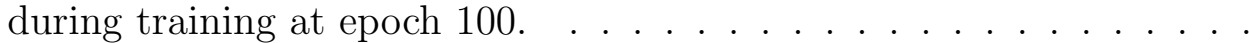

8.22 Samples of generator output with $|\mathbf{z}|=8$ and random values of $z_{i}$ during training at epoch 100.

8.23 Variations of output of the GAN generator by varying the values of $\mathbf{z}$ where $|\mathbf{z}|=2$. The columns represent the range of $z_{1}$, and the rows for $z_{2}$

8.24 Close up of some samples generated by the GAN generator. . . . . . 335 
8.25 Two samples of gestural motions produced by the GAN motion generator model (Top and bottom). Each sample consists of 100 frames; each pose shown here are taken one for every 10 poses. . . . . . . . 335

8.26 Video demonstrations of the motion samples produced by the GAN model (https://youtu. be/Km0x3jNr45k). . . . . . . . . 335 


\section{CHAPTER 1 INTRODUCTION}

This dissertation is focused on the processes and methods for creating expressive animations for emotionally-engaging humanoid robots.

Robots in social settings with humans need to be emotionally engaging to make the social interaction effective [1] [2]. This feature is important as there is increasing demand for new kinds of robots in close proximity with humans in social settings such as: collaborative robotics (Universal Robotics, Baxter), assistive robotics (assisted living facilities, healthcare, hospitals (coronavirus robot)), robots in therapy for autism [3]. Additionally, there is a class of robots which primary function is not to engage humans in social interactions, but these robots are operating in such close proximity to people's social environment (as opposed to strictly work environment like a factory) that they will occasionally encounter social interactions with humans such as robots in supermarkets (Walmart), urban delivery robots (Amazon, competitors).

People experience better engagement and interaction with a robot when the robot performs expressive gestures [4] [5]. Takayama et. al show that when robots can show emotional expressions and behaves in an expected way in social settings, such as being sad if the robot done something wrong, or showing happiness when the robot was successful in completing a task, people viewed the robot to be more trustworthy and intelligent and are more comfortable to be near the robot [6]). In that work, the animations of the robot in their experiments were created by a professional animator from Pixar animation studio. The robot KISMET was designed with a toddler-like

personality (curious, sad when not given enough attention) and modality ('cooing' 


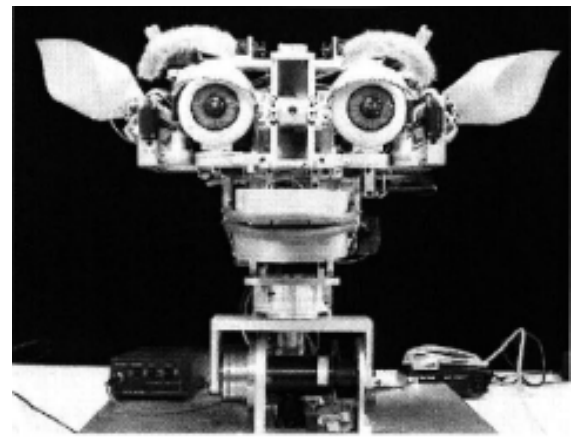

(a)

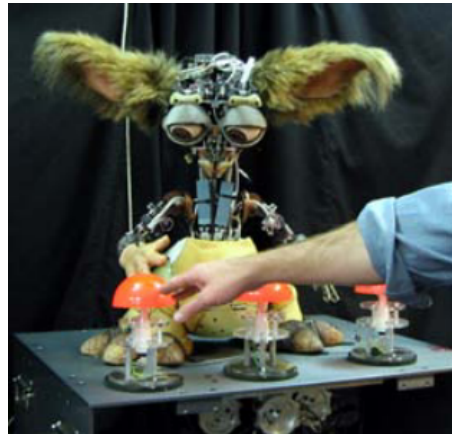

(b)

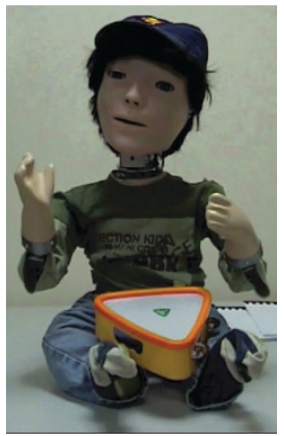

(c)

Figure 1.1: Some examples of expressive robots: (a) KISMET [7], (b) LEONARDO [9], (c) KASPAR [3]

voices instead of speech) [7]. Because of its design, people are more inclined to engage and interact with KISMET as if they are interacting with a toddler (playful, caregiver role). The work with KISMET shows how having the ability of performing expressive gestures helps in the regulation of its interaction with people (e.g. turn-taking conversation) [8]. Breazeal et. al investigated the benefit of expressive behaviors in human-robot collaborative task using the LEONARDO robot [1]. In that work, LEONARDO performs behaviors such as eyes blinking, shifting posture or looking at the person to show 'liveness'. Other times, LEONARDO performs shrugging and other facial expression to show confusion when it does not understand the user. We consider these nonverbal behaviors as 'expressive behaviors' whereas Breazeal et. al refers to the expressive behaviors as 'implicit behaviors'. That study shows that when LEONARDO performs these nonverbal behaviors, the collaborative task can be done in less time compared when LEONARDO only responds to explicit user inputs.

The works in this dissertation are focused on developing new methods and approaches to synthesize expressive animations on humanoid robots - such as the ones demonstrated by Takayama et al. - so future robots can synthesize their own expressive animations instead of relying of hand-made animations and ultimately be better 


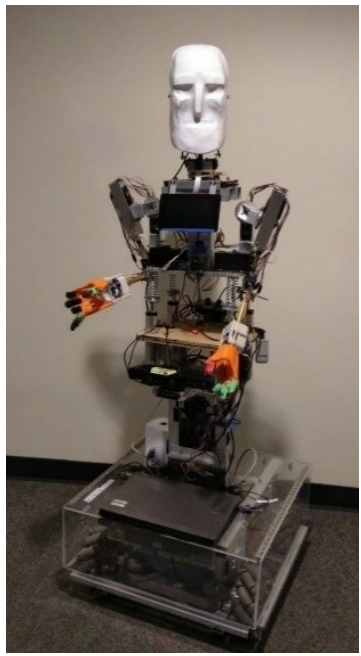

(a)

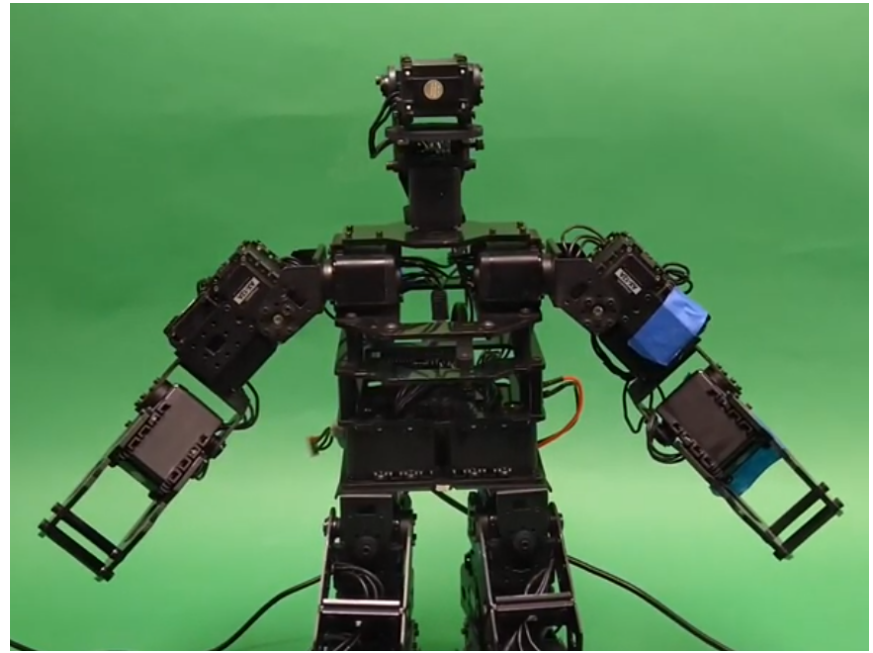

(b)

Figure 1.2: Robots used in this dissertation: (a) Mr. Jeeves robot, (b) HROS-1 mini humanoid robot.

at emotional engagements in human-robot social settings. Figure 1.2 shows the two humanoid robots we used to implement our methods: Jeeves - an adult-sized, mobile humanoid robot, and HROS-1 a miniature humanoid robot. Jeeves was built as a tour guide robot for the Maseeh College of Engineering and Computer Science (MCECS) at Portland State University. Thus, it is important for Jeeves to have expressive non-verbal behaviors to be emotionally engaging with guests who visit MCECS. The HROS-1 robot is a humanoid robot platform that is used as the main robots for the Robot Theater project [10]. Similarly, the it is important that the HROS-1 robot can perform believable and convincing acting using non-verbal actions. More details about these robots are discussed in Chapter 3.

In this dissertation, we use the term "robot behavior" as an action involving the robot's movements that is performed when the robot is in some state. From this point forward, we will use the word "behavior" in place of "robot behavior" for brevity, unless it is necessary to make the context clear. A behavior is considered 'expressive' 
when an observer can attribute some emotion as the robot executes the behavior. Otherwise, the behavior is considered 'neutral'. A person can easily perform the same behaviors using combinations of hand gestures, postures, and body language without requiring much thinking, and in different ways for any kinds of situations. In contrast, creating all these dynamic, expressive behaviors for humanoid robots remain difficult to do by hand or by a computer program.

Problem Area of Contribution in this Thesis - We identified four areas which make synthesis of expressive behaviors for humanoid robots challenging: tuning of timing parameters, high number of degrees of freedom (DOF), creating variety of behaviors, and reusability. First, finding the right timing parameters and poses to get the desired expressiveness can be very time consuming. The user may have to go through many iterations to get the desired expression. Granted, there are gestures that are relatively easy to create such as hand waving or head shaking. But having the correct timing of the movements can make the difference between head shaking for saying "No" angrily, saying "No" calmly, or looking left and right before crossing the street. Second, high number of degrees of freedom (DOF) or joints makes it difficult to create new motions. A typical commercial mini humanoid robot consists of about 20 DOFs (HROS-1, HROS-5, KHR-series, DARWIN OP, etc.). As the number of DOFs increases, the more joints the user must manage when creating each pose in a behavior. Third, regarding the creation and storage of the variety of behaviors. To make each reaction appear genuine, it is important to have varieties of reaction to use. The variety can come from the way a gesture is executed (e.g. speed, range of motion, timing) or a set of different actions that convey the same type of emotion or sentiment. It would be very expensive to create different versions of one behavior such as "happy hand waving" vs. "sad hand waving". We can parameterize the behaviors to create different expressions [11] [12] [13], and some behaviors have some natural groupings 
with other behaviors which convey the same type of reaction or sentiment. Typically, each behavior is stored as a single file, but as the number of behaviors grows, more storage space will be required. Fourth, reuse of existing behaviors onto another robot. Suppose a set of expressive behaviors have been programmed for a robot (e.g. HROS1). In order to create the same set of behaviors on a different robot with a different DOF configuration or different actuator properties, each behavior must be recreated from scratch for the new robot.

\subsection{Contribution}

This work presents my contributions as follows:

\subsubsection{Primary contributions}

- Created a new method for composing humanoid robot motions using algebraic expressions modeled after regular expressions called Behavior Expressions (BE).

- Introduced new operators for motion composition based on regular expression operators: concatenation, union/choice, and repetition.

- Extended regular expression operators with probability and introduced new operators: parallelism and subtraction to create families of behaviors, allowing real-time generation of behavior variants for humanoid robots.

- Created a software tool called Robot Behavior Expression Language (REBeL) to implement, experiment, and evaluate families of behaviors from BEs on two different humanoid robots.

- Proposed a new method to extract musical information that can be used for 
generation of new robot motion data and to provide automatic timing specifications for predefined motion sequences.

- Performed rigorous hypothesis testing by analyzing survey data from the musicto-motion work (Chapter 5) using repeated-measure ANOVA and Friedman tests. Additionally, post-hoc analysis using Scheffe and Wilcoxon Signed-Rank tests to find the significant ranking difference between categories.

- Showed that using Kochanek-Bartels interpolation and wavelet analysis can produce principles from traditional animation: anticipation, exaggeration, slowin/slow-out, and overlapping and followthrough.

- Introduced a more efficient way to perform motion texturing - styling of predefined motion trajectories such that the identity of the original motion sequence is still recognizable. In contrast to previous works which require manual local (per frame) modifications by the user, this method allows global modifications with only a few parameters that give users sufficient flexibility to control a wide variety of effects which are difficult to accomplish using manual methods. This method also contrasts with other works by using established KB interpolation and wavelet analysis methods instead of using custom equations of motions [14] $[12]$.

- Showed using classifier models of Naïve Bayes, Decision Tree, Support Vector Machine (SVM), and long short-term memory (LSTM) network that classifier models without much fine-tuning can be used to evaluate quality of robot motions with over $98 \%$ accuracy and thus show potential as alternative method to human subject evaluation methods which are commonly done in the area of social and entertainment robotics. 
- Showed comparison of the performance of three generative models using LSTM network, variational autoencoder (VAE) and generative adversarial networks (GAN) in the novel task of generating new robot gesture motion data. The generated motion data from the three models are novel (not seen in training data or previously known) and verified as having human-motion-like qualities using our trained motion evaluator model, showing potential as automatic gesture generators for future humanoid robots. To the best of my knowledge, the task of producing new robot motion and comparing the three different generative models has not been done in previous works.

\subsubsection{Auxiliary contributions}

- Primary contributor to the Mr. Jeeves robot:

- Designed and proposed the original concept.

- Managed multiple teams of students during its development and construction phase, and integrated the source codes developed by the various teams.

- Designed and built the head and neck mechanism that can show various emotive and expressive head movements and facial animations.

- Designed and built the web user interface for Jeeves.

- Developed speech-based user interaction feature.

- Demonstrated Jeeves at various public events at PSU (MESA Day 2017, PSU Maker Expo 2017)

- Built a new dataset combining human motion capture dataset from CMU and humanoid robot HROS-1 motion dataset that were developed by our team. 
- Showed additional benefit of using wavelet analysis in reducing jerk in concatenated motions.

- Introduced new method for discretizing continuous joint angle values using Haar wavelet for use in the motion dataset for machine learning. The discretization enabled reduction of unique values by 4 orders of magnitude (over 2 million unique values to just over 200 values), the discretized data show good reconstruction $\left(\mathrm{MSE} \leq 10^{-3}\right)$ with respect to the original data compared to standard discretization methods, and significantly improved the generated data of the LSTM motion generator network.

\subsection{Overview of Contributions}

In order to facilitate a more powerful and expressive way to compose motions for humanoid robots, I introduced new composition operators which semantics are extended from operators from regular expressions, in particular: concatenation, union, and repetition. Additionally, these new operators can have probabilistic behaviors. These operators are used with a set of robot poses (or motion library) such that the poses are arranged in a particular order (i.e. sequence), which the sequence defines a robot motion. Composing motions in this manner using the new operators and poses from the motion library is done by writing a script similar to an algebraic expression which we call Behavior Expression (BE) which is parsed with a tool I developed called Robot Behavior Expression Language (REBeL). Using BE allows specification of deterministic robot motions but more importantly, the probabilistic behaviors of each operator allows specification of families of robot motions. The latter, is an important feature for social and entertainment robots, which current motion editor tools do not yet have. Beyond the operators from regular expression, I also introduced the sub- 
traction operator to remove unwanted elements in the sequence. Future works may expand the set of operators in REBeL to include other operations such as concurrency and logical operations. Specifying robot motions using BEs allows more ways to compose motions using current motion editor tools. Furthermore, composing complex motions using BEs are more efficient and easy to read compared to using standard syntax in programming languages like Python or $\mathrm{C} / \mathrm{C}++$. In my experiments, using BEs was proven to be the most effective way to prototype new motion variants and families of motions.

REBeL significantly reduced the amount of work needed to compose new motions than the current methods, as demonstrated on the HROS-1 and Mr. Jeeves robots. Using current motion editor tools for humanoid robots, users are limited to create a single motion data with each session of using the tool. Creating motion variants in current tools involve many copy-and-pasting steps from the original motion. Lastly, each motion data is saved in individual files or a large file of multiple motion data. Users have greater flexibility to compose motion sequences in different order, to create variants of existing motions, and to define families of motions in a single BE than using current motion editor tools or using general-purpose programming languages like Python and $\mathrm{C} / \mathrm{C}++$. The probabilistic operators of REBeL lets the robot also give the robots probabilistic behaviors during runtime, giving a better illusion of 'intelligence' than simple randomized choice. REBeL also allows hierarchical composition, where a predefined BE can be used inside another BEs, so complex behaviors can be constructed by composing simple behaviors. Moreover, the concept of Behavior Expressions is easily generalizable to not just motions, but also animations (e.g. facial animations on Jeeves), voice/sounds, and other modalities on the robot to compose complex and expressive behaviors.

Providing timing information is one of the tasks that requires a lot of trial-and- 
error in composing expressive robot motions. I proposed a method to use music information to provide timing for a predefined sequence of actions. As demonstrated in Chapter 5, this method produces motions that are viewed as expressive by people, and they are at least comparable or better to motions that were timed manually. The advantage of this method over what is available in current tools is twofold: 1) it eliminates the need for users to manually specify timing for each pose, which reduces the number of parameters need to be adjusted, ultimately reducing the trial-anderror iterations. 2) different expressions for the same sequence of actions can easily be modified by providing different music data, thus it can be done in real-time. In the current motion editor tools, modifying expressions will require redefining the sequence, which the new sequence needs to be saved in separate files.

Using a combination of Kochanek-Bartels interpolation and wavelets, I demonstrated that motion textures can be added or modified by a set of parameters that the user can adjust either off-line (i.e. predefined motions) or on-line (i.e. adjusted during runtime). The current methods emphasize on manual tweaking [15] [16] or local adjustments using custom equations [12] [14]. In contrast, this method applies the 'texture' globally across the whole motion data while maintaining the quality and identity of the modified motion (average MSE $\leq 10^{-3}$ ). Some of the effects that can be achieved using this method is: stuttering, tremor/shaking, jolt, exaggeration, followthrough, and anticipation from the Disney Principles of Animation. In contrast to accomplishing the textures using current tools, this method circumvent the need for the user to specify in-between frames/poses to stylize each motion, thus reducing the motion editing iteration time. In addition, this method also shown to be more effective in reducing jerk at the joins of concatenating multiple motion data compared to using transition filter [17]. As additional benefit, the transitions produced at the joins using this method consistently exhibit more natural transitioning motions than 
transition filter. To the best of my knowledge, this method is the most comprehensive and flexible motion texturing method for social and entertainment humanoid robots.

I introduced a new method to evaluate robot motions by using trained classifiers as motion evaluators as an alternative to subjective evaluations (e.g. using surveys). Most works that evaluates robot motions in the area of social and entertainment robotics rely on human evaluations (questionnaires, surveys, interviews). The new idea behind my method is to instead of relying on human opinion to determine whether a motion sample appear natural or expressive, which can be inconsistent from one person to another, is to use a reasonably accurate classifier which determines if a motion sample belongs in the class of motion capture data or not. The method assumes human motion as the "golden standard" for expressive, natural-looking motions and the "baseline" of robot motions is where the motions are linearly-interpolated. In other words, "good" motions are the ones that have similar qualities to human motions (as in motion capture data), and "bad" motions are the ones with 'mechanical' qualities as in typical robot motions. I compared the performance of four classifier models as motion evaluators: Naïve Bayes, Decision Tree, SVM, and a long shortterm memory (LSTM) network. All models were able to achieve above $98 \%$ accuracy on average with little tuning, indicating that each one of them is a viable model for motion evaluators. I further showed that motion texturing is an effective way to make robot motions be classified as natural and expressive, and verified by executing the motions on the HROS-1 robot.

Lastly, I presented experimental results on a new approach to produce expressive motions on humanoid robots by using generative models. In Chapter 8, I show comparisons of the motion data generated from three different models that I designed: an long short-term memory (LSTM) network model, a variational autoencoder (VAE) model, and a generative adversarial network (GAN) model. The results show that 
these models could serve as promising vital components for future humanoid robots in the application of social and entertainment robotics, as it could reduce the need of manual programming of gestures. To the best of my knowledge, using these three generative models to produce expressive gestures on humanoid robot is a novel work, as other generative models focused only on image and sound/music generation. As preliminary results, over $90 \%$ of the motions generated by these three motion generators were classified as having human motion qualities using the motion evaluators described earlier.

\subsection{List of Publications}

- Peer-reviewed:

- M.Sunardi and M. Perkowski, "Animating behaviors of humanoid robots using probabilistic algebraic operations" - submitted to International Journal of Social Robotics, May 2020. (pending review)

- M. Sunardi and M. Perkowski, "Behavior Expressions for Social and Entertainment Robots" - accepted February 2020 to International Symposium of Multi-Valued Logic (ISMVL) 2020.

- M.Sunardi and M.Perkowski, "Music to motion: Using music information to create expressive robot motion,"International Journal of Social Robotics, vol. 10,no. 1, pp. 43-63, 2018.

- M. Perkowski, A. Bhutada, M. Lukac, and M. Sunardi, "On synthesis and verification from event diagrams in a robot theatre application," in 2013 IEEE 43rd International Symposium on Multiple-Valued Logic, IEEE, 2013, pp. 77-83. 
- Reviewed on abstracts:

- Andy Goetz, Camille Huffman, Kevin Riedl, Mathias Sunardi and Marek Perkowski, Artistic Robots through Interactive Genetic with ELO rating system, Proc. 22nd International Workshop on Post-Binary ULSI Systems, May 21, Toyama International Conference Center, Toyama, Japan, 2013, pp. $27-35$.

- Quay Williams, Scott Bogner, Michael Kelley, Carolina Castillo, Martin Lukac, Dong Hwa Kim, Jeff Allen, Mathias Sunardi, Sazzad Hossain, and Marek Perkowski, An Emotional Mimicking Humanoid Biped Robot and its Quantum Control Based on the Constraint Satisfaction Model, Proceedings of ULSI 2007. May 13, 2007, Oslo, Norway.

\subsection{Scope and Limitations}

Expressive behaviors cannot be isolated from the human-robot interaction (HRI) context, which often includes technologies such as natural language processing, action recognition, facial recognition, and many other. These technologies are related to issues that need to be addressed in HRI such as: correctly identifying user response and input, maintaining physical balance, inter-body collisions (e.g. robot's gesture causing the arm hitting its own body), coordination between movements, speech, light, and sound, and so on. These issues and their respective technologies are beyond the scope of this dissertation and would be reserved as the subject of future studies but may be occasionally discussed when relevant in subsequent chapters.

In this dissertation there is less emphasis on developing the cognition capabilities for determining the correct expressive behavior response given some input. Instead, the focus is on how to make the gesture executed in a way that shows emotion, i.e. 
'expressive' given a robot gesture behavior. Additionally, I discuss the incorporation of principles from traditional animation to guide the definition and creation of behaviors that are considered 'expressive', 'natural', and 'believable'.

\subsection{Overview of Dissertation}

This dissertation is organized as follows. Chapter 2 covers the related works in literature. Chapter 3 describes the hardware and software used in this work. Chapter 4 introduces a framework called Robot Expressive Behavior Language (REBeL) for composing complex expressive behaviors using simple algebraic operations and expressions as the improvement over the current methods. In Chapter 5, we show how music can be used as a source for generating motion data and for determining timing information for the animation. Chapter 6 discusses parameter-based methods using interpolation and filtering, and other parameters related to the hardware of the robot. In Chapter 7, we introduce our approach of building a new dataset comprised of human motion capture samples and robot motion samples. Then we use several learning methods to discriminate motion capture samples from robot motion samples as an approach to evaluate quality of synthesized robot motion data. While in Chapter 7 the

focus is on discriminative models, in Chapter 8 the focus is on generation/synthesis of motion data. We discuss using two generative models using variational autoencoder (VAE) and generative adversarial network (GAN) to synthesize motion data. We discuss conclusions and contributions of this dissertation in Chapter 9.

All source codes I created and used in this dissertation will be made available in the following GitHub repository: https://github.com/msunardi/dissertation2020. 


\section{CHAPTER 2 RELATED WORKS}

This chapter presents the works in the area of social robotics and realistic/expressive motion synthesis/generation. We discuss related works from the social robotics point of view because expressive behaviors play an important role in social interaction. Section 2.1 discusses the role of expressive robot behaviors in the area of social robots. Section 2.2 discusses the guiding principles from traditional animation to make objects appear as believable characters. Section 2.3 discusses the current tools available for

creating motion behaviors (e.g. gestures) on humanoid robots. Section 2.4 discusses procedural methods and learning methods for synthesizing motion data.

\subsection{Expressive Behaviors of Social Robots}

Social robots or socially interactive robots are robots which social interaction is the primary function for the robots [18]. This definition includes human-robot social interaction and robot-robot social interaction. For the purpose of this dissertation, we focus on research on social robots with the goal of being adept in human-robot interactions in social settings.

Being adept in social situations means that the robot needs to be able to communicate using human communication modalities such as: speech, facial expressions, and hand gestures. On one hand, the robot must be able to recognize those modalities from a user. On the other hand, the robot is expected to also be able to communicate to the user using the same modalities, such as: expressive speech, expressive gestures, gaze/eye contact, and to respond in a congruent way with response time that is com- 
parable to normal human-human conversations. Studies have suggested that people view the robot more favorable when it can exhibit those abilities [19][20] [6] [21] [22] [4] [5]. By favorable, it means people feel more comfortable and confident interacting with the robot, people understand the robot more easily, people remember the messages conveyed in the interaction, and people feel the interaction was more enjoyable (Cassell, Embodied conversational interface agents, 2002). By having robots that are socially competent, the hope is that the robots can operate within humans' personal space for tasks such as entertainment, cooperation, companionship, caretaking, and therapy.

Many aspects of social behaviors benefit from technologies such as speech recognition, speech synthesis, image/object recognition, facial recognition, autonomous navigation, and so forth. For example, attention is where the robot directs its gaze which is important in interaction and cooperative/collaborative tasks [23] [1]. When a humanoid robot is interacting with a person, or even any object in general, if the robot directs its gaze towards the person or object, the gaze shows the observer where the robot attention is. Conversely, without the gaze, the robot's actions and intent will be difficult to read. Having certainty of the state or behavior of the robot, i.e. what the robot is currently doing gives the person a sense of security and safety in their interaction with the robot. Conversely, the ability to anticipate what the human would do in a human-robot collaborative task, increases the task efficiency [24]. Speech recognition and synthesis technologies have reached an astounding level of sophistication as shown with modern auto-transcription [25] and conversational agents such as Meena [26]. Parsing speech signals into text is now quite reliable. The difficult part is how to combine these input-output modalities to develop the cognitive skills for social robots to learn and understand the intent behind the message and respond accordingly in a socially intelligent manner. The work of Cynthia Breazeal is one of 
such attempts using models from human psychology on the Kismet robot [7].

Dautenhahn et al showed that robots can be useful in therapy for children with autism [27]. Takeyama et al showed that when a robot can show what it is thinking (expressing thought), it helps the person to understand/anticipate the robots' intentions, and consequently be more comfortable around the robot [6]. Showing intent helps set the expectations for the person in the robot's space, such as how to approach a person in a manner that is comfortable to the person [22] or doing cooperative tasks [28]. According to one study, people tend to prefer robots that always appear happy and confident rather than moving erratically or suddenly as it creates uncertainty and fear [21].

Expressive behaviors in the context of this dissertation are behaviors that express intent and personality. The ability to show intent and personality through non-verbal behaviors are among the most desired features in social robots [21]. Research in expressive behaviors of social robots can be categorized into three kinds: classification, utility, and generation/synthesis. Classification is the study of how well/easily people can recognize which expression the robot is showing. Utility is the study of how useful or effective is the robot in accomplishing a task by having expressive behaviors. Generation/synthesis is the study of tools and methods to generate expressive behaviors on robots. We distinguish the works in this dissertation (expressive gestures) from works involving natural-looking, 'dynamic' actions in robots such as: locomotion [29] [30] [31] [32], recovery actions [33], backflips [34], bipedal balance [35] [36], and similar actions. Dynamics control of robot actions often yields "natural-looking" motions such as the gain achieved by [29] and [31]. However, dynamics control does contribute to creation of emotional expressions for instances like dangling arms and slumping body to show exhaustion.

One example of classification studies using LMA on robots was done by Knight, 
which uses Laban Effort to parameterize the movements of low degree of freedom robots and show that people can recognize what is being expressed by the robot is consistent with the expressiveness defined by the Laban Effort parameter [12]. Ribeiro used the robot EMYS to create a range of facial expressions and evaluated how well each expression is recognized by people [37]. Breazeal also did similar study with the robot Kismet [7]. The primary interest in this dissertation is on full body gestures and not on facial expressions, therefore our discussions below will focus more on the former rather than the latter.

Works in utility studies include Takayama et. al. [6] which shows that when the robot can indicate its intent (i.e. "expressing thought"), people can understand the robot better, thus perceiving the robot as 'intelligent'. In collaborative tasks, when robots use hand gestures to point where an object is to be placed, people also view the robot as more pleasing to work with[38]. Intent is also useful in collaborative task where both the robot and person must work together to complete a task [39]. By designing a control system such that the robot's arm reaching motion is legible to the person, this legible motion shows the intent of the robot's reach which helps the person understand where the robot is about to reach and ultimately helps completing the task more efficiently.

One way to show personality is by showing the robot's reaction such as surprise, sad, happy or angry when encountering certain situations. Emotional facial expressions occur only at the initial reaction when an event occurs [7] [2]. This reaction can also be interpreted as the robot's assertion whether the event or situation is perceived by the robot positively or negatively. Therefore, the way the robot moves, and its choices of future behaviors should reflect this sentiment. Personality such as extroversion or introversion [40] can give the robot a closer connection with the person and establish some expectations for the person on how to approach the interaction. 
Saerbeck and van Breemen suggested a guidelines to designing behaviors for personal robots [41]. They categorized three types of methods for designing motions for robot behaviors. Trajectory Design Methods refers to generating motion from motion capture, keyframed animation, and scripted animations. Motion Editing Methods refers to editing existing motion data using techniques such as filtering, retargeting, and constraints. Finally, High Level Behavior Design Methods refers to generating motion behaviors based on some abstract parameters such as emotions. The motivation of the work in this dissertation is similar to those of [41] in that we address the issues of designing expressive behaviors and the parameterization of their execution at runtime.

Finally, this dissertation is also focused on humanoid robots. We define humanoid robot as robot that has at least one of these human-like embodiments: head, neck, torso, arms, hands, and we leave legs as optional. We distinguish humanoid robots from anthropomorphic robots in that anthropomorphic robots are not required to have human-like embodiment, but have features in their embodiment that people can attribute to human features, e.g. an arm robot can be anthropomorphized as neck and the end effector/hand as "head/face".

\subsection{Motion Vocabulary from Performance Arts and Traditional Anima- tion}

The two popular methods of analyzing motions are Laban Movement Analysis (LMA) [42] and Principles of Animation developed by animators at Walt Disney animation studios [43]. LMA and the Principles of Animation comes from two different needs; LMA was developed as a common language to describe motions, the Principles of Animation method was developed as a guideline for animators to make believable 
animations. The main motivation behind LMA was so that motions for dance choreographies can be described precisely so that the motions can be reproduced exactly from the LMA terminologies. In contrast, the Principles of Animation method was developed by observations by animators in order to provide guidelines as to what are the things animators should look for when creating believable and entertaining animations. In practice, these two methods can be used in conjunction with each other such as when the animation director describes to the animators some kind of emotion the character must convey.

In the following sections we give a brief introduction to LMA and the Principles of Animation.

\subsubsection{Laban Movement Analysis}

Using LMA, precise descriptions of movements, and consequently a choreography can be done such that the dance or acting performance is repeatable even by different people. However, LMA was found to be useful in describing motions outside the arts and for analysis such as to assess workers health e.g. due to repetitive physical tasks.

There are many facets of LMA, but the most used concept in motion synthesis for social and expressive robotics is the Effort concept as it directly assesses motion quality. Arguably, among the other LMA concepts, Effort is the easiest to understand and associate directly with motion parameters. Effort consists of four categories: Weight, Time, Space, and Flow. Each category is described with a spectrum. Weight is explained with the spectrum of Strong vs. Light, as the effort exerted in the motion of lifting a heavy object versus a light one. For a person, a 'Strong Weight' appears as visibly engaging his muscles and exerting work as he lifts a heavy box. A 'Light Weight' may be illustrated by the lack of exertion to lift a piece of paper. Time refers to the amount of time spent to perform the motion. The spectrum for Time is Sudden 
vs. Sustained. 'Sudden Time' is characterized by a quick, jerk-like motion, such as when a person quickly retracts her hand after touching a hot kettle. Conversely, 'Sustained Time' is taking more time - as if lazily - to perform the motion. Space refers to the amount of physical space used to move from one position to the target position. The spectrum for Space is Direct vs. Indirect. A 'Direct Space' refers to motions that take the shortest path, like running a sprint. An 'Indirect Space' refers to motions that explores the space between the starting position and the target position, like meandering. Flow refers to the 'continuous-ness' of the motion. The spectrum for Flow is Bound vs. Free. 'Bound Flow' motion appears as if the person is restricted, reduced range of motion, or in a small, confined space, while 'Free Flow' motions have more seamless continuity like a palm tree being blown by the wind.

Computational models for the Effort concept have been developed in works such as EMOTE [14] and [12]. The EMOTE model was applied to a 3D humanoid model, demonstrated by setting the different effort parameters, and validated the resulting motions to be consistent with LMA definitions by evaluation from a certified Laban Practitioner [14]. In another work by Knight [44], their model was validated using a series of A-B testing employing anonymous workers ${ }^{1}$ on a simulated model of a simple robot without humanoid features but evaluated by the way it moves between two points. Knight showed that even with a simplified, non-humanoid model, the different Effort categories can still be observed only from the way the robot moves in a round trip between two points.

\subsubsection{Principles of Animation}

Animators and artists are able to create believable characters out of ordinary objects by animating them in certain ways that follow a set of guiding principles using

\footnotetext{
${ }^{1}$ Workers that can be hired through Amazon Mechanical Turks service.
} 
media like hand-drawn animations, stop-motion animations, or 3D animations [45]. Many of these guiding principles were derived by the animators' observations of motions of humans and animals [46], including inanimate objects and how they behave in the physical world. One such guidelines that is commonly used by animators is the Principles of Animation developed by animators at Walt Disney Studios in the 1930's. There are twelve principles: Staging, Squash and Stretch, Anticipation, Exaggeration, Arcs, Slow-in and Slow-out, Pose-to-pose vs. Straight-ahead animation, Timing, Follow Through and Overlapping Actions, Secondary Actions, Appeal, and Solid Drawing [43]. In this dissertation, we only focused on a select few principles: Anticipation, Timing, Exaggeration, Slow-in and Slow-out, Secondary Actions, and Follow Through and Overlapping actions. These principles were chosen by us due to their relative importance in animation [47] and relevancy to expressive motion behaviors. In contrast, principles like solid drawing are not applicable to our application in robotics. We can argue that staging and appeal can be important to a design in our social robots, but for now those principles are reserved for future works. In the future, we could also benefit from a validation study, i.e. which principles are more important in social robotics applications.

The principles in the Principles of Animation can be grouped in four categories: simulating physics, designing aesthetically pleasing actions, effectively presenting action, and production technique [48]. Principles in the category of simulating physics are squash and stretch, timing, secondary action, slow in and slow out, and arcs. Proper application of these principles ensures the physical properties of the object being animated is clearly conveyed, e.g. the material and weight of a falling ball, the material of clothing worn by a character, etc. "Aesthetically pleasing action" is important to capture the audience's attention and interest in the final product. Principles that are involved in designing aesthetically pleasing actions include: appeal, 
solid drawing, and follow through and overlapping action. Often, the principles in the simulating physics category also contributes to the "aesthetically pleasing action". "Effectively presenting action" refers to delivering the action in such a way that is clear and unambiguous to the audience. The principles in this category are exaggeration, anticipation, and staging. Finally, the only principle associated with the "production technique" category is the straight ahead vs. pose-to-pose animation.

The Anticipation principle refers to creating an action that prepares the audience for what is about to happen next. The first kind of anticipation is purely for plausibility of performing a physical action. For example, the anticipation action of leaping is by crouching like the loading of a spring before the leap (Figure 2.1). In this type of anticipation, the anticipation motion is typically in the opposite direction as the main action (crouching - downwards, leaping - upwards). The second kind is to tell a story to the audience, such as by increasing tension between characters which tells the audience some conflict is about to happen between them. In other words, Anticipation is used to manage the audience's expectations. Anticipation can be used to create comedy when the following action is not what the audience expects. For example, when Daffy Duck points a gun labeled as "Disintegrating Pistol" at Marvin the Martian, instead of firing a beam that disintegrates Marvin, the gun itself disintegrates as Daffy pulled the trigger. When used incorrectly, breaking expectations can cause a jarring, uncomfortable experience to the audience. Takayama et. al. demonstrated the utility of anticipation as "expressing thoughts" [6]. Their study showed that people rate the robot as more understandable and more intelligent when the robot can express to the person what it is about to do next. In one scenario, their robot (in simulation) is about to open a door. "Expressing thought" is done by having the robot look at the door and have its hand ready to reach the knob, but then briefly look at the person and then look back at the door. This behavior tells 


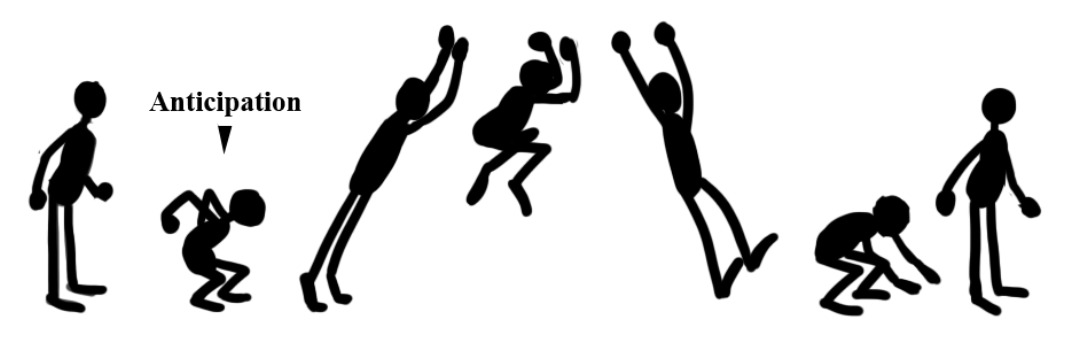

Figure 2.1: Example of Anticipation principle in a jumping action [47]. Anticipation happens at the second frame when the character crouches downward, indicating he is about to leap upward.

the person that the robot intends to perform a task on the door.

The Timing principle can refer to several things. The first is related to frame rates. Because traditional animation is using hand-drawn frames, the quality of an animation is directly influenced by the frame rates used; the more frames used per second, the smoother the animation appears. In practice, animations are recorded at 24 frames per second, but some animation uses only 12 frames per second for economic reasons (fewer frames, lower cost). Therefore, each frame is shot twice in this approach. In the movie SpiderMan: Into the Spider-verse, it is said that animators used timing on ones (each frame is shot once) versus on twos (each frame is shot twice) to create a contrast between a character who is confident and skilled in his acrobatic movements versus someone who is more clumsy [49]. Timing can also refer to the artistic direction of when to do which action. Delaying an action or prolonging a shot can induce anticipation to the audience, so these concepts can be related. However, if the delay is too long or too short, then it can also cause a jarring experience to the audience. For a social robot, timing can refer to delaying a response, or doing some 'waiting behavior' when the robot needs a long time to respond and give the illusion of 'thinking'. But if the delay or wait is too long, then the user/person may think that the robot is malfunctioning. 
Exaggeration in animation is usually displayed as an action of the character which often breaks physical constraints, such as when the character's jaw drops to the floor or eyes that grew twice their normal size to show extreme surprise. However, the true purpose of Exaggeration is not simply to make something looks so distorted for the sake of creating comedy, instead it is used to make the story being conveyed unambiguously clear to the audience, sometimes to the point of comedy [43]. This idea is similar to how actors in a live stage performance have to exaggerate their acting because the distance between the stage and the audience [50]. In contrast, acting in front of a camera for films and movies need to be more subtle and controlled as the audience can view the actors/actress in a close-up. In my discussion with a professional stop-motion animator Brooke, she said that exaggeration can be done on real, physical models such as stop motion puppets or robots by first establishing a baseline of the 'normal'. Exaggeration can then be shown by going beyond this baseline. For example, the robot moves its arms at a certain speed that is not too fast or too slow. Then when the speed suddenly becomes faster or slower than normal, the person who observes this change will notice that something different is happening. However, in order for this trick to work, there must be enough difference between the 'normal' versus the more intense or less intense action. In other words, if exaggeration is shown through speed, then the difference of speed that is noticeable to the observer must first be determined. Finding the right difference may require trial and errors. Exaggeration also must be performed sparingly for it to be recognized; if every action is done exaggerated, none of the actions appear exaggerated. In addition to range of motion, exaggerated can also be shown via speed.

The Slow-in and slow-out principle refers to creating acceleration and deceleration when starting or finishing a movement when appropriate. Without acceleration and deceleration, the movement will appear abrupt and sudden. Movements that 
are 'robotic' are often characterized by their abrupt-ness and sudden-ness, and they appear unsafe when done by robots near people because it indicates lack of control. Secondary Actions are additional actions that complement the character's main action. For example, wiping sweat from the forehead while running. Actors perform secondary actions to express the behavior and personality of their character to the audience, such as nervousness, confidence, and thought process. For example, in a scene where two persons are having a conversation, one of the persons keeps playing with a pen with his fingers, loses control, drops it, and picks it up with a nervous laugh. All these are secondary actions that show to the audience that his character is nervous, clumsy, or not paying attention to the other person. Secondary actions must not be too much or too prominent so that they do not overtake the main action, otherwise the audience could get confused. Agents that do not perform any secondary actions appear too stiff, rigid, and emotionless. Similarly, if social robots can perform secondary actions, this will give much more personality to the robot, and perhaps would make humans have a better emotional engagement experience with the robot.

Follow Through and Overlapping Actions principle refers to the emulation of physical dynamics in animation. This principle is important for creating a sense of realism and plausibility in hand-drawn animation, but also makes the motion appear more fluid and organic. An example of Follow Through action is the way the clothes on a character moves with the character. The fabric of the character's dress should flow with the direction of the character's movements and drags/lags behind the movement of the character. On the other hand, Overlapping Action refers to creating a dynamic motion by initiating motion as a kinematic chain. Figure 2.2 shows an example of Overlapping Action in the motion of raising an arm [47]. Williams showed how Overlapping Action can be created by consecutively delaying the motions of the extremity of the kinematic chain of the arm: the shoulder moves while the elbow and wrist 


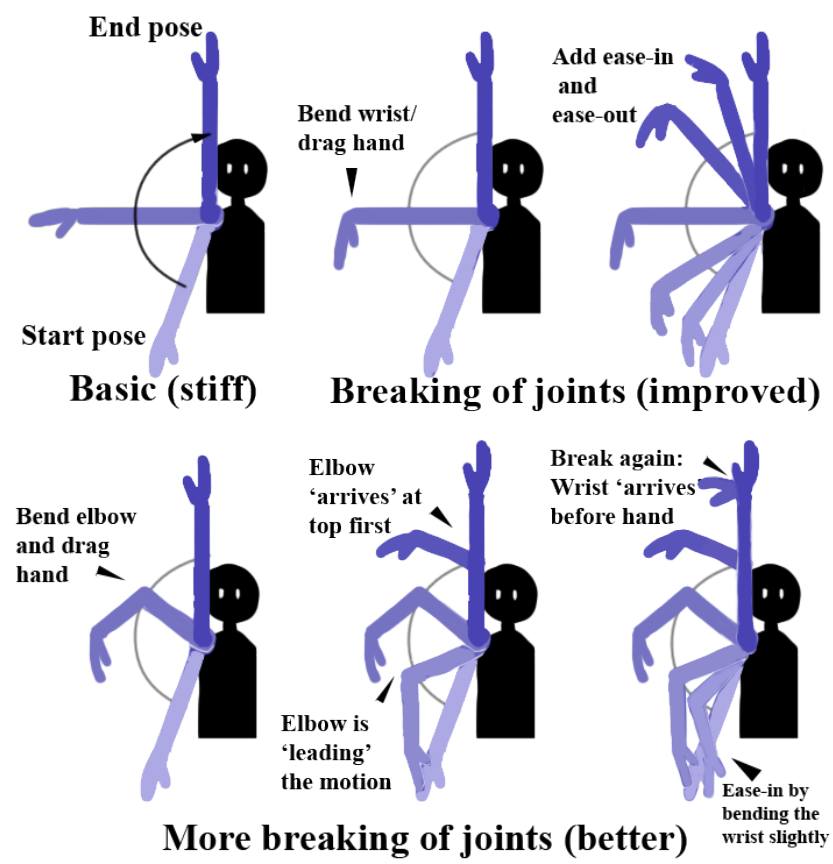

Figure 2.2: Example of Overlapping Action. In creating the animation of 'raising an arm', instead of only rotating at the shoulder, adding successive bending to the wrist and elbow (a technique called 'breaking of joints') makes the raising arm motion appear more organic rather than mechanical [47]

are lagging behind, as the shoulder reaches the apex, the elbow straighten first, and then followed by the wrist. Without Overlapping Action, the arm, elbow, and wrist remain in the straightened pose and only the shoulder is moving. This 'chain' of actions is what is referred by overlapping action. Most actions that are self-initiated usually initiated from the head or the extremities such as hands and feet. Once again, this principle addresses the issue of motions that look 'robotic'. Therefore, if a humanoid social robot can perform its behavior by applying the Follow Through and Overlapping Action, its behavior can appear more dynamic and natural.

The challenge is that these principles are difficult to implement onto a humanoid robot's animations. In order to enhance a robot's behaviors with these principles, it usually requires a skilled animator(s) to create these behaviors. Moreover, the robots 
will need to have a very capable actuators and electromechanical systems to support these movements. Robots which movements are controlled by dynamical models show much more natural-looking motions such as Boston Dynamics's Big Dog robot [31] and Agility Robotics' Cassie robot [32] but they excel in agility/mobility movements and behaviors. Our focus is in communicative animations like gestures which are difficult to model as dynamical systems. In the next section, we discuss a few tools for animating humanoid robots to understand what the limiting factors and gaps are for creating expressive behaviors and gestures on humanoid robots.

\subsection{Humanoid Robot Motion Editing Tools}

Creating expressive motions for robots can be done manually by skilled animators and programmers using special software and manual inputs; we refer to this approach as authoring and the tools as motion editors. The advantage of this approach is its low learning curve, and when done right by skilled animators, the motions can be legible and easy to recognize. The downside is that this process may require the animator a lot of iterations to get the right motion, and the tools available can be limiting. In this section we discuss some motion editors that are used with popular humanoid robots and other editors that were developed by other researchers.

When using motion editors, typically motions are created pose-by-pose by editing each joint angle. Then, for each pose the animator must 'guess' how much delay to add, and how fast the motion should be. Some tools provide a graphical user interface (GUI) with a 3D model of the robot so the motion can be simulated and thus improving iteration time. These tools are usually proprietary, and the 3D models are limited to the robots made by the same manufacturer.

We studied several commercial humanoid robot motion editors to understand the 
procedures the users go through and the features of the motion editors used to make new gesture animations. The following are the motion editors we studied: Kondo HeartToHeart4 [51], Lynxmotion Visual Sequencer [52], Robotis RoboPlus Motion 1.0 and 2.0 [53], ROBOTIS-OP3 [54], Robot Motion Editor (RME) ${ }^{2}$ [15] and its Windows variant WinRME ${ }^{3}$ [55], Choreographe [16], Lego Mindstorms EV3 ${ }^{4}$ [56], and Pololu Maestro [57]. In addition, we also look at several humanoid robot motion editors that were developed in research and academic environments such as Improv [11], EMOTE [14], Choreonoid [58] [59]. In this discussion, we exclude robot software that are only intended for end-user consumption such as mobile apps.

To understand the animation process using the motion editors listed above, we studied the written documentation of each editor and any video instructions we could find on the internet (e.g. YouTube, official websites of the editors). Additionally, we also evaluated the project reports written by students who did projects involving animating humanoid robots from the Intelligent Robotics courses at PSU. The list of motion editors that we selected was also determined by selecting the editors with sufficient documentation available in English. We use the term author to refer to the person who is performing the animation process. To focus only on the animation process, we set the discussion with the following scope:

- Exclude the set-up process

- Assume the system (robot and software) are ready to use by the author.

- Exclude human-robot interactivity

\footnotetext{
${ }^{2}$ The RME tool was developed based on the features of ROBOTIS-OP3 action editor tool.

${ }^{3} \mathrm{~A}$ version of the RME tool for the Windows operating system made by a member of the community.

${ }^{4}$ Built using National Instrument's LabView platform
} 
- Only animation on one robot (exclude multi-robot configurations and coordination)

- Include experimental/research-based motion editors in addition to commercial ones

\subsubsection{Animation Process using Motion Editors}

We identified the common process of creating robot animations using motion editors and illustrated in the flowchart in Figure 2.3. First, the author creates an initial keypose of the robot ("Create keypose' block). This initial pose is typically the robot's resting or home pose. Then, the author creates one or more poses to create a sequence ('Enough keyposes?'). At this point, the author may want to check how the sequence looks when executed on the actual robot or a 3D model of the robot ('Check sequence'). Once the sequence is played, the author decides whether the resulting motions are satisfactory ('Animation achieved?'). If the motions are satisfactory, the author saves the motion data and the process is complete ('Finish'). Otherwise, the author modifies the sequence by modifying one or more poses currently in the sequence ('Modify keyposes/timing/sequence'), or even adding new poses ('Create keypose'). This process is repeated until the desired animation is achieved ('Finish').

From the animation process flowchart in Figure 2.3, the three main tasks that the author repeatedly performs can be immediately seen: creating keyposes ('Create keypose' block), modifying the sequence or keyposes ('Modify keyposes/timing/sequence' block), and checking the motion sequence ('Check sequence' block). Motion editors facilitate the first two tasks using two tools: the pose editor for creating keyposes, and the sequence editor to arrange the keyposes in a sequence to get the desired motion/animation. Below, we start discussing with the pose editor, followed by the 


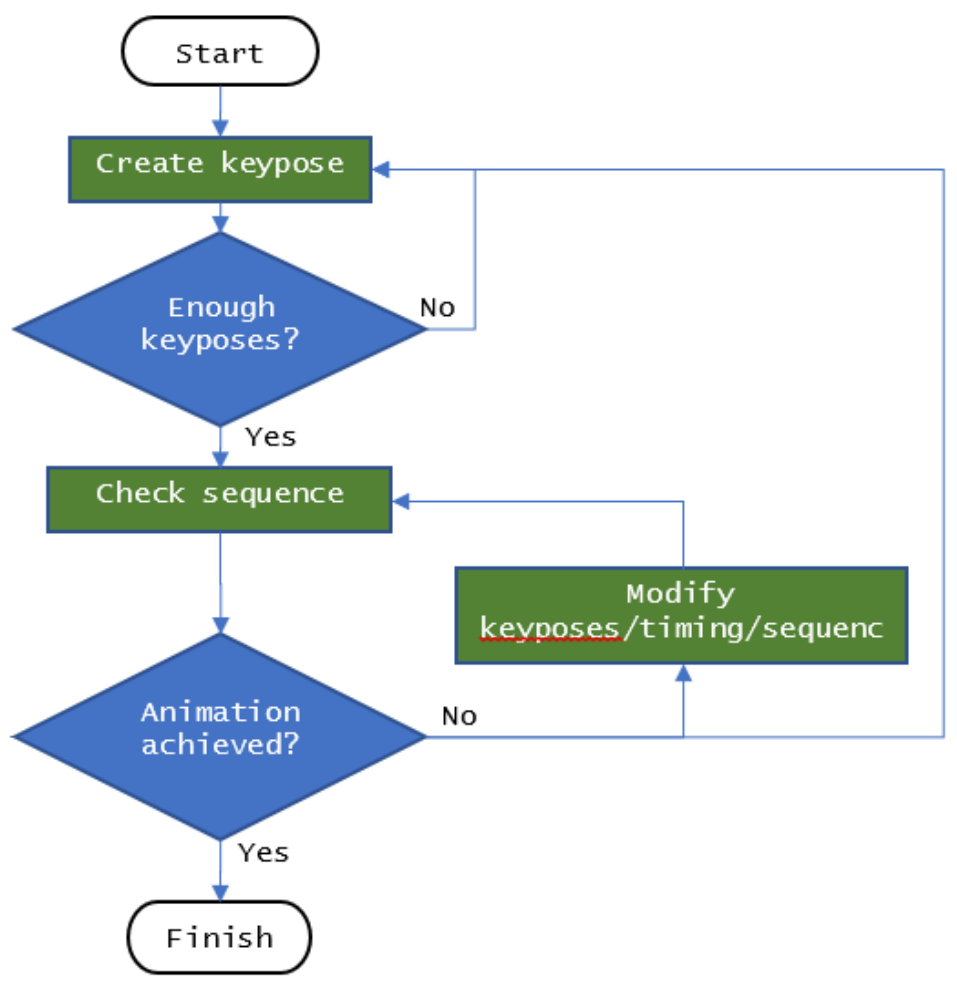

Figure 2.3: The general animation process using motion editor tools for humanoid robots. 
sequence editor. For the third task, some motion editors provide a view with an animated 3D model of the robot to test the motion sequence, while others can only test by directly executing the sequence on the robot. Within pose editors and sequence editors, there are a variety of tools and visualizations to aid in performing modifications which we discuss in the following sections. We discuss the tools for checking a motion sequence in section 2.3.3.

\subsubsection{Pose Editor}

The first common tool we identified and encountered in most of the editors studied is called the pose editor. This tool is primarily used in the "Create keypose" and "Modify keypose" steps in the animation process (Figure 2.3). This tool can be found in WinRME, Lynxmotion SSC-32 Servo Sequencer (Lynxmotion SSC32), Choreographe, HeartToHeart4, RoboPlus Motion 2.0, Pololu Maestro software, and editors developed from research such as Choreonoid [59], SDR Motion Creating Sys-

tem [60], and Kouretes [61]. The pose editor lets the author create a pose on the robot by adjusting the positions of the robot's DOFs, saving the pose, and add the pose into the sequence editor. We define a pose editor as an editor that shows a complete or partial list of the robot's DOF positions at one moment in time.

A pose editor has some means/tools to let the author modify the DOF values called widgets. There are four types of widgets that are commonly used in a pose editor to adjust joint angle values:

- Textbox: a text field for manual entry of values.

- Sliders: a handle that can be moved in a linear slot.

- Knobs: a circular version of a slider. 
- Spinner: a textbox with up and down arrow buttons that lets users enter a value by typing in the textbox or increment/decrement values using the up/down buttons.

In many pose editors, a combination of two or more widgets are provided at the same time to give authors more options. Table 2.1 summarizes the list of pose editors and the widgets that they used to modify joint angle values. From the table, it shows that spinner and slider combination is the most common among motion pose editors. This shows that editors are designed with as many options as possible to let users choose which widget they are most comfortable with. Figure 2.4 shows a few examples of pose editors from different motion editors we studied.

If the robot's hardware supports real-time reading of the servo positions, the DOF values in the pose editor may reflect the current positions of the servos. This feature exists for robots such as Nao, Pepper, Bioloid, DarwinOP, HROS-1, and HROS-5. In this case, the author can create the pose by physically moving the robot into the desired pose as shown in Figure $2.5^{5}$. This method of directly posing the robot to capture a pose is called puppeteering. Because servos hold their positions when powered, the puppeteering feature usually requires a button in the GUI or command to turn the servos on and off momentarily so the author can move the joints to create the pose. RME allows the author to arbitrarily choose the set of servos to activate/deactivate by ID or by kinematic chains like left_arm, right_arm, left_leg, right_leg, and head [15]. Choreographe only allows switching power by kinematic chains (e.g. left arm). Even when puppeteering is available, pose editors usually allow author to switch on-demand between puppeteering method and entering joint angle values using the widgets when creating a pose.

\footnotetext{
${ }^{5}$ Screenshot taken from https://youtu.be/1Ot36Otpp4Y.
} 
Table 2.1: Pose editors and their widgets (note: Spinners always include editable textbox)

\begin{tabular}{|l|c|c|c|c|}
\hline \multirow{2}{*}{ Editor } & \multicolumn{4}{|c|}{ Widgets } \\
\hline & Spinner & Slider & Knob & Textbox \\
\hline RME (text-based/no GUI) & & & & $\checkmark$ \\
\hline $\begin{array}{l}\text { ROBOTIS-OP3 (text- } \\
\text { based/no GUI) }\end{array}$ & & & & $\checkmark$ \\
\hline RoboPlus 1.0 & & & $\checkmark$ & $\checkmark$ \\
\hline RoboPlus 2.0 & & & $\checkmark$ & $\checkmark$ \\
\hline WinRME & & $\checkmark$ & & $\checkmark$ \\
\hline LEGO Mindstorms EV3 & & $\checkmark$ & & $\checkmark$ \\
\hline Kouretes & $\checkmark$ & & & $\begin{array}{l}\text { in- } \\
\text { cluded w/ } \\
\text { spinner in- }\end{array}$ \\
\hline Choreographe & $\checkmark$ & $\checkmark$ & & $\begin{array}{l}\text { in- } \\
\text { cluded w/ } \\
\text { spinner }\end{array}$ \\
\hline Chorenoid & & & & $\begin{array}{l}\text { in- } \\
\text { cluded w/ } \\
\text { spinner in- }\end{array}$ \\
\hline HeartToHeart4 & $\checkmark$ & $\checkmark$ & & $\begin{array}{l}\text { in- } \\
\text { cluded w/ } \\
\text { spinner in- }\end{array}$ \\
\hline Pololu Maestro & & & & $\begin{array}{l}\text { in- } \\
\text { cluded w/ } \\
\text { spinner }\end{array}$ \\
\hline Lynxmotion Flowbotics & $\checkmark$ & $\checkmark$ & &
\end{tabular}


When the author is satisfied with the pose, there is a button or a command in the pose editor to save the current pose. Some editors use the term "frame" instead of "pose". In Choreographe and RoboMotion Plus, the pose editor is in a section in the complete GUI, so the pose-saving button is located outside the pose editor or can be invoked using a keyboard shortcut. The saved poses can then be arranged in a sequence to create a motion using a sequence editor which we will discuss next. Some pose editors also provide additional parameters like speed (WinRME, Pololu Maestro), acceleration (Pololu Maestro), and commands to switch the servos on/off (RME/WinRME, Choreographe, RoboPlus, Pololu Maestro) for puppeteering.

Some pose editors provide additional features when posing, such as: mirroring which will apply the same joint angle values on a different joint, for example mirroring the motion of the left arm to the right arm. Other editors have a complete kinematic model of the robot, so some constraints can be enabled to avoid collisions as the author is positioning the robot into various poses.

\subsubsection{Sequence Editor}

The second common tool in a robot motion editor is what we call the sequence editor. ${ }^{6}$ This interface is used to arrange the poses created using the pose editor into a sequence, thus creating a motion. For this reason, we consider the sequence editor as the core tool of a motion editor. We found four different types of tools which are used as a sequence editor: tabular, timeline, curve editor, and flow graph. Below we discuss the advantages and disadvantages of tabular, timeline, and curve editors. The flow graph editor is a general GUI that can be used for more than arranging the sequences for a motion. For this reason, we discuss the flow graph editor in its own

\footnotetext{
${ }^{6}$ There is no standardized term; some tools call it 'motion editor' while others call it 'sequence editor'. We choose to use "sequence editor" because of it is the least unambiguous in our opinion.
} 

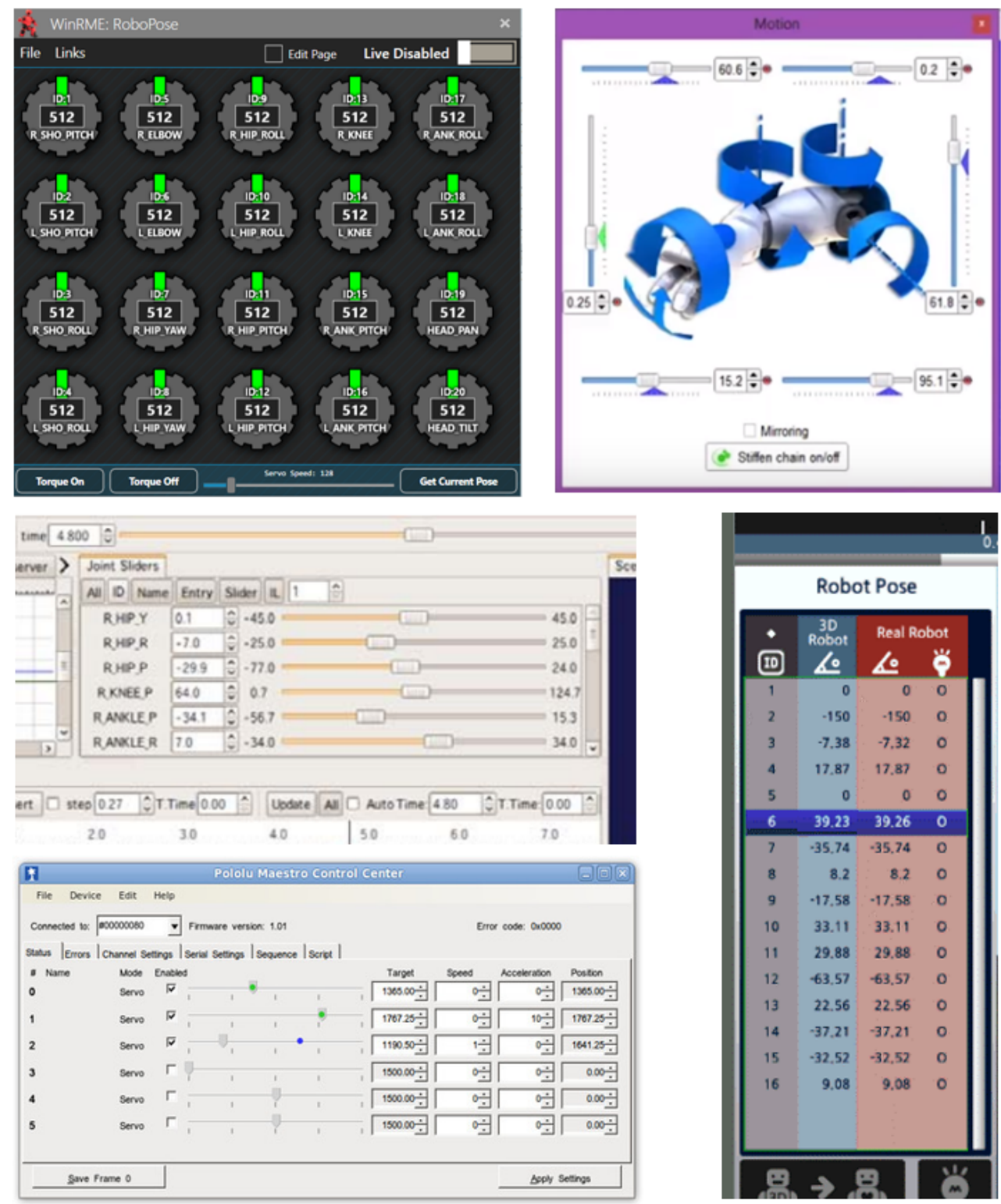

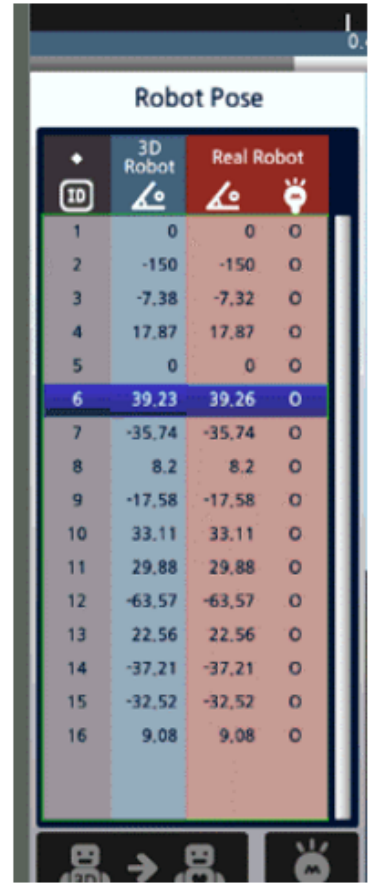

Figure 2.4: Various pose editors (From top left, clockwise): WinRME, Choreographe (for Nao robot), RoboPlus Motion 2.0, Pololu Maestro, Choreonoid [59] 


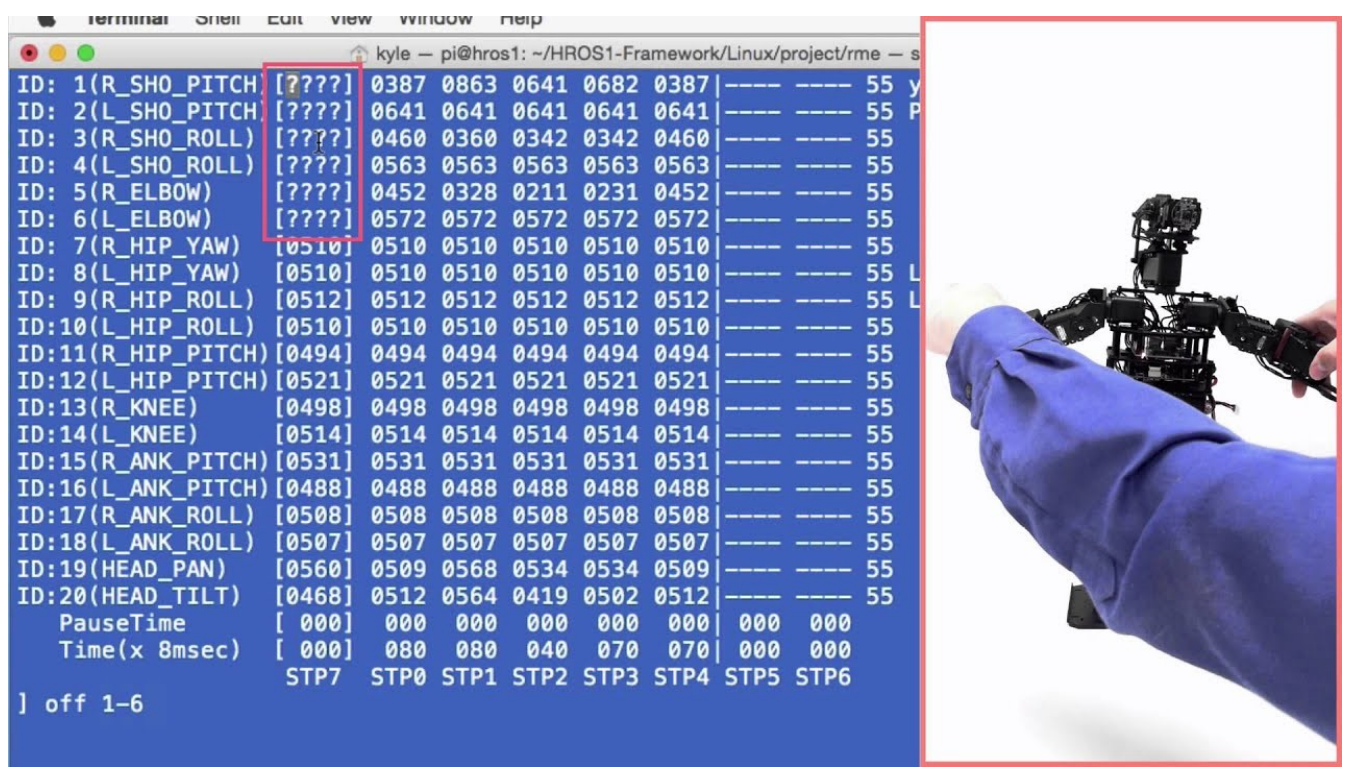

Figure 2.5: Puppeteering on the HROS-1 robot. The servos with ID 1-6 have values "????" because those servos are turned off so the author can move them into the desired pose by moving the robot (right).

section outside the context of sequence editors.

\subsubsection{Tabular Sequence Editor}

Figure 2.6 shows the examples of tabular sequence editors. In the tabular form, the motion data is organized with each row representing one DOF and each column representing one pose of the robot. In other words, when the motion data is viewed by row, each row displays the trajectory or motion of one DOF over time. When the motion data is viewed by columns, each column represents a full pose of the robot at a moment in time.

Creating a motion using the tabular sequence editor is done by creating one pose at a time and can be facilitated by a pose editor. Since RME is a text-based editor, each pose is created directly in the sequence editor. In WinRME, the author can either use the pose editor or directly create the pose using the puppeteering method and directly adds a pose entry in the sequence editor (as in RME). In RoboPlus 

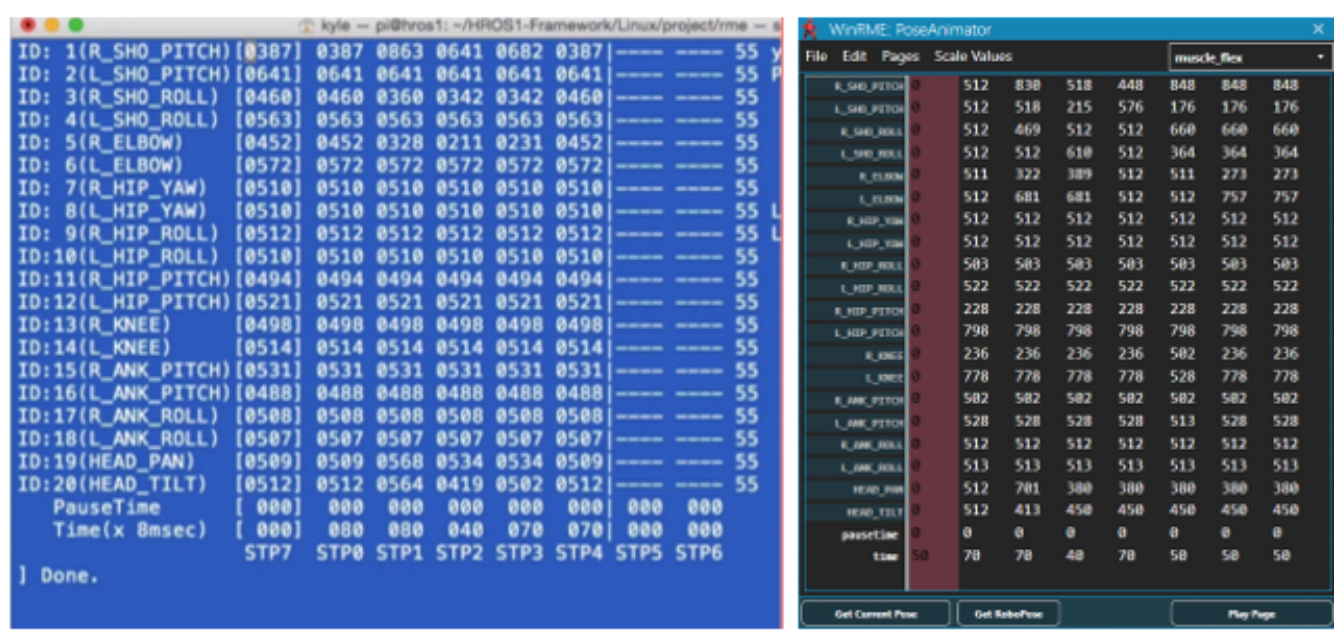

Figure 2.6: Interface of the RME tool (left) and WinRME tool (right) for creating motions on HROS-1 humanoid robot [55]

Motion 2.0, the pose is shown in a tabular format (Figure 2.4 bottom right), but the sequence of poses is represented in the timeline editor (Figure 2.8 middle). The Pololu Maestro sequence editor is a special case of the tabular format since each 'pose' (or 'frame' in Pololu terminology) is given a name e.g. 'Frame 1', 'home', etc. and the sequence is arranged in a list (Figure 2.7). In the tabular format, each pose can also be given additional parameters such as indicating the speed for the stroke for that pose using timing parameters (RME, WinRME) or speed parameter (Pololu Maestro).

RME and WinRME only support up to seven poses in a motion sequence ('page'). When a longer sequence is desired, a page can be directed to execute a "next page". Unfortunately, we could not find a specific reason for this limitation in any of RME/ WinRME documentations.

The main advantage of the tabular sequence editor is that it is both a pose editor and a sequence editor; the poses and sequences can be modified from the table. We can also argue that this method has a low learning curve because of its logical nature of construction (one pose at a time) and is easy to use for simple motions with few 


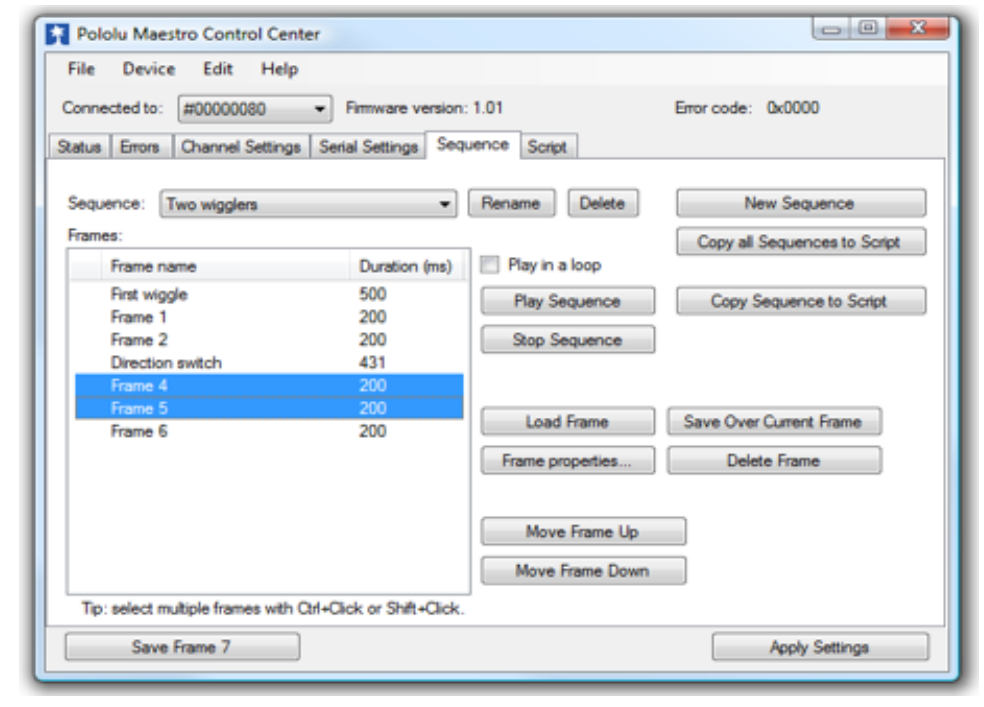

Figure 2.7: Pololu Maestro Control Center sequence editor[57].

poses. The main disadvantage of this tool is that it is difficult to make fine-grained movements such as making motions that smoothly accelerate and decelerate or where one DOF's oscillating motion is on a slightly different phase than another DOF's oscillation. Because of this disadvantage, motions created using a tabular sequence editor often suffer from the 'robotic motion' look. This issue can be addressed using another hybrid tool that can simultaneously edit pose and sequence which is called the curve editor and discussed in section 2.3.3.3. Another disadvantage of the tabular editor is that when creating a motion with many poses (e.g. 10 or more), the tabular view can be overwhelming for the author due to the large number of values displayed in the editor and makes editing/modifying the motion difficult. Another disadvantage is that it may require many iterations to get the timing parameters correct to get the desired speed and effect of each stroke in the motion. The timeline editor alleviates this issue by visually showing the distances between poses which give some indication of the speed and duration of every stroke. We discuss the timeline editor in the next section. 


\subsubsection{Timeline Editor}

Another form of motion editing GUI that we often encounter is the timeline editor. A timeline editor shows time in the horizontal or $\mathrm{x}$-axis direction. Each location on the timeline is a point in time and often referred to as a frame (in reference to traditional animation). Timeline editors are commonly found in animation software like Autodesk Maya, Blender, and video editing applications like Adobe Premiere, iMovie and Final Cut Pro. Figure 2.8 shows the timeline editor from (a) Choreographe, (b) RoboPlus Motion 2.0, and (c) Lynxmotion Flowbotics. The timeline editors are very similar to the one found in computer animation software like Blender (Figure 2.9). The sequence of poses that creates a motion on the robot can be arranged on this timeline by placing each pose at a particular frame. A frame that contains a pose created using a pose editor is called a keyframe. The frames between two keyframes are called the in-betweens and typically generated using linear interpolation. The motion between two keyframes is called a stroke. The distance between keyframes on the timeline indicates the speed of the stroke; the smaller the distance, the faster the stroke will be and vice versa. The timeline editor also gives an idea of the duration of the whole sequence. Thus, using the timeline editor the author has a better estimate on how the motion will appear on the robot rather than using the tabular sequence editor.

In Choreographe, there is a more detailed view of its timeline editor where instead of just one row with keyframes, there are several rows according to the number of DOF on the robot where each row shows the sequence of key frames for each DOF (Figure 2.10). In the detailed timeline view, the author can now modify the motion sequence by moving, or copy-pasting the keyframes of individual DOFs to their liking. 


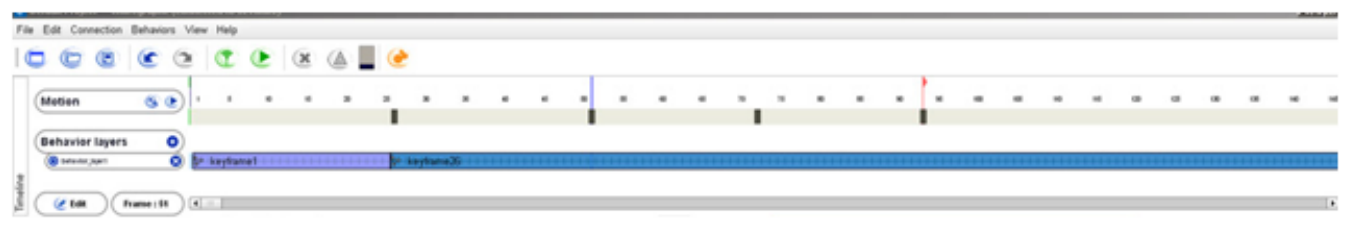

a
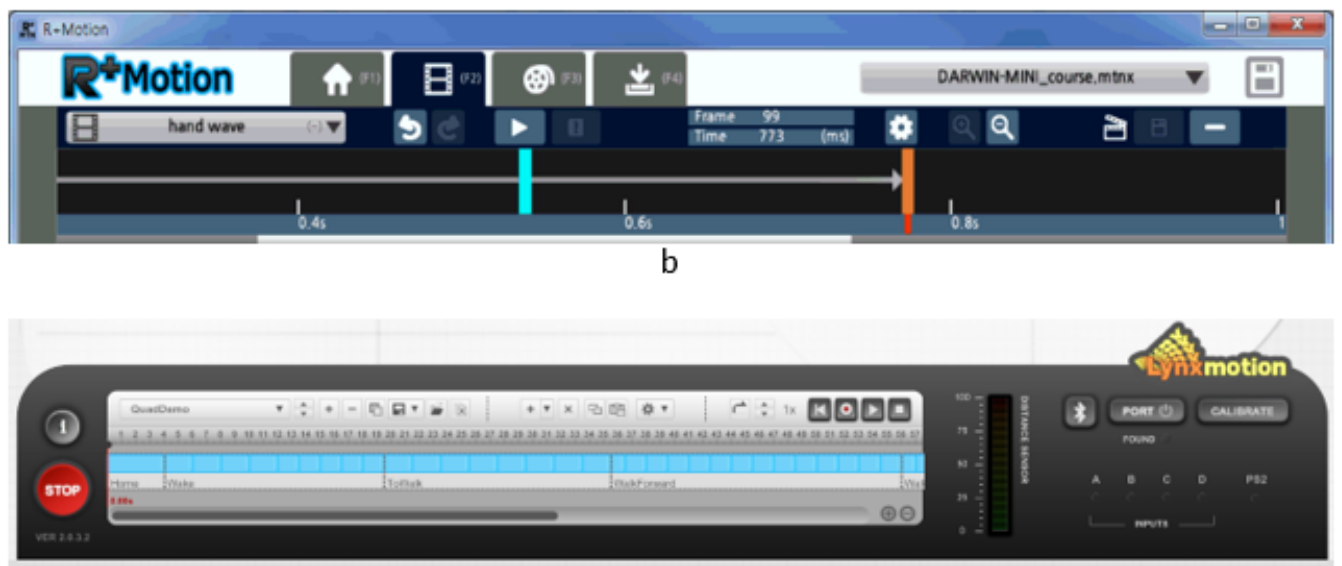

$\mathrm{C}$

Figure 2.8: Timeline editors: (a) Choreographe, (b) RoboPlus Motion 2.0, and (c) Lynxmotion Flowbotics

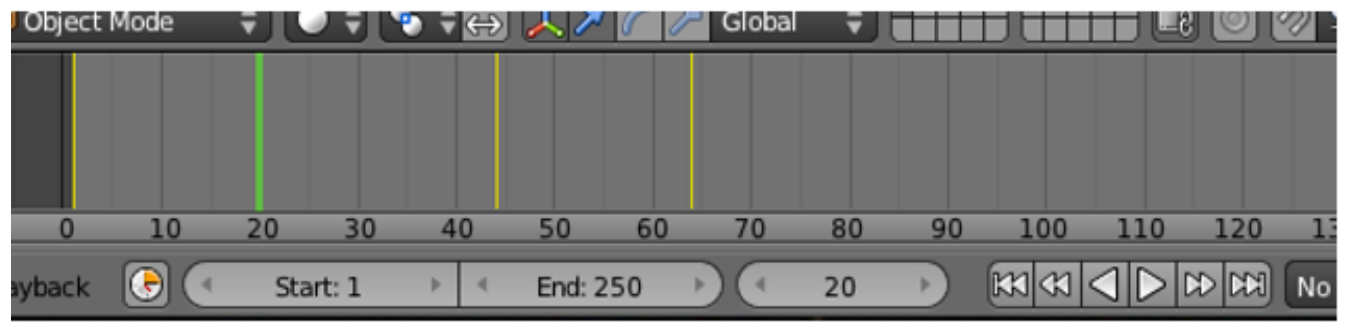

Figure 2.9: Timeline editor in Blender 


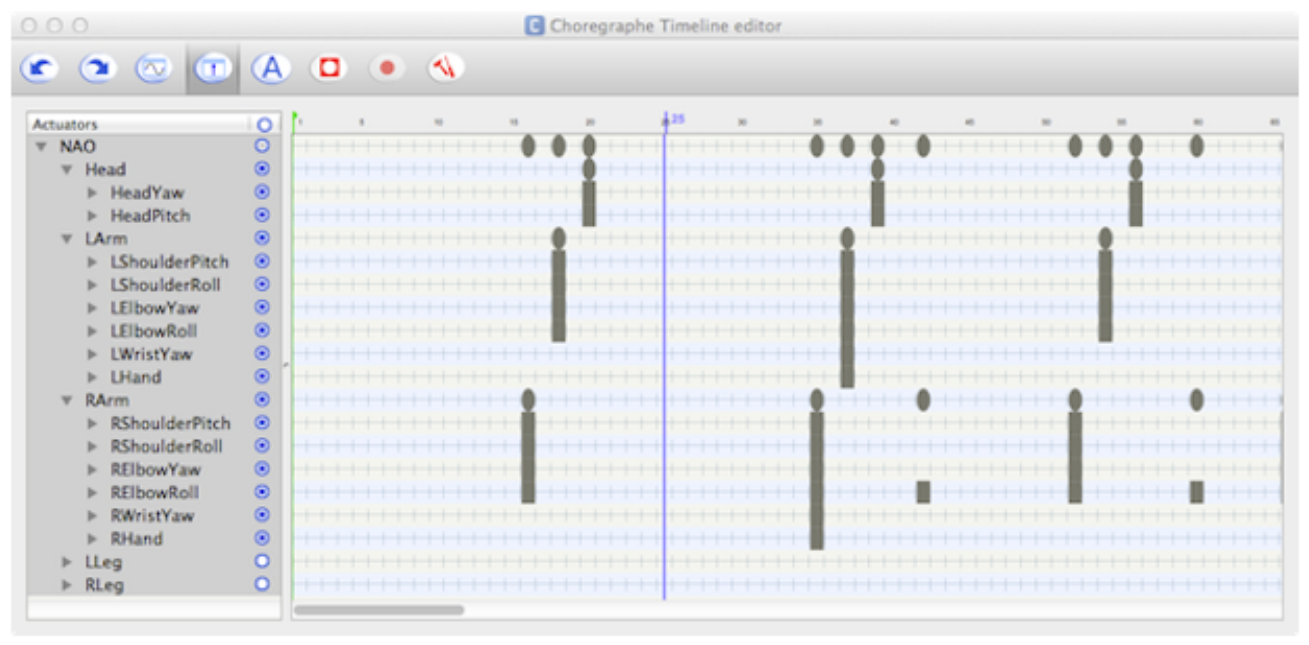

Figure 2.10: Choreographe detailed timeline editor [16]

\subsubsection{Curve Editor}

A curve editor is a GUI that is a standard in most computer animation software like Blender and Autodesk Maya to give animators the ability to fine tune animations to appear more fluid and natural. The x-axis of a curve editor represents time - like in a timeline editor - but the $\mathrm{y}$-axis represents joint angle values. The motion data is represented as a set of waveforms (signals) where each waveform represents the motion a single DOF and typically differentiated using different colors (Figure 2.11). Among the motion editors we studied, only Choreographe provides this feature.

In this form, the animator has a visual sense about the motion joint angle data: a slope indicates a movement, a flat line is a constant over time and indicates a still pose, the transition from a flat line to a slope indicates the beginning of a motion, and the transition between a slope to a flat line indicates a motion coming to a stop. The animator can adjust the shape of the signals to get the intended effect. For example, speed is determined by adjusting the steepness of the slopes in the signals. A signal is typically adjustable using Bézier curves (Figure $2.12^{7}$. Bézier curve is a

\footnotetext{
${ }^{7}$ Curves are generated from the website: https://pomax.github.io/bezierinfo/
} 


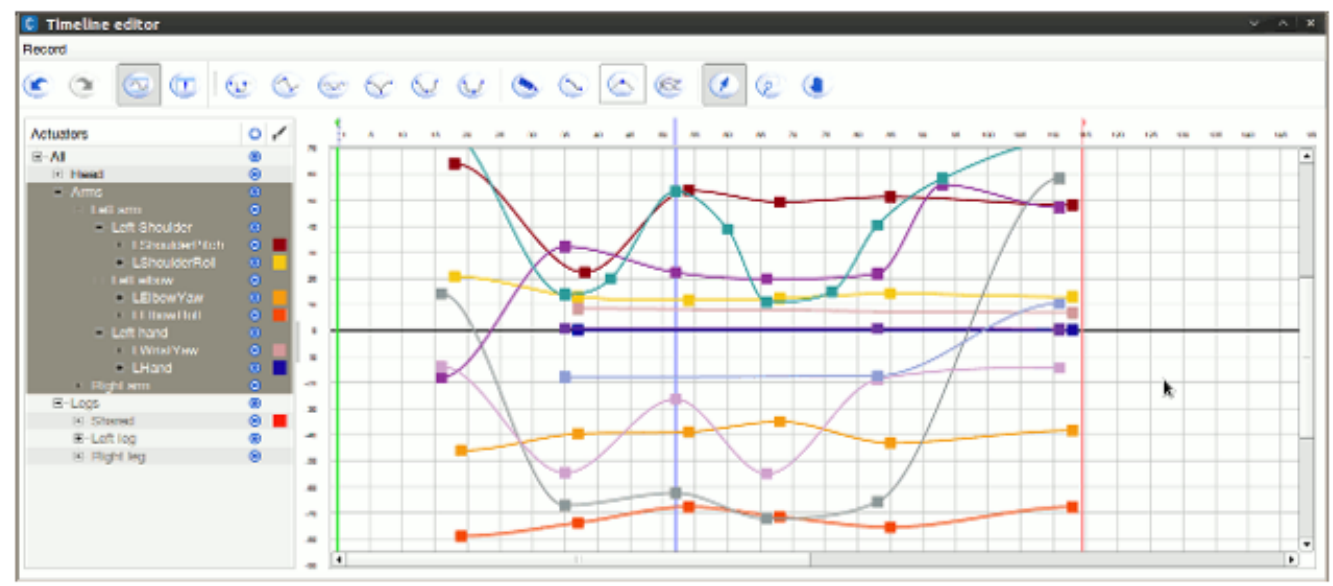

Figure 2.11: Choreographe curve editor interface [16]

parametric curve used to interpolate two points $P_{0}$ and $P_{n}$, where $n$ indicates the degree of Bézier curve. A Bezier curve of degree one $(n=1)$ is equivalent to linear interpolation, degree two $(n=2)$ is quadratic or parabolic, degree three $(n=3)$ is cubic, and so on. The points $P_{1}$ through $P_{n-1}$ are the control points of the curve, which determine the shape of the curve, but are not part of the interpolated points on the curve itself. Animators use the control points to shape the waveform to get the desired effects, e.g. smooth or abrupt motions, fast or slow motions, etc. Therefore, the curve editor becomes a standard in animation tools to make intricate, expressive motions in addition to the timeline editor.

We found that very few robotics GUI software provide curve editors such as Choreographe [16], and the iCat animation tool [17][62].

\subsubsection{Simulated/Virtual Robot}

After creating a sequence of poses, the author would want to check whether the sequence achieves the desired motion. A simulated/virtual robot is a tool as part of the motion editor which shows an animated 3D representation of the robot in the GUI. The virtual robot tool provides a safe way to evaluate the sequence that has 

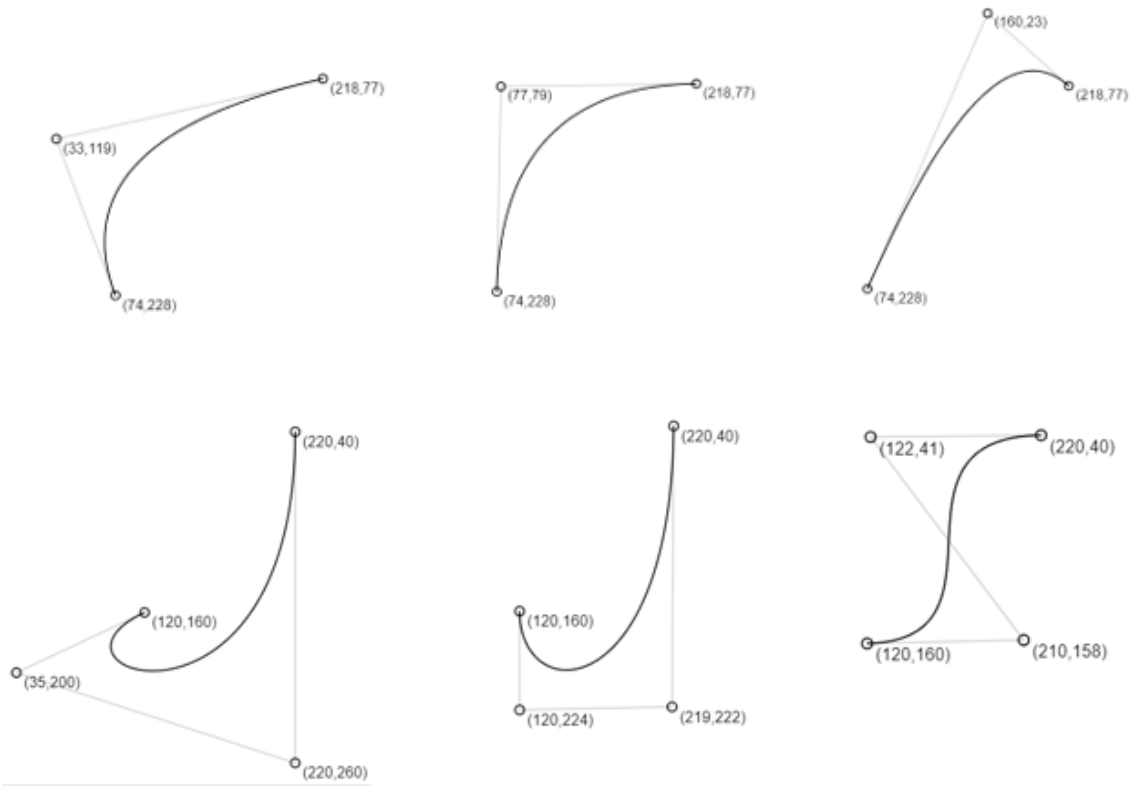

Figure 2.12: Examples of Bezier curves [63]. The nodes that do not lie on the curve are the control points. Top row: one control point creates quadratic Bezier curves. Bottom row: two control points create cubic Bezier curves. Notice that the end points in the quadratic and cubic curves remain in the same coordinates, and the shapes of the curves are determined by the positions of the control points. 


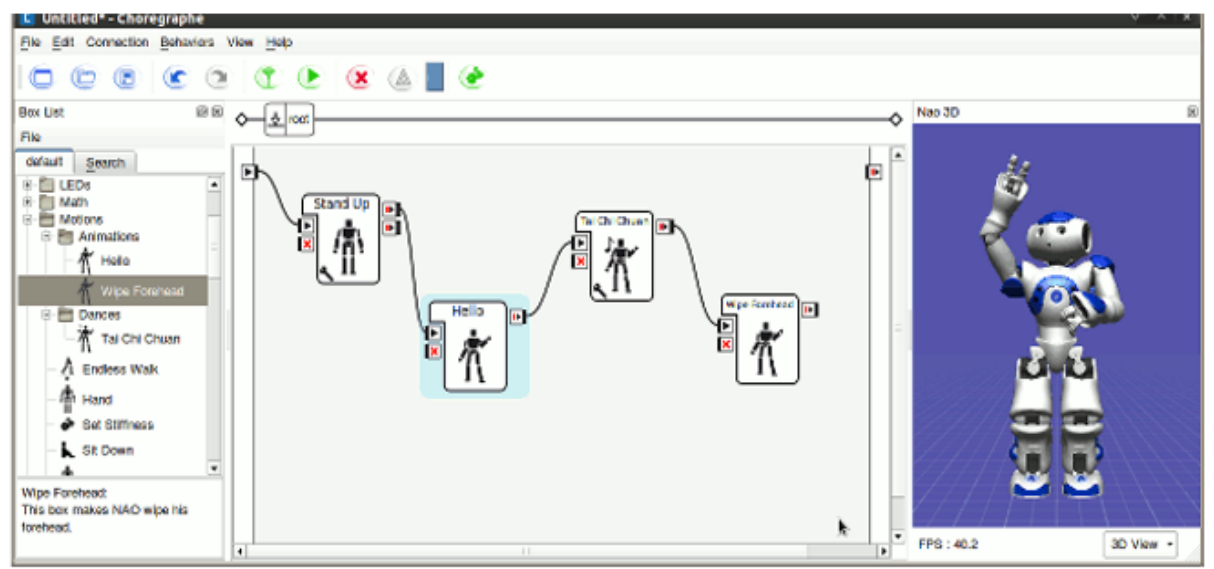

a

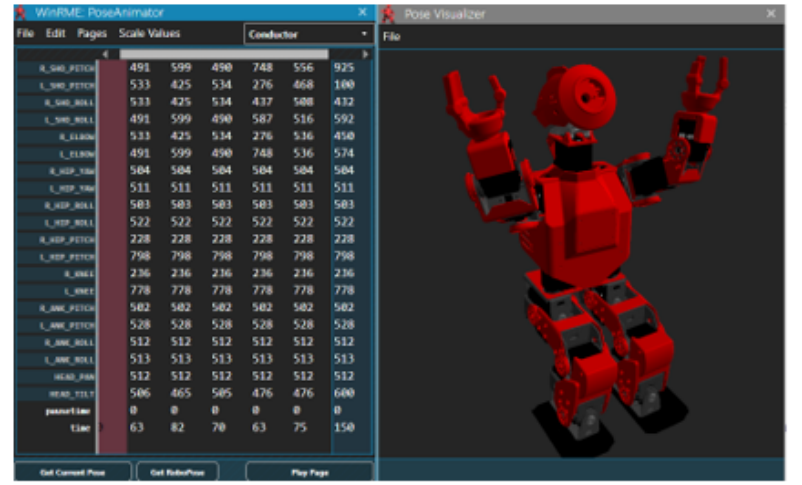

b

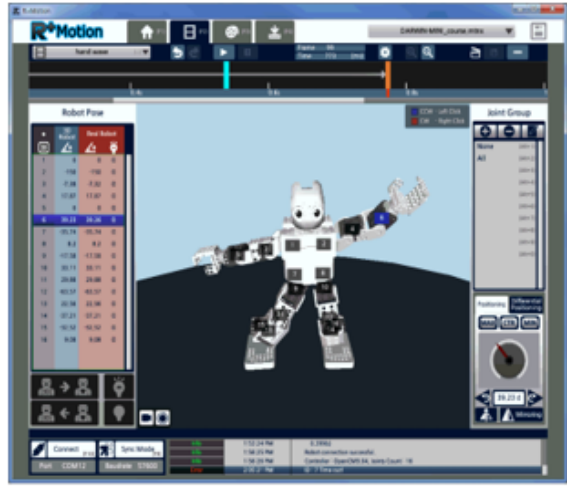

C

Figure 2.13: Virtual robots in (a) Choreographe, (b) WinRME, (c) RoboPlus Motion 2.0

been created since there is no risk of damaging the robot hardware. The author can safely evaluate whether a motion will cause collision with another body part of the robot and make the necessary adjustments. We found the virtual robot tool on Choreographe, WinRME, and RoboPlus Motion 1.0 and 2.0 (Figure 2.13).

The simulated/virtual robot tool in Choreographe can provide the author with dynamic simulation indicators when a pose or motion (e.g. walking) will cause imbalance and potentially cause the robot to fall down. In WinRME and RoboPlus Motion 1.0 and 2.0, there does not seem to have the same indicators as in Choreographe. In the former tools, the robot's 3D model appears to be suspended in the air so the 
torso remains upright and the legs can move freely. Therefore the author cannot tell whether the motion or pose will cause the robot to fall down.

\subsubsection{Dynamics Simulator and Inverse Kinematics}

Dynamics simulation in a motion editor is used to aid the author to check whether the motion that was created will cause the robot to fall down or will it remain upright. Inverse kinematics calculation is useful when the author wants to create a motion where the specific placement of the end effector is desired such as the positioning of the feet in a walking motion. Among the motion editors we evaluated, only Choreographe, RoboPlus Motion 1.0 and 2.0 provide the dynamics simulation feature as these editors have the set of complete kinematic models of the robots each respective editor supports.

\subsubsection{Flow Diagram Editor}

Another tool that can be used to create a sequence of poses is the flow diagram editor. The motion sequence is arranged by connecting two motion blocks together (Figure 2.8). Each motion block represents a pose of the robot. In HeartToHeart4 and Choreographe, this connection is shown as a directed edge. In Lego Mindstorms EV3 editor, two blocks are connected by placing them adjacent and aligned with each other. In editors like Choreographe and Lego Mindstorms EV3 editors, flow diagrams are their main mode of programming the robots' behaviors. There are various types of action blocks, such as:

- Audio: for voice, speech, sound effects

- Script: for writing custom code

- Logic/decision: for behavior branching/conditionals or loops 
- Sensor: for reading sensor inputs

- Motor: for controlling actuators/servos and thus, motion

Thus, the flow diagram is a general tool can be used to describe not only motion, but also high-level intelligent behaviors which combine motion, sensing, voice, and decision making. Here, we are only concerned with the 'motion blocks' used for creating motion. In these kinds of 'motion blocks' in addition to storing the robot's pose, there may be other parameters that the author can set such as number of repetitions, speed, time duration, or pause time.

The advantage of a flow diagram editor is its generality as we mentioned above. Another advantage is that unlike the timeline editor where a frame is only represented as an abstract marker, the flow diagram shows the names of the poses arranged in the sequence, giving the author a sense of how the motion would be executed. In Choreographe, a motion created using flow graph editor can also be viewed in the timeline and curve editor. In other words, the author can design a motion from a highlevel view (flow graph) then make detailed adjustments later in the curve editor. We found that this high-to-low design construct (flow graph to curve editor) is common in animation and video game tools such as Blender, Autodesk Maya, and Unity, but in our observation so far, the only robot motion editor that has implemented this feature is Choreographe.

Among the robot motion editors we studied, only the HeartToHeart4, Lego Mindstorms EV3, RoboPlus Motion 2.0, and Choroeographe provide flow diagram editor (Figure 2.14). Flow diagram is more suitable for designing sequences of actions and behaviors rather than delicate, complex, and intricate motions. 


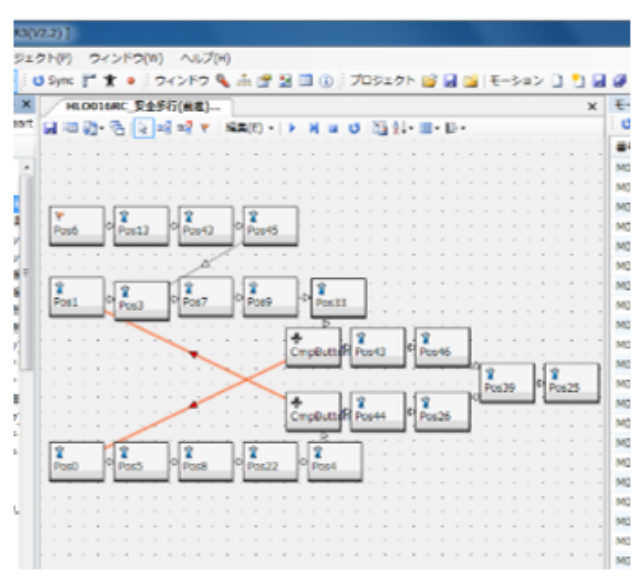

a

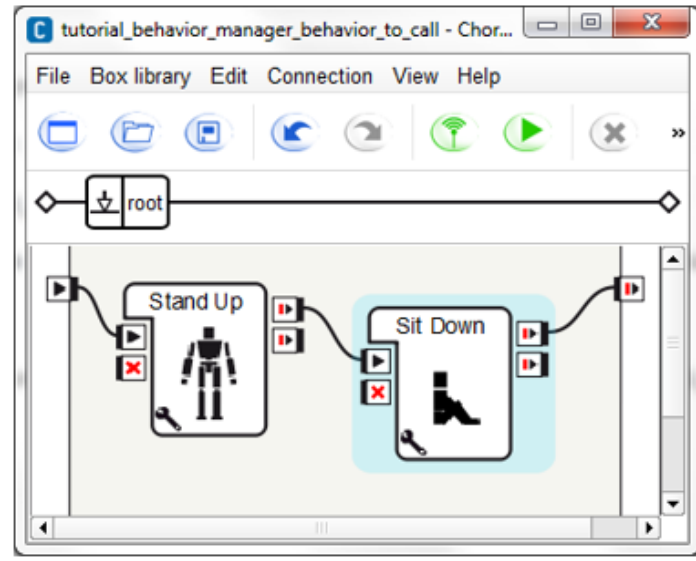

b

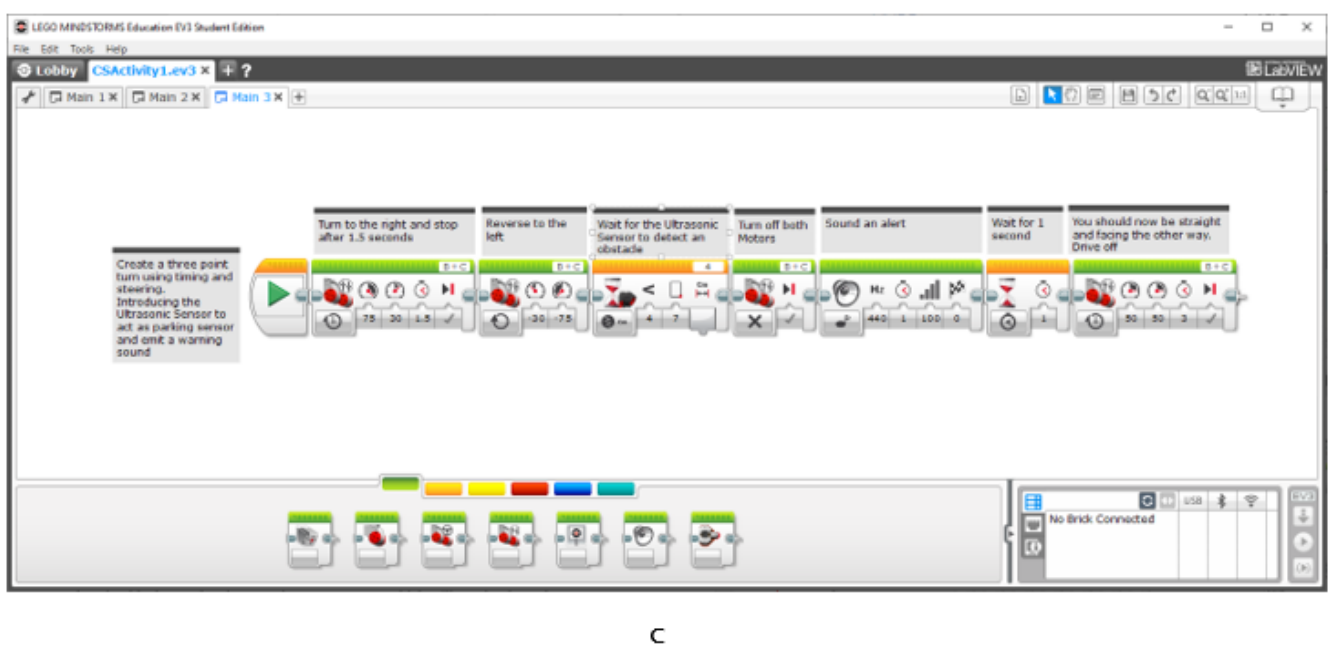

Figure 2.14: Flow diagram editor from (a) HeartToHeart4 [51], (b) Choreographe [16], and (c) Lego Mindstorms EV3 [56] 


\subsubsection{Scripting}

Often, robots are pre-programmed with several behaviors and each behavior is executed deterministically based on the input to the robot. After some time, people quickly notice that the robot is behaving deterministically and has very limited skills. Scripting features are developed into motion editors so the author can program the robot to exhibit more complex behaviors that are produced algorithmically. Many of the properties of scripting schemes exhibit very similar properties to the flow diagram tool discussed above, such as branching/conditionals, loops, and coordination of multimodal actions (speech + motion). We found there is no standard scripting scheme that is preferred by all the motion editors we studied; each editor that supports scripting has developed their own schemes (Pololu, Choreographe, Improv). The Robot Expressive Behavior Language (REBeL) which is one of the main contributions in this dissertation, is also a scripting framework. We discuss REBeL in more detail in Chapter 4.

In the Robot Theater capstone project [10], a scripting tool allows the authors to create a "script" of a sequence of actions to happen in a scene of the play. The authors needed to coordinate the stage lighting, music and recorded speech in addition to executing motions on the robot "actors." Each of these elements are controlled by individual programs. The authors used the scripting tool to enable them to create a sequence of actions that describes a scene they desired. Each command is encoded using a number as shown in Table 2.2.

An example of a script using this scheme is shown in Table $2.2^{9}$. While the script scheme is very simple (e.g. no logical/conditional or repetition command), the script gives the authors a high-level control of the stage elements (actors, lighting, sounds),

\footnotetext{
9 "mp3" indicates a sound file, and "midi" refers to the MIDI message format often used for sounds/music control (MIDI, 2020).
} 
Table 2.2: Scripting command code from [10]

\begin{tabular}{|c|l|l|}
\hline Code & Command & Parameter \\
\hline 1 & Robot walk & Duration in seconds \\
\hline 2 & Robot turning & Turning angle in degrees \\
\hline 3 & Play motion page ${ }^{8}$ & Page ID/number \\
\hline 4 & Sleep & Duration in milliseconds \\
\hline 5 & $\begin{array}{l}\text { Play MP3 (music, } \\
\text { speech) }\end{array}$ & ID/name of .mp3 file to play \\
\hline 6 & Send MIDI command & $\begin{array}{l}\text { MIDI command format: port : } \\
\text { event }: \text { param } 1: \ldots \text { param }(n)\end{array}$ \\
\hline 7 & Exit program & N/A \\
\hline
\end{tabular}

Table 2.3: Example of actual script from [10].

\begin{tabular}{|c|c|}
\hline Script/code & Meaning \\
\hline 15 & Walk forward for 5 seconds \\
333 & Play motion 'Karate Kick' (page 33) \\
545 & Play 'Kungfu' .mp3 (file '45.mp3') \\
36 & Play motion 'Fight' (page 6) \\
329 & Play motion 'Fall' (page 29) \\
6 128:F8:C9:00:12 & Send midi command to fade lights \\
513 & Play 'Dying' .mp3 (file '13.mp3') \\
4150000000 & Play 'dying .mp3 (file '33.mp3') \\
7 & Sleep for 15 seconds \\
\hline
\end{tabular}

which greatly simplifies the planning of a scene.

Improv uses a custom language where behaviors and animations can be described in the same structure [11]. It also supports hierarchical structure; a behavior description can be represented with a name, and another behavior can include the former behavior by referencing its name. Improv includes features for controlling multiple characters, randomization, behaviors, and grouping of animation scripts, to name a few. Figure 2.15 shows a sample ACTION script which defines the low-level specifications (i.e. Pitch, Roll, Yaw rotations) of an action called "Talking Gesture 1". Figure 2.16 shows an example of a SCRIPT script, which specifies a sequence of high-level actions for an agent for a behavior called "greeting". The animation of the characters 


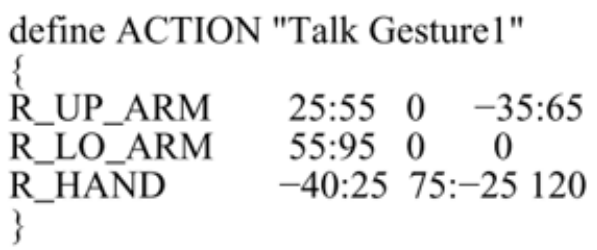

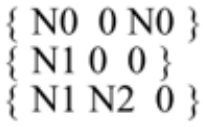

Figure 2.15: The Improv ACTION script defines low-level motion of the DOF of the agent (example from [11]). Each row in the "Talk Gesture1" ACTION block refers to a DOF (R_UP_ARM, R_LO_ARM, R_HAND). The three columns of numbers refer to the rotation in Pitch, Roll, and Yaw rotations in degrees, respectively. The number 25:55 refers to range of motion for the Pitch, 0 for Roll, and -35:65 for Yaw rotations of R_UP_ARM. The numbers in braces refer to interpolation using Perlin noise [64]. N0, N1, N2 refers to different configurations of Perlin noise.

can be synchronized with speech, music, and coordinated with the actions of other characters in the system. Among the unique things about Improv is the inclusion of Perlin noise in its animation description. Perlin noise was first introduced as a technique to produce noise which has the property of being seemingly random but smooth (i.e. small value difference between neighboring points) [64]. Perlin noise can be used for creating 1-D, 2-D, and 3-D objects. It also has been found useful to create textures not only on the surface but also inside of 3D computer-generated objects, such as stone, smoke, and ground. By adding Perlin noise to motion produced by Improv, the noise added some imperfection to the motion that makes it look more natural than without the noise, or with just random noise. Improv allows defining choices of actions which can be specified with probabilities, coordination of speech and action, and more. Improv is also equipped with an animation engine that prevents animation artifacts, for example: interpolating hand trajectory that causes the character hands to go around the body instead of passing through the character's body. The work presented in this dissertation for creating behaviors using REBeL is similar to Improv but differs by using probabilistic algebraic operators and expressions to describe behaviors instead of using keywords. 


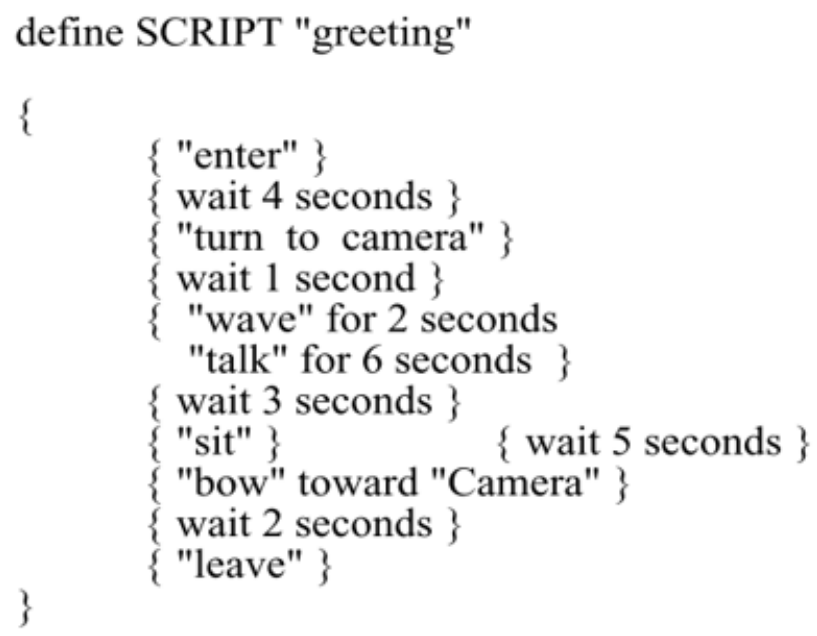

Figure 2.16: The Improv SCRIPT "greeting" defines a complete sequence of high-level actions for an agent's greeting behavior (example from [11]).

\subsection{Behavior Markup Language and its Related Frameworks}

The eXtensible Markup Language (XML) has been the preferred technology to create behavior generation frameworks for embodied conversational agents (ECAs) for human interaction with computer-generated human characters [65] [66] [67] [68] [69]. Behavior Markup Language (BML) is a domain-specific language for describing multimodal behaviors of computer-generated agents (i.e. ECAs) based on the eXtensible Markup Language (XML) specifications [70] [67]. Figure 2.17 shows an example of a BML script that synchronizes speech with a head nodding gesture. BML was developed as a multinational joint effort from embodied conversational agent (ECA) researchers from Germany, Austria, France, USA, and Iceland to create a unified framework for ECAs. These authors wanted to create a unified framework because they noticed there are multiple different ECA frameworks that were developed independently by separate groups but each uses their own domain-specific languages [71] [68]. A large portion of the works using BML are dedicated to generation of synchronization of gestures with speech content [67] [71] [72] [73] [74]. 


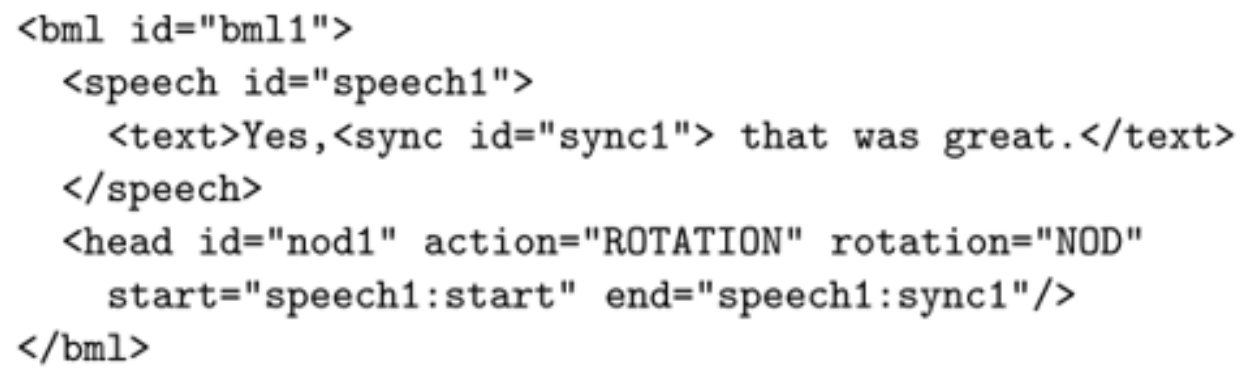

Figure 2.17: An example of a BML script (example from [70]). The speech "Yes, that was great" is synchronized with a head nod which starts at the beginning of the speech and ends after saying "Yes" at the "sync1" mark.

The goals of the work in this dissertation are similar to the BML architecture with respect of building an intelligent system for generating behaviors and gestures for believable agents. My work contrasts with BML where I focus more on humanoid robot applications, and BML focuses on computer-generated agents. I believe the systems developed in BML and my work can be easily adapted for either applications in the future.

Specifically for the motion editing feature, a user or artist can create new gestures for an agent using the BML Sequencer tool [75]. Thus, the BML Sequencer is the closest equivalent to a motion editor in the BML framework, and thus comparable with the RME tool of HROS frameworks, and REBeL. However, the majority of a character's body animation is performed using pre-recorded animation clips. A character's gesture in BML is composed of three parts: body animation, facial animation, and gaze animations. Fig. 2.18 shows the interface of the BML Sequencer.

Sometimes we want to be able to tell the robot to move with certain expressions using descriptive words from our spoken language. For a human, this is relatively easy to do, but the robot does not know the meaning of these words. Therefore, ideas from performance arts such as Laban Movement Analysis (LMA) have been used to map high-level descriptions of motions as parameters of motions [14]. Other works 


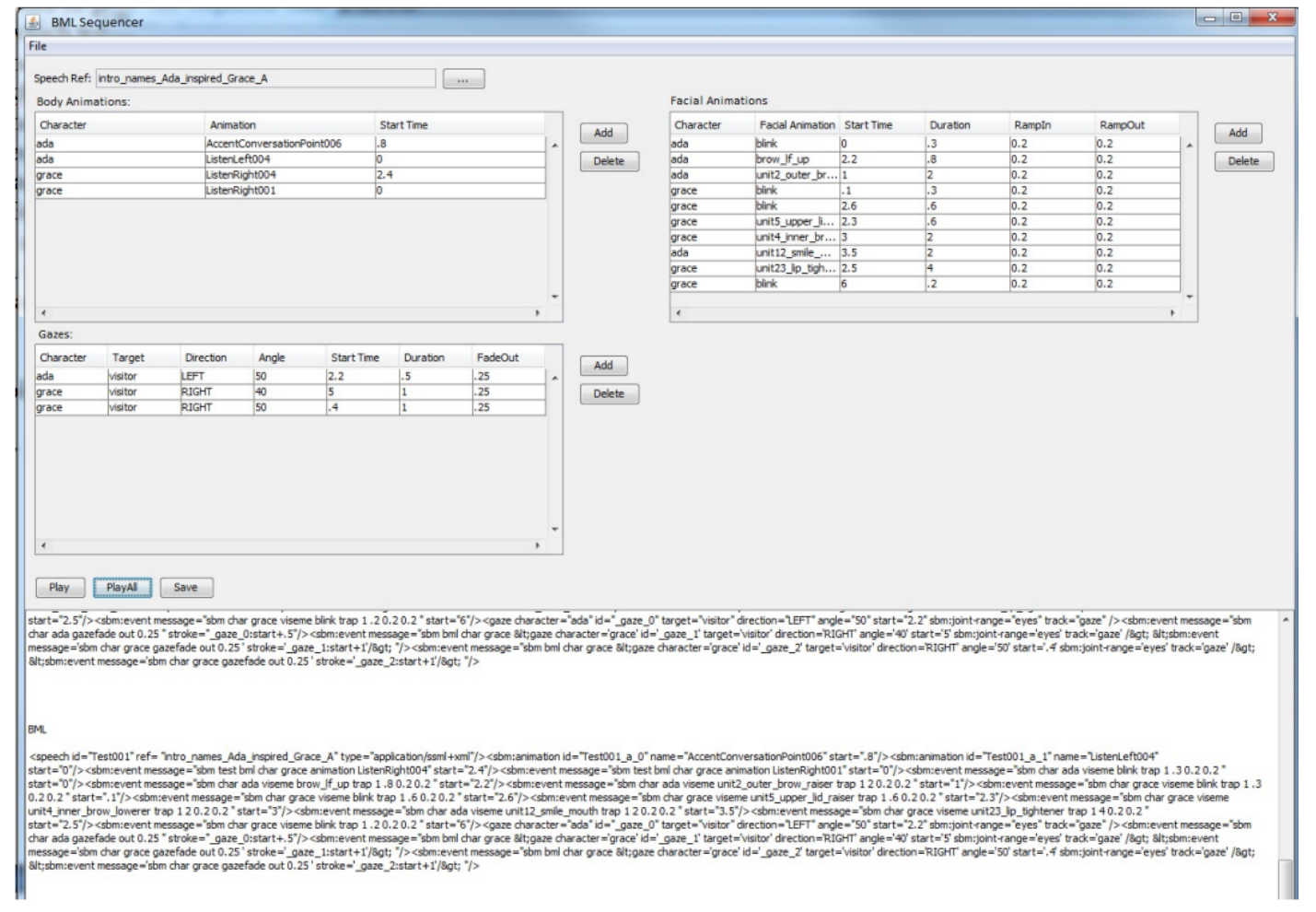

Figure 2.18: The BML Sequencer interface (Image from [75]). 
also developed specifications for multimodal robot behaviors using markup languages such as MultiML [66], MURML [68], and APML [65].

Neff et. al. developed a system that can imitate the gesticulation characteristics of a person [76]. The system involves a manual process for annotating 10-minute video clips of two talk show hosts with distinct gesticulation styles. Statistical information collected includes: the type of gesticulations, the number of strokes per gesture,

and the type of words and context the gestures were used. Neff et. al. showed that given text scripts that are different from the sample videos, their system can produce animations on a 3D computer-generated person that people can recognize as exhibiting gesticulations that mimics the style of the two model talk show hosts. The Beat toolkit [71] can generate communicative facial and hand gestures from input text of a sentence to be spoken by an ECA and synchronized with the speech during execution. Beat generates the communicative gestures from text using heuristics from a knowledge base that was created by analyzing examples of speech performances to obtain the relationships between types of utterance and context in a speech and the accompanying gestures.

\subsection{Synthesis Using Motion Capture Data}

Using motion capture data is another approach that can be used to quickly generate motion data for robots. Originally, motion capture data is more commonly used to animate computer-generated (CG) characters so their movements will appear humanlike and natural. This method is often used in movies and video games. Figure 2.19 shows an example of motion capture data visualized in two different formats. In Figure 2.19 left, the motion capture data consist of marker positions in 3D space that were placed on the performer's body. On Figure 2.19 right, the motion data consist 

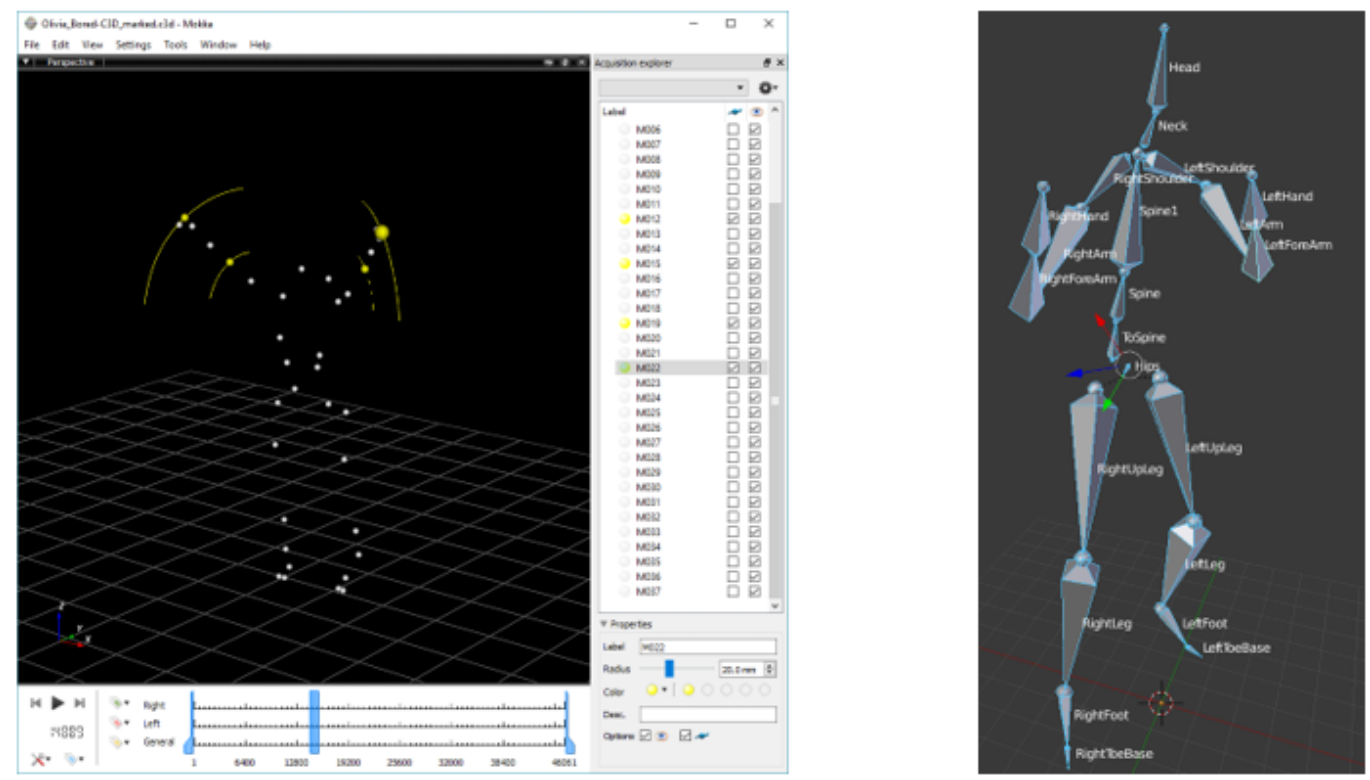

Figure 2.19: Visualization of motion capture data in Mokka tool (left) [77] and Blender (right) $[78]$

of joint angle values in $\mathrm{x}, \mathrm{y}$, and $\mathrm{z}$ axes for each joint/DOF of the motion capture model.

In practice, human motion capture data is difficult to use on a robot unless the robot has similar proportions and articulations as the motion capture model. The problem of mapping motion data of one model to another model with different proportions and kinematics is called motion retargeting. Often, manual edits must be done to the motion data, so the movement appear in a consistent way on the target model. Sometimes the motion capture data is discarded altogether in favor of manual animation [79]. Other big challenges of using motion capture data are: removing artifacts (e.g. sliding foot), to create smooth transitions between actions [80], and adapting the motions to various situations (e.g. uneven terrain) [29]. Many of these challenges of using motion capture data pertains to computer animation. Motion retargeting in robotics is often a challenging problem since the robots usually have different kinematics than the motion capture model, and limitations of the actuators 
that cannot keep up with the movement speed in the motion capture data [81] [82].

In video games where the character's movements are controlled by the player, the character's animation must exhibit realistic and 'fluid' movements. Most of the motions for the character such as walking, running, climbing, jumping, punching, kicking, etc. are obtained from motion capture. Since the player is controlling the characters, the challenge is how to create seamless and plausible/realistic transitions between the different actions as the player commanded, but with quick response time, at the same time without undesired artifacts such as foot sliding, and mesh clipping. Motion Graph is a method to find the optimal transition points between two motion capture clips which would give the smoothest transitions from one action to another [83]. Improvements to Motion Graphs address undesired motion artifacts, creating transitions for animations of quadrupedal animals, and creating optimal transitions regardless in which part of the clip the transition is initiated (by a user/player) [84].

Spectral methods have been used to modify motion data to create different expressions. For example, wavelet decomposition can be used to remove jitter (i.e. noise), increase or decrease the range of motion [13]. Fourier transform can be used to transfer styles of one motion to another [85] [86]. Both wavelet and Fourier transform can be used to blend two motion data together and create interpolation between the two (Figure 2.20).

\subsection{Dynamic Models and Biologically-inspired Methods}

Spacetime Constraints (SC) and its extensions allow animators to create animations by only specifying the key points (keyframes) and some dynamic properties (e.g. stiffness), SC then generates motion trajectories that exhibit animation principles like anticipation, squash and stretch, and follow-through [87] [88] [89]. Hoshyari et al 


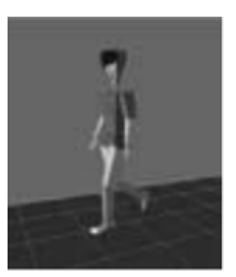

a

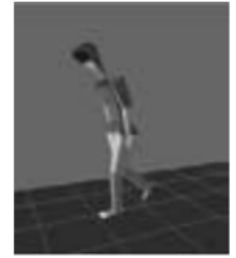

b

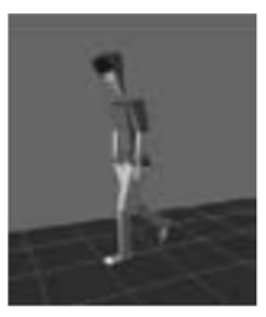

c

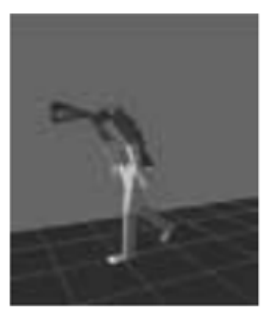

d

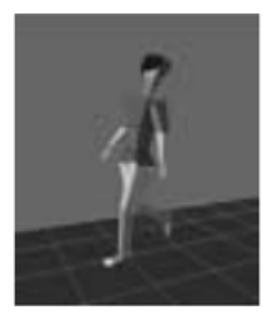

Figure 2.20: Interpolation and extrapolation using Fourier transform on two different styles of walking animation (image from [85]). The two reference animations are: (a) "Normal" walk, and (b) "Tired" walk. Adjusting the interpolation coefficient s in the Fourier domain, (c) shows a "slightly tired" walk $(\mathrm{s}=0.5),(\mathrm{d})$ "very tired" walk $(\mathrm{s}=2.0)$, (e) "brisk" walk $(\mathrm{s}=-0.5)$.

introduced a system that uses a dynamics simulator that is able to transfer dynamic, fast motions from the Autodesk Maya animation software which minimizes jerk and vibrations to match the input motion data onto a stationary robotic figure with limbs made of rigid and flexible parts [90]. The motion data used by Hoshyari et al were created by professional animators, so the motions are very dynamic, such as boxing, drumming, and dancing.

In neurobiology, there is a theory that biological motions are generated by some combination of nonlinear dynamical movement primitives called motion pattern generators (MPG). Schaal developed Dynamic Movement Primitives (DMP) which method took the idea of MPG and added control so movement primitives can be combined to perform movements for a specific task [91]. Lim et. al used Principal Component Analysis (PCA) to collect motion primitives from a collection of motion capture data [92]. Using just linear combination of the principal components (motion primitives) 
they were able to create new motions with natural-looking motion.

Sims used Genetic Algorithm to evolve 'creatures' from a set of components such as motor control and limbs, and certain rules on how limbs can be connected to each other (Sims, 1994). The fitness functions for the individuals are goals such as traversal distance and securing resources (e.g. 'food') against another creature. The result produced individuals that resemble organic 'creatures' in the way they move such as walking, swimming, or capturing resources despite their unusual shapes. Geijtenbeek evolved musculoskeletal models using Genetic Algorithm which evolves the placements, length, and size of muscles to produce a stable bipedal gait, where the shape and size of the bipedal creature is given by the user [29]. Without telling the model how a gait should look like, the evolved models were able to produce stable walking gaits that are resilient to perturbations, user controllable, and the results appear like natural gait. Besides walking, other bipedal models were successfully evolved to produce other gaits such as running, trotting, and jumping. Figure 2.19 left shows some examples of "creatures" generated by Sims' algorithm. Figure 2.19 right shows a few different pre-defined biped models on which gait was evolved using Geijtenbeek's algorithm.

The Braitenberg vehicle is a conceptual vehicle used to illustrate that a simple sensor-actuator system can make a robot appear to have complex behaviors [94]. Figure 2.22 shows a model of the classic Braitenberg vehicle which is a robot with two wheels on each side, each actuated by a motor. In front of the robot there are two light sensors, one on each side. The sensors are hardwired to the actuation of the motors that drive the wheels. In one configuration, the sensor on the left side directly controls the motor on the left side, similarly on the right side. As the light intensity detected by the sensor increases, the motor will spin faster. Therefore, when the light is on the left side of the robot, the robot's left wheel will turn much faster than the 

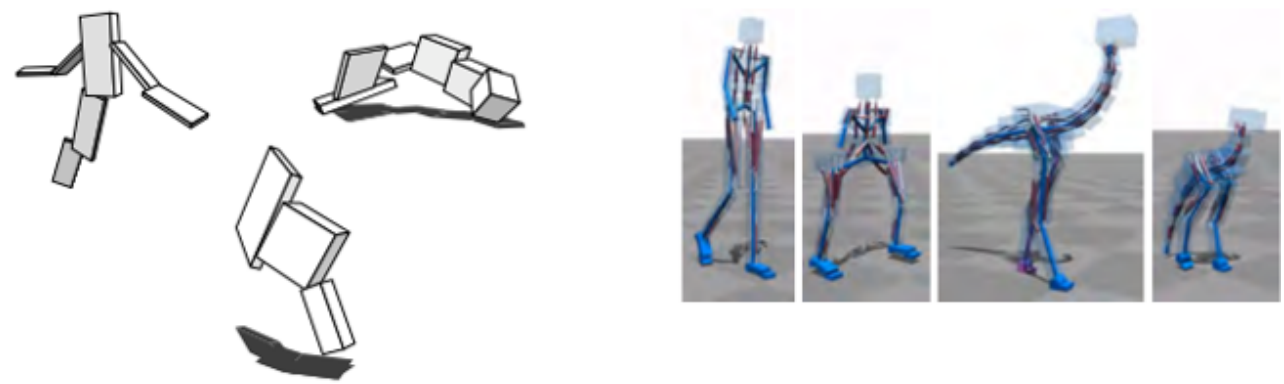

Figure 2.21: Examples of applying genetic algorithms for gait generation without prior knowledge. On the left, examples of "creatures" created by Sims' algorithm for different kinds of locomotion. From top left, clockwise: for swimming, walking, and jumping [93]. (right) Some of the different bipedal models used by Geijtenbeek to develop walking, jumping, trotting gaits using genetic algorithm without being given gait examples [29]

right side, causing the robot to turn away from the light. This is often referred as shy behavior. Whereas if the wiring is crossed - left sensor to right motor, and vice versa - the robot will turn towards the light source. This behavior is considered aggressive behavior. The intensity of the stimuli can also influence how fast the vehicle will move; effectively giving the illusion of levels of aggressiveness. Thus, Braitenberg showed that a simple hardwired connection between input and output can exhibit seemingly complex behaviors.

\subsection{Synthesis Using Machine Learning Methods}

Motion graphs uses clustering method to find relationships between motion data in a collection, which allows finding and creating natural transitions when switching between different motions [95]. Arikan and Forsyth extended motion graphs so user can interact with and impose constraints to the animated figure, and the system synthesize motions from the motion clips in a natural way [96]. Learning algorithms have been used to train models that can exhibit natural-looking motions. Gaussian process has also been used to model human motion dynamics and used for generation 


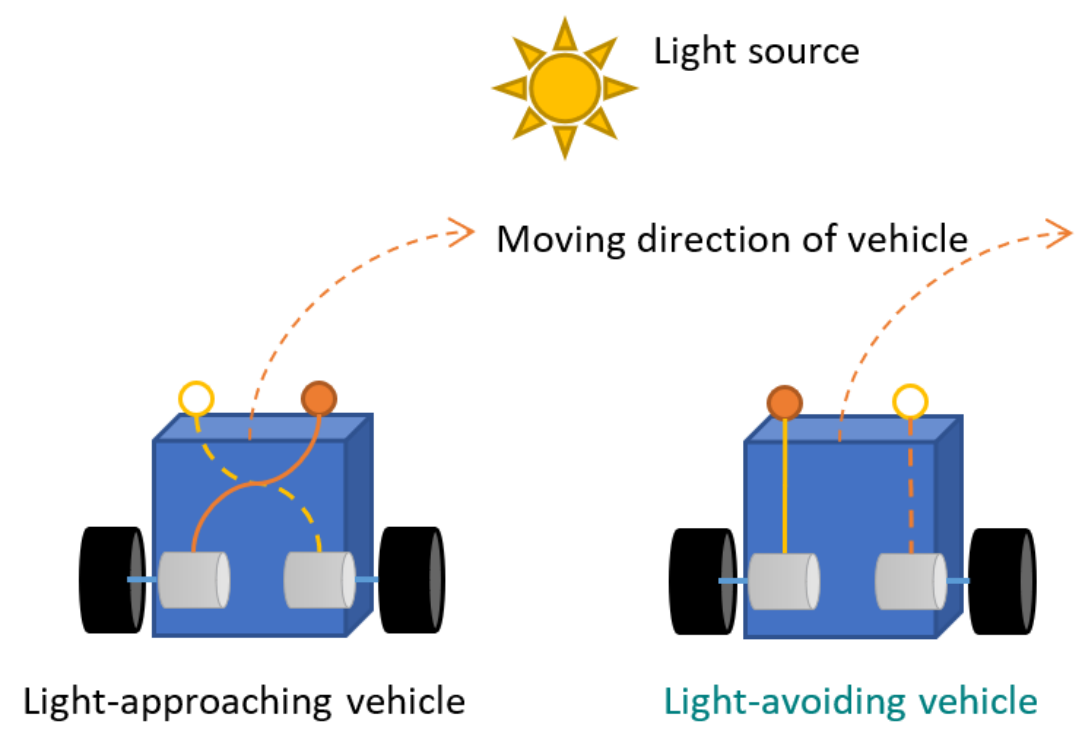

Figure 2.22: Braitenberg vehicles light-approaching behavior (left) and light-avoiding behavior (right) are created by the hard-wiring the light sensors (circles) to the motors (grey cylinders) to turn the wheels (black cylinders). Filled circles indicate high intensity at the sensor. Solid and dashed lines are just to differentiate how the left and right motors are connected to the sensors.

[97]. Deep reinforcement learning algorithm had been used to develop models that can produce various gaits that can adapt to its environment as shown in Figure 2.23 [98]. It is important to note that the gaits were learned by the algorithm and not by prior examples of bipedal walking.

Another interesting models of synthesis are generative models. Generative models are models of computation that can produce new data or samples that have some similarities with the data the model was trained on. In other words, generative models learn the distribution of its training data and thus, new samples can be obtained by sampling the learned distribution. The new samples are not merely a reproduction of the samples in the training data, but entirely new samples that do not exist in the training data. Unfortunately, generative models are difficult to evaluate objectively as there is no ground truth to check the generated samples against [99]. So far the best method to evaluate the generated results are by human evaluation. 


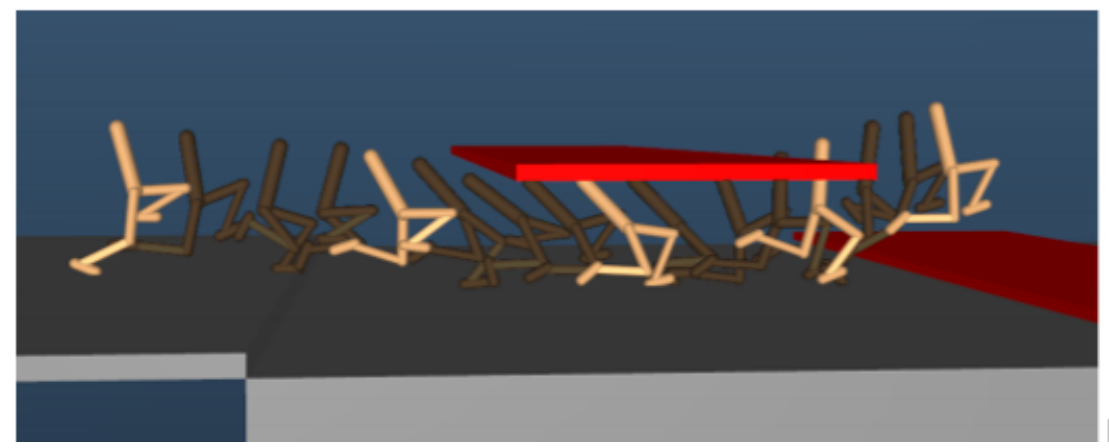

Figure 2.23: Walking gait on dynamic terrains and obstacles learned using deep reinforcement learning [98].

Deep recurrent networks with Long Short-term Memory (LSTM) modules can produce handwritten-like texts that are comparable to human handwriting [100], images like MNIST digits and house numbers [101], and robotic head motion for accompanying speech [102]. Figure 2.24 shows an example of handwritten-like texts generated by such networks. The first line in Figure 2.24 is the only true handwriting done by a person; the remainder were generated by Graves' method. The handwriting synthesis model was trained using the IaM Online Handwriting dataset [103] made of the handwriting examples of people which comprised of sequences of pen locations in $\mathrm{x}$ and $\mathrm{y}$ axes and binary value of whether the pen touches the surface or not. This dataset is similar to a motion capture dataset except that motion capture data consists of positions of the motion capture markers in 3D space. Other works used special architecture like variational autoencoder (VAE) [104] and generative adversarial networks (GAN) [99] as generative models that can be combined with LSTM networks to produce short, multi-instrument musical sequences [105] or convolutional layers to generate novel images [106]. Augello et. al used a VAE network to make a humanoid robot NAO dance in real-time with music [107]. 


$$
\begin{aligned}
& \text { from his travels it might have been } \\
& \text { from his travels it might have been } \\
& \text { from his travels it might have been } \\
& \text { from his travels itcmightmave boen } \\
& \text { fromer sel tirals it might have ben } \\
& \text { from hes fravels it might have been }
\end{aligned}
$$

Figure 2.24: Handwriting generation from an LSTM (recurrent) network of a given text "from his travels it might have been". The first line is from real handwriting data, the remaining lines were generated using recurrent neural network [100].

\subsection{Computational Creativity}

In 1950, Alan Turing asked the computing community to consider the question: "Can machines think?" and suggested that computers can simulate any human reasoning [108]. Turing's question lead to the birth of the field of artificial intelligence a few

years later. Advances in the field of AI have produced many systems that are very good at decision-making, classifications [109] [110], and new solutions [93] which seems to cover most of human reasoning. In 1998, Boden prompted the AI community to address the problem of creativity [111].

'Creativity' is the term used to describe the cognitive process wherein novel ideas emerge as the result of combining disparate concepts from different domains to form a new idea [111]. The term "conceptual blending" was first used in human cognitive sciences to formalize how humans think creatively. The activity of "telling jokes, creating idioms, using analogy, creating metaphors. or conveying wisdom using parables", are all based on this mental process of conceptual blending where "two or more 


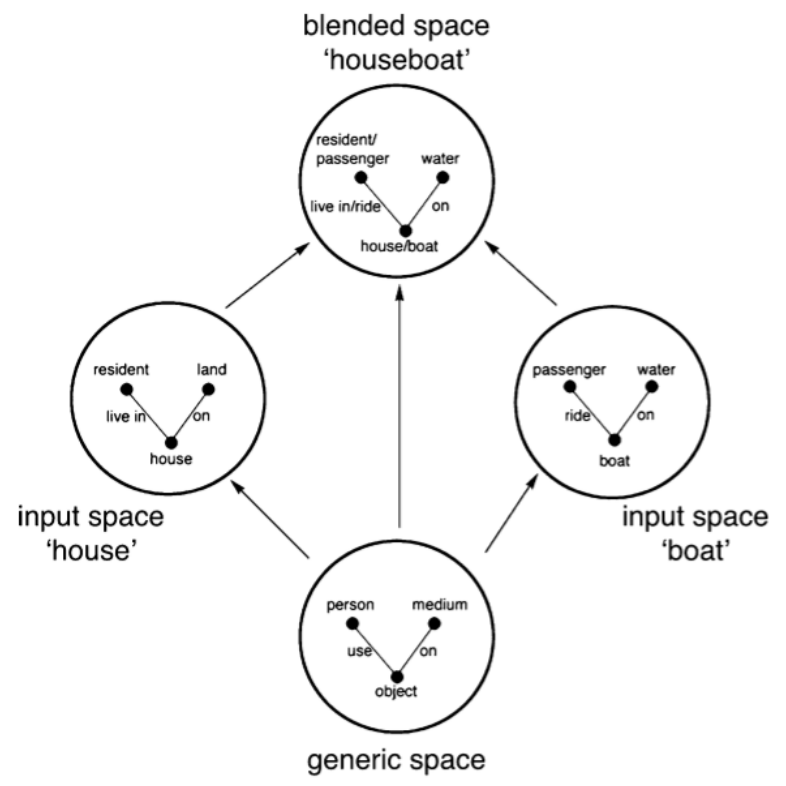

Figure 2.25: A Conceptual Blending Network for 'houseboat' [115]

ideas that are seemingly different are combined to produce novel ideas. We have myriad examples of this cognitive process being the basis of advances in every field, including the arts, humanities, mathematics, and sciences [112] [113]. And, until very recently, this process was thought of as being uniquely human.

Out of this question has emerged an exciting area of research in which computational models have been developed that combines concepts from different domains to create novel ideas [111] in order to approximate human "creativity" through the use of neural networks. One prominent cognition model used in computational creativity is Conceptual Blending Theory, also called the Conceptual Integration Network (CIN) [112] [114]. The Conceptual Integration Network is constructed of structures called "mental spaces" (Fig. 2.25).

These spaces are, as follows:

- Input spaces: two (or more) domains of ideas or concepts.

- Generic space: a generalization space between the elements in the two input 
spaces to find commonalities.

- Cross-space mapping/counterpart connections: a mapping of counterparts between elements of one input space and elements in other input spaces.

- Blend space: the space where the elements from the input spaces are combined to make new ideas

In these mental spaces, ideas are represented as network structures, similar to a semantic network [116] where properties in an idea are nodes and edges connecting these nodes describe the relationship between the properties. Fauconnier and Turner suggests that "creativity" takes place in the blend space by taking select properties from the input spaces through a process called selective projection to produce a new network structure which they call "emergent structure" and this new structure represents the new idea [112].

There are two main types of blending: single-domain and cross-domain blending. Single-domain blending is when the ideas in the input space come from the same type of domain, such as: paintings and photographs, group theories in mathematics, and styles of chord progressions in music. However, the most interesting type of blending and is the more compelling argument for creativity is cross-domain blending, where the inputs came from two different domains and the blending produce a new structure (idea) - usually as new ideas in the domain of one of the ideas in the input space. For example: blending cyclic groups theory from mathematics with the musical theory of chord progressions produces new musical chord progressions that are never encountered in practice but still sound pleasant and interesting [115]. Below are some examples of single-domain blending and cross-domain blending. 


\subsubsection{Single-domain Blending}

Examples of models that exhibit this form of artificial, computer-based 'creativity' using 'blending' include Google Deep Dream, which uses deep convolutional networks [117] to meld two images: Van Gogh's Starry Night painting, and a picture of a dog, to produce a final 'blended' output, (referred to as "style transfer") with the final product being a new picture, featuring the original image of the dog, but now altered by the use of brush strokes similar to the patterns from the Starry Night painting. Other examples include computational models that learn the audio signatures of one musical instrument and then apply those musical notes to the sound, thus creating novel sound output as if the music was played using a different instrument [118]. Eppe et. al implemented the Fauconnier and Turner's conceptual blending theory using category theory and a declarative language called Answer Set Programming (ASP) [115]. In one example, the authors demonstrated single-domain blendings using their system where the input domains are from the natural number theory and lists theory from mathematics to produce a new mathematical lemma for the lists theory . In a second example, the input domains are two types of chord progressions called cadences, where the blending produces a new cadence.

\subsubsection{Cross-Domain Blending}

Eppe et. al also demonstrated cross-domain blending using their system by blending concepts from cyclic groups theory and music theory to produce new musical chords and cadences [115]. Dr. Divago is a system that performs cross-domain blending [119] based on the Metaphor Theory of Veale and Keane [113]. Pereira and Cardoso used Dr. Divago to find and map overlap between properties of music, such as tension and theme, to properties of images, such as form and shape. In Dr. Divago, an input 
domain of "sonata" (music) is represented with attributes such as "rest" and "tension" where "tension" consists of the properties of, "theme" and "cadence", with each of these attributes having either numerical values or text descriptions. Dr. Divago also performs Cross-Domain linking, which is equivalent to cross-space mapping in CIN, to find the associations between properties of the input domains.

There are many similarities between the concepts of metaphor of Veale and Keane used in Dr. Divago [113] and the Conceptual Integration Network (CIN) [112]. For example, the "Generic Space" in CIN is similar to the "Domain Concept Maps" in Dr. Divago, in that the latter represents the general knowledge about the input domain and the relationships between the properties in that domain. In the input domain "house", a "person" is an object for the "resident" attribute of "house".

\subsubsection{Other models of cognition}

The Four Strategy Model by Tubb and Dixon [120] was based on the Creative Systems Framework by Wiggins [121]. In the Four Strategy Model, creative thinking is modeled as navigating between four spaces of thinking: convergent vs. divergent and implicit vs. explicit thinking. The Four Strategy Model was implemented by Tubb and Dixon as a collaboration tool where a human works with the system to guide the invention of new ideas in the context of music creation.

The Psi model was developed by Bartl and Dörner[122] and used by Augello et. al with the humanoid robot NAO to complete three tasks: collaborative painting, photo collage creation, and dancing in real-time listening to music [123]. In the Psi model, an agent's decision is modeled like a dynamical system that is driven by a set of motivations such as: energy, pain avoidance, certainty, external inputs, and finally short-term and long-term memory [123]. What makes the Psi model unique compared to the blending model, are: 1) the inclusion of user input and 2) the use 
of short and long-term memory to make decisions, which makes the Psi model more similar to classical dynamical systems than the other models.

\subsubsection{Intersections Between My Work and Computational Creativity: Similarities and Differences}

There are numerous opportunities for concepts and ideas, derived from models and frameworks used in computational creativity, to overlap in the research and development of technology used in social, entertainment, and assistive humanoid robots. If "Artificial Intelligence" is a larger general field of research acting as an umbrella category within which the field of Computational Creativity is a subset, it would be located closely adjacent that of humanoid robotics. The tie between the two areas of study exists particularly when the focus of research is exploring specific themes in the Arts and Humanities. Therefore, It is incumbent upon me to discuss briefly where my work may inform or be informed by the field of computational creativity and whether my research could be built upon for use in computational creativity modeling and research in the future.

Some of the methods used in my approach share a degree of conceptual similarity with certain models and theories described in the literature on Computational Creativity. In Chapter Four, for example, I used the analogy that robot poses or behaviors are equivalent to symbols in an alphabet, and composing robot behaviors/motions is the same as composing words/strings using the same set of operators used in regular expressions. In Chapter Five, when I use the sound frequency (aka "note") and the timing of the music (aka "rhythm") progression between intervals (aka "melody") to control the motions of the robot). Expanding on my work with the

use of systems like Dr. Divago could explore the creative potential of novel mappings between the algebra of regular expressions and motion composition, and mappings of 
the structure and elements of music (e.g. cadence, chord progressions, dynamics) to motion properties (e.g. timing, range of motion, trajectories) for executing gestures.

But despite this incidental conceptual overlap, the significant difference is that my work in its present stage of development is intended to be operated by human consciousness and does not explore development of cognitive functions. The primary goal of my research in this dissertation was to find ways to make robotic movement appear more 'natural' and 'expressive' to the human eye, while also improving upon the current methods for creating motion data output, advancing it from a tedious and time-consuming process in which motion parameters are painstakingly entered manually for each movement of the robot, to a more efficient method that obviates the need for manual entry of parameters input detail replacing it with a more efficient form of data input, based upon using heuristics and rules instead of manual instructions for each movement. This method not only not dramatically reduces the labor and time ( and presumably cost ) previously required to create motion output data in humanoid robots, it also has the improved outcome of increased nuance, variance, flexibility, and range in the motion output resulting in gestures and movement of the robot being described as looking more 'natural' and 'human'.

While my work was not produced with the goal of developing cognitive function, in many ways, this would be a natural expansion. Due to incidental overlap between my approach and frameworks used in the field of Computational Creativity, my work would be easily adaptable to be used as a jumping off point to facilitate further study and development in the field of Computational Creativity.

Moreover, in many papers in the literature on computational creativity, examples are overwhelmingly focused on semantic research, and surprisingly few papers in the field use robotics as a platform for research in this area [123] [124]. Metaphor and analogy would be a very natural framework lending itself to research in the field of 
social and entertainment robotics.

\subsection{Concluding Remarks}

I am interested in technologies that would enable real-time, dynamic generation of gestures for humanoid robots so these robots can show social aptitude. We discussed how expressive behaviors are important in the area of social robotics. Concepts from animation and dance discussed to provide some common vocabulary to describe expressive behaviors. We also discussed works from the area of computer animation that were also interested in creating agents for intelligent social interaction with people and show some overlap with the works in robotics. A review of humanoid robot animation process and tools were also presented. Finally, we also discuss machine learning techniques that have been used to produce novel samples like images and music, which we believe can be leveraged to also produce robot motions that are expressive. 


\section{CHAPTER 3 \\ HARDWARE AND SOFTWARE PLATFORMS}

In this chapter we introduce the software and hardware platforms used in this dissertation as a high-level overview: the Robot Operating System (ROS) middleware, the Jeeves robot, HROS-1 robot, KHR-1 robot, and Lynxmotion robot. In the interest of keeping the size of this document manageable, in this chapter we only provide cursory information about the hardware and software systems used in this work. More detailed information will be provided as references to separate documents.

\subsection{Robot Operating System (ROS)}

The Robot Operating System (ROS) is a middleware framework for developing robotics applications. ROS was originally developed by WillowGarage that perhaps is more well known as the company that produced the PR2 robots [125]. ROS has been used both by robotics hobbyists as well as the Robonaut humanoid robot built by NASA [126].

Although the name is "operating system", ROS is not an operating system in the truest sense. ROS provides a framework to build complex robotics systems by compartmentalizing functionalities as separate packages. A ROS package may consist of one or more ROS nodes and services. Nodes are a ROS concept of a long-running process that performs a certain task. Nodes run continuously as a process until the user sends a shutdown signal or when the program runs into a catastrophic error. There are two types of communication between nodes in ROS: by using publishersubscriber model and by services. Nodes in the publisher-subscriber model, one node 


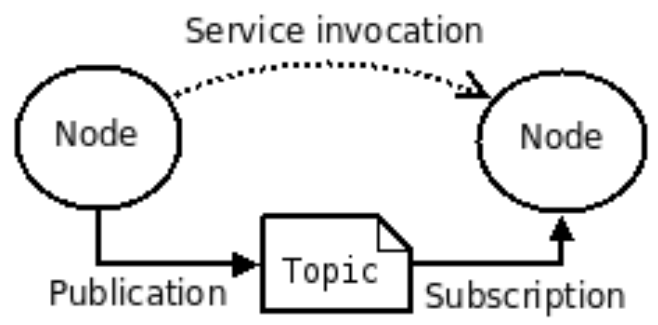

Figure 3.1: Two types of interaction between two nodes in ROS: by service and by publisher-subscriber model via Topics (image from: https://wiki.ros.org/ROS/Concepts).

usually continuously publishes messages and another node subscribes (or listens) to messages to accomplish a certain task from the former node. A node may perform a single task such as interfacing with a web camera directly and publish the video frame images into an data object communication channel called topic (in ROS terminology). Each topic belongs to a specific type of message object. A message in ROS is a data structure object for a particular type of information. The most basic types of ROS messages are equivalent to common data types in programming languages such as string, integer, and floating-point numbers. But messages can be as complex as a custom object which contains information such as timestamps, metadata, nested structures, and images or pixel values. Service is another type of program in ROS that runs in the background. Nodes and services are very similar; in fact, a service program is written like a node, but the way to utilize it is different. Unlike the publish-subscribe model a node which process runs continuously, services are typically reserved used for performing a single computation by demand by making a service call and the service will immediately return a value. Some examples where a service is used instead of a node are for tasks like: performing a computation like addition for a set of input arguments and return a single value, to perform a table lookup given a query and return the corresponding entry, and other one-shot tasks like changing the speed setting on a servo. 
As an example, a mobile robot ROS system may consist of a ROS navigation package for finding the best route along with obstacle avoidance, another ROS package for interfacing with the robot's sensors like LIDAR (laser scanner), and another package for accepting user keyboard input for selecting destinations. Each ROS package runs as a separate process in the host operating system (in this case, Linux) and may have one or more nodes and services. This enables the ROS robotic system to keep running should any of the packages fail, except for a complete system failure. This framework also makes developing the robotics system more flexible, allowing building more functionalities for the robotics system much easier than a monolithic program. ROS also has a very active community that have built many useful packages, which makes it easier to quickly build a robotic system. For these reasons, all the software for the robots used in this dissertation were written using ROS, leveraging several third-party packages, and our own packages written in Python language or $\mathrm{C}++$.

\subsection{The Robot Jeeves}

Jeeves is a 5 foot 9 inches tall mobile humanoid robot (Figure 3.2, 3.3). Jeeves was built as a guide robot for the Maseeh College of Engineering and Computer Science (MCECS) with the original name "PSU MCECSBot" and was designed with a personality of a butler. Thus, we renamed him to "Mr. Jeeves." More details are given in a separate document called MCECSBot Manual v2.0 which can be obtained at the GitHub repository (https://github.com/msunardi/jeevesrobot).

\subsubsection{Contributors}

My contribution with respect to the Jeeves robot:

- Built the head and facial animation system 


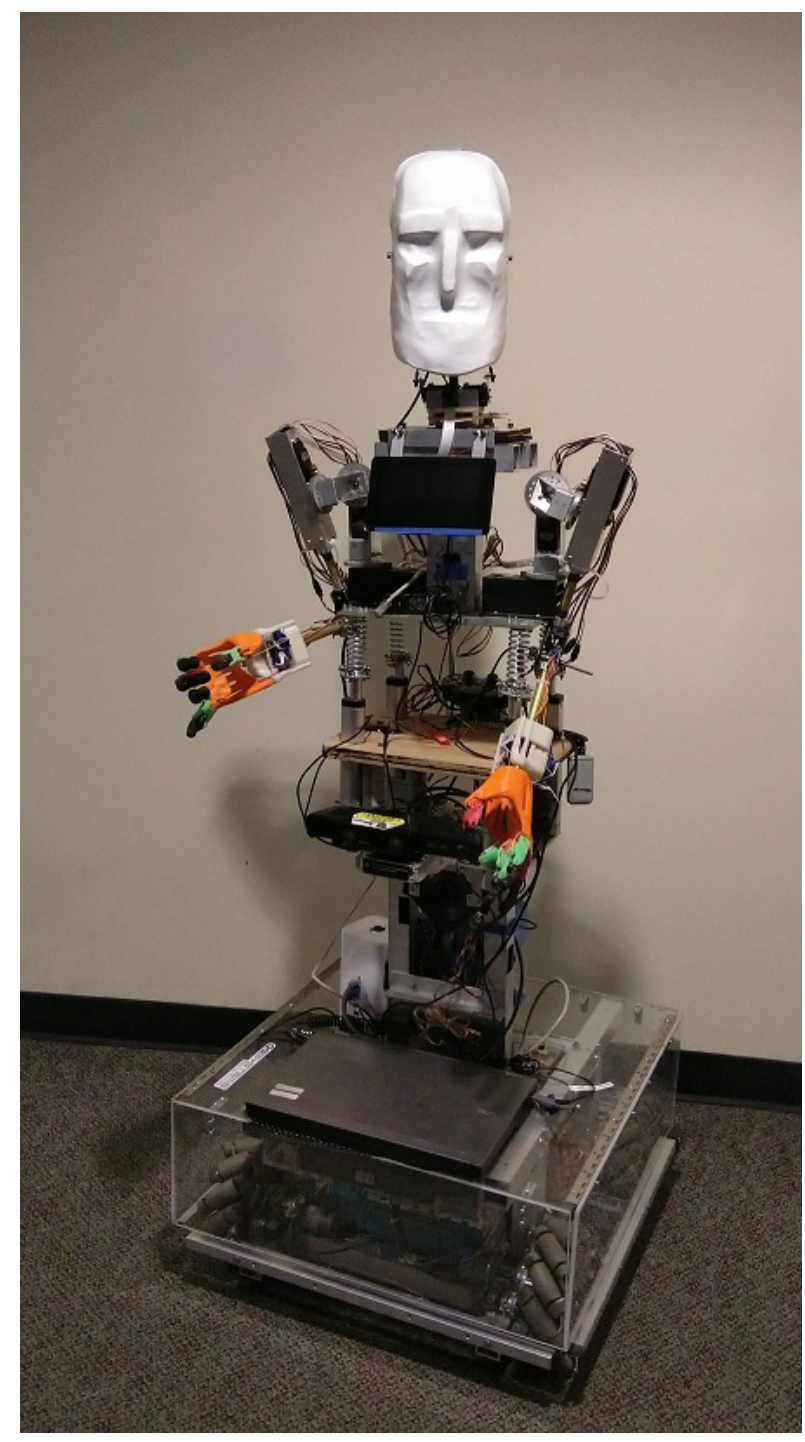

Figure 3.2: The Mr. Jeeves robot.
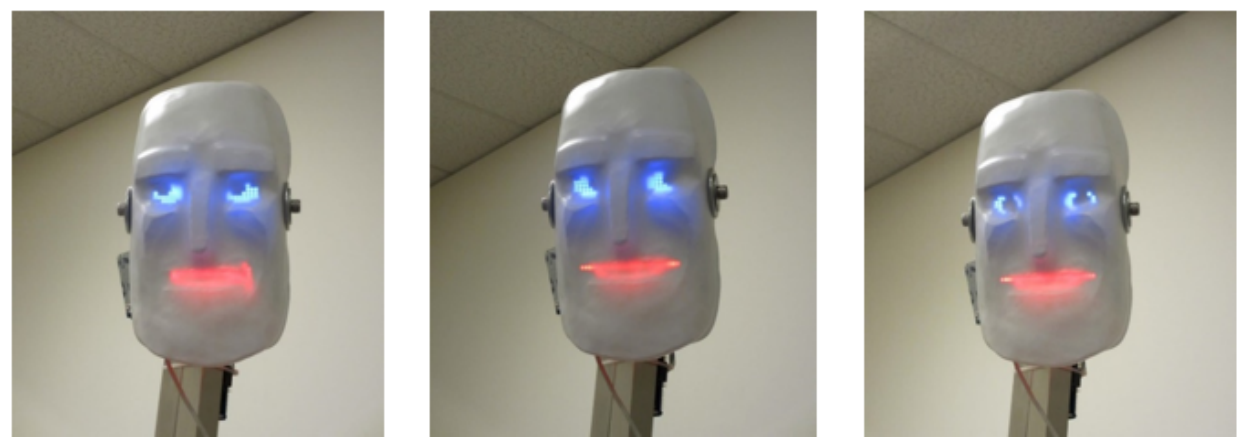

Figure 3.3: Various facial expressions of Jeeves with our modifications from the original code by Burgess 
- Improved the neck mechanism

- Developed the web-based user interface

- Developed the speech system and integration with Mycroft speech processing

- Integrated the user interface with navigation, speech system, and head and arm gesticulations

The following people are contributors to the development of the Jeeves robot project:

- Omar Mohsin (Electrical and Computer Engineering (ECE)):

- Built the base frame

- Designed the power system

- Implemented the first version of working mobile base movements

- Designed and implemented distance sensing using ultrasound sensors

- Richard Armstrong (Mathematics Dept.):

- Lead the transition from using Mobile Robot Programming Toolkit (MRPT) framework to ROS.

- Developed and implemented the navigation system

- Designed and implemented the main power distribution system

- Managed the teams of students working on Jeeves

- Dakota Ward (ECE): Web-based user interface

- Brendan Ball (ECE): Built, installed, and tested 3D printed hands 
- Josh Sackos:

- Developed the speech recognition and dialog system using CMU Sphinx and AIML

- Developed the QR code reader module

- Jeff Fischer (ECE):

- Designed and implemented the improved power switching and battery recharging system

- Fabrication of shoulder mechanism redesign

- Yoseph Ukbazghi (Mechanical Engineering (ME)): Built and installed the cover for the mobile base

- Julien-Granich-Young (ME): Fabrication of fiberglass torso cover

- Tiago Denczuk (ME): Fabrication and installation of arms and upper torso

- Evan Wellington (3D art student group):

- Fabrication of aluminum face mask

- Fabrication of fiberglass torso cover

- Mario Bitzer (ECE): Waist control system

- Paul Long (ECE): Prototype of facial animation system

- Rami Alshafi (ECE):

- Prototype of facial animation system

- Prototype of user interface 
- Mike Lowe (ECE): Prototype of navigation system using Mobile Robot Programming Toolkit (MRPT)

- Cody Hanks (ECE): Prototype of backend information system and networking

- Peter Depeche (ECE): Obstacle avoidance and people following using Kinect

There are many others that have made other contributions to the Jeeves project. We apologize for not being able to list all of them here.

\subsubsection{Mobile Base}

Jeeves' mobile base houses all of the hardware used for navigation, his main power supply, and the main PC where the primary ROSCore server is running. Jeeves' mobile base is equipped with four mecanum wheels where each wheel is independently actuated by a DC motor, allowing it great mobility for moving sideways on top of the usual forward/back and rotational movements. Jeeves is equipped with a SICK 200 laser scanner for its navigation and obstacle avoidance.

\subsubsection{Torso}

The torso is actuated with four Firgelli linear actuators allowing side-to-side tilting, bowing (forward), or arching (back). The linear actuators are controlled using two RoboClaw motor controllers and an Arduino MEGA that is directly connected to the main PC. A ROS node running on the main PC can take input from the user (e.g. from gamepad controller, depth image) and apply the input values to some function which outputs control the movements of the linear actuators by sending the commands to the Arduino MEGA.

Jeeves has two humanoid arms each with 3 degrees of freedom at the shoulder

for pitch, roll, and yaw rotations. At the end of each arm, there is a 3D-printed 


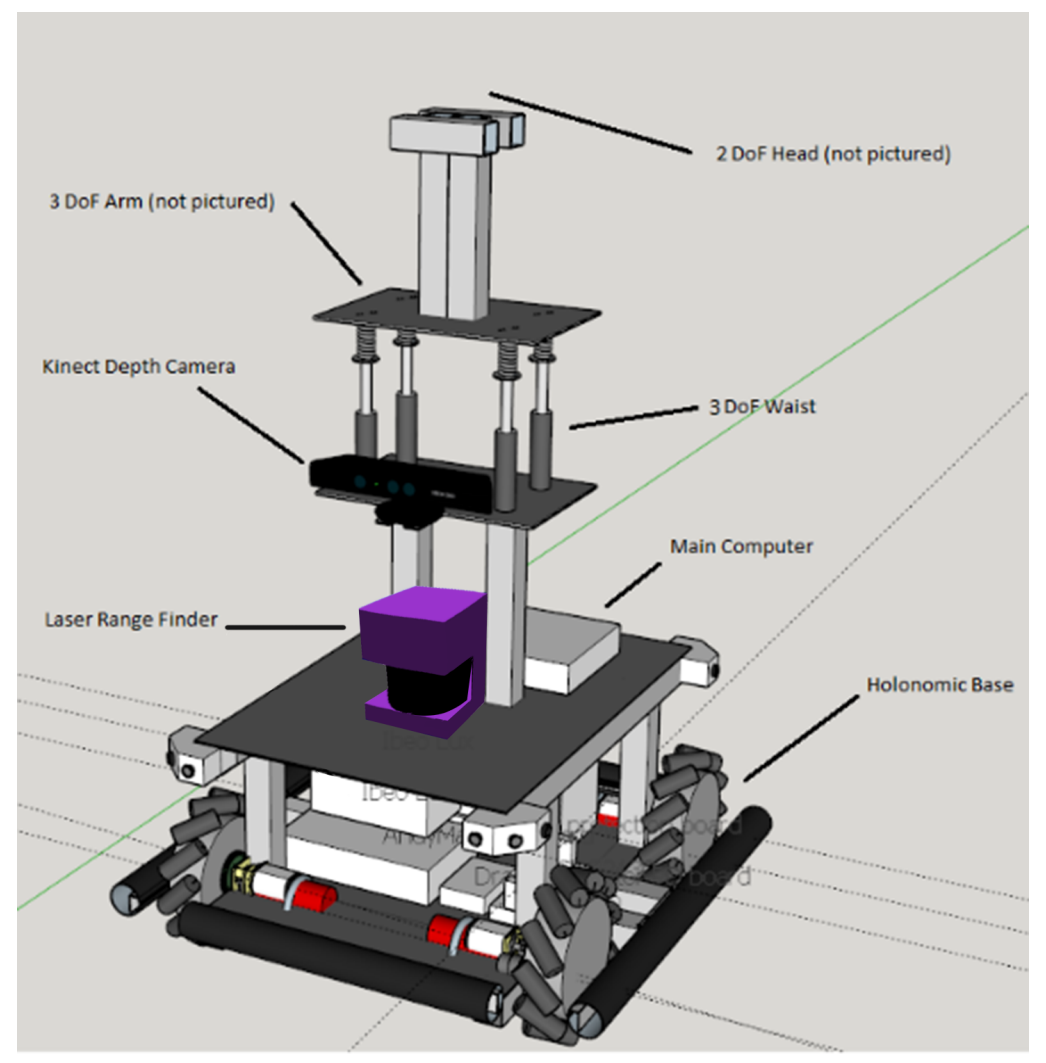

Figure 3.4: 3D model of Jeeves' mobile base core components 
five-fingered hand which each finger is actuated using one micro servo. The hands were built and installed by Brendan Ball. Currently, the elbows are affixed with a flexed/bent position, and there is no actuation at the elbows. The right elbow is bent at about 80 degrees while the left elbow is bent at about 100 degrees. This difference of angles is to avoid the symmetrical look to help the robot appear more organic and less mechanical.

\subsubsection{Neck and Head}

Jeeves' neck has 3 degrees of freedom allowing expressive head gestures such as tilting sideways, looking up and down, and turning to the side (Figure 3.5 left). Finally, Jeeves' face was created using acrylic plastic that was molded by us using the vacuumforming process. A positive mold (template) of the face was first created from an aluminum cast. Then a sheet of white acrylic plastic was heated until it begins to soften and pliable. At this point, the sheet is quickly placed and pressed down over the positive mold that was placed over a vacuum form platform. The vacuum form platform is a bed with vents on the top surface which is connected to a vacuum machine. Once the heated plastic sheet is placed over the mold on the platform, the vacuum machine is activated, and the vacuum suction pulls the pliable plastic to tightly conform to the contours of the mold.

Jeeves face is animated using a pair of 8x8 LED matrices for the eyes, and three 8x8 LED matrices for the mouth which are connected together to display a wide variety of mouth shapes. By coincidence, we discovered that the LED lights can shine through from behind the plastic mask. This creates a 'softer' and more integrated look for the eyes and mouth compared to cutting the mask and letting the LED matrices be visible in its entirety.

Jeeves has a camera placed on the neck which is used for face detection and 
recognition (Figure 3.5 right). The face detection is used to control the gaze system which moves the 'pupils' of the eyes guided by the relative position of the detected face in Jeeves' field of view. Using this approach, Jeeves has a very expressive and natural way of doing 'person following' by a combination of gaze and head movements, as opposed to the naïve method where only the head is turning/following and the eyes are not directed at the person. We found that the latter method creates an expressionless (or "deadpan") look for the robot at which most people say the robot becomes creepy.

The facial animation loop is running on an Arduino MEGA board (Figure 3.6) and was based on the sample code provided by Philip Burgess from AdaFruit, the manufacturer of the LED matrix units [127]. We expanded from this sample code by adding two different eye shapes: slightly closed, and mostly closed. We also enlarged the size of the pupils from $2 \times 2$ pixels to $3 \times 3$ pixels so the eyes looked more 'friendly' whereas the smaller pupil size creates a more cartoonish/crazy look. Additionally, we also created an interface to the animation loop to manually control the eyes and mouth shapes, and positioning of the pupils of the eyes to support the gaze-based people following feature.

The head unit is controlled separately using a Raspberry Pi module which runs as a ROS node connected to the main node running on the laptop located at the base (Figure 3.6. The separation is done due to the position of the camera used for face detection. The camera is placed at the base of the neck for two reasons:

1. Face level: the camera is placed at a height that is aligned with the height of the average person for optimal face detection.

2. Stability: ideally the camera would be placed near Jeeves' eyes, but expressive head movements would cause the camera to shake too much interfering with the face detection. For this reason, the camera is placed at the panning base of 
the neck so pan-tracking can still be performed while the camera is at a more stable mount.

Because of the camera placement, connecting the camera with the main PC located at the base becomes impractical. Therefore, the camera is connected to a Raspberry Pi mounted at the "chest" of Jeeves. The Raspberry Pi is running a set of ROS nodes that perform:

- Face detection

- Streaming of video frames to a web server

- Control of eye/pupil and neck movements for face tracking

The Raspberry Pi is connected via ethernet cable to a router located at the mobile base to connect it to the same network as the main PC. By connecting to the same network, the ROS node running on the Raspberry Pi can communicate directly with the main ROS core on the main PC by providing the correct host name of the main PC.

\subsubsection{Speech and Dialogue System}

For speech recognition, Jeeves uses Mycroft.ai (https://mycroft.ai) Application Programming Interface (API), and speech synthesis is partly done by Mycroft.ai and on-board Festival text-to-speech software. The speech system enables Jeeves to answer simple questions such as telling time, and other facts that can be retrieved by Mycroft's interface with the Wikipedia web API. Additionally, Mycroft comes with a set of skills such as to do simple calculations of numbers. Users can ask simple mathematical operations on arbitrary numbers such as "what is two plus two?" or "what is the square root of eleven thousand seven hundred and thirty-five?". We use 


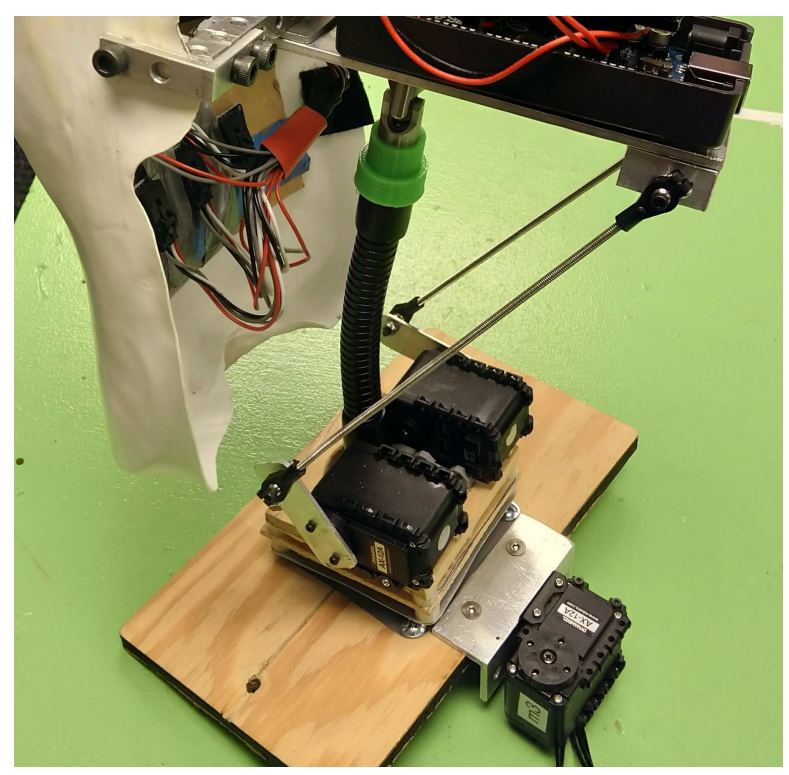

(a) Close-up of the neck mechanism

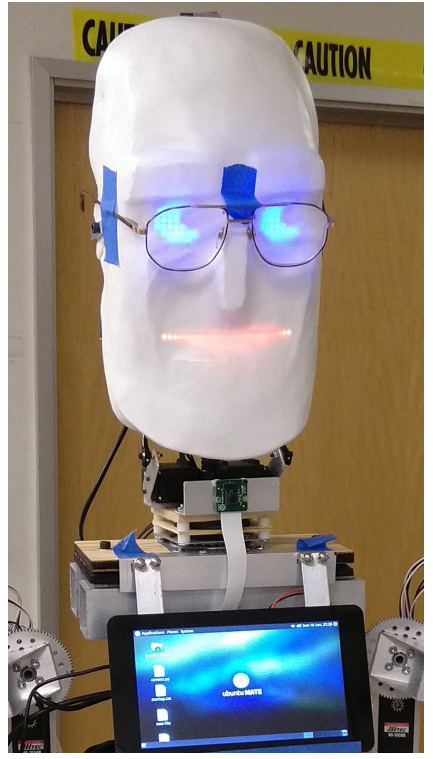

(b) Jeeves head mounted on the body

Figure 3.5: Jeeves' head and neck mechanism.

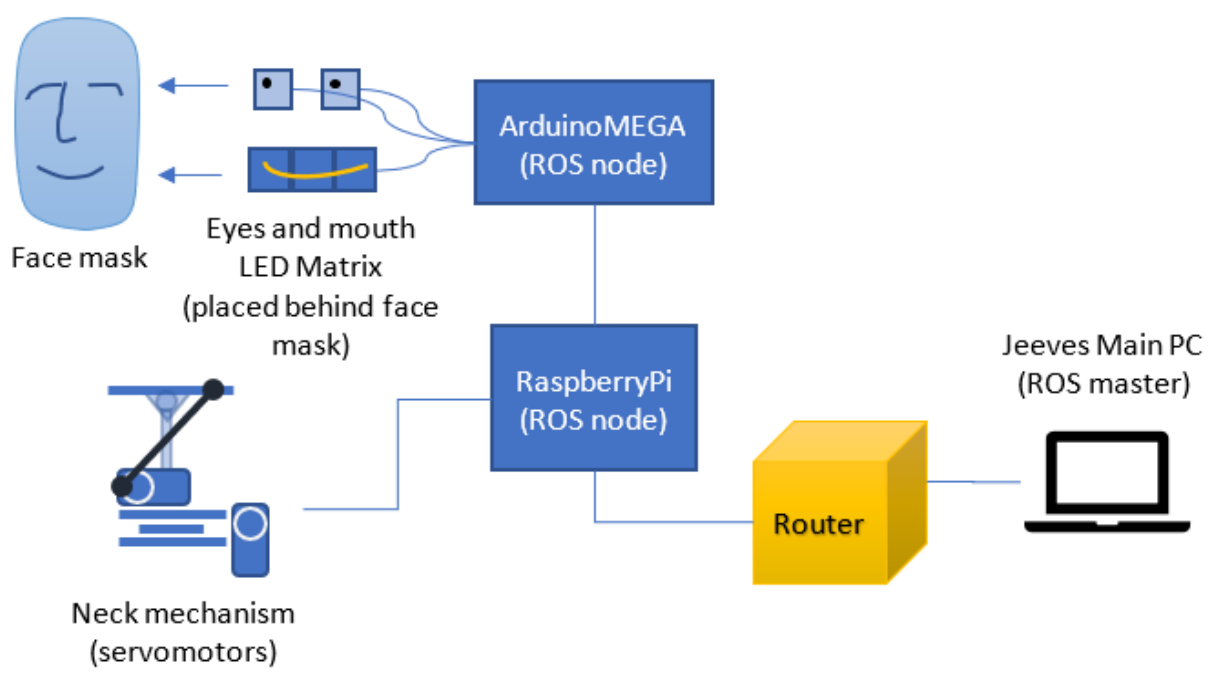

Figure 3.6: Jeeves' head/neck and face system. The animation of the eyes and mouth is controlled by an Arduino program from AdaFruit [127] that has been modified to be a ROS node, and the neck servo movements are controlled by a ROS node in the RaspberryPi. The head/neck and face system is connected to the Jeeves Main PC by connecting both in the same network via ethernet to a router. 


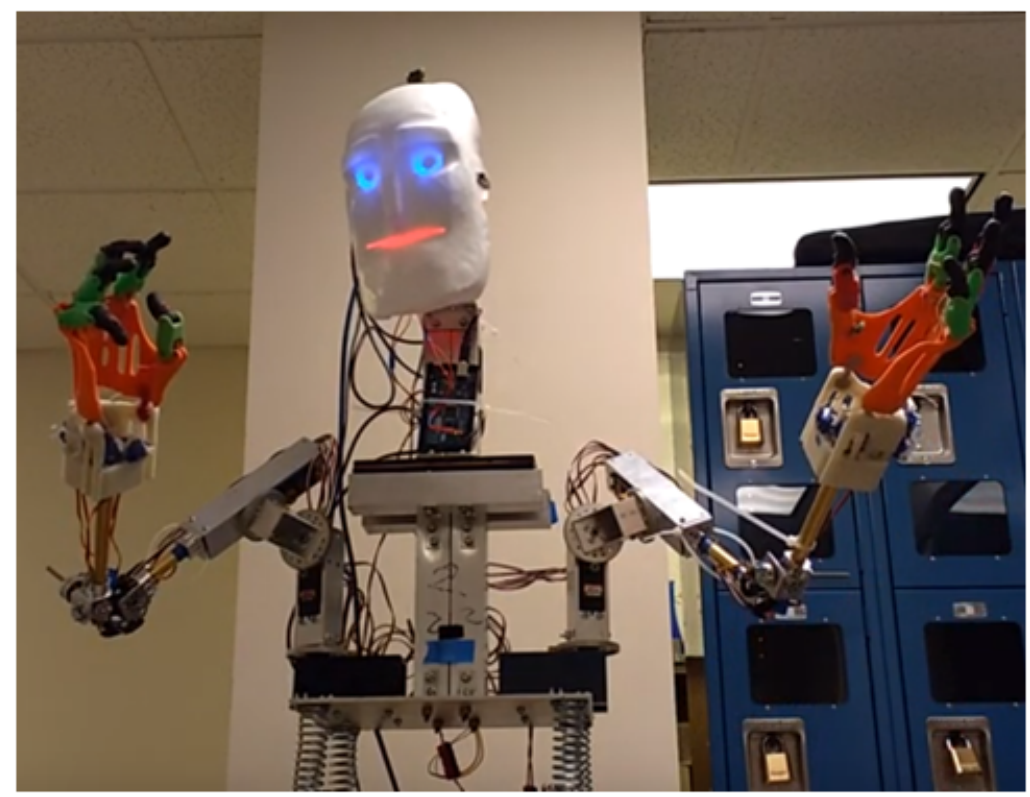

Figure 3.7: Jeeves expressing the number 8 using hands ${ }^{1}$.

the answer returned by Mycroft so that Jeeves will say the answer by speech, and also use his hands to indicate the numbers (Figure 3.7).

\subsubsection{User Interface}

We built Jeeves user interface (UI) from the ground-up using Django web framework written in the Python language and the UIKit JavaScript framework for the UI components (buttons, grids, etc.). An example of the Jeeves' UI is shown in Figure 3.8 .

\subsubsection{Source Code}

The source codes for Jeeves can be found on the following GitHub repositories:

- Navigation, speech, waist control, gamepad control:

\footnotetext{
${ }^{1}$ Video: https://youtu.be/zdUzF2lUT3o. Jeeves is shown with the old neck mechanism in this picture.

${ }^{2}$ Video: https://youtu.be/D_AnmYoSXjA
} 


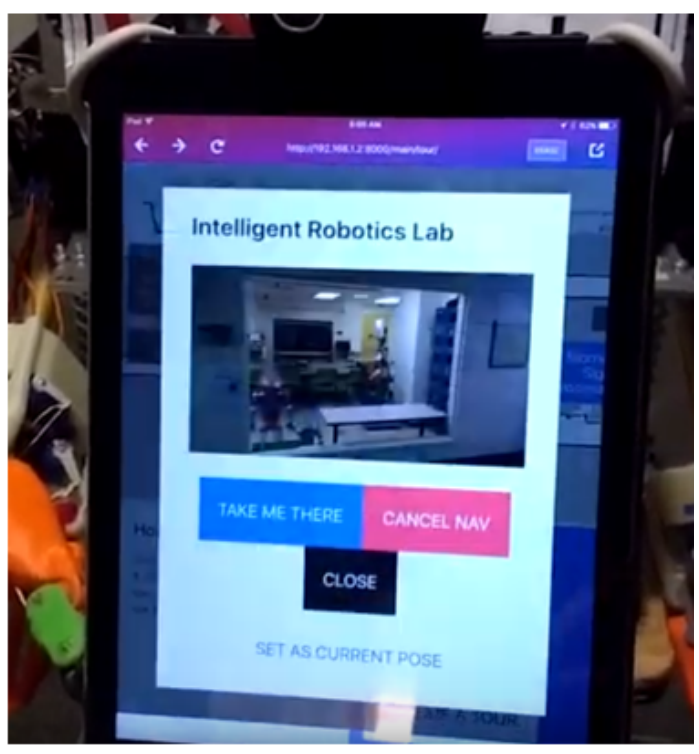

Figure 3.8: Jeeves' navigation user interface running on an Apple iPad ${ }^{2}$.

https://github.com/msunardi/jeevesrobot

- Gaze control: https://github.com/msunardi/jeeveseyecontrol

- Neck control: https://github.com/msunardi/jeeves_neck

- Face detection: https://github.com/msunardi/jeeves_facedetect

- Graphical user interface: https://github.com/msunardi/jeeves_ui

- Tour dialog: https://github.com/msunardi/jeeves_arbiter

\subsection{HROS-1}

The HROS-1 is a small humanoid robot about 12 inches in height sold by Trossenrobotics $^{3}$ (Figure 3.9). The HROS-1 robot has 20 DOFs: two DOFs for the head/neck, three DOFs for each arm, and 5 DOFs for each leg. All the actuators on the HROS-1 use the Dynamixel AX-12A servos [128]. The AX-12A servo is a reduced-gear DC

\footnotetext{
${ }^{3}$ https://www.trossenrobotics.com/HR-OS1. Currently discontinued
} 


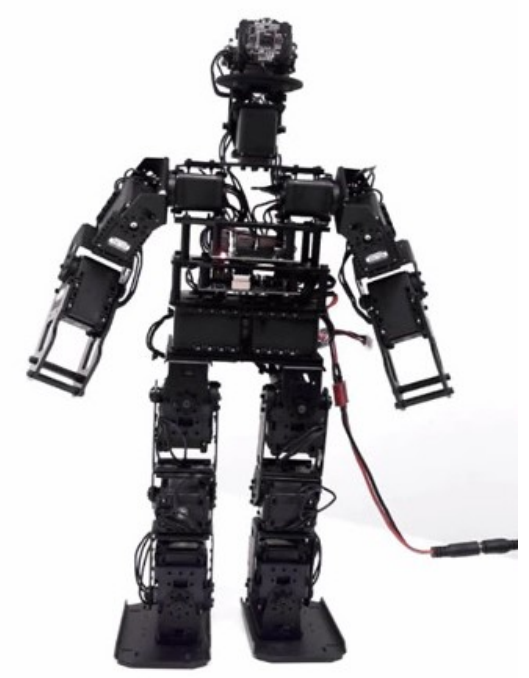

Figure 3.9: HROS-1 robot

motor with half duplex asynchronous serial bus and an integrated controller. Multiple servos can be daisy-chained and be controlled individually by referring to the correct servo IDs stored in the integrated controller. The user can change various settings of the servo by modifying parameter values stored in the ROM of the integrated controller, such as: maximum torque, slope (for acceleration and deceleration), motor speed, servo ID, and switching between continuous rotation or 300 degrees rotation. Real-time values can also be read from the servo such as: current load, temperature, error codes, current position target/command, current servo position, and error/difference between target position and current position. The servos can be controlled via USB from a computer system such as a PC, Raspberry Pi, or Intel Edison.

The HROS-1 package comes with a Raspberry Pi and an Intel Edison board which are pre-installed with Linux and the HROS-1 framework software. The HROS1 framework is an open-source software ${ }^{4}$ that provides a set of sample animations

\footnotetext{
${ }^{4}$ https://github.com/Interbotix/HROS1-Framework
} 
and programs to let users quickly get started working on the robot. The HROS-1 framework comes with a program that allows controlling the robot using a Playstation controller to perform simple actions such as sitting, standing, a few simple gestures, and directional walking. Additionally, the framework provides a command-line tool called Robot Motion Editor (RME) to create new gestures.

In this dissertation, we do not use the HROS-1 framework. Instead, we use ROS to write all the control software for the HROS-1 robot using the ROS package arbotix_ros (http://wiki.ros.org/arbotix).

\subsection{KHR-1}

The KHR-1 is another small humanoid robot about $34 \mathrm{~cm}$ tall (about the same size as the HROS-1 robot) made by the Japanese company Kondo Kagaku [51] (Figure 3.10). The KHR-1 robot has 17 DOFs: 1 DOF for the neck, 3 DOFs for each arm, and 5 DOFs for each leg. The robot's DOF configuration allows us to program simple gestures and some dancing motion using the Heart2Heart software that was developed by Kondo Kagaku for the KHR-1 robot. I used the KHR-1 robot in past works [129] and in Chapter 5 when we discuss our Music to Motion approach.

At the time of this writing, the KHR-1 robot had been discontinued by Kondo Kagaku making references about the robot, the HeartToHeart software, and spare parts for the robot difficult to find ${ }^{5}$. However, the servos used on the KHR-1 robot are the standard 3-pin connector RC servos so it would be trivial to use off-theshelf parts such as the Pololu Maestro servo controller boards made by Pololu [57]. The control software for the KHR-1 robot in the Music to Motion experiments was developed by us using the Python language (without ROS).

\footnotetext{
${ }^{5}$ The most current version of HeartToHeart software does not work with the KHR-1 robot
} 


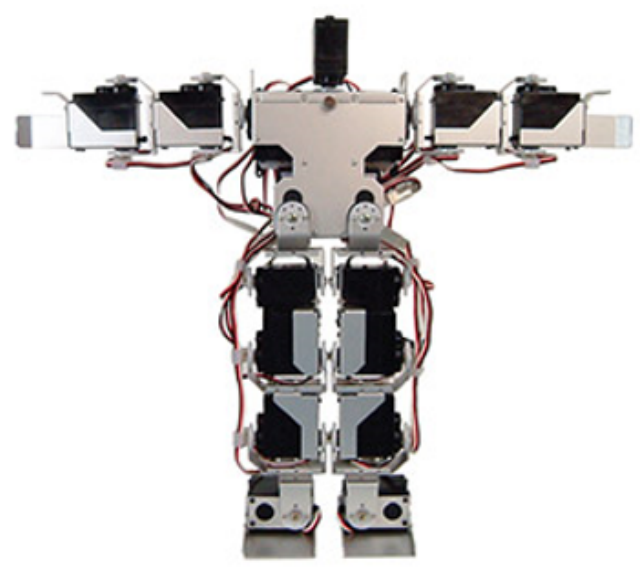

Figure 3.10: KHR-1 robot.

\subsection{Lynxmotion Arm}

A second robot we used in our Music to Motion method (Chapter 5) [130] is an arm robot based on the Lynxmotion AL5A arm robot [131] (Figure 3.11). We replaced the gripper of the AL5A robot with a Sharp IR sensor to make the robot appear to have a face with a pair of eyes due to the shape of the sensor. By replacing the gripper with the IR sensor, the robot now appears as a 'neck/head' robot instead of an 'arm' robot. The sensor itself was not activated. The robot has a total of 4 DOFs: 1 DOF of yaw rotation at the base, 1 DOF for pitch from the base, 1 DOF at the "elbow" also for another pitch movement, and 1 DOF for roll at the end of the arm. The two pitch DOFs enables a 'chicken neck' motion where the neck can extend forward, raised up, or retracted backwards. The robot is controlled using an SSC-32 servo controller board and connected to a PC via USB. Figure 3.12 shows the DOFs of the robot. The control software for the Lynxmotion robot in the Music to Motion experiments was developed by us using the Python language (without ROS). 


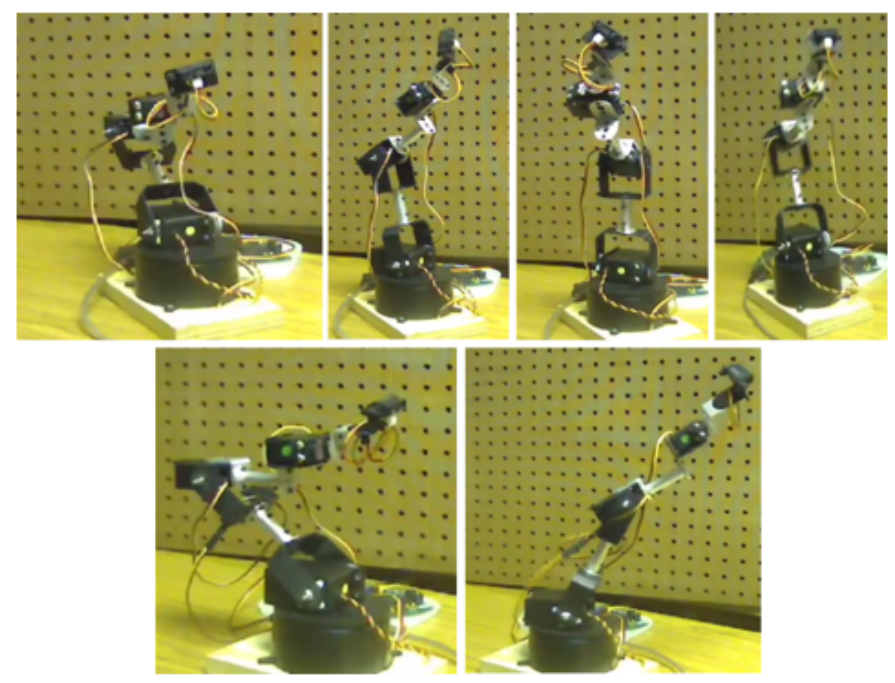

Figure 3.11: Sample poses of the modified Lynxmotion AL5A Arm generated by music data [130].

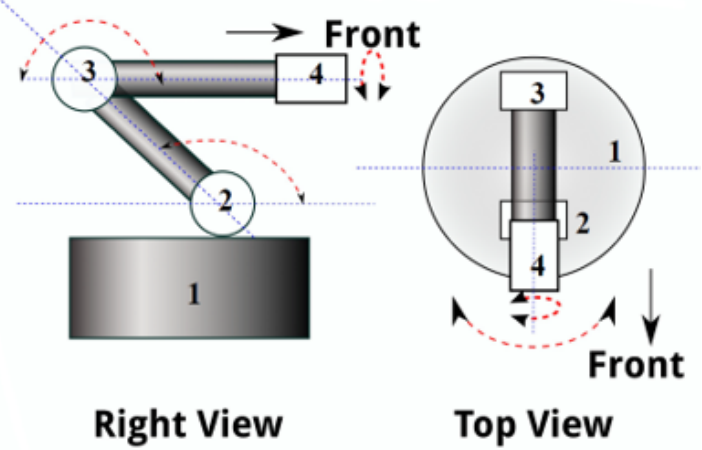

Figure 3.12: DOFs of the Lynxmotion robot. DOF 1 is the yaw rotation, DOF 2 and 3 perform pitch rotation and together creates the 'chicken neck' motion, DOF 4 is used to perform a roll to tilt the 'face' sideways. 


\subsection{Representation of Motion Data}

In this section we discuss the format of motion data representation used for the gesture data for the Jeeves, HROS-1, and KHR-1 robots. In practice, the motion capture and robot motion data used in this dissertation are saved in the text-based, comma-separated value (.csv) file format. Motion data for gesture behaviors are commonly represented as a matrix $M$ with $N \times T$ dimensional data where $N$ is the number of degrees of freedom (DOFs) and $\mathrm{T}$ is the number of timesteps. This data represents the robot's pose at each time step $t \in T$ for each DOF $n \in N$. The values in these data are typically joint angle values but can also be locations in space. Let's annotate this value as $\phi_{n, t}$ where $\phi$ can be joint angle or position values, $n$ as the index of DOF, $t$ as index for timestep, and the complete gesture data as $M$.

$$
M=\left[\begin{array}{ccc}
\phi_{0,0} & \cdots & \phi_{0, t-1} \\
\vdots & \ddots & \vdots \\
\phi_{n-1,0} & \cdots & \phi_{n-1, t-1}
\end{array}\right]
$$

We observe that the gesture data $\mathrm{M}$ (eq. 3.1) can be viewed in two ways. The first is as a sequence of poses or frames which we refer as F-mode (for 'frame'), and the second is as a stack of motion signals for all DOFs which we refer as S-mode (for 'signal'). In F-mode, the gesture data $M$ is created and edited per frame; that is, by creating the robot's pose one timestep at a time. The robot's pose at each frame is also called the robot's joint configuration, and we can represent each frame as the vector $f_{t}$ of size $N$ for the number of DOFs and the subscript $t$ indicates the timestep index. So in F-mode, $M=\left[f_{0}, f_{1}, \cdots, f_{t-1}\right]$ and $f_{t}=\left[\phi_{0}, \cdots, \phi_{n-1}\right]^{T}$ where $\phi$ can be joint angle or position values and $\mathrm{n}$ is the index of the DOFs. In S-mode, the gesture data $M$ is represented as a set of sequence data $s_{n}$ for the $n$ DOFs. 


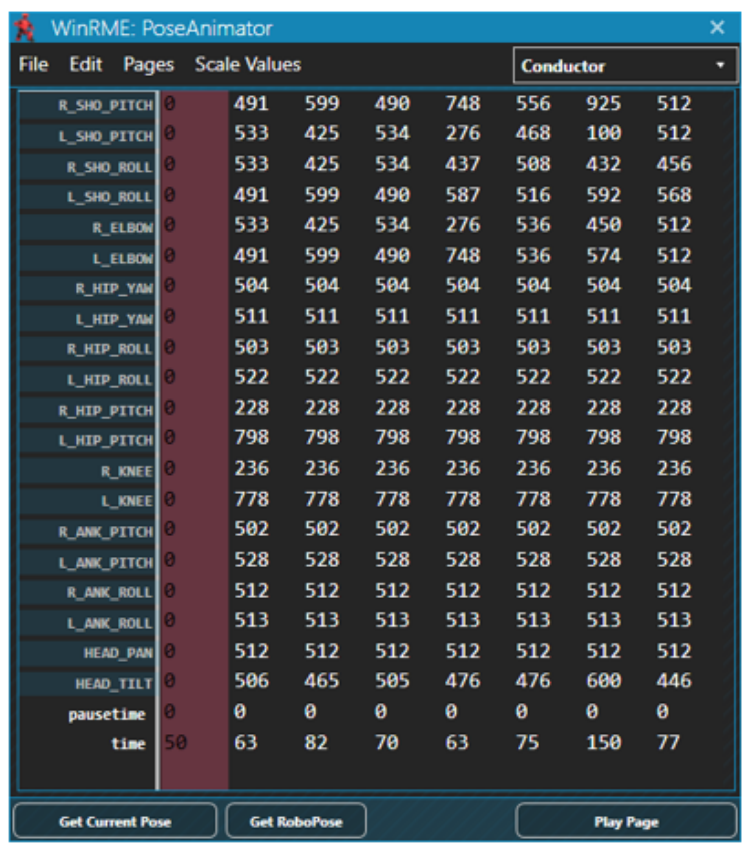

Figure 3.13: Motion data in WinRME PoseAnimator (sequence editor) [55].

Each $s_{n}$ is a vector of size $t$ of a sequence of values such as joint angle, torque, or position values representing the motion of the DOF $n$ for $t$ timesteps. So, in S-mode, $M=\left[s_{0}, \cdots, s_{n}\right]^{T}$ where $\mathrm{n}$ is the index for DOFs and $s_{n}=\left[\phi_{0}, \cdots, \phi_{t}\right]$ where $\phi$ is the same as before but now indexed over $t$ timesteps. Figure 3.13 shows an example of one motion data representation in the WinRME tool.

There are strengths and weaknesses in either F-mode or S-mode. Working in F-mode is best for creating a new gesture because it corresponds directly with the pose-to-pose method of animation [43] and the process is easy to understand. Sometimes other information can be added to each frame $f_{t}$. For example, in the HROS-1 framework used for the HROS-1 robots from Trossenrobotics [15], each frame $t$ consists of $N$ joint angle values plus two pieces of timing information. The first information is simply called Time and represents the desired amount of time to reach the pose from the previous pose which determines the speed of the motion. The second time information is called PauseTime and represents the amount of time to hold the 
current pose. In the Pololu Maestro Sequencer tool [57], each DOF at each timestep has speed and acceleration values, and each timestep has a duration value . However, when using F-mode it is difficult to create the in-between poses to make the motion appear more dynamic and expressive. Using F-mode is also prone to creating animation where only a small subset of the robot's DOFs are moving and the rest remain still which creates a 'stiff' and 'mechanical'-looking motion.

In the S-mode, $s_{n}$ (the motion of DOF $n$ ) can be visualized as a signal. The signal visualization gives the user a better idea of how the resulting motion will look like. A smooth curve corresponds to a motion with a low acceleration or deceleration, so the motion is not abrupt. Conversely, when there are sharp corners along the signal, the motion will abruptly change direction at those points. Users can change the smoothness or sharpness of the points in $s_{n}$ manually or apply any signal processing methods to remove or add noise, to smooth the signal using interpolation methods, to increase or decrease amplitude, superpose or merge with another signals, and so forth [13]. Each $s_{n}$ is not required to have the same number of timesteps, whereas in F-mode all DOFs will have the same number of timesteps. The weakness of this approach is that it is difficult to use for building new gestures or motions. This is because building a complete motion by determining the movements of one DOF at a time is not very intuitive and can be difficult to debug when adding the next DOF.

In practice, professional animators will first build the keyframes of the gesture motion (like in F-mode), then refine the motion using a curve editor (in S-mode). Unfortunately, most robotic softwares only offer inexpensive F-mode editors like the RME in the HROS-1 Framework, or proprietary to a specific robot like the Choreographe tool for the NAO robot [16]. In this dissertation, we use a similar mixed approach. Gesture motion data are first composed using a library of poses, then refined using filtering and other signal processing methods. For the HROS-1 robot, 
we use the library of poses that was created by Mekana Burch ${ }^{6}$ using the WinRME tool [55]. This library uses the HROS-1 format, so each pose also contains duration and pause timing information in addition to the joint configuration. The duration and pause timing information are used to determine the interpolation between two poses to create in-between poses. The motion is executed on the robot by sending each frame at a fixed rate (about $19 \mathrm{~Hz}$ ). Issues related to sending the motion data to the actual robot will be discussed further in Chapter 6 .

A "wave left hand behavior" can be defined by the data in Table 3.1 where each row is one pose, and the second column onward indicates the set of joints/DOFs. The data in this table is a transposed version of the data shown in Figure 3.13. The joint angle values are $[0,1024]$ corresponding to 0-300 degrees of rotation for the actuators. The value 512 correspond to the servo center/home position (therefore the can move 150 degrees clockwise and counter clockwise. L_SHO_PTCH starts from 512 and goes to 598 showing that it is being raised to the its maximum range. The robot is at its 'home' position when all joint angle values are 512. The L_SHO_ROLL DOF moves the arm away or closer to the torso. Table 3.1 shows that it alternates between 567 to 401 to 441 indicating a part the waving movement. The value 512 indicates the home $_{\text {pose }}$ for the DOF. The value -1 indicates that the DOF is not being used for the behavior. In the wave left hand data, R_SHO_PTCH, R_ELBOW joints are not being used so their values are -1 throughout the data. DOFs with -1 values will not move and remain at their previous position when the motion data is executed.

Some behaviors are not ideal to be represented as tabular data. For example, traveling behaviors for wheeled robots where the robot moves from one location to another, are best represented as sequences of commands (e.g. move forward $5 \mathrm{ft}$, turn left, etc.). If the robot can perform autonomous navigation (e.g. SLAM), all it needs

\footnotetext{
${ }^{6}$ Unpublished work
} 
Table 3.1: Example motion data representation for a "wave left hand" gesture. Each row is the robot's pose at one time step and each column is a DOF.

\begin{tabular}{cccccc}
\hline Time & R_SHO_PTCH & L_SHO_PTCH & R_SHO_ROLL & L_SHO_ROLL & R_ELBOW \\
\hline 0 & 512 & 512 & 512 & 512 & -1 \\
1 & 512 & 598 & 456 & 567 & -1 \\
2 & 512 & 179 & 456 & 401 & -1 \\
3 & 470 & 272 & 456 & 401 & -1 \\
4 & 470 & 207 & 456 & 441 & -1 \\
\hline
\end{tabular}

is the sequence of waypoints or target locations. Complex behaviors that require controls that involve dynamical models (e.g. balancing, walking) are often represented using a combination of state machines, differential equations, and dynamics simulators [29]). There are also other kinds of robot behaviors that do not explicitly use state machines and do not require sequential commands but purely as function of its inputs and is called behavior-based robotics. For example: Braitenberg vehicles [94] are robots that can exhibit seemingly complex behaviors such as light avoiding or approaching, but the behavior is only determined by how the light sensors are wired to the motors on the wheels. The speed of the motors is directly a function of the intensity of light detected by its sensors.

\subsection{Summary}

In this chapter we described the robot platforms used in this study: the Mr. Jeeves robot, the HROS-1 robot, the KHR-1 robot, and the Lynxmotion AL5A robot. Additionally, we also discussed how the robot motion data are represented which are used in the works in chapters 6,7 and 8 . 


\section{CHAPTER 4}

\section{ALGEBRAIC EXPRESSIONS FOR EXPRESSIVE BEHAVIORS}

The work presented in this chapter is an extension to my work published in the following publication: M. Perkowski, A. Bhutada, M. Lukac, and M. Sunardi, "On synthesis andverification from event diagrams in a robot theatre application," in 2013 IEEE 43rd International Symposium on Multiple-Valued Logic, IEEE, 2013, pp. 77-83.

\subsection{Introduction}

In this chapter, the concept of expressing behaviors using algebraic expressions called Behavior Expressions (BEs) is presented as a method to address the challenges of building motion repositories for humanoid robots. Creating new motion data for humanoid robots is often challenging because the user has to manage the high number of degrees of freedom (DOFs) on the robot, and the many poses required to make the desired expressive motions such as: energetic vs. lethargic hand waving, and emotional communicative gestures such as deictic and metaphoric gestures [132]. Expressive motions involve detailed animations that require many subtle pose changes and precise timing to achieve the desired expressions [47]. One particular issue addressed in this chapter is the creation of new gestures and variety of motions which is time consuming and inefficient in terms of storage space. Creating variety of motions is taking one motion and creating different variants, such as "waving hand" which can be done with either the right or left arm. With current methods, each motion is saved as a separate record (motion record) either in its own file, or a new record in a database of motions. Each of this record is of size $N \times M$ where $N$ is the number of DOF 
and $M$ is the number of poses. As will be demonstrated later in this chapter, specifying motions using the new composition operators and expressions allows creation of families of motions, where a single expression can represent a multitude of motion variants, instead of just a single motion. In some cases, there can be theoretically infinitely many motion variants for certain BEs. Furthermore, using BEs, motions can be constructed hierarchically by reusing BEs in other BEs with arbitrary depth. In addition to a more compact way to represent motions, the probabilistic behavior of these operators allows the robots to also exhibit probabilistic behaviors, which in turn gives the illusion of "intelligence".

This chapter is organized as follows: Section 1 discusses the idea behind using algebraic expressions for behaviors. Section 2 presents representation of behaviors at the lowest levels e.g. vectors of joint angles. Section 3 presents the formalism of the framework. Examples are given and discussed in Section 3. Section 4 discusses some experiments and evaluations, and conclusions and discussions are in Section 5.

\subsection{Composing Expressive Behaviors}

Creating expressive gestural animations for humanoid robots requires a lot of time and effort primarily due to the iterations to find the correct timing parameters and coordinating high number of DOFs. Consequently, creating variety of gestures for different kinds of expressions and different situations is notoriously time consuming. The current processes typically involve: creating a new motion record for each motion variant, copy-and-pasting joint angle values to create repetition, and re-adjusting the timing parameters. The question is: what can be done to improve this process?

In this chapter, we focus on improving the composition process using a new notation we call behavior expression which was derived from regular expression. In- 
formally, regular expression is a notation to describe the finite set of strings that is accepted by some language $L(\mathbf{E})$ where $\mathbf{E}$ is a regular expression. The regular expression $\mathbf{E}$ is constructed from a set of operators: concatenation, union, and closure which operands come from a finite set of symbols called the alphabet [133]. In Chapter 5 and 6 we describe our methods for adding expressiveness to robot motion.

Let's look at an example gestures can be composed from a set of simpler actions using simple composition operations which we 'borrow' from regular expressions. The main idea of this chapter is my work on using and expanding these composition operations from regular expressions for use in behavior expressions. Fig. 4.1 shows five poses for the HROS-1 robot: HOME - for the robot's default/home pose (typically chosen as the robot's resting position when no other actions is given), R_ARM_RAISE a pose where the robot's right arm is swung forward and upwards, placing the hand above its head, R_ARM_OUT - a pose where the right arm is rotated at the shoulder and pointing in the rightside direction, and similarly for L_ARM_RAISE and L_ARM_OUT poses for the left arm. These poses are what we call the basic actions or atomic actions of the robot - the smallest unit of actions that cannot be broken down further. A collection of these atomic actions is stored in what we call a motion library, but the motion library can also contain non-atomic actions or compound actions which we will show later. Now, from these atomic actions (poses) in Fig. 4.1 we can create some hand-waving gestures by composing the poses using some simple operations. The first operation we borrow from regular expression is concatenation, which denotes that when some arbitrary action $A$ is 'concatenated' with another arbitrary action $B$, it means the action $A$ is executed first, followed by action $B$. We will denote the concatenation operation with the dot symbol: ‘. Let's first define a hand-waving gesture using the right arm where the arm is waved twice written as the following expression: 


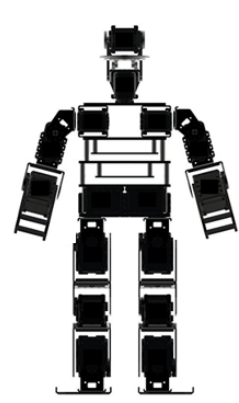

HOME

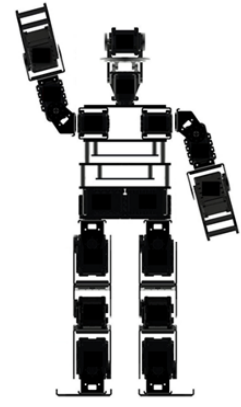

R_ARM_RAISE

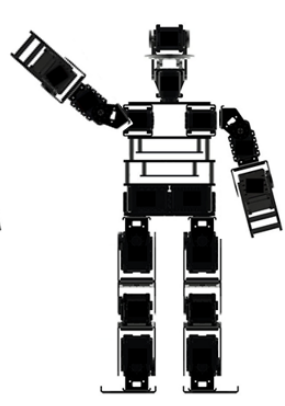

R_ARM_OUT

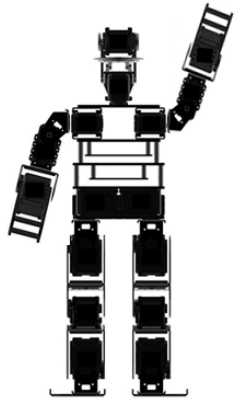

L_ARM_RAISE

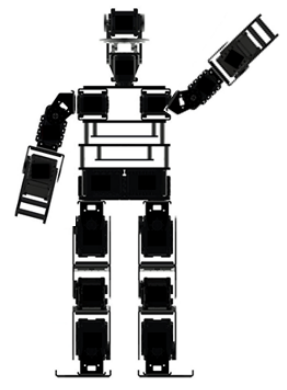

L_ARM_OUT

Figure 4.1: Five basic poses of the HROS-1 robot for hand-waving gestures: HOME, R_ARM_RAISE, R_ARM_OUT, L_ARM_RAISE, L_ARM_OUT.

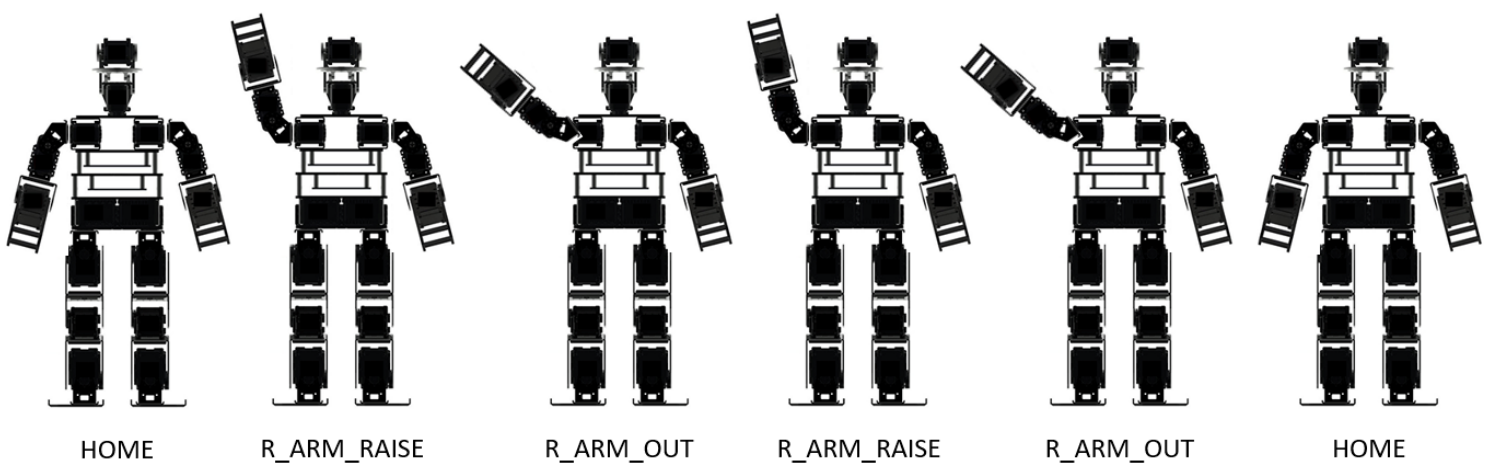

Figure 4.2: The sequence of poses (motion) of the WAVE_RIGHT_ARM gesture.

$W A V E \_R I G H T \_A R M=H O M E \cdot R \_A R M \_R A I S E \cdot R \_A R M \_O U T \cdot$

$$
\text { R_ARM_RAISE } \cdot R \_A R M \_O U T \cdot H O M E
$$

When executed on the robot, the hand-waving gesture defined in eq. 4.1 will wave the arm twice because the sequence $R_{-} A R M_{-} R A I S E \cdot R_{-} A R M \_O U T$ appears twice. Finally, the gesture ends with the HOME pose (Fig. 4.2). Similarly, we can define the hand-waving gesture for the left arm using the following expression: 
$W A V E \_L E F T \_A R M=H O M E \cdot L_{-} A R M_{-} R A I S E \cdot L_{-} A R M \_O U T \cdot$

$$
L_{-} A R M \_R A I S E \cdot L_{-} A R M \_O U T \cdot H O M E
$$

Now if we want the hand-waving to be done more than twice, the sequence R_ARM_RAISE - R_ARM_OUT must be repeated as many times as the number of waving desired. This is impractical. This time, we borrow the closure operation from regular expression to denote repetition which denoted with the symbol star: *. Informally, the closure operation in regular expression can be interpreted as "repeated zero or more times" ${ }^{1}$. Because we only want to repeat the hand-waving poses, to use the repetition operator, the alternation of R_ARM_RAISE - R_ARM_OUT must first be enclosed in parenthesis to indicate that the sequence is to be repeated. We can now update the hand-waving expressions using the repetition operation as follows:

$$
\begin{aligned}
W A V E \_R I G H T \_A R M= & H O M E \cdot R \_A R M \_R A I S E \cdot\left(R \_A R M \_O U T\right. \\
& \left.R_{-} A R M_{-} R A I S E\right)^{*} \cdot H O M E \\
W A V E \_L E F T \_A R M= & H O M E \cdot L_{-} A R M_{-} R A I S E \cdot\left(L_{-} A R M \_O U T .\right. \\
& \left.L_{-} A R M_{-} R A I S E\right)^{*} \cdot H O M E
\end{aligned}
$$

The updated expressions in eq. 4.3 do change the definition of the hand-waving gestures slightly. In eq. 4.1 and 4.2 , the hand-waving is done exactly twice. In the updated expressions, for either the left and right hand-waving gesture, the arm is at least raised first, but each time the gesture is executed, the number of actual waving can be zero or more times. Later, we will introduce our version of the repetition

\footnotetext{
${ }^{1}$ For the formal definition, see [133]
} 
operator where the user can explicitly denote the number of repetition desired. Notice, that with the repetition operation, the repeated action or sequence does not have to be explicitly written repeatedly in the expression. The operator makes the expression shorter and more succinct, yet expresses many possibilities on how the right or left hand-waving gestures can be executed.

Lastly, let us imagine further that the robot will use the hand-waving gestures in its repertoire of greeting behaviors. Additionally, for variety, we want the robot to perform the hand-waving gestures sometime with the left arm, and other times with the right arm. To express this kind of choosing behavior, we can use the union operator from regular expression to denote choice. Classically, the symbol for the union operator is the plus sign: + which we will use in the examples below. We can now create a new expression to denote that the hand-waving gesture can be done with either the right arm or the left arm that were already defined in the expressions WAVE_RIGHT_ARM and WAVE_LEFT_ARM, respectively:

$$
H A N D \_W A V I N G=W A V E \_R I G H T \_A R M+W A V E \_L E F T \_A R M
$$

The examples above illustrate some of the common operations for composing robot's gestures and creating variety of robot gestures: 1) the concatenation operation to arrange actions in a particular order/sequence, 2) the repetition operation to vary the number of times an action is repeated, and 3) the union/choice operation to vary the action to execute each time. In the following sections, we introduce our system called Robot Expressive Behavior Language (REBeL) which implements this method of composing robot actions and behaviors as shown from the examples above. As will be shown below, the set of REBeL operators is inspired from the operators of 
regular expressions to include concatenation, union/choice, and repetition. But we also introduced the subtraction operation, and added probabilistic features to the set of REBeL operators to increase the expressiveness of the behavior expressions to create families of behaviors. REBeL was implemented using the Python programming language, and we used a slightly different notation than the above examples to write the expressions for convenience.

There are still other aspects of creation of expressive behaviors that are not addressed by these composition operators. Among other things: timing - how to set delays or scheduling actions, reach/range of motion - how big a movement is done, continuity - how smooth or sharp are the transitions between movements, and so on. Some of these properties have been addressed in other works using parameters from concepts such as Laban Movement Analysis (LMA) [134], [12], or spectral transforms [135]. However, these approaches require manual adjustments of some parameters to change the styles of emotional expression.

In Chapter 5 we introduce a method to create the timing and range of motion (reach) modifications using information from music as a parameter-free alternative. In that work, music data is used in two ways: 1) to generate motion data directly, and 2) modify an existing motion data using the properties of the music data (e.g. tempo, dynamics, melody). In chapter 6 we show methods for further stylistic modifications using user-tuned parameters such as: interpolation parameter and filtering coefficients. The remainder of this chapter discusses the use of composition operators to compose robot behaviors.

Although the proposed composition method can be generalized to non-movement behaviors such as speech, sounds, or lights, the discussions in this work focus more on gestural and movement-based expressive behaviors. 


\subsection{Robot Expressive Behavior Language (REBeL)}

In this section, we introduce the Robot Expressive Behavior Language (REBeL) as one of the main contributions of this dissertation.

REBeL is the system that we developed to generate sequences of robot behaviors. The term 'robot behavior' is used as a general term to refer to actions that can be performed by a robot such as, but not limited to: full body motion, facial expressions, and speech. In this dissertation, we only focused on the motion-based behaviors of gestures for humanoid robots.

There are two main components of REBeL: 1) the library of basic actions, and 2) behavior expressions.

\subsubsection{Library of Basic Actions}

The library of basic actions is the collection of actions that the robot can perform. As the name says, the library generally contains actions that are simple called basic actions or atomic actions - the smallest unit of action that cannot be deconstructed further. Because the main interest in this work is on robot gestures, the basic actions for robot gestures consist mainly of individual robot poses like in the examples in Fig. 4.1. A pose is a set of joint angle values for the all degrees of freedom (i.e. joints) on the robot.

At the end of this chapter we show that the library of basic actions is not limited to robot poses, and can be expanded to include other modalities such as commands to control facial animations like on the robot Jeeves. In practice, it is not required that every item in the library is an atomic action; compound actions can also be defined and included in the library. Compound actions are actions that consist more than a single pose, and defined using behavior expressions (explained next). Allowing 
to include compound actions Additionally, new actions can be added to the library dynamically during the robot's runtime.

Each member in the library of basic action is represented by a descriptive name of the action it represents (i.e. a string), such as: HAND_WAVING, L_ARM_RAISE, L_ARM_OUT, and so on. It is important that each item in the library has a unique name. Just as in regular expressions a finite set of symbols called the alphabet from which to construct the regular expressions, the behavior expression of REBeL (explained in the next section) also depends on a finite set of names for the basic actions as the "alphabet" to construct behavior expressions which represents the behaviors of the robot. In other words, the names of the actions in the library of basic actions compose the alphabet for the behavior expressions.

Complex behaviors are created by combining basic actions and other behaviors using sequencing, repetition, or choice operations written in a notation we call behavior expression. In the next section, we will discuss behavior expressions and the process of composing complex behaviors in more detail. The operators for composing behaviors are: concatenation, union, repetition, and subtraction, which are discussed below.

\subsubsection{Behavior Expressions}

Behavior expressions are notations to describe robot behaviors. Behavior expressions consists a finite set of names of basic actions from the library of basic actions, and a set of operators. Currently, we have implemented four operators for behavior expressions: concatenation, union, repetition, and subtraction. Future works can add other operations such as: concurrency to allow executing two or more behaviors at the same time, and inversion to reverse the order of actions in a sequence.

When writing a variable that represents a behavior expression, the variable is 
written in bold, capital letters. The default variable for behavior expressions is $\mathbf{B}$. The following are some definitions in behavior expressions:

- Alphabet - The finite set of names of the basic actions is commonly referred to as the alphabet and represented by the symbol $\Lambda$.

- $\Lambda^{*}-$ All possible combinations of all the basic actions in $\Lambda$ is denoted as $\Lambda^{*}$.

- Behavior - The sequence of basic actions that is generated by a behavior expression $\mathbf{B}$ is called a behavior. If $a, b$ are names of some basic actions, and a behavior expression generates the sequence $a b$, then $a b$ is a 'behavior'. If a behavior expression generates only $a$ (a sequence of length 1), then $a$ is also a 'behavior', because it was generated from a behavior expression. 'Behaviors' in behavior expressions is the analog to 'strings' or 'words' in regular expressions.

- Family of behaviors - The set of all possible behaviors that can be generated by a behavior expression $\mathbf{B}$ is called the family of behaviors of $\boldsymbol{B}$ and denoted as $F(\mathbf{B})$. If $a, a b, c d$ are behaviors that can be generated by a behavior expression $\mathbf{B}$, then the family of behaviors $F(\mathbf{B})=\{a, a b, c d\}$.

The following are valid behavior expressions:

- $\emptyset$ : the empty set, or containing no behavior.

- $\epsilon$ : the No Action $(N A)$. A 'No Action' is a behavior with no basic action nor compound action, i.e. a behavior with zero length sequence, and $\epsilon \neq \emptyset$.

- a: is a singleton; is a behavior expression consisting of a single basic action $a$ without using any operators. 


\subsubsection{Notation}

Behavior expressions are written in the prefix form, where the operator is written first, followed by the operands. For operations that can accept more than one operands, the operator is only written once. Each operation of behavior expressions must be enclosed with parentheses, except for singletons. By writing each operation in parentheses, behavior expressions explicitly group each operation so there is no operator precedence. When specifying a singleton behavior expression, no parentheses are used.

To illustrate the examples below, let's assume some basic action names like: $a$, $b, c, H O M E, L_{-} A R M_{-} R A I S E, W A V E_{-} R I G H T_{-} H A N D$, etc. Below are some examples of correct and incorrect behavior expressions formatting (the operators: concatenation $(\&)$, union $(+)$, repetition $(*)$ are explained in the next section):

- Correct format:

- Singleton: $a, L_{-} A R M \_R A I S E$

- Concatenation: (\& $a b),\left(\& H O M E L \_A R M \_R A I S E H O M E\right)$

- Union: $(+a b c),\left(+W A V E \_R I G H T \_H A N D W A V E \_L E F T \_H A N D\right)$

- Repetition: $(* a),\left(* W A V E_{-} L E F T_{-} H A N D\right)$

- Multiple operations: (+ (\& $\left.a b)\left(\& H O M E L_{-} A R M \_R A I S E H O M E\right)\right)$

- Incorrect format:

- Operator after operand: $(a \& b)$

- Missing parentheses: \& $a b$

- Incomplete form in multiple operations: $(+a(\& b c)$ 
Our REBeL program is implemented in Python programming language ${ }^{2}$. The parenthesis-enclosed format is used to write behavior expressions as Python strings.

\subsubsection{Generating Behaviors}

In general, a behavior expression $\mathbf{B}$ denotes a set of behaviors - all the possible behaviors the behavior expression can produce, which we call family of behaviors or $F(\mathbf{B})$. To produce a behavior to be executed on a robot from a behavior expression B, we say: "we evaluate the behavior expression B" or "we instantiate the family of behaviors $F(\boldsymbol{B})$ " or simply "instantiate $F(\boldsymbol{B})$ " and can be written as $\operatorname{eval}(F(\mathbf{B}))$. The evaluation of behavior expressions is performed by the behavior expression interpreter that we developed in Python programming language.

Each evaluation of a behavior expression returns only one of the behaviors from the family of behaviors. To get a different behavior from $F(\mathbf{B})$, do another evaluation of $F(\mathbf{B})$. For example, if $F(\mathbf{B})=\left\{W A V E_{-} R I G H T \_A R M, W A V E_{-} L E F T \_A R M\right\}$, $\operatorname{eval}(F(\mathbf{B}))$ will return one of the behaviors in $F(\mathbf{B})$.

$$
\begin{gathered}
\text { First call: } \operatorname{eval}(F(\mathbf{B}))=W A V E \_L E F T \_A R M \\
\text { Second call: } \operatorname{eval}(F(\mathbf{B}))=W A V E \_R I G H T \_A R M \\
\text { Third call: } \operatorname{eval}(F(\mathbf{B}))=W A V E \_R I G H T \_A R M
\end{gathered}
$$

And so on ...

Notice that the second and third call to $\operatorname{eval}(F(\mathbf{B}))$ return the same behavior twice. This is because evaluation of the behavior expressions to generate one of the behaviors in $F(\mathbf{B})$ is performed by the REBeL interpreter probabilistically. We explain how the probabilities for generating behaviors from behavior expressions when we discuss each

\footnotetext{
${ }^{2}$ Our behavior expression interpreter is written in Python based on the Lisp interpreter by Peter Norvig [136].
} 
operator of behavior expressions below.

Although behavior expressions do not have operator precedence, evaluation of behavior expressions is recursive so the innermost operation is evaluated first. Because behavior expression operators can have probabilistic behaviors, each evaluation can produce different behaviors depending on how the probabilities in the behavior expression are specified. We will explore the behavior expression operators and their probabilistic behaviors in the next section.

\subsubsection{Similarity and Differences with Regular Expression}

While largely inspired by regular expressions [133], the main difference between the behavior expressions of REBeL and regular expressions is that behavior expressions are used to generate a sequence of symbols (i.e. strings), while regular expressions describe the set of strings that is accepted by the regular expressions. Another difference is with respect to the alphabet. In regular expression, the alphabet is typically the latin characters $\{a, \cdots, z\}$, or numbers $\{0,1\}$. In behavior expressions, the set of symbols refers to the set of basic/atomic actions or compound actions of a robot, stored in the library of basic actions described above. Therefore, the set of unique names of the basic actions in that library defines the alphabet of behavior expres-

sions. However, for the sake of brevity and readability, when showing examples of behavior expressions operators, we will often use a single character to represent an action, such as ' $a$ ', ' $b$ ', and so on. In the following discussions, we will use the symbols $\{a, b, c, \cdots\}$ to represent basic actions from the library of basic actions, and simplify the explanation to illustrate how the operators work.

A regular expression take a string as input, and either accepts or rejects the input string, depending on whether or not the input string belongs to the set of strings defined by the regular expression; in other words, if the input string belongs in the 
language of the regular expression. In contrast, a REBeL behavior expression does not take any input, but produces a sequence of symbols which denotes a behavior for a robot, e.g. a hand-waving gesture as shown in the examples above.

We summarize the similarities and differences between behavior expressions and regular expressions in Table 4.1.

\subsubsection{Behavior Expression Operators}

There are three basic operations from regular expressions that are adopted by behavior expressions: concatenation, union, and repetition (closure). Additionally, we introduce the subtraction operator. In this section, we discuss the main behavior expressions operators, their properties, and probabilistic behaviors.

4.3.2.4.1 Concatenation Concatenation is the operation that creates a sequence in the order the operands given. REBeL uses the symbol \& (ampersand) as the concatenation operator. If $\mathbf{C}$ and $\mathbf{D}$ are behavior expressions, the concatenation of $\mathbf{C}$ and $\mathbf{D}$ is a behavior expression.

$$
\mathbf{B}=(\& \mathbf{C} \mathbf{D})
$$

Let's suppose the family of behaviors for $\mathbf{C}$ is $F(\mathbf{C})=\{a, b c\}$, and the family of behaviors of $\mathbf{D}$ is $F(\mathbf{D})=\{a c, b d\}$, where $a, b c, a c, b d$ are some behaviors, then the concatenation of $\mathbf{C}$ and $\mathbf{D}$ gives the family of behaviors which elements are the 
Table 4.1: Similarities and differences between behavior expressions of REBeL and regular expressions. ${ }^{\dagger}$ Read: subtract $b$ from $a$. See section 4.4 .4 for more details. ${ }^{\ddagger}$ Assumed groupings of operands unless explicitly stated using parentheses.

\begin{tabular}{|c|c|c|}
\hline & Behavior expression & Regular expression \\
\hline Denotes & $\begin{array}{l}\text { The family of behaviors } F(\mathbf{B}) \\
\text { that can be generated from be- } \\
\text { havior expression } \mathbf{B} \text {. }\end{array}$ & $\begin{array}{l}\text { The set of strings accepted by } \\
\text { the language } L(\mathbf{E}) \text { where } \mathbf{E} \text { is a } \\
\text { regular expression. }\end{array}$ \\
\hline $\begin{array}{l}\text { Set of symbols (alpha- } \\
\text { bet) }\end{array}$ & $\begin{array}{l}\text { A finite set of names of } \\
\text { basic/atomic } \\
\text { HAND_WAVING, L_ARM_RAISE, etc. } \\
\text { Symbol: } \Lambda \\
\end{array}$ & $\begin{array}{l}\text { A finite set of characters, e.g. } \\
\text { alphanumeric } \quad(\text { a..zA..Z } Z 0 . .9), \\
\text { numbers }\{0,1\} \text {, etc. Symbol: } \Sigma\end{array}$ \\
\hline $\begin{array}{l}\text { A sequence of symbols } \\
\text { from the alphabet }\end{array}$ & Called a "behavior" & Called a "string" or "word" \\
\hline $\begin{array}{l}\text { Set of operators and } \\
\text { their symbols }\end{array}$ & $\begin{array}{l}\text { concatenation }(\&) \text {, union }(+) \text {, } \\
\text { repetition }(*) \text {, subtraction }(-)\end{array}$ & $\begin{array}{l}\text { concatenation }(\cdot \text { or omitted }) \text {, } \\
\text { union }(+),(\text { Kleene }) \text { closure }\left({ }^{*}\right)\end{array}$ \\
\hline Notation style & $\begin{array}{l}\text { Prefix (operator is written } \\
\text { first followed by arguments). } \\
\text { Every operation is written } \\
\text { in parentheses. } \\
\text { Concatenation: }(\& a b) \\
\text { Union: }(+a b) \\
\text { Repetition: }(* a) \\
\text { Subtraction }{ }^{\dagger}:(-a b)\end{array}$ & $\begin{array}{l}\text { Infix (operator is written } \\
\text { between every two argu- } \\
\text { ments, except for closure) } \\
\text { Concatenation: } a \cdot b \text { or } a b \\
\text { Union: } a+b \\
\text { Closure: } a^{*}\end{array}$ \\
\hline $\begin{array}{l}\text { Associativity of concate- } \\
\text { nation operation }\end{array}$ & $(\& a(\& b c))=(\&(\& a b) c)$ & $a(b c)=(a b) c$ \\
\hline $\begin{array}{l}\text { Associativity of union } \\
\text { operation }\end{array}$ & $(+a(+b c))=(+(+a b) c)$ & $a+(b+c)=(a+b)+c$ \\
\hline $\begin{array}{l}\text { Commutativity of con- } \\
\text { catenation operation }\end{array}$ & $(\& a b) \neq(\& b a)$ & $a b \neq b a$ \\
\hline $\begin{array}{l}\text { Commutativity of union } \\
\text { operation }\end{array}$ & $(+a b)=(+b a)$ & $a+b=b+a$ \\
\hline Operator precedence ${ }^{\ddagger}$ & $\begin{array}{l}\text { Deepest level of parenthesis is } \\
\text { evaluated first regardless of the } \\
\text { operation. }\end{array}$ & $\begin{array}{l}\text { Highest to lowest: Closure, con- } \\
\text { catenation, then union [133]. }\end{array}$ \\
\hline
\end{tabular}


concatenation between the elements in $F(\mathbf{C})$ and $F(\mathbf{D})$.

$$
\begin{aligned}
F(\mathbf{C}) & =\{a, b c\} \\
F(\mathbf{D}) & =\{a c, b d\} \\
\mathbf{B} & =(\& \mathbf{C} \mathbf{D}) \\
F(\mathbf{B}) & =\{a a c, a b d, b c a c, b c b d\}
\end{aligned}
$$

A behavior expression which performs multiple concatenations where each one is a concatenation of two behavior expressions is equivalent to a single concatenation with multiple operands. Suppose the following are behavior expressions: B, C, D, E, F, G, $\mathbf{H}, \mathbf{I}, \mathbf{J}$.

$$
\begin{aligned}
& \mathbf{C}=(\& \mathbf{E} \mathbf{F}) \\
& \mathbf{D}=(\& \mathbf{G} \mathbf{H}) \\
& \mathbf{H}=(\& \mathbf{I} \mathbf{J}) \\
& \mathbf{B}=(\& \mathbf{C} \mathbf{D})=(\& \mathbf{E} \mathbf{F} \mathbf{G} \mathbf{I} \mathbf{J})
\end{aligned}
$$

The expression $\mathbf{B}$ denotes a family of behaviors which consists of one behavior composed of two basic actions: $a$ and $b$, and the two actions are to be executed in the following order: first, action $a$ is executed, followed by action $b$.

In general, there can be more than two operands, and each operand is a behavior expression. Therefore, the family of behaviors of concatenation behavior expressions consists of all possible concatenations of the members of the family of behaviors of its operands. For example:

Probabilistic Behavior The probabilistic behavior of the concatenation operator is that with some probability $p$, the REBeL parser will evaluate the next item in 
Table 4.2: Probabilistic Concatenation

\begin{tabular}{lll}
\hline & Behavior expression $(\mathbf{B})$ & Family of behaviors $(F(\mathbf{B}))$ \\
\hline 1 & $(\& \mathrm{a} b \mathrm{~b}$ d e 0.5) & $\{\epsilon, \mathrm{a}, \mathrm{ab}, \mathrm{abc}, \mathrm{abcd}, \mathrm{abcde}\}$ \\
2 & $(\& \mathrm{a} \mathrm{b} c \mathrm{~d}$ e & $\{\epsilon, \mathrm{a}, \mathrm{ab}, \mathrm{abc}, \mathrm{abcd}, \mathrm{abcde}\}$ \\
& $[0.5,0.5,0.4,0.7,0.85])$ &
\end{tabular}

the sequence. The default value (i.e. no probability argument is provided) is $p=1$ so the default behavior is that all items in the sequence are evaluated. So when $p<1$ there is some probability the evaluation will stop before going through the whole sequence. The probability argument can be given in two ways: 1) a single value $p$, or 2) as a list of real numbers $p$ where the length is equal to the number of arguments minus 1 . When a single value is given, every time before an argument in the sequence is evaluated, there is $p$ probability that the argument will be evaluated. This includes the very first argument. When a list $p$ is given, and given an input sequence $c$, the probability of an argument $c_{i} \in c$ be evaluated is $p_{i}$ where $p_{i} \in p$. Table 4.2 shows some examples of the probabilistic use with concatenation, where $\{a, b, c, d, e\} \in \Lambda$.

There are a few things to notice in Table 4.2. First, the list of probabilities in the second expression does not sum to 1 . This is because the probability of evaluating the next symbol is only computed from the current symbol and independent from the other symbols. Second, both expressions have the same output set. The difference between the two output sets is the probability of each outcome as illustrated Fig. 4.3. The probability for the occurrence for each output can be calculated by simple multiplication. The probabilistic concatenation behavior can be illustrated in Fig. $4.3^{3}$. Each node represents an occurrence of a symbol, and the directed edges point to the next possible symbols. The symbol $\varepsilon$ represents the empty string/sequence which means no symbol is evaluated. The symbol <EOS> stands for "End of Sequence"

\footnotetext{
${ }^{3}$ Graphs are created using Graphviz tool [137].
} 
Table 4.3: Calculating concatenation Probabilities for Behavior Expressions from Table 4.2

\begin{tabular}{ccc}
\hline Behaviors & Behavior expression 1 & Behavior expression 2 \\
\hline$\epsilon$ & $P(\epsilon)=0.5$ & $P(\epsilon)=0.5$ \\
$\mathrm{a}$ & $P(a)=0.5$ & $P(a)=0.5$ \\
$\mathrm{ab}$ & $P(a b)=P(a) \times 0.5=0.25$ & $P(a b)=P(a) \times 0.5=0.25$ \\
$\mathrm{abc}$ & $P(a b c)=P(a b) \times 0.5=0.125$ & $P(a b c)=P(a b) \times 0.4=0.1$ \\
abcd & $P(a b c d)=P(a b c) \times 0.5=0.0625$ & $P(a b c d)=P(a b c) \times 0.7=0.07$ \\
abcde & $P(a b c d e)=P(a b c d) \times 0.5$ & $P(a b c d e)=P(a b c d) \times 0.85$ \\
& $=0.03125$ & $=0.0595$ \\
\hline
\end{tabular}

and indicates the termination of the process. Since the probability value given as argument is the probability of evaluating the corresponding symbol, the probability for the alternative is simply $1-p_{i}$. In this case, the alternative is always $\langle$ EOS $>$.

Given a concatenation expression $S=\left(\& s_{1} s_{2} \ldots s_{t}\right)$, another variant of probabilistic behavior is instead of going to $\langle$ EOS $>$ as the alternative for each step $t$, the alternative is to skip the next symbol $s_{t+1}$ and evaluate the probabilities for the symbol $s_{t+2}$ (Fig. 4.4). The output set will be different than before. Table 4.4 shows the output set for the second probabilistic concatenation variant, where $\{a, b, c, d, e\} \in \Lambda$.

Currently, we only implemented the first probabilistic concatenation variant. The second variant will be incorporated in future works.

Table 4.4: Output set of the second variant probabilistic Concatenation

\begin{tabular}{lll}
\hline & Behavior expression $(\mathbf{B})$ & Family of behaviors $(F(\mathbf{B}))$ \\
\hline $1 \quad(\& \mathrm{a} b \mathrm{~b}$ $\mathrm{e} \mathrm{0.5)}$ & $\begin{array}{l}\text { \{abcde, abcd, abce, abd, acde, acd, } \\
\text { ace, bcde, bce, bde, bcd, bd }\}\end{array}$ \\
$2 \quad(\& \mathrm{a} b \mathrm{~b}$ e & $\begin{array}{l}\text { abcde, abcd, abce, abd, acde, acd, } \\
\text { ace, bcde, bce, bde, bcd, bd }\}\end{array}$ \\
\hline
\end{tabular}

4.3.2.4.2 Union Union operation denotes choice, and often understood as "choose" operation. In behavior expressions, the symbol '+' (plus) represents the union op- 


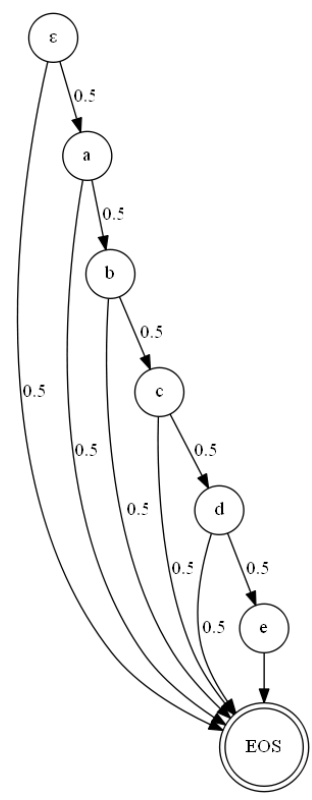

(a)

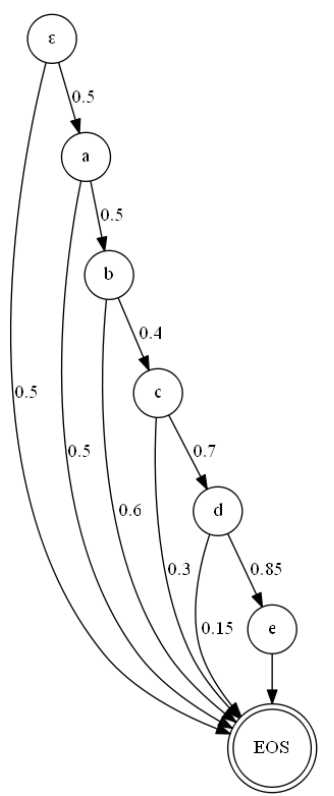

(b)

Figure 4.3: Concatenation with probability for the two expressions in Table 4.2.

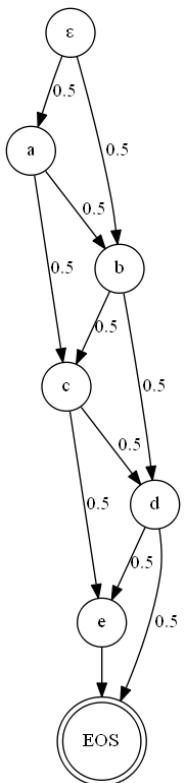

(a)

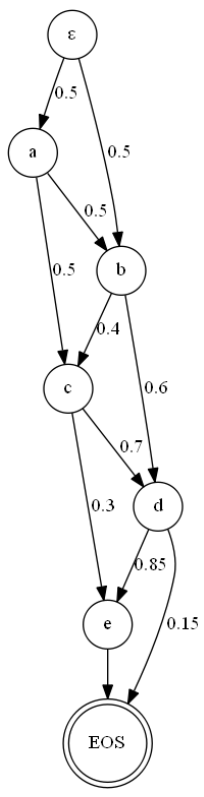

(b)

Figure 4.4: Concatenation with probability alternative version for the expressions in Table 4.4. 
erator. The operator takes two or more behavior expression operands. The union operation of behavior expressions $\mathbf{C}$ and $\mathbf{D}$ is written as the following behavior expression $\mathbf{B}$ :

$$
\mathbf{B}=(+\mathbf{C ~ D})
$$

The family of behaviors $F(\mathbf{B})$ for the expression in eq. 4.9 is the set containing the elements of the family of behaviors $F(\mathbf{C})$ and $F(\mathbf{D})$. For example, if $F(\mathbf{C})=\{a, b\}$ and $F(\mathbf{D})=\{a x, c y\}$, where $a, b, a x, c y$ are some behaviors, and $\mathbf{B}=(+\mathbf{C} \mathbf{D})$, then $F(\mathbf{B})=\{a, b, a x, c y\}:$

$$
\begin{aligned}
F(\mathbf{C}) & =\{a, b\} \\
F(\mathbf{D}) & =\{a x, c y\} \\
\mathbf{B} & =(+\mathbf{C} \mathbf{D}) \\
F(\mathbf{B}) & =\{F(\mathbf{C}), F(\mathbf{D})\}=\{a, b, a x, c y\}
\end{aligned}
$$

A behavior expression that performs union operations of two or more behavior expressions that also perform union operations can be written as one union behavior expression. Suppose the following are behavior expressions: $\mathbf{B}, \mathbf{C}, \mathbf{D}, \mathbf{W}, \mathbf{X}, \mathbf{Y}, \mathbf{Z}$, then:

$$
\begin{aligned}
\mathbf{C} & =(+\mathbf{W} \mathbf{X}) \\
\mathbf{D} & =(+\mathbf{Y} \mathbf{Z}) \\
\mathbf{B} & =(+\mathbf{C} \mathbf{D})=(+\mathbf{W} \mathbf{X} \mathbf{Y} \mathbf{Z}) \\
F(\mathbf{B}) & =\{F(\mathbf{W}), F(\mathbf{X}), F(\mathbf{Y}), F(\mathbf{Z})\}
\end{aligned}
$$

If the family of behaviors: $F(\mathbf{W}), F(\mathbf{X}), F(\mathbf{Y}), F(\mathbf{Z})$ are defined, the family of behaviors for $\mathbf{B}(F(\mathbf{B}))$ can be derived like in Eq. 4.10. 


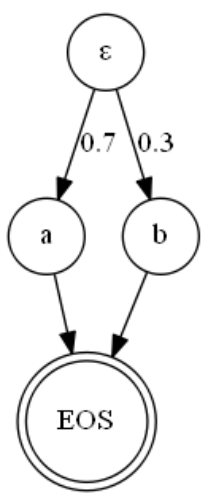

(a)

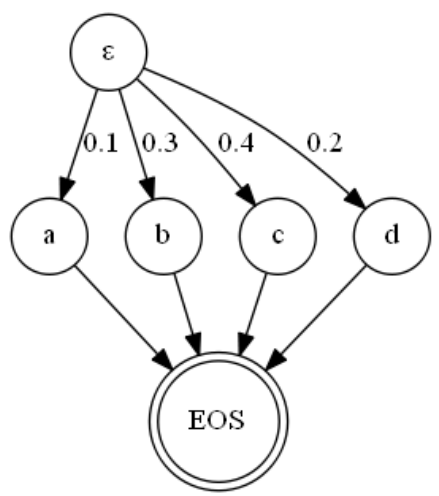

(b)

Figure 4.5: Graph for the first and third expressions in Table 4.5: (a) $(+a b)$, (b) $(+a b c d[0.1,0.3,0.4,0.2])$

Probabilistic Behavior The probabilistic behavior of the union operator is that each element in the output set is assigned some probability of being chosen. If the probability argument is not given, then REBeL assumes each argument has equal probability. For convenience, when there is only two arguments, it is enough to provide one probability value $p$. That value will be assumed for the first argument, and the probability for the second argument can be calculated as $1-p$. When there are three or more arguments, the probability argument should be given as a list $p$ and the number of elements equal the number of arguments, and $\Sigma_{i} p_{i}=1.0$. Table 4.5 shows some examples, where $\{a, b, c, d\} \in \Lambda$.

Table 4.5: Union probability usage examples

\begin{tabular}{ll}
\hline Behavior expression $(\mathbf{B})$ & Family of behaviors $(F(\mathbf{B}))$ \\
\hline$(+\mathrm{a} b)$ & $P(a)=0.5 ; P(b)=0.5$ \\
$(+\mathrm{a} \mathrm{b} 0.7)$ & $P(a)=0.7 ; P(b)=0.3$ \\
$(+\mathrm{a} \mathrm{b} \mathrm{c}$ d $[0.1,0.3,0.4,0.2)$ & $P(a)=0.1 ; P(b)=0.3 ; P(c)=$ \\
& $0.4 ; P(d)=0.2$ \\
\hline
\end{tabular}


4.3.2.4.3 Repetition Repetition is a unary operator which means it only accepts one behavior expression operand. The repetition operation works by evaluating the behavior expression operand or ending the evaluation with <EOS $>$ with some probability. In behavior expressions, the repetition operator is represented with symbol '*'. Like in regular expression, the operation can be interpreted as 'zero or more repetition'. Eq. 4.12 shows a behavior expression $\mathbf{B}$ where its operand is a behavior expression $\mathbf{C}$.

$$
\mathbf{B}=(* \mathbf{C})
$$

The family of behaviors of a repetition behavior expression is a set of behaviors where each behavior is some number of repetition of the operand and the empty string $\epsilon$ (some exceptions depending on the probability). For example: if the operand is a behavior expression $\mathbf{C}$ where its family of behaviors $F(\mathbf{C})=\{a, x\}$ and where $a, x$ are some behaviors (e.g. robot pose), then the family of behaviors $F(\mathbf{B})$ is the set union $(\cup)$ of 0 and more repetitions of $F(\mathbf{C})$ i.e.: $F(\mathbf{C})^{0} \cup F(\mathbf{C})^{1} \cup F(\mathbf{C})^{2} \cup \cdots F(\mathbf{C})^{n}$. Note that for any behavior expression $\mathbf{B}, F(\mathbf{B})^{0}=\epsilon$.

$$
\begin{aligned}
\mathbf{B} & =(* \mathbf{C}) \\
F(\mathbf{C}) & =\{a, x\} \\
F(\mathbf{B}) & =\left\{F(\mathbf{C})^{0}, F(\mathbf{C})^{1}, F(\mathbf{C})^{2}, \cdots, F(\mathbf{C})^{n}\right\} \\
& =\{\epsilon, a, x, a x, x a, a a, x x, a a x, a x a, \cdots\}
\end{aligned}
$$

The family of behaviors $F(\mathbf{B})$ where $\mathbf{B}$ is a repetition behavior expression and the operand $\mathbf{C}$ is a non-singleton behavior expression that uses operators like concatenation, for example: $\mathbf{C}=(\& a b)$, every repetition re-evaluates $\mathbf{C}$. Therefore, in general, the family of behaviors $F(\mathbf{B})$ consists of repetitions of the members of $F(\mathbf{C})$. 
Table 4.6: Repetition usage example

\begin{tabular}{cc}
\hline Behavior expression $(\mathbf{B})$ & Family of behaviors $(F(\mathbf{B}))$ \\
\hline$(*$ a $)$ & $\left\{\epsilon, a, a a, a a a, \cdots, a^{n}\right\}$ \\
$(*(\&$ a b $))$ & $\left\{\epsilon, a b, a b a b, a b a b a b, \cdots,(a b)^{n}\right\}$ \\
$(*(+$ a b $))$ & $\left\{a, \cdots, a^{m}, b, \cdots, b^{n}, a^{m} b^{n}, b^{m} a^{n}, a b a, b a b, \cdots\right\}$ \\
\hline
\end{tabular}

For example:

$$
\begin{aligned}
\mathbf{C} & =(\& a b) \\
F(\mathbf{C}) & =\{a b\} \\
\mathbf{B} & =(* \mathbf{C}) \\
F(\mathbf{B}) & =\{\epsilon, a b, a b a b, a b a b a b, \ldots\}
\end{aligned}
$$

Theoretically, the repetition operation can produce behaviors with an infinite number of actions.

This repetition operator only takes one behavior expression operand and optionally a probability value $p$. The repetition operator is by default probabilistic, with default probability of 0.5 for evaluating the argument and 0.5 for ending the evaluation $<$ EOS $>$ (End of Sequence), and is repeated if the argument is evaluated. Table 4.6 shows some example of usages, where $\{a, b\} \in \Lambda$.

Probabilistic Behavior Sometimes it is desired to have a minimum number of times that the argument be evaluated. For this reason, the probability argument can accept values greater than 1 , where the whole number is the number of times the argument will be evaluated. After the minimum number of repetitions is achieved, the decimal part denotes the probability of repetition as usual. For example: $p=2.6$ means that the argument will be repeated at least twice. Table 4.7 shows a few more 
Table 4.7: Repetition with Probability usage examplesge

\begin{tabular}{cc}
\hline Behavior expression $(\mathbf{B})$ & Family of behaviors $(F(\mathbf{B}))$ \\
\hline$(*$ a 2.6$)$ & $\left\{a a, a a a, \cdots, a^{2+n}\right\}$ \\
$(*(\& \mathrm{a}$ b $) 3.7)$ & $\left\{a b a b a b, \cdots,(a b)^{3+n}\right\}$ \\
$(*(+\mathrm{a}$ b $) 2.0)$ & $\{a a, a b, b a, b b\}$ \\
$(* \mathrm{a}[2 \quad 1.0])$ & $\{a a, a b, b a, b b\}$ \\
\hline
\end{tabular}

examples. Fig. 4.6 shows the graph representations of the repetition operation ${ }^{4}$.

Notice in the third example: when the decimal point of the probability argument is zero, then the evaluation stops after the number of minimum repetitions is achieved. Therefore, this property can be used to generate a sequence of fixed length.

The probability of generating a sequence of length $n$ can be calculated as $p^{n}$. Notice that when the probability argument is a whole number without decimal component, it behaves as combination with replacement. While theoretically the repetition operator can produce a sequence with up to infinite length, very long sequences are not desirable in normal use cases for our Robot Theater. An extremely long sequence can be induced by setting $p$ very close to 1.0 such as 0.9999999 . To avoid this extremely long sequence, REBeL rounds up when $p>0.9$ to 1.0, and caps the maximum number of repetitions to 10 times per expression. If for some reason a certain high number of repetitions is desired, then this can be done using the whole numbers for the probability argument or concatenating copies of the expression.

Repetition probability can also be defined using a normal distribution by specifying the mean and standard deviation as parameters. The last example in table 4.7 shows the syntax, with mean $=2$ and standard deviation $=1.0$. This means most of the time the expression will generate the string ' $a a^{\prime}$, but sometimes ' $a$ ' or ' $a a a^{\prime}$, and very rarely generates the empty string or a sequence longer than 3 .

\footnotetext{
${ }^{4}$ Due to limitations of the Graphviz tool, no two nodes can have the same labels.
} 


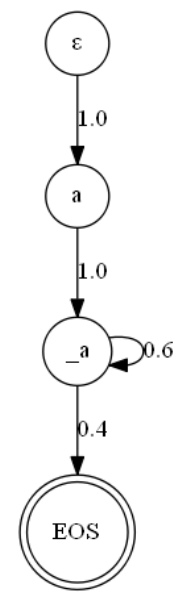

(a)

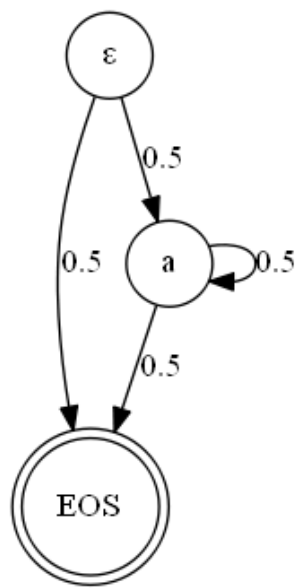

(b)

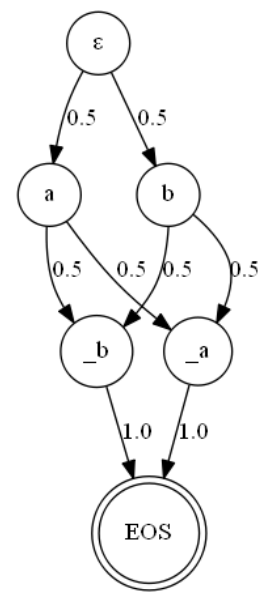

(c)

Figure 4.6: (a) Graph for expression 1 from table 4.6, (b) graph for expression 1 from Table 4.7, (c) graph for expression 3 from Table 4.7. The symbols _ $a$ and $\_b$ denote the symbols a and $\mathrm{b}$ are the second instances in the sequence.

\subsection{Subtraction}

Sequences and Subsequences To discuss subtraction, we must first discuss the concept of sequences and subsequences in behaviors of behavior expressions. Recall that a behavior is a sequence of basic actions. If $B$ is a behavior in a family of behaviors $F(\mathbf{B})$ and can be broken up into a concatenation three 'sub-behaviors': $X, Y, Z$, such that: $B=X Y Z$. The length of $X, Y$, or $Z$ can be anywhere between 0 and the length of $B$ or $|B|$, i.e. $B$ can be broken up into sub-behaviors of various sizes as long as the order is maintained. Therefore, if $B$ is a sequence of basic actions, $X, Y, Z$ are sub-behaviors of $B$. In the discussion of subtraction that follows, we use the term 'subsequence' and 'sub-behavior' interchangibly as they refer to the same thing. Subtraction is the removal of any of the subsequences or the sub-behavior of $B$. 
Function The behavior to subtract/remove is also called the "subtrahend behavior" and the behavior to be subtracted by the subtrahend is called the "minuend behavior". The subtraction operation removes a subsequence of a minuend behavior if and only if the subsequence matches the subtrahend behavior. If the minuend behavior is exactly the same as the subtrahend behavior, then the result of subtraction is the family of behaviors: $\{\epsilon\}$ or the $N A$ (No Action) behavior. Otherwise, the minuend behavior is unchanged. The symbol for the subtraction operator is the minus sign "-". Given two behavior expressions $\mathbf{C}$ and $\mathbf{D}$, the subtraction of $\mathbf{C}$ by $\mathbf{D}$ is written as the behavior expression $\mathbf{B}$ as follows:

$$
\mathbf{B}=(-\mathbf{C} \mathbf{D})
$$

The family of behaviors of a subtraction behavior expression is the set which consists of three kinds of behaviors from the family of behaviors of the minuend: 1) behaviors that is unchanged because no subtrahend behaviors is a subset of those behaviors, 2) behaviors from which the subsets of behaviors (i.e. the sequence of basic actions) that matches the behaviors of the subtrahend have been removed, and 3) $N A$ (No Action) if the minuend behavior is exactly the same as the subtrahend 
behavior.

$$
\begin{aligned}
\mathbf{B}=(-\mathbf{C} \mathbf{D}) & \left\{\begin{array}{l}
\{C \mid C=X Y Z \text { and } D \notin\{X, Y, Z\}\} \\
\{X Y \mid C=X Y Z \text { and } D=Z\} \\
\{X Z \mid C=X Y Z \text { and } D=Y\} \\
\{Y Z \mid C=X Y Z \text { and } D=X\} \\
\{\epsilon \mid C=D\}
\end{array}\right.
\end{aligned}
$$

Where: $C \in F(\mathbf{C})$ and $D \in F(\mathbf{D})$

$X, Y, Z$ are sub-behaviors of $C$

To illustrate how subtraction works, let's simplify our notation for concatenation for a moment. Given two behavior expressions $\mathbf{C}, \mathbf{D}$, and $\mathbf{E}$, normally we write the concatenation of $\mathbf{C}$ and $\mathbf{E}$ as $(\& \mathbf{C} \mathbf{E}$ ), and the concatenation of three behavior expressions with the order: $\mathbf{C}$ first, $\mathbf{D}$ second, and $\mathbf{E}$ third as (\& $\mathbf{C} \mathbf{D} \mathbf{E}$ ). For now, let's simplify the concatenation of the former to be written as $\mathbf{C E}$, and the latter be written as CDE . Subtraction of the behavior expression CDE (minuend) with the behavior expression $\mathbf{D}$ (subtrahend) can now be written as the behavior expression B:

$$
\begin{aligned}
\mathbf{B} & =(-\mathbf{C D E} \mathbf{D})=\mathbf{C E} \\
F(\mathbf{B}) & =F(\mathbf{C E})
\end{aligned}
$$

For a more concrete example, let's define the families of behaviors $F(\mathbf{C})=\{a, b\}$, $F(\mathbf{D})=\{x, y\}$, and $F(\mathbf{E})=\{i, j\}$, where $a, b, x, y, i, j$ are some behaviors. The subtraction operation in eq. 4.17 has the minuend behaviors in $F(\mathbf{C D E})$ and the 
subtrahend behaviors in $F(\mathbf{D})$. The subtraction means: any occurences of behaviors in $F(\mathbf{D})$ either $x$ or $y$ is removed from the behaviors in $F(\mathbf{C D E})$. The family of behaviors from a subtraction operation is shown below:

$$
\begin{aligned}
F(\mathbf{C}) & =\{a, b\} \\
F(\mathbf{D}) & =\{x, y\} \\
F(\mathbf{E}) & =\{i, j\} \\
\mathbf{C D E} & =(\& \mathbf{C} \mathbf{D} \mathbf{E}) \\
F(\mathbf{C D E}) & =\{a x i, a x j, a y i, a y j, b x i, b x j, b y i, b y j\} \\
\mathbf{B} & =(-\mathbf{C D E} \mathbf{D}) \\
F(\mathbf{B}) & =\{a i, a j, b i, b j\}=F(\mathbf{C E})
\end{aligned}
$$

If subtraction is written with multiple behavior expressions as operands, the subtraction is assumed to be performed sequentially between every two behavior expressions starting from the first and second operands. For example: given the behavior expressions $\mathbf{C}, \mathbf{D}, \mathbf{E}, \mathbf{F}$ and the subtraction behavior expression $\mathbf{B}=(-\mathbf{C} \mathbf{D} \mathbf{E} \mathbf{F})$, the subtraction operation is performed first between the behaviors in $F(\mathbf{C})$ (as minuend) and $F(\mathbf{D})$ (subtrahend). Then, a second subtraction is performed on the family of behaviors $F(-\mathbf{C} \mathbf{D})$ as the minuend, and $F(\mathbf{E})$ as the subtrahend, i.e. $(-(-\mathbf{C} \mathbf{D}) \mathbf{E})$. The process is repeated for subsequent operands.

$$
\begin{aligned}
\mathbf{B} & =(-\mathbf{C} \mathbf{D} \mathbf{E} \mathbf{F}) \\
& =(-(-(-\mathbf{C} \mathbf{D}) \mathbf{E}) \mathbf{F})
\end{aligned}
$$

Table 4.8 shows some more example of subtraction operations, where $\{a, b, c\} \in \Lambda$. 
Table 4.8: Additional Subtraction Example Usage

\begin{tabular}{cc}
\hline Behavior expression (B) & Family of behaviors $(F(\mathbf{B}))$ \\
\hline$(-a b)$ & $\{a\}$ \\
$(-a a)$ & $\{\epsilon\}$ \\
$(-(\& a b c) b)$ & $\{a c\}$ \\
$(-(+a b c) c)$ & $\{c\}$ \\
$(-(\& a b c)(\& a b))$ & $\{a c b\}$ \\
$(-(\& a c b)(\& a b))$ & $\{b c b, a c a\}$ \\
$(-(\& a b c b a)(+a b))$ & \\
\hline
\end{tabular}

Probabilistic Behavior Currently, subtraction operation is deterministic. Subtraction only appears probabilistic when the subtrahend expression is probabilistic like union or repetition.

\subsubsection{Other/Future Behavior Expression Operations}

We envisioned new operators can be developed to expand behavior expressions in future works. Below we discuss one such operation that we have begun designing, but not yet implemented in the current version of REBeL: the concurrency operation.

4.3.2.5.1 Concurrency In the context of composing robot motions and behaviors, we define concurrency as: two or more motions or behaviors to appear to be executed at the same time. For example: suppose there are two robot behaviors: $W A V E \_R I G H T \_A R M$ and $W A V E \_L E F T \_A R M$ from eq. 4.3. "Concurrency" means both behaviors are executed in such a way that it appears the robot is waving with both arms. Future works would look to implement concurrency by using methods and formalisms such as interleaving processes [138] or Communicating Sequential Processes (CSP) [139].

The concurrency operation can accept any number of arguments, and if the arguments are actionable, then they will be executed at the same time. Each of the 
Table 4.9: Example usage of concurrency operation

\begin{tabular}{cc}
\hline Behavior expression $(\mathbf{B})$ & Family of Behaviors $(F(\mathbf{B}))$ \\
\hline$(\% \mathrm{a} b)$ & $\{\mathrm{a} \% \mathrm{~b}\}$ \\
$(\%(\mid \mathrm{a} b) \mathrm{b})$ & $\{\mathrm{a} \% \mathrm{c}, \mathrm{b} \% \mathrm{c}\}$ \\
$(\%(\mid \mathrm{a} b) \mathrm{b}) \mathrm{d})$ & $\{\mathrm{a} \% \mathrm{c} \% \mathrm{~d}, \mathrm{~b} \% \mathrm{c} \% \mathrm{~d}\}$ \\
\hline
\end{tabular}

argument is an expression. The output of this operation is a sequence where each item in the sequence is a combination of the symbols at each index, or the result of conflict resolution (explained below). This operation is useful to compose a set of simple behaviors into a complex behavior.

There are two main coordination nuances to concurrency in REBeL:

- Start and end times of behaviors

- Priority/conflict of resources between behaviors

Because each behavior may have different duration, there are several options to handle the start and end times of events. The simplest option is to start all behaviors at the same time and let each behavior finish on their own. The second option is to have all behaviors end at the same time. This can be done by simply end-aligning the motion behaviors and zero-padding in the beginning of the shorter behaviors in the set. The third option is to have triggers where one or more behaviors start only after a behavior reaches a certain point. The last option is for the user to explicitly give the start times (or delays) for each behavior.

Additional arguments are:

- Delay: this argument denotes the delay time of starting the execution of the expression-arguments. This can be a single value or a list of values. The default value is 0 and all items are executed immediately at the same time. With a single value, all items are executed at the same time after the delay. 
Table 4.10: Concurrency Delay Argument Usage and Examples

\begin{tabular}{|c|c|c|}
\hline Behavior expression $(\mathrm{B})$ & $\begin{array}{l}\text { Family of } \\
\text { Behaviors } \\
(F(\mathbf{B}))\end{array}$ & Expected Result \\
\hline$(\%$ a $\quad b)$ & $\{a \% b\}$ & $\mathrm{a}$ and $\mathrm{b}$ executed at the same time \\
\hline$(\%$ a b $\{$ delay: 0.5$\})$ & $\{a \% b\}$ & $\begin{array}{l}\mathrm{a} \text { and } \mathrm{b} \text { executed after } 0.5 \text { seconds } \\
\text { delay }\end{array}$ \\
\hline $\begin{array}{l}(\%(\mid \text { a b }) \text { c }\{\text { delay: } \\
[0.5,0.5]\})\end{array}$ & $\{a \% c, b \% c\}$ & $\begin{array}{l}\text { a executed after } 0.5 \text { seconds de- } \\
\text { lay; c executed } 0.5 \mathrm{~s} \text { after a is ex- } \\
\text { ecuted. OR } \\
\mathrm{b} \text { executed after } 0.5 \text { seconds de- } \\
\text { lay; } \text { executed } 0.5 \mathrm{~s} \text { after b is exe- } \\
\text { cuted. }\end{array}$ \\
\hline $\begin{array}{l}(\%(\&(\mid a b) c) d \\
\{\text { delay: }[0.0,0.7]\})\end{array}$ & $\begin{array}{l}\{a c \% d\} \\
\{b c \% d\}\end{array}$ & $\begin{array}{l}\text { The sequence ac executed imme- } \\
\text { diately; d executed after } 0.7 \text { sec- } \\
\text { onds delay OR } \\
\text { The sequence bc executed imme- } \\
\text { diately; d executed after } 0.7 \text { sec- } \\
\text { onds delay }\end{array}$ \\
\hline
\end{tabular}

With a list of delay values, the number of values must be equal to the number of expression-arguments, where each value denotes the start delay of the expression-arguments. Table 4.10 shows some examples on using the start delay arguments.

- Priority: this argument denotes the priority of the concurrent behaviors and used to resolve conflicts. By default, the order of priority from high to low is first to last expression-arguments, respectively. Table 4.11 shows some example of using th priority argument, where behaviors $\mathrm{a}$ and $\mathrm{b}$ have conflicts.

Notice the expression $(\%(\&(\mid a b) c) d\{$ delay $:[0.0,0.7]\})$. Although it appears long, there are only two behavior expressions: (\& ( $\mid$ a b b c) and d. Therefore, only 
Table 4.11: Concurrency Priority Argument Usage and Examples

\begin{tabular}{|c|c|c|}
\hline Expression & $\begin{array}{l}\text { Output } \\
\text { Set }\end{array}$ & Expected Result \\
\hline$\left(\begin{array}{lll}\% & a & b\end{array}\right)$ & $\{a \% b\}$ & $\begin{array}{l}\mathrm{a} \text { and } \mathrm{b} \text { executed at the same } \\
\text { time; will be executed as-is even } \\
\text { with conflicts }\end{array}$ \\
\hline $\begin{array}{l}(\% \text { a b fpriority: }[0, \\
1]\})\end{array}$ & $\{a \% b\}$ & $\begin{array}{l}\text { a has higher priority than } \mathrm{b} \text {; if } \\
\text { there is conflict, only a is exe- } \\
\text { cuted }\end{array}$ \\
\hline $\begin{array}{l}(\% \text { a b fpriority: } \\
0]\})\end{array}$ & $\{a \% b\}$ & $\begin{array}{l}\mathrm{b} \text { has higher priority than } \mathrm{a} \text {; if } \\
\text { there is conflict, only } \mathrm{b} \text { is exe- } \\
\text { cuted }\end{array}$ \\
\hline
\end{tabular}

two start-delay values are needed. Start delay values are delays in seconds from the start of execution (absolute).

Conflict Resolution Executing multiple behaviors concurrently poses a risk that the behaviors need the same resources on the robot causing conflict of resources and need for resolution. By resources, we mean joints, audio output, LED, wheels, etc. Here, we are focusing on motion-based behaviors, so the relevant resources are joints/actuators. Because of this, concurrency will result in the merging of the joint angle values for all the joints on the robot of the concurrent behaviors.

One way to resolve the conflict is to give one behavior higher priority than another. Another way is to combine the actions of the resources in the concurrent behaviors in a coherent manner. There two types of conflict:

- Compatible: two or more behaviors using the same resource that the net results are the same. Suppose two behaviors are executed concurrently: behavior A $\left(b_{A}\right)$ and behavior $\mathrm{B}\left(b_{B}\right)$. $b_{A}$ only has one step and turns the head to the right for 45 degrees. $b_{B}$ turns the head to the right for 45 degrees and then raise 


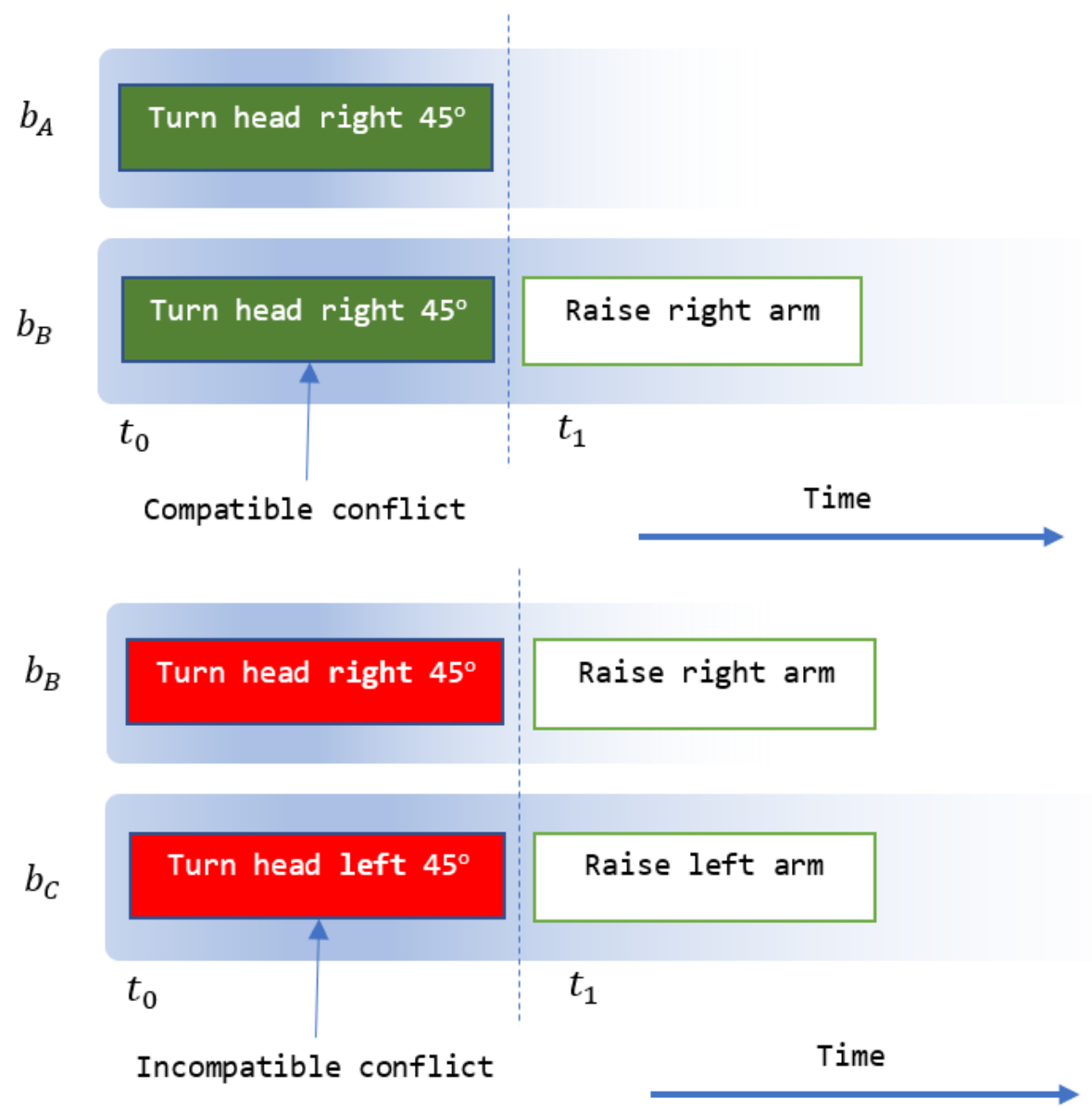

Figure 4.7: Two types of conflicts: compatible (top) and incompatible (bottom).

the right arm. The there is a conflict on the use of the neck DOF, but since the two actions are the same, they can be merged.

- Incompatible: two or more behaviors using the same resource with differing actions. Suppose two behaviors $b_{B}$ and $b_{C}$ where $b_{C}$ turns the head left and raise the left arm. Since $b_{B}$ turns the head to the right, i.e. in the opposite direction, an incompatible conflict occurs. 


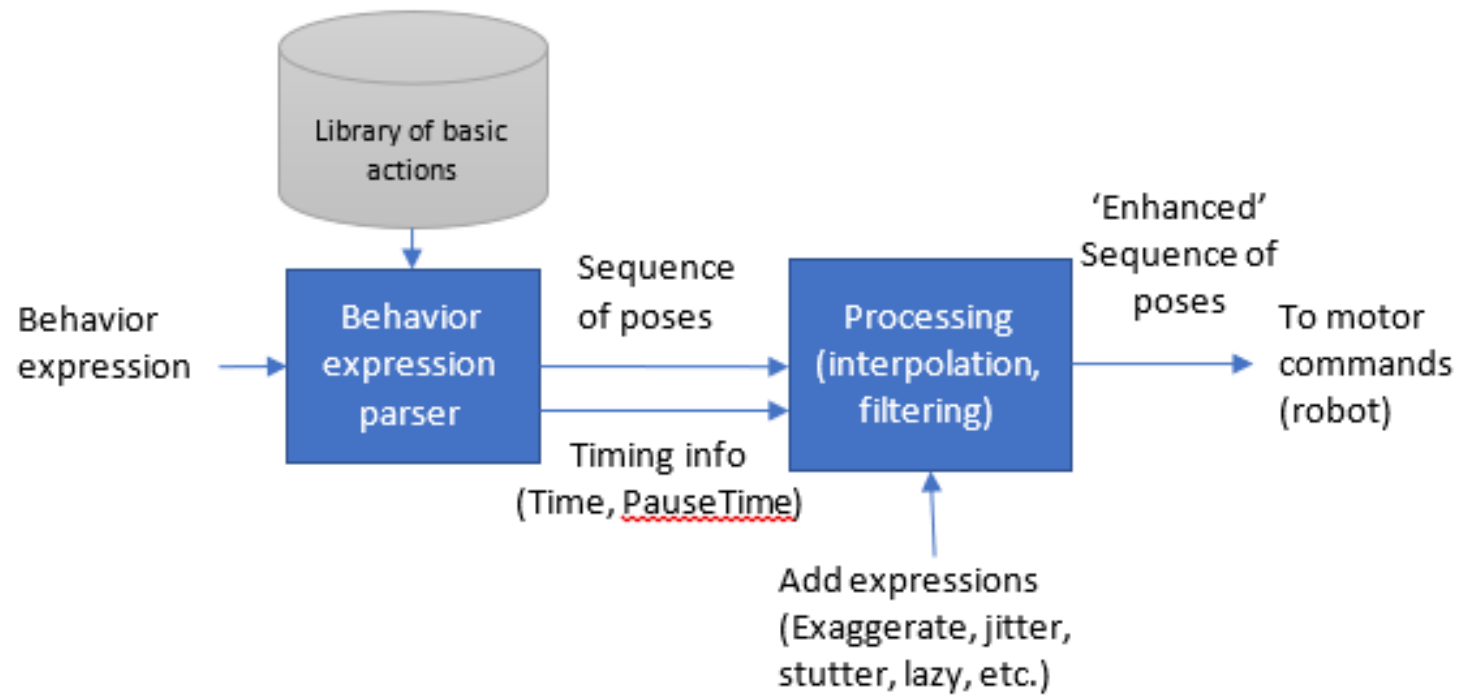

Figure 4.8: The REBeL pipeline. A behavior expression is given as input to the behavior expression parser. The behavior expression parser will evaluate the expression and produce an instance of behavior sequence. Each symbol in the behavior sequence is converted into their corresponding motion data from the Database of Poses. The output of the behavior expression parser is a sequence of numbers which correspond to joint angle positions (i.e. sequence of poses) and some timing information (i.e. Time, PauseTime). The timing information is used to interpolate the poses to create the desired motion dynamics. Additionally, expressions can be added by applying filtering methods [13]. The interpolated and filtered motion data is then converted into motor commands.

\subsubsection{The REBeL Pipeline}

We have developed a system for robot motion generation using REBeL in the form of a pipeline shown in Fig. 4.8. The pipeline takes a behavior expression as input, which then evaluated by the behavior expression parser (i.e. eval() call) to produce a new instance of motion data (sequence of poses). We currently use this pipeline to generate motions on the HROS-1 and Jeeves humanoid robots but the pipeline can be extended for other robots as well.

Motions produced by REBeL consists of sequences of poses where each pose de- 
fined using the format used by the Robot Motion Editor for Windows (WinRME) tool for HROS-1 [55] and the database of HROS-1 motions that was created by Mekana Burch $^{5}$. Each pose consists of 20 joint angle values for the 20 DOFs on the HROS-1 robot, with additional two values for timing: Time and PauseTime for a total of 22 values per pose. Using REBeL, we can produce motions with sequences of arbitrary number of poses, as opposed to RME which limit each motion to a sequence of 7 poses at a time. REBeL also allows us to quickly create many different varieties of motions using its probabilistic operators by arranging motions of gestures in different combinations and order, and with different number of repetitions.

To add motion texture [140], which are styles of motion such as: jitter/tremor, stutter, smoothing, or exaggeration, before the motion data is sent as a sequence of commands to the robot, the sequence of poses and the timing information for each pose are passed through the Processing block (Fig. 4.8). Later in Chapter Six, we introduce our methods used in this Processing block to create motion textures using a polynomial interpolation called Kochanek-Bartels interpolation [141] and multiresolution filtering using wavelets [142]. After the desired motion texture is applied, the processed motion data only consists of pose data which can be sent to the robot to be executed.

\subsection{Results}

We evaluated REBeL by creating several expressions to compose composite behaviors using library of behaviors created from past works ${ }^{6}$ and new ones. We tested several expressions that utilize the operators concatenation, union, repetition, and subtraction in a multitude of ways. The expressions are tested on the robot HROS-1 and a

\footnotetext{
${ }^{5}$ Unpublished work

${ }^{6}$ Motion library for HROS-1 robot created by Makana Burch (unpublished work).
} 
simulated environment using the Gazebo simulator.

\subsubsection{Probabilistic Behaviors}

We evaluated the behavior of the probabilistic concatenation, union, and repetition operations. In particular, we are interested to see what is the frequency of occurrence of each possible output from the output set given different probability values on each operator. For each operator, we evaluated a simple expression with no nesting one thousand times $(N=1000)$, and this is repeated one hundred times $(M=100)$. Therefore, the sum of all the occurrences in each plot should be 1000. The numbers of occurrences for each operator shown below are the average of the one hundred runs.

\subsubsection{Concatenation}

Fig. 4.9 shows the results of concatenation operation for the expression ' $\& a b c \mathrm{p})$ with three symbols $\{a, b, c\}$ and three different probabilities p: $0.5,0.25$, and 0.7 . The output set of this probabilistic concatenation is $\left\{{ }^{\prime} a^{\prime},{ }^{\prime} a b^{\prime},{ }^{\prime} a b c^{\prime}\right\}$ In this case, the concatenation probability is the probability of continuing to concatenate the next symbol or otherwise ends the sequence.

If the full length of the concatenation sequence is $K$ (e.g. for the expression

above, $K=3$ ), and $\mathcal{M}$ is the number of occurrence of sequences of length $k$ with the probability of concatenation $p$ is:

$$
\mathcal{M}(k)=\left\{\begin{array}{l}
0 \quad \text { if } \quad k=0 \\
(1-p)\left(M-\sum_{i=1}^{k} \mathcal{M}(i)\right) \quad \text { if } \quad 0<k<K \\
M-\sum_{i=1}^{K-1} \mathcal{M}(i) \quad \text { if } \quad k=K
\end{array}\right.
$$



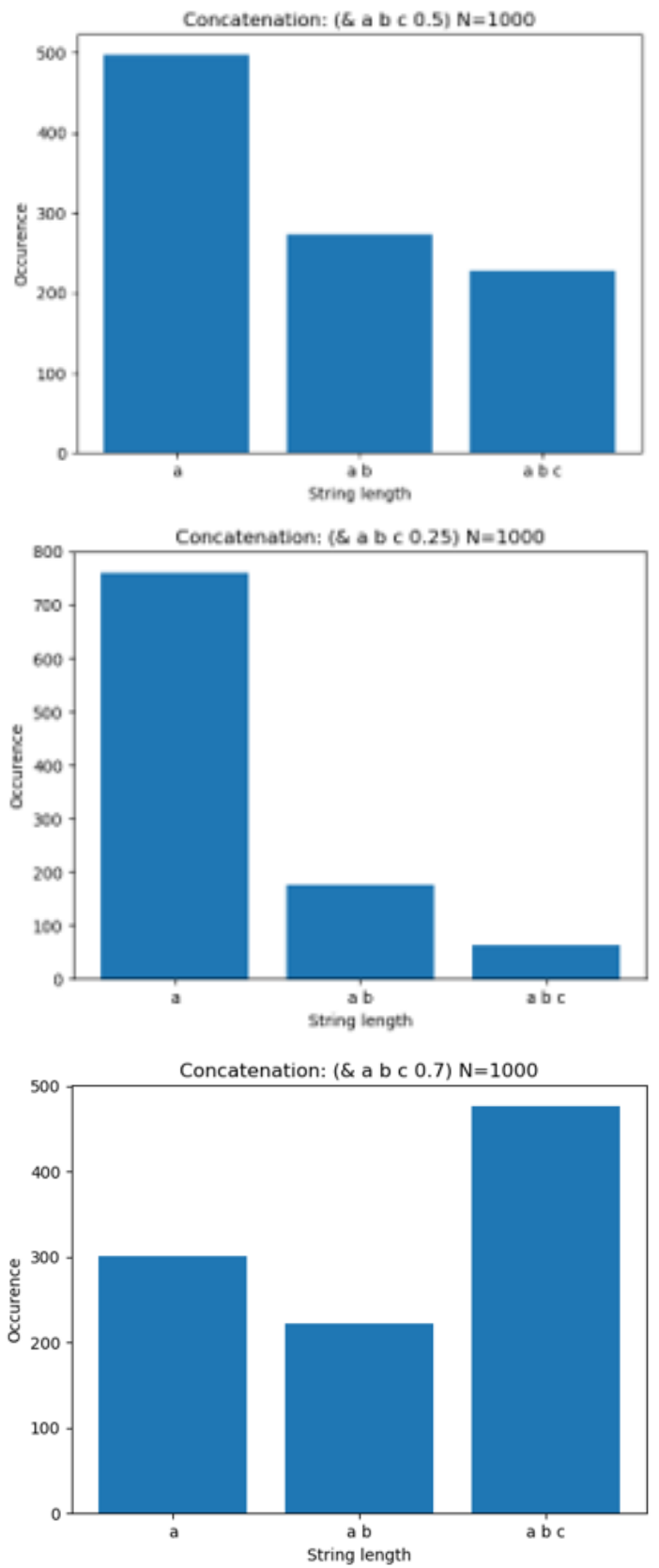

Figure 4.9: Occurrences of the output set from probabilistic concatenation operation for the expression: (\&abc p) with $p$ is the probability value, where: (top) $p=0.5$, (middle) $p=0.25$, (bottom) $p=0.7$. 


\subsubsection{Union}

Fig. 4.10 shows the occurrences of each element of the output set from the expression: $(+A B C \mathrm{p})$ where $p$ is the probability value. In the first top and bottom plots in Fig. 4.10 , only one probability value is given. This means the value $p$ only applies to the first argument in the expression (i.e. the symbol $A$ ). The remainder of the arguments in that expression have the probability $1-p$ distributed equally among each argument. The bottom plot in Fig. 4.10 shows the occurrences when the probabilities for each argument is provided.

\subsubsection{Repetition}

Fig. 4.11 shows the results of various ways of using the repetition operator with a sample expression $(* G)$. Fig. 4.11a shows the result when no probability value is given, the operator assumes $p=0.5$. Thus, $50 \%$ of the time, the expression will be evaluated to the empty string. Fig. $4.11 \mathrm{~b}$ and $4.11 \mathrm{c}$ show the results when $p$ is specified. In Fig. 4.11c in particular, with $p=2.6$ the REBeL repetition operator repeats at least twice, and applies $p=0.6$ for the subsequent repetitions. In Fig. $4.11 \mathrm{~d}$, probability is given as a Gaussian distribution by specifying mean $(\mu)$ and standard deviation $(\sigma)$. Using this distribution as repetition probability gives the user the ability to specify behaviors that "approximately repeats $x$ number of times" where $x$ is determined by the value $\mu$. However, when using the Gaussian probability, there are very low chances that the sequence generated will be very short or very long. In Fig. 4.11d the shortest sequence is 2 and the longest sequence is 19, but as shown, their occurrences are very low. 

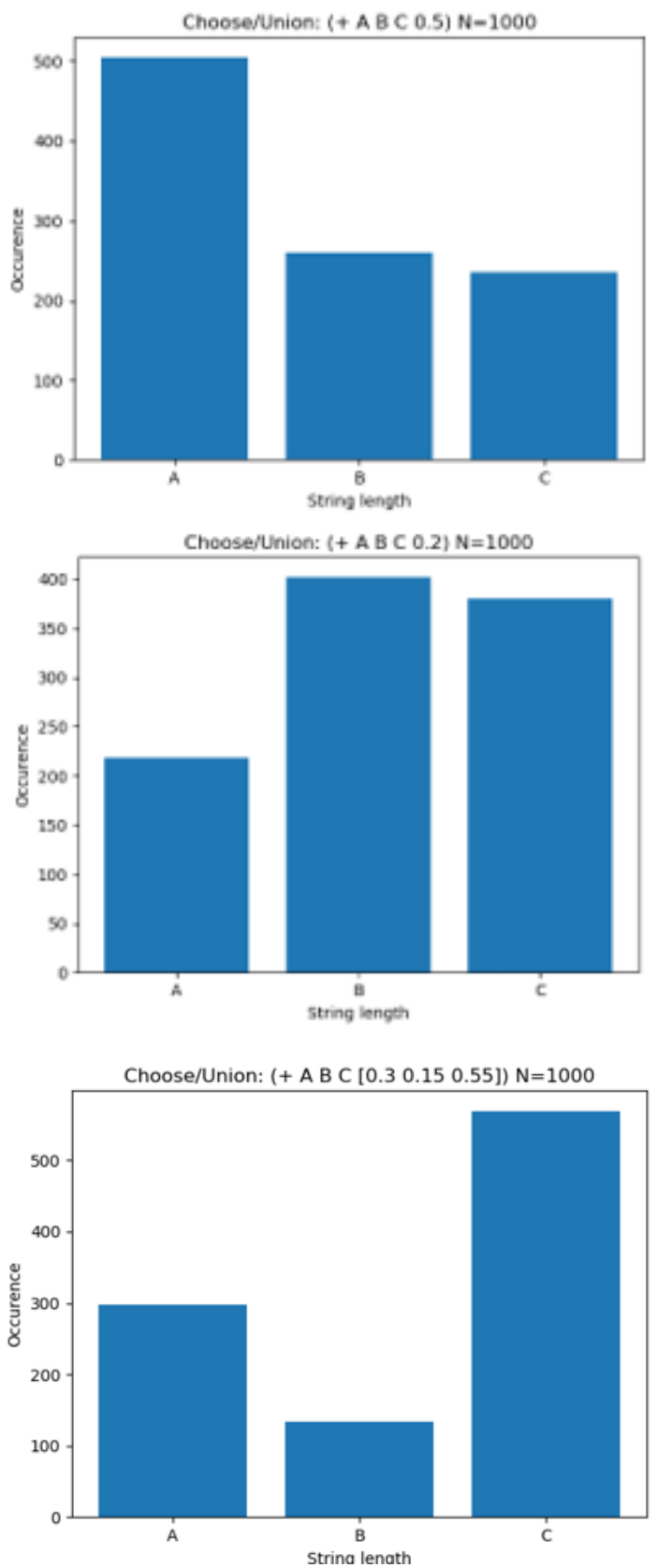

Figure 4.10: Occurrences of the output set from the probabilistic union operation for the expression: ( $+A B C \mathrm{p}$ ) with $p$ as the probability value, where: (top) $p=0.5$, (middle) $p=0.2$, (bottom) $p=[0.3,0.15,0.55]$. 


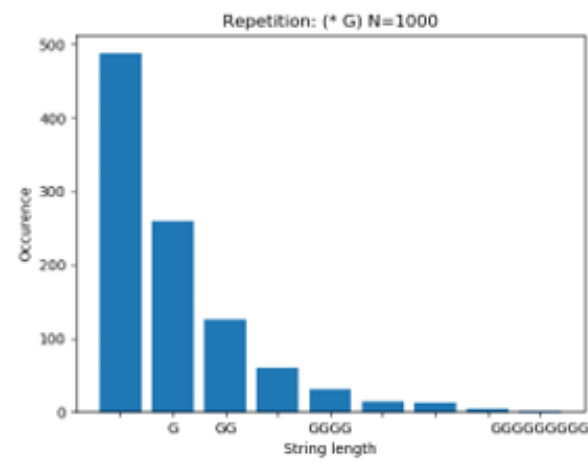

(a)

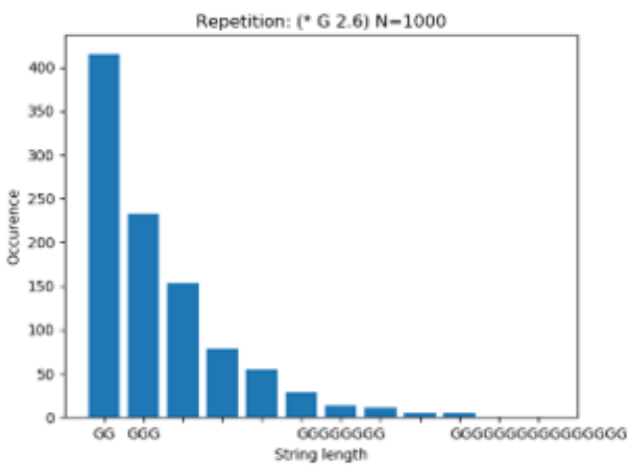

(c)

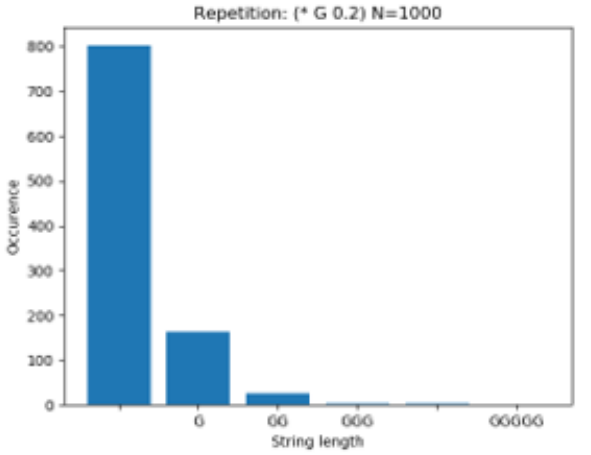

(b)

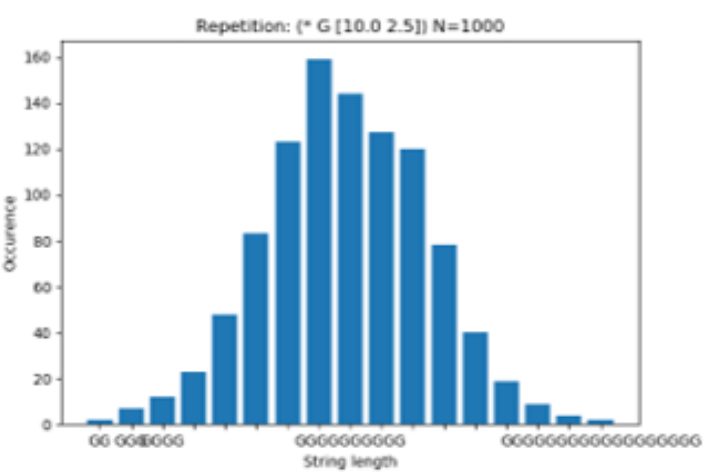

(d)

Figure 4.11: Occurrences of output set of a repetition expression $(* G)$ with different probability values $p$. (a) With default probability 0.5 , (b) $p=0.2 ; 80 \%$ of occurrences is the empty string, (c) $p=2.6$ repeats at least twice, (d) with Gaussian probability distribution by specifying mean and standard deviations $\mu=10, \sigma=2.5$ produces sequences of symbol $G$ with length around 10 . 

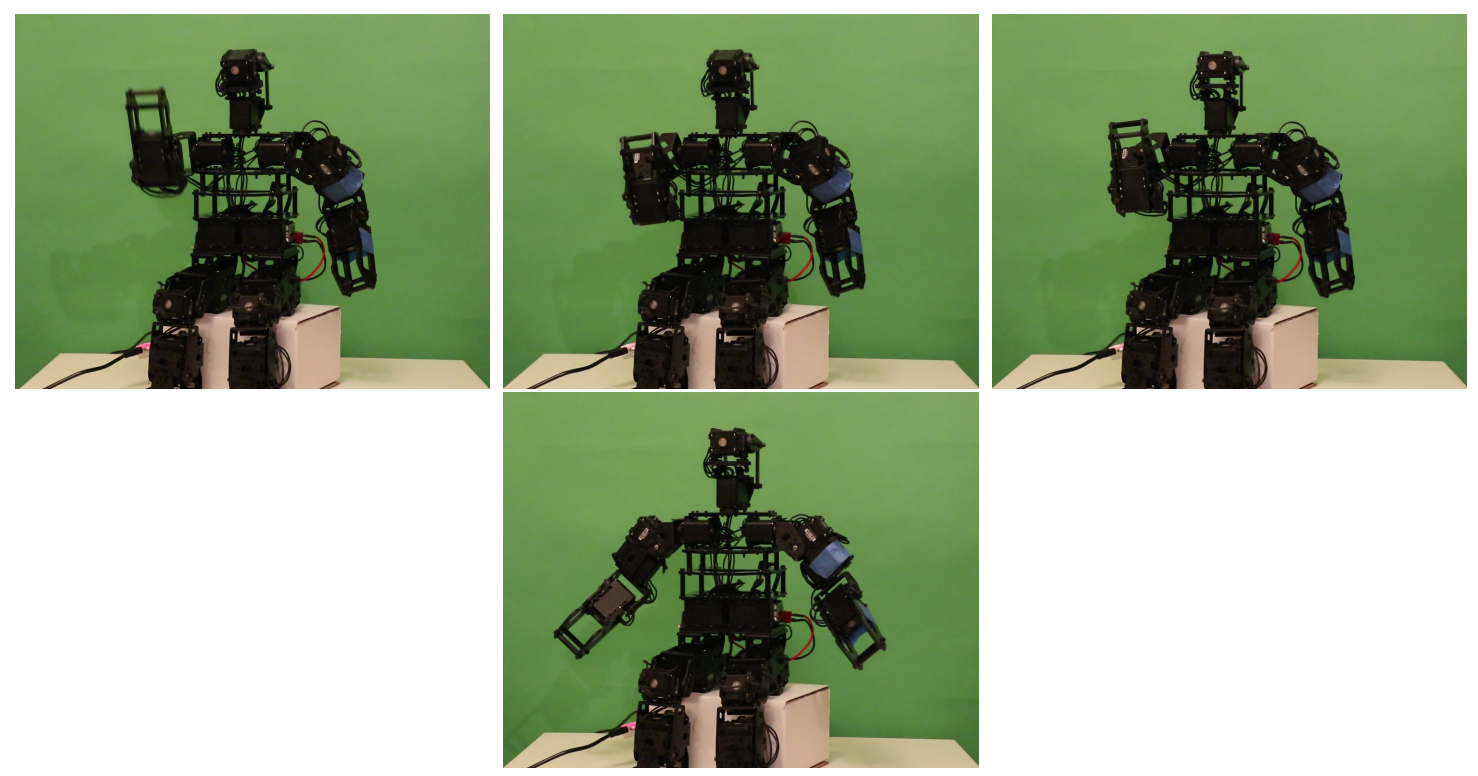

Figure 4.12: The sequence of poses of the "Yes" gesture on HROS-1 robot. Order: left to right, top to bottom.

\subsubsection{On HROS-1 Robot}

We selected a handful of pre-programmed individual behaviors that we use to compose more complex behaviors with longer sequences. The behaviors are:

- "Oops" - a gesture showing regret or remorse

- "Wave" - a hand-waving gesture

- "Clap" - a hand-clapping gesture

- "Wow" - a gesture showing awe

- "Yes" - a 'fist-pump' gesture

Figures 4.12 and 4.13 show the poses on the robot for the "Yes" and "Wow" gestures, respectively. Fig. 4.14 shows the HROS-1 model in the Gazebo simulation environment. We tested the generated motions both on the robot and in Gazebo. 


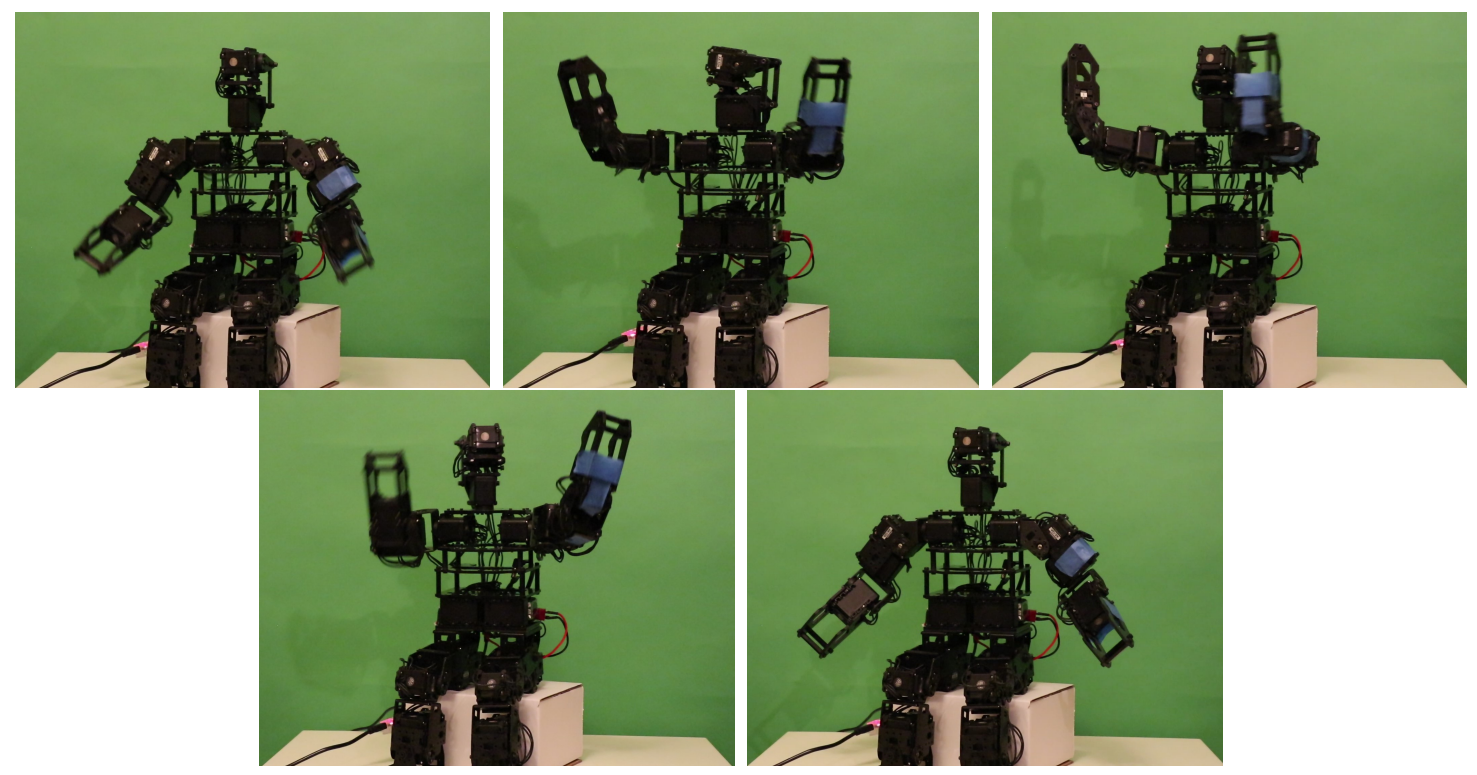

Figure 4.13: The sequence of poses of the "Wow" gesture on HROS-1 robot. Order: left to right, top to bottom.

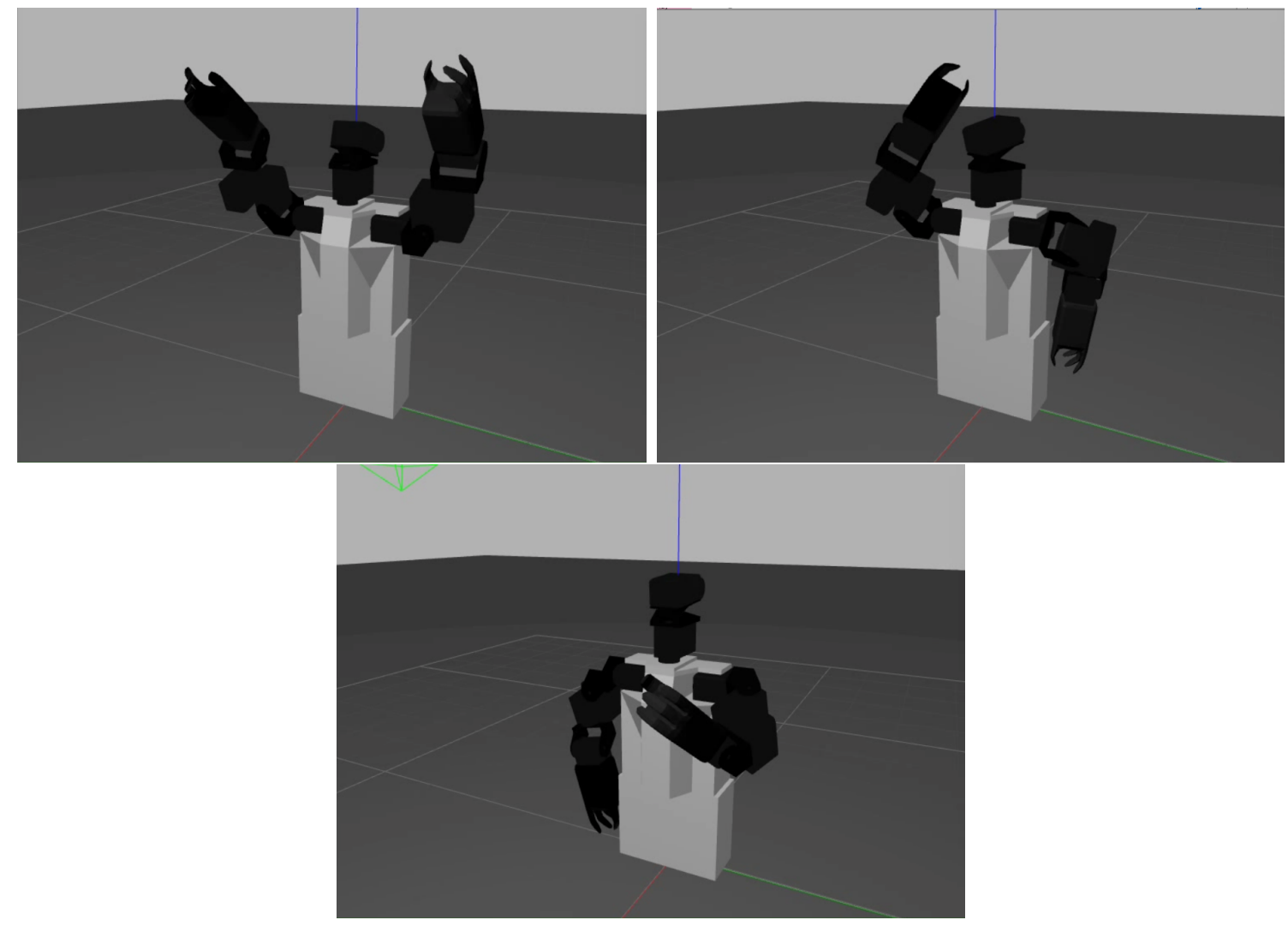

Figure 4.14: HROS-1 model in the Gazebo simulation environment. (Snapshots at various moments in simulation) 
The concatenation and union operations by themselves are very straightforward in producing a sequence and alternatives/choices, respectively. For example, the expression with concatenation (\& oops wave) is performed on the robot with first the "Oops" gesture until completion then followed by the "Wave" gesture also until completion. The union operation (+ oops wave) will only perform the "Oops" gesture half of the time, and the "Wave" gesture the other half of the time.

A more complex behavior can be obtained by using slightly more complex expressions, for example suppose a "Happy" behavior is written as:

$$
" \text { Happy" }=(+(\& \text { wow clap })(\& \text { wave clap }) \text { yes })
$$

Each time this expression is called and evaluated, the robot performs one of the three possible behaviors: \{Wow Clap", "Wave Clap", "Yes"\} which are "Wow" followed by "Clap", "Wave" followed by "Clap", or only the "Yes" gesture. Because there is no explicit probability given to the union operation, each behavior have equal probability of being executed; for any number of executions, each behavior will occur on average about $30 \%$ of the time.

The repetition operator can be used for creating extremely complex behaviors. For example, the "Happy" behavior above can be made more complex by adding repetition:

$$
" \text { VeryHappy" }=(*(+(\& \text { wow clap })(\& \text { wave clap }) \text { yes }) 2.5)
$$

Which will repeat evaluating the "Happy" expression at least twice. If the "Happy" behavior is registered in the REBeL symbol database, then the "VeryHappy" expres- 
sion can be made even simpler by using the "Happy" symbol instead:

$$
" \text { VeryHappy" = (* Happy 2.5) }
$$

It is then very easy to create even more complex behaviors by making what we call "composite expressions" which are expressions that consist of symbols that represents other expressions.

\subsubsection{Subtraction}

Here we discuss how the subtraction operator can be handy. Most of the gestures in our motion library starts and ends with the home pose. This makes the result of concatenating multiple gestures a little awkward because there are moments where the robot is moving towards the home pose in between gestures. Gestures are specified as REBeL expressions. At the lowest level, a non-operator symbol used in the expressions represents the series of joint angle values for the robot' ${ }^{7}$ The 'home' pose is represented by the 'stand' symbol. Most gestures starts and ends with the 'stand' symbol. For example:

$$
\begin{aligned}
& " Y e s "=\left(\& \text { yes } \text { yes }_{2} \text { yes } 3 \text { stand }\right) \\
& " W o w "=\left(\& \text { wow }_{1} \text { wow }_{2} \text { wow }_{3} \text { wow }_{4} \text { stand }\right)
\end{aligned}
$$

Where $y e s_{i}$ and $w_{0}$ are poses that defines the "Yes" and "Wow" gestures, respectively. At the end of each gesture, the robot should return to its 'home' pose indicated by the stand symbol. When a series of such gestures are concatenated together, the stand symbol will appear multiple times in the middle of the sequence. We can use the subtraction operator to remove these stand symbols so the concate-

\footnotetext{
${ }^{7}$ A symbol may also represent other types of commands e.g. play sounds, but currently we only represent poses.
} 
nated sequence appears as one contiguous sequence. If it is desired to have the robot return to the home pose at the end of the sequence, the stand symbol can be added at the end by using the concatenation operation. For example, we create a sequence that performs the "Yes" gesture followed by the "Wow" gesture that repeats twice:

$$
" Y e s W o w "=(*(\& Y e s \text { Wow }) 2.0)
$$

This expression will produce the sequence:

"YesWow" =yes yes $_{2}$ yes 3 stand wow $_{1}$ wow $_{2}$ wow $_{3}$ wow $_{4}$ stand yes $_{1}$ yes $_{2}$ yes 3 stand wow wow $_{2}$ wow $_{3}$ wow $_{4}$ stand

Notice that the symbol stand appears four times in the sequence. The plot of such sequence is shown in Fig. 4.15a.

We can get rid of the stand poses by using the subtraction operator:

$$
" Y e s W o w 2 "=(-(*(\& \text { Yes Wow }) 2.0) \text { stand })
$$

which will give the following sequence:

$$
\begin{aligned}
\text { "YesWow2" }= & y_{1} \text { yes }_{2} \text { yes }_{3} \text { wow }_{1} \text { wow }_{2} \text { wow }_{3} \text { wow }_{4} \\
& \text { yes }_{1} \text { yes }_{2} \text { yes }_{3} \text { wow }_{1} \text { wow }_{2} \text { wow }_{3} \text { wow }_{4}
\end{aligned}
$$

Fig. 4.15 shows the plots of the motion data before and after the stand symbol is removed from the sequence. The top plot (Fig. 4.15a) shows the motion data with the stand poses still in place; they are visible around frames 110, 290, 430, and 600 onward. The bottom plot (Fig. 4.15b) shows the motion data after the subtraction 
operation. The resulting motion on the robot appear more as a single, contiguous motion sequence after the intermediate stand poses are removed and the rest of the sequence remain unchanged. The side effect of this operation is that the end of the sequence does not end with the home position.

If it is desired for the robot to return to the home position, the stand symbol can be added at the end using concatenation:

$$
" Y e s W o w "=(\&(-(*(\& Y e s \text { Wow }) 2.0) \text { stand }) \text { stand })
$$

Video demonstration of results of using the subtraction operation can be viewed here: https://youtu.be/FgocbQAp91Y.

The above are only a few examples of the features of REBeL that we have not seen in other robot motion editing tools. There are more ways REBeL can be used to create complex motions and behaviors. However, the main limitation of the current version of REBeL is that there is no feature in REBeL that lets users manipulate the timing information or manipulation of the existing data directly. To address these, in the next chapter we present our method of using MIDI music data to obtain timing information for expressive motion without requiring the user to manually enter timing information. In the chapter following that, we present our method of using interpolation and wavelet transforms to change the shapes of the motion data, again to create interesting effects that can enhance the basic motion data to be more expressive.

\subsection{Multimodality}

REBeL can easily be extended to support not just motions, but also other modalities such as facial animation, or voice. This feature is demonstrated on the robot Jeeves' 


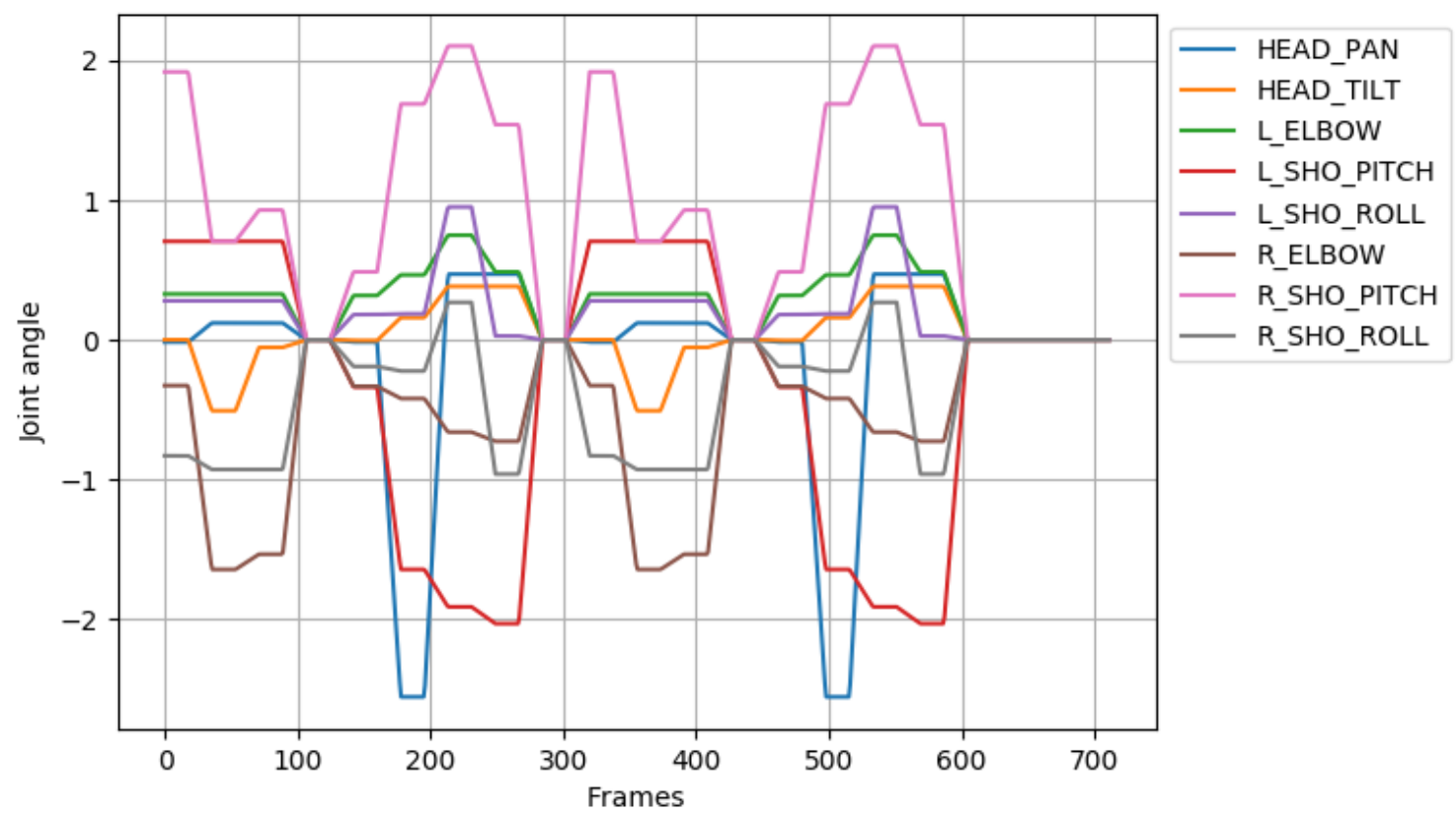

(a)

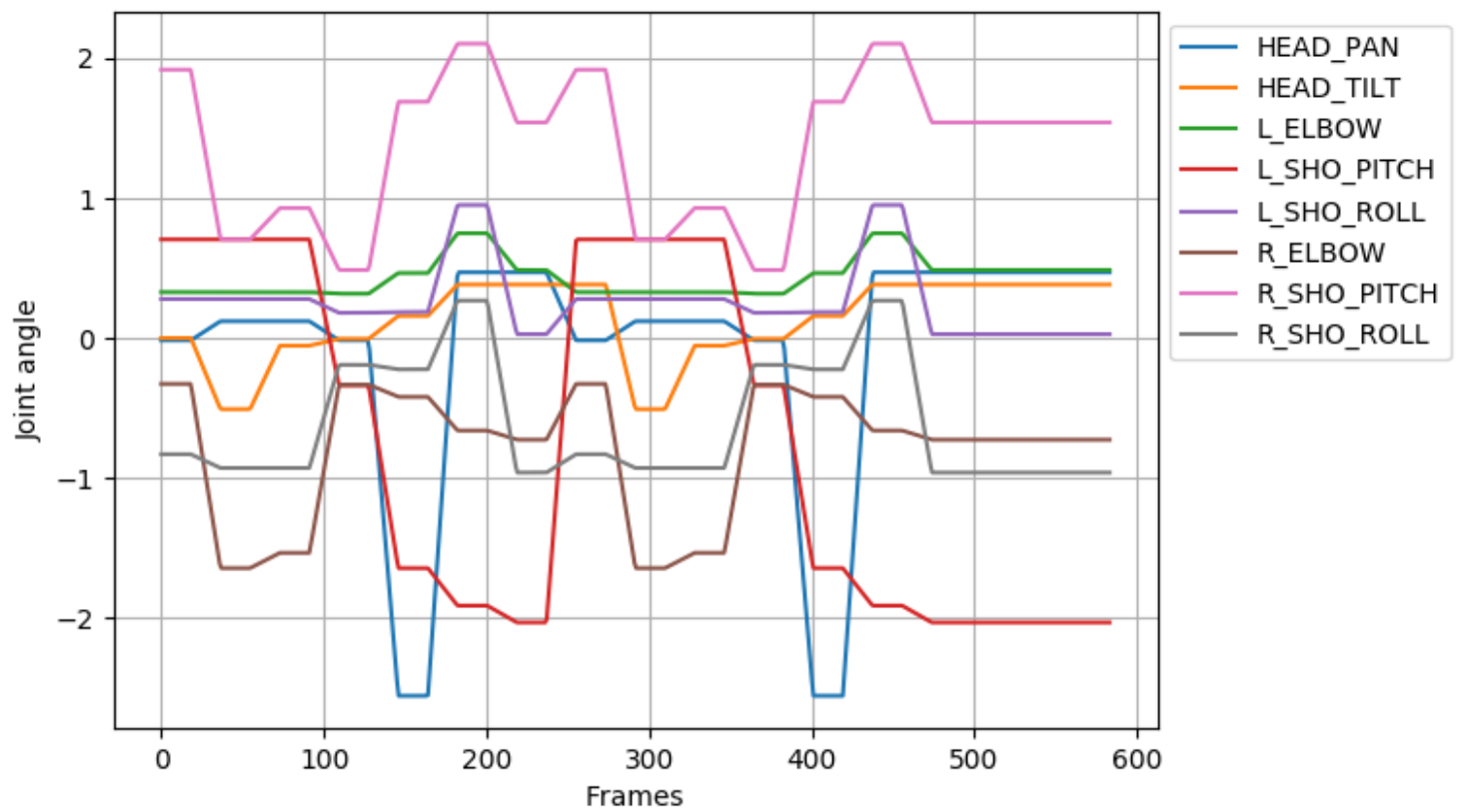

(b)

Figure 4.15: Motion data of the expression 4.25: (a) the original sequence (Eq. 4.26) with the stand poses at around frames 110, 290, 430, and 600 onward., (b) after the subtraction of stand pose (Eq. 4.28). 


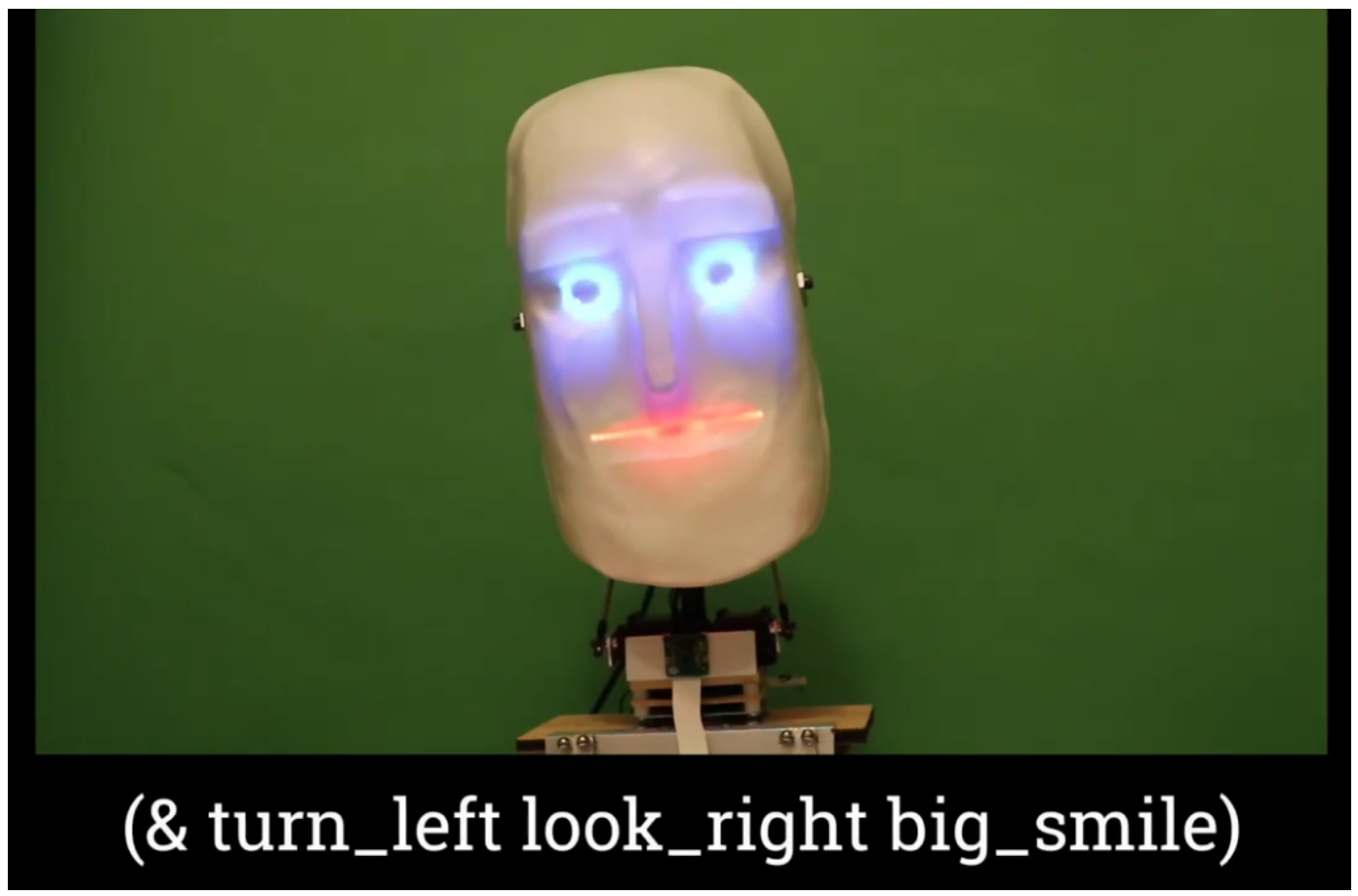

Figure 4.16: Video of animating Mr. Jeeves' head using REBeL.

head by controlling both the neck movements and the facial animations of eyes and mouth using LEDs simultaneously (Fig. 4.16). Video of this demonstration can be viewed on the internet: https://youtu.be/ENZxQLOVgIQ.

\subsection{My Contributions}

In this chapter I introduced REBeL, a tool I created to compose robot motions using algebraic expressions called Behavior Expressions (BE). I also introduced new probabilistic variants of the composition operators: concatenation, union, and repetition. In addition, I have also implemented the new operator: subtraction. To address the current issues and limitations with the current methods and tools, REBeL provides the following benefits:

- Quick prototyping: motions can be quickly prototyped by modifying BEs op- 
erators and arguments. In contrast, using the currently available motion editing tools, prototyping motions require creating a new motion record, duplicating joint angle and timing values from other motion records, and rearranging the order of poses requires multiple steps and is cumbersome.

- Families of behaviors: a single BE can represent a family of motions for a type of behavior, which can produce very many variants of individual motions by choosing different poses or number of repetitions by using the probabilistic operators. In contrast, current motion editors must specify individual behavior as separate motion records [15][16], and scripting tools only allows random choice between a set of behaviors [11][143].

- On-line generation of behaviors: BEs are evaluated at runtime so different sequences for motions, gestures, and behaviors can be generated before loading the actual poses/commands (pre-loading). In the current methods, complete motion records or files must be loaded for each motion to be executed. Changing the order in a sequence of poses is done after the sequence is loaded (postloading), which require more complex manipulations.

- Expressiveness: the semantics of REBeL operators used in BEs make them easier for the user to understand the expected outcomes of behavior expressions and the corresponding family of behaviors.

- Hierarchical composition: complex motions and gestures can be constructed by referencing previously-defined motion names/symbols and composing them in a BE.

The features of REBeL (at its current state) is compared with current tools of RME [15] representing the most basic tool, Choreographe [16] representing the more 
advanced tool, and the more esoteric solutions of Improv [11], and BML Sequencer [75] and shown in Table 4.12: 

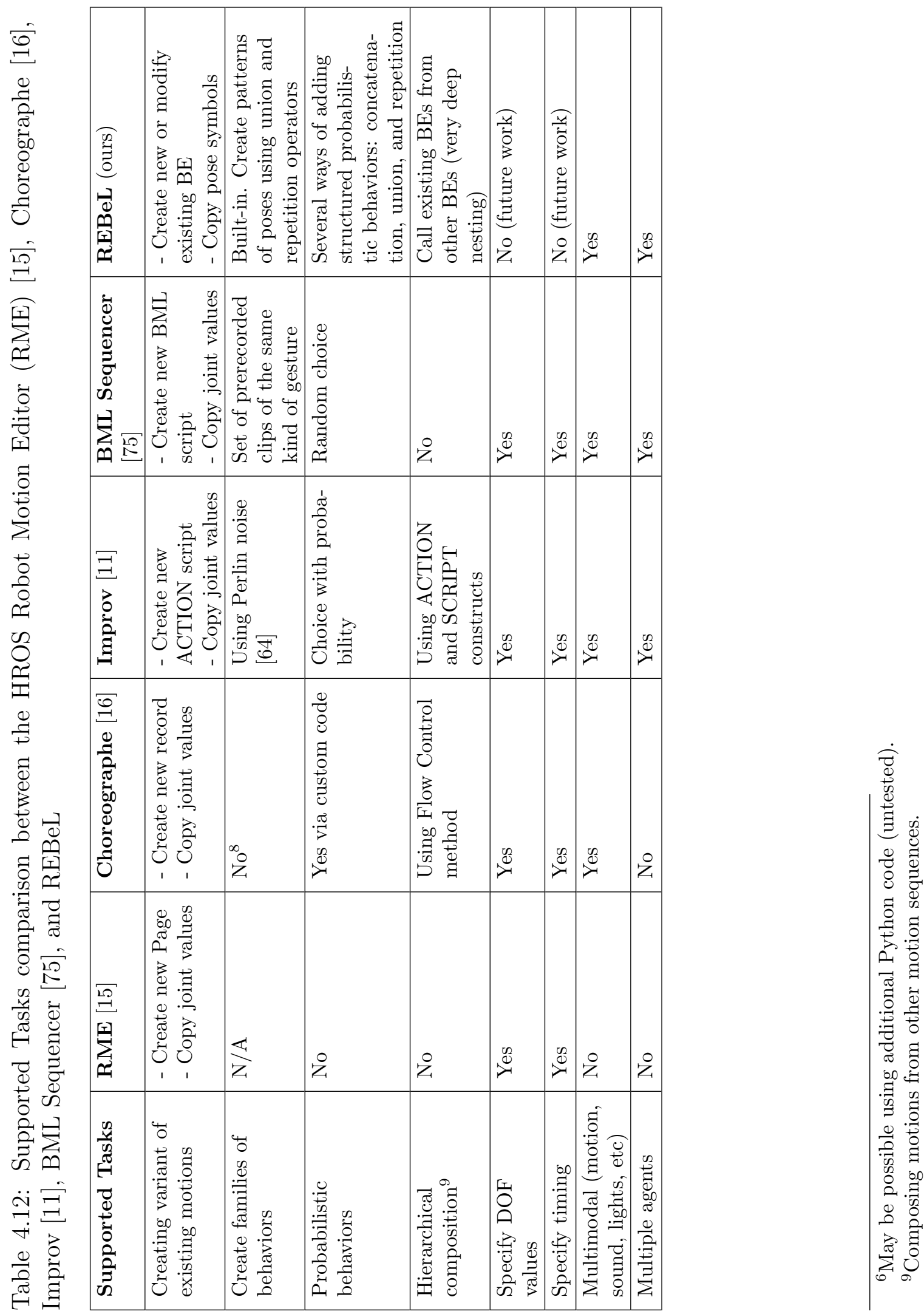


\section{CHAPTER 5 MUSIC TO MOTION}

This work has been published in the following publication: M. Sunardi and M. Perkowski, "Music to motion: Using music information to create expressive robot motion," International Journal of Social Robotics, vol. 10, no. 1, pp. 43-63, 2018 $[130]$.

\subsection{Introduction}

In this chapter, I introduce a method to use music information as a way to create expressive robot motions. Timing is one of the most crucial components in creating emotive and expressivity in motions [71] [69]. In the current methods of animating humanoid robots, timing information has to be done manually for each pose and requires many iterations with trial and errors [15][55][16]. The method proposed in this chapter showed that timing and therefore, expressivity for the entire duration of motions can be created by using timing and note interval information from music that embodies the desired expression e.g. happy, sad, energetic, or lazy, as an alternative to manually editing the timing on each pose of a motion data.

Music can influence its listeners emotionally. Music that possesses certain structure or dynamic properties may invoke certain kinds of emotions. For example, songs written in the major scale tend to be perceived as happy or bright, while music in minor scale tends to be perceived as sad or tragic [144]. Harmonics and melody may also induce emotional effects. Other properties such as tempo, timbre (distinguishes the instrument), and volume can influence excitement to the audience; sometimes referred 
to as the energy in the music. The energy in a rock-and-roll music is very different from a slow blues music. Tempo and rhythm were shown to also have physical effects on the audience [145].

Dancers can express these emotions or dynamics in the music through the movements of their bodies. A 'happy' tune can be expressed by movements that appear smooth, effortless, with wide range of motion, and the dancer seemingly 'light on their feet.' A 'somber' tune on the other hand, is often expressed in movements that appear heavy and slow, as if the dancer is fighting gravity. While these vocabularies are not very technical, we believe they sufficiently convey our point. Unfortunately, to use a more specific vocabulary to describe motions requires the reader to first be familiar with a specific motion theory. There are many different methods from performance arts that attempt to describe the properties of movements from dance or general motion theories both for analysis and synthesis, but we mainly adopt the vocabulary used in [146] and [43].

Our vision is to develop the field of robot theater perkowski2013synthesis, [147]. In a robot theater, there are multiple robots that portray the characters in the theater play. We call these robots as robot actors [148]. We envision the robot actors do not have to be of the same kind; some can be humanoid, others can be wheeled, animal-like, or even flying. Even the stage itself can be considered as a robot. Our long-term goal is to build a complete system to manage the complete robot theater. Here, we focus mainly on the motions of humanoid and anthropomorphic robots. We consider a robot humanoid if it has a human-like structure: head, shoulders, arms, torso, legs. Anthropomorphic are robots that possess parts or limbs that resemble or behave like a human limb, e.g. a robot arm can behave like an arm, or a head with a long neck.

The problem being addressed by this study is as follows. We observed that even 
with the guide of a script, creating motions for a humanoid robot in our robot theater requires a lot of effort. In particular, it is a difficult and time consuming task to manually fine-tune the motions so they appear more convincing, and believable to the audience. The few gestures that were eventually made, often only have short, discrete sequences of poses, and the result appears too robotic and mechanical. Therefore, we are looking into ways to make it easier to create many robot motions with longer, continuous sequences, in such a way that the produced motions appear expressive and natural without requiring a lot of manual refinement work by the animator of the robot theater. This work introduces an approach to exploit dynamics information from music to generate and enhance robot motions for a robot theater based on the work by Sunardi [149].

This work asks two questions: 1) can the dynamics information in music be used directly to produce robot motions that can be perceived as expressive by people, and 2) can the dynamics information in music be used to enhance an existing motion sequence by making the motion more expressive? Motions are considered expressive if the audience can agree that the movement corresponds to the music dynamics, similar to the dancers mentioned above. It must be emphasized that the goal of this approach is not to have a dancing robot per se, where the music plays as the robot moves, but to have the robot's motion system utilize the dynamics information in music data internally to produce dynamic-looking motions. Beyond robot theater, interactive robot applications such as a tour guide can also benefit from more expressive motions for a more natural human-robot interaction.

The opposite of what we consider as expressive movements are mechanical movements. Movements that appear mechanical have several characteristics. The movements are sudden, and seemingly have constant speed where acceleration and deceleration are hard to observe. There is also an observable starting and stopping of 
actions; typically there is a brief moment where the robot completely stops moving. This property is used by dancers who do 'The Robot' dance and 'popping' in hip-hop dances to make their movements look robotic. Conversely, typical human movements are continuous, with varying speeds, and the transitions between one action to the next are seamless. Sometime some parts of the body will move in succession instead of always start and stop together, as indicated by the Overlapping Action principle in the Disney animation principles [43].

Based on the desired movement qualities mentioned above, we focused our surveys on two adjectives: expressive and dynamic. We adopt the definition from the Merriam-Webster dictionary for "expressive": "showing emotions and feelings clearly and openly" [150]. For the word "dynamic", we adopt the definition also from Merriam-Webster dictionary: "always active or changing" [151].

We performed two experiments to evaluate this approach using two robots. One of the robot performs 'dancing' to music, and the other performs a Scenario. In the Dancing task, the robot will perform movements while MIDI music is being played, where the movements are created directly from the music data (offline, not in real time). A Scenario is a pre-programmed sequence of Gestures. A Gesture consists of a sequence of poses or joint configurations, but can be generalized to other activities such as blinking LEDs, playing a sound, etc. The Scenario will be executed by modifying the pre-programmed motion data with the dynamics extracted from music data. The goal of this experiment is to evaluate if dynamics information in music can be used to make robot movements that were designed by a person appear more expressive. The audience is asked to give their ratings on the performance of the robots in a survey questionnaire. The results suggest that applying dynamics information in music into robot motions using similar translations/rules in dance makes the robot appear more dynamic yet in a coherent manner. 
The remainder of this study is organized as follows. Section 5.2 discusses past and related works in similar area. Section 5.2.1 describes the details of our system to exploit music information for robot motion. Section 5.4 explains the experiment setup, followed by the results in Section 5.5. Section 5.6 discusses analysis of our data using non-parametric hypothesis testing methods. Included in this section is the analysis of data from a second survey, and comparison of results from using parametric versus non-parametric methods. Some discussion points are presented in Section 5.7. Finally, we close with conclusions and future works in Section 5.8.

\subsection{Related Works}

Manipulation of motion and animation data to show expressive qualities has been shown to be possible by treating the motion data as signals, using common signal processing methods [64] [152] [143]. The idea of the relationship between symbolic and prosodic qualities in sound and motion is found to hold a big potential in improving human-robot interaction experience. Prosody (defined as the study of suprasegmental characteristics of speech such as: vocal pitch, loudness, and rhythm [153]) has been shown to give enough affective (e.g. emotional) effects in communication [85], and an even better interaction experience when prosody is complement-ed with gestures [154] [155] [156]. In most verbal conversations, gestures are often synchronized with the prosody in speech to create emphasis, clarify a concept by adding meaning, show emotions, and so forth.

\subsubsection{Music and Motion}

Michalowski et. al suggested that when a robot's movements are synchronized with the beats of music, people are more likely to have a higher engagement with the 
robot, e.g. by starting to dance in rhythm with the robot [152]. Conversely, when the robot is out of sync with the rhythm of the music, people are less likely to engage the robot. However, their robot did not move according to the beat or rhythm of music; instead, their system matched the robot's movements to the beat and rhythm of the movements of the person in its view.

Conversely, Camurri et. al used information from human motion to control the dynamics in music, such as tempo, pitch, and intensity [143]. The motions of a user are captured by camera, and the dynamics of the motions (such as: acceleration/deceleration and breaks/ pauses) are analyzed using a technique called Silhouette Motion Images (SMI). SMI calculates a sum of a series of the person's silhouettes minus the current silhouette (a variation of Motion History Images) and extracts motion information such as sequences of pause (rest) and motion (stroke) phases. The motion information is then mapped to certain controls of musical notes, such as pauses or duration of the note, the note pitch, and intensity or loudness (volume).

Bhutada developed a user interface where the user can create a segment of MIDI music to play along a sequence of motions for multiple robots [157]. Bhutada's interface allows user to compose a sequence of pre-made gestures for a small humanoid robot. The music does not directly influence the motion of the robot, but only plays along as the robot performs its movements.

\subsubsection{Motion as Signals}

Bruderlin and Williams showed that by representing motion data as signals, common signal processing techniques such as multiresolution filtering, waveshaping, timewarping, and interpolation can be applied to the motion data [13]. Using their approach, motion data can be exaggerated, subdued, or blended with other motions while to an extent maintaining the characteristics of the original motion. 
Unuma et. al used Fourier Analysis to create transformations between two periodic motions using normalized coefficients multiplied with the Fourier coefficients of the two motions [85]. Their approach uses characteristic functions which can be extracted by calculating the difference between the coefficients of a 'neutral' motion, and its variations. The extracted characteristic function can then be applied to other motion to create a motion with similar characteristics. For instance, the characteristic function 'tired' can be extracted from 'tired walking' and applied to 'running' to create 'tired running.' Amaya et. al used a similar concept to extract emotional transforms from one motion and apply them to a different motion [86]. Unlike Unuma et. al, their method performs calculations in the time domain but the emotional transforms are transferable between non-cyclic motions. In a sense, the approach presented in this work is similar to Unuma's; by taking characteristic functions from a reference source and applying them to robot motions. In this case, the characteristic function is obtained from the dynamics information in a music piece instead of from another motion data.

Ken Perlin developed a method to generate pseudorandom noises that can be used for creating noise in animation data (one-dimension), textures (two-dimensions), and solid textures (three-dimensional solids) [64]. Perlin's noise-generating method (popularly known as Perlin Noise) has been used to add noise in the movement data of virtual/computer-generated characters [156] [158] or robots [159] to simulate little movements such as breathing, blinking, fidgeting, and was also often used to produce animation of textured objects in nature such as water, clouds, fire, and other elements [160]. However, indiscriminately adding noise to motion data can make the character appear to be experiencing seizures. 


\subsubsection{Synchronized Speech and Gestures}

Beat gestures that are synchronized with speech can make a virtual character appear convincing [158]. The beat gestures (e.g. moving the hands up and down) and their mappings to certain prosodic characteristics features are done using Hidden Markov Model (HMM). Levine used motion capture data synchronized with speech, and made his system learn the probabilities of observing a beat gesture when a prosodic features is detected. Using the derived model, the prosodic feature from an arbitrary speech input (e.g. internet chat) are used to synthesize the beat gestures on the 3D human model arms and hands, such that the arm and hand gestures occur in certain parts of the speech to create stresses and emphases, as indicated by McNeill [132].

Others used prosodic features of pitch and intensity to control the animation of a 3D head model. Busso [156] and Sargin [158] focused on the control of the animated head gesture of a computer-generated character also synchronized with prosody from speech. All these prosody-driven animation methods used a very similar approach. The mappings of prosody and head gesture of a person are learned using HMM, then the learned mappings are applied to arbitrary speech and the animation of a 3D head model.

Breazeal showed that using prosody in human- computer interaction is enough to create affect, although the prosody information is not directly matched to the motions of Kismet [154]. Except for Kismet, all the above works are applied on computer-generated 3-D models.

In summary, there are many studies in the computer animation domain that try to produce more dynamic, convincing motions on the computer-generated characters without hand-tuning the motion data. The method of extracting characteristic function is promising. Unfortunately, one cannot simply produce characteristic func- 
tions without any human movement reference data, for example from motion capture data. We believe music contains rich information similar to characteristic functions or emotional transforms which can be used to produce novel motions (e.g. dancing) and modify existing motion data to be more expressive in its execution.

\subsection{The Melodic Motion System}

To apply dynamics of a musical piece to robot motion, the system takes a sound file as its input, and outputs a set of movement instructions for the robot. The system focuses on using the timing and volume of the pitches (notes) that constitute the prominent melody in a music piece.

Extraction of dynamics (e.g. timing) in music and its components (e.g. instruments/timbre) is a complex task that is beyond the scope of this study. Thus to simplify the extraction process, we used music encoded in MIDI format where the properties of the musical piece are laid out in plain text and thus easier to extract its dynamic information. The extracted information is then used to produce motion data on a robot using a set of production rules. In this work, the input used is a .med file, which represents the MIDI information of a song in XML format. The software tool used to convert MIDI into .med is called MusE [161].

Dynamics information from the music file is extracted, such as: pitch (musical note), note-on velocity (the volume of the note as it is being played), tempo (in beats-per-minute or BPM), rests (moments when no note is being played), sustains (duration of a note or rest), and so on. These bits of information are annotated in the MIDI format or can be extracted from it, and used to either: a) generate new motion data or b) modify an existing sequence of movements (i.e. Scenario) in terms of range of motion and timing. Fig. 5.1 presents a high-level view of the system. 


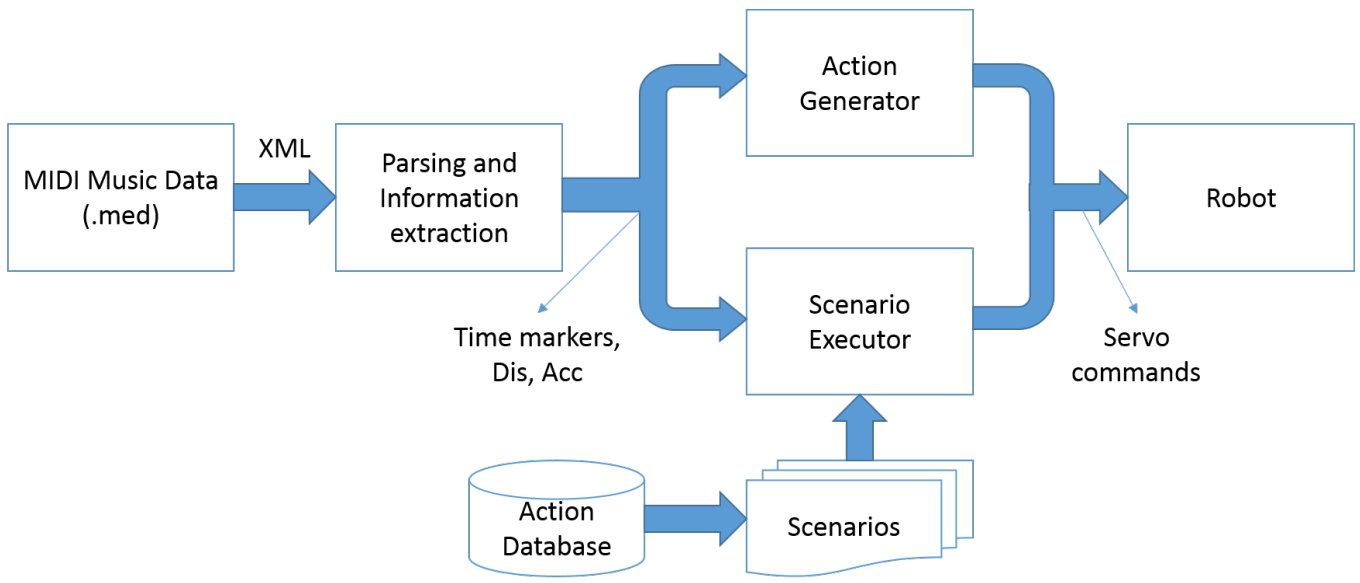

Figure 5.1: High-level view of the Melodic Motion System. Input to the system is a MIDI information of a song represented in .XML format. Music information such as beat, pitch, note-on velocity, etc. are extracted as Time markers, Dis and Acc. This information is used to generate a sequence of actions by the Action Generator block, or regulate the execution of a Scenario by the Scenario Executor block. A scenario is a sequence of motions or gestures. The Gesture Library stores a set of gestures or poses that the user can use to create Scenarios.

The main idea of the system is to have robot gestures executed according to the dynamics in music. For example: when a note abruptly stops (i.e. a 'strong' rest), a dancer would also stops her movement abruptly. This example also shows that in addition to the synchronized timing between the stop event in the music and the dancer's movement (i.e. on time), the dynamics of the stop in the movement of the dancer is also synchronized with the dynamics of the stop event of the music (i.e. abruptly). Similarly, when the change is gradual, the dynamics of the movement also changes gradually.

\subsubsection{Parsing and Information Extraction of Music Data}

The parsing of the MIDI music data is done in two stages. The first stage extracts the events that occur on every beat from the MIDI format and collects them into an array. The second stage compiles the results from the first stage into two components: 
Time Markers, and Timed Events.

\subsubsection{First Stage - Parsing/Collecting Note Events per Beat}

Tempo is one of the main properties in a music that people immediately recognize when they first listen to a song. Therefore, we use tempo as the backbone information to regulate our system. In music, there could be multiple events that occur within a beat, such as: a chord, different instruments, and vocals. In this work, we are only using instrumental music, and only analyzing the events of one MIDI channel (i.e. instrument). In future works, we shall consider the other musical structures/elements, including rhythms, and taking into account simultaneous events.

At this stage, the events that occur within one beat of music are collected into bins where each bin corresponds to one beat and is indexed by the time marker (tick) for the beat ${ }^{1}$. There are three pieces of information recorded in each bin: the beat time marker, the number of events in the beat, and the array of the events in the beat.

The event when a note first occurs is called a note event. The following detail about a note event is specific to the .med file used by the MusE Sequencer tool [161]. Each note event consists of five pieces of information: start time, duration, note code, note-on velocity, note-off velocity. Start time refers to the time marker or moment when the note starts. The unit is in ticks. Duration indicates the length of time of the note being played in unit of ticks. Note code is the MIDI code for the note pitch. Note-on and note-off velocities describes the volume when the note is being played initially or when it finishes, respectively [162]. An example of a note event is shown in Table 5.1.

Events are only recorded in the beat where they first occur. For example, if a note

\footnotetext{
${ }^{1}$ The MIDI beat resolution used by MusE is 384 ticks per beat.
} 
Table 5.1: One of the note events in beat 3

\begin{tabular}{lllll}
\hline $\begin{array}{l}\text { Start } \\
\text { time }\end{array}$ & Duration & Note code & $\begin{array}{l}\text { Note-on } \\
\text { velocity }\end{array}$ & $\begin{array}{l}\text { Note-off } \\
\text { velocity }\end{array}$ \\
\hline 1532 & 568 & 92 & 110 & 64 \\
\hline
\end{tabular}

first occurs on beat 5 and lasts for two beats, the note event will only be recorded in the bin for beat 5 . When a beat does not contain any new note event, it is stored as an empty array. Fig. 5.3 shows an example of the output of this first stage. Fig. 5.2 shows the raw data of six events of the MIDI music in the format of .med file. In Fig. 5.3, Beat 3 starts at time marker 1152 and has two note events.

Notice that while the actual time marker of Beat 3 is 1152, the start time for the first note event in that beat is 1532. The note event has a duration of 568 ticks (which lasts more than 1 beat), note code (pitch) is 92, with note-on velocity of 110 , and default note-off velocity of 64 . If there are more than one note events that occur within the time window of a beat (within 384 ticks), those note events are stored as the events for that beat. These stored note events will be used to create motion data, which will be described below. If a note event lasts for more than 1 beat, it will not be recorded as a note event in subsequent beat bins.

\subsubsection{Second Stage - Information Extraction}

At this stage, the parsed MIDI data from the first stage is processed to obtain values for acceleration and displacement. In this work, acceleration is used to set the acceleration parameter specifically for the ASC16 servo controller board used on the robots [163]. As another approach, acceleration can be created using some interpolation methods (e.g. linear, cubic). Displacement determines the direction and amount of movement the robot should make. The formulas to calculate acceleration 


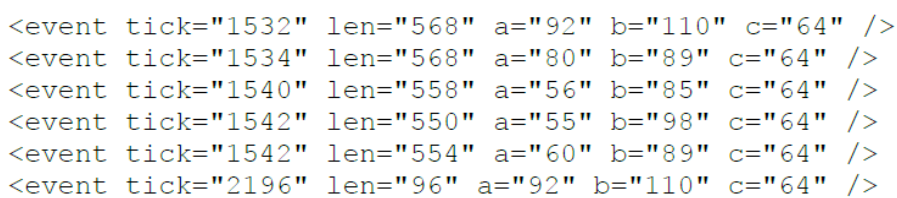

Figure 5.2: MIDI music data in XML format from .med file

and displacement are given below.

The collection of note events in a beat is called Timed Events. To calculate acceleration and displacement, we must first determine which of the note events in each beat is going to be used, since each beat may have multiple note events. The strategy to choose the note event for a given beat is in two steps with the following order: 1) select the note event with the earliest start time in the event_beat, or 2) if there are more than one note events which have the same start times and the start times are the earliest, select the one with the highest note-on velocity value (the loudest note). If somehow there are still more than one simultaneous note events with the exact start time and note-on velocity, then random choice is used. This strategy is applied to each beat and produces a list where there is only one note event per beat.

The strategy above was derived from observations of dance performances. Typically, the audience can notice when the dancer creates stylistic movements that matches timing and dynamics at different moments in the music. This is often referred to as hitting the music. While there could be many sounds happening at any given moment (e.g. vocals, strings, percussion, bass), it is more appealing to the audience when the 'hit' corresponds to only one of the sounds, otherwise the movements will become too busy and confusing. It is up to the dancer's artistic taste to choose which part of the music he/she wants to accentuate. Therefore, the strategy chooses only 


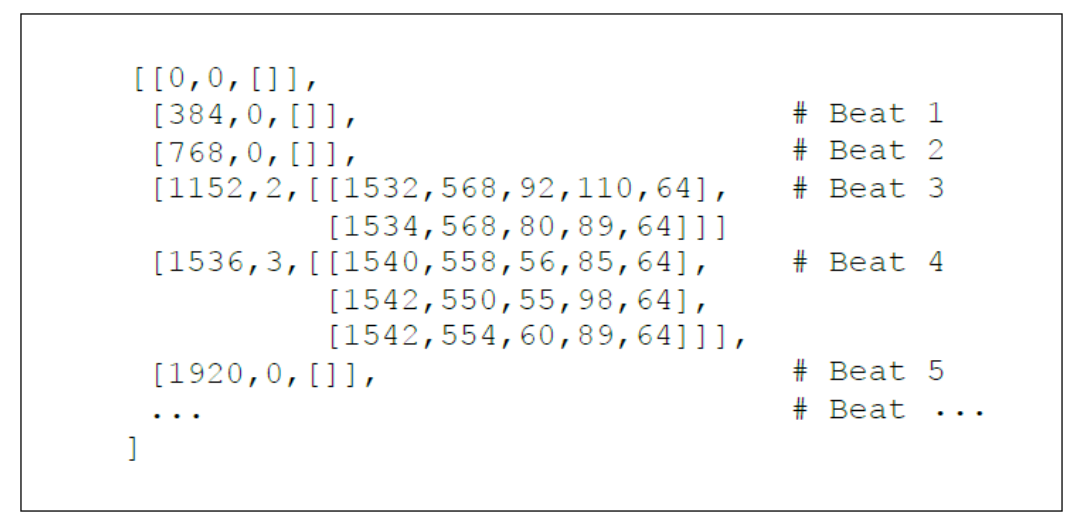

Figure 5.3: Output of the first stage of parsing. Each bin (row) is a list with the entries: tick/time mark, number of note events in this beat, the list of note events in this beat. Beat 3 and 4 each have multiple note events, while Beat 1-3 and 5 have none. Each note event is a list with entries: tick/time mark, duration (in ticks), note code, note-on velocity, note-off velocity.

one note event in any given beat.

The final output of the Parsing and Information extraction block are three lists: Time Markers, Acc and Dis. Time Markers is a list of the start times of the selected note events from each Timed Event. Likewise, Acc and Dis are lists of acceleration and displacement values, respectively, produced by the selected note events. An example of the application of this policy is shown on the first row in Table 5.2. In that row, the first note event from beat 4 in Fig. 5.3 is selected since both events have the same note-on velocities, but the first note event has the earliest time marker for that beat.

From the note events in each Timed Event $e_{i}$, acceleration and displacement data at beat $i$ for joint $j$ are produced by equations 5.1 and 5.2, respectively. A high note-on velocity indicates the loudness of the note being played initially. We assume this property makes the note with high note-on velocity more prominent than the other notes in a Timed Event. The duration of the note event with the highest noteon velocity is obtained by $D\left(c_{i}\right)$, where $c_{i}$ is the note event with the highest note-on 
velocity. Equation 5.3 defines $c_{i}$, where $N_{i}$ is the list of note events in Timed Event $e_{i}$. It is possible to have more than one note events that have the same highest note-on velocities. In such case, the note event to be used is chosen at random. Thus, the duration is used to determine the acceleration of the movement for that event; the longer the note duration, the higher the value for the acceleration will become, and vice versa.

$$
\begin{gathered}
A c c_{i, j}=\frac{D\left(c_{i}\right)}{384} * \operatorname{maxacc}_{j} \\
\operatorname{Dis}_{i, j}=\frac{a b s\left(c_{i}-c_{\text {base }}\right)}{a b s\left(C_{i}-c_{\text {base }}\right)+1} * \text { maxrange }_{j} \\
c_{i}=\max _{\text {nov }}\left(N_{i}\right) \\
C_{i}=\max _{\text {code }}\left(N_{i}\right)
\end{gathered}
$$

The value 384 at the denominator is the beat resolution (ticks/beat). $\operatorname{maxacc}_{j}$ is a parameter for the maximum allowable value for the acceleration parameter of the ASC16 servo controller board. From our observations, acceleration values higher than 30 did not give any visible difference in the movement of the robot. Therefore, the value for $\operatorname{maxacc}_{j}$ is set to 30 in the experiments. In the future, each joint $j$ may have different values of $A c c_{i, j}$ and $D i s_{i, j}$, but in this version we applied the same values for all joints $j$ on the robot.

The Home position of the joint is mapped to the basenote variable. Displacement at beat $i$ for joint $j$ (eq. 5.2) is obtained by taking the difference of the notecode of a note event and the basenote. The difference is then used as the displacement of the 
movement relative to the Home position of the joint.

In eq. $5.2, C_{i}$ is the highest note code value among the collection of note events $N_{i}$ at beat $i$ as defined in 5.4. The variable $c_{b a s e}$ is the base note which value is the constant 60 and corresponds to the MIDI note code for the middle $\mathrm{C}$ note in the Western musical scale. The parameter maxrange $j$ is the constraint for maximum allowable movement range for the joint $j$. The absolute value of the difference is used because we want to create alternating motions (up-down, left-right) on the robot, since they are more observable as distinct events than consecutive motions in the same direction (e.g. turning right followed by another right turn). Moreover, alternating the motion avoids the risk of reaching one extreme of the joint's range of motion too soon or too often. The displacement equation is designed so that if the selected note event is also the note with the highest pitch, then the generated displacement range should be equal to maxrange. The denominator is added by 1 to avoid division by zero when $C_{i}=c_{b a s e}$.

Table 5.2: Choosing Note Events Information and Production of Dis $_{i, j}$ and $A c c_{i, j}$ for a joint $j$ with maxrange $_{j}=1400$ and $\operatorname{maxacc}_{j}=30$

\begin{tabular}{lllllll}
\hline Note Event & $\begin{array}{l}\text { Highest } \\
\text { note-on } \\
\text { velocity }\end{array}$ & $\begin{array}{l}\text { Highest } \\
\text { note } \\
\text { code }\end{array}$ & $\begin{array}{l}\text { Selected } \\
\text { Note }\end{array}$ & $\begin{array}{l}\text { Selected } \\
\text { Note } \\
\text { Dura- } \\
\text { tion }\end{array}$ & & \\
& & & s $_{i, j}$ & & \\
\hline$[2196,96,92,110,64]$ & 110 & 92 & 92 & 96 & 1357.58 & 7.5 \\
\hline$[[3242,64,83,98,64]$, & 98 & 83 & 83 & 64 & 1288 & 5 \\
\hline $3446,102,82,98,64]]$ & & & & & & \\
\hline
\end{tabular}

The motivation for the above choices is as follows. Most of the melodic notes in musical scores are played near or around the middle scale. Under this assumption, the Home position of each joint is mapped to the basenote so that the generated 
displacements are always relative to the Home position of the joint in order to prevent the joint to be overextended. Therefore, if the selected note is equal to the basenote, then the displacement is zero (i.e. in the Home position). A note higher than the basenote is a rotation in counter-clockwise direction, and conversely clockwise for lower notes. This choice was arbitrary. In the experiments, we only allow a range of approximately 60 degrees. The additional +1 in the denominator is to avoid division by zero when the $\max ($ notecode) is equal to basenote. Table 5.2 shows an example of the generated acceleration $\left(A c c_{i}\right)$ and displacement $\left(D i s_{i}\right)$ values using equations 5.1 and 5.2, respectively. Arguably, there may be many more ways to map the dynamics information from music to motion. The method presented here is only one of them. In future works, we will explore other possible mappings.

\subsubsection{Free Mode and Scenario Mode}

The information extracted from the music data as Time Markers, Acceleration, and Displacements is used in two execution modes: Free/Dance and Scenario modes.

\subsubsection{Free/Dance Mode}

In Free/Dance Mode, the music data is directly converted into motion data by the Action Generator. The displacement information is used to create the poses of the motion, while the acceleration information is used to set the acceleration for transitioning between poses. Every displacement and acceleration data are stored in one Time Marker. The time information in the Time Marker determines the timing when a movement should be executed.

Currently, the Free/Dance Mode is only applied to our custom Lynxmotion robot. The two main reasons for this are: 1) the configuration of the robot is relatively simple

compared to our second robot, the KHR-1 where there are many positions that can 
cause the robot arms or legs to collide with other parts of its body, and 2) having fewer degrees of freedom and non-humanoid form will help to keep the viewer from bias of looking for human-like gestures. This way, we can see if the system is able to generate expressive motions directly from music data, and if the concept is applicable to non-humanoid robots.

The configuration of the Lynxmotion robot is shown in Fig. 5.4. The robot has four degrees of freedom (DOFs): one at the base to turn left and right, one directly on top of the base, one at the elbow, and one at the end of the arm. The robot only uses normal RC/Hobby servos. The Home position of the base DOF is at the center and can turn approximately 90 degrees in either left or right directions (the bottom two-directional arrows in the Top View in Fig. 5.4). The second DOF is limited to a range of 0 to approximately 115 degrees, with the Home position at 115 degrees. The third DOF has a full range of motion of 180 degrees with Home position at 180 degrees. The Home position of the fourth DOF is at the center, thus the DOF can be rotated 90 degrees to either left or right. Because of this configuration, some constraints are applied when generating the motion data for the robot. Fig. 5.5 shows some of the poses that were produced by the Free/Dance Mode. For simplicity, the term 'DOF' will be used interchangeably with the term 'joint' from this point on.

The displacement values are used to create absolute position values for the servos. To calculate the position values, the displacements are added or subtracted from the Home position of each joint. Thus, to generate position data for each joint, only certain movements (i.e. displacement from the Home position) are allowed as shown in Table 5.3. In the demonstrations, the range of movement of each joint is limited so the robot will operate within safe limits. These values are specific to the servo controller used on the robot.

Because the first and fourth joint can be moved to either direction, the generated 


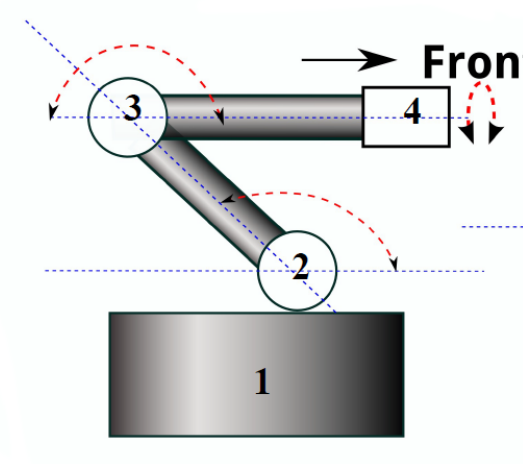

Right View

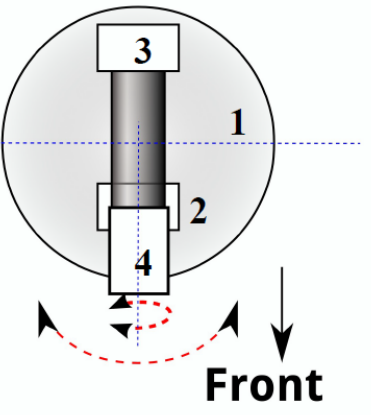

Top View

Figure 5.4: Configuration of the Lynxmotion robot. The degrees of freedom (DOFs) on the robot are: base (1), lower arm (2), upper arm (3), and 'face' (4). The twoheaded arrows near each DOF indicate the directions for the motions of the DOF.

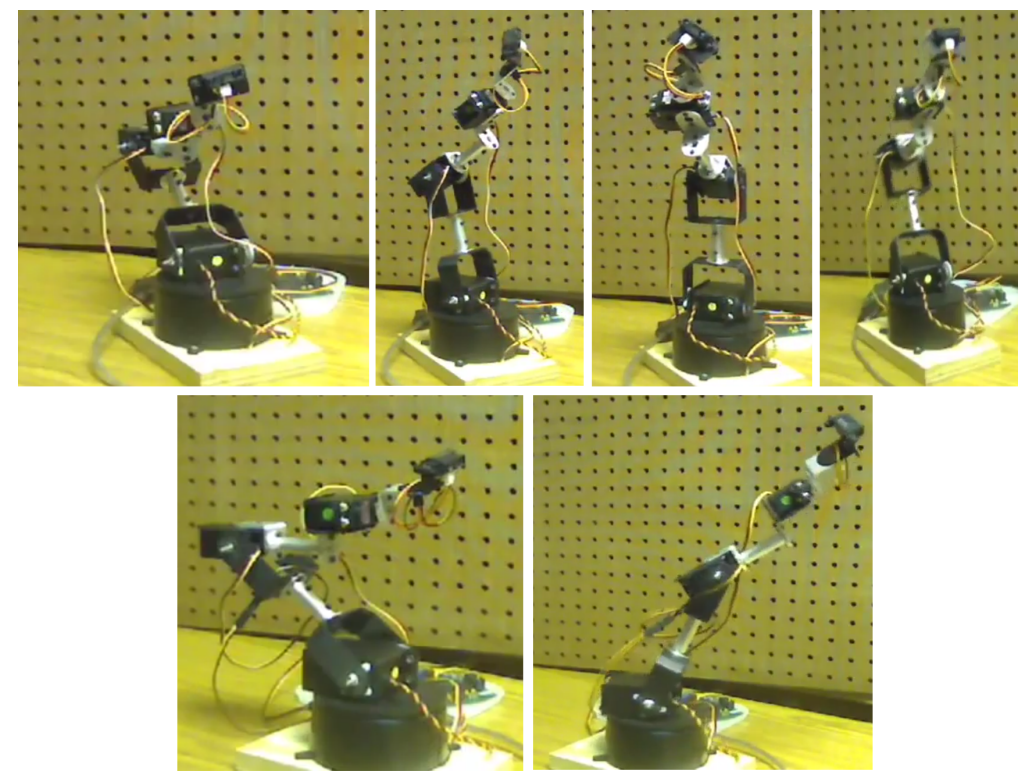

Figure 5.5: Some sample poses of the Lynxmotion robot which are generated by the Action Generator in Free/Dance mode using the displacement information extracted from music data. 
Table 5.3: Allowable Movements for the Lynxmotion Robot DOFs

\begin{tabular}{ll}
\hline DOFJoint & Allowable Movement \\
\hline 1 (Base) & Home +/- displacement \\
2 & Home - displacement \\
3 & Home - displacement \\
4 (Face) & Home +/- displacement \\
\hline
\end{tabular}

displacement value for these joints is alternated between being added to and subtracted from the Home position with every new event. The same acceleration values are applied to all DOFs.

Since displacement and acceleration values are generated in each beat of the music where there exists a note event, no new values are generated when there is no note event in the beat. The timing of each movement is determined by the time marker of the selected note event in a beat of the music. Table 5.4 shows some of the motion data generated using the displacement data under the constraints in Table 5.3. The same acceleration and displacement values produced by equations 5.1 and 5.2 using the selected note event for each beat are applied to all joints of the robot. Each line in Table 5.4 is mapped to a time marker for a synchronized execution of the motion with the music data.

Table 5.4: A few examples of generated motion data for the Lynxmotion robot

\begin{tabular}{lllllll}
\hline $\begin{array}{l}\text { Time } \\
\text { Marker }\end{array}$ & Dis & Acc & $\begin{array}{l}\text { Joint1 } \\
\text { (base) }\end{array}$ & Joint2 & Joint3 & Joint4 \\
\hline 1.99 & 1357.58 & 44.4 & 2660 & 1202.42 & 1202.42 & 2660 \\
2.01 & 5600 & 43.59 & 2660 & 2660 & 2660 & 2660 \\
2.86 & 1357.58 & 7.5 & 2660 & 1486.67 & 1486.67 & 2660 \\
4.01 & 1341.67 & 9.38 & 1000 & 1486.67 & 1486.67 & 1000 \\
4.6 & 1344.5 & 5 & 2660 & 1218.33 & 1218.33 & 2660 \\
\hline
\end{tabular}

As seen in table 5.4 at Time Marker=2.01, some times a large displacement value 
is produced (5600). In such case, the final output is capped to the maximum limit of the joints. The minimum and maximum limits for all four joints are 1000 and 2660, respectively.

\subsubsection{Scenario Mode}

In this work, a Scenario is defined as: a sequence of Gestures arranged in a particular order of execution. A Gesture is defined as a sequence of poses/strokes. A Scenario is analogous to a directed, planned scene such as the one in a theatrical play, which is a particular order of actions that the actor performs to convey a story. Note that a Scenario can also consist only of one gesture or motion. The sound information will be used to control the execution of each gesture in the Scenario. For instance, a Gesture in the Scenario may be executed in one musical phrase, one beat, half of a phrase, and so forth depending on the timing information of the music.

The information extracted from the music input is used to control the timing and execution of a Scenario data without re-arranging the sequence of Gestures in the Scenario. Specifically, the timing information from Time Markers determines when a movement (or pose) is going to be executed, while the Acceleration (Acc) and Displacement (Dis) information will be used to alter how each pose in each Gesture in the Scenario is executed in terms of speed and range of motion, respectively. A weighting factor (labeled as Scaling Factor) $\alpha$ is added to control the influence of the displacement modifications to the original pose values.

Fig. 5.6 shows the basic idea of applying information in Time Markers, Acceleration, and Displacement to a Scenario data. Each gesture $\mathbf{G}_{m}$ consists of $p$ poses/ strokes $\mathbf{S}_{m, p}$. Each pose consists of the joint angle values for all the degrees of freedom of the robot, i.e. $s_{m, p, j}$ where $j$ is the index of the robot's DOF. The modifications are applied on the robot as follows: 
1. The moment of execution for each pose $\mathbf{S}_{m, p}$ of a Gesture $\mathbf{G}_{m}$ is determined by a Time Marker $\left(t_{i}\right)$.

2. The acceleration of the transition from poses/strokes $\mathbf{S}_{m, p}$ to $\mathbf{S}_{m, p+1}$ is determined by the Acceleration value $A c c_{i}$ at time $t_{i}$, where $A c c_{i} \in$ Acc. Each $t_{i}$ is aligned with one $\mathbf{S}_{m, p}$.

3. The value for each joint of the pose $s_{m, p, j}$ is modified by the value of the Displacement $D i s_{i}$ multiplied by a scaling factor $\alpha$, where $D i s_{i} \in$ Dis. This is expressed in eq. 5.5. To avoid a pose change that is too large, $\alpha$ is set to a small value, e.g. 0.1 .

4. The values for each joint post-modification is capped to its safe maximum/ minimum limit to avoid damage to the robot.

5. The Acceleration (Acc) and Displacement (Dis) are applied to all joints that are involved in the pose.

$$
s_{m, p, j}^{\prime}(i)=s_{m, p, j}(i)+\left(\alpha * D i s_{i}\right), \forall j \text { in } \mathbf{S}_{m, p}
$$

The lengths of the Time Markers and Timed Events are always the same, but not necessarily the same length as the Scenario. For example: suppose the Scenario consists of $\mathrm{m}$ number of Gestures, each with a different number of strokes. Then suppose the total number of strokes in the Scenario is S. Therefore, if the Time Markers and Timed Events are of length N, and the Scenario consists of S strokes, only S number of Time Markers and Timed Events information is used. In most cases the number of strokes $\mathrm{S}$ is far less than the length of Time Markers/Timed Events $\mathrm{N}$. Currently, the system will fail if $\mathrm{S}$ is larger than $\mathrm{N}$ because there are no more 
Table 5.5: KHR-1 Pose and Gesture Library

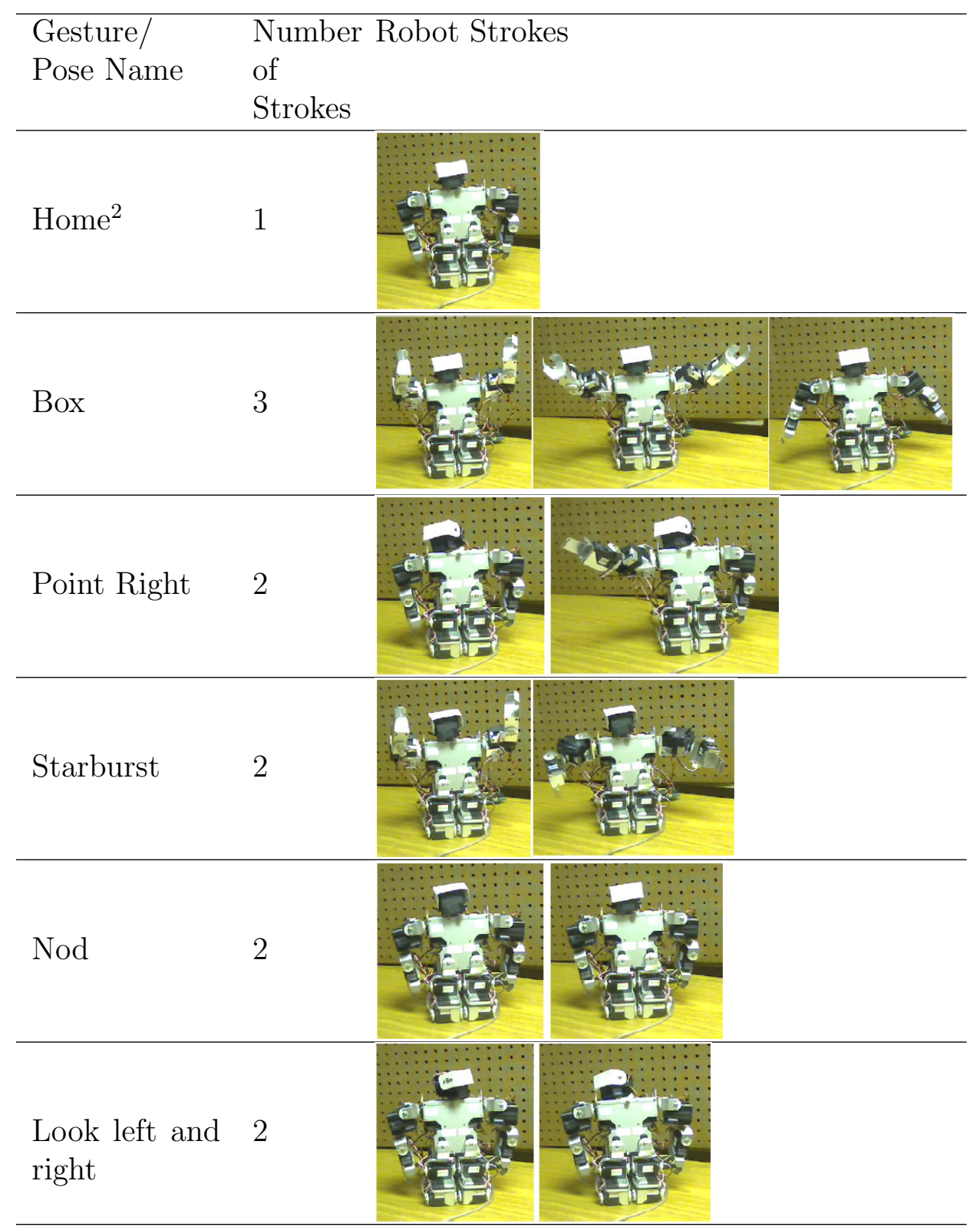




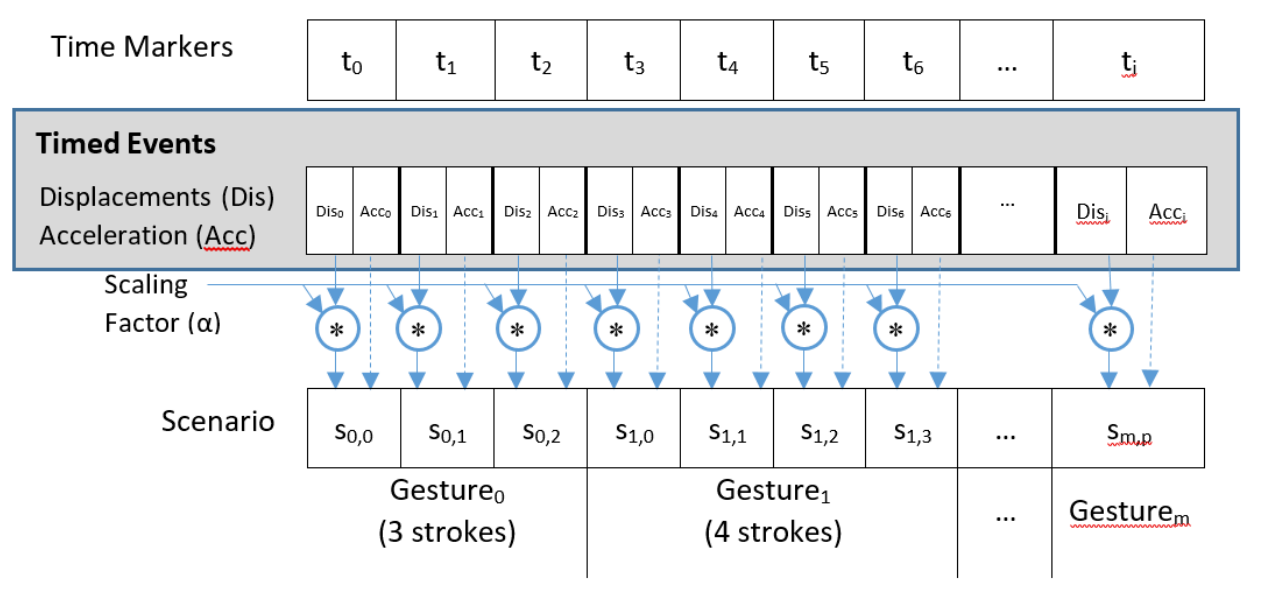

Figure 5.6: Timed Events (Displacement and Acceleration) are applied to gestures by first associating each stroke of the gesture to a time marker. The Timed Events on each time marker are then applied to each stroke. Application of displacement value to stroke is scaled by a factor $\alpha$.

Time Marker/Timed Events information to apply. A possible solution is to go back to the beginning of the Time Markers/Timed Events list and add each value of Time Markers to the last time marker (repeat the pattern). Fig. 7 shows the effect of applying Timed Events to a Scenario data.

The movement of the robot starts at every time marker. The system takes priority of each stroke to be executed on time (according to the assigned time marker) over completion of the stroke. Therefore, if there is enough time between time markers, a stroke can be completed. Otherwise, if a stroke is not yet complete but the next time marker has arrived, then the current stroke is interrupted by executing the next stroke.

\subsection{Experiment}

To validate the main hypothesis of this chapter, we conducted a survey with two experiments: one for the Free Mode and another for the Scenario Mode. Two sets of 
videos were shown to an audience, one for each experiment. The first set of videos is used to evaluate the audience's perception on the expressive and dynamic qualities of the Lynxmotion robot's movements with respect to four different songs. The second set of videos is done to evaluate the audience's perception on the contribution of timing and melodic information in music data to the expressive and dynamic qualities in the execution of a Scenario. In this video we used the humanoid robot KHR-1 ${ }^{3}$. The KHR-1 robot has 17 DOFs (1 neck, 3 each arm, 5 each leg). We added one DOF to the neck for nodding movements, and one on each elbow for pronation/supination movements. The video used in this survey is available on the YouTube website ${ }^{4}$.

Because it is often difficult to articulate the dynamic qualities in music and motion, and their perceived effect on people, the surveys only ask the participants whether or not there exists affect and did not try to specify what type of affect it was. In other words, the experiments are designed to capture whether or not the robot motions generated by the proposed system using information from music data have emotional influence.

There were 31 participants in this survey who were all undergraduate students in electrical and computer engineering. They were informed that their participation in the survey is entirely voluntary. The majority of the participants are male (28 out of 31), with ages ranging from 21 through 30 years of age (20 out of 31). Two participants are younger than 20 years old, five participants are between 31 and 40 years old, two participants are older than 41 years old, and one did not specify. Besides age and gender, no other information about the participant is collected.

The first video consists of five clips of the Lynxmotion robot. In the first four clips, the robot is moving to the motion data generated from a MIDI music file. The robot

\footnotetext{
${ }^{3}$ The KHR-1 robot has since been discontinued by its manufacturer. Information about the robot can still be found here: [164]

${ }^{4}$ URL: https://youtu.be/9QHp9B-0QK0
} 
motion is not generated in real-time. The timing of the music and the robot's motion sequence are arranged so they start at the same time. The contents of the music files played in the clips are not manipulated in any way, but only the MIDI channel which is used to generate the motion data is played while the other channels are muted, with exception of the Mozart Sonata No. $16^{5}$. The same setup is used with the Scenario experiment. The MIDI music files are: Beethoven's Symphony No. 5, Mozart Sonata No. 16, Lullaby of Birdland, and Mancini's Pink Panther Theme song. These songs are chosen for their prominent melodic content from musical instruments as opposed to vocals. The additional consideration for these songs is that we consider them to be familiar and easy to digest for most people.

In the fifth clip, the robot is moving to a motion data that was generated manually by us to the Beethoven's Symphony No. 5 music. In the survey, each clip is referred to as Robot A, Robot B, Robot C, Robot D, and Robot E in the following order: Beethoven's Symphony No. 5, Mozart's Sonata No. 16, Lullaby of Birdland, the Pink Panther Theme song, and Beethoven's Symphony No.5. The last clip used manually created motion data as control. In the survey, participants were asked to rate in a 5-point Likert scale to the following questions:

1. How expressive are the robot's movements? This question is asked to evaluate if the participant perceive the robot as being expressive with respect to the music.

2. How dynamic are the robot's movements? This question is asked to evaluate if the participant can perceive interesting movement dynamics such as speed, range of motion, transitions/continuity, and variety of motions without getting into the specifics of what makes the movements appear more dynamic.

\footnotetext{
${ }^{5}$ The whole music is played for the Mozart Sonata No. 16 because the channel used to generate the motion data does not sound good when played by itself - the main melody is constructed by the combination of multiple channels.
} 
3. Overall, how do you like the robot's performance? This question asks the participants' perception on the performance as a whole.

In addition to the three questions above, the participants are asked to choose which robot performance they like best. The participants are allowed to choose more than one robot performance. Finally, the participants were encouraged to include comments on why they choose a particular robot performance as the one they like, and also to give general comments on both experiments.

In the second video, four clips of the KHR-1 robot performing a Scenario were shown to the audience. There are six gestures used in the Scenario, each of them performed at least once. The six gestures are shown in Figure 6. The Scenario consists of a total sequence of nineteen gestures.

The first clip shows the KHR-1 robot executing the Scenario without modification from any music data as control. The remaining three clips show the KHR-1 robot executing the same Scenario with the addition of three music data (in order): Beethoven's Symphony No. 5, the Pink Panther Theme song, and Lullaby of Birdland. The same set of questions as above is used for the survey in this second experiment.

\subsection{Results}

We first analyze the survey results of each experiment using repeated-measure ANOVA [165]. The null hypothesis for the Free/Dance Mode experiment is that there are no statistical differences between the results of RobotA, RobotB, RobotC, RobotD, and RobotE. That is to say, the information from music did not have a significant effect to make the movements of the robot more expressive than the movements created by a person. The null hypothesis for the Scenario Mode experiment is similar: there is no statistical differences between the results of RobotA, RobotB, RobotC, and RobotD 
which indicates that the information extracted from music did not have a significant effect to make the movements of the robot more expressive than the movements in the Scenario without the influence of the information extracted from music. The alternative hypothesis for both experiments is that there are differences between the survey results. If the result of ANOVA shows that we can reject the null hypothesis, then a post-hoc analysis is performed using pairwise Scheffe's test [166] to identify where the differences occur.

\subsubsection{Free/Dance Mode}

Due to several participants did not answer all the questions in the survey completely, only partially, or a combination of both, the actual sample size is lower than the total number of participants of 31. For Question 1 (Q1) and Question 2 (Q2) there are 26 samples $(N=26)$, while there are only 24 samples for Question $3(\mathrm{Q} 3)(N=24)$ and 30 samples for Question 4 (Q4) $(N=30)$.

The results of the Free/Dance Mode survey are shown in Fig. 5.7. With respect to Q1: "How expressive are the robot's movements?" $(N=26)$, we are able to reject the null hypothesis with $F(4,100)=23.01, p<0.0001$. Next, we performed pairwise Scheffe test to analyze the statistical difference between pairs of categories, e.g. RobotA vs RobotB, RobotA and RobotC, and so on. The significant differences in the result of Question 1 appear between RobotB and RobotD with $F(4,100)=3.37$, $p<0.05$, and RobotE with the rest of the categories.

Since the means of the RobotA, B, C, and D are higher than RobotE, the result of our analysis indicate that our system is able to produce robot motions that are perceived by the audience as being more expressive than the handcrafted motion (RobotE).

With respect to Q2: "How dynamic are the robot's movements?" $(N=26)$, 


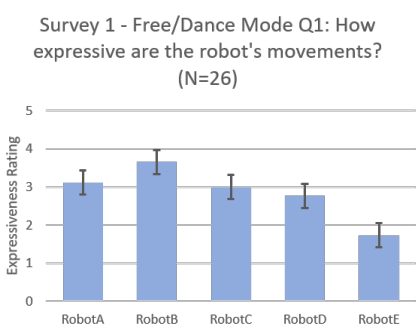

(a) Perceived Expressiveness

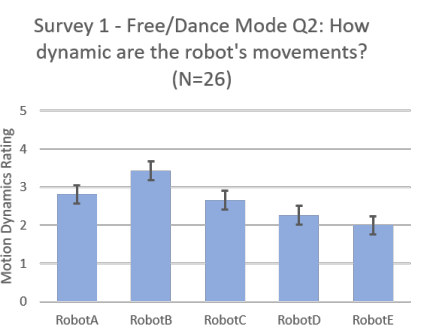

(b) Perceived Motion Dynamics

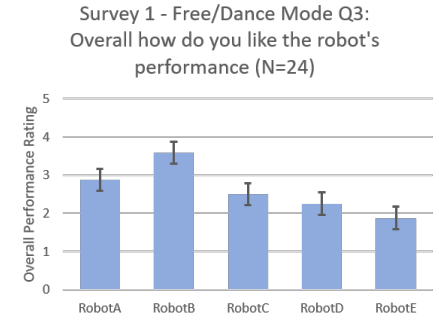

(c) Perceived Overall Performance

Figure 5.7: Means and standard error for Free/Dance Mode from the first survey.

we are able to reject the null hypothesis with $F(4,100)=12.08, p<0.0001$. The significant differences in the result of Question 2 only appear between RobotB and D with $F(4,100)=4.38, p<0.05$, and RobotB and E with $F(4,100)=6.67, p<0.05$.

With respect to Q3: "Overall, how do you like the robot's performance?" ( $N=$ 24 ), we are able to reject the null hypothesis with $F(4,92)=20.21, p<0.001$. The significant differences in the result of Q3 is apparent in RobotB versus all other categories. RobotB has the highest mean among the other categories $(\mathrm{M}=3.65$, $\mathrm{SD}=0.75)$.

The result for Q2 suggests that RobotB appears more dynamic to the audience than the other Robots. There are no differences between the performances of the other Robots. Looking at the mean values, only RobotB is rated above 'OK'. It is difficult to draw any conclusion from the result of Q2. We must take into account that the audience may have different interpretation of dynamic movement. We used the word 'dynamic' because the it is often used when people make qualitative comments on artistic performances. Technical movement terminologies such as the ones used in dancing, acting, or animation are unusable because the participants were not required to be knowledgeable in any of those domains. Therefore, Q2 uses the word 'dynamic' to assess the quality of the robot's movements as produced by the system under the 


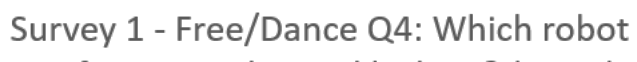

performance do you like best? $(\mathrm{N}=30)$

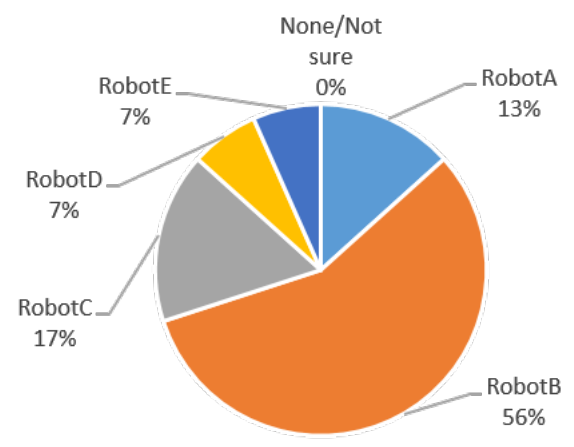

Figure 5.8: Survey result for the question: "Which robot performance did you like best?"

assumption that it is a qualifier with which most people are familiar.

Fig. 5.8 shows the survey result of Q4: "Which robot performance did you like best?" $(N=30)$. The score for each performance is calculated as the number of votes for that performance over the total number of votes (Eq. 5.6). Most participants chose RobotB as the one having the best performance (56\%). RobotC is rated as the second best (17\%), and RobotA rated as the third preferred performance (32\%). None of the participants expressed that they do not like any of the performances nor unable to pick a performance they liked among the five performances (None/Neither $=0 \%)$.

$$
\text { Performance }_{r}=\frac{\text { votes }_{r}}{\sum_{r} \text { votes }_{r}}
$$

Where $r \in\{$ RobotA, RobotB, RobotC, RobotD, RobotE\}.

\subsubsection{Scenario Mode}

Due to several participants did not answer all the questions in the survey completely, only partially, or a combination of both, the actual number of samples for Q1, Q2, 
and Q3 in this experiment is 29 samples $(N=29)$. There are 26 samples for Q4 $(N=26)$.

Adding music data to Scenario seems to only have little effect to improve the robot's movements. The results of the Scenario Mode survey are shown in Fig. 5.9. The audience perceived a difference in expressiveness (Q1, $F(3,84)=8.07, p<$ $0.05)$ and overall performance $(\mathrm{Q} 3, F(3,84)=5.73, p<0.05)$ but post-hoc analysis shows only RobotB that stands out over the other categories. There is no perceived difference of dynamics in the motion between all five categories $(\mathrm{Q} 2, F(3,84)=1.87$, $p=0.14)$

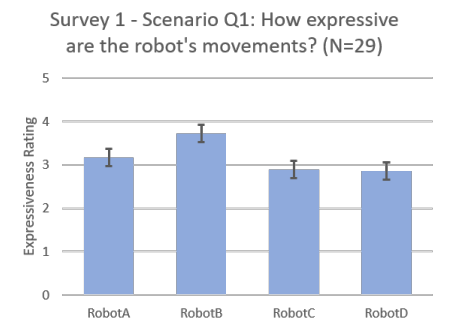

(a) Perceived Expressiveness

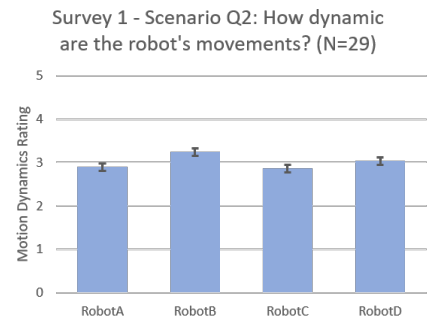

(b) Perceived Motion Dynamics

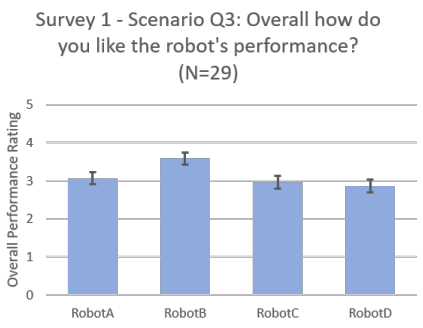

(c) Perceived Overall Performance

Figure 5.9: Means and standard error for Scenario Mode from the first survey.

Fig. 5.10 shows the survey result of Q4: "Which robot performance did you like best?" $(N=26)$. The score for each performance is calculated in the same way in Eq. 5.6, with the difference where $r \in\{$ RobotA, RobotB, RobotC, RobotD $\}$. Overall, the participants seem to prefer the Scenario performances with the addition of the music data (RobotB: 46\%, RobotC: 15\%, RobotD: 23\%) over the Scenario without the addition of the music data (RobotA: 4\%), and $12 \%$ of the participants said that they either cannot decide which they liked best (all equally good) or none of the performances are liked at all.

The participants seem to disagree on the performance of RobotC. One participant 


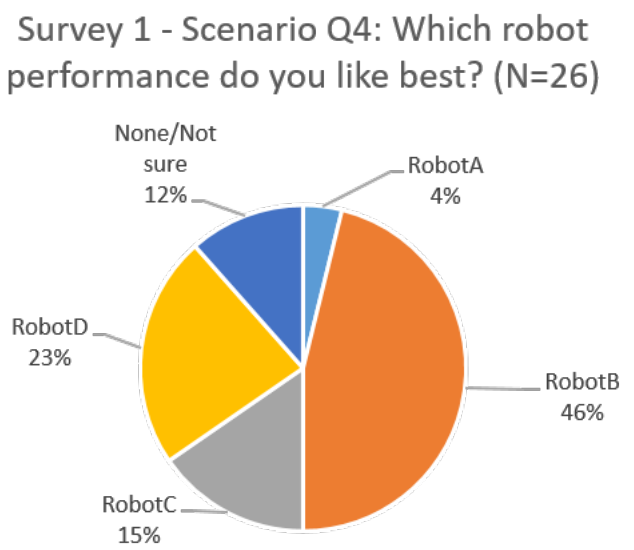

Figure 5.10: Survey result for the question: "Which robot performance did you like best?"

commented that RobotC was:

"Very static"

Another participant commented on Robot C as:

"The movements were a bit confusing for some reason."

While others commented:

"Robot C matched with the music,"

"You can see the reaction of the robot to the music which added dynamics,"

"Robot C was as fluent as Robot A and B and expressed well near the end."

\subsection{Results from Non-parametric Analysis}

Since the measurements were done using Likert-scale, there are concerns that the data does not meet the requirements for a parametric method such as ANOVA where the data is assumed to fit a normal distribution, have homogeneity of variance, and no extreme outliers. Moreover, the sample size was relatively small $(N<30)$ which typically is not sufficient to draw statistical significance. To address these concerns, we re-analyzed the data from the previous survey using Friedman test as the non- 
parametric test for within subjects measurements, and Wilcoxon Signed-rank test for post-hoc analysis since non-parametric methods do not have the aforementioned requirements [167]. Additionally, we conducted a second survey and analyzed the data also using Friedman and Wilcoxon Signed-rank tests.

The population size of the second survey is thirty-five people. Participants are undergraduate engineering students and were not intentionally chosen because of their field. Seventy-four percent of the participants are in the age range of 18-28 years old, $23 \%$ in 29-39 bracket, and $3 \%$ in the above 50 bracket. The same set of questions and measures as the earlier survey were used for Free/Dance and Scenario modes.

Due to several participants who did not completely answer all questions, or erroneous/invalid answers, the number of valid samples is lower than 35 samples. For the Free/Dance mode, we collected 31 valid samples for Q1 and Q2 , 30 samples for Q3, and 33 samples for Q4. For the Scenario Mode, there are 32 valid samples for Q1, Q2, Q3 and 33 samples for Q4.

To give the participants some directions to the meaning of the terms "expressive" in question 1 "How expressive are the robot's movements?" in the second survey, we added a description on the questionnaire based on the definition from the MerriamWebster dictionary "showing emotions and feelings clearly and openly" [150] as follows: "Expressive means when you see the movements, you can associate them with some emotions or adjectives, e.g. happy, strong, confident, shy, sad, frantic, graceful, etc." For question 2 "How dynamic are the robot's movements?" in the second survey, we give the following description: "Dynamic means you think that the robot's movements exhibit changing speed, directions, shapes that does not appear as just random, uncontrollable or seizure-like movements." The latter is based on the definition from Merriam-Webster dictionary: "always active or changing" [151].In contrast, no descriptions were given in the first survey. 
We added one question to collect data about people's opinion about the music used in the study as mentioned earlier. The question was "Please rate how much you like you like each music" and measured using 5-point Likert-scale with labels: "I do not like it at all", "I do not like it", "It is OK", "I like it", and "I like it very much". The ordering of the music was unknown to the user, and different from the order they are used in the videos. Each music piece is played for 30 seconds. The collection for this data was done at the start of the experiment.

A few notes regarding the video clips used in the second survey. First, the same set of video clips are used and no new motion data are produced for the second survey both for the Free/Dance mode and the Scenario mode. The order which each video clip appears is also unchanged. However, in the video clips used in the first survey, there is a considerable amount of background noise including noise from the environment and the servos as the robot is moving due to the fact that the video and sound were recorded using a web camera. We suspect that these noises create a distraction for the user and may influence their evaluations. Therefore, for the second survey, the original sound (noise and music) was completely removed and each music was overlaid onto the corresponding video clip using Adobe Premiere software. The start times of the music clips are aligned as close as possible with the start times of the music in the original video clips. In addition, the music played for each corresponding video clip uses the whole music from the MIDI file; that is, all the MIDI channels are played but the playback duration is matched with the length of the video clip. In contrast, only the MIDI channel that was used by the system was played in the video clips of the first survey ${ }^{6}$. The video used in this second survey is also available on the YouTube website ${ }^{7}$.

\footnotetext{
${ }^{6}$ URL: https://youtu.be/9QHp9B-0QK0

${ }^{7}$ URL: https://youtu.be/ASWy61H14zs
} 
The remainder of this section presents the result of the re-analysis of the first survey followed by the results from the second survey.

\subsubsection{Results from First Survey}

\subsubsection{Free/Dance Mode}

The critical value for Friedman test is determined by the Chi-squared $\left(\chi^{2}\right)$ table determined by $\alpha$ and degree of freedom $(d f)$. Degree of freedom is dependent to the number of categories $(k)$ as $d f=k-1$. Since Free/Dance Mode survey has five categories, the degree of freedom is $d f=5-1=4$. For Q1, Q2, Q3 of Free/Dance Mode, the critical value is $\chi^{2}(4)=9.488$ for $\alpha=0.05$. When the result of Friedman test shows that the calculated $\chi_{r}^{2}$ value is larger than the critical value $\left(\chi_{r}^{2}>\chi^{2}\right)$ which consequently indicates that $p<\alpha$, then we reject the null hypothesis. Post-hoc analysis of the Free/Dance Mode results takes into account the Bonferroni correction for ten pair-wise measurements. With $\alpha=0.05$, the adjusted value with correction is $\alpha_{a d j}=0.05 / 10=0.005$. Results are considered statistically different if $p<\alpha_{a d j}$. For each of Q1, Q2, and Q3 the null hypothesis to test is: there is no difference between the ratings of all categories: RobotA through RobotE. The same null hypotheses also apply to the Free/Dance Mode survey in the second survey below.

For Q1: "How expressive are the robot's movements?" $(N=26)$ we reject the null hypothesis with $\left.\chi_{r}^{2}(4)=37.4, p<0.00001\right)$. Post-hoc analysis reveals the rating differences occur between RobotB with RobotA, RobotC, RobotD, and RobotE, and between RobotE and all other categories. When the average rating/scores are considered, RobotB is rated significantly higher than other categories (MEAN=3.65, STD.DEV =0.75), while RobotE is rated as significantly lower than the other categories $(\mathrm{M}=1.73, \mathrm{SD}=0.87)$. 
For Q2: "How dynamic are the robot's movements?" $(N=26)$ we reject the null hypothesis with $\left.\chi_{r}^{2}(4)=22.85, p=0.000135\right)$. Post-hoc analysis reveals the rating differences occur between RobotB with RobotC, RobotD, and RobotE, and between RobotE and RobotA and RobotB. When the average rating/scores are considered, RobotB is rated significantly higher than other categories $(\mathrm{M}=3.42, \mathrm{SD}=0.76)$, while RobotE is rated as significantly lower than the other categories $(\mathrm{M}=2, \mathrm{SD}=1.06)$.

For Q3: "Overall how do you like the robot's performance?" $(N=24)$ we reject the null hypothesis with $\left.\chi_{r}^{2}(4)=39.93, p<0.00001\right)$. Post-hoc analysis reveals the rating differences occur between RobotB with RobotC, RobotD, and RobotE, and between RobotE and RobotA and RobotB. When the average rating/scores are considered, RobotB is rated significantly higher than other categories $(\mathrm{M}=3.58, \mathrm{SD}=0.65)$ while RobotE is rated as significantly lower than the other categories $(\mathrm{M}=1.88, \mathrm{SD}=0.8)$.

The re-evaluation of the first survey data using non-parametric methods of Friedman test yields similar results as using ANOVA; for all Q1, Q2, and Q3 the null hypothesis were rejected; however, post-hoc analysis shows some different outcomes. For Q1, RobotB is now reported as significantly different with respect to RobotC and RobotD instead of just RobotD from Scheffe. RobotE still reported as significantly different with the other categories and consistent with the result from the Scheffe test. For Q2, RobotB is reported as significantly different to RobotC, RobotD, and RobotE, while the result for RobotE differs from RobotA and RobotB. This result is different from Scheffe test where the differences are only between RobotB against RobotD and RobotE. For Q3, RobotB is found to be different from RobotC, RobotD, and RobotE, and RobotE is also different from RobotA. This result differs from the Scheffe test results where significance was found between RobotB and all other categories. No re-analysis was done for Q4, so the results are the same as previously reported. 


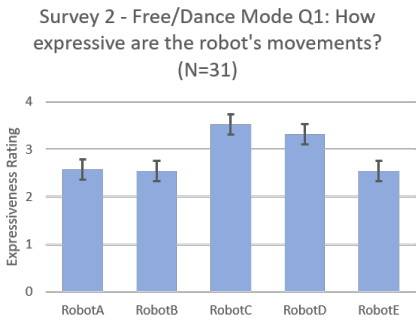

(a) Perceived Expressiveness
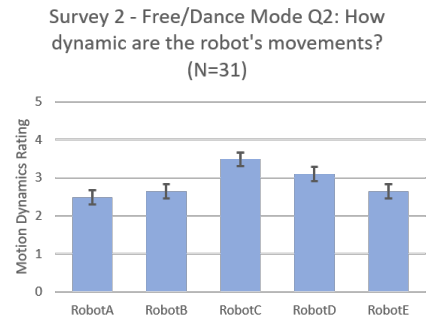

(b) Perceived Motion Dynamics

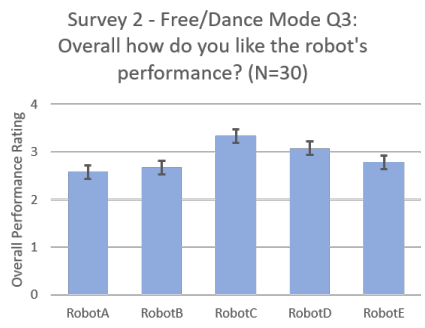

(c) Perceived Overall Performance

Figure 5.11: Means and standard error for Free/Dance Mode for the second survey.

Survey 2 - Free/Dance Q4: Which

robot performance do you like best?

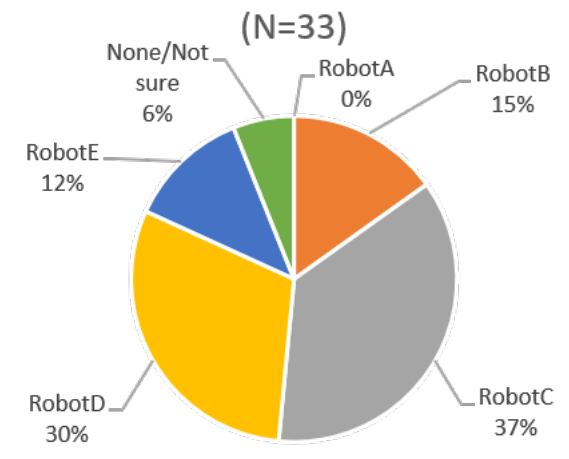

Figure 5.12: Survey result for the question: "Which robot performance did you like best?" 


\subsubsection{Scenario Mode}

Since the Scenario Mode survey has four categories, the degree of freedom is $d f=$ $4-1=3$. For Q1, Q2, Q3 of Scenario Mode, the critical value is $\chi^{2}(3)=7.815$ for $\alpha=0.05$. Post-hoc analysis of the Free/Dance Mode results takes into account the Bonferroni correction for six pair-wise measurements where the adjusted value with correction is $\alpha_{a d j}=0.05 / 6=0.008$. Results are considered statistically different if $p<\alpha_{a d j}$. For each of Q1, Q2, and Q3 the null hypothesis to test is: there is no difference between the ratings of all categories: RobotA through RobotD. The same null hypotheses also apply to the Scenario Mode survey in the second survey below.

For Q1: "How expressive are the robot's movements?" $(N=29)$ we reject the null hypothesis with $\left.\chi_{r}^{2}(3)=12.67, p=0.005\right)$. Post-hoc analysis shows the rating differences occur between RobotB with RobotC, and RobotD. When the average rating/scores are considered, RobotB is rated significantly higher than other categories $(\mathrm{M}=3.72, \mathrm{SD}=0.59)$ and the lowest rated is $\operatorname{RobotD}(\mathrm{M}=2.86, \mathrm{SD}=0.92)$.

For Q2: "How dynamic are the robot's movements?" $(N=29)$ we cannot reject the null hypothesis with $\left.\chi_{r}^{2}(3)=3.8, p=0.28\right)$.

For Q3: "Overall how do you like the robot's performance?" $(N=29)$ we reject the null hypothesis with $\left.\chi_{r}^{2}(3)=12.07, p=0.007\right)$. Post-hoc analysis shows the rating differences occur between RobotA and RobotB, and RobotB with RobotC, and RobotD. When the average rating/scores are considered, RobotB is rated significantly higher than other categories $(\mathrm{M}=3.59, \mathrm{SD}=0.63)$ and $\operatorname{RobotD}$ rated the lowest $(\mathrm{M}=$ $2.86, \mathrm{SD}=0.99)$

The non-parametric analysis of the Scenario Mode from the first survey yields similar results as using ANOVA as reported earlier. 


\subsubsection{Summary}

The null hypothesis testing using ANOVA and Friedman tests on the First Survey dataset yield identical results for both the Free/Dance Mode and the Scenario Mode: The null hypothesis was rejected for Q1, Q2, and Q3 in the Free/Dance Mode survey, also for Q1 and Q3 in the Scenario Mode survey, and accepted in Q2 of the Scenario Mode survey. However, the post-hoc analysis shows slightly different results between the Scheffe test and Wilcoxon Signed-rank test.

\subsubsection{Results from Second Survey}

\subsubsection{Music Survey}

Performing Friedman test over the music survey data $(N=35)$ shows that there are no differences of preferences between the four music pieces used in the study $\chi_{r}^{2}(\alpha=0.05, d f=3)=7.01,\left(\chi^{2}<7.82\right)($ n.s $)$.

Figures 5.13 and 5.14 show the results of three samples selected from our dataset as example. The top row (Figures 5.13a, 5.13b, 5.13c) shows the ratings of subjects $\mathrm{A}, \mathrm{B}$, and $\mathrm{C}$ with respect to the music and Free/Dance Mode questionnaires. The first group of bars is labeled 'Music' indicating the subject's ratings for music pieces M1, M2, M3, and M4 which represents: Beethoven's Symphony No. 5, Lullaby of Birdland, Mozart Sonata No. 16, and the Pink Panther Theme song, respectively. The five bars over the labels 'DQ1', 'DQ2', and 'DQ3' represents the ratings for RobotA through RobotE for Q1, Q2, and Q3 of the Free/Dance Mode questionnaire. Recall that the music M1 is used both RobotA and RobotE; hence, there are four bars for the 'Music' group and five bars for the groups 'DQ1', 'DQ2', and 'DQ3'. The first four bars in every group are arranged with respect to the order of the

music pieces in the music questionnaire. Each bar is labeled as 'the music piece/video 
clip', i.e. 'M1/RobotA', 'M2/RobotC', 'M3/RobotB', 'M4/RobotD', and the fifth bar refers to 'M1/RobotE' as indicated by the legend on each graph. The bottom row (Figures 5.14a, 5.14b, 5.14c) shows the ratings of subjects A, B, and C with respect to the music versus Scenario Mode questionnaire. The labels 'SQ1', 'SQ2', and 'SQ3' represent the subjects' responses to Q1, Q2, and Q3 of the Scenario survey, respectively. Since the Scenario Mode survey only uses three music pieces: M1, M2, and M4, there are only three bars shown for each group representing only the video clips in the survey that use music. The three bars in 'SQ1', 'SQ2', 'SQ3' represents the ratings for 'M1/RobotB', 'M2/RobotD', and 'M4/RobotC'.

We do not find significant evidence that participants' ratings of the individual music pieces directly influence their ratings towards the video clips for either the Free/Dance or Scenario Modes. For example, Subject B in Figure 5.13b rated M3 the lowest, but gave M3/RobotC in DQ1, DQ2, and DQ3 a high rating. Similarly, there is no significant correlation between the music scores and the participants' ratings in the Scenario Mode (Figure 5.14). With these results, we tentatively conclude that how much a person likes a music piece does not affect how the person rates the robot performance that is based on that music piece.

\subsubsection{Free/Dance Mode}

As before, the Free/Dance Mode survey has five categories, the degree of freedom is $d=5-1=4$. For Q1, Q2, Q3 of Free/Dance Mode, the critical value is $\chi^{2}(\alpha=$ $0.05, d f=4)=9.5$. Post-hoc analysis of the Free/Dance Mode results takes into account the Bonferroni correction for ten pair-wise measurements. With $\alpha=0.05$, the adjusted value with correction is $\alpha_{a d j}=0.05 / 10=0.005$. Results are considered statistically different if $p<\alpha_{a d j}$. The mean and standard errors for Q1, Q2, and Q3 are shown in Fig. 5.11. Fig. 5.12 shows the result for Q4. 


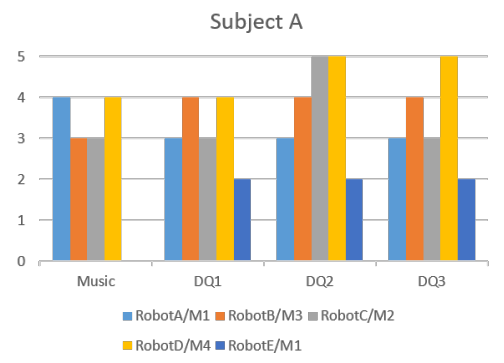

(a)

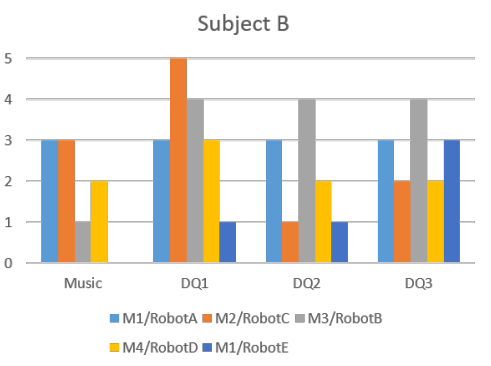

(b)

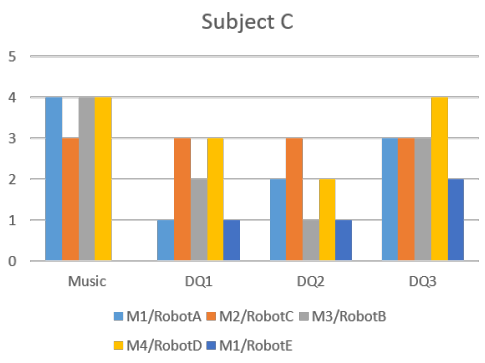

(c)

Figure 5.13: Ratings of music versus Free/Dance Mode video clips of three subjects (A, B, and C). The four bars over 'Music' represent the ratings for four music pieces used: M1, M2, M3, M4. The five bars over 'DQ1', 'DQ2', and 'DQ3' represent the five video clips in each Free/Dance Mode survey question and labeled as 'music piece/video clip': 'M1/RobotA', 'M2/RobotC', 'M3/RobotB', 'M4/RobotD', 'M1/RobotE'

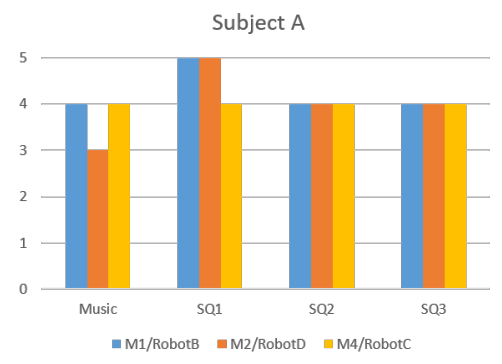

(a)
Subject B

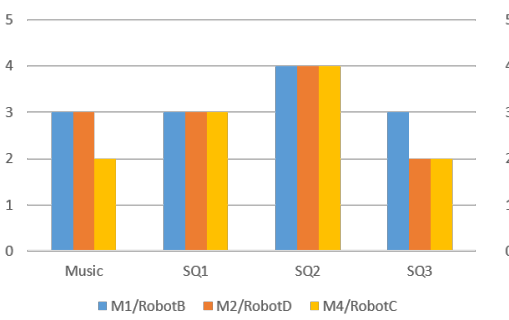

(b)

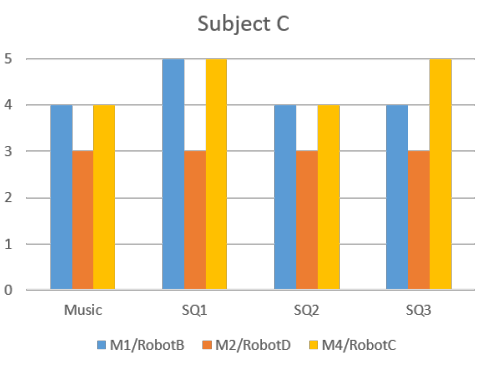

(c)

Figure 5.14: Ratings of music versus Scenario Mode video clips of three subjects (A, $\mathrm{B}$, and $\mathrm{C}$ ). These responses correspond to the same subjects in Figure 5.13. The three bars over 'Music' represent the ratings for three music pieces used: M1, M2, M4. The three bars over 'SQ1', 'SQ2', and 'SQ3' represent only the three video clips in each Scenario Mode survey question which uses music and labeled as 'music piece/video clip': 'M1/RobotB', 'M2/RobotD', 'M4/RobotC' 
For Q1: "How expressive are the robot's movements?" $(N=31)$ we reject the null hypothesis with $\chi_{r}^{2}(4)=23.05, p=0.00012$ ). Post-hoc analysis reveals the rating differences occur between RobotA with RobotC and RobotD, between RobotB with RobotC, and RobotD, and between RobotC and RobotE. When the average rating/ scores are considered, RobotC is rated significantly higher than other categories $(\mathrm{M}=$ $3.48, \mathrm{SD}=1.39$ ), while Robot $\mathrm{A}$ is rated as significantly lower than the other categories $(\mathrm{M}=2.55, \mathrm{SD}=1.12)$.

For Q2: "How dynamic are the robot's movements?" $(N=31)$ we reject the null hypothesis with $\chi_{r}^{2}(4)=13.48, p=0.0092$ ). Post-hoc analysis reveals the rating differences occur between RobotA with RobotC and RobotD, and differences between all other pairs of categories are not significant. When the average rating/scores are considered, RobotC is rated significantly higher than other categories $(M=3.48$, $\mathrm{SD}=1.39$ ), while RobotA is rated as significantly lower than the other categories $(\mathrm{M}=2.48, \mathrm{SD}=0.81)$.

For Q3: "Overall how do you like the robot's performance?" $(N=30)$ we reject the null hypothesis with $\left.\chi_{r}^{2}(4)=39.93, p<0.00001\right)$. Post-hoc analysis reveals the rating differences occur between RobotA with RobotC, and differences between all other pairs of categories are not significant. When the average rating/scores are considered, RobotC is rated significantly higher than other categories $(\mathrm{M}=3.34, \mathrm{SD}=1.008)$, while RobotA is rated the least $(\mathrm{M}=2.58, \mathrm{SD}=0.71)$.

For Q4: "Which robot performance do you like best?" $(N=33)$ RobotC has the highest number of votes at 37\%, followed by RobotD at 30\%, while no votes were given for RobotA (0\%). Six percent of the participants voted "None/Not sure". 


\subsubsection{Scenario Mode}

Since Scenario Mode survey has four categories, the degree of freedom is $d f=4-1=$

3. For Q1, Q2, Q3 of Scenario Mode, the critical value is $\chi^{2}(3)=7.82$. Post-hoc analysis of the Free/Dance Mode results takes into account the Bonferroni correction for six pair-wise measurements. With $\alpha=0.05$, the adjusted value with correction is $\alpha_{a d j}=0.05 / 6=0.0083$. Results are considered statistically different if $p<0.008$. The mean and standard errors for Q1, Q2, and Q3 are shown in Fig. 5.15. Fig. 5.16 shows the result for Q4.

For Q1: "How expressive are the robot's movements?" $(N=32)$ we cannot reject the null hypothesis with $\left.\chi_{r}^{2}(3)=7.43, p=0.06\right)$. When the average rating/scores are considered, RobotB is rated the highest $(\mathrm{M}=3.78, \mathrm{SD}=0.75)$ and the lowest rated is $\operatorname{RobotA}(\mathrm{M}=3.31, \mathrm{SD}=0.86)$.

For Q2: "How dynamic are the robot's movements?" $(N=32)$ we cannot reject the null hypothesis with $\left.\chi_{r}^{2}(3)=4.88, p=0.18\right)$. With respect to raw scores, RobotC has the highest rating $(\mathrm{M}=3.69, \mathrm{SD}=1.06)$, and RobotA with the lowest rating $(\mathrm{M}=$ $3.34, \mathrm{SD}=0.97)$.

For Q3: "Overall how do you like the robot's performance?" $(N=32)$ we also cannot reject the null hypothesis with $\left.\chi_{r}^{2}(3)=6.67, p=0.083\right)$. When the average rating/scores are considered, RobotB has the highest rating $(\mathrm{M}=3.59, \mathrm{SD}=0.87)$ and RobotD rated the lowest $(\mathrm{M}=3.19, \mathrm{SD}=0.99)$.

For Q4: "Which robot performance do you like best?" $(N=33)$ RobotC has the highest number of votes at $37 \%$, followed by RobotD at 30\%, while RobotA received the least number of votes $(3 \%)$. 


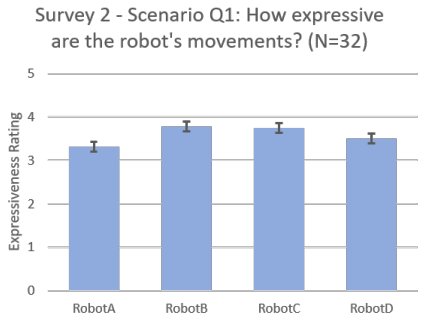

(a) Perceived Expressiveness

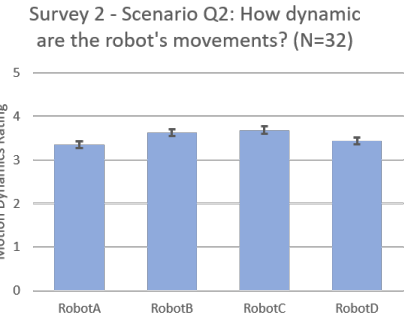

(b) Perceived Motion Dynamics

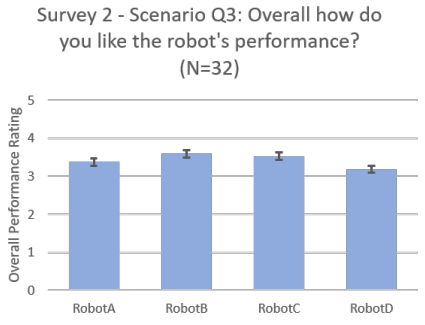

(c) Perceived Overall Performance

Figure 5.15: Means and standard error for Scenario Mode from the second survey

Survey 2 - Scenario Q4: Which robot

performance do you like best? $(\mathrm{N}=33)$

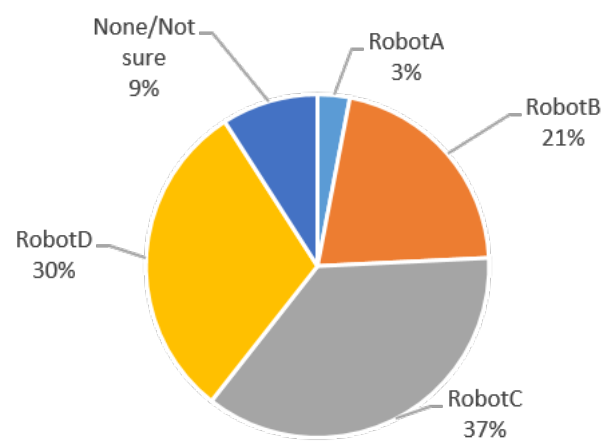

Figure 5.16: Result for Q4: "Which robot performance did you like best?" from the second survey 


\subsubsection{Summary}

For the Free/Dance Mode survey, the null hypotheses were rejected for all three questions Q1, Q2, Q3. For the Scenario Mode survey, the null hypotheses for all three questions are accepted; that is to say that there are no differences between the ratings for the four categories. There are two participants that commented that they liked RobotE in the Free/Dance Mode (manually-created motions).

\subsubsection{Comparison of Results from Repeated-measure ANOVA versus Friedman Test}

We summarize the results of using repeated-measure ANOVA and Friedman test in Table 5.6 for to the $F$ and $\chi^{2}$ values, respectively, and Table 5.7 for acceptance/ rejection of the null hypothesis. From the data of the first survey, both methods yield identical results for both the Free/Dance and Scenario modes for Q1, Q2 and Q3. In the second survey, differences occur on Scenario Mode Q1 and Q3 where the null hypotheses are rejected by repeated-measure ANOVA but accepted by Friedman test. Post-hoc analysis using Scheffe test found no difference between any of the categories for Q1 and Q3, but Wilcoxon signed-rank test found difference between RobotA and RobotB for Q1, and found nothing for Q3. Assuming no false positives/negatives in the post-hoc analysis, these results indicate a possible Type II error (false negative) on the Friedman test for Q1 and Type I error (false positive) for the repeated-measure ANOVA for Q3.

Post-hoc analysis using Scheffe versus Wilcoxon Signed-rank tests yield a few

differences in the results as shown in Table 5.8. Differences occur on Free/Dance Mode Q1 in Survey 1 and 2, Free/Dance Mode Q2 in Survey 2, Free/Dance Mode Q3 in Survey 1, and Scenario Mode Q3 in Survey 1. Notice that although the Friedman 
test failed to reject the null hypothesis for Scenario Mode Q1 in Survey 2, posthoc analysis shows a difference between RobotA and $\operatorname{RobotB}\left(\chi_{r}^{2}(3)=0.0062, p<\right.$ 0.0083), suggesting a possible Type II error as mentioned earlier.

Table 5.6: Compilation of hypothesis testing results of the first and second survey

\begin{tabular}{|c|c|c|c|c|c|}
\hline \multirow{2}{*}{ Mode } & \multirow{2}{*}{ Item } & \multicolumn{2}{|c|}{ Survey 1} & \multicolumn{2}{|c|}{ Survey 2} \\
\hline & & $\begin{array}{l}\text { Repeated- } \\
\text { measure } \\
\text { ANOVA }(\alpha= \\
0.05)\end{array}$ & $\begin{array}{l}\text { Friedman } \\
\text { test } \\
(\alpha=0.05)\end{array}$ & $\begin{array}{l}\text { Repeated- } \\
\text { measure } \\
\text { ANOVA } \\
(\alpha=0.05)\end{array}$ & $\begin{array}{l}\text { Friedman } \\
\text { test } \\
(\alpha=0.05)\end{array}$ \\
\hline Free/Dance & Q1 & $\begin{array}{l}F(4,100)= \\
23.01, \quad p< \\
0.0001\end{array}$ & $\begin{array}{l}\chi_{r}^{2}(4) \quad= \\
37.4 \\
p<0.00001\end{array}$ & $\begin{array}{l}F(4,120)= \\
9.11, \quad p< \\
0.0001\end{array}$ & $\begin{array}{l}\chi_{r}^{2}(4) \\
23.05 \\
p<0.001\end{array}$ \\
\hline Free/Dance & $\mathrm{Q} 2$ & $\begin{array}{l}F(4,100)= \\
12.08, \quad p< \\
0.0001\end{array}$ & $\begin{array}{l}\chi_{r}^{2}(4) \quad= \\
22.85, \\
p=0.00014\end{array}$ & $\begin{array}{l}F(4,120)= \\
5.34, \quad p< \\
0.001\end{array}$ & $\begin{array}{l}\chi_{r}^{2}(4) \\
13.48 \\
p<0.001\end{array}$ \\
\hline Free/Dance & Q3 & $\begin{array}{l}F(4,92)= \\
20.21, p< \\
0.0001\end{array}$ & $\begin{array}{l}\chi_{r}^{2}(4) \quad= \\
39.93 \\
p<0.00001\end{array}$ & $\begin{array}{l}F(4,112)= \\
4.92, \quad p< \\
0.01\end{array}$ & $\begin{array}{l}\chi_{r}^{2}(4) \\
39.93 \\
p<0.00001\end{array}$ \\
\hline Scenario & Q1 & $\begin{array}{l}F(3,84)= \\
8.073, \quad p< \\
0.0001\end{array}$ & $\begin{array}{l}\chi_{r}^{2}(3) \quad= \\
12.67, \\
p=0.0054\end{array}$ & $\begin{array}{l}F(3,93)= \\
4.78, \quad p< \\
0.05\end{array}$ & $\begin{array}{l}\chi_{r}^{2}(3)= \\
7.43, \\
p=0.059 \\
\text { (n.s.) }\end{array}$ \\
\hline Scenario & Q2 & $\begin{array}{l}F(3,84)= \\
1.87, \quad p= \\
0.14(\text { n.s. })^{*}\end{array}$ & $\begin{array}{l}\chi_{r}^{2}(3)=3.8 \\
p=0.28 \\
\text { (n.s.) }\end{array}$ & $\begin{array}{l}F(3,93)= \\
2.65, \quad p= \\
0.053 \text { (n.s.) }\end{array}$ & $\begin{array}{ll}\chi_{r}^{2}(3) & = \\
4.875, & \\
p \quad= & 0.18 \\
\text { (n.s.) } & \end{array}$ \\
\hline Scenario & Q3 & $\begin{array}{l}F(3,84)= \\
5.72, \quad p< \\
0.01\end{array}$ & $\begin{array}{l}\chi_{r}^{2}(3) \quad= \\
12.07 \\
p=0.0071\end{array}$ & $\begin{array}{l}F(3,84)= \\
2.93, \quad p< \\
0.05\end{array}$ & $\begin{array}{l}\chi_{r}^{2}(3)= \\
6.67 \\
p=0.083 \\
\text { (n.s.) }\end{array}$ \\
\hline
\end{tabular}


Table 5.7: Null hypothesis acceptance/rejection using ANOVA versus Friedman test for data in the first and second survey (see Table 5.6)

\begin{tabular}{|l|l|l|l|l|l|}
\hline \multirow{2}{*}{ Mode } & \multirow{2}{*}{ Item } & \multicolumn{2}{|c|}{ Survey 1 } & \multicolumn{2}{c|}{ Survey 2 } \\
\cline { 3 - 6 } & & $\begin{array}{l}\text { Repated- } \\
\text { measure } \\
\text { ANOVA }\end{array}$ & Friedman & $\begin{array}{l}\text { Repated- } \\
\text { measure } \\
\text { ANOVA }\end{array}$ & Friedman \\
\hline Free/Dance & Q1 & Reject & Reject & Reject & Reject \\
Free/Dance & Q2 & Reject & Reject & Reject & Reject \\
Free/Dance & Q3 & Reject & Reject & Reject & Reject \\
Scenario & Q1 & Reject & Reject & Reject & Accept** \\
Scenario & Q2 & Accept & Accept & Accept & Accept \\
Scenario & Q3 & Reject & Reject & Reject & Accept** \\
\hline
\end{tabular}

$* *$ Result difference

Table 5.8: Post-hoc analysis using Scheffe versus Wilcoxon Signed-rank (WSR) tests for data in the first and second survey to find pairs of categories with significantly different ratings $(\mathrm{A}=\operatorname{Robot} \mathrm{A}, \mathrm{B}=\operatorname{Robot} \mathrm{B}, \mathrm{C}=\operatorname{Robot} \mathrm{C}, \mathrm{D}=\operatorname{Robot} \mathrm{D}, \mathrm{E}=\operatorname{Robot} \mathrm{E})$

\begin{tabular}{|c|c|c|c|c|c|}
\hline \multirow{2}{*}{ Mode } & \multirow{2}{*}{ Item } & \multicolumn{2}{|c|}{ Survey 1} & \multicolumn{2}{|c|}{ Survey 2} \\
\hline & & Scheffe & $\begin{array}{l}\text { WSR (w/ } \\
\text { Bonferroni) }\end{array}$ & Scheffe & $\begin{array}{l}\text { WSR (w/ } \\
\text { Bonferroni) }\end{array}$ \\
\hline Free/Dance & Q1 & $\begin{array}{ll}\text { A-E, } & \text { B-D, } \\
\text { B-E, } & \text { C-E, } \\
\text { D-E } & \end{array}$ & $\begin{array}{l}\text { A-E, B-C }{ }^{* *}, \\
\text { B-D, B-E, } \\
\text { C-E, D-E }\end{array}$ & $\begin{array}{l}\text { A-C, } \quad \text { B-C, } \\
\text { C-E }\end{array}$ & $\begin{array}{l}\mathrm{A}-\mathrm{C}, \quad \mathrm{B}-\mathrm{C}, \\
\mathrm{B}-\mathrm{D}^{* *}, \mathrm{C}-\mathrm{E}\end{array}$ \\
\hline Free/Dance & Q2 & $\begin{array}{l}\text { A-E, } \quad \text { B-C, } \\
\text { B-D, B-E }\end{array}$ & $\begin{array}{l}\text { A-E, } \quad \text { B-C, } \\
\text { B-D, B-E }\end{array}$ & $\mathrm{A}-\mathrm{C}$ & $\mathrm{A}-\mathrm{C}, \mathrm{A}-\mathrm{D}^{* *}$ \\
\hline Free/Dance & Q3 & $\begin{array}{ll}\mathrm{A}-\mathrm{B}^{* *}, & \mathrm{~A}- \\
\mathrm{E}, \mathrm{B}-\mathrm{C}, & \mathrm{B}- \\
\mathrm{D}, \mathrm{B}-\mathrm{E} & \end{array}$ & $\begin{array}{l}\text { A-E, } \quad \text { B-C, } \\
\text { B-D, B-E }\end{array}$ & $\mathrm{A}-\mathrm{C}$ & $\mathrm{A}-\mathrm{C}$ \\
\hline Scenario & Q1 & $\mathrm{B}-\mathrm{C}, \mathrm{B}-\mathrm{D}$ & $\mathrm{B}-\mathrm{C}, \mathrm{B}-\mathrm{D}$ & $\mathrm{N} / \mathrm{A}$ & $\mathrm{A}-\mathrm{B}^{* *}$ \\
\hline Scenario & Q2 & $\mathrm{N} / \mathrm{A}^{*}$ & $\mathrm{~N} / \mathrm{A}$ & $\mathrm{N} / \mathrm{A}$ & $\mathrm{N} / \mathrm{A}$ \\
\hline Scenario & Q3 & $\mathrm{B}-\mathrm{C}^{* *}, \mathrm{~B}-\mathrm{D}$ & B-D & $\mathrm{N} / \mathrm{A}$ & $\mathrm{N} / \mathrm{A}$ \\
\hline
\end{tabular}

* Not Available.

** Result difference between Scheffe versus WSR per survey 


\subsection{Discussion}

Both the Free/Dance Mode and Scenario Mode results provide interesting insights to the issue of synthesis of expressive movements for a Robot Actor in a Robot Theater. The insights are the following:

- The results show that there indeed exists information of expressiveness in a musical score (i.e. song). This information of expressiveness can be extracted and translated into robot motions which exhibit dynamic qualities that match the dynamic qualities in the music, e.g. large change of note/pitch translated as large motions on the robot.

- The proposed system was able to capture some of the expressive information in terms of timing, note-on velocity, note duration and pitch, and translate these information into motion properties of: time of execution, range of motion, and acceleration. In other words, the proposed system was able to use the dynamic information in music and produce motions which matched with the music. Some participants were able to articulate that some of the robots performed according to the affect the participants perceived in the music.

- At a glance, the proposed system scored better overall both over manually created dance sequence, and normal Scenario execution (without music information). However, there was only one example of manually created performance for each experiment. Further studies need to be done to analyze how useful is the system to improve or create expressiveness by comparing the performances generated by the proposed system with more manually-created performances.

- We expected that the final Preference score could be predicted from the Perceived Expressiveness, Dynamism, and Overall Performance scores. The actual 
Preference scores show that this is not always the case. Fig. 5.7 and Fig. 5.9 show the average scores between the three questions for each robot in the Free/Dance and Scenario surveys, respectively. In both surveys, the performance that consistently scored the best in both surveys did emerge as the best preferred performance. However, the robot performance that has been a runnerup from the results of the three questions did not end up being the runner-up in the Preference score.

- We found no indication that when a user likes a particular music piece, the user will also favor the robot performance that was created by our system based on that music piece.

- In the Free/Dance Mode, the Lynxmotion robot produces motion artifacts in the form of jitters and strong whiplashes on fast movements. These artifacts may negatively influence how the audience perceive the quality of movement of the robot, and consequently the survey scores.

- When executing a Scenario, the addition of music information only slightly helps improving the performance of the Scenario. This could be caused by the limited range of motion and degrees of freedom of the robot, and the set of gestures that does not convey any strong meaning, at least as perceived by the survey participants.

- On faster music, the RC servos are unable to keep up with the speed required to reach the desired range of motion. This results only in small movements on the robot, similar to a seizure, which may be confusing for the observer.

- The current system only extracts information from one channel out of the several channels in the MIDI music data where each channel typically correspond to one 
musical instrument. This limits the number of Timed Events that is extracted which creates several long pauses in the movements, whereas when the whole music is played, the melody continues to play. This is because the melody of the music may be created by the interplay of multiple instruments.

- Removal of the noise from the video clips may seem like a good idea since it focuses the participant only on the movements versus music, but in actual/live performances, the servo noises cannot be avoided (unless using some specialized, noiseless actuators is possible).

- Several studies suggested that some parametric methods are not sensitive to data that violates the assumptions e.g. normal distribution, homogeneity of variance, and extreme outliers, even for Likert-scale (ordinal) data and small sample size [168]. In general, it is still safer to use non-parametric methods as suggested in [169] to avoid Type I or Type II errors. As shown in Tables 5.7 and 5.8, there are a few disagreements between the results of the parametric versus non-parametric methods. We recommend future researchers whose data is collected using similar surveys, measurements, and sample size as the one used in this study to analyze their data with both parametric and non-parametric methods for a more thorough report, when possible.

- Throughout the experiment, the factor parameter that determines how much the music data will influence the original Scenario data was set to 0.1. The results of using different factor values have not been observed, and should be investigated in future experiments.

- In the second survey, there are two participants that commented that they like the performance of RobotE in the Free/Dance Mode (manually created motion). 
Arguably, with enough time and effort, one could definitely manually create an excellent robot 'dance' performance. However, the results show that our system is able to produce outcomes that are at least as good as hand-crafted motions with much less time and effort.

\subsection{Conclusions and Future Works}

The problem addressed by the methods proposed in this chapter is:

- Current method of finding the right timing information when executing robot motion to exhibit affect (e.g. emotions) is done by trial-and-error. Timing determines the dynamic changes in acceleration, speed, and range of motion.

The main contribution of the work in this chapter is proposing using music information to provide the timing information needed to exhibit affect in robot motion. I show that music information can be used to:

\section{Produce complete motion data}

2. Change the performance of a predetermined sequence of motion

The benefit of this method is that the user no longer need to modify timing for each pose by trial-and-error. Instead, to change the affect of the motion, the user only need to provide the type of music that represent the desired affect, e.g. 'upbeat' music for more energetic motion, 'slow' music for a more calm/controlled motion.

The dynamic qualities in music can be translated into similar expressive and dynamic qualities in the motions of robots of different form. The music data was used to synthesize motion data directly from the note code values. The result is a expressive and dynamic-looking robot motion. Additionally, the timing of note events in the music can be used to time the execution of a pre-determined sequence of 
gestures in a Scenario. Note events such as note-on velocity (loudness), note duration, and note code (pitch) in the music data can be used to control the execution of the gesture motion data. This gives the motion some expressive dynamics in terms of velocity, acceleration, range of motion.

The approach presented here shows that music data can be used as some kind of a characteristic function that can be applied to different motions that works in a similar way as the work done by Unuma [85]. Whereas events in speech information have been used to execute beat gestures [158], the rich melodic information in music was shown to allow expressive movements on the Lynxmotion robot, which occasionally resemble deictic and iconic gestures. However, there are many more ways to map the melodic events to the gesture selection, only guidelines. For example: for a melody with an increasing pitch trend, deictic gestures that move upwards such as pointing up are appropriate. A melody that has repetition in some intervals is suited for repeating gestures such as beat gestures. One of the survey participant was a musician and suggested that since music can be marked with down beat and up beat (odd and even beats, respectively), typically a downward movement is done on the down beat, and an upward movement on the up beat. These insights can also be taken into consideration in the future.

The use of the word "dynamic" in Q2 of the surveys may be ambiguous for some participants. In music, the term "dynamics" is strictly used to refer to loudness of the notes being played, e.g. pianissimo (very softly/quietly) or fortissimo (very loudly) [170]. In motion, the term "dynamic" as in "dynamic motion" refers to continuously changing or moving with some energy (e.g. "energetic" or "forceful" [151]). In this case, because the interest is on the robot motion, descriptions must be added to the respective questions based on the definitions from Merriam-Webster dictionary with respect to motion [151]. 
This work can be extended in several ways. First, there is still no reliable method to extract rich melodic information from sampled music signals. This is the main reason MIDI was used as input in this study. Moreover, the extraction and conversion of music information to motion was generated offline, not in real time. Better methods need to be investigated to enable both extraction of melodic information and motion generation in real-time. The current program is limited to offline processing of the music data, thus cannot be used for generating motion data in real-time with active listening.

Second, there are several MIDI control events that are not being evaluated in the proposed method. The contributions of those events as additional motion control parameter may be explored. When fully explored, the MIDI Show Control (MSC) protocol [171] may be used to control the whole Robot Theater.

Third, simultaneous note events are consolidated into a single event in the proposed method. Most likely, the simultaneous note events represent a musical chord. Musical chords have interesting properties such as creating harmonics in the sound frequency. A study can be done to analyze simultaneous note events into chords, and then analyze the chords information to be used as a control parameter for motion data. More musical pieces could be used to test the effectiveness of the current version of the program to generate expressive motion data with respect to different types and genre of music. Many other properties in the music still need to be explored such as: scales, and cadence.

Fourth, the typical RC servos used low-cost robots used in this study are limited in speed and response time, making execution of fast, short-duration movements caused by quick succession of notes intangible. A smarter approach is needed so only Timed Events which corresponding movements would be visible (i.e. within the capabilities of the servos) are executed. 


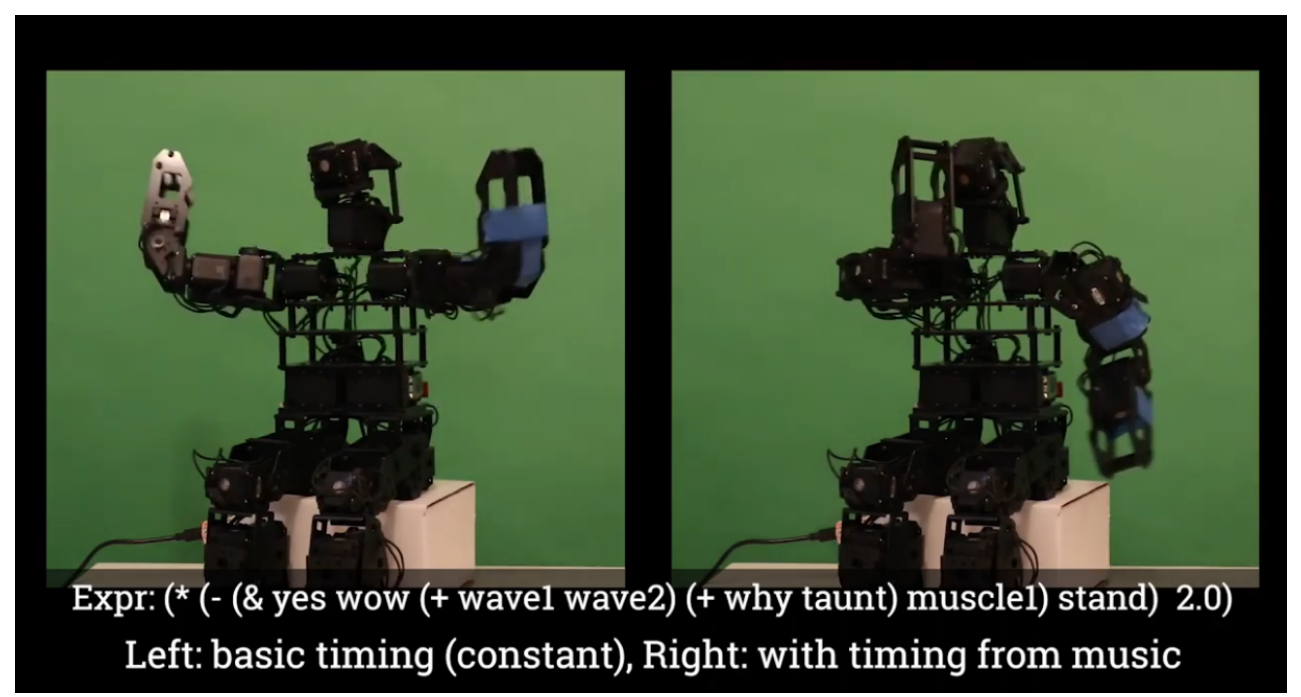

Figure 5.17: Video demonstration of motions timed by music information from Lullaby of Birdland (https://youtu.be/JuDXhHRo2Kw)

The proposed system could be improved by using forward or inverse kinematics to use the melodic information on the robot's paths instead of joint angles. Dynamics simulation can also be used to allow animations that appear to be reacting to external and internal forces.

\subsection{Additional Videos}

Additional videos demonstrating using the music timing information to change motion expressions (Fig. 5.17) can be viewed on the internet: https://youtu.be/6vp7YNxESY, https://youtu.be/JuDXhHRo2Kw.

\subsection{Contributions}

The contributions of my work presented in this chapter are as follows:

- Introduce a new method to extract information from music to produce timing and stroke data for new motion generation, and automatic application of the 
extracted timing data to pre-defined motions, resulting in motions that can have different expressions depending on the styles of music provided.

- Performed user study that show the validity of this method in producing robot motions that are scored as at least as expressive or better compared to motions which timing were designed by hand.

- Performed rigorous hypothesis testing analysis from survey data by comparing results from repeated-measure ANOVA with the non-parametric Friedman test. Post-hoc analysis was also performed on the survey data using Scheffe and Wilcoxon Signed-Rank tests to find pairs of categories with significantly different rankings.

\subsection{Future Works}

The work presented in this chapter is limited to only using timing and note interval information to guide the execution of humanoid robot motion data. There are many other musical elements that can be used that have not been utilized in this work, such as: cadence to manage the completion or start of a musical phrase ${ }^{8}$, chord $^{2}$ progressions to create the sensation of tension and release, musical dynamics that varies when a note is being played loudly or softly (fortissimo vs. pianissimo), or musical scales where major scale indicates a 'happy' or 'bright' feeling while the minor scale indicates a 'foreboding' or 'brooding' feeling. These elements of music can be further analyzed and investigate their mapping to motion data either using some heuristics or rules, or using 'creative systems'[119].

\footnotetext{
${ }^{8}$ Often equated with punctuations in a sentence.
} 


\section{CHAPTER 6}

\section{PARAMETERS FOR MOTION TEXTURING}

In this chapter, I present new methods for motion texturing using established methods of Kochanek-Bartels interpolation [141] and wavelet analysis [172] [173]. The term "motion texture" has been used to refer to variability in motions which can be applied to motions of human-like characters [140] [174]. In the context of this work, the term "motion texture" is used to refer to additional modifications to add expressivity to a "base" motion data like emotions, stuttering effect, exaggeration and other effects. A base motion datum/trajectory only consists of primary or key poses that represent the characteristics of the motion e.g. hand waving. Current methods emphasize on manual editing of individual poses in the motion data [13] or customized local modifications [14] [12]. The method presented here provide global modifications to the entire motion data which can produce many variety of "textures" while maintaining the identity of the motion (minimal deviation from the base motion trajectories up to an extent) by modifying a few parameters instead of modifying individual frames/poses. Near the end of this chapter, I also reported which types of wavelets are effective in producing certain kinds of motion textures. In contrast, previous methods only focused on producing a limited set of expressions [13] [175] [12].

In hand-drawn or computer animation each rendered frame contains a pose, and each frame lasts only a fraction of a second, depending on the rate of frames per second (fps). Therefore, for a 1-second animation clip at $24 \mathrm{fps}$, there are twentyfour distinct poses. In contrast, one robot motion data contains very few poses and 
its execution could last for a few seconds. Each pose is analogous to a keyframe in hand-drawn animation. To execute the motion, the joint angles or position values in each pose are given as a command to the actuators (i.e. servos) to be executed simultaneously. Unlike hand-drawn animation, there is no 'in-between' frames/poses in robot motion because they naturally occur as the actuators move. A motion from one pose to the next is called a 'stroke'. Therefore, depending on the distance of the stroke and the actual speed of the actuator, the amount of time to complete the stroke can vary. To ensure the robot reaches the desired pose before sending the next pose command, the animator can implement a delay by estimating how much time the stroke will take, or if the actuator has position feedback, perform a check until the desired pose is achieved. In some tools like RME, the animator can specify the speed of a stroke by declaring the amount of time the stroke should take. A shorter period would imply a faster stroke, and a longer period implies a slower stroke. The user can also specify the amount of pause or hold time for the pose, so the robot maintains the pose for a moment before moving to the next pose.

Based on the above, the main differences between how motions are specified in the 'traditional' keyframe method and how motions are typically specified for robots is in the way timing is specified for the motion. These differences are summarized in Table 6.1. In the keyframe method, the timing between each pose is fixed by the framesper-second rate. In robot motion, the user has the freedom to specify the timing between each pose. In the former, speed, acceleration, and dynamics of movements are created by the animators by how much change occurs between two poses. In the latter, the user fixes the amount of change between two poses and controls the dynamics of movements via timing. It is important to note that animating fluid, dynamic movements can be a labor-intensive task for an animator because he/she must draw each frame and there are very many frames to create. Moreover, the 
Table 6.1: Differences between traditional keyframe animation and robot animation.

\begin{tabular}{ll}
\hline Traditional animation & Robot animation \\
\hline $\begin{array}{l}\text { Timing between poses is fixed by fram- } \\
\text { erate }\end{array}$ & $\begin{array}{l}\text { Timing between poses determined by } \\
\text { user-specified parameter }\end{array}$ \\
\hline $\begin{array}{l}\text { Motion dynamics created by amount } \\
\text { of change between two consecutive } \\
\text { poses/frames }\end{array}$ & $\begin{array}{l}\text { Motion dynamics determined by speci- } \\
\text { fying speed or time constraint }\end{array}$ \\
\hline $\begin{array}{l}\text { Does not have to obey laws of physics } \\
\text { but physical realism must be created } \\
\text { manually }\end{array}$ & $\begin{array}{l}\text { Constrained by laws of physics in the } \\
\text { robot's kinematics and performance of } \\
\text { the actuators }\end{array}$ \\
\hline $\begin{array}{l}\text { Animator creates key poses and in- } \\
\text { between poses }\end{array}$ & Animator specifies only key poses \\
\hline Easy to make dynamic movements & Difficult to create dynamic movements \\
\hline
\end{tabular}

animator needs not to worry of violating laws of physics in the animation. However, when an animation requires physical realism, the animator must create that physical realism manually. Robot motion - on the other hand - is impractical to create by specifying small, incremental movements by hand. Like in computer animation, interpolation methods are used to create the incremental movements for a desired trajectory.

Creating in-between frames is one of the most labor, time, and cost-intensive process in animation. Therefore, modern 3D and computer animation software like Blender and Autodesk Maya provide interpolation features so animators only need to work with keyframes and do not need to manually create each in-between frames.

Robot motion data are often represented as tabular data where the rows represents the degrees of freedom (DOFs) and columns represent the sequence of poses. Every column defines a pose of the robot, which is the positions for all DOFs of the robot at one unit of time. Each row represents the motions of one DOF over time. The datapoints in this row can be plotted as a curve where the $\mathrm{x}$-axis is time and $\mathrm{y}$-axis 
is the axis for joint angle or position in space. Because of this, motion data can be viewed and treated as signals.

Gestural motion data are typically defined by a sequence of key poses; specific poses that define the shape of the gesture. For example, a waving gesture is defined by two key poses: the hand raised up slightly angled to the right, and the same raised hand but angled slightly to the left. The waving gesture is accomplished by alternating between these two poses. To make the gesture executed with some (emotional) expressiveness, additional poses must be given in between the two poses. For hand-drawn animation, this is done by the process called in-betweening, which refers to adding the "in-between frames" between the key poses. In computer animation or robot animation, these additional frames are commonly generated using interpolation methods.

\subsection{Motion Texture}

The term "motion texture" was first introduced by Pullen [140] and has been used to describe changes/different motion characteristics in various context, from human motion synthesis [174], general video querying/retrieval [176], and analyzing physical systems [177].

In this work, I use the term "motion texture" like [140] [174] to refer to effects that are added to change motion trajectories to change the expressions of the motion. The "normal" or baseline of the motion is when the motion is linearly interpolated, or executed using the do-and-delay/bang-bang method (explained below). Examples of motion textures are: smoothing, smear, stutter, shaky/tremor, and exaggeration. In general we call 'smoothing' as introducing acceleration and deceleration when the motion starts, stops, or changes direction. We call 'smear' when the motion data is 
overly-smoothed such that it cause some loss of detailed movements from the original motion. 'Stutter' is where the motion progression towards a pose but consists of visibly discrete steps. 'Shaky/tremor' is when the motion appear shaky as having noise in the motion data. We use the term 'exaggeration' to refer to when motions are executed with reduced (subdued) or increased (exaggerated) range of motion with respect to the joint angle values in the original data.

In the following section, we discuss the process of producing motion textures on a robot on a sequence of gestures that was specified using a REBeL's behavior expression. For now, the specific kind of gesture is not important. The following discussion will be general for any motion-based gesture behaviors specified using REBeL.

Let's assume a robot gesture that is defined by a probabilistic REBeL behavior expression $G$. Evaluating (sampling) $G$ yields an instance of the gesture $\hat{G}$ and consists of a sequence of length $\mathrm{m}$ of primary actions $P_{i}$ so $\hat{G}=\left[P_{0}, \cdots, P_{m}\right]$. For now, let's assume that each primary action is a pose of the robot which may be repeated within the sequence. Let's further assume that each pose $P_{i}$ is a key pose and the sequence does not contain transient/in-between poses. If the sequence is executed as it is, when a pose is given as a command to the robot, the robot's actuators will move at their maximum speed from pose $P_{i}$ to $P_{i+1}$. The resulting motion will appear 'mechanical' because every part moves and stops at the same time for each pose, and 'lifeless' because the robot appears to perform the motions without any pauses which would indicate thinking. To avoid this, each $P_{i}$ is assigned Timing and PauseTime parameters. Timing is a real-valued variable which defines the amount of time to execute the stroke from $P_{i-1}$ to $P_{i}$. High Timing value indicates a slow stroke, while a low value indicates a fast stroke. PauseTime indicates the amount of time to hold the pose $P_{i}$. These timing parameters are used to determine how many additional data points should be added before applying interpolation. After the data are interpolated, 
Table 6.2: Parameters to tune for executing expressive motions using our method

\begin{tabular}{lll}
\hline Parameter name & Context & Description \\
\hline Tension & Interpolation & $\begin{array}{l}\text { One of the parameters of Kochanek- } \\
\text { Bartels (KB) interpolation [141] }\end{array}$ \\
\hline Bias & Interpolation & KB interpolation parameter \\
\hline Continuity & Interpolation & KB interpolation parameter \\
\hline Bandpass coefficients & Filtering & $\begin{array}{l}\text { Magnitude of bandpass components } \\
\text { from multiresolution filtering (MRF) } \\
{[13]}\end{array}$ \\
\hline Time window & Filtering & Parameter for transition filter $[17]$ \\
\hline
\end{tabular}

multiresolution filtering can be applied for further processing to reduce noise, creating exaggeration, and so on. The set of parameters used in our method is summarized in Table 6.2.

\subsection{Approaches of Executing Motion Data}

We now know how gestural motion data for a humanoid robot are represented. How do these data get executed on the robot? Based on literature, my experience and previous works, I identified three basic approaches: "do-and-delay", reactive, and streaming.

- Do-and-delay - What we call as the "do-and-delay" approach is where each pose is being sent to the robot's hardware (e.g. servo controller board) without a feedback system to indicate when the pose has been reached. This method is also sometimes called the 'bang-bang' approach, as the commands are given suddenly without considering what the system (robot) is currently doing. In this method, the user must guess and set an estimate how much time it will take to move from one pose to the next and delays sending the next target 
pose until that time has passed. This approach is relatively simple to use but finding the right amount of delays between poses can be difficult to do. When the delay is too short, the actuator may start moving towards the new target pose before reaching/completing the current target pose. For example: armwaving gesture has two key poses of the arm with distance $\Delta \theta$ at the shoulder repeated alternatingly. When the delay is too short, the arm will appear to oscillate very quickly without completing the distance $\Delta \theta$. When the delay is too long, the distance $\Delta \theta$ may be reached with time to spare. Therefore, there will be periods where the robot appears to stop moving completely at each pose, creating the common "robotic motion" effect. Thus, it is difficult to create a smooth, natural-looking motion using this approach.

- Reactive - The "reactive" approach is when there is some feedback loop between the robot's actuators and the control system. The feedback system allows the control system to wait until the feedback indicates that the previous pose command had The control system sends the new target pose, and the feedback loop continuously updates the control system with the information of the instantaneous positions of the actuators giving the difference $\Delta \theta$ between the target pose and the actuator's current pose. Once the feedback information indicates that the target pose has been reached $\Delta \theta \leq 0 \pm \epsilon^{1}$, then the control system can send the next target pose. This approach eliminates the need for the user to guess how much delay must be given between poses. Naturally, some additional hardwares are required to create the feedback loop such as: adding encoders to the actuators, limit switches, potentiometers, and some additional wirings. An alternative would be to use actuators which are already equipped with a

\footnotetext{
${ }^{1}$ The error term $\epsilon$ is added to take into account inaccuracies of measurements.
} 
feedback system such as the Dynamixel servos [128]. This approach works well for simple motions or when precise placements are required like pick-and-place tasks. However, from our initial experimentations using the Dynamixel servos, "waiting" for each pose to complete before sending the next target pose often results in pauses at each pose, causing the "robotic motion" effect, which is not conducive for creating expressive motions. This is partly because the Dynamixel servos use the same line to send and receive information to and from the servo, which causes some additional delays between getting the feedback and sending the target positions.

- Streaming - The "streaming" approach refers to continuously sending pose data onto the robot at a fixed transfer rate. For this approach to work well, it is highly dependent on the compatibility between the frequency of publishing new target pose, the speed of the actuator, and the amount of travel demanded by the new target pose. This compatibility issue is discussed in more detail in Section 6.4. Given that the compatibility requirements are met, there are two advantages with the "streaming" approach over the previous two approaches. First, there are no delay mismatch (too short or too long) causing the "oscillating" or "robotic motion" effect despite using a fixed transfer rate. Second, there is greater control over motion dynamics such as speed, acceleration, transitions between actions, and even actualizing animation principles which are important for achieving natural-looking, expressive gestures. The other requirement for this approach is that the motion data must be interpolated such that the interpolated points (i.e. in-between poses) create the desired expressiveness on the robot by considering timing and animation principles. The "streaming" approach is used in the HROS-1 Framework [178] where the motion data is in- 
terpolated using linear interpolation. Our implementation uses a variant of the cubic spline interpolation method called Kochanek-Bartels interpolation [141].

In the next section, we discuss the Kochanek-Bartels interpolation. Following it, we discuss Multiresolution Filtering and its utility for editing and creating expressive motions.

\subsection{Interpolating Gesture Data}

When motion data for a robot are created using the keyframe method, the standard way to interpolate the values between poses is using linear interpolation. Linear interpolation is undesirable for creating expressive gestures as the motions tend to appear discontinuous. Cubic interpolation gives a very smooth, continuous function which translates to smooth motions. From our experiments, cubic interpolation is often enough to make gesture movements appear smooth and natural-looking when executed on the robot. However, the Kochanek-Bartels (KB) interpolation method gives additional parameters which the user can use to control how the interpolation is performed [141]. These parameters allow us to create different kinds of expressions on the gestures compared to the cubic interpolation. More explanations and some derivations of the interpolation methods mentioned here are provided in Appendix A.

\subsubsection{Kochanek-Bartels Interpolation}

KB-interpolation is a cubic polynomial based on the scaled sum of four segments of Hermite interpolation basis functions [141] as shown in Figure 6.1. Two of the 'scales' or 'coefficients' are determined by the constraint that the basis functions must pass through $P_{i}$ and $P_{i+1}$. KB interpolation chose to use the tangent vectors of adjacent 
points, $P_{i-1}$ and $P_{i+2}$ to calculate the other two coefficients; $D S$ (derivative from source) and $D D$ (derivative to destination). Where:

$$
\begin{aligned}
& D S_{i}=\frac{(1-t)(1-c)(1+b)}{2}\left(P_{i}-P_{i-1}\right)+\frac{(1-t)(1+c)(1-b)}{2}\left(P_{i+1}-P_{i}\right) \\
& D D_{i}=\frac{(1-t)(1+c)(1+b)}{2}\left(P_{i}-P_{i-1}\right)+\frac{(1-t)(1-c)(1-b)}{2}\left(P_{i+1}-P_{i}\right)
\end{aligned}
$$

Where $t$ is tension, $c$ is continuity, and $b$ is bias parameter.

The point $P(s)$ can then be determined by:

$$
P(s)=P_{i} h_{1}(s)+P_{i+1} h_{2}(s)+D D_{i} h_{2}(s)+D S_{i+1} h_{4}(s)
$$

Where $s \in[0.0,1.0]$ and $h_{i}(s)$ are:

$$
\begin{gathered}
h_{1}(s)=2 s^{3}-3 s^{2}+1 \\
h_{2}(s)=-2 s^{3}+3 s^{2} \\
h_{3}(s)=s^{3}-2 s^{2}+s \\
h_{4}(s)=s^{3}-s^{2}
\end{gathered}
$$

What makes KB interpolation attractive for humanoid robot motion creation in 

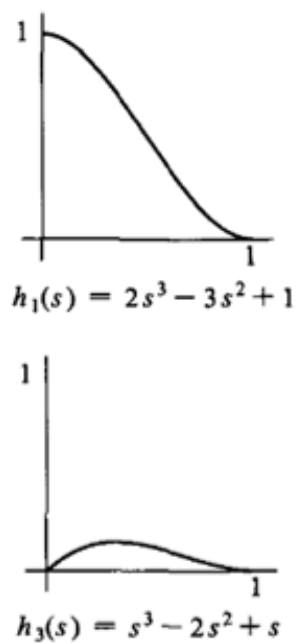

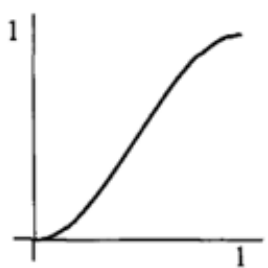

$h_{2}(s)=-2 s^{3}+3 s^{2}$

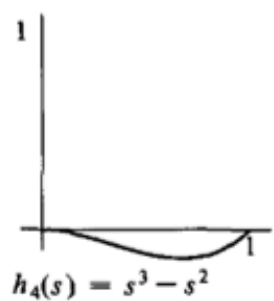

Figure 6.1: Four Hermite basis functions $h_{1}(s), h_{2}(s), h_{3}(s), h_{4}(s)$ (image from: [141]), where $s$ is on the x-axis, $h_{i}(s)$ is on the y-axis, and $s \in[0,1]$.

this dissertation is because it provides three interpolation parameters: bias, tension, and continuity that have human-friendly meanings to them. Moreover, when all three parameters are set to $0.0, \mathrm{~KB}$ interpolation is equivalent to Catmull-Rom interpolation which is commonly used in computer animation [179]. Typical range of values for the three parameters are in interval $[-1.0,+1.0]$. Values bigger than this range are possible but should be used with caution as large values may yield undesirable outcomes.

The bias parameter determines the location of the 'peak' of the curve around a data point. Suppose a one-dimensional data like the motion data and a data point $d_{t}$ on the motion data, a negative bias puts the peak to the left of $d_{t}, 0$ puts the peak exactly at $d_{t}$, and values greater than zero put the peak to the right of $d_{t}$. These effects are illustrated in Figure 6.2. The continuity parameter controls the angles between the tangents on each side of $d_{t}$. At 0 , the tangents are aligned and the curvature at $d_{t}$ is continuous/smooth. For value 1.0 of continuity, the tangents form an acute angle causing a 'cusp' (overshoot on either sides) and sharp corner at $d_{t}$. When continuity 

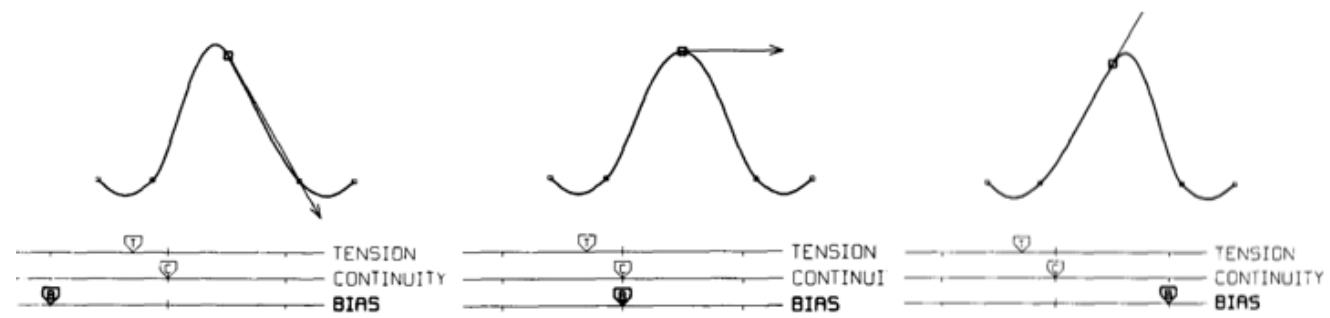

Figure 6.2: The effects of different Bias values at a single point (image from: [141]). (Tension $=-0.2$, Continuity $=0.0$ ) (left) Bias=-1.0, (middle) Bias=0.0, (right) Bias= 1.0 .

value is -1.0, the tangents form an obtuse angle, and create an angular corner at $d_{t}$. These effects are illustrated in Figure 6.3 Tension controls how sharp the curve bends at $d_{t}$. When tension value is 1.0 the curvature at $d_{t}$ becomes an angular corner while with tension $=-1.0$ the length of the tangents at $d_{t}$ is doubled, causing overshoots near the previous point $d_{t-1}$ and the next point $d_{t+1}$. These effects are illustrated in Figure 6.4.

At a glance, the shapes of the curves at continuity $=-1.0$ and tension $=1.0$ in Figure 6.5 appear similar. However, the dynamics of the two curves are different. At tension $=1.0$ there exists deceleration as the motion approaches $d_{t}$ and there exists acceleration when leaving $d_{t}$ because the interpolated data points near $d_{t}$ are spaced closer together. On the other hand, with continuity $=-1.0$, the interpolated data points around $d_{t}$ are spaced at equal distances, so there is no acceleration and deceleration. These operations are performed locally for each data point.

\subsubsection{Applying KB Interpolation to Motion Data}

Figure 6.6 shows application of KB interpolation on a dummy motion data for one joint consisting of five poses/frames. The first graph on the left shows the KB interpolated data with tension, bias, and continuity parameters set at 0.0 (solid line) overlaid on the linear interpolation (dashed line) of the five poses. In this configu- 

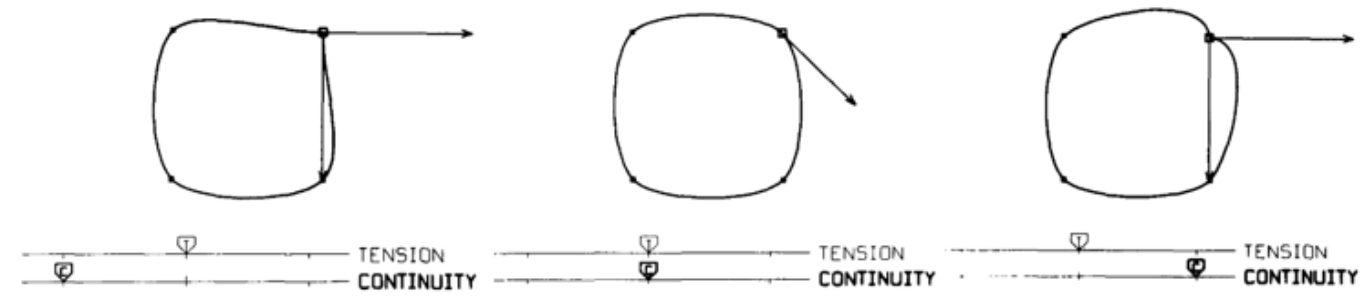

Figure 6.3: The effects of different Continuity values at a single point (image from: [141]). (Tension=0.0, Bias=0.0) (left) Continuity=-1.0, (middle) Continuity=0.0, (right) Continuity $=1.0$
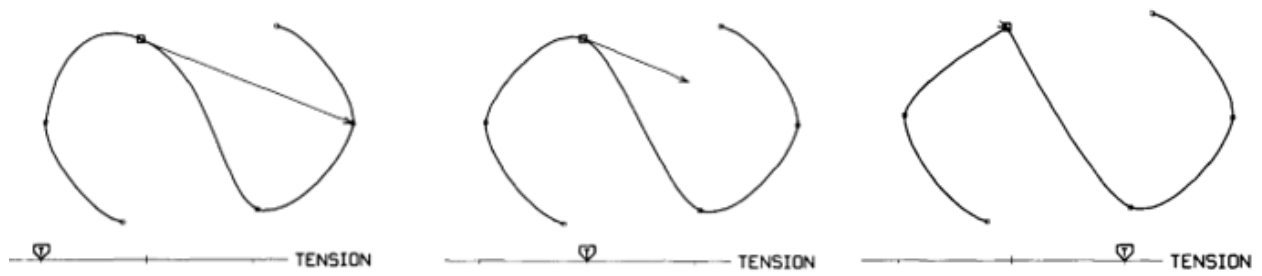

Figure 6.4: The effects of different Tension values at a point on a curve (image from: [141]). (Bias=0.0 and Continuity $=0.0$ ). (left) Tension=-1.0, (middle) Tension=0.0, (right) Tension $=1.0$

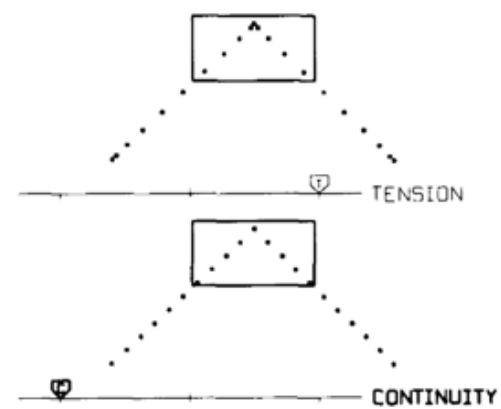

Figure 6.5: Comparison of results between setting Tension to 1.0 while keeping the other parameters to 0.0 and setting Continuity to -1.0 and other parameters to 0.0 (image from: [141]). The shape of the curve looks the same, but the spreads of the interpolated points are different. With Tension 1.0, interpolated points near the data points are spaced closer together which creates an acceleration and deceleration effects. With Continuity -1.0, the interpolated points are evenly spaced thus will not exhibit acceleration and deceleration. 

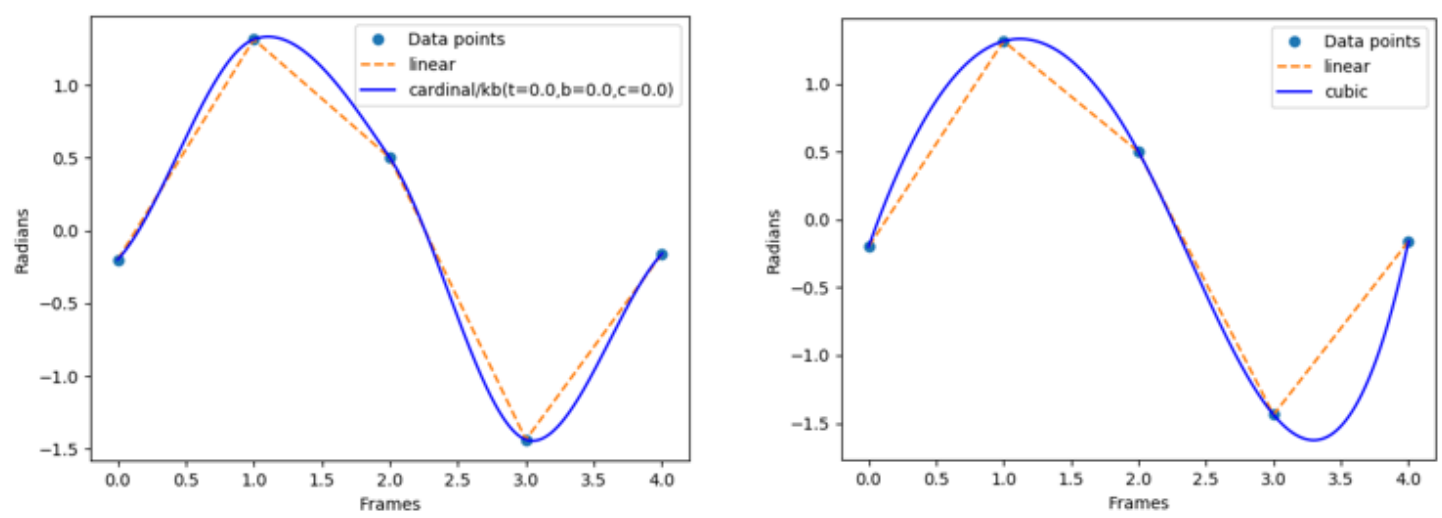

Figure 6.6: Comparison of (left) Kochanek-Bartels interpolation with tension, bias, and continuity parameters set to 0 , and (right) cubic interpolation. The dots represent the original data points and the dashed line is linear interpolation.

ration, the $\mathrm{KB}$ interpolation is equivalent to the Catmull-Rom interpolation in the 'cardinal' mode [179]. The second graph on the right shows the result of applying cubic interpolation. Both curves resulting from $\mathrm{KB}$ and cubic interpolation pass through all data points. The main difference is apparent where near the first and last data points, $\mathrm{KB}$ interpolation results in the curve aligning with the tangents in the direction of the adjacent data points while the cubic interpolated curve does not exhibit the same property. The bends at the inflection points ('peak' and 'valley' in 2nd and 4th frames) in the cubic interpolated curve are more rounded and blunter than the KB interpolated curve. These differences do not affect the shape of the motion as the curves still fit through all the original data points but will affect the way the motion accelerates and decelerates.

It should be noted that the type of data points in the motion data is different than the data points used in the original paper by Kochanek and Bartels [141]. In the original paper, the data is in two-dimensional spatial $\mathrm{x} / \mathrm{y}$ dimensions where the data points can create loops like in Figure 6.3 and 6.4. In the motion data, the x-axis indicates the timeline and so the order of poses and y-axis is the joint angle, and thus 
loops cannot appear in the motion data representation. In the spatial dimensions, accelerations are represented by the distances between data points like in Figure 6.5 (top). In the motion data representation, the timeline dimension has a fixed distance (i.e. discrete steps), so acceleration is represented by the changes on the y-axis. Visually, acceleration appears as smooth curves, while the jagged (noisy) curves and lines with sharp corners result with sudden or jerky movements.

Changing the bias, continuity, and tension parameters changes the motion data more than just the acceleration, but also introduces side effects such as overshoots (Fig. 6.7 (continuity=1.0 and 2.0), Fig. 6.8 far left, Fig. 6.9 far right), undershoots (Figure 6.8 far right frame 2, Fig. 6.9 far left). These side effects may change the shape of the motion (i.e. gesture) altogether, and the intended gesture may no longer be recognizable.

The differences between the effects of continuity $=-1.0$ vs. tension $=1.0$ in Fig. 6.5 are more apparent with the motion data. As indicated by Kochanek and Bartels, with continuity $=-1.0$ the motion does not exhibit acceleration and only moving with constant velocity. This is shown in Fig. 6.7 in the second graph from the left where the shape of the curve is now similar to the linear interpolation. In contrast, with tension $=1.0$ the motion data in Fig. 6.8 the third graph from the left does not appear 'linear' like in Fig. 6.5. Recall that in Fig. 6.5 acceleration exists in the spacing of the in-between points near the data points. Likewise, acceleration exists in the third graph in Fig. 6.8 but is shown as a curve which is continuous and differentiable everywhere because the $\mathrm{x}$-axis is the timeline which has discrete steps.

In summary, KB interpolation provides the robot animator with parameters that enable acceleration changes, and desirable effects such as overshoots and undershoots to make a motion (gesture) appear more dynamic. The overshoots and undershoots are creating effects that are consistent with the follow through and anticipation prin- 

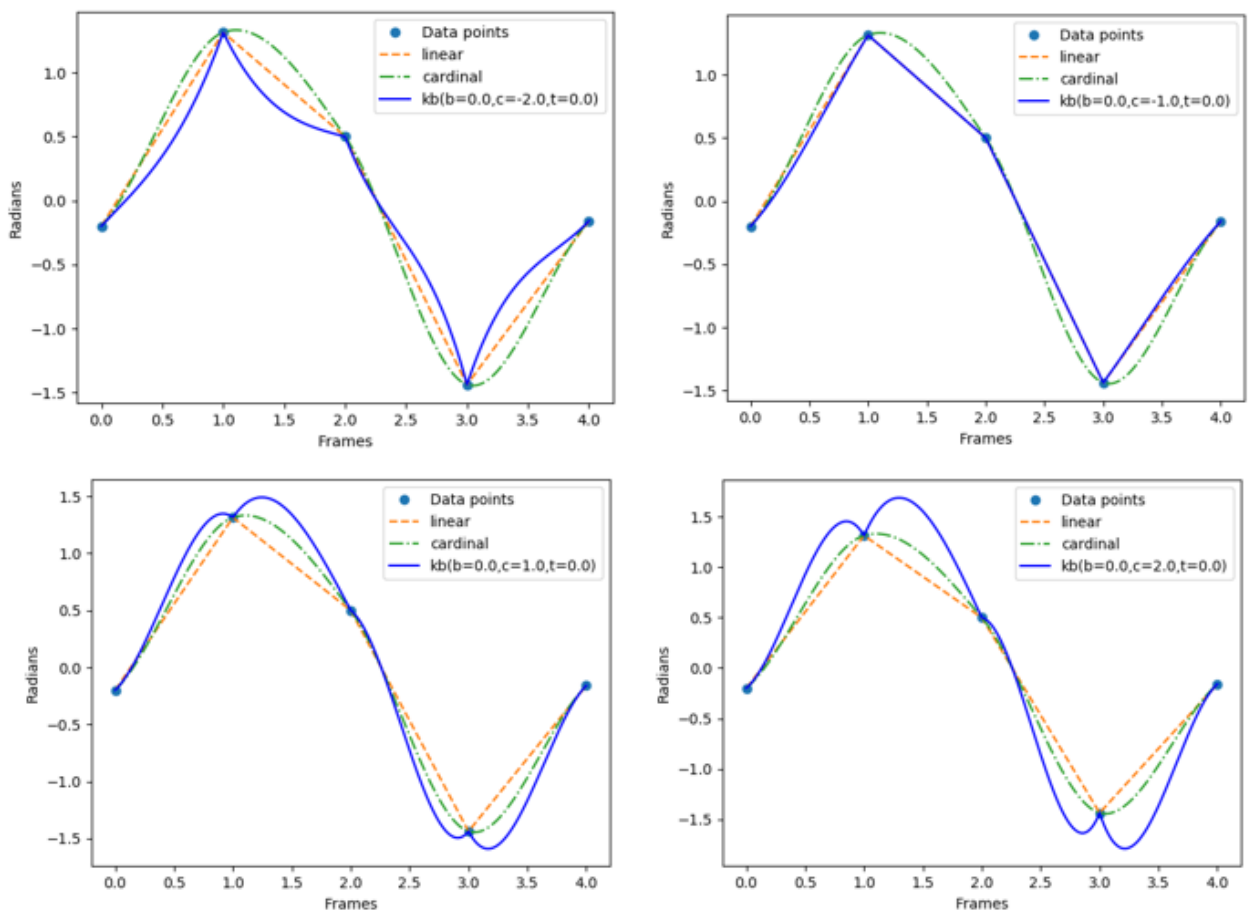

Figure 6.7: Results of KB interpolation results with tension and bias set to 0.0, and continuity (from left to right, top to bottom): -2.0, -1.0, 1.0, 2.0 (solid line). Dashed line is the result of linear interpolation. Dash-dot line is the result of KB interpolation with tension, bias, and continuity set to 0.0 (equivalent to Catmull-Rom 'cardinal' interpolation). 

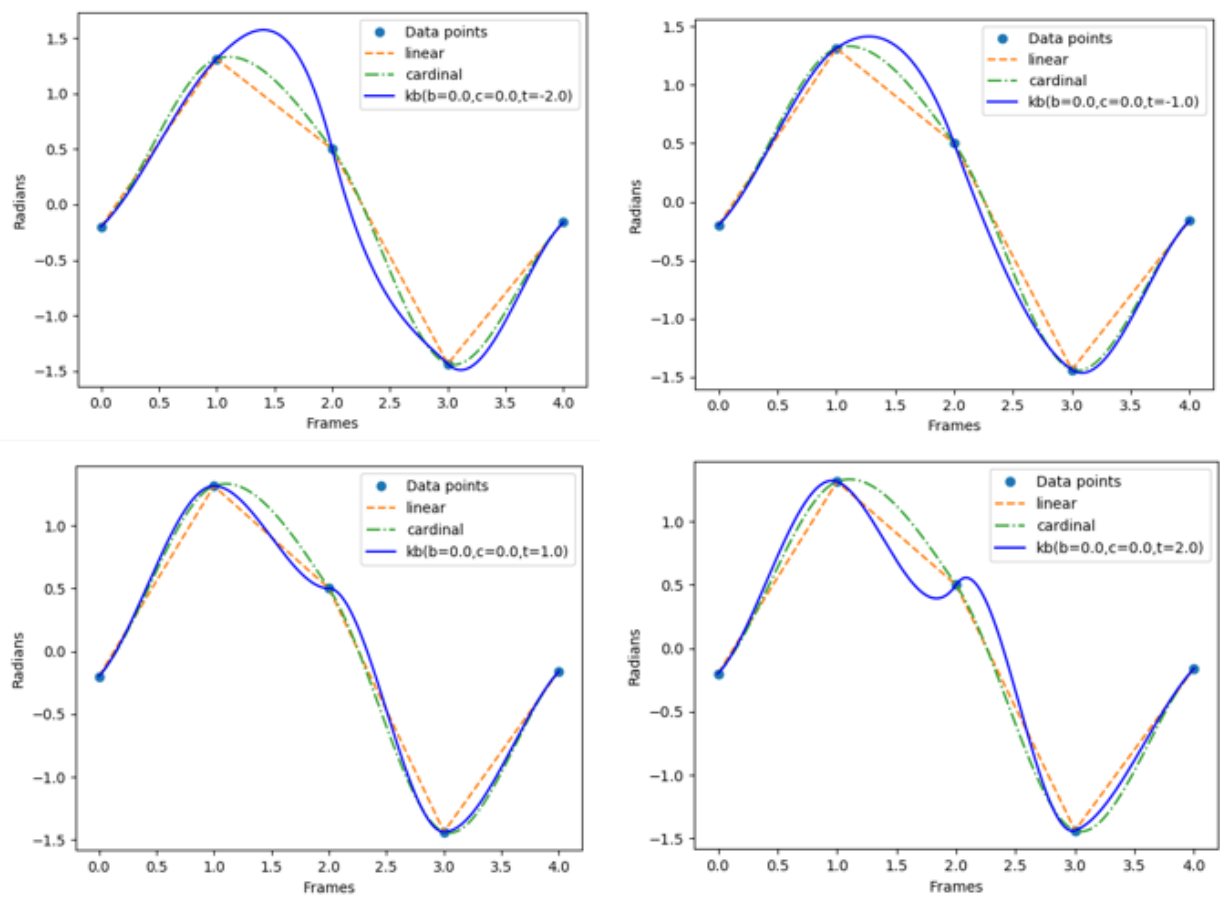

Figure 6.8: Results of $\mathrm{KB}$ interpolation with continuity and bias set to 0.0, and tension (left to right, top to bottom): -2.0, -1.0, 1.0, 2.0.

ciples from the Principles of Animation. However, the animator must still use caution when using KB interpolation such that the intended gesture is still recognizable from the interpolated motion data, unless desired otherwise. We use KB interpolation to pre-process robot motion data prior to concatenating or merging with other motion data when composing long-running, complex behaviors.

\subsection{Multiresolution Analysis}

Multiresolution analysis (MRA) is a method for analyzing an arbitrary input signal by convolving the signal with some orthogonal basis function successively at different resolution levels [173]. Bruderlin showed that by manipulating the magnitude of the coefficients of the bandpass filter bands from the result of MRA they can exaggerate or reduce of range of motions to create different emotional expressions (e.g. taking 

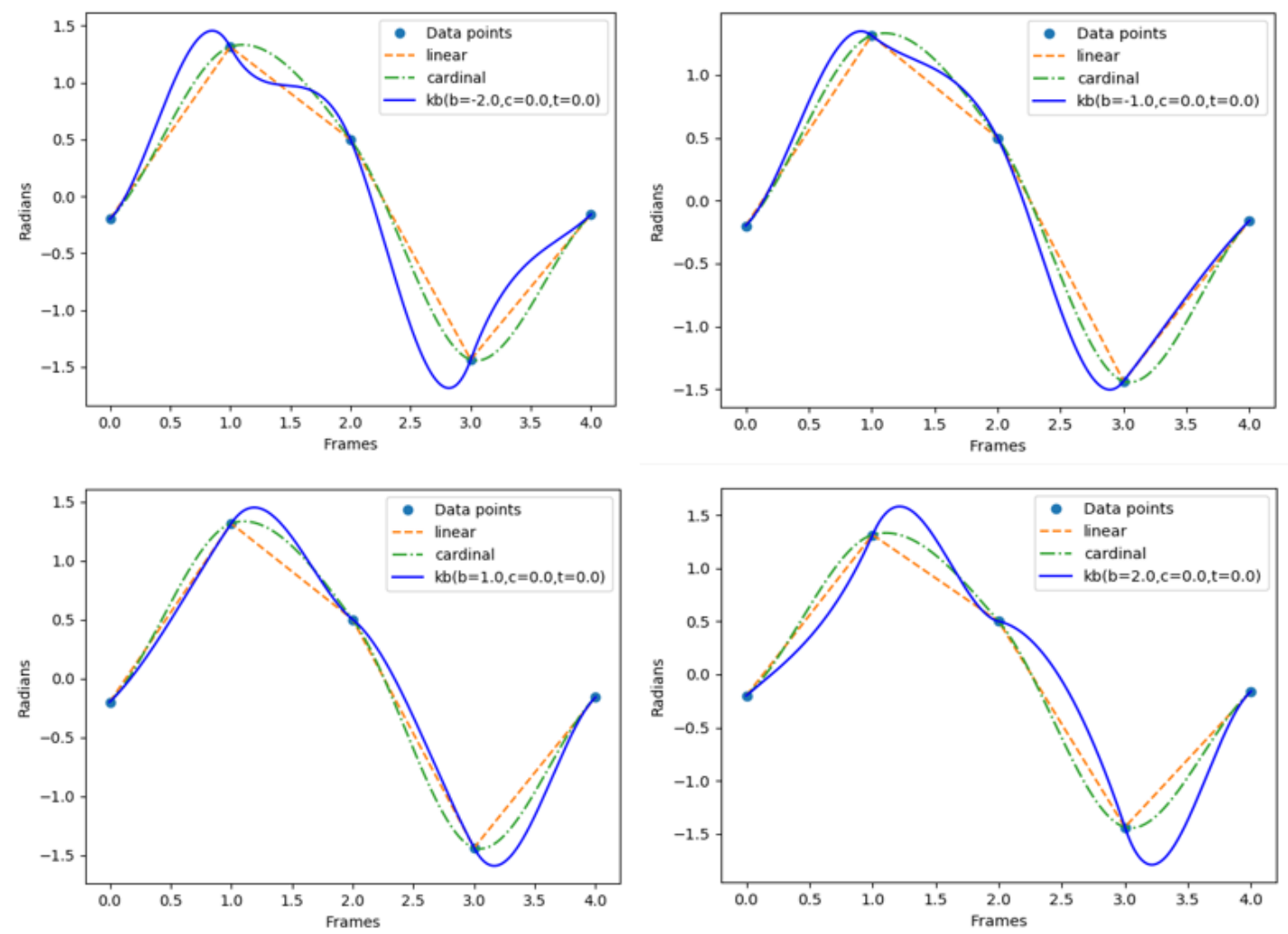

Figure 6.9: Results of KB interpolation with tension and continuity set to 0.0, and bias (left to right, top to bottom): -2.0, -1.0, 1.0, 2.0. 
a bigger/smaller step in a walk cycle, raising the arm higher for a waving motion) (e.g. excited vs. tired). Additionally, two motions can be 'merged' using MRA into one motion by adding the bandpass coefficients in frequency space (e.g. drumming while waving arm). When weights are added to the two motion data coefficients, one can create an interpolation between the two motions. However, the naive merging by adding the bandpass coefficients of the two motion data can sometimes create unnatural-looking motion. Dynamic timewarping can help to better synchronize the merged motions, especially when the motions only have a slight phase difference.

Processing of the motion signal can then be done using the filter bands, for example: noise can be reduced by removing the high frequency filter bands. To create the first level of the cascade, first the input signal $G_{0}$ is convolved with a kernel $w$ resulting with the signal $G_{1}$ (eq. 6.8). The Laplacian $L_{0}$ is the first filter band which is obtained by $L_{0}=G_{0}-G_{1}$. At this time, $G_{1}$ still has the same number of data points as $G_{0}$. To proceed with the second level of the cascade, $G_{1}$ is downsampled to half the resolution. Once $G_{1}$ is downsampled, the process repeats starting with the convolution with kernel $w$ and the next $L_{k}$ (eq. 6.9). The process is repeated until $G_{k}$ cannot be downsampled anymore (i.e. only has one value - the DC component). Therefore, the number of levels of the cascade $K$ is determined by the length of the signal $N$, where: $2^{K} \leq N \leq 2^{K+1}$.

$$
G_{k+1}=w * G_{k}
$$

Where $w$ is a function that has compact support ${ }^{2}$ called a filter kernel. For example, a function of size five was used in [135] where $w=[c, b, a, b, c]$ where $a=$

\footnotetext{
${ }^{2}$ The function has non-zero values in one location (i.e. bounded), and zero everywhere else
} 


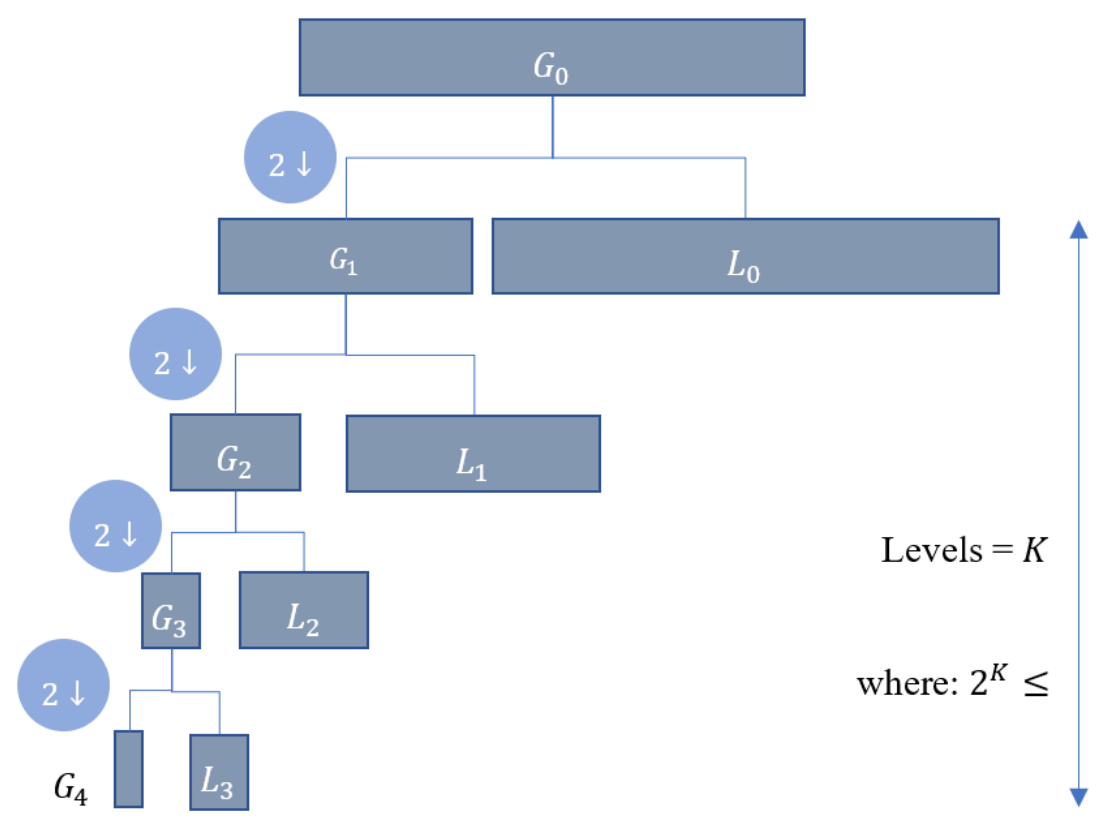

Figure 6.10: Cascades of multiresolution filtering. The symbol $2 \downarrow$ indicates downsampling to half the resolution (the width of the blocks illustrates the size of the data).

$3 / 8, b=1 / 4, c=1 / 8$ and $\operatorname{sum}(w)=1.0$.

$$
L_{k}=G_{k}-G_{k+1}, k \in[0, \cdots, K]
$$

The signal can then be manipulated by modifying the magnitudes of each $L_{k}$. The original signal $G_{0}$ can be reconstructed by adding the DC component $G_{K}$ with all the filter bands components $L_{k}$ :

$$
G_{0}=G_{K}+\sum_{k=0}^{K-1} L_{k}
$$

In addition to modifying the expressions of the motion and merging of multiple motion data [13], below we show that multiresolution filtering is useful to smooth transitions on concatenated motion data, and using different kinds of wavelets can 
also transform the motion data into different expressions.

\subsubsection{Changing Motion Expressions}

Bruderlin and Williams demonstrated that manipulating the magnitudes of the coefficients of the different bandpasses from multiresolution filtering (MRF) can change emotional expressions in the resulting motion. For example: reducing the low frequency bandpass reduces the range of motion, making the motion appear more constrained. Conversely, amplifying the same bandpass makes the motion appear exaggerated. Removing the high frequency bandpass removes the noise and jitter in the motion data, resulting in a smoother motion. Later we show that MRF is also useful for making smooth transitions when concatenating multiple motion data.

We can map a set of expressive qualifiers in REBeL to the coefficient values for the bandpass in any motion resulting from sampling a REBeL behavior expression $B$. Currently, we have implemented four qualifiers: tired, energetic, nervous, and cool. The 'tired' qualifier is applied to $B$ written as: $\operatorname{tired}(B)$ which will cause reduced range of motion with respect to the original data. This is done by reducing the low frequency bandpass of the motion to be reduced by a predetermined factor of $0.0<\alpha<1.0$. The values of $\alpha$ determined by trial-and-error. The 'energetic' qualifier is applied to $B$ as: energetic $(B)$ with the opposite effect of 'tired'. In this case, the range of motion will be increased (within the allowable limits) by increasing the low frequency bandpass coefficient. 'Nervous' and 'cool' qualifiers add and remove noise and jitter from the motion data, respectively. Adding noise and jitter is done by increasing the high frequency bandpass coefficient(s) and reducing the coefficients of the high frequency bandpass is done for reducing noise and jitter.

One advantage of using MRF versus other filtering methods like Fourier transform is that filtering using Fourier transform may introduce disruptive changes to the orig- 
inal motion data which cannot be directly attributed to some expressive behaviors. Fig. 6.11 shows the result of removing high frequency noise from actual motion data using the Fourier transform ${ }^{3}$. While the signals filtered using Fourier transforms are smooth and will not exhibit jerky motions, because of the sinusoid basis function, removing the high frequency components introduces sinusoid shapes of medium frequencies in the second half of the signal. This effect is apparent in the first and third graphs in the left column in Figure 6.11.

Next, we focus on using MRF as our primary method of combining motion data. We show that using MRF provides a nice way of combining/merging motion data, and useful for creating natural transitions when concatenating multiple motion data while maintaining similarity to the shape of the original motion data.

\subsubsection{Merging of Motion Data}

Combining two motion data can be done by simply adding the respective filter bands of the two motions. If there are two motions $M_{1}$ and $M_{2}$, and the filter bands for $M_{1}$ are $\left.\left[L_{0}, 1, \cdots, L_{(} K, 1\right)\right]$ and for $M_{2}$ are $\left.\left[L_{0}, 2, \cdots, L_{(} K, 2\right)\right]$. Fig. 6.12 shows an example of the merging of two motion data using MRF. Each of these motions corresponds to motion of one degree of freedom over time. For this example, the kind of gestures these motions represent and DOFs they are from are not important. The two motions are merged by some weighting coefficients $a$ for the first motion and $b$ for the second motion. The merging is calculated as in Eq. 6.11. The plots in Fig. 6.12 are as follows:

- Top left: the two motion data we chose arbitrarily to merge as an example, and let's call them 'motion 1' and 'motion 2'. There is no merging in this plot.

\footnotetext{
${ }^{3}$ We used the Fast Fourier Transform implemented in Scientific Python package scipy.fftpack.
} 

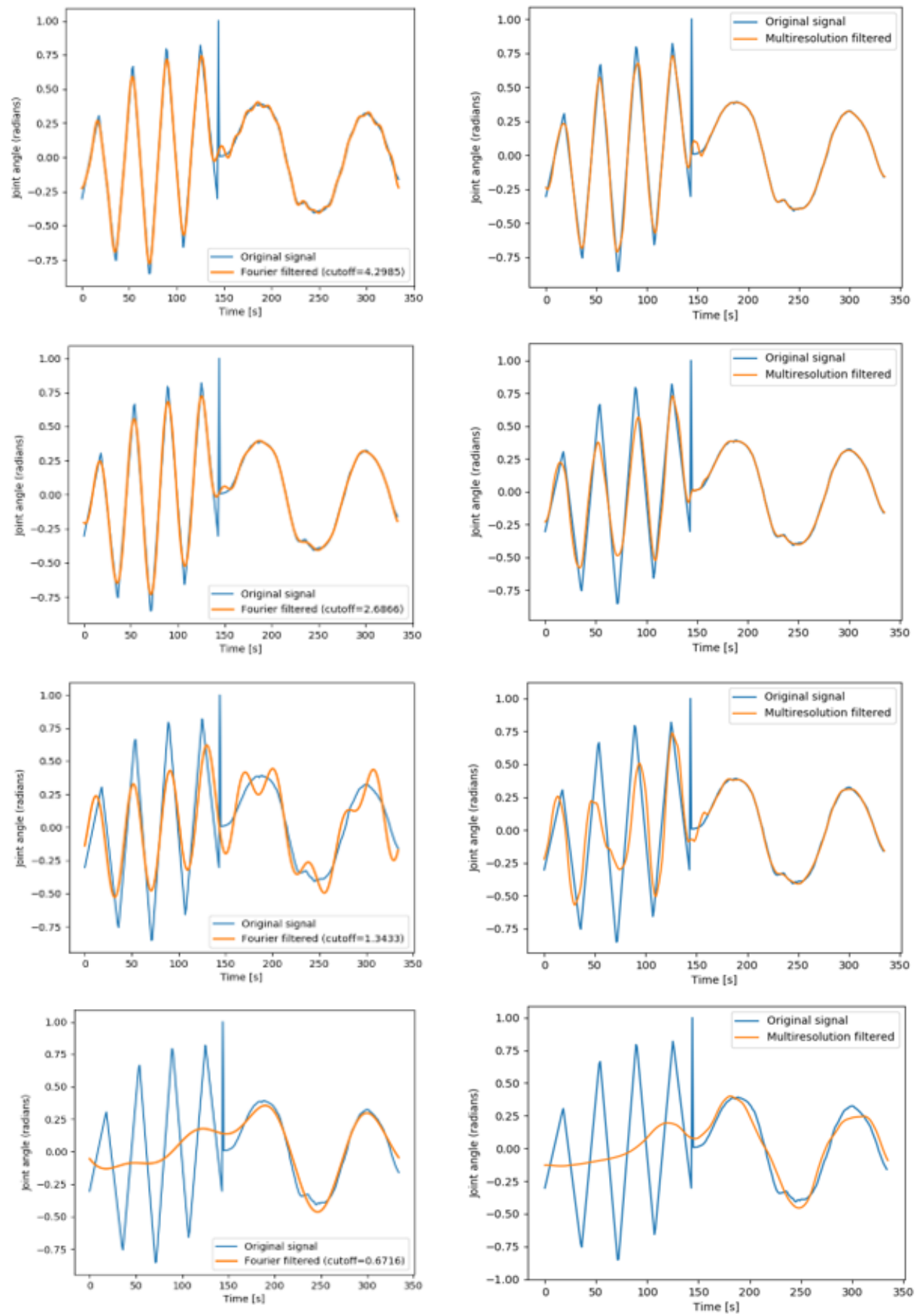

Figure 6.11: Comparison of filtering motion data using Fourier transform (left column) and multiresolution filtering (right column). From top to bottom: more high frequency components (least filtered) to fewer high frequency components (most filtered). The cutoff value indicates the threshold where frequency components with magnitude higher than this value are removed (set to zero). 
- Top right: the solid blue line as the result of merging with coefficients $a=1.0$ and $b=1.0$.

- Bottom left: the result of merging with $a=0.5, b=0.5$.

- Bottom right: the merging was done with $a_{K}=1.0, b_{K}=1.0$ and the other $a_{0 . . K-1}=0.0$ and $b_{0 . . K-1}=0.0$. This is equivalent to only summing the lowest frequency bandpass of the two motion data. The result is a smoother data (signal) because the high frequency components were removed. Notice that the result is similar to the plot on the top right.

$$
M_{1}+M_{2}=G_{K, 1}+G_{K, 2}+\sum_{i=0}^{K-1}\left(a_{i} L_{i, 1}\right)+\sum_{i=0}^{K-1}\left(b_{i} L_{i, 2}\right)
$$

However, while combining two or more motion data is possible with MRF, and with additional freedom of controlling the combination, the user must visually check whether the resulting motion is useful or meaningful. As Bruderlin and Williams noted that sometimes the combined motion is unnatural and nonsensical ${ }^{4}$ and had to be augmented or constrained using other methods such as dynamic timewarping [13].

In the work of Bruderlin and Williams, they used the filtering kernel that was used by Burt [180] where the kernel is approximately Gaussian. This Gaussianapproximate kernel has been shown to be sufficient to combine images with seamless transition. We extend this idea of using multiresolution filtering to manipulate motion data by using the wavelet transform. In wavelet transform, there are many different types of kernels which are also referred to as wavelet bases. In the following section, we give a brief overview of wavelet transform, and introduce new things we can do to motion data by using different wavelet bases.

\footnotetext{
${ }^{4}$ For example: combining walking and running may have moments where both feet are floating above ground but not like part of a natural gait.
} 

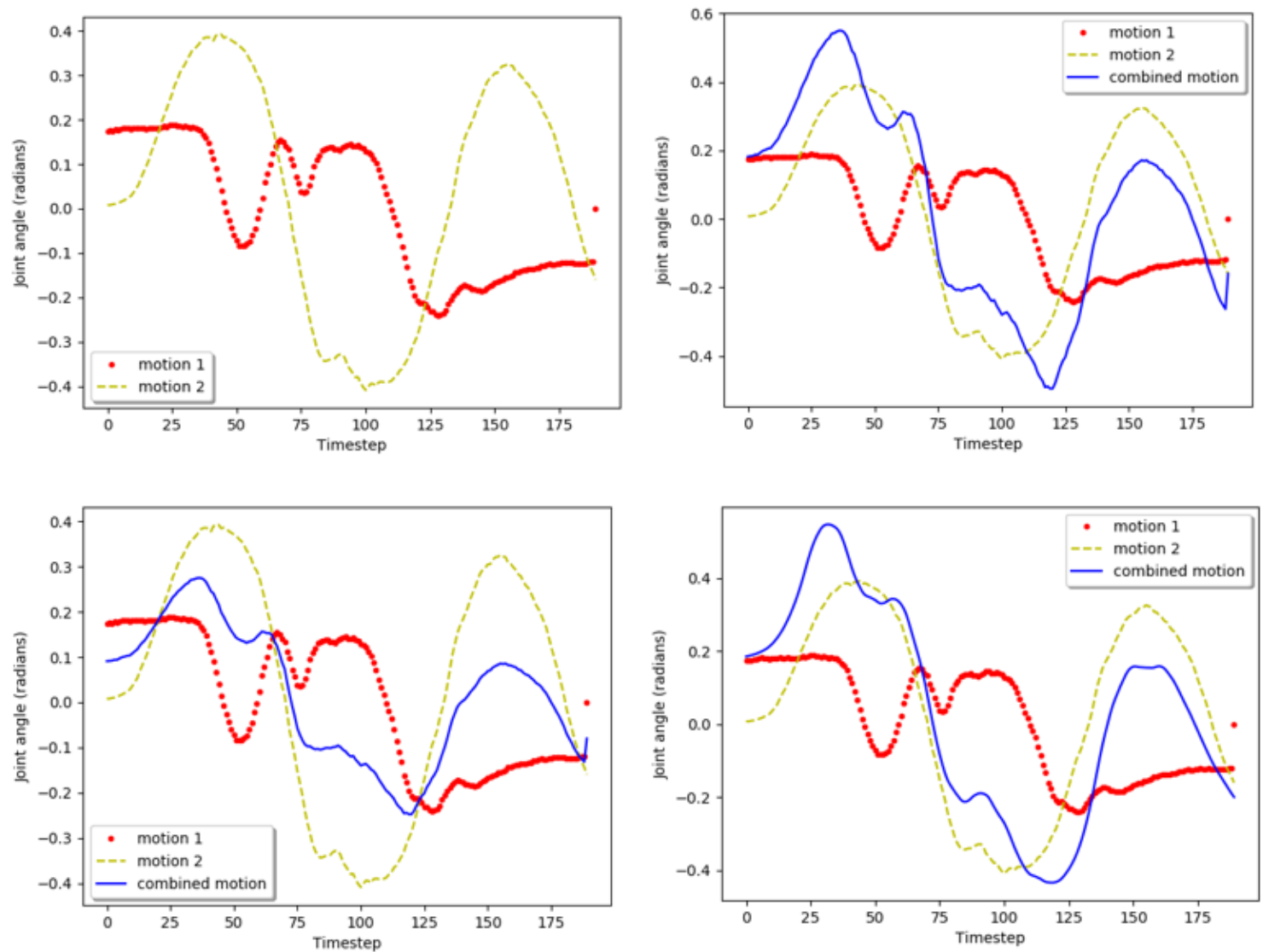

Figure 6.12: Top left: two motion data to merge: red-dotted line (motion 1) and yellow-dashed line (motion 2). Top right: solid blue line shows the combined motion with magnitudes of 1.0 on all filter bands for both motion 1 and motion 2. Bottom left: the combined motion (solid blue line) obtained with magnitudes of 0.5 for all filter bands for both motion 1 and motion 2 creates an 'average' motion. Bottom right: the combined motion obtained by adding only the last Laplacian of motion 2 $L_{K, 2}$ with magnitude 1.0 to the last Laplacian of motion $1 L_{K, 1}$. 


\subsection{Using Wavelets}

Fourier transform (FT) is widely used to analyze an input signal in terms of the frequency components that exist within the signal. The result of the FT of a signal is in the frequency domain (spectrum). In sound signals, FT can be used to remove noise to make the sound clearer or to add noise of certain frequency to block ambient noise (noise cancelling). Eq. 6.12 shows the Fourier transform to convert a signal from time domain $f(x)$ to frequency domain $\hat{f}(\omega)$ by deconstructing $f(x)$ into sine and cosine components, while eq. 6.13 shows the inverse Fourier transform to convert the signal from spectral domain back to the time domain. In $2 \mathrm{D}$ data like images, Fourier transform can also be used to denoise the image, or find features like edges in the image. Fourier transform works best with stationary signals, that is, signals that repeat infinitely many times. With such signals, FT can give precise information about the frequency components in the signal, shown as large spikes in the frequency domain. Non-stationary signals refer to signals that do not appear to have recurring patterns or consisting of short burst of activities for a short period of time. FT can be used to analyze non-stationary signals, but the spectra will appear 'noisy' as more frequency components are needed to converge to the original signal. In other words, FT has good localization in frequency but poor localization in time. FT cannot give information where in time a certain frequency occurs. Wavelet transform remedies this problem.

Wavelets are often used to denoise signals, anomaly detection, and image compression [181] [182]. Fig. 6.13 shows some examples of common uses for wavelet analysis.

$$
\hat{f}(\omega)=\int_{-\infty}^{\infty} f(x) e^{-2 \pi i x \omega} d x
$$




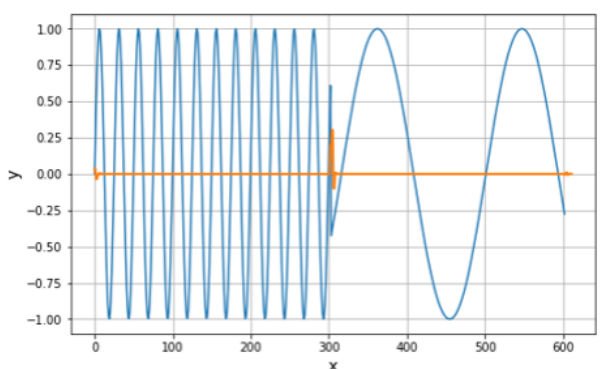

(a)

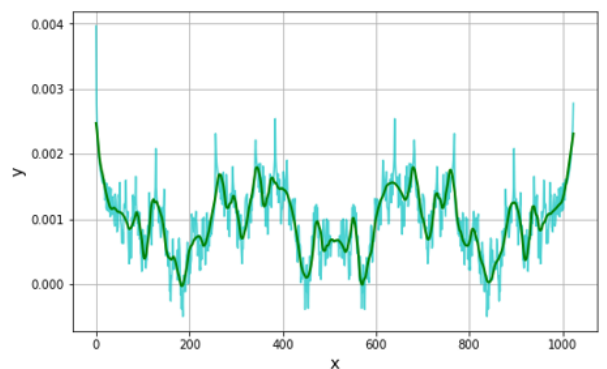

(b)

Figure 6.13: Examples of wavelet usage on time series data $(x=$ time, $y=f(x))$ : (a) detecting anomalies/change (spike of orange line indicates where frequency change happens), (b) denoising data (green line is the denoised data). The wavelet called Debauchies5 was used in both cases.

$$
f(x)=\int_{-\infty}^{\infty} \hat{f}(\omega) e^{2 \pi i x \omega} d \omega
$$

A wavelet $\psi$ is a oscillating wave function which only has non-zero values within a short period of time and zero everywhere else. Due to this property, functions like wavelets are said to have compact support. The main idea behind wavelet transform is to decompose an arbitrary input signal by translating and scaling the wavelet basis. Increasing and decreasing the scaling value lengthen and shorten the width (i.e. support) of the wavelet, respectively ${ }^{5}$. Thus, scaling corresponds to the inverse of frequency of the wavelet; a large scaling value (wide support) captures the low frequency components, while a small scaling value captures the high frequency components of the input signal. The wavelet transform returns the coefficients of the correlation between the wavelet and the input signal.

Given a wavelet $\psi$ and input signal $f$, then the wavelet transform $W_{\psi}$ is defined

\footnotetext{
${ }^{5}$ Analogous to 'zoom in' and 'zoom out' of an image.
} 
as:

$$
W_{\psi}[f](a, b)=\left(f, \psi_{a, b}\right)=\int_{-\infty}^{\infty} f(t) \bar{\psi}_{a, b}(t) d t
$$

Where $\bar{\psi}$ is the complex conjugate of the wavelet, $a$ is the scaling parameter, and $b$ is the translation parameter, and $a, b \in \mathbb{R}$. Eq. 6.14 is also called the continuous wavelet transform (CWT).

Functions $\psi$ is called mother wavelet because it can generate families of functions $\psi_{a, b}$ by scaling and translation operations:

$$
\psi_{a, b}(x)=\frac{1}{\sqrt{|a|}} \psi\left(\frac{x-b}{a}\right)
$$

where $a, b \in \mathbb{R}, a \neq 0$ (from [172]).

Functions $\psi_{a, b}$ that qualify as wavelets that can be used for the wavelet transform are called analyzing wavelets or mother wavelets must satisfy the so-called admissibility constraint $c_{\psi}<\infty$ (Eq. 6.16) which indicates that the Fourier transform of the mother wavelet must be finite [172][183].

$$
c_{\psi}=\int_{-\infty}^{\infty} \frac{|\psi \hat{(\omega)}|^{2}}{|\omega|} d \omega<\infty
$$

where $\hat{\psi}(\omega)$ is the Fourier transform of the wavelet $\psi$ :

$$
\hat{\psi}(\omega)=\frac{1}{\sqrt{2 \pi}} \int d x e^{-i x \omega} \psi(x)
$$

The admissibility condition implies that the Fourier transform of $\hat{\psi}(\omega)$ at frequency zero has zero magnitude. Consequently it also means that the average value of the wavelet in the time domain is zero, which suggests that the wavelet is oscilla- 
tory.

$$
\int \psi(x) d x=0
$$

The original signal can be reconstructed with the inverse wavelet transform with wavelet $\psi_{a, b}$ by integrating over the bounds $[a, b]$ and defined as:

$$
f(t)=\frac{1}{c_{\psi}} \iint \frac{1}{a^{2}}\left(W_{\psi}[f](a, b) \psi_{a, b}(t) d b d a\right.
$$

When the wavelet does not satisfy this admissibility constraint $(\psi \longrightarrow \infty)$, the inverse transform is undefined.

Because of the shifting and scaling operation, wavelet transform can give the information of which frequency component occur in time, subject to the Heisenberg uncertainty principle. The Heisenberg uncertainty principle states that the momentum and position of a moving particle in space cannot be known for certain simultaneously; at any given time one can know the momentum of the particle precisely but not its exact position and vice versa. Similarly in signals with respect to frequency and time. This can be illustrated in Fig. 6.14. In FT, the resolution in frequency is high, but time localization is not possible. In wavelet transform, at low frequency the frequency resolution is high, but the time resolution is poor. This means it is easier to get a precise frequency of the low frequency component, but difficult to determine its location in time. At high frequency, the frequency resolution is lower, but the time resolution is high. At higher frequencies, it is more difficult to get the precise frequency, but it is easy to determine where in time the frequencies occur.

The families of wavelets generated by scaling and translating in CWT are highly redundant, which means they are not independent bases. Moreover, CWT requires a significant amount of computation, which makes CWT not practical to use. When 

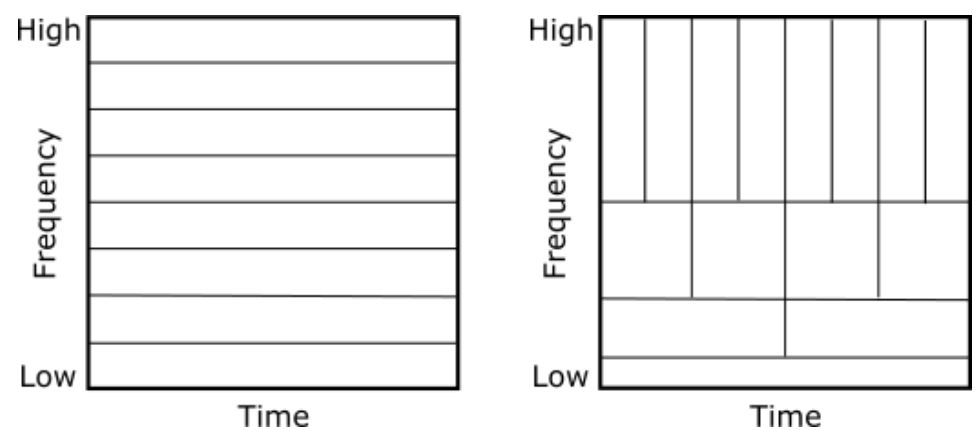

Figure 6.14: Time vs. frequency resolutions for Fourier transform (FT) (left) and wavelet transform (WT) (right). In FT, there is only frequency resolution, while in WT the time-frequency resolution changes with frequency. Note that the area of the inner rectangles for WT are the same but at different widths and heights, showing the trade-offs between time and frequency resolutions at different frequency levels.

using wavelet bases that are orthogonal, one can perform the discrete wavelet transform (DWT) [172]. With orthogonal bases, redundancy is removed and the forward wavelet transform can be done in discrete steps and computed efficiently using multiresolution analysis [173]. The inverse DWT also reproduces the original signal. A wavelet $\psi$ is orthogonal when:

$$
\int \psi_{j, k}(t) \bar{\psi}_{m, n}(t) d t= \begin{cases}1 & \text { if } j=m \text { and } k=n \\ 0 & \text { otherwise }\end{cases}
$$

The formulation of discrete wavelets are [172]:

$$
\psi_{m, n}(x)=a_{0}^{-m / 2} \psi\left(a_{0}^{-} m x-n b_{0}\right)
$$

where $m, n \in \mathbb{Z}$, which gives orthonormal wavelet bases by choosing values for $a_{0}$ and $b_{0}$. A typical choice is $a_{0}=2$ and $b_{0}=1$. For example for the Haar basis function 
[172]:

$$
\begin{gathered}
\psi(x)= \begin{cases}1, & 0 \leq x<\frac{1}{2}, \\
-1, & \frac{1}{2} \leq x<1, \\
0, & \text { otherwise }\end{cases} \\
\psi_{m, n}(x)=2^{-m / 2} \psi\left(2^{-m} x-n\right)
\end{gathered}
$$

The DWT is formed from multiresolution analysis (MRA) space in $L^{2}(\mathbb{R})^{6}[172]$. The main idea behind MRA is to analyze/decompose an input signal successively, at different resolution at each step. MRA also let the original signal be reconstructed. The properties of MRA are:

- There is a sequence of subspaces, where each subspace contains the subspace before it, where $V_{m} \subset L^{2}(\mathbb{R}), m \in \mathbb{Z}$ (monotonicity) i.e.

$$
\cdots \subset V_{2} \subset V_{1} \subset V_{0} \subset V_{-1} \subset V_{-2} \subset \cdots
$$

- The union of all subspaces is dense in $L^{2}(\mathbb{R})$, i.e. the union of all subspaces gives the entire space of $L^{2} \mathbb{R}$ :

$$
\bigcup_{m=-\infty}^{\infty} V_{m}=L^{2} \mathbb{R}
$$

- The intersection between subspaces is the empty set, i.e. every subspace is

${ }^{6} L^{2}(\mathbb{R})$ is the space of square integrable functions, where the integral of the square of any function in that space is finite: $\int|f(x)|^{2} d x<\infty, f \in L^{2}(\mathbb{R})$ 
independent:

$$
\bigcap_{m=-\infty}^{\infty} V_{m}=\emptyset
$$

- Dilation property: $f(x) \in V_{m}$ iff $f(2 x) \in V_{m-1}$

- There exist a scaling function $\phi \in V_{0}$ where $\phi$ such that the set $\left\{\phi_{0, k}\right\}_{k \in \mathbb{Z}}$ is orthonormal. The scaling function is called the father wavelet.

The scaling function is such that:

$$
\phi(x)=\sum_{n} c_{n} \phi_{1, n}(x)=\sqrt{2} \sum_{n} c_{n} \phi(2 x-n)
$$

And the mother wavelet for discrete multiresolution analysis :

$$
\psi(x)=\sum_{n}(-1)^{n} c_{n+1} \phi(2 x+n)
$$

where:

$$
c_{n}=\left(\phi, \phi_{1, n}\right)=\sqrt{2} \int_{-\infty}^{\infty} \phi(x) \bar{\phi}(2 x-n) d x
$$

And the inverse DWT:

$$
f(x)=c_{\psi} \sum_{a} \sum_{b} W_{\psi}[f](a, b) \psi_{a, b}(x)
$$

With the orthogonal wavelets, the wavelet transform can be done in discrete steps in a recursive way using multiresolution analysis.

There are many different kinds of wavelets and choosing which wavelet basis to use depends on the type of the problem and data being analyzed (e.g. for smoothing/denoising, anomaly detection, pattern detection, etc.). Fig. 6.15 shows some examples of a few wavelets. In this work, we applied different wavelet bases to vari- 

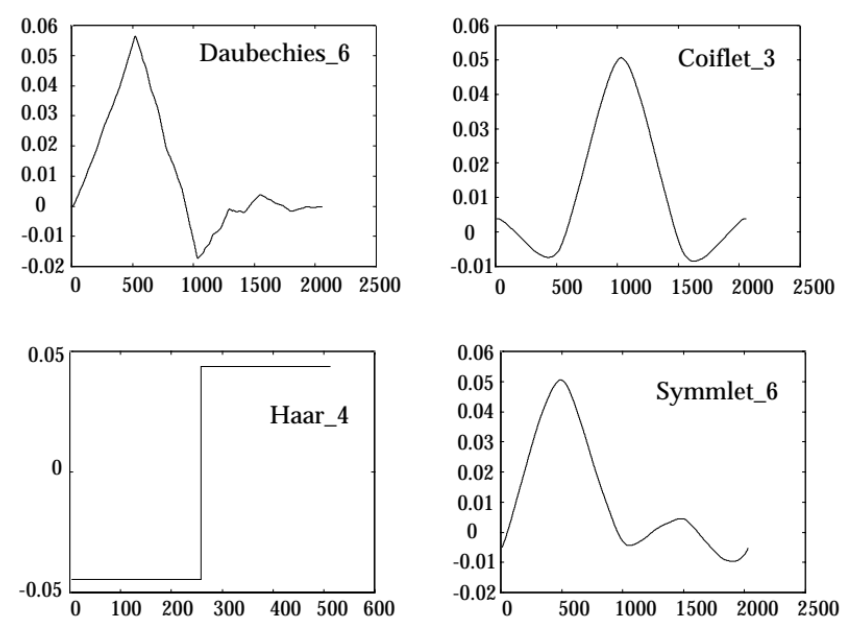

Figure 6.15: Plots of wavelets: Debauchies6, Coiflet3, Haar, and Symlet6 (image from $[184])$.

ous sequences of motion data and we look for the wavelets that are useful for creating expressive robot motions. We found that wavelet transform can help create smooth transitions when concatenating disjoint motion data, but also can create more natural transitions like undulations that exhibit the "anticipation" and "follow through" principles from animation. We also found that, reconstructing motion data at different levels of filtering can create interesting motion quirks, such as twitch, exaggerated robotic motion, and other effects which we describe below.

Future works may look into finding new wavelet bases that can be used to synthesize a specific type of emotional expressions and autonomous expressive motion compositions for robots. For more theoretical discussions on wavelets, more information can be found in [172] [173] [183].

Next, we discuss our findings in using different wavelet basis to introduce artifacts to an existing motion data to create different motion styles. We used the same motion dataset as in the previous section. In all our works using wavelets, we used the Python wavelet package PyWavelets [185]. 


\subsubsection{Transitions in Concatenation of Motion Data}

A contribution of this dissertation is demonstrating the use of multiresolution filtering as the method to create smooth transition between two or more motion data.

MRA has been used to create composite photographs such that the transition boundaries between the two photographs are unnoticable and seamless [180]. The blending is done at the lower scale of the bandpass filterbank, such that the lower frequency components between the two images are smoothly blended together, while the higher frequency components retain the details in the two images.

Robot motion data typically start and end with the robot's home position. When two or more motion behaviors are arranged sequentially without any modifications, the resulting motions appear awkward as the robot returns to its home position repeatedly during the behavior transitions. In another situation, when the end pose of one behavior is significantly different from the start pose of the next behavior, the robot may produce a significant 'jerk' - a sudden motion - during the transition. Fast or sudden movements from robots are undesirable because they create uneasiness and discomfort to the user as they are perceived to be out of control [186]. Additionally, any method that attempts to join the transition points in a smooth way, must ensure that the rest of the motion data should not be affected significantly. For a more natural looking behavior, motion transitions between behaviors should be smooth yet clearly indicate to the user when one behavior ends and another one starts. Moreover, the transition method must maintain the original shape of each motion segment without too much change such that the motion segments remain recognizable as its original intent.

Van Breemen used a method called transition filter to create transitions from one

movement to the next [62]. In his method, to create a smooth transition from one 


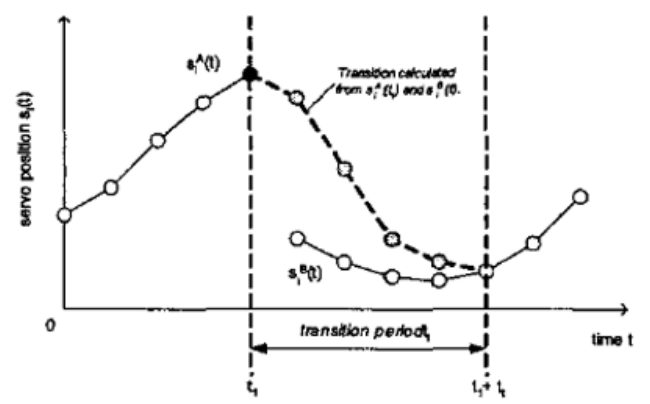

Figure 6.16: Transition filter (image from [187]).

motion to the next the transition is assumed to be a blending from the last pose of the previous motion into the next motion by some amount of transition period. An illustration of how the transition filter works is shown in Fig. 6.16.

The transition filter works by giving a transition period with $j$ timesteps which starts at $t_{1}$ and ends at $t_{k}=t_{1}+j$. Where $s_{i}(t)$ is the pose of servo $s_{i}$ at time $t$, $s_{i}^{A}(t)$ and $s_{i}^{B}(t)$ are the motion $A$ and motion $B$ for $s_{i}$. At $t_{1}$ in the transition filter, $s_{i}\left(t_{1}\right)=s_{i}^{A}\left(t_{1}\right)$ where $s_{i}^{A}\left(t_{1}\right)$ is the last pose of motion $A$. The transition filter linearly interpolates from the last pose of motion $A$ to the $j$-th pose of motion $B$ using the following rules [17]:

$$
s_{i}(t)= \begin{cases}s_{i}^{A}(t) & t<t_{1} \\ \alpha(t) \cdot s_{i}^{B}(t)+(1-\alpha(t)) \cdot s_{i}^{A}(t) & t_{1} \leq t<t_{1}+j \\ s_{i}^{B}(t) & t \geq t_{1}+j\end{cases}
$$

Where:

$$
\alpha(t)=\frac{t-t_{1}}{j}
$$

Using multiresolution filtering creates a better transition between two or more 
motion data by concatenating the motion data as usual, followed by removing the high frequency components (setting the filter band coefficients to 0.0). From our experiments, keeping the last $\lfloor K / 2\rfloor$ of the filter bands (the half of the bandpass consisting of the lower frequency components) was sufficient to remove the jarring transitions at the joins between two motion data, while at the same time reducing noise, and minimizing disruption or changes to the original data.

Fig. 6.17 shows the comparison between creating transition with Van Breemen's transition filter (left column) and using multiresolution filtering (right column). Throughout these examples, we only use the Debauchies5 wavelet as the kernel function due to its smooth shape.

We found that using the multiresolution filter method introduces some changes to the original data, whereas using transition filter only modifies the data during the transition period. However, when executed on the robot, we found that the changes introduced by the multiresolution filter give a more naturally looking motion than the transition filter. Moreover, since most of the high frequency bandpass components were removed, the resulting motions appear smoother overall. As seen in Fig. 6.17, using transition filter still produces sudden, jerky motions at the joins. Sudden, jerky transition is shown as large changes in the y-axis in a short time period (few timesteps) which can be seen in Fig. 6.17 (first row: timestep 190, second row: timestep 110, third row: timestep 145). The right column of Fig. 6.17 shows three different kinds of transitions as the result of filtering which are smoother motions than without the filtering:

- Top right: gradual transition

- Middle right: moving in the opposite direction of the start of the next motion (anticipation principle) 

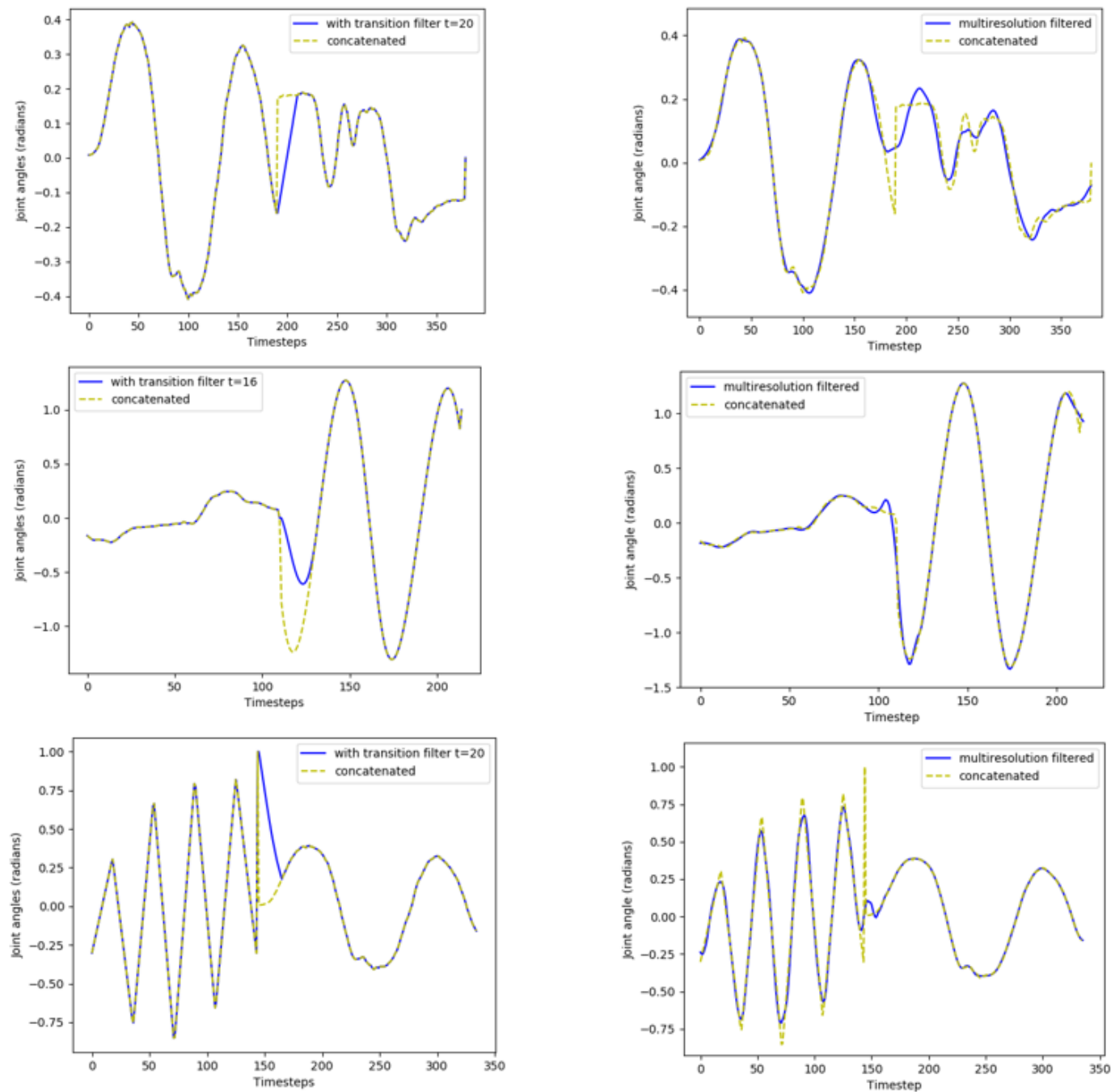

Figure 6.17: Concatenation of two different motion data using transition filter[187] (left column) vs. multiresolution filtering (right column). The pairs of figures in each row show the same concatenation of two motion data. The kernel Daubechies5 was used for all multiresolution filtering results. The lines labeled "concatenated" show the two motion data concatenated without any modifications. 
- Bottom right: small undulations

The jerk (rate of acceleration) $\frac{d^{3} s}{d t^{3}}$ performance with respect to the three examples shown in Fig. 6.17 is shown in Fig. 6.18. We consider motions with the least amount of jerk as optimal. Therefore, we want to find a way to minimize jerk in the concatenation.

We show that using multiresolution filtering gives significantly less jerk than using the transition filter. For conciseness, let's refer to the concatenated motion data in the first row of Fig. 6.17 as Concatenated 1, in the second row as Concatenated 2, and the third row as Concatenated 3. Each plot in Fig. 6.18 shows three plots: the plot of the jerk without any filtering (solid yellow), jerk in the result of using transition filter (red dotted triangle marker), and jerk in result of using multiresolution filtering (dashed blue circle marker). The original motion signal is plotted with black dots to show the location of the joins. Table 6.3 shows that using multiresolution filtering yields the least amount of jerk. Additionally, the filtered motion data using multiresolution filtering also gives the least deviations from the original motion data compared to transition filter (measured as mean squared errors) as shown in Table 6.4.

Applying the same multiresolution filtering method after applying transition filter produces good results as well. Fig. 6.19 shows the comparison of results from applying transition filter $(\mathrm{TF})$, multiresolution filtering $(\mathrm{MRF})$ and multiresolution filtering after transition filter $(\mathrm{TF}+\mathrm{MRF})$. The pair of plots in each row shows the plot of two concatenated motion data in the same order as in Fig. 6.17. The plots on the left column show the actual joint angle values on the y-axis, and timesteps on the $\mathrm{x}$-axis. Unlike in Fig. 6.17, the plots are zoomed in on the joins/transition points to focus on the different results of applying TF, MRF, and TF + MRF filters. Specifically, the transition locations are: Concatenated 1 at $t= \pm 185$, Concatenated 2 at $t= \pm 110$, 

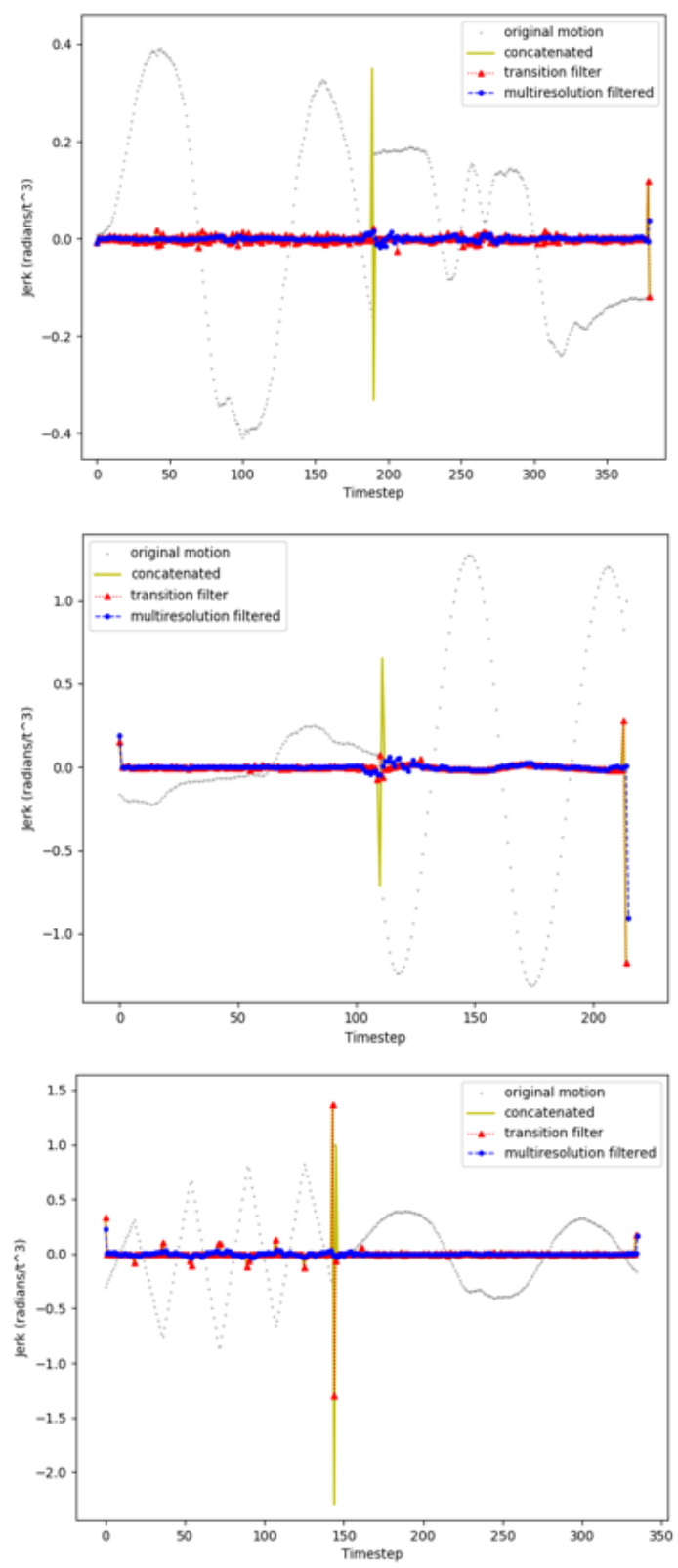

Figure 6.18: Three examples of jerk plot from concatenated motions of two motion data from Fig. 6.17. The first plot (top) is for Concatenated 1, the second plot (middle) is for Concatenated 2, and the third (bottom) is for Concatenated 3. The joins of the two motion data in each plot can be seen by the highest jerk amplitude near the middle of each plot. The solid yellow plot is the jerk of the concatenated motion without filtering, the red dotted plot with triangle markers is the jerk from transition filter, and the blue dashed plot with circle marker is the jerk from multiresolution filtering. 
and Concatenated 3 at $t= \pm 145$. The plots on the right column show the comparison of the jerk profiles for each method. The y-axes in the jerk plots are shown on a logarithmic scale. In all these examples, multiresolution filtering is done in the same way as previously: by keeping the last $\lfloor K / 2\rfloor$ of the filter bands (the lower half of the bandpass components) with kernel Debauchies5.

As expected, applying MRF smoothed the remaining jerk in the results of transition filter $(\mathrm{TF})$. In the Concatenated 1 and Concatenated 2 datasets, $\mathrm{TF}+\mathrm{MRF}$ filtering create smoother motions with respect to TF only but also removing the undulations that occur when using MRF only. The loss of the undulations is somewhat undesired because they represent the Overlapping Action and Anticipation principles. But perhaps this result may be appropriate for some specific artistic reasons, where motions just seamlessly blend from one to the next. This can be an option for the robot animator. On the other hand, the TF+MRF filter is able to capture the motions in Concatenated 3 around $t=140-160$ in a smoothed way whereas TF resulted in a high jerk, while MRF filtered out the jerk along with the other motions within that period. Table 5.2 shows the range of jerk values as the result of not applying any filter, with transition filter, with multiresolution filter, and with the combination of the transition filter with the multiresolution filter. Table 5.3 shows the mean squared error of the filtered data with respect to the non-filtered concatenated data as a rough measure on how much the filtered motion data deviates from the original.

This concatenation method can be generalized to any number of motion data in the sequence. As an example, we picked arbitrary motion samples in Fig. 6.20 to be concatenated. The motions are chosen so that the joins have very abrupt transitions to show the effects of the transition filter compared to multiresolution filtering more obvious. Fig. 6.21a shows the results of applying only transition filter and Fig. 6.21b shows the result of using multiresolution filtering for comparison. Notice that the big 

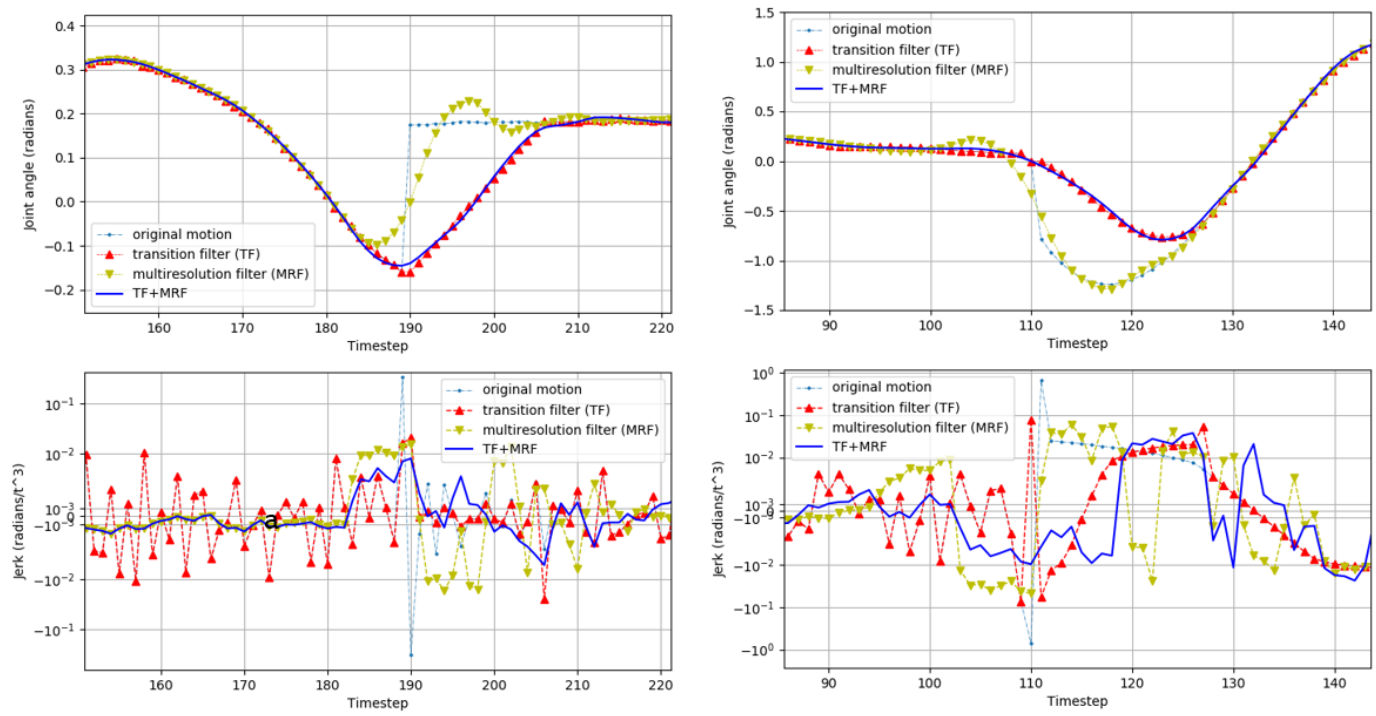

(a)

(b)
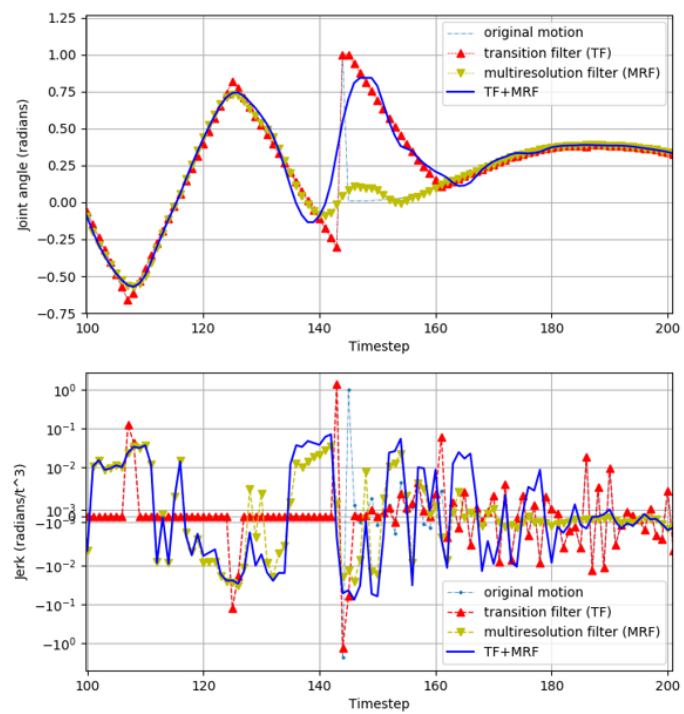

(c)

Figure 6.19: Comparison plots of applying transition filter (TF), multiresolution filter $(\mathrm{MRF})$, and multiresolution filter after transition filter $(\mathrm{TF}+\mathrm{MRF})$. Each stacked pair of plots shows the concatenated motion data as in Fig. 6.17: (a) Concatenated 1, (b) Concatenated 2, and (c) Concatenated 3. The plots on the top are joint angles over time, while the plots on the bottom show the jerk profiles. Unlike Fig. 6.18 where the whole length of the motion data is shown, here the plots are zoomed in around the joins/transition points where the differences are significant. 
Table 6.3: Jerk range in concatenation results $\left(\frac{d^{3} s}{d t^{3}}\right)$ (lower is better).

\begin{tabular}{|c|l|l|l|l|}
\hline Motion data & No filter & $\begin{array}{l}\text { Transition } \\
\text { filter (TF) }\end{array}$ & MRF & TF + MRF \\
\hline Concatenated 1 & -0.3323 & -0.1193 & -0.01685 & $\mathbf{- 0 . 0 0 9 7 1 2}$ \\
& $(0.3496)$ & $(0.1187)$ & $(0.03719)$ & $\mathbf{( 0 . 0 3 7 1 9 )}$ \\
\hline Concatenated 2 & -1.172 & -1.172 & $\mathbf{- 0 . 9 0 6 5}$ & $\mathbf{- 0 . 9 0 6 5}$ \\
& $(0.6542)$ & $(0.2799)$ & $\mathbf{( 0 . 1 8 6 9 )}$ & $\mathbf{0 . 1 8 6 9 )}$ \\
\hline Concatenated 3 & $-2.294(1.366)$ & $-1.303(1.366)$ & $\mathbf{- 0 . 0 3 4 9 7}$ & -0.07637 \\
& & & $\mathbf{( 0 . 2 2 5 6 )}$ & $(0.2256)$ \\
\hline
\end{tabular}

Table 6.4: Mean squared error of filtering with respect to original data (lower is better).

\begin{tabular}{|l|l|l|l|}
\hline Motion data & $\begin{array}{l}\text { Transition } \\
\text { filter }(\mathbf{T F})\end{array}$ & MRF & TF + MRF \\
\hline Concatenated 1 & $1.746 * 10^{-3}$ & $2.951 * 10^{-4}$ & $1.791 * 10^{-3}$ \\
\hline Concatenated 2 & $3.073 * 10^{-2}$ & $1.769 * 10^{-3}$ & $3.079 * 10^{-2}$ \\
\hline Concatenated 3 & $1.695 * 10^{-2}$ & $4.211 * 10^{-3}$ & $1.896 * 10^{-2}$ \\
\hline
\end{tabular}

spike at around timestep 142 is retained when using transition filter and removed when using multiresolution filtering. While this removal can be considered as a significant disruption to the original data, the resulting motion does not have the jarring motion and appears more natural.

We collected 240 motion samples where each sample is a concatenation of two or three motion sequences from our motion dataset. Our motion dataset consists of 100step motion samples collected from motion capture and HROS-1 robot motion data
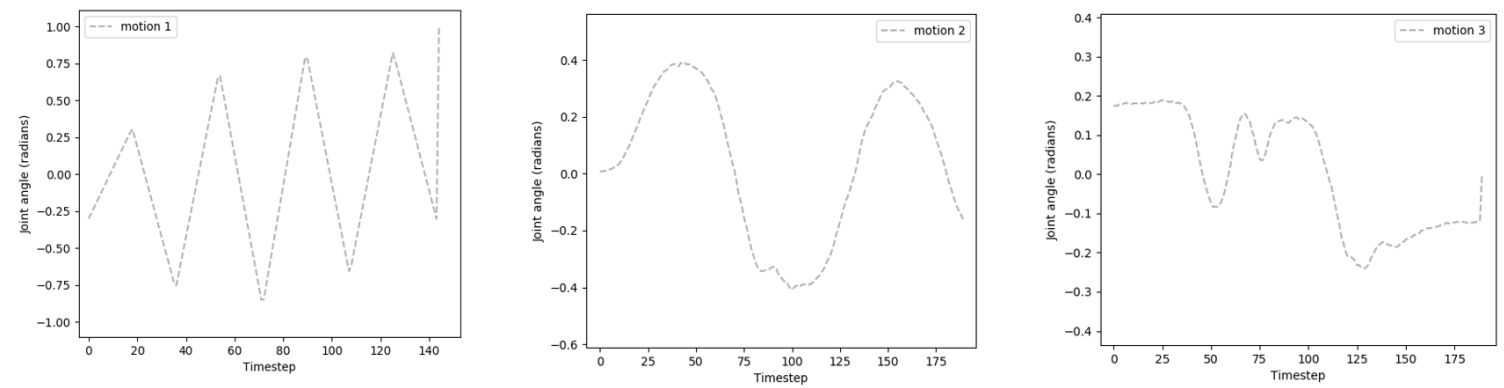

Figure 6.20: Three arbitrary motion data to concatenate 


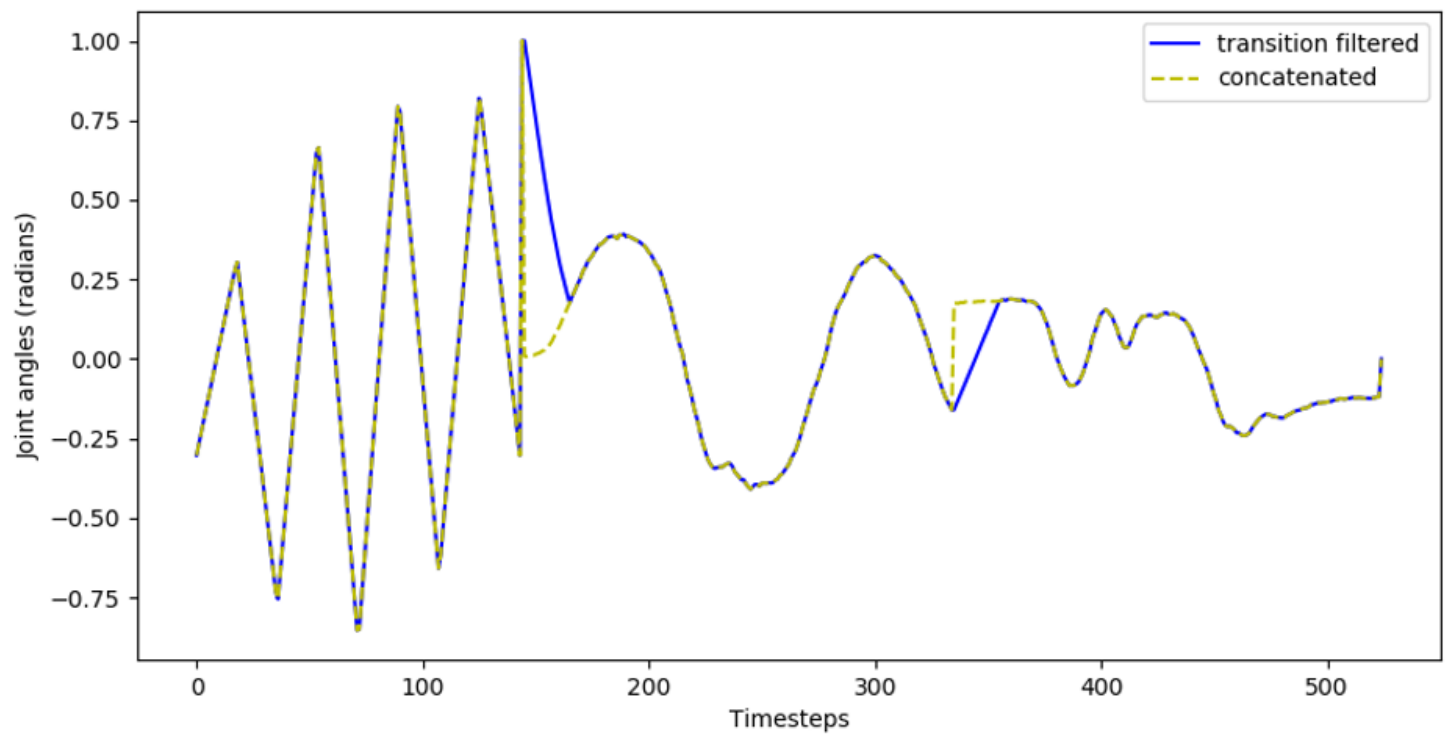

(a)

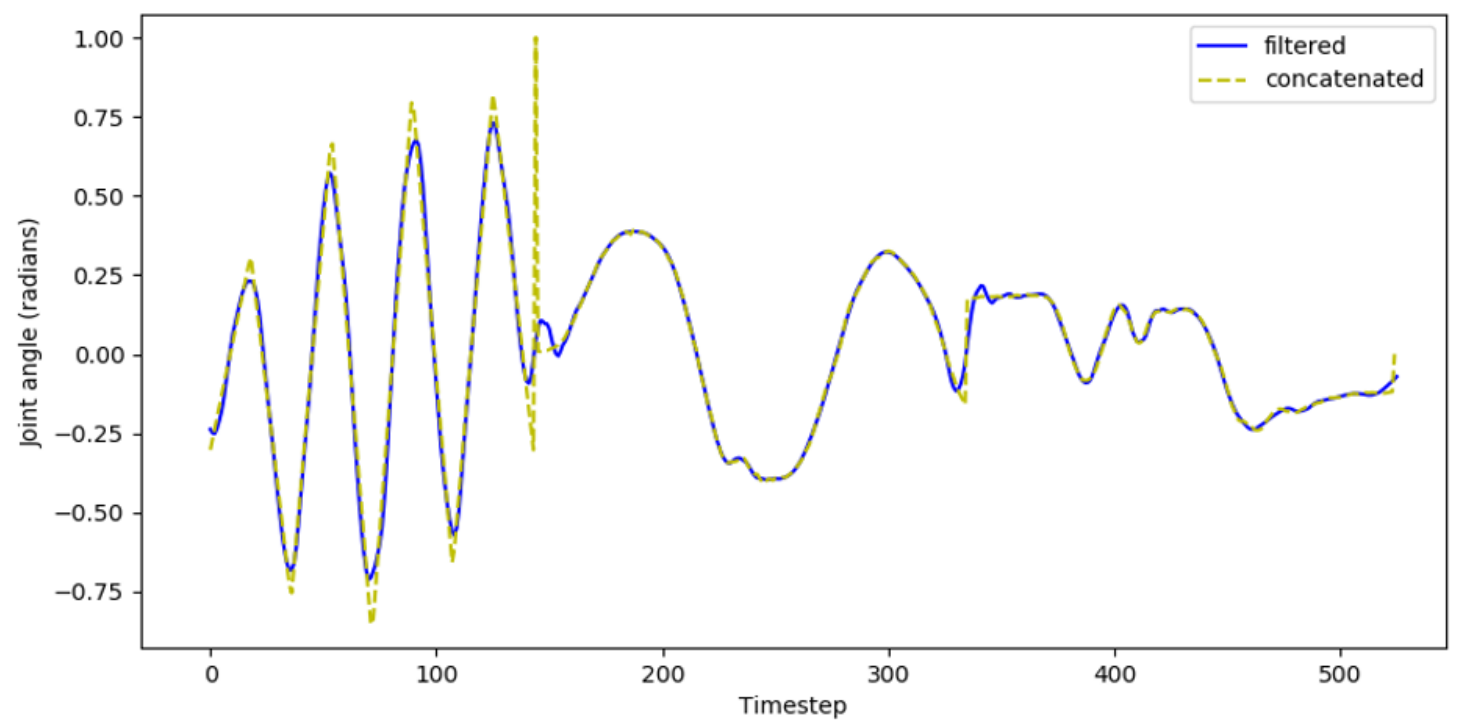

(b)

Figure 6.21: Concatenation of 3 motion data from Fig. 6.20: (a) Using van Breemen's transition filter ('transition filtered' line) to concatenate three motion data, (b) using multiresolution filtering ('filtered' line) to concatenate the same set of motion data. Joins between motion data are at Timesteps 140 and 330. 
that has been linearly interpolated. These samples are filtered using the different wavelet bases and different filtering levels. The filtered/reconstructed signals are applied to our simulated HROS-1 robot and some on the real HROS-1 robot to evaluate the effects. Below we list the resulting transitions, the wavelet bases used, and the filtering levels that we deemed useful and natural both on the simulation and the real robot. Our criteria for choosing the good and usable results are: 1) results which reduce the jerk overall but particularly around the concatenation points, and 2) minimal disruption to the the original motion (i.e. gesture).

There are several effects that we observed:

- Simple, smooth transitions. Transitions are blended seamlessly and the motion appears as one continuous action. The wavelets that support this effect are the biorthogonal3.x, and some of the symlet wavelets. For most of these wavelets, we accomplish this effect by using only the lower half of the filterbank coefficients.

- Followthrough. Followthrough is the effect where there is an overshoot added after the transition. This could be desirable as it indicates the transition that one motion ends and the next one begins. The Debauchies and coiflet wavelets can create this effect.

- Anticipation. Anticipation creates additional motion prior to the transition point that is usually in the opposite direction of the next pose. Anticipation is a preparation action, and can be a clear indication of a change (i.e. transition). This effect can be produced using some Debauchies wavelets, symlets wavelets, and the biorthogonal3.3 wavelet.

To achieve the effects above, the motion data must be reconstructed at certain filtering levels. We summarize our findings in Table 6.5. Here, we assume a filterbank 
level is used as is by keeping the coefficient values or removed completely by changing the coefficient values to zero. The number of filterbanks from the wavelet transform varies depending on the length of the input signal and the width of the wavelet basis. For this reason, we denote the number of filterbanks as $N$ and each filterbank is denoted as $f_{n}$. To indicate which filtering level was used to produce the effects, we write $f_{0}, f_{1}, \cdots$ when referring to individual levels, $f_{0-2}$ to refer to a range of levels from 0 to 2 , or $f_{0-\frac{N}{2}}$ to indicate the lower half of the filterbanks. We summarize our

findings in Table 6.5. The names of the wavelets are listed using their commonly used abbreviations: Debauchies (db), symlets (sym), Haar (haar), coiflets (coif), biorthogonal (bior), reverse biorthogonal (rbio), and discrete Meyer (dmey).

The results summarized in Table 6.5 are qualitative and compiled based on our observations on the motions performed in simulation and the real robot.

\subsubsection{Discretized Steps}

Discretization can be done using wavelets which reconstruction kernels are rectangular, such as: Haar, Debauchies1 (db1), Biorthogonal1.1 (bior1.1), Biorthogonal1.3 (bior1.3), and Biorthogonal1.5 (bior1.5). When filtering is done too aggressively where only the lowest filterbank component (lowest frequency) is retained, the reconstructed signal loses most of the details of the original motion and consists only of mostly constant values. On the other extreme, when most of the filterbank components are used, the reconstructed signal becomes very close to the original signal. To create the more interesting effects mentioned above, $f^{\prime}(t)$ must be reconstructed using a number of filterbank components somewhere in between these two extremes. Figure 6.22 shows using the bior1.5 wavelet and reconstructed using only two of the lowest filterbanks and other filterbanks set to zero. Sudden movements are removed, but detailed movements like in around frame 200 are also removed. To obtain the de- 
Table 6.5: Wavelets, and the filterbanks to use to create transition effects. $N$ is the number of resulting filterbanks from the discrete wavelet transform (DWT). ( $N / 2$ is usually rounded down to the nearest integer)

\begin{tabular}{|c|c|c|}
\hline Effect & Wavelet & Filterbanks $\left(f_{n}\right)$ \\
\hline Simple & $\begin{array}{l}\text { - bior }(3.1,3.3,3.5,3.7 \text {, } \\
\quad 3.9), \\
\text { - } \operatorname{sym}(6,9,11,13,15), \\
\text { - } \operatorname{db}(5-25)\end{array}$ & $\begin{array}{l}\text { - bior: } 0-N / 2 \\
\text { - sym: } 0-N / 2 \\
\text { - db: } 0-N / 2\end{array}$ \\
\hline Followthrough & $\begin{array}{l}\text { - } \mathrm{db}(15-26) \\
\text { - } \operatorname{bior}(3.3) \\
\text { - } \operatorname{coif}(4,5)\end{array}$ & $\begin{array}{l}\text { - db: } 0-1 \\
\text { - bior: } 0-N / 2 \\
\text { - coif: } 0\end{array}$ \\
\hline Anticipation & $\begin{array}{l}\text { - } \operatorname{db}(15,24,26) \\
\text { - } \operatorname{sym}(7,10,11-18) \\
\text { - } \operatorname{bior}(3.3)\end{array}$ & $\begin{array}{l}\text { - } \mathrm{db}: 0 \\
\text { - } \operatorname{sym}: N / 3 \\
\text { - bior: } N / 2\end{array}$ \\
\hline
\end{tabular}


tailed movements, one can simply gradually add another filterbank for reconstruction until the desired level of detail is achieved.

The reconstructed signal will have the same length as the original signal. As a step function, there are fewer discrete steps than the original signal and the remaining frames are constants of those discrete values. These constant values give pauses to the motion, creating a stuttering effect. We consider the motion to 'stutter' when

moving from pose $\mathrm{A}$ to pose $\mathrm{B}$, and there are visible pauses taken between poses A and B making the motion appear to be taking discrete steps. In stuttering, these discrete steps are poses that incrementally progress from pose A to pose B in contrast to jitter where the motion plot looks like a noisy signal.

Without the filtering, the stuttering effect can occur when the robot pose data is being interpolated at a rate that is slower than the response of the controller and the robot's hardware (servo controller, servo).

We can create the stutter effect by using one-third to half of the lowest filterbanks. We observed that using more than half of the filterbanks the reconstructed data gets closer to the original data and the stutter effect becoming less apparent.

\subsubsection{Reduction}

Since the reconstructed signal gives a step function, we can remove the repeating values and only collect the discrete 'steps' values to get the 'reduced' signal. Figure 6.23 shows an example that the motion data is shortened from 773 frames (top) to 24 frames (bottom). This method is similar to 'downsampling' the signal, but our reduction method seems to retain the details of the original motion slightly better. Figure 6.24 shows a comparison between the original data, the reconstructed reduction signal and a downsampled result.

This method is useful in three ways. First, the signal reduction method can be 


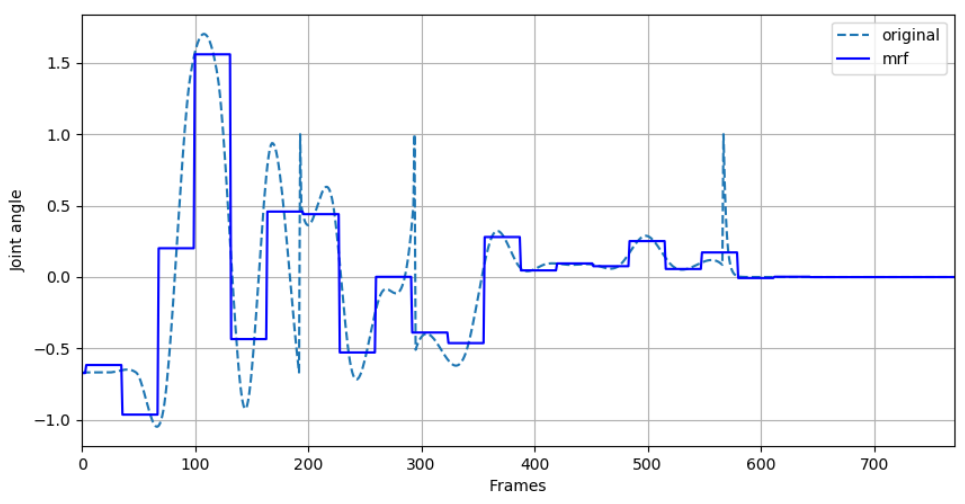

(a)

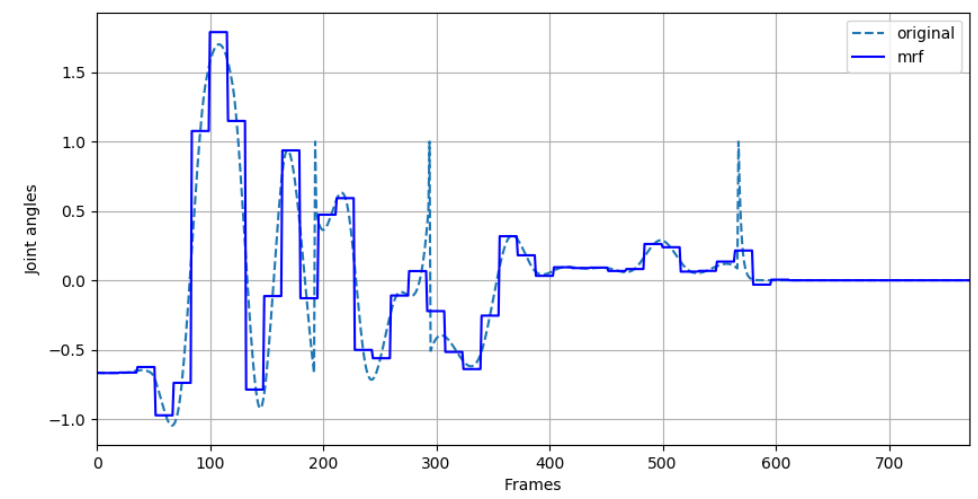

(b)

Figure 6.22: Stuttering effect using the bior1.5 wavelet. The dashed line is the original motion data, the solid line is the reconstructed data. (a) shows reconstruction using two lowest filterbanks, (b) uses three filterbanks and captures more details but higher number of 'stutters'. 

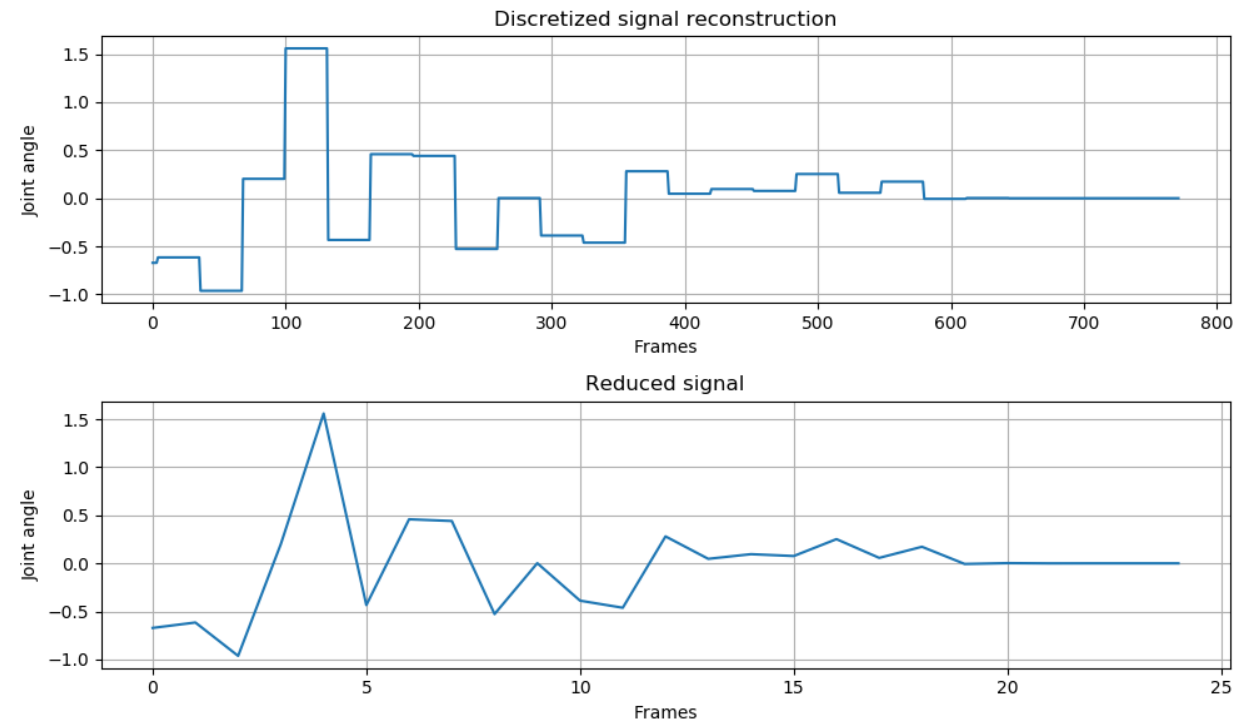

Figure 6.23: The top plot is a signal reconstructed using the bior1.5 wavelet. The bottom plot is the reduced signal: the same signal but only consisting of values when they change.

used to convert motion capture data into standard robot motion data format such as HROS-1. Second, by removing the repeating values, we shorten the length of the sequence, effectively speeding up the motion (assuming each pose in the sequence is being sent at a constant rate). If desired, the reduced signal can be interpolated to change the speed of the motion. Finally, we also found this method to be useful when training a generative model using neural networks. When training a generative model for motion data, the goal is to generate a sequence of pose data (i.e. joint angles) at the output of the trained model. Since the output values are usually real valued, training such networks can be difficult - the generated sequence tends to be noisy and does not represent the properties of the reference motion data (e.g. smooth trajectory, mostly consist of low frequency components). 

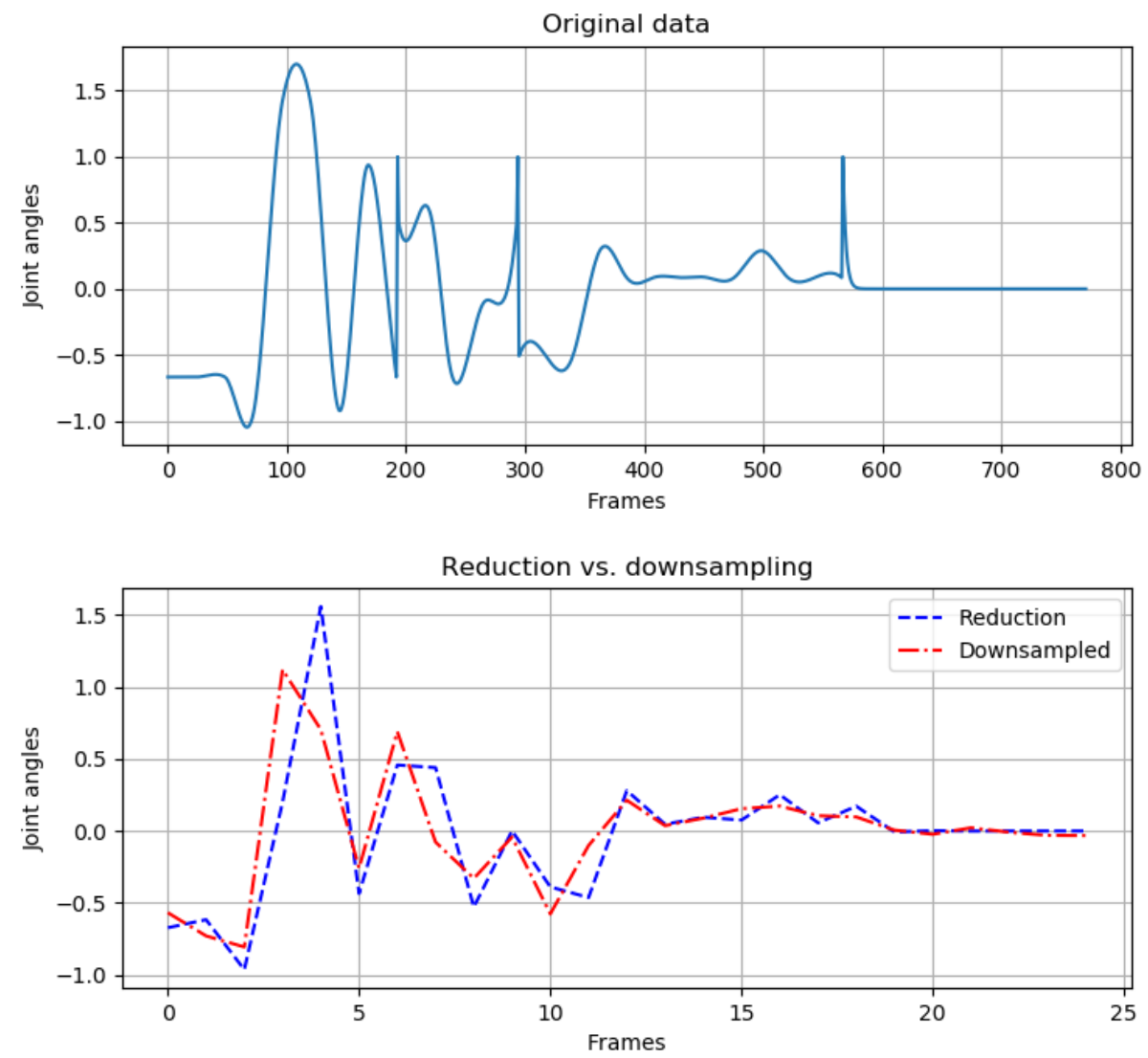

Figure 6.24: Comparison of 'reduction' versus 'downsampling'. The 'reduction' method captures details such as the peak near frame 100 in the original signal in frame 4 of the reconstructed signal, whereas the dowsampled signal misses the peak in frame 3. The reduction signal is the same as in Figure 6.23. 

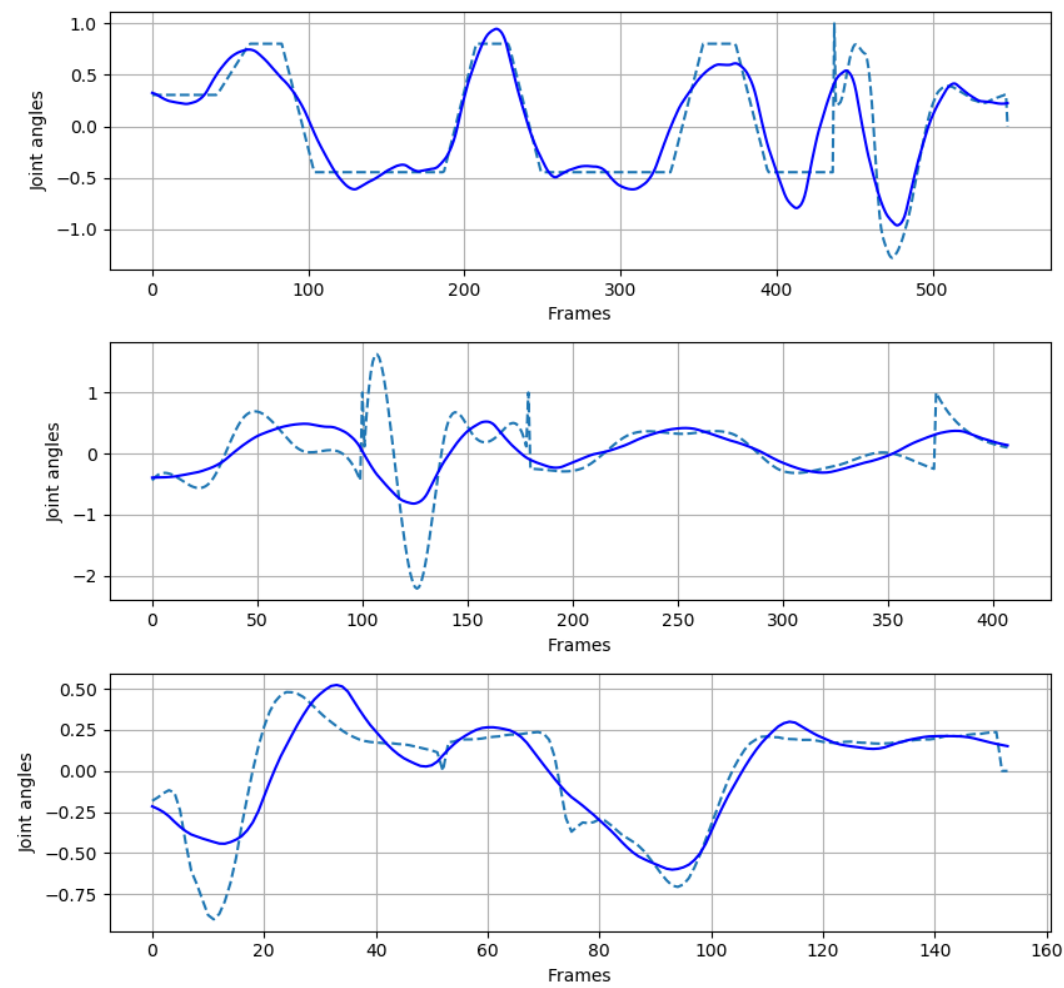

Figure 6.25: 'Lazy' effect. The three motion data were randomly picked from our concatenated motion dataset. All motion data were filtered using the symlet5 wavelet and reconstructed with only the lowest filterbank and other filterbanks set to zero. Many detailed parts are missing in the reconstructed data, but retain the overall 'shape' of the original data.

\subsection{4 'Lazy' or 'Bounded' Effect}

Certain levels of filtering may cause the reconstructed signal to lose many of the detailed movements in the original signal such as small undulations and reduced range of motion. We call this loss of detailed motion as 'lazy' or 'bounded' (Figure 6.25). In contrast, with the stutter effect most of these details are retained.

We can produce this 'lazy/bounded' effect by using wavelets which are continuous (not CWT), or wavelets which reproduction functions are continuous (in the case for biorthogonal and reverse biorthogonal wavelets) with, for example: wavelets sym5 through sym12. 
We can use the typical energy measure of a signal $E_{x}$ (eq. 6.33 for discrete signals) and show that the 'lazy' reconstruced signals indeed have lower energy than the original data.

$$
E_{x}=\sum_{n=-\infty}^{\infty}|x(n)|^{2}
$$

\subsubsection{Noise/jitter}

One could ask the question: why do we want to add noise to the motion data now whereas typically filtering is used to denoise the signal? Jitter or noise can add expressiveness to the robot motion such as depicting a character with tremors. We found that using certain wavelets can create the jittery effect in a more coherent manner than simply adding random noise or amplifying certain frequency components like was done by Bruderlin and Willams (adding jitter was mentioned but not demonstrated in the paper [13]).

Figure 6.27 shows the comparison of adding random noise and using the rbio3.3 wavelet to a concatenated motion data (join around frame 140). The one added with random noise has jitter added everywhere in the signal and slightly amplified the

discontinuity (i.e. jerk) at the concatenation join around frame 140. When using the rbio3.3 wavelet, some noise appear along the signal but not everywhere, so the executed motion has moments where the motion appears relatively smooth and easier to follow by the audience. Moreover, the reconstruction removes the discontuity at frame 140 making the motion appear as one contiguous piece.

Adding noise using the appropriate wavelets adds jitter only to some parts of the motion data in addition to making smoother transitions on concatenated data. We argue that these properties make the motion more coherent and understandable to 

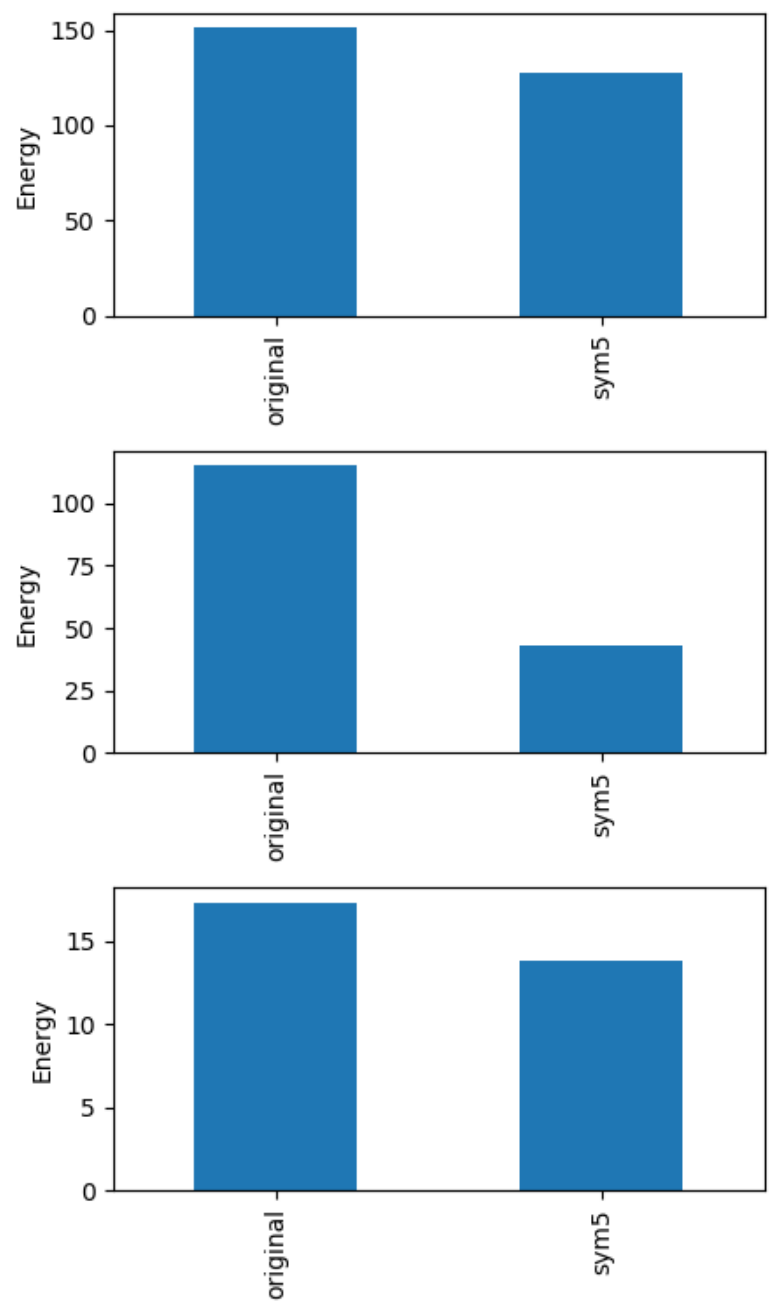

Figure 6.26: Energy level comparison of the original signal ('original') versus the reconstructed signal ('sym5') for the 'lazy' effect using sym5 wavelet. Each plot corresponds to each of the figures in Figure 6.25 in the same order top to bottom. 

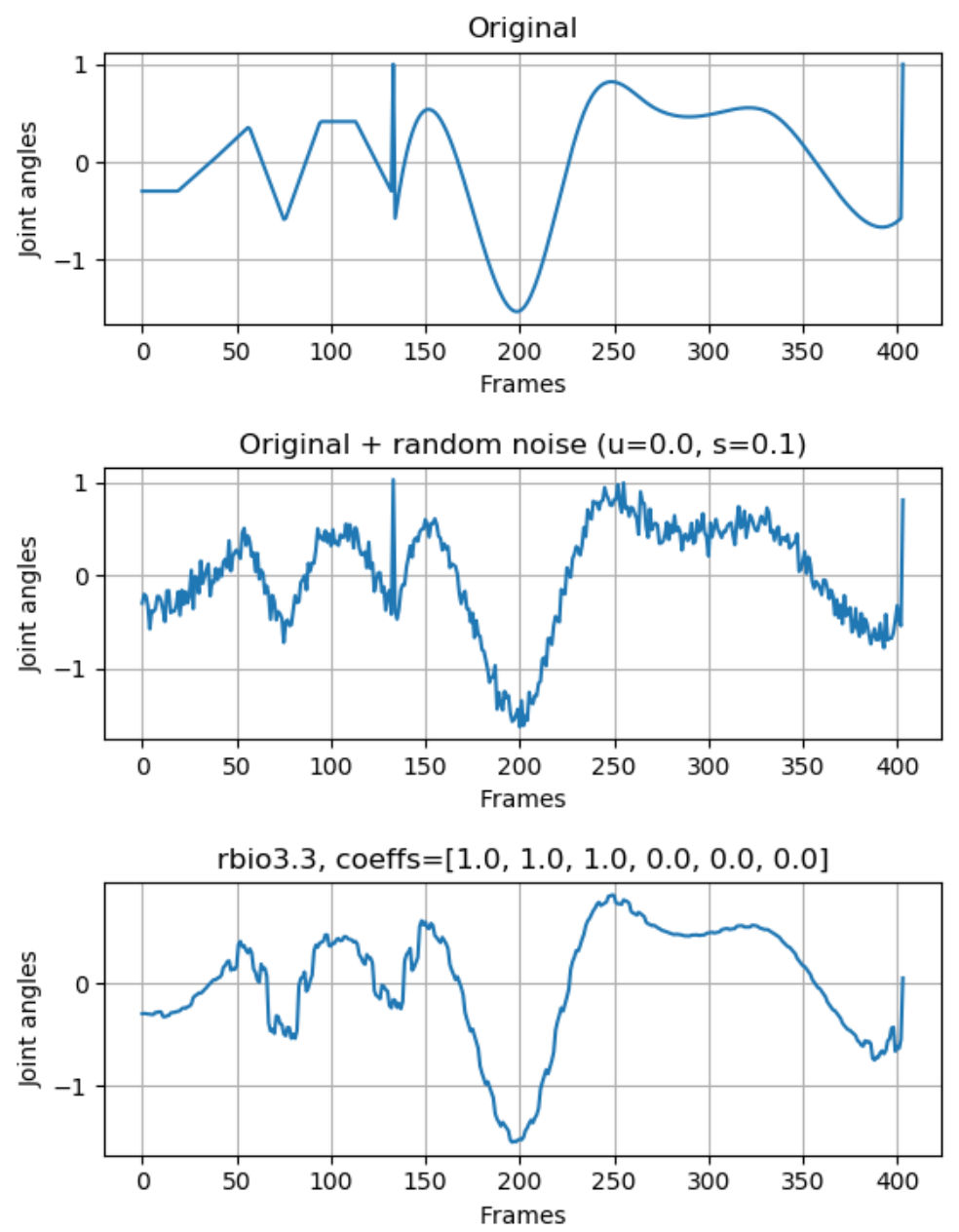

Figure 6.27: Concatenated motion data (top), with added random noise $(\mu=0.0, \sigma=$ 0.1 ), and using rbio3.3 wavelet reconstructed with lowest half of the filterbanks. 
the audience compared to adding random noise. We look to verify this with user studies in the future. Other wavelets that exhibit these properties are $\operatorname{rbio}(3.1,3.5$, 3.7, 3.9). Figure 6.28 shows some of the results from using these other wavelets.

The result from rbio3.1 shows some regularly-shaped oscillating noise which amplitude seems to dependent on the slope of the signal; the steeper the signal, the bigger the amplitude and vice versa. The result of rbio3.5 is similar to rbio3.3 from Figure 6.27 but slightly smoother. The results from rbio3.7 and 3.9 actually do not introduce much jitter, but they rather smooth the linear part in the first third of the signal. The smoothing effect is caused by the smoother reconstruction wavelets of rbio3.7 and 3.9 .

\subsubsection{Linearize}

We found that some of the biorthogonal wavelets can be used to reconstruct a signal making the signal appear to be linearly interpolated. The most effective wavelets to create this effect are bior2.2, bior2.4, bior2.6, bior2.8. Figure 6.29 shows some example of linearizing motion data using various bior wavelets.

Note that this result is different from the results of the reduction method discussed earlier. In the reduction method, the length of the reduced signal is significantly shorter than the original signal. In the linearize method, the length of the reconstructed signal remains the same as the input signal.

\subsubsection{Miscellaneous Effects}

There are other wavelets besides the ones mentioned above that can also create interesting 'textures' on the reconstructed signals. Wavelets that create jitter can produce different patterns of jitter when plotted, but when applied on a robot's movements, the jitter created using one wavelet is difficult to distinguish from jitters created by 

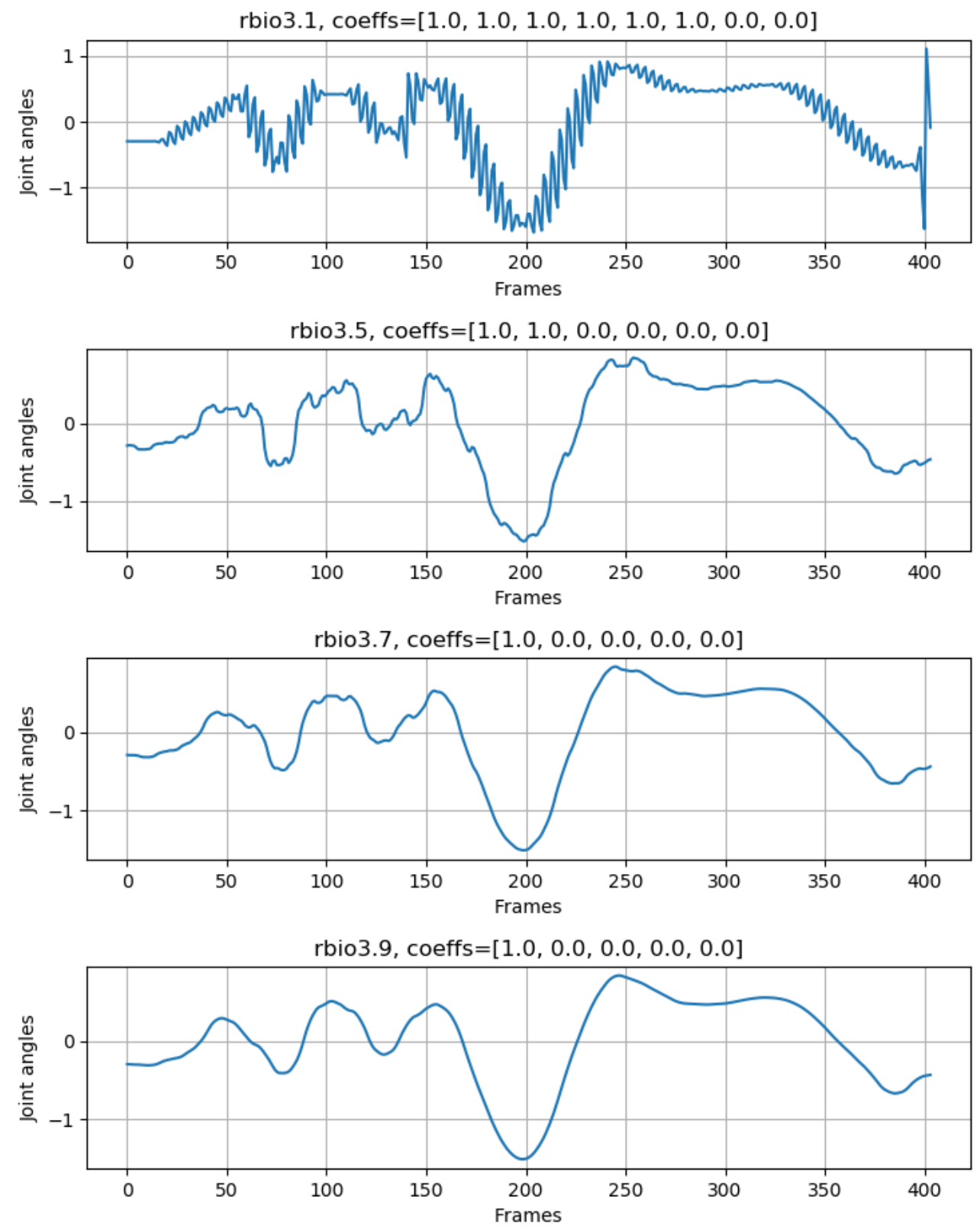

Figure 6.28: Effects of applying various rbio wavelets on a motion data. In the title of each plot, 'coeffs' indicate the number of bandpass as the result of the multiresolution analysis using wavelet transform. coeffs $_{i}=1.0$ indicates the bandpass being used for reconstruction, and coeffs $_{i}=0.0$ indicates the bandpass are not being used (the bandpass coefficients are set to zero). 

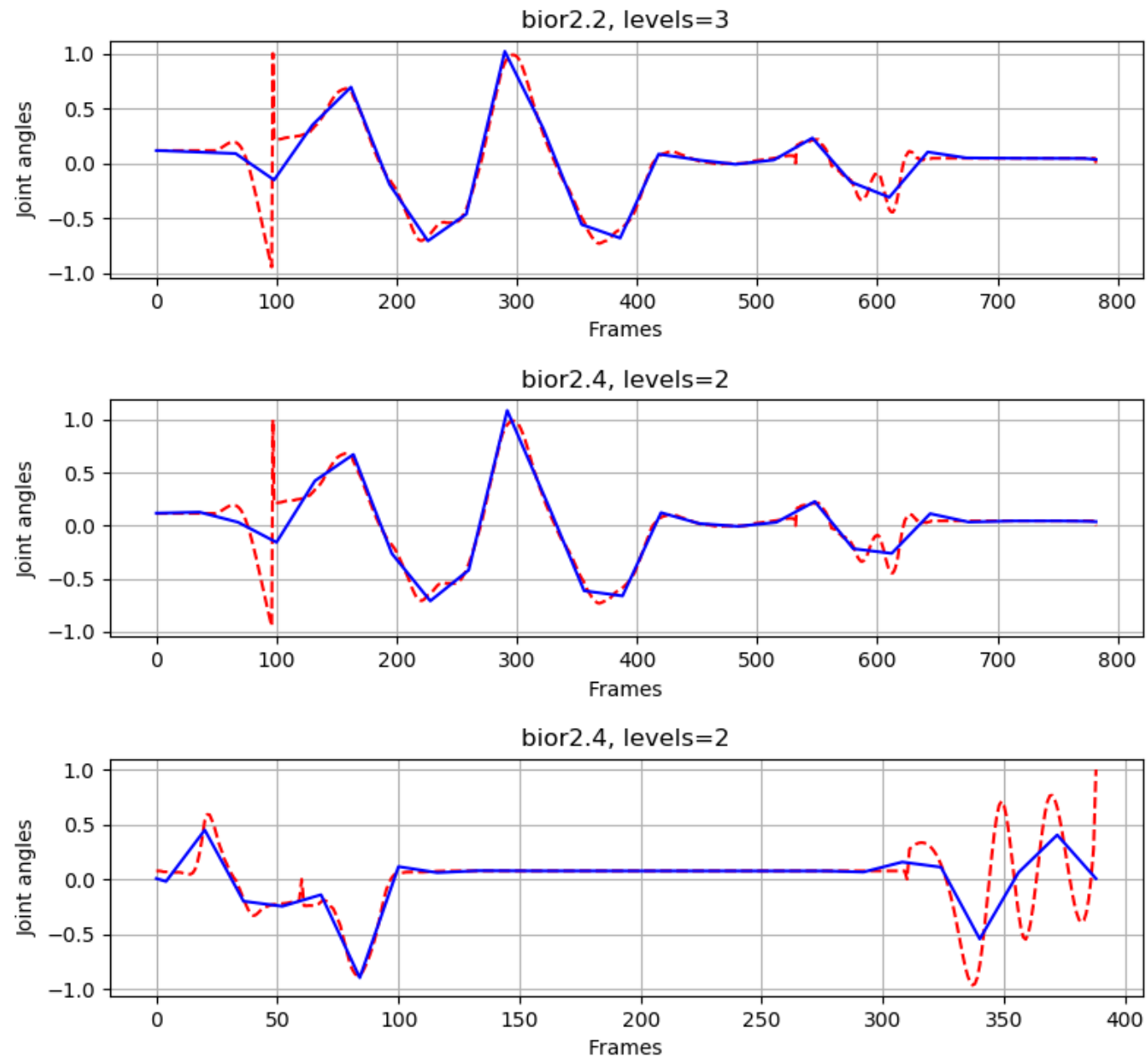

bior2.6, levels $=2$

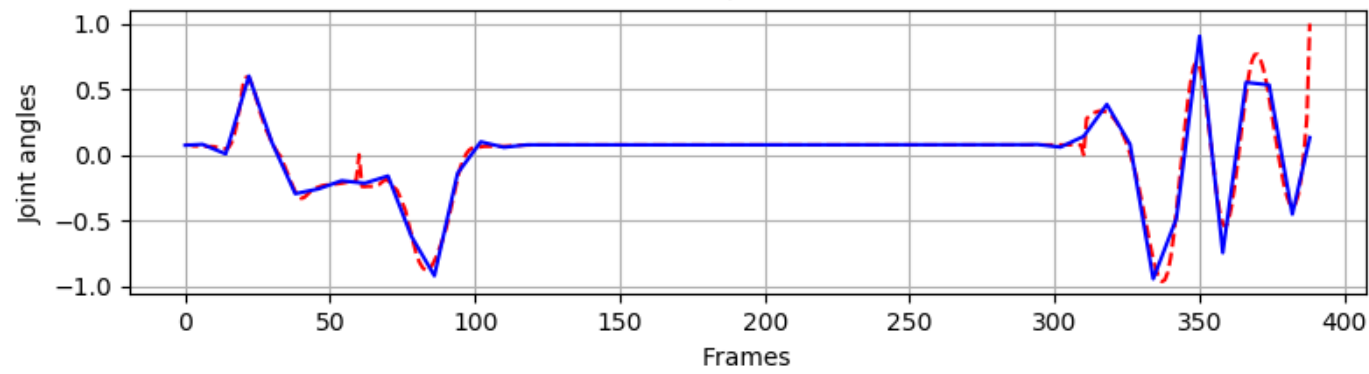

Figure 6.29: Using the bior wavelets to 'linearize' motion data. The dashed red line is the original data, the solid blue line is the reconstructed data. 'levels' indicate the number of bandpass banks used in the inverse transform to reconstruct the data, starting from the lowest bandpass. 
other wavelets. Most of the textures created by these other wavelets also appear similar to the textures already mentioned above: smoothing/lazy, adding jitter, discretizing, or linearizing.

\subsection{Legibility of Wavelet Motion Effects on Actual Robot}

We applied the method of using wavelets for creating expressive motion effects on the HROS-1 robot.

Our experiments show that the effects on the motion signals that are visible on the plots (such as the ones above) often are not immediately apparent when executed on an actual robot. However, these motion effects are more visible when the motion data is played on a simulated, 3D model of the robot. One of the reasons that the effects are not visible on the actual robot is due to the response time of the servos on the robot. The movements in the motion data are being sent to the robot at $1 \mathrm{Mbit} / \mathrm{second}^{7}$. As new position values are received, the controller in the servo immediately changes the servo's target position regardless whether or not the previous target position has been reached. Meanwhile, there is a delay between the time the servo's target position is updated and when the motor starts to move towards the new target. Due to these factors, tiny, incremental movements in the motion data as the result of the wavelet processing, often are not apparent when executed on the real robot (HROS1). Although all data points sent to the servos are received, we cannot confirm that each data point will be reached to completion.

In addition to setting the baud rate to $1 \mathrm{Mbit} / \mathrm{sec}$, we adjusted the slope parameter of all servos on HROS-1 to $70 \%$ (or 178/255). The slope parameter on the AX12A servo allows a slight acceleration as the servo begins moving towards a new

\footnotetext{
${ }^{7}$ We found this baud rate to be the most stable to use with the Dynamixel AX-12A servos and the U2D2 interface board.
} 
target position, and deceleration when approaching the target position. With this configuration, we are able to achieve relatively smooth motions on the robot, although sacrificing the ability to execute sudden motions. Reducing the slope value will cause the 'stutter' effect on the robot.

The stutter effect is one of such cases where the effect is visible in simulation, but still appears as a smooth continuous motion on the actual robot. The user will have to experiment with different filtering levels (how many bandpass components to use to reconstruct the signal for the inverse wavelet transform) to achieve the desired effect. We found that using a lower slope value (for AX-12A servos) works better than the wavelet transform with our robot. We also revise our claim about producing the 'lazy' effect. Instead of using the symlet5 wavelet, the Haar wavelet (or similar wavelets like db1 and bior1.1) should be used instead where the motion signal is reconstructed with very few bandpass components. We are able to produce the jitter effect as reported above, and the motion appears as being done by a person with tremors syndrome.

We claimed that the stutter effect can be used as a discretization/reduction tool. One use of the 'discretized' data is to reduce the length of the sequence, hence increasing the speed of the motion. In practice, we found that this reduction operation is more suitable to apply to motion capture data rather than robot motion data. In Chapter 8 we will show that the discretization method is useful for building our onehot encoded motion dataset for training generative models. Robot motion data are typically only stored with very few key poses and very short sequences. To make the robot motion appear smooth, the motion data is typically interpolated using linear or other interpolation methods and the interpolated data is streamed onto the robot at a constant rate. To manipulate the speed of robot motion data, we found it is more effective to use the resolution of the interpolation, which refers to the number 


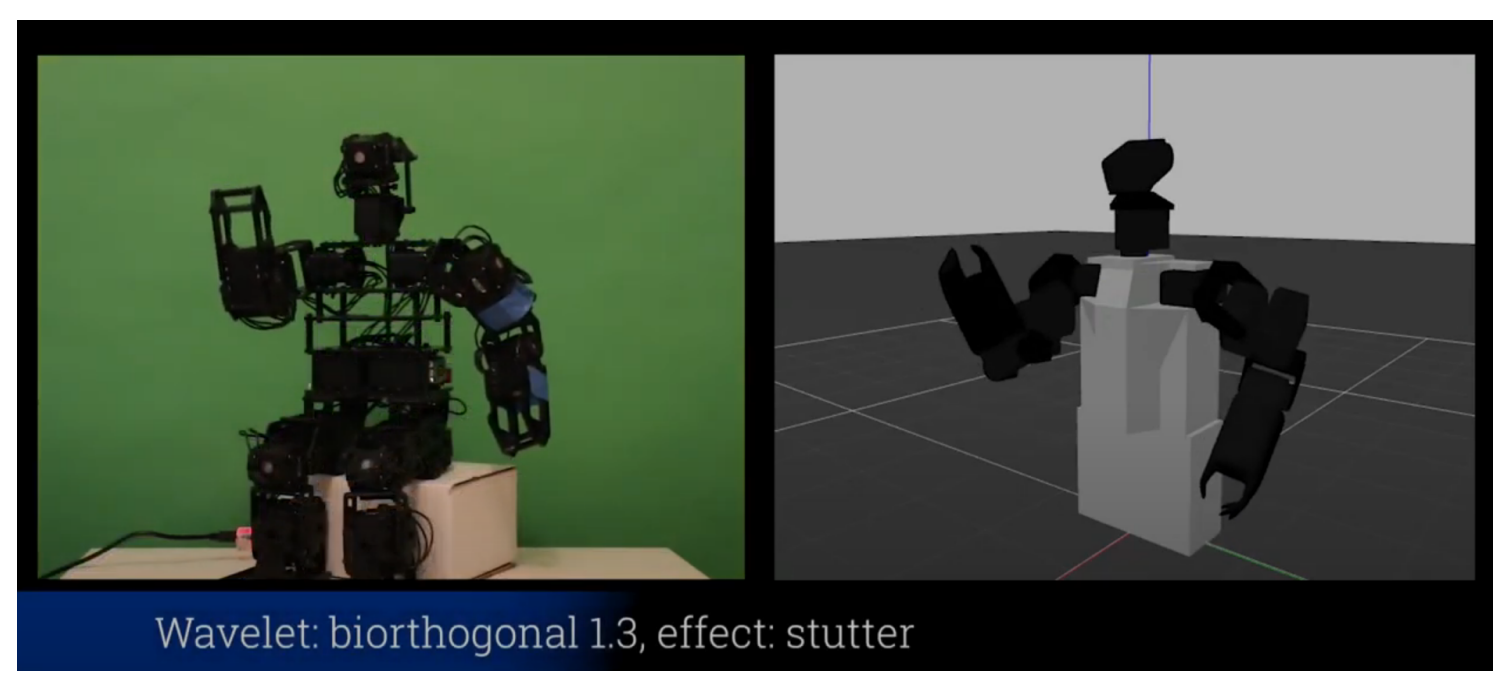

Figure 6.30: Video demonstration on the effects of using different wavelets (https: //youtu.be/9LoDtXDUNC8).

of interpolated points between two known points. The higher the resolution, the more 'in-between frames' are created between two key points, and vice versa. With the constant transfer rate of the data to the robot, the higher the resolution, the slower the motion will appear, and the lower the resolution, the faster the motion will appear.

Videos of these demonstrations can be found on the internet as shown in Fig. 6.30 :

- Different wavelet effects: https://youtu.be/9LoDtXDUNC8

- Varying speeds: https://youtu.be/kGuWbF1YROo

\subsection{Contributions of This Work}

In this chapter, I addressed the following challenges in creating motion textures for robot motion:

- Creating different motion textures such as jitter vs. smoothing require manually 
editing motion data locally, either by modifying each pose, or adding in-between poses in addition to finding the correct timing for each pose.

- Implementing principles of animation of Anticipation, Followthrough and Overlapping action, and Slow-in and slow-out to make motions appear more natural and believable are difficult due to the above reasons.

To address the above issues, I proposed the following methods:

\section{- Use Kochanek-Bartels interpolation tension and continuity parame-} ters. My experiments demonstrated that using the tension and continuity parameters is an efficient way to produce principles of animation on robot motion data such as Anticipation, Followthrough and overlapping action, and Slowin/out principles. Modifying the bias parameter does not produce discernable effects consistent with the original paper [141]. In contrast, using the standard cubic interpolation can produce Slow-in/out principle with reasonable effort, but cannot be tweaked further. Producing other principles of animation require fine tuning of many additional in-between poses. In contrast, previous works [14] [12] use custom equations of motion that had to be developed to create the desired stylings for only a limited set of textures (i.e. expressions).

\section{- Using different wavelet kernels to produce different motion textures.}

This work extends the work of Bruderlin and Williams [13] where they only used a single Gaussian-like kernel with multiresolution analysis and show effects only in terms of exaggeration vs. restrained motions, and using manual methods (e.g. motion waveshaping) to create motion textures. In contrast, I showed that various motion textures can be created effectively and globally on a motion data using the appropriate wavelets instead of applying manual, local adjustments to the motion data. 
- Wavelet smoothing for concatenated motions. Using wavelet filtering also performs better in creating smooth transitions between two (or more) concatenated motion data compared to the transition filter method [17]. In the former, the amount of jerk on average is two orders of magnitude lower than without or with transition filter method, while global disruption to the motion data is kept minimal $\left(\mathrm{MSE}<10^{-} 2\right)$ and an order of magnitude lower MSE on average than using only transition filter.

The benefit of the methods I proposed is that users do not have to manually create additional poses or perform local editing of the motion data to create these effects as in the traditional animation method or using the motion editors like RME. Users can use the parameters of the KB interpolation and wavelet multiresolution filtering levels to produce the motion "textures". Furthermore, the motion "textures" would be able to be produced programmatically for example, based on user input or the robot's sensor inputs.

In this section, we presented our contribution of this dissertation by extending the idea from using multiresolution filtering that was presented by [13] and used wavelets to: 1) reduce jerk in concatenation of highly disjoint motion sequences, and 2) using various wavelets to create more expressive versions of robot motions. We show our results on a real robot (HROS-1) and also in simulated environment (Gazebo). 


\section{CHAPTER 7 \\ MACHINE LEARNING MODELS FOR EVALUATING SYNTHESIZED ROBOT MOTION}

Humanoid robot motions created by hand or by procedural methods often suffer from looking too mechanical, while the desired outcome is a more natural, humanlike motion. However, it is difficult to objectively determine when the motion has the desired natural, human-like quality. Subjective evaluation by humans are commonly done to assess the quality of the robot's motions. However, the results of subjective evaluations can sometimes be inconsistent or unreliable. In this chapter, I present an alternative approach to evaluate the quality of robot motion by using motion evaluators a classifier that has been trained to label motion data that belong in the motion capture dataset as ' 1 ' and motions that belong in a robot motion dataset as '0'. This method assumes human motion as the "golden standard" for expressive, natural-looking motions and the "baseline" of robot motions is where the motions are linearly-interpolated. In other words, 'good' motions are the ones that have similar qualities to human motions (as in motion capture data), and 'bad' motions are the ones with 'mechanical' qualities as in typical robot motions. New motion data produced by some motion generator system for a humanoid robot can then be given to the classifier and be labeled ' 1 ' if the motion data exhibit the qualities of motion capture data and '0' otherwise. In other words, the classifier gives us the confidence to consider the motion data produced by the motion generator system is 'good' because the motion data exhibits the qualities of motion capture data, and 'not good' if otherwise. In this work, I describe the process of building a new dataset for training 
the classifier by consolidating a motion capture dataset with robot motion dataset. To demonstrate this new idea of motion evaluators, I trained and compared the performances of four classifier models: Naïve Bayes, Decision Tree, Random Forest, Support Vector Machine (SVM) and a LSTM network. In this chapter, I also discuss the issues and challenges of building such a system.

The main motivation of the work in this chapter is to find an alternative to subjective evaluation methods such as surveys and questionnaires with human subjects to evaluate the quality of the motion data produced by some robot motion generation system. Normally, the validation of the system would be done by having a person observe a robot performing the motions (e.g gestures) produced by the motion generator, then the person would give his/her answers regarding the quality of the motion in a survey or questionnaire. In the questionnaire there will be qualitative questions such as whether the motions of the robot look 'natural/human-like' or 'unnatural' [188]. 'Unnatural' can be interpreted as having mechanical qualities, or 'zombie-like' as the term used in the Uncanny Valley theory [189]. In our observation, this method is unreliable because the person often cannot articulate using precise vocabulary what makes the motion 'natural' or 'unnatural' or there may be questions that cause inconsistencies in the survey results [190]. In our own experience when showing our robots to people, some of the descriptive terms that we have observed used by people when commenting on a robot's gesticulations include: 'creepy', 'sluggish', 'awkward' but also occasionally 'cute'. However, it is rare that people can comment on specific motion qualities. Animators often use vocabulary from the principles of animation [43] $\mathrm{m}$ such as: 'The motion needs more anticipation' , '(the motion) can use better timing', 'there is no follow through' and so forth. Another factor that people commented was that the actuator (servo) noise can be distracting and gives an unpleasant experience being around the robot. We believe this is an important issue to address in future 
works despite not directly related to the motions themselves.

In this work, we propose using motion capture data as the benchmark when evaluating the quality of humanoid robot motion produced by some motion-generating system $S$. To do this, we propose using a classifier that is trained on a dataset of motion capture data and robot motion data to learn the correct labeling for a motion data given at the input as shown in Figures 7.1 and 7.2. A diagram of the training of the classifier $C$ is shown in Figure 7.1, where $C$ can be of any of the models we mentioned. The dataset for training the classifier $C$ is the set of labeled data pairs $(\mathbf{X}, Y)$ of motion capture data and artificially-made robot motion data where $\mathbf{X}$ is the vector of motion data and $Y$ is the true labels for the motion data. The motion data vector $\mathbf{X}$ is of some size $T \times N$ where $T$ is the length of the sequence with each timestep contains one pose, and $N$ for number of degrees of freedom (DOFs). In the dataset, the motion capture data is labeled ' 1 ', and the robot motion data is labeled '0'.

The classifier is trained by iteratively updating its parameters so its output $\hat{Y}$ matches the true labels of the input motion data $\mathbf{X}$ with high accuracy. Once the classifier is trained and tested, the classifier $C$ is presented with new motion data $\mathbf{X}^{\prime}$ that were generated by $S$. Assuming the classifier $C$ exhibits good accuracy during training and testing phases, when the classifier outputs '1' $(\hat{Y}=1)$ for the newlygenerated motion data $\mathbf{X}^{\prime}$, then we can confidently say that the motion data produced by $S$ is 'good'. This process is illustrated in Figure 7.2. $S$ can refer to any methods for generating motion data such as: using robot motion editors or other procedural methods. It is important to note that the goal is not to evaluate whether $S$ can reproduce a specific motion capture data, but rather to evaluate whether the motion data generated by $S$ exhibit the qualities of motion capture data. In this work we evaluate the motions generated by the REBeL motion generation system that was 

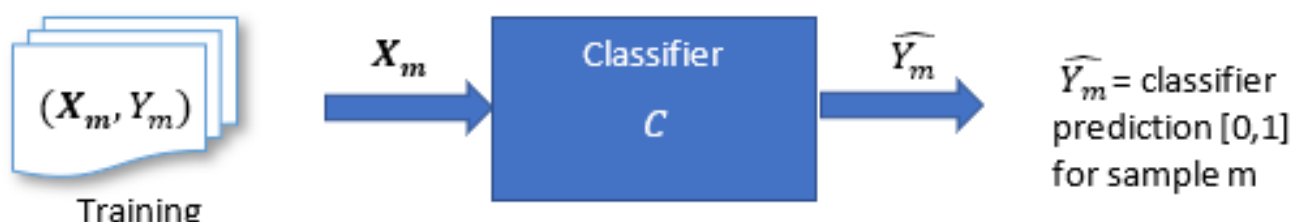

Training

dataset

Training dataset:

$X_{m}=$ motion sequenœ,

$Y_{m}=$ true label (0: artificial data, 1: motion capture data)

Figure 7.1: Training the classifier of some model $C$. Given a dataset of size $M, m \in M$, $\mathbf{X}_{m}$ is the vector of motion sequence, $Y_{m}$ is the true label, and $\hat{Y}_{m}$ is the output (prediction) of the classifier.

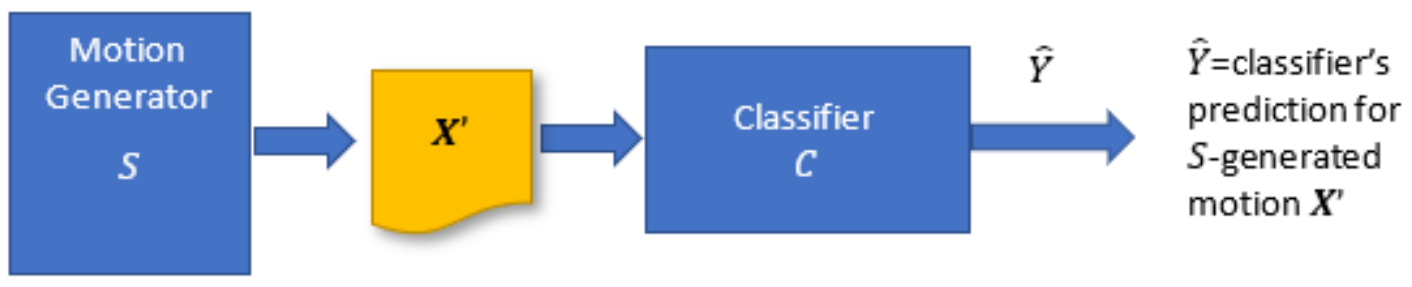

Figure 7.2: Using a classifier model as a way to measure the performance of the motion generator $S$.

developed by us. As another contribution of this work, we developed the dataset that is used to train this motion classifier $C$ because such dataset has not been created by previous authors to the best of our knowledge.

The remainder of the section is organized as follows. Next, we discuss related works in humanoid robot motion using motion capture data followed by a brief discussion of some factors for better robot motion we obtained from discussions with animators. Following it, we discuss our approach to build our dataset. Then, we discuss the design of our classifiers. Afterwards, we show some results and finally, conclude with discussion on the choices made and future directions. 


\subsection{Related Works}

Motion capture data can be applied to humanoid robots. However, it is often the case that the robot has very different kinematic configurations than the human (e.g. fewer degrees of freedom, proportions) such that some mapping (retargeting) method is needed. Pollard et al. introduced a system that can directly transfer the pose of an actor to the pose of a PR2 robot with varying degrees of success [191]. For a bipedal humanoid robot, balance is also an issue when performing human motion capture data. The human actor inherently moves in a balanced way for him/her, but that balance does not always translate to the robot. In such case, a separate balancing method is needed to adjust the position of the robot body while the upper body performs the actions of the human actor [82] [192]. Boutin et al. use motion capture to capture human walking gait and apply the gait on multiple humanoid robots while maintaining balance [193]. Because the model in motion capture is often different than the model of the robot which to apply the motion capture data, Gartner et al. proposed using an intermediate kinematic model called Master Motor Map (MMM) specifically for use with humanoid robots [194]. The MMM itself is a humanoid kinematic model with 54 DOF but designed to be easily modified for differing limb length, sizes, and moments of inertia so it can be adapted for different humanoid robots.

Machine learning approaches have been used to learn from human motion capture data for generating new animations on 3D characters. Wang et. al used recurrent neural network and adversarial training to train a generator to improve the quality of synthesized human motion data by removing undesired artifacts such as foot sliding [195]. Combinations of generative adversarial networks and reinforcement learning have been used to learn control policies for generating simple motions by imitating 
from examples [196] [197].

To the best of our knowledge, there is no standard benchmarks or quantitative measuring instrument that can determine whether the motions produced by generative models for a humanoid robot looks 'good enough'. Existing measurements depend on the subjective assessments of the human audience and the motion designer [21] [188] [6] [130]. There are tools that have been designed to make subjective assessments of robots more robust such as the Godspeed questionnaire [188]. To ensure the robot motion has good quality, it can be developed with someone who is an expert and/or trained in animation or artist to be able to identify specific aspects in the motion that need improvement [6]. After the changes are applied, the animation is run, and the expert will refine the motion as necessary. The process is repeated until the motion is deemed satisfactory. Often, the expert resource is not always available to the robot animator. In this dissertation, we are focusing only on conversational gestures, although our results should be generalizable to manipulation tasks, but not locomotion.

Included in the category of artificial/synthetic motion are motions created using motion editor tools [16] [53] [15] [55] and motions produced procedurally using algorithms such as inverse/forward kinematics, and other robotics manipulation algorithms. For the synthetic dataset in our experiments, we use motion data created using the WinRME tool for HROS-1 [55].

\subsection{The Dataset}

To train a classifier, a dataset of both the motion capture and artificial/synthetic motions are required. To our best knowledge, currently such dataset is not available. Therefore, in this section we explain our approach to building the dataset. See 
Appendix A for more information on the dataset files that accompany this document.

For motion capture data, there are several datasets publicly available and downloadable from the internet. Several research labs provide motion capture data which are free to use for research purposes (some even for commercial purposes) such as the Carnegie Mellon University Graphics Lab Motion Capture Database ${ }^{1}$. We used the alternative file format for the CMU dataset that is provided by Bruce Hahne using the Biovision Hierarchy (BVH) format [198]. On the other hand, there are no large dataset of humanoid robot motion available. For the latter, we develop the samples using REBeL. While both the motion data in the BVH format and the WinRME format can be read in a regular text editor, the two datasets have considerably different structure and motion representation.

The BVH format specifies the motion data in three parts. The first part describes the structure of the bones to construct the rig/skeleton in a 3D animation tool (e.g. Blender, Maya) as shown in Figure 7.3. Each bone may have three to six degrees of freedom $(\mathrm{DOF})$ : three for rotations in $\mathrm{x}, \mathrm{y}, \mathrm{z}$ axes, or six for location and rotation each in $\mathrm{x}, \mathrm{y}, \mathrm{z}$ axes . The second part provides frame information (number of frames, frame rate). The third part is the actual motion data. Each line of the motion data represents a frame where the first value denotes the time of the frame, and the remaining values are the location and rotation values for all DOFs in the model. There are 31 bones total in the .bvh model of the CMU motion dataset, with the root at the hip joint having six values (3 axis for location, 3 axis for rotation) and the remaining 30 bones with 3 values (3 axis of rotation). With the inclusion of the timing of each frame as the first value and all the DOFs of the model, there is a total of 97 values per frame. Because we used Blender tool, the location values were set in Blender's internal units for location (neither metric nor imperial units). The values

\footnotetext{
${ }^{1}$ http://mocap.cs.cmu.edu/
} 


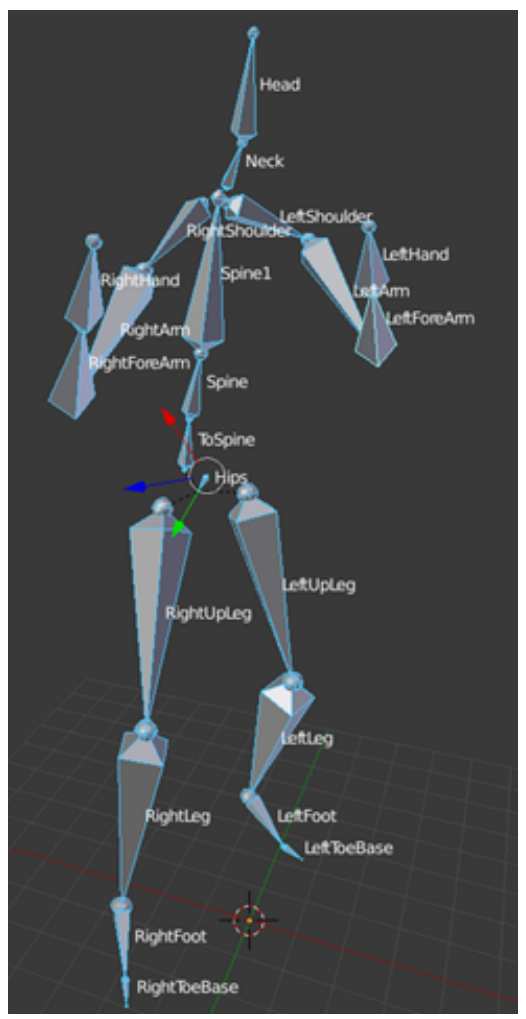

Figure 7.3: The skeleton model from the motion capture dataset in BVH format.

of the rotations are in degrees. The motion data were recorded at 120 frames per second.

The artificial motion data were created using the pose format of the WinRME tool for the HROS-1 robot shown in Fig. 7.4. WinRME motion files are described using XML format. Each pose is described as an array of 20 joint angle values representing all the DOFs on the HROS-1 robot. The HROS-1 robot has two DOFs for the head/neck, three DOFs for each arm, and 6 DOFs for each leg. The joint angle values are in the range of integers $0-1024$ corresponding to 300 degrees of rotation as the units used by the AX-12A Dynamixel servos which are the actuators on the HROS-1 robot. By default, each servo's home position is set at 512 where the servo horn is centered, and the robot is in its home pose (Fig. 7.4 right). Consequently, values 511-0 represent up to 150 degrees of counterclockwise rotation, and values 512- 


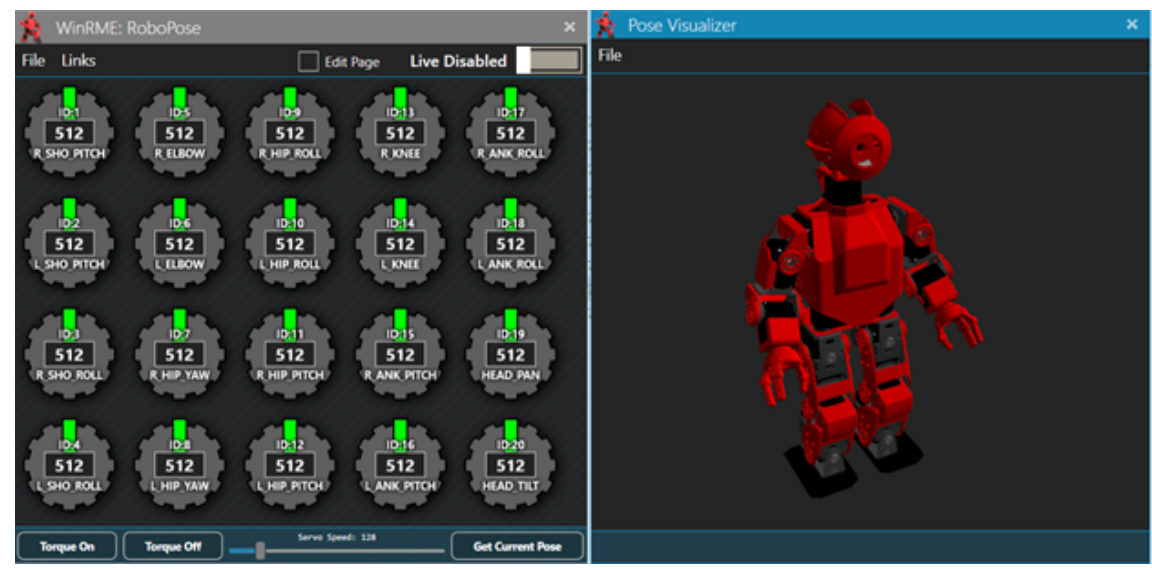

Figure 7.4: Interface of the WinRME tool for creating motions on HROS-1 humanoid robot.

1024 represent up to 150 degrees clockwise rotation. Additionally, for each pose there are two timing parameters: PauseTime for a duration to hold the pose, and Time for the desired time it takes to complete the pose from a previous pose. The Time parameter directly affects the speed of the motion between two poses; the smaller the value, the faster the motion will be.

Because the motion capture dataset and the artificial motion data have very different representations, we must consolidate them into a single format. Our consolidated dataset consists of samples with features: 100 timesteps of poses for 8 DOFs for both the motion capture data and the artificial motion data: HEAD_PAN, HEAD_PITCH, LEFT_SHOULDER

PITCH, LEFT_SHOULDER_ROLL, LEFT_ELBOW, RIGHT_SHOULDER_PITCH, RIGHT_ SHOULDER_ROLL, and RIGHT_ELBOW. The values for the $100 \times 8$ vector are real numbers representing the angular velocities in radians/timestep. Finally, the last value of each sample is the label for the sample: '0' for artificial motion data and '1' for motion capture data. The next section discusses how the issues in building the dataset are addressed. 
To consolidate the motion capture and artificial robot motion dataset into a single dataset, we address the challenges with regards to 1) different number of DOFs, 2) different joint angle units, 3) the number of frames per second, 4) the size of the dataset, and 5) clean-up.

\subsubsection{Degrees of Freedom (DOF)}

The number of degrees of freedom (DOFs) on the motion capture data is significantly different from the DOFs available on the robot. Because we are interested only in gestural motions, and the fact that the robot cannot perform bending at the torso, we made the decision to only consider the motion of the upper body for both motion capture and the artificial motion data. Thus, we reduce our focus to only the movements of the arms and head. Because the artificial (robot) motion data has fewer DOFs, we determine that the best approach is to choose a subset of the DOFs from the motion capture data that are most similar in representing the movements of the DOFs of the head and arms on the robot to obtain the same number of DOFs for the dataset. After observing several motion-capture clips, and analyzing the skeleton/rig in Blender, we decided on the mappings presented below.

The HROS-1 robot has two DOFs for the head/neck; one for panning (turning left and right) and one for pitch (move up and down). We consolidate the DOFs on the motion capture model by selecting the $\mathrm{Z}$ rotation axis of the Neck bone to represent the head panning, and X rotation axis of the Head bone to represent the head pitch. For each arm, the HROS-1 robot has three DOFs: two DOFs at the shoulder for pitch (swing forward and back) and roll (swing sideways in and out), and one at the elbow. We consolidate the DOFs of the motion capture data as follows. To represent the shoulder pitch, we chose the X rotation axis of the LeftArm and RightArm bones for each left and right arm. Similarly, for both arms, we chose the Z rotation axis to 
represent the shoulder roll motion. For the elbow motion, we select the $\mathrm{X}$ rotation axis of the LeftForeArm and RightForeArm bones for the left and right arms, respectively.

Therefore, we selected 8 DOF total as one of the dimensions of the feature vector for the dataset: HEAD_PAN, HEAD_PITCH, LEFT_SHOULDER_PITCH, LEFT_SHOULDER_ ROLL, LEFT_ELBOW, RIGHT_SHOULDER_PITCH, RIGHT_SHOULDER_ROLL, and RIGHT_ELBOW.

We acknowledge that this approach is not ideal due to the motion retargeting problem, where some movements in the motion capture data space may involve rotations from multiple rotation axes which has no correspondence with the DOFs available on the robot. The ideal condition would be where the DOFs of the model in the motion capture data is identical to the DOFs on the robot. However, our goal is not so that the robot's motions mimic exactly the motions of the motion capture data. Instead, we are interested to make the robot's motions have similar dynamics of motion as the motion capture data. Therefore, we believe that this approach is adequate for now.

\subsubsection{Joint Angle Units}

Now that we have determined a subset of DOFs to use, we can focus our attention to only the range of motion of the DOFs that matter. We observed that the range of motion of the arms and neck from several samples of the motion capture data, rarely have large deviations, e.g. +/- 100 degrees or more. In other words, the movements of the arms in the motion capture data rarely, if ever, goes beyond the range of motion of the arms on the robot which is $+/-150$ degrees. Since the values for rotation in the .bvh files is in degrees, we can convert the values to radians. Similarly, the values for the joint angles on the artificial motion data can also be converted to radians, so the values between the two datasets are compatible. In the case of the artificial motion data, values $0-1024$ are converted to $-2.618-+2.618$ radians where the 
positive values indicate counter-clockwise rotation up to 150 degrees (2.618 radians), and negative values indicate clockwise rotation.

For the final dataset, we converted the absolute joint angle values into angular velocities by taking the difference between the current and the previous joint angles: $\Delta \theta_{t}^{i}=\theta_{t}^{i}-\theta_{t-1}^{i}$ for DOF i at timestep $t$. Since we are interested in the reproduction of motion dynamics instead of mimicking the motions exactly, we believe information contained in velocity data is more useful for capturing motion dynamics than the absolute joint angle data.

\subsubsection{Frame Rate}

The rate of recording of the motion capture data has implications on how we interpolate the robot's motion data. Normally, motions created using the RME tool consist of very few poses. The tool itself only allows a sequence of up to 7 poses per one motion

file or 'page' in RME terms. A gesture is typically created as a single page, therefore RME only allows creation of one simple gesture per page. In comparison, one clip of motion capture data can consist anywhere between 100 frames to several thousand frames. We must first augment the artificial motion data to make it comparable to the motion capture data. First, we used REBeL to create longer sequences and more variety of motions. These motions are more complex than a single page because they can consist of concatenation of multiple gestures of different lengths. Moreover, because of the probabilistic operations in REBeL, there are different permutations in the order of concatenations of the gestures. Second, we interpolated the sequence of poses in the motions generated by REBeL. Using the Time and PauseTime information in the RME pose data, we perform linear interpolation which produces additional pose data such that the number of data points is approximately equivalent to the frame rate of the motion capture data. The CMU dataset that we use was recorded at 120 
frames per second (fps). For example, if the Time parameter indicates 2 seconds and PauseTime is 1 second, the linear interpolation will produce 240 pose data between the previous pose and the current pose, and an additional 120 pose data of the current pose to indicate the 1-second pause.

For the combined dataset, we assume the rate of $30 \mathrm{fps}$ of motion capture data by taking one of every four frames in each motion capture clip. This is done for two reasons: 1) to reduce the dimensions of each training sample thus saving the amount of computation during training of the classifier, 2) to ensure that when training a recurrent neural network (RNN) model, the sequence is not too long as RNN is difficult to train with extremely long sequences. Moreover, in motions that are recorded at $120 \mathrm{fps}$ there is not enough useful information between consecutive poses because the differences would be very small. Also, in the linearly interpolated artificial motion data, there would be too many samples with constant values due to pauses and idle DOFs when interpolated for 120 fps.

\subsubsection{Dataset Size}

The number of motion clips in our dataset is relatively small - about 2500 clips for motion capture data, and about 900 clips for the artificial motion data. Meanwhile, each motion clip from the motion capture data and the artificial motion data can have different number of timesteps (length). In order to use such data to train a classifier, each record in the dataset must have the same length. To address these issues, we augment our dataset by taking slices of the motion clip with a length of 100 timesteps (about 3 seconds at $30 \mathrm{fps}$ ). This length was chosen by us because we observed that

it is long enough to capture variety of motions per sample. Additionally, we use a step size of 15 (about 0.5 seconds) when taking the next slice from the same motion clip. We repeat this slicing until reaching the end of the motion clip. Note that each 
motion clip may contain a sequence of several different gestures. After slicing and clean-up, we collected about 100,000 motion capture clips and 50,000 robot motion clips.

For each training run, an equal number of motion clips are randomly selected from the sliced motion capture clips and the robot motion slices so the dataset consists of equal distribution of the two classes of motion data. The collected clips are then separated into training, validation, and test sets, where each set consists of equal number of clips from both classes. The training set is used to train the classifier model. The validation set is used to check whether the classifier generalizes well and avoid overfitting. The test set is to test the performance of the classifier with data it has not seen during training. Furthermore, the training, validation, and test may be repeated many times by selecting different subsets of the dataset to provide statistical data on the performance of the chosen algorithm.

\subsubsection{Dataset Cleanup}

Due to the slicing of motion clips, there are some samples in the dataset that only consist of constant values (i.e. no movement) like the one showed in Figure 7.5. These kinds of data often appear in the interpolated robot motion data due to pauses but occasionally appear at the end or beginning of a motion capture clip. Preliminary tests on recurrent neural network model show that these kinds of data cause the network to focus on such features to make its decision too often. Training a generative model where these kinds of data occur often in the training dataset yields a model that often generates constant-valued data like in Figure 7.5, which is undesirable. Therefore, we remove such data points from our dataset.

To remove the constant-valued samples from the dataset, we performed a scan through the dataset. For each sample, we analyze the variations of the motion signal 


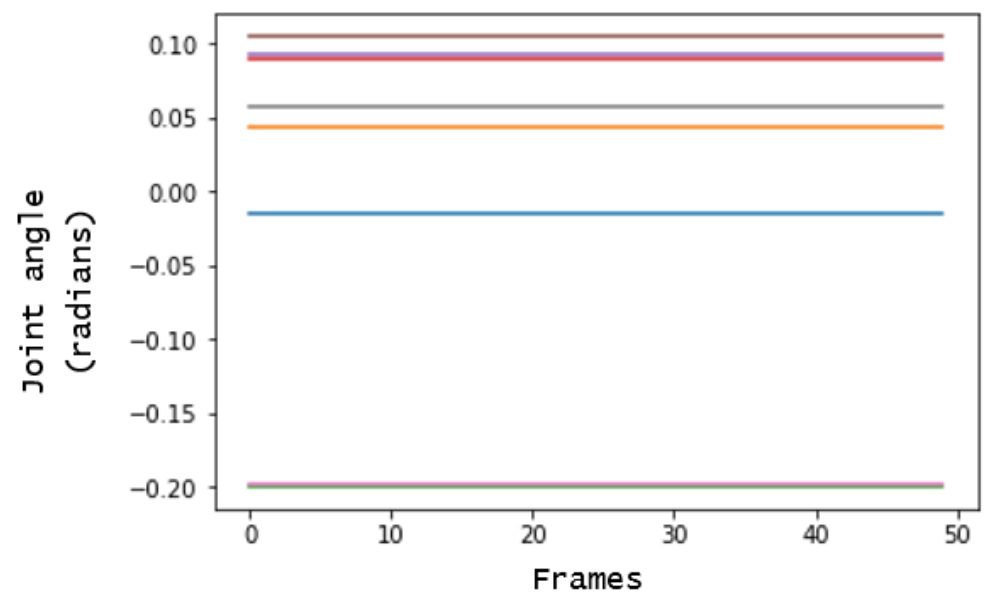

Figure 7.5: A sample with only constant values in all timesteps indicates a static pose. X-axis: time/frames, y-axis: joint angle in radians.

for each degree of freedom for the length of the clip. We used the $\operatorname{std}()$ method from the Numerical Python (NumPy) package to obtain the standard deviation of the motion signal of each degree of freedom $j$. Let's denote the standard deviation for each DOF in a sample as $\sigma_{i, j}$, where $i$ is the index of the sample, and $j$ is the index of the DOF. The mean of the standard deviation of all the DOFs in sample $i$ is denoted as $\bar{\sigma}_{i}$, and let $J$ be the number of DOFs in the sample $i$ such that:

$$
\bar{\sigma}_{i}=\frac{1}{J} \sum_{j} \sigma_{i, j}
$$

We then keep samples which $\bar{\sigma}_{i}$ exceeds a certain threshold $T_{\sigma}$. A sample with low $\bar{\sigma}_{i}$ indicates that the motion signals in the sample do not vary much, indicating the samples may contain DOFs with constant values, or static poses like in Figure 7.5 .

To choose $T_{\sigma}$, we trained several generative models using Variational Autoencoder (VAE) and Generative Adversarial Network (GAN) using multiple versions of the dataset that have been "cleaned-up" with different $T_{\sigma}$ values. We explain how we 
use these generative models in more details in Chapter 8. We then use the trained models to generate several batches of motion data. We analyzed each batch of the generated data and chose $T_{\sigma}$ values that yield motion generator models which can produce 'interesting' data - that is, the generated batch that has a low number of constant-valued motion signals.

A $T_{\sigma}$ value that is too small would keep many samples in the training dataset that have only constant-valued motion signals. A larger threshold value will more aggressively filter the training dataset by only selecting samples that have a large amount of movements and reduces the number of samples, ultimately reducing the size of the dataset. We also do not want to entirely exclude actions and gestures with subtle movements. We want to have enough variety in the training dataset and avoid homogeneous samples. In other words, the dataset should have a distribution that is interesting enough from which both large and small movements can be drawn.

From this exercise we determined that $T_{\sigma}$ values between 0.2 or 0.3 to give good results; there are varieties of motion samples in the training dataset and in the generated samples from the trained models. When $T_{\sigma}<0.2$, the generative models produce constant-valued samples about $30 \%$ of the time, while $T_{\sigma}>0.3$ yields generative models that produce noisy samples which noise is of high frequency and magnitude. We also noticed that with $T_{\sigma}=0.2$, what remains of the dataset is roughly $50 \%$ the size of the original dataset due to the removal of the constant-valued samples. Since our collection of motion capture data is limited, we do not want a $T_{\sigma}$ value that will aggressively reduce the size of the training dataset. 


\subsection{The Discriminator/Classifier Models}

We used the Orange Data Mining tool (www.orange.org) to train several classifier models using Naïve Bayes, Decision Tree, and Support Vector Machine (SVM) using the built-in modules provided in Orange. In addition to providing many different machine learning models, the Orange Data Mining tool also provides data processing tools, data visualization tools, and evaluation tools. The drag-and-drop interface of Orange makes experimenting with multiple machine learning models easy. Therefore, we can experiment with multiple models at the same time to find a discriminator model that performs well on our motion dataset.

The decision tree model could provide an indication which set of features contributes most to the motion capture quality. Because motion data is inherently time series data, models that could process temporal information such as recurrent neural network can be used. SVM is used as it is known to be an optimal binary classification algorithm. For the LSTM recurrent network model, we built our own model using Keras which is a high-level wrapper for Tensorflow and PyTorch (www.keras.io) for the Python language because the LSTM model does not currently exist in the Orange tool yet.

Each motion data point in the dataset for the non-recurrent network models of the classifier is the flattened motion data for 8 DOFs and 100 timesteps, resulting in a vector of 800 values. Only for the recurrent network model the size of the input vector is 8 for the number of DOFs and a sequence size of 100 . For all models of the classifier there is only one output with values ' 0 ' or ' 1 '. In this case, ' 0 ' corresponds to the robot motion class and ' 1 ' for the motion capture class. For the neural network models (one recurrent and one feedforward), the softmax layer with two values can be used as the output layer where $[1,0]$ corresponds to the value ' 0 ' and indicate 
the artificial motion class, and $[0,1]$ corresponds to the value ' 1 ' which indicate the motion capture class. However, we found no significant difference in the resulting accuracy for both the recurrent and feedforward networks when using the softmax output layer or a single value output.

The total size of our motion dataset is about 80,000 samples with training:test split 80:20, which equates to roughly 64,000 training samples and 16,000 test samples. The models reported here were trained using 10-fold cross-validation. The performance reported for each model are in terms of their accuracy on the test set.

\subsection{Naïve Bayes Classifier}

The Naïve Bayes algorithm is a probabilistic model that uses Bayes rule to make predictions [199]. Naïve Bayes is called "naïve" because the algorithm strongly assumes that the features that are used to make the predictions are independent of each other. When the features are not independent, Naïve Bayes may perform poorly. In the simplest form, the Bayes rule is as follows:

$$
P(H \mid D)=\frac{P(D \mid H) P(H)}{P(D)}
$$

$H$ is called the hypothesis or the thing/event in question and $D$ is data or evidence or input. $P(H \mid D)$ is called the conditional probability which is read as "the probability of the hypothesis is true given the evidence." Equivalently, the probability $H$ occurs if $D$ occurs. $P(D \mid H)$ is called the likelihood and can be interpreted as "the likelihood of seeing the evidence $D$ when $H$ occurs". $P(H)$ is called the prior indicating our "prior knowledge or guess" of the probability of $H . P(D)$ is called the marginal probability of the evidence $D$.

For multi-featured data like motion capture data, the Bayes rule can be written 
as follows:

$$
P\left(Y=y_{k} \mid x_{1}, \cdots, x_{n}\right)=\frac{P(Y) \prod_{i=1}^{n} P\left(x_{i} \mid Y=y_{k}\right)}{\prod_{n=1}^{n} P\left(x_{i}\right)}
$$

The class $\hat{Y}$ for a new data $\mathbf{X}^{\prime}$ (in Figure 7.2) can be computed as:

$$
\hat{Y} \leftarrow \underset{y_{k}}{\operatorname{argmax}} P\left(Y=y_{k}\right) \prod_{i} P\left(x_{i}^{\text {new }} \mid Y=y_{k}\right) \quad x_{i}^{\text {new }} \in \mathbf{X}^{\prime}
$$

In this motion classification case, $Y$ is the class of the motion sample, $y_{k}$ is one of the classes, and the set of evidence $x_{i}$ can be considered as features which are joint angle values. Since the $x_{1}, \cdots, x_{n}$ have continuous values, the likelihood is computed by assuming each feature $x_{i}$ follows a normal (Gaussian) distribution:

$$
P\left(x_{i} \mid Y=y_{k}\right)=\frac{1}{\sqrt{2 \pi \sigma_{i k}^{2}}} e^{\frac{-\left(x_{i}-\mu_{i k}\right)^{2}}{2 \sigma_{i k}^{2}}}=\mathcal{N}\left(x_{i} ; \mu_{i k}, \sigma_{i k}\right)
$$

Where $x_{i}$ is some value of feature $i, \mu_{i k}$ is the mean and $\sigma_{i k}^{2}$ is the variance for the feature $x_{i}$ for $\mathrm{Y}$ with class $y_{k}$. In this kind of calculation (continuous), the marginal is often ignored because it is a normalization constant. The prediction $Y^{\text {new }}$ for a new input can then be calculated using maximum likelihood, that is the prediction is the value of $y_{k}$ that has the highest value for $P(Y) \prod_{i} \mathcal{N}\left(x_{i}^{n e w} ; \mu_{i k}, \sigma_{i k}\right)$ :

$$
\hat{Y} \leftarrow \underset{y_{k}}{\operatorname{argmax}} P\left(Y=y_{k}\right) \prod_{i} \mathcal{N}\left(x_{i} ; \mu_{i k}, \sigma_{i k}\right)
$$

Where $x_{i}^{\text {new }}$ is the value for feature $i$ of $\mathbf{X}^{\text {new }}, y_{k}$ is the one of the possible outputs, $\mu_{i k}$ and $\sigma_{i k}$ are the mean and standard deviation of feature $i$ for output $y_{k}$ from the training data. $P\left(Y=y_{k}\right)$ is a scaling factor. In practice, the value $Y^{\text {new }}$ is sometimes obtained by class with the highest posterior value (maximum likelihood), but in other cases $Y^{\text {new }}$ is sampled from the posterior distribution, making the result 


\begin{tabular}{rr|r|r|r} 
& \multicolumn{3}{|c|}{ Predicted } \\
& & $<0.5$ & $\geq 0.5$ & $\Sigma$ \\
\hline Actual & 0.5 & 7894 & 0 & 7894 \\
$\geq 0.5$ & 268 & 7623 & 7891 \\
$\Sigma$ & 8162 & 7623 & 15785
\end{tabular}

Figure 7.6: Confusion matrix for Naïve Bayes. The class 0 is represented by label $"<0.5 "$ and class 1 is labelled " $\geq 0.5 "$.

truly probabilistic.

\subsubsection{Naïve Bayes Classification Results}

A Naïve Bayes model was trained using the model provided by the Orange data mining tool. The trained model achieved $98.30 \%$ accuracy. Figure 7.6 shows the confusion matrix of the trained Naïve Bayes model on the test set. The model was able to predict all class 0 (robot motion data) correctly and misclassified about $3 \%$ of the motion capture data.

\subsection{Binary Decision Tree}

A tree is a kind of graph called directed acyclic graph (DAG). A graph, and hence a tree is constructed by a set of nodes and edges, where edges connect two nodes. A DAG is a special kind of graph which "directed" means each edge connecting pair of nodes can only go in one direction, and "acyclic" means the graph does not have any node where there exist a path through the graph to return to the same node. A tree is commonly drawn from the top down, where the first node on the top is called the root node, and nodes that do not have further branches are called leaf nodes. The other nodes inside the tree that are not root nor leaf nodes can be referred to as interior nodes. The subsequent nodes of a root node or interior node are called 
child nodes. In a binary tree, each node can only have two child nodes, but there are trees can have more than two child nodes. Because the model used in this work is the binary decision tree, the discussion that follows pertains to binary decision trees.

A binary decision tree models data by systematically choosing a set of deciding features at each level of the tree. Inference for a new data can be done by traversing the tree from the top called the root node. Each node can only have two child nodes. At each node, a feature in the new data is evaluated; if the feature value meets a condition imposed by the node, the inference then proceeds to one of the children of the node. This process is repeated until an output is obtained at a leaf node - a node which does not have child nodes. The advantage for using a decision tree is that the model is easy to understand, and the tree reveals which features in the data are important in making the decisions.

In general, each interior node of a decision tree represents a decision point for an input variable and the output class (or target variable) are on the leaf nodes. Each interior node performs a check on an input variable, and depending on the value of the input variable, the tree traverses to one of the child nodes of that node. For discrete variables, each node asks a 'Yes' or 'No' question such as "is fruit $==$ orange" where 'fruit' is an input variable and 'orange' is one of the possible values of the input variable. For continuous variables, each node checks whether the value of the input variable is below or above a certain threshold, e.g. $x>=0.5$.

Decision tree learning is the algorithms to construct a tree from the training data and determine which feature to use to make a decision on each node. To determine which feature to use, decision tree learning compute a measure called Information Gain. Information Gain uses entropy to choose features that can best split the data into two parts. Entropy is a measure of uncertainty of a discrete random variable and 
computed as:

$$
H(T)=\operatorname{Entropy}(S)=-\sum_{i=1}^{J} p_{i} \log _{2} p_{i}
$$

Where $\mathrm{S}$ is the set of training examples, and $p_{i}$ is the proportion of number of examples of class $i$ in the set $S$. For binary classification where there are only two classes on the output, the entropy is:

$$
H(T)=\operatorname{Entropy}(S)=-\left[p_{+} \log _{2} p+p_{-} \log _{2} p_{-}\right]
$$

Information gain is calculated as:

$$
I G(T, a)=H(T)-H(T \mid a)=-\sum_{i=1}^{J} p_{i} \log _{2} p_{i}-\sum_{a} p(a) \sum_{i=1}^{J}-\operatorname{Pr}(i \mid a) \log _{2} \operatorname{Pr}(i \mid a)
$$

Where $I G(T, a)$ is the information gain for the set of training examples $T$ given the value of attribute $a, H(T)$ is the entropy and $H(T \mid a)$ is the conditional entropy or the weighted sum of entropy of the children.

\subsubsection{Decision Tree Classification Results}

Figure 7.7 shows the resulting binary decision tree produced by the Orange tool. The training data set contains equal number of robot motion class and motion capture class, so in the root node it shows $50 \%$. Feature number 76 was chosen as the first feature that splits the data best with threshold value -0.00539481 . The first level of the tree shows that splitting the dataset on feature number 76 with the threshold was able to split the data into two subsets. The first subset on the left $\left(X_{787} \leq-1.02871\right)$

contains 5830 data points with 5780 of those data points belonging to class 0 (99.1\%), 


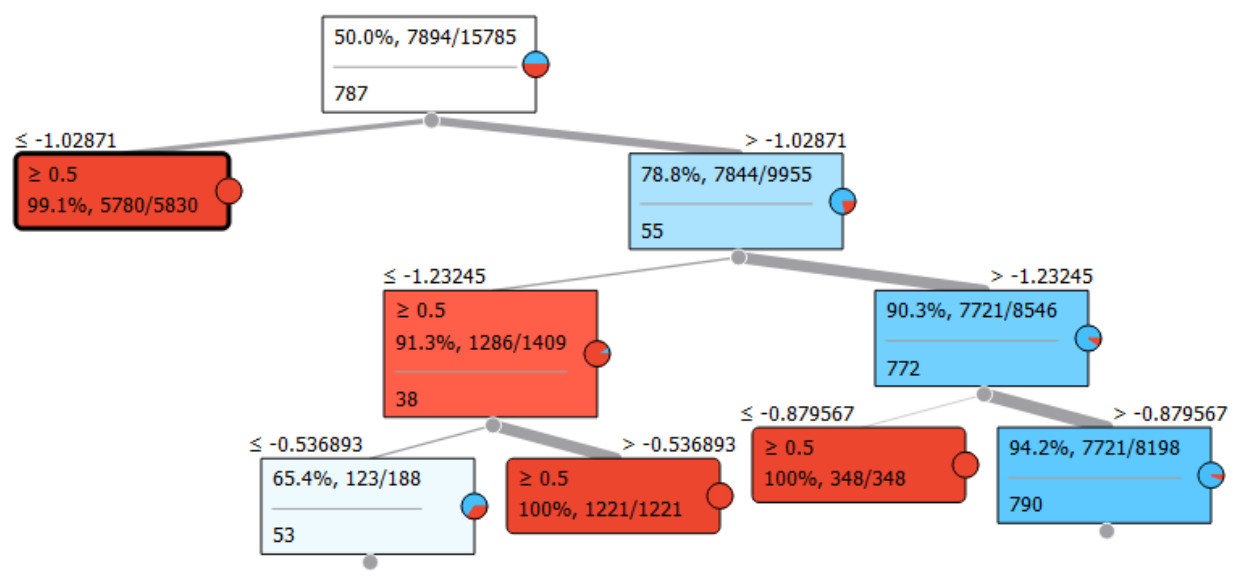

Figure 7.7: Trained decision tree model (shown only 4 out of 10 levels)

and the second subset $\left(X_{787}>-1.02871\right)$ containing 9955 data points with 7844 of them have the class 1 (78.8\%). The left subtree is not split any further because it explains the majority of class 0 in that subset $(>95 \%)$. This threshold is a parameter for the Tree model in the Orange tool. The second level on the right subtree uses feature number 55 with threshold value -1.23245 . The subset is split into two more subsets, where the left subset contains $91.3 \%$ of class 0 or 1286 out of 1409 data points and the right subset contains $90.3 \%$ of class 1 or 7721 out of 8546 data points. And so forth.

Figure 7.8 shows the confusion matrix for the decision tree. 154 motion capture samples were misclassified as robot motion, while 136 robot motion samples were misclassified as motion capture. The model achieved $98.16 \%$ accuracy.

\subsubsection{Support Vector Machine}

Support Vector Machine (SVM) is a supervised learning algorithm for two-class classification problem by finding a hyperplane that creates the largest margin between the two classes [110]. Linear regression method fits a hyperplane that separates two 


\begin{tabular}{rr|r|r|r} 
& \multicolumn{4}{c}{ Predicted } \\
\hline & $<0.5$ & $\geq 0.5$ & $\Sigma$ \\
\hline Actual & $<0.5$ & 7758 & 136 & 7894 \\
$\geq 0.5$ & 154 & 7737 & 7891 \\
$\Sigma$ & 7912 & 7873 & 15785
\end{tabular}

Figure 7.8: Confusion matrix for a trained decision tree model. The class 0 is represented by label "<0.5" and class 1 is labelled " $\geq 0.5 "$.

classes, but SVM finds the optimal hyperplane called the "maximum-margin hyperplane". the hyperplane with which the data points of either class that are nearest to the hyperplane are separated with the largest margin. These data points are called support vectors. While SVM can be a powerful classifier, its learning can be slow because the algorithm must first find the support vectors out of the large training samples. A hyperplane can be defined as:

$$
\mathbf{w} \cdot \mathbf{x}+b=0
$$

Where $\mathbf{w}$ is the vector normal (perpendicular) to the hyperplane $\mathbf{x}$ is a set of points, and $b$ is the offset of the hyperplane from the origin.

Given a set of labeled dataset $\left(\mathbf{x}_{1}, y_{1}\right), \cdots,\left(\mathbf{x}_{n}, y_{n}\right)$ and $y_{n} \in-1,1$, is linearly separable if the following inequalities are satisfied:

$$
\mathbf{w} \cdot \mathbf{x}+b\left\{\begin{array}{l}
\geq 1 \text { if } y_{i}=1 \\
\leq-1 \text { if } y_{i}=-1
\end{array}\right.
$$

And can be written as:

$$
y_{i}(\mathbf{w} \cdot \mathbf{x}+b) \geq 1
$$


For all $1 \leq i \leq n$.

The name 'support vectors' are the vectors $\mathbf{x}_{i}$ such that $y_{i}\left(\mathbf{w} \cdot \mathbf{x}_{i}+b\right)=1$. These vectors lie at the boundaries between the group of $y_{i}=-1$ and $y_{i}=1$ in feature space. These vectors are used as guides to optimally place the linear separating hyperplane. There is a unique optimal hyperplane:

$$
\mathbf{w}_{0} \cdot \mathbf{x}+b_{0}=0
$$

Where $\mathbf{w}$ and $b$ defines the slope and position of the hyperplane. The distance of every data point $x_{i}$ to this hyperplane is:

$$
\rho(\mathbf{w}, b)=\min _{\{x: y=1\}} \frac{\mathbf{x} \cdot \mathbf{w}}{|\mathbf{w}|}-\rho(\mathbf{w}, b)=\max _{\{x: y=-1\}} \frac{\mathbf{x} \cdot \mathbf{w}}{|\mathbf{w}|}
$$

The optimal hyperplane parameters $\left(\mathbf{w}_{0}, b_{0}\right)$ (eq. 7.13) are the parameters that maximizes the distance in eq. 7.14 :

$$
\rho\left(\mathbf{w}_{0}, b_{0}\right)=\frac{2}{\left|\mathbf{w}_{0}\right|}
$$

The SVM algorithm is formulated as finding the minimial $\mathbf{w}$ to maximize eq. 7.15. An important feature of SVM when it was introduced was the concept of kernel functions $\phi$ which maps the $n$-dimensional input vector into higher $N$-dimensional feature vector space which allows creating a linear separating hyperplane w. An example of such $\phi$ is the Gaussian kernel also known as radial basis function.

$$
\phi: \mathbb{R}^{n} \rightarrow \mathbb{R}^{N}
$$

An unknown input $x$ is then classified by applying the function $\phi$ and taking the 


\begin{tabular}{rr|r|r|r} 
& \multicolumn{3}{|c|}{ Predicted } \\
& & $<0.5$ & $\geq 0.5$ & $\Sigma$ \\
\hline Actual & $<0.5$ & 7855 & 39 & 7894 \\
$\geq 0.5$ & 139 & 7752 & 7891 \\
$\Sigma$ & 7994 & 7791 & 15785
\end{tabular}

Figure 7.9: Confusion matrix for a trained SVM classifier. The class 0 is represented by label "<0.5" and class 1 is labelled " $\geq 0.5 "$.

sign of the function:

$$
f(\mathbf{x})=\mathbf{w} \cdot \phi(\mathbf{x})+b
$$

\subsubsection{SVM Classification Results}

We used the SVM module from the Orange Data Mining Tool with the RBF kernel to train a SVM classifier for our motion dataset. Figure 7.9 shows the confusion matrix from the trained SVM model. The trained model reported $98.87 \%$ accuracy with 139 motion capture samples misclassified as robot motion and 39 robot motion samples misclassified as motion capture motion.

\subsection{Long Short-Term Memory Network}

The Long Short-Term Memory (LSTM) network is a recurrent neural network where each recurrent node consists of a 'memory cell' and three 'gates' that control how the contents of the memory cell get updated [200]. LSTM is a significant improvement over the standard recurrent neural network (RNN) model which was difficult to train because RNN is susceptible to the vanishing and exploding gradient problem [201]. Vanishing and exploding gradient problem occurs in deep networks when performing the backpropagation computation. As gradients are multiplied over each 
layer during backpropagation, the gradients can quickly reduce to very small values when close to zero (vanishing gradient) or grow to very large values when bigger than 1 (exploding gradient). Vanishing and exploding gradient problem have been addressed using normalized weight initializations [202] and batch normalization layers [203]. Other models achieved continuous learning improvements with very deep feedforward networks by using 'skip connections' where some inputs are connected directly to deeper layers by 'skipping' a few layers [204]. The skip connection is identity, which means information is propagated into the deeper layers undisturbed, and helped with the learning in deeper layers. By having the memory cell, LSTM can retain information about long-term dependencies in sequence data and overcome the vanishing/exploding gradient problem.

Figure 7.11 shows a model of an LSTM node. The three gates in LSTM are: input gate, forget gate, and output gate. Each of the three gates is made of a feedforward network with output vector of values zeros and ones. A parameter of a LSTM node is the number of hidden units. The number of hidden units in each gate in the LSTM node is determined by this parameter. The input gate is trained to create a filter that selectively accepts new input values and the values of the previous state of the cell. The forget gate is trained to selectively choose which values of the memory cell to 'forget', and the output gate is trained to selectively choose which values from the memory cell to output.

The LSTM node used in this work as shown in Figure 7.11 is based on the implementation in (Graves, 2008) and the functions are defined as follows:

$$
i_{t}=\sigma\left(W_{x i} x_{t}+W_{h i} h_{t-1}+W_{c i} c_{t-1}+b_{i}\right)
$$




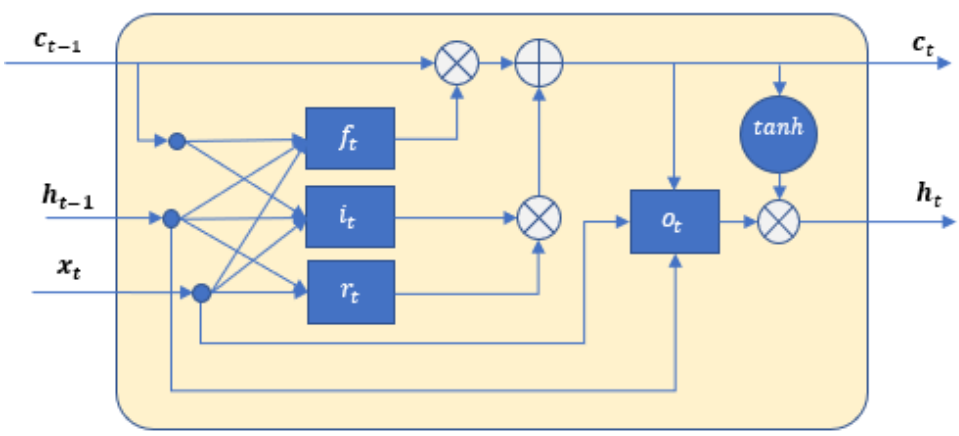

Figure 7.10: An LSTM node. $\mathbf{c}_{t-1}$ is the contents of memory cell from the previous timestep. $\mathbf{h}_{t-1}$ is the output of the node at the previous timestep. $\mathbf{x}_{t}$ is the current input to the node. $f_{t}, o_{t}, i_{t}$ are forget gate, output gate, and input gate, respectively. $r_{t}$ is an intermediate network for regularizing the new values for the memory cell. tanh indicates the tanh activation function. $c_{t}$ and $h_{t}$ are the state of the memory cell and output of the node at the current timestep. $\otimes$ indicates element-wise multiplication, $\oplus$ indicates element-wise addition.

$$
\begin{gathered}
f_{t}=\sigma\left(W_{x f} x_{t}+W_{h f} h_{t-1}+W_{c f} c_{t-1}+b_{f}\right) \\
r_{t}=\tanh \left(W_{x c} x_{t}+W_{h c} h_{t-1}+b_{c}\right) \\
o_{t}=\sigma\left(W_{x o} x_{t}+W_{h o} h_{t-1}+W_{c o} c_{t}+b_{o}\right) \\
h_{t}=o_{t} \tanh \left(c_{t}\right)
\end{gathered}
$$

Where $\sigma(\cdot)$ is the sigmoid activation functions $W_{*}$ are weight matrices, and $b_{*}$ are bias values. $W_{x i}$ refers to the weight matrix of the input vector $\mathbf{x}_{t}$ to the input gate $\left(i_{t}\right)$. $W_{h i}$ refers to the weight matrix of the hidden state from the previous timestep $h_{t-1}$ to the input gate, and so on. The LSTM network can be trained using backpropagation through time (BPTT). BPTT calculates the backpropagation of errors of a recurrent 

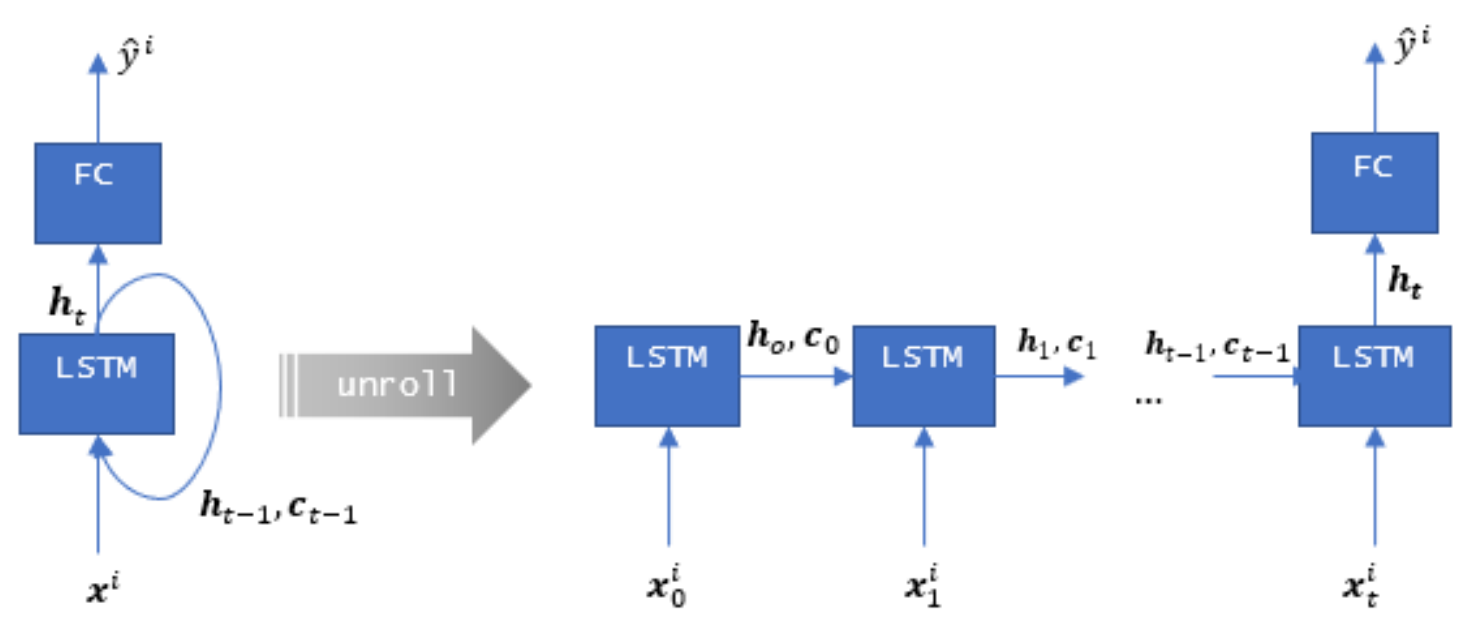

Figure 7.11: The basic LSTM motion classifier network. The left image is the compact representation of the recurrent network, and the loop at the LSTM layer indicates recurrent process. $\mathrm{FC}=$ fully connected output layer. The image on the right is the 'unrolled' depiction of the same classifier network. $\mathbf{h}_{t}$ and $\mathbf{c}_{t}$ indicates the output and cell state at time $t$ of the LSTM layer, respectively. The index $i$ indicates the index of samples, e.g. the labeled sample $\left(x_{i}, y_{i}\right)$. Notice that only the last output of the sequence of the LSTM layer $\mathbf{h}_{t}$ is used by FC to make the class prediction $\hat{y}^{i}$.

network by 'unrolling' the network and lays out the sequential process in space as shown in Figure 7.11. The error propagations are calculated backwards from the end of the sequence to the beginning of the sequence while keeping the weights for the recurrent layer fixed for each timestep. The error is propagated between timesteps $t$ and $t-1$ via the cell state $c_{t}$ and current output $h_{t}$. The final $\Delta w$ to update the weights of the recurrent layer is obtained after the backpropagation is done for the whole sequence.

\subsubsection{The Model}

We built a two-layer classifier network using LSTM nodes in the first hidden layer and a fully connected network (FC) at the output layer which outputs one value as shown in Figure 7.11. The input to the network is the dataset of motion data which consists of samples from motion capture data and robot motion data as described in 
the previous sections. Each sample is given a label; 0 for robot motion sample and 1 for motion capture sample. Recall that each sample is a two-dimensional data where the columns are the degrees of freedom, so each row is a pose, and therefore the data is a sequence of poses. In our dataset, the length of the sequence for each sample is 100. The LSTM layer processes the data one row at a time for the length of the sequence. Although when processing each row, the LSTM layer produces an output, in this classifier model, only the output at the last timestep is used as input to the fully connected (FC) output layer. This way, the LSTM layer acts as an encoder and only the output at the last timestep contains encoded information about the whole sequence in some new feature space. The output layer then performs linear regression over this feature space with sigmoid activation.

In our experiments, the LSTM motion classifier network was set with the following parameters:

- Input size: 100 timesteps x 8 DOF flattened to 800-valued vectors

- LSTM hidden nodes: 512

- Total number of trainable weights: 1 million parameters

- Optimizer: Adam (learning rate: 0.001, $\beta_{1}=0.9, \beta_{2}=0.999$ )

- Loss function: binary cross-entropy

- Training set size: 2560

- Validation set size: 640

- Test set size: 800

- Ratio of samples for each type: 1:1 

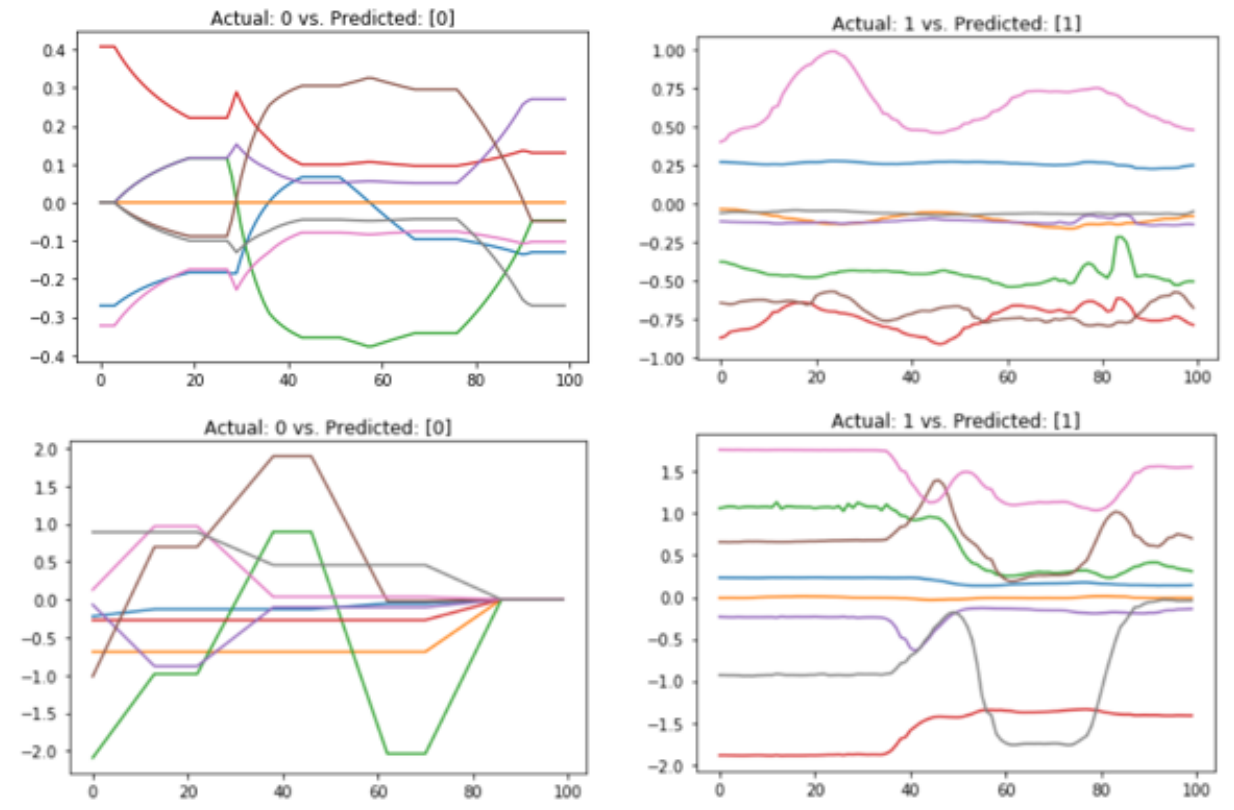

Figure 7.12: Four randomly-selected samples from the test set and their classification results from the trained LSTM motion classifier. Class: $0=$ robot motion, $1=$ motion capture.

- Epoch: 100

The above parameters were selected arbitrarily as a starting point.

\subsubsection{Classification Results}

Our LSTM classifier model was able to achieve $99.6 \%$ accuracy on the test set. Figure 7.12 shows the classification results of four randomly selected samples from the test set predictions. For each figure, the x-axis indicates timesteps/frames, and y-axis indicates the joint angle values.

The classifier made two false positive predictions and one false negative prediction. Figure 7.13 shows the samples where the classifier failed to make the correct predictions. The two figures at the top of Figure 7.13 shows that the classifier misclassified the robot motion data (class 0) as belonging to the motion capture data 

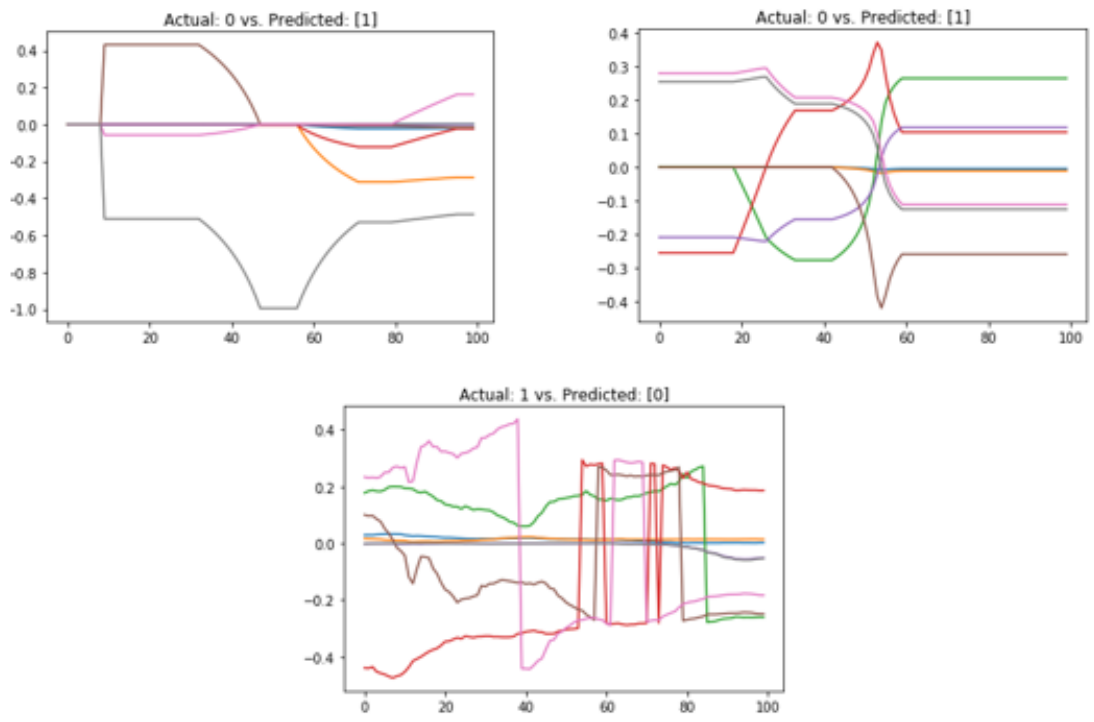

Figure 7.13: False positives predictions (top), and false negative prediction (bottom). Class: $0=$ robot motion, $1=$ motion capture.

(class 1). The bottom figure in Figure 7.13 shows a motion capture sample that was misclassified as robot motion data.

\subsection{Contributions}

My contributions in relation to the work presented in this chapter are as follows:

- Developed a new dataset of motion data consisting of human motion capture data, and robot motion data of various upper body gestures and actions. This is a new dataset that we have not found in other works. Future works can expand this dataset to add more samples.

- Demonstrated that classical machine learning models such as Naïve Bayes, Decision Tree, and SVM and also modern model of LSTM network were able to achieve high accuracy ( $\geq 98 \%$ on average) in discriminating human motion data and robot motion data. By using the assumption that human motion is the "golden standard" of natural-looking motions, and expressive robot motion 
should have similar qualities as human motions, these results indicate that machine learning models can be used as a method to evaluate the quality of robot motions generated by some method S (either manual or procedurally) as an alternative to subjective methods such as surveys, interviews, and questionnaires. 


\section{CHAPTER 8 GENERATIVE MODELS}

In the previous chapters, we focused on approaches to produce expressivity on humanoid robot motions using methods that are slightly better than manual editing of poses. In the future, fully autonomous humanoid social and entertainment robots may need the ability to produce novel gesticulations outside of their knowledge base in unexpected circumstances. Therefore these humanoid robots may need some kind of intelligent motion generators that can produce meaningful, yet natural-looking motions and gestures. Thus, the goal of work presented in this chapter is to design a good motion generator for humanoid robots that can produce novel gestures that are interesting, meaningful, while having natural-looking motions.

In this chapter, we explore different generative models trained on our motion dataset to produce new motion data. In particular, we built three generative models: a Long Short-Term Memory (LSTM) network model, a Variational Auto Encoder (VAE) model, and a Generative Adversarial Network (GAN) model that was based the Deep Convolutional GAN (DCGAN) model. The motivation behind the generator approach is motivated by the work of Graves, who trained a recurrent network model to produce renderings of text that are comparable to the handwritings of a person [205]. In his work, Graves used the IAM on-line handwritten dataset [103] which consists of a set of handwriting data of multiple people that was recorded on a digital whiteboard. The handwriting data are represented as sequences of three values: $\mathrm{x}$ and y positions of the pen, and a value to indicate when the pen is lifted or touching the whiteboard. Given that motion data also consist of a sequence of coordinate values, 
thus are similar to the handwriting dataset, we ask the question: can a similar method be applied to motion capture data? In other words, can a neural network model be trained using a human motion capture dataset so that it can learn to generate new motion data that possess the same qualities as the motion capture data? Given that the generative model can produce new motion data with comparable qualities as the motion capture data, what features of motions did the model learn, and can the model be used to generate motion data for a humanoid robot, such that the motions of the robot will exhibit qualities of motion capture data and appear more human-like?

There are similarities between a person's handwriting and a person's movements and gesticulations. To the human eye, it is easy to recognize when a text written in cursive was printed or handwritten by a person. In the former, the shapes of each letter are consistent at every occurrence throughout the text. In the latter, each letter may not look exactly the same at every occurrence in the text, but a person can recognize that there is a consistency of writing style that belongs to the same writer. We believe that the same phenomenon also applies to a person's gesticulations: a person can recognize another person by the way he/she gesticulates even without seeing their faces. We want to apply this idea of gesticulation styles to humanoid robots since a robot always performs a gesture exactly the same way every time, but a person never performs the same gesture in exactly the same way every time, even under the same conditions.

Therefore, we hypothesize that if variations of handwriting styles can be learned and reproduced by a machine learning model like recurrent neural networks (RNNs) [205] and styles of music can be produced by variational autoencoder [25], similarly styles of motions should also be learnable and reproduceable using a machine learning model. Additionally, Graves created a variant of his generative model with the addition of constraints such that the output of the model is conditioned on an input 
text, which produces renderings of the input text in some handwriting style. Graves showed that the same RNN architecture was able to learn to generate different kinds of samples depending on the type of dataset it was trained on. The model was trained on different kinds of datasets: a corpus of literature, Wikipedia articles, Linux source codes, and handwriting dataset. In terms of motion-generating models, Generative Adversarial Imitation Learning (GAIL) is a model of inverse reinforcement learning (i.e. learn from examples or 'expert') that is trained using generative adversarial networks (GAN) that have shown to produce human-like movements in tasks like locomotion [196] and hand manipulation [206]. Wang, Chai and Xia used a combination of a LSTM-based RNN and GAN to synthesize humanoid walking animation that can be controlled by a user [207]. The RNN component was trained using motion capture data and generates new walking animation. The GAN model was trained as a "refiner network" which function is to remove unwanted artifacts in the generated data such as noise, and foot sliding. Holden, Saito and Komura used an autoencoder model with convolutional layers to create a similar system as Wang, Chai and Xia for synthesis and control of human actions where the model was trained using human motion capture data [208]. Another interesting model is the Sketch-RNN model that is a VAE model with RNNs for its encoder and decoder with mixture density network layer [209] for the output of the decoder, and was trained on the QuickDraw dataset [210]. The QuickDraw dataset consists of sequences of pen strokes of sketches done by people from an online game called Quick, Draw! The game prompts the player to draw a certain object in less than 20 seconds. Thus the results are messy line illustrations of the objects. From this dataset, Sketch-RNN model is able to synthesize new samples that are similar and recognizable, yet does not exist in the dataset. Additionally, Sketch-RNN was able to extrapolate/complete a sketch from an incomplete or partial sketch, interpolate between two sketches using a weighting parameter, and 
perform algebraic operations to add or subtract features from different sketches.

The work in this chapter is focused only on building generative models to produce novel motion data for the HROS-1 humanoid robot such that the robot's motions exhibit human-like motion qualities by training the model on human motion capture dataset. Our work is different from the works mentioned earlier in that we are focused on expressive behaviors - nonverbal actions that aids emotional engagement of people with the robot instead of functional actions like walking, punching, jumping, or object manipulation.

In this chapter we discuss three motion generator models. The first motion generator is using a LSTM network (introduced in Chapter 7), the second is a Variational Auto Encoder (VAE) model [104] and the third model is a Generative Adversarial Network (GAN) model [99] [106]. All models were trained using the motion dataset that we developed, as described in the section 7.4. The input vector for the models is a sequence of motion data points from the DOFs of the motion capture model of joint angle values in radians.

The LSTM model produces motion data that have similar properties as the training data; the produced sequences have smooth contours with very few places experiencing large discontinuities. To be useable, these discontinuities may need to be reduced e.g. using wavelet transform as in Chapter 6. The GAN model was able to produce motion data that have similar characteristics and variety as the training data. However, the data from the GAN model tend to exhibit a little bit of noise, making the data resemble Brownian motions. We observed that even when training the generative models on the linearly-interpolated robot motion dataset, the trained models do not yield linear data like the samples in the training data. Instead, the output produced is similar to the ones produced by the models trained on motion capture data that we characterize as: "wavy with noise". Our current VAE model 
was not able to produce usable motion data; the sequences produced tend to have too many oscillations to make any meaningful gestures. We discuss our methods and results with more details below.

The results will be shown at the end of this section. We used the generated motion data on the HROS-1 and Jeeves humanoid robots to evaluate the quality of the motions on the robots. Finally, section 8.5 summarizes and compares the performances of the two models for the task of generating motion data.

This chapter is organized as follows. Each section begins with a discussion on the machine model used and the theories related to it. Then, we describe the model that we implemented, the experimental results, followed by discussions on the results. All models discussed here were implemented using Python programming language and Keras machine learning library for Python (https://keras.io/ ) with Tensorflow backend (https://www.tensorflow.org). The Keras library provides high-level wrappers written in Python for machine learning components such as neural network layers and related operations, which greatly simplifies the process of building machine learning models.

\subsection{The LSTM Model}

In this section, we explain our generator network built using the LSTM recurrent network model. We have introduced the LSTM layer model in Chapter 7 so the details of the LSTM layer are not repeated here.

\subsubsection{Dataset Preprocessing}

Our preliminary experiments in building generative models with feed forward and recurrent neural networks suggest that training networks to generate sequences of 
continuous values is difficult. The generated sequences tend to have values that oscillate wildly, or a signal with high frequency noise. These sequences do not create natural-looking motions, difficult to interpret their meaning, and in general look out of control. We believe using one-hot encoding would make the network easier to train. However, since the values for the feature vectors are continuous, we need some systematic way to discretize the values.

We encoded our training data using one-hot encoding by discretizing using the Haar wavelet that was introduced in Chapter 6. We first trained two models, one using only the motion capture data and the second using only robot motion data. This is done to evaluate the generation capability of the networks, i.e. will it generate data that are mostly continuous when trained with the motion capture data, and will it generate linear data when trained with the robot motion data?

First, the dataset is scanned to collect the unique feature values. Strictly for motion capture dataset, there are nearly 2.1 million unique values, whereas for the robot motion dataset there are about twenty-six thousand values. Let's call these sets as $\xi_{t}$ where $t \in$ 'mocap',' robot' with 'mocap' referring to motion capture and 'robot' to robot motion datasets. Second, the set of unique values is sorted so the values are monotonically increasing. Next, the sorted values are discretized using wavelet transform using Haar wavelet at $40 \%$ filtering level; this means the data is reconstructed with only 40 percent of the filterbanks obtained by the wavelet transform. This filtering level parameter is chosen so that the number of discrete 'bins' are at a 'sweet spot' where it is not too big for the one-hot encoding of the feature vector for training, but big enough to still retain the resolution for smooth motion trajectories. We found that $40 \%$ is a fair number with the following results:

- motion capture data: 2,097,359 values $\rightarrow 257$ values/bins 

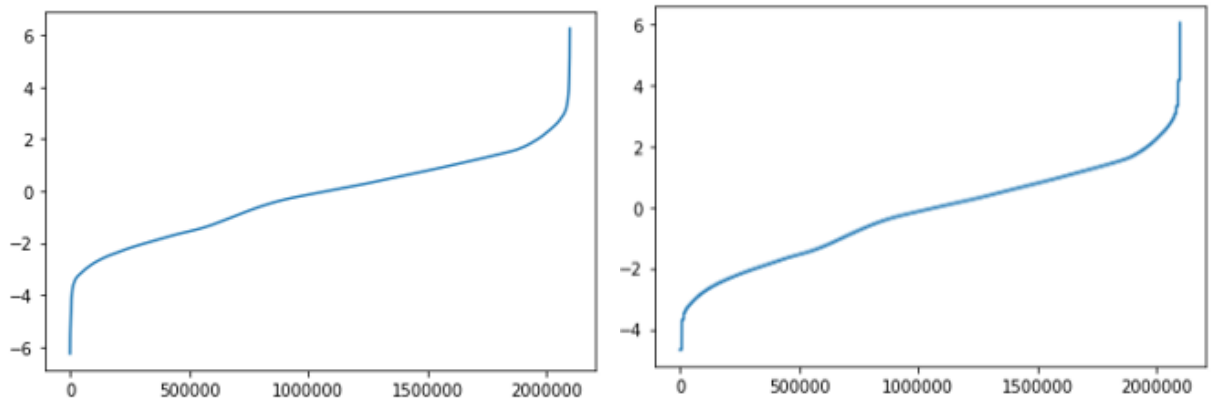

(a)
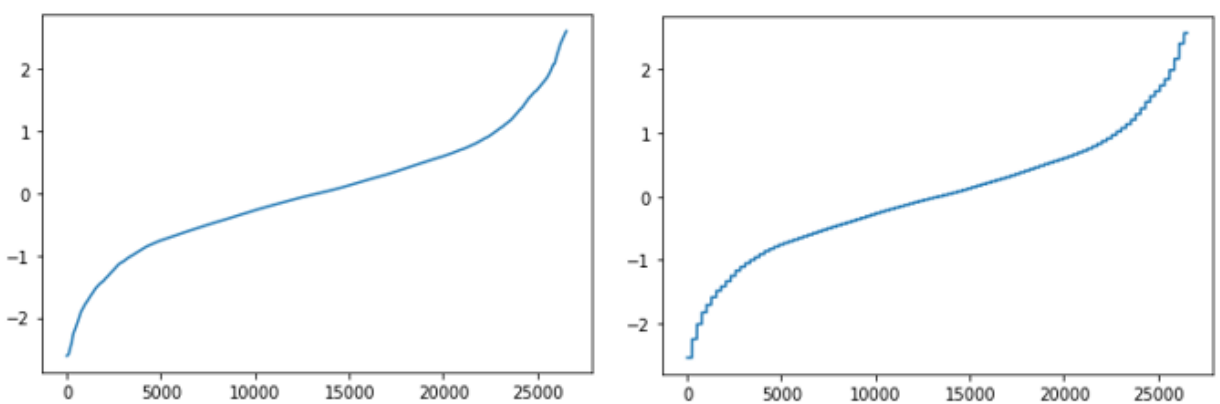

(b)

Figure 8.1: Plots of the range of unique values in our motion dataset. The x-axis shows the number of unique values in the dataset. The y-axis shows the range of values. The values are sorted from low to high. (a) There are over 2 million unique values in the motion capture dataset which ranges from -6 to 6 . (b) There are over twenty-six thousand unique values in the robot motion dataset which ranges from -2.5 to 2.5. Left: unique values in the original dataset, right: the unique values after discretization.

- robot motion data: 26546 values $\rightarrow 104$ values/bins

We validated these 'reduced' values to be sufficient to encode motion data by comparing the distribution of unique values between the real values and discretized values (binned). We also validated the discretization by reconstructing a few samples from both the motion capture and robot motion dataset (Fig. 8.2). We consider the reproduction is good since the reconstructed data retains most of the details from the original data. All of the samples in the respective datasets are then converted into one-hot encoding representation using the 'bins' that are created using this method. 


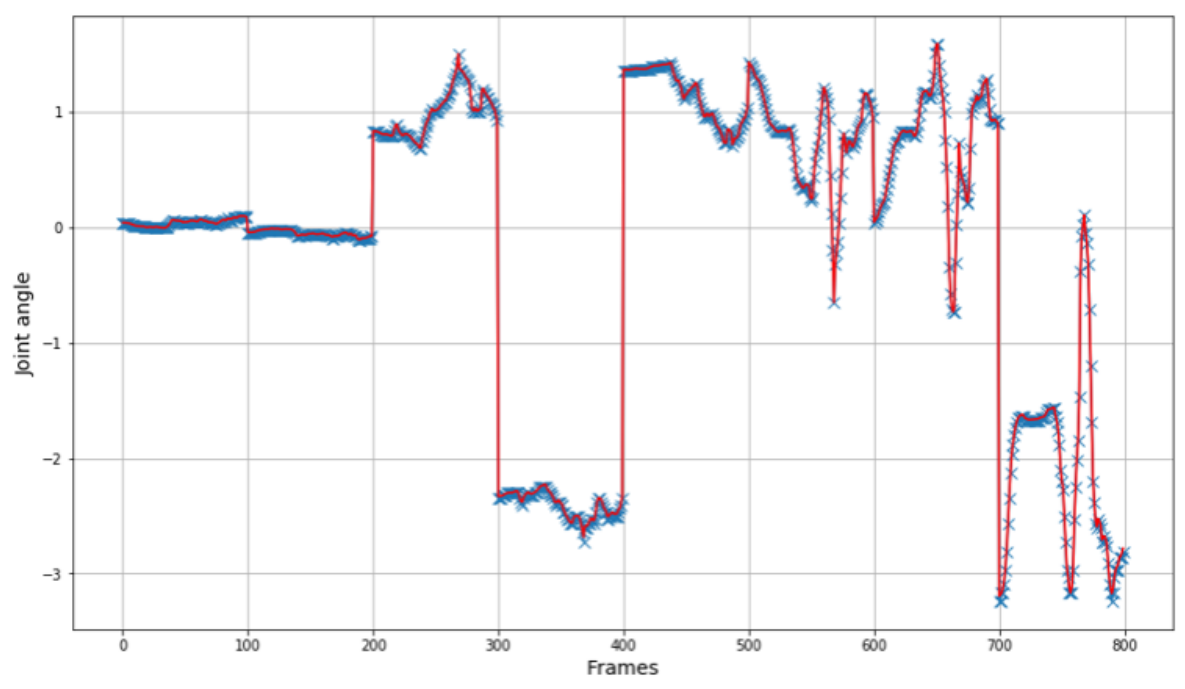

(a)

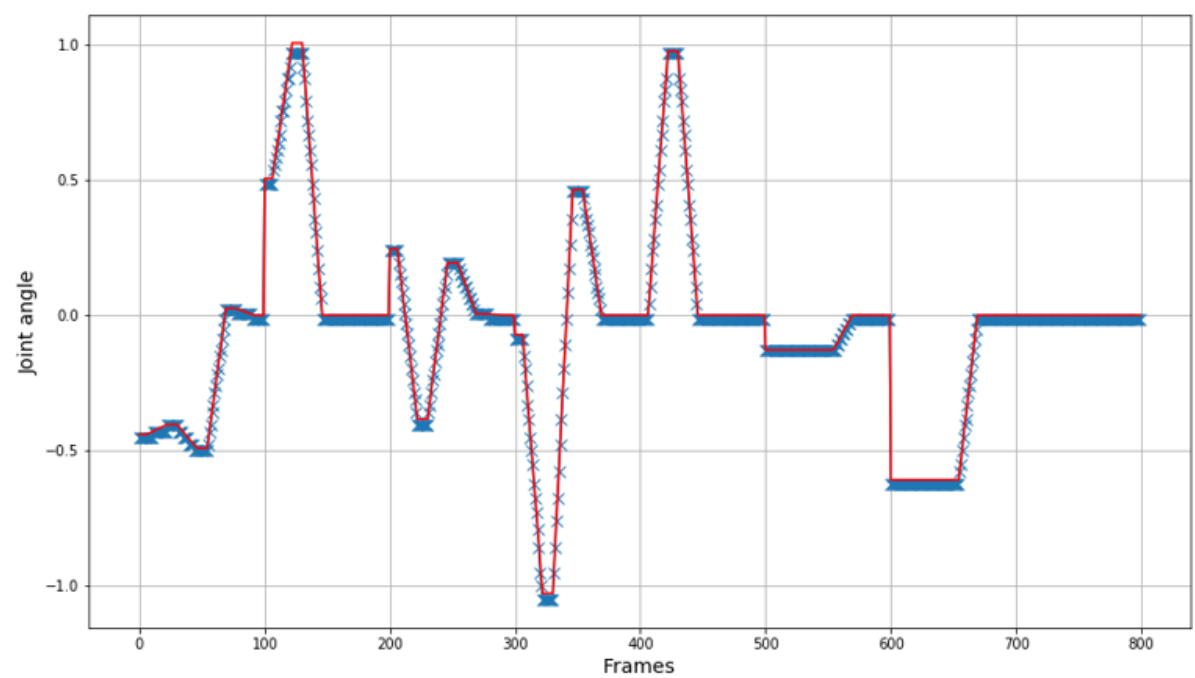

(b)

Figure 8.2: Reproducing sample data using discretized values. Solid red line is the real data, ' $\mathrm{X}$ ' marks represents the discretized values. (a) is a sample from the motion capture dataset, (b) is a sample from the robot motion dataset. 
Table 8.1: LSTM motion generator parameters

\begin{tabular}{ll}
\hline Layer & Parameters \\
\hline Input $(X)$ & Dimension $/$ size $=1$ \\
Embedding layer & embedding dimensions $=256$ \\
LSTM layer & units $=1024$ \\
Fully connected $(\mathrm{FC})$ layer & units $=200$, activation $=$ ReLu \\
\hline
\end{tabular}

The benefit of this discretization method is that it captures the distribution of continuous values in the dataset better than the naïve method of pre-determining the number of bins and taking uniform intervals from the minimum and maximum values. With this method, the number of bins and the value each bin represented are determined from the data, instead of the other way around. This means that values within the mean of the distribution are given more bins than values on the outlier, giving the ability to capture more resolution/details - and consequently, reproduction - for these values.

\subsubsection{The LSTM Network Model}

Our LSTM generator model is the same as the one shown in Fig. 7.11, with the parameters listed in Table 8.1.

\subsubsection{LSTM Generation Results}

With the one-hot-encoded data and the embedding layer, our LSTM network is able to produce data that exhibit similar properties as the samples in the training dataset. Fig. 8.3 shows an example of the data that were generated by our LSTM network. To produce the output sequence, the network must be given some initial sequential data.

We consider the motion data produced by our LSTM model to have good prop- 


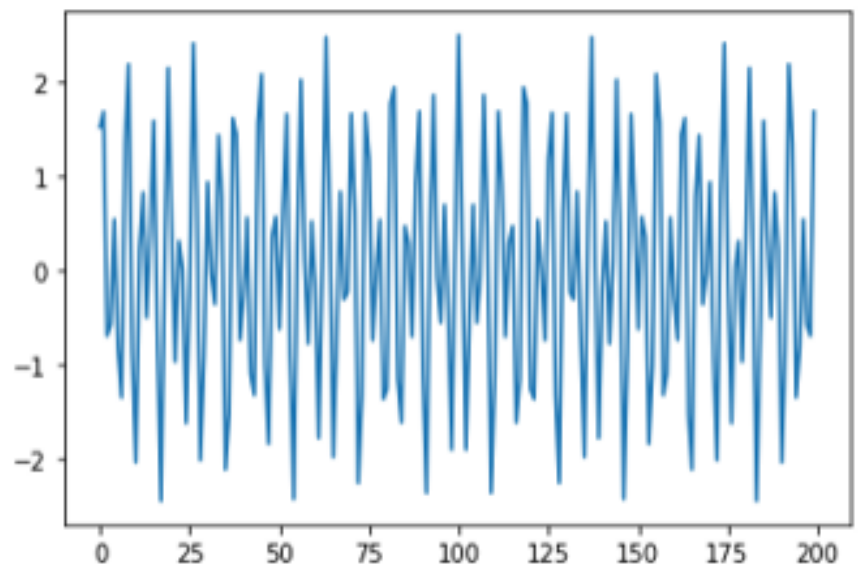

(a)

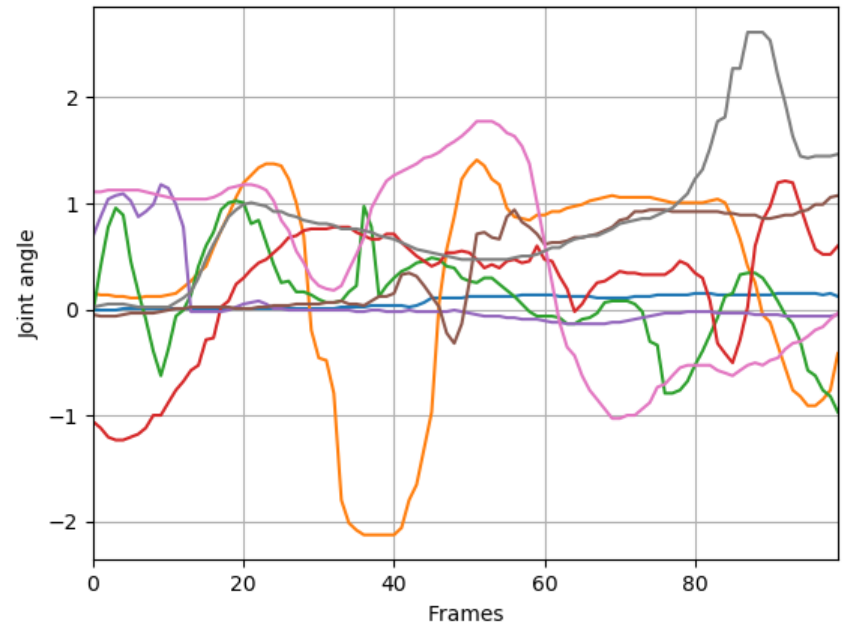

(b)

Figure 8.3: (a) Input to the trained LSTM generator, (b) the output produced by the LSTM generator. 

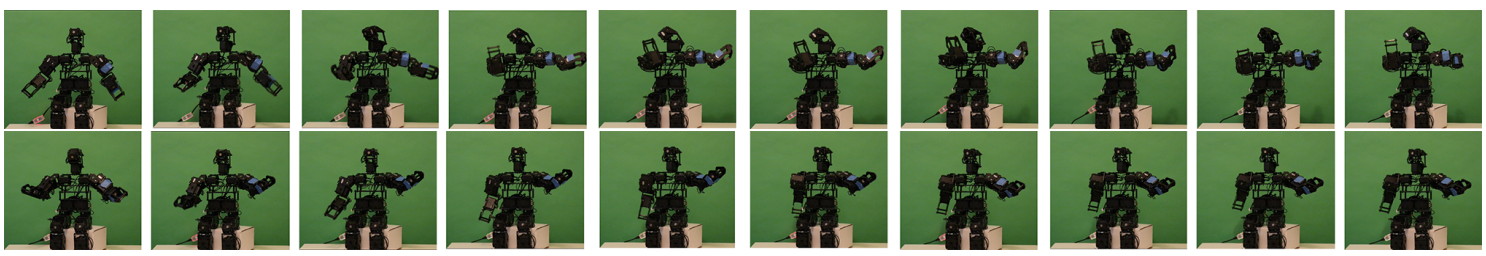

Figure 8.4: Two samples of gestural motions produced by the LSTM motion generator model (Top and bottom). Each sample consists of 100 frames; each pose shown here are taken one for every 10 poses.

erties: no high frequency (e.g. Gaussian) noise throughout the trajectories, having smooth progressions, and also arm and head poses that shows discernable gestures. The top row of the sample in Fig. 8.4 show a gesture that is like a 'shy' gesture: the head turned to the right and looking down, the right arm raised to cover the face, and the left arm extended to the left as if 'pushing' someone away. The second sample in Fig. 8.4 bottom shows a gesture that appears like diectic gestures that normally used in conversations: in the first frame both arms are extended forward and out as an "open arms" gesture, then the gesture progresses to lower the right arm and pointing with the left arm. However, we noticed in several occasions where the elbow are bent unnaturally in the reverse direction, and the head overturned to be looking behind the robot. Currently, we have no way to direct specific output for the LSTM model to produce certain kinds of gestures from the input. This will be a topic for future study.

Video demonstrations of the motions produced by the LSTM generator (Fig. 8.4, Fig. 8.5) can be found on the internet: https://youtu.be/pIFBIe4HxyU.

\subsection{Variational Auto Encoder}

To discuss the variational auto encoder (VAE) model, let's first briefly discuss the auto encoder model without getting into much detail. An auto encoder (AE) is an artificial 


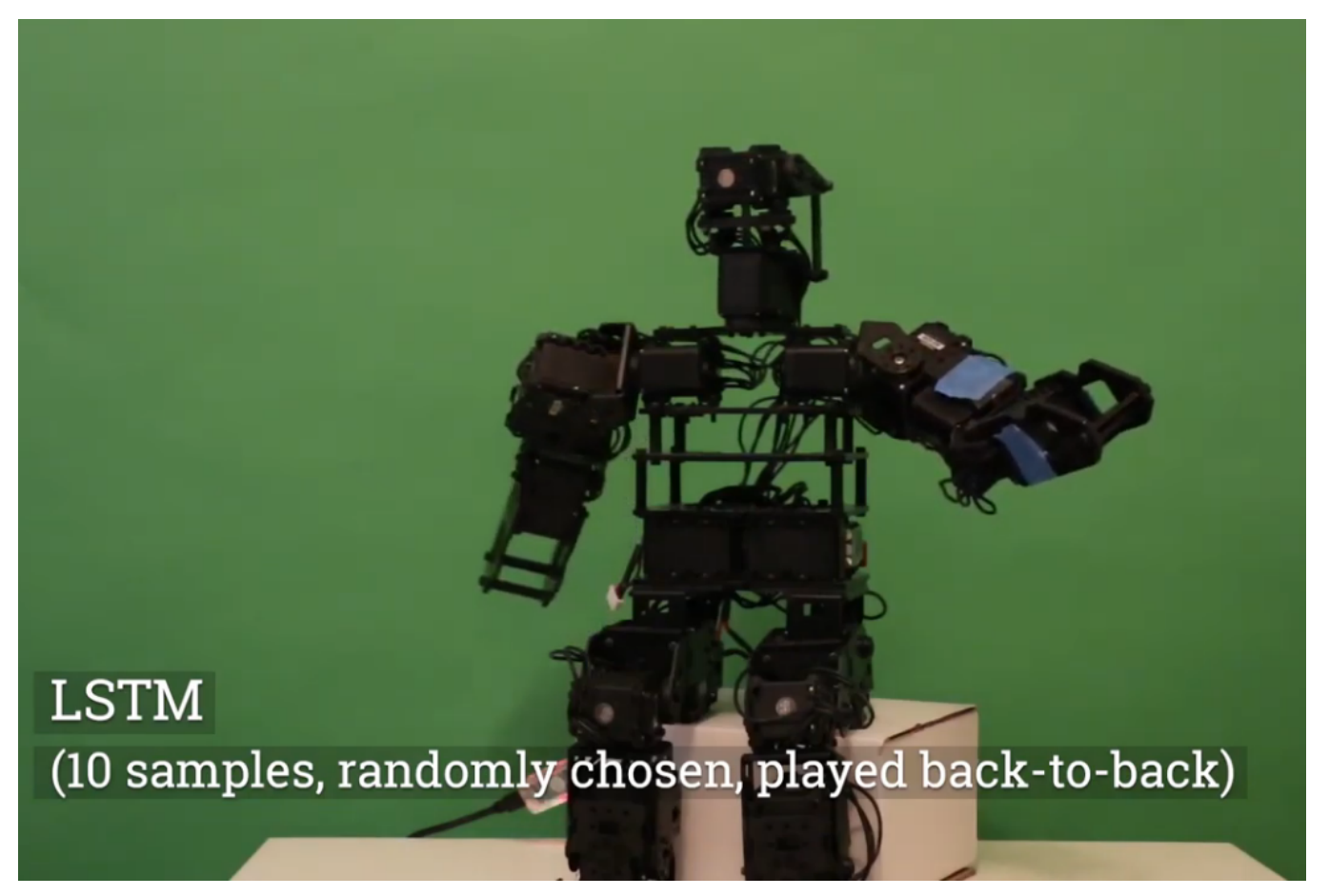

Figure 8.5: Video demonstrations of the motion samples produced by the LSTM network (https://youtu.be/pIFBIe4HxyU).

neural network model which consists of two parts: an encoder network and a decoder network (Figure 8.1). The main idea for the AE model is that the encoder network can learn a compact representation (i.e. encoding or code) of the training data $\mathbf{X}$ in some parameter space Z, which then the decoder network can learn to 'decode' to produce some output $\hat{\mathbf{Y}}$ that matches some desired/true output Y. Formally, the encoder network learns the mapping $E: \mathbf{X} \rightarrow \mathbf{Z}$, and the decoder network learns the mapping $D: \mathbf{Z} \rightarrow \mathbf{Y}$. The encoder network $E$ takes as its input the feature vector of the training data $\mathbf{X}$. The output of the decoder network $\mathbf{D}$ is $\hat{\mathbf{Y}}$. The encoder and the decoder networks are connected by a hidden layer bottleneck where the number of the hidden units is much smaller than the size of the input or output feature vectors. This hidden layer is referred to as the latent variables $\mathbf{z}$, and sometimes also referred to as code. Thus, the auto encoder is the pipeline of the encoder network $E$, latent 


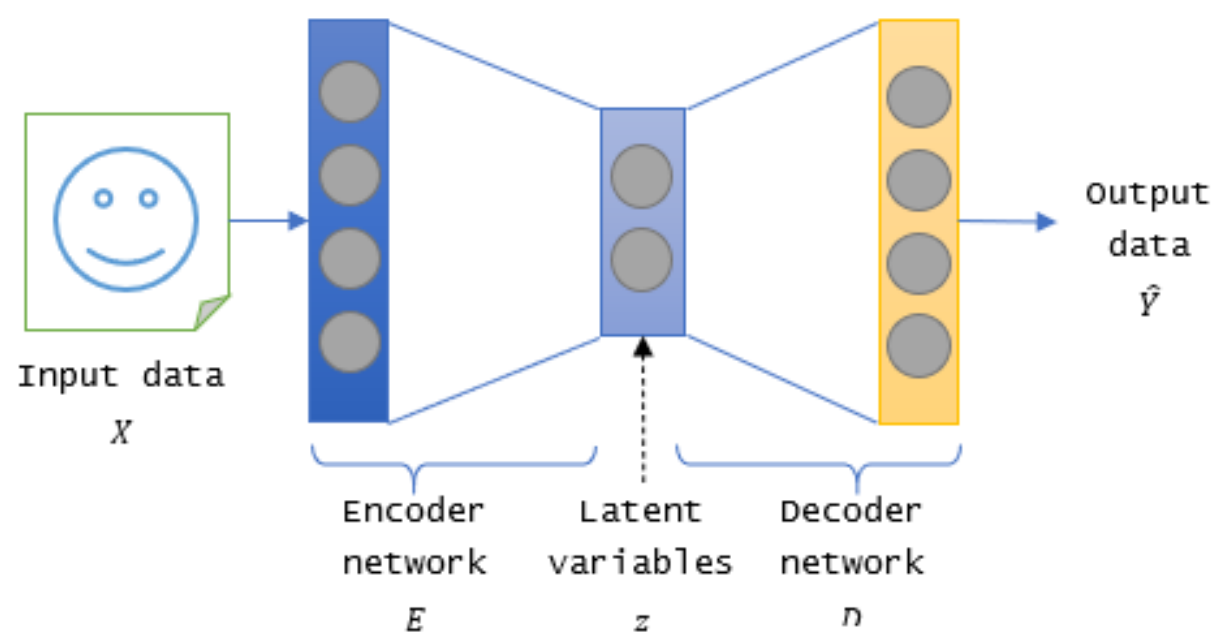

Figure 8.6: The basic architecture of an auto encoder model.

variables $\mathbf{z}$, and the decoder network $D$. The auto encoder is trained by feeding the training data $\mathbf{X}$ through the encoder $E$, and the error is calculated as the difference between the output of the decoder $\hat{\mathbf{Y}}$ and the true sample $\mathbf{Y}$ for the input $\mathbf{X}$. Using the backpropagation algorithm, the weights of the network are adjusted as usual.

The Variational Auto Encoder (VAE) is a special form of the auto encoder network that utilizes variational Bayes method [104]. VAE has a similar structure as an auto encoder in terms of consisting of an encoder network and a decoder network, but the latent variables $\mathbf{z}$ represent some distribution. Figure 8.7 shows the architecture of the VAE network. The VAE is mainly used for generative tasks, for instance generating images of MNIST, and producing music from MIDI music loops [105]. The goal of VAE is to maximize the probability of observing the training data $P(X)$ using continuous latent variables as parameters that approximates the distribution $P(X)$

Suppose there is an arbitrary function $f(\mathbf{z} ; \theta)$ which takes $\mathbf{z}$ as input and with some parameters $\theta$, where $\mathbf{z}$ is a random variable with prior $P(\mathbf{z})$ and in some high dimensional space $\mathcal{Z}, \theta$ is fixed and in the space $\Theta$, and $X$ is in the space $\mathcal{X}$, such 


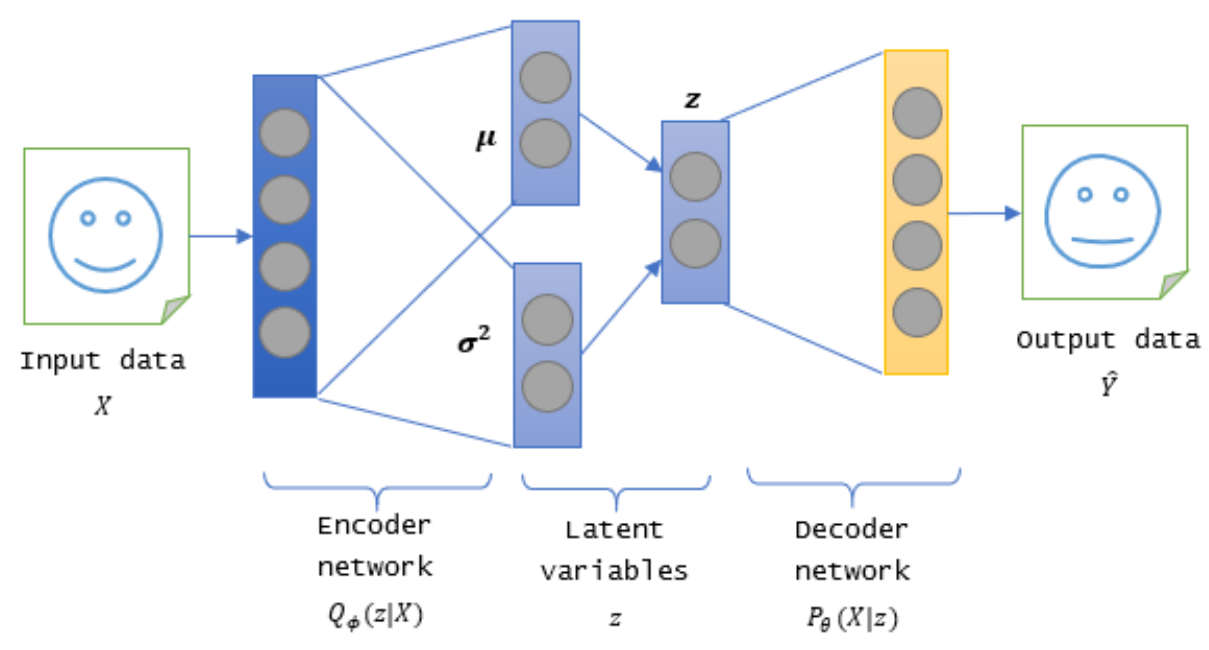

Figure 8.7: Variational Auto Encoder model. The latent variables z are produced by sampling a set of Gaussian distributions parameterized by mean $\mu$ and variance $\sigma^{2}$.

that $f: \mathcal{Z} \times \Theta \rightarrow \mathcal{X}$, then $f(z ; \theta)$ is a random variable in $X$. We want to find $\theta$ that maximizes the likelihood that $f(\mathbf{z} ; \theta)$ will be similar to $\mathbf{X}$. Since $\mathbf{z}$ is random, $f(\mathbf{z} ; \theta)$ can be written as some conditional probability $P(X \mid \mathbf{z} ; \theta)$. In other words, we want to model $P(X)$ using some prior distribution $P(\mathbf{z})$ and conditional $P(X \mid z ; \theta)$ according to the generative process:

$$
P(X)=\int P(X \mid z ; \theta) P(z) d z
$$

The computation of the integral to find $\mathrm{P}(\mathrm{X})$ can become intractable when the random variable has very many states, and worsen for high dimensional variables. The two approaches to overcome this intractable computation is by stochastic approximation of Markov Chain Monte Carlo (MCMC) and deterministic approximation of Variational Inference (VI). MCMC approximates the distribution by performing sequential conditional sampling where each sampling is conditioned only by the result 
of the previous sampling:

$$
q(z \mid x)=q\left(z_{0} \mid x\right) \prod_{t=1}^{T} q\left(z_{t} \mid x, z_{t-1}\right)
$$

With enough sampling, MCMC can converge such that $q(z \mid x)$ (eq. 8.2) matches the exact posterior $p(z \mid x)$. The main drawback of MCMC is that it is generally unknown how many times to perform the sampling described in eq 8.2 to get the true likelihood distribution. Variational Inference (VI) uses a parameterized posterior approximation $q_{\psi}(z \mid x)$ where $\psi$ is the parameter of $q$, and $z$ is a random latent variable with prior $q(z)$. VAE uses the variational inference approach to find $P(X)$. Later, we show the role of $\mathrm{q}$ in VAEs after the derivation of the objective function. In Figure 8.7, $P(X \mid z ; \theta)$ is written as $P_{\theta}(X \mid z)$.

\subsubsection{Loss Function}

The objective function of VAE is the Kullback-Leibler divergence or KL divergence $\left(D_{K L}\right)$ [104]. KL divergence is a measure of divergence between two distributions $g(x)$ and $h(x)$ measured as the loss of information in units of bits (when using $\log _{2}$ ) or nats (when using natural $\log (\ln )$ ), and defined as:

$$
\left.D_{K L}(g \| h)\right)=\mathbb{E}[\log g(x)-\operatorname{logh}(x)]
$$

Which can be expressed as a sum:

$$
D_{K L}(g \| h)=\sum_{i=1}^{N} g\left(x_{i}\right) \log \frac{g\left(x_{i}\right)}{h\left(x_{i}\right)}
$$

In eq. 8.4 , the divergence is measured for $h(x)$ with respect to $g(x)$. Note that $D_{K L}(g \| h) \neq D_{K L}(h \| g)$. When two distributions are equivalent, the KL divergence 
is zero. If the two distributions are significantly different, $D_{K} L$ will be very large. For example: when a value $x_{i}$ is within the distribution of $h$, but an outlier of $g$ such that $g\left(x_{i}\right)$ is very close to zero, then $\log \left(g\left(x_{i}\right)\right) \approx \log (0)=-\infty$.

Variational inference pose the problem of finding the approximation of $P(X)$ in equation 8.1 as an optimization problem by establishing the relationship between $P(X)$ and $\left.E_{(z} Q\right)[P(X \mid z)]$ by minimizing the KL divergence between the distribution $Q(z)$ and $P(z \mid X)[211]$ :

$$
D_{K L}(Q(z) \| P(z \mid X))=\mathbb{E}_{z q}[\log Q(z)-\log P(z \mid X)]
$$

Where $Q(z)$ is the distribution of some latent variables, and $P(z \mid X)$ is the distribution of $\mathrm{z}$ that is conditioned on the training data X. A quick note on the expected value notation $\left.\mathbb{E}: \mathbb{E}_{(} z Q\right)[P(X \mid z)]$ is read as the expected value for $P(X \mid z)$ where $z Q$ means that $z$ is sampled from the distribution $Q$. The expected value for a function $f(x)$ where $x$ is discrete and sampled from a distribution $P(x)$ is computed as:

$$
\mathbb{E}_{x p}[f(x)]=\sum_{x} P(x) f(x)
$$

And computed as an integral when $x$ is continuous variable:

$$
\mathbb{E}_{x p}[f(x)]=\int p(x) f(x) d x
$$

And applying Bayes rule to $P(z \mid X)$ in eq. 8.5:

$$
\begin{gathered}
\log P(z \mid X)=\log \left(\frac{P(X \mid z) P(z)}{P(X)}\right) \\
\log P(z \mid X)=\log P(X \mid z)+\log P(z)-\log P(X)
\end{gathered}
$$


Now eq. 8.5 can be written as:

$D_{K L}(Q(z) \| P(z \mid X))=\mathbb{E}_{z q}[\log Q(z)-\log P(X \mid z)-\log P(z)+\log P(X)]$

Since $P(X)$ does not depend on $z$, it can be taken out of the expectation term:

$D_{K L}(Q(z) \| P(z \mid X))=\mathbb{E}_{z Q}[\log Q(z)-\log P(X \mid z)-\log P(z)]+\log P(X)$

Rearranging the terms:

$$
\begin{aligned}
D_{K L}(Q(z) \| P(z \mid X))= & -\mathbb{E}_{z Q}[\log P(X \mid z)]-\mathbb{E}_{z Q}[\log Q(z)-\log P(z)] \\
& +\log P(X) \\
D_{K L}(Q(z) \| P(z \mid X))= & -\mathbb{E}_{z Q}[\log P(X \mid z)]+D_{K L}(Q(z) \| P(z))+ \\
& \log P(x) \\
\log P(X)-D_{K L}(Q(z) \| P(z \mid X))= & \mathbb{E}_{z Q}[\log P(X \mid z)]-D_{K L}(Q(z) \| P(z))
\end{aligned}
$$

Note that $Q(z)$ can be any distribution. Since $Q(z)$ is in some high dimensional space Z, $P(X \mid z)$ will be close to zero for most $z$. It would make sense to use a distribution $Q$ that depends on $X$, so we replace $Q(z)$ with $Q(z \mid X)$ such that eq. 8.12 becomes:

$$
\log P(X)-D_{K L}(Q(z \mid X) \| P(z \mid X))=\mathbb{E}_{z Q}[\log P(X \mid z)]-D_{K L}(Q(z \mid X) \| P(z))
$$


Eq. 8.13 forms the main formulation of VAE. The term $\log P(X)$ is the distribution to approximate with some error term $D_{K L}(Q(z \mid X) \| P(z \mid X))$. The right-hand side consists of two parts. The first part $\mathbb{E}_{z} Q_{Q}[\log P(X \mid z)]$ is the decoder part of the VAE that reconstructs data in the likelihood of $X$ given some latent variable $z$. The second part: $D_{K L}(Q(z \mid X) \| P(z))$ is the part of the encoder that maps $X$ to $z$. For VAE, $P(z)$ is chosen to be Gaussian with zero mean with one standard deviation, $z N(0, I)$.

So how to find the subspace of $Z$ that would maximize the likelihood $P(X \mid z)$ ? It is possible to map one distribution $z$ to another distribution $X$ by creating a new function $g$ as a random variable such that: $X=g(z)$, where $g$ can be learned from training data. VAE assumes that $z$ comes from a random normal distribution of mean zero and unit standard deviation. VAE uses the encoder network to find the function $g$ in the form of $Q_{\psi}(z \mid X)$ where $\psi$ is the parameter of the encoder network (i.e. weights). Notice that $Q_{\psi}(z \mid X)$ corresponds to the $q_{\psi}$ of the variational inference method as mentioned previously.

The VAE is trained by minimizing the loss function, which is the right-hand side of eq. 8.13:

$$
\mathcal{L}(\phi, \theta, x)=\mathbb{E}_{z} Q_{\phi}(Z \mid x)\left[\log P_{\theta}(x \mid z)\right]-D_{K L}\left(Q_{\phi}(z \mid x) \| P(z)\right)
$$

Eq. 8.14 is called the evidence lower bound (ELBO). Maximizing $\mathcal{L}$ is done by minimzing the KL divergence $D_{K L}\left(Q_{\phi}(z \mid x) \| P(z)\right)$. The first term of eq. 8.14 is called the reconstruction loss. It is read as the expected value of $\log P_{\theta}(x \mid z)$ where $\mathrm{z}$ comes from the distribution $Q_{\phi}(z \mid x)$.

The first term in eq. $8.14 E_{q_{\phi}(z \mid x)}\left(\log P_{\theta}(x \mid z)\right)$ is the term to maximize and is 
computed as a maximum likelihood estimation:

$$
\mathbb{E}_{z Q_{\phi}}\left[\log P_{\theta}(X \mid z)\right]=\frac{1}{2}\left(\frac{\|X-f(z)\|^{2}}{\sigma_{\phi}^{2}}\right)
$$

Where $X$ is the original data, $f(z)$ is the output of the decoder network obtained by sampling $Q_{\phi}(z \mid X)$ and $\sigma_{\phi}^{2}$ is the variance of $Q_{\phi}$. The second term is the $\mathrm{KL}$ divergence between the distribution of the encoder network $Q_{\phi}(z \mid X)$ and $P(z)$ and computed as:

$$
D_{K L}\left(Q_{\phi}(z \mid X) \| P(z)\right)=\frac{1}{2}\left[-\sum_{i}\left(\log \sigma_{i}^{2}+1\right)+\sum_{i} \sigma_{i}^{2}+\sum_{i} \mu_{i}^{2}\right]
$$

Where $\sigma^{2}$ and $\mu$ are the variance and standard deviation of $Q_{\psi}(z \mid X)$ (the output of the encoder network). The summation over $i$ is the number of latent variables $[212]$

$$
\begin{gathered}
z=\mathcal{N}(\mu, \sigma) \\
z=\mu+\sigma * \varepsilon
\end{gathered}
$$

The sampling of a probability distribution to compute $P_{\theta}(X \mid z)$ in eq. 8.15 is an operation which cannot be differentiated; thus, the gradient cannot be computed for the backpropagation algorithm. The reparameterization trick circumvents this problem by instead of sampling directly from the distribution (eq. 8.17), the sampling is done as a linear operation as shown in eq. 8.18, where the sampled value is the $\mu$ plus $\sigma$ multiplied by a random number from a zero-mean, one standard deviation 


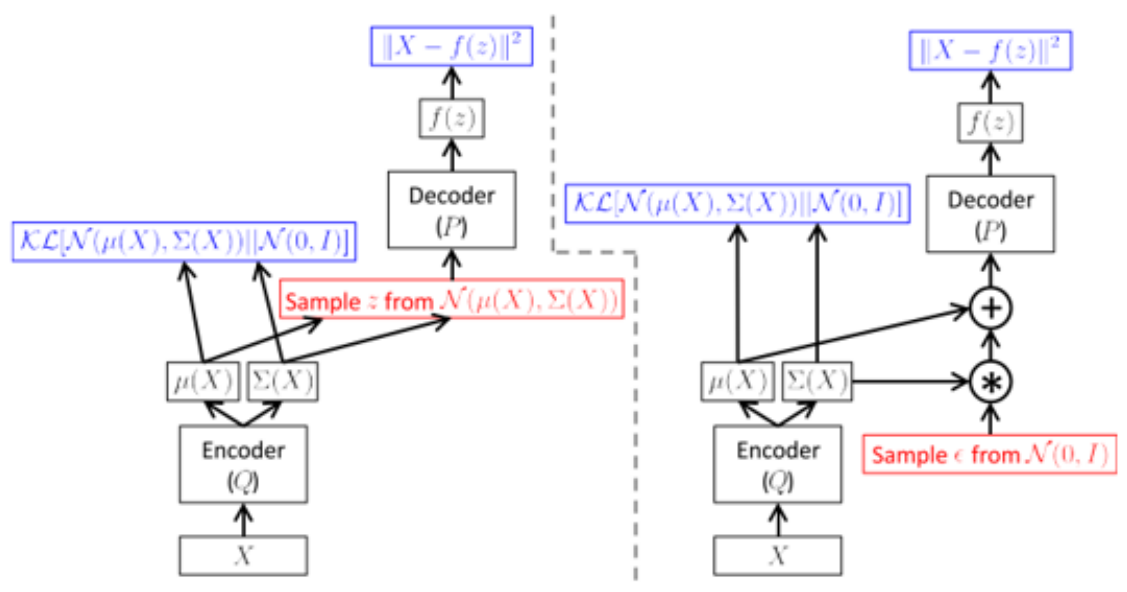

Figure 8.8: Training of variational autoencoder without the reparameterization trick (left), and with the reparameterization (right). Image from [212].

Gaussian distribution $\varepsilon=\mathcal{N}(0, I)$. The gradients $\frac{\delta z}{\delta \mu}$ and $\frac{\delta z}{\delta \sigma}$ can now be computed since the random variable $\varepsilon$ can be regarded as a constant. Figure 8.8 shows how the reparameterization trick allows the backpropagation path [212]. The red box in figure 8.8 shows the sampling operation which is not differentiable. On the left, is the path without the reparameterization, while on the right it is shown that the reparameterization allows a direct path for computing backpropagation from the output of the decoder back to the encoder.

\subsubsection{Motion Generation using VAE}

We built a VAE model to obtain a motion data generator. The goal is to investigate if various kinds of gestural motions can be produced using few parameters rather than using the manual pose-by-pose process.

The training dataset for this model is the set of motion clips that we built as described in Section 6.3.

The input to the VAE model (i.e. the input to the encoder network) is a vector of size 800, which is the flattened vector of the motion clips of 8 DOF x 100 timesteps. 
Table 8.2: Encoder parameters

\begin{tabular}{ll}
\hline Layer & Parameters \\
\hline Input $(X)$ & Dimension/size $=800$ \\
Fully connected (FC1) & units $=200$, activation $=$ ReLu \\
Fully connected (FC2) & units $=200$, activation $=$ ReLu \\
Fully connected (z_mean) & units $=2$, activation $=$ linear \\
Fully connected (z_var) & units $=2$, activation $=$ linear \\
\hline
\end{tabular}

The output of the VAE (i.e. the output of the decoder network) is also a vector of size 800 for the same reason as the input. The size of the latent variables was varied between 3 and 5 . The encoder network consists of three layers of fully connected feedforward network. The two hidden layers have 200 hidden nodes for each layer. The third layer is the "output" layer for the encoder which consists of two networks; one for the mean and another for the variance for the latent variables. These networks have the same size as the number of latent variables. In total, the motion generator VAE has about 400,000 trainable weights. The activation functions for the last hidden layer of the encoder network and the output layer of the decoder network is the hyperbolic tangent ( $\tanh$ ) to allow for positive and negative values. The activation functions for the remaining layers are set to the rectified linear units (ReLU). The network was implemented using Keras and Python. The network was trained using Adam optimizer with default parameters of learning_rate $=0.01, \beta_{1}=0.9$, and $\beta_{2}=0.999$ as recommended in the original paper [213]. Table 8.2 and 8.3 list the parameters for the encoder and decoder networks. Our model was based on the example from the Keras website [214].

\subsubsection{Results}

The main idea behind VAE is that once the training is complete, new samples can be generated by giving values of $\mathbf{z}$ directly to the decoder network. By varying the values 
Table 8.3: Decoder parameters

\begin{tabular}{ll}
\hline Layer & Parameters \\
\hline Input $(X)$ & Dimension/size $=2$ \\
Fully connected (FC3) & units $=200$, activation $=$ ReLu \\
Fully connected (FC4) & units $=200$, activation $=$ ReLu \\
Fully connected (Output) & units $=800$, activation $=$ tanh \\
\hline
\end{tabular}

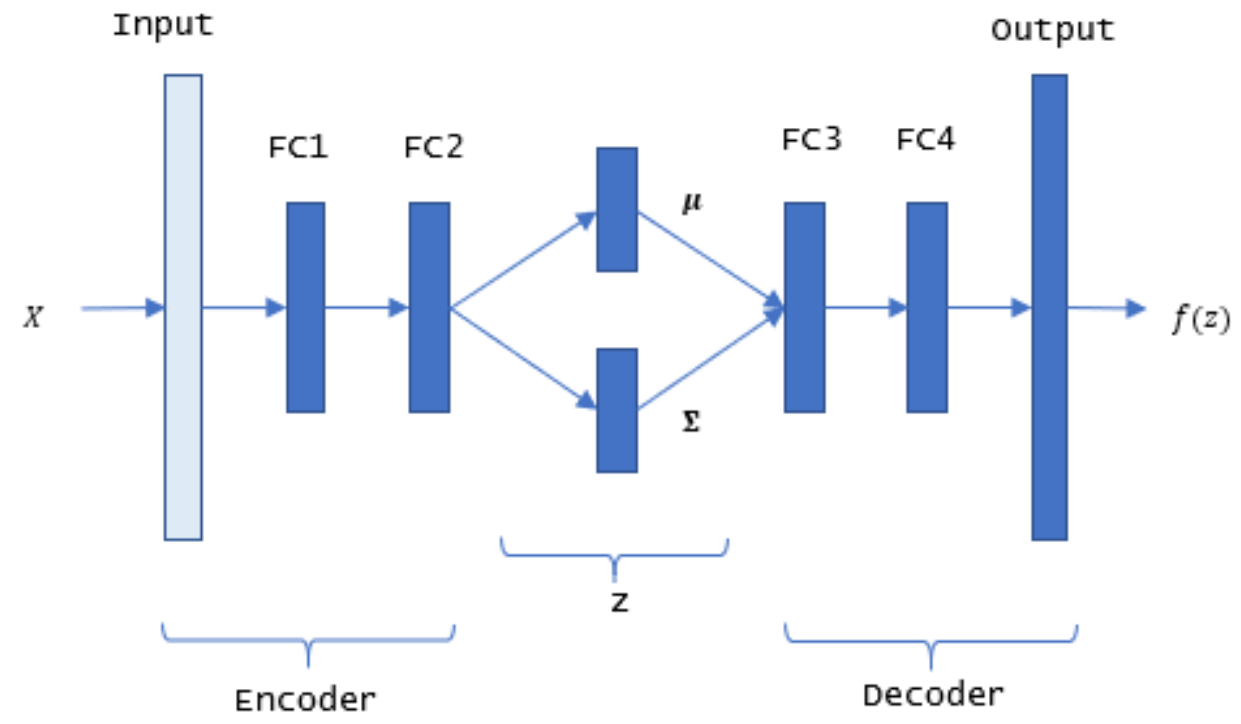

Figure 8.9: The motion generator VAE that uses only fully-connected, feed-forward network layers. The parameters are given in Tables 8.2 and 8.3. $\left(\mu=z_{-}\right.$mean, $\Sigma=$ Z_var) 
of the latent variable space $\mathbf{z}$ we should be able to see how the sample is produced and how its shape gradually changes. Ideally, each $z_{i}$ would correspond to specific properties of the output sample.

The output of the VAE tend to be very noisy. Fig. 8.10 shows some examples of the data generated by the decoder network by varying the values of the latent variables z. The columns are the variations for the latent variable $z_{1} \in[-5.0, \cdots, 5.0]$ (left to right), and the rows are the variations for $z_{2} \in[-5.0, \cdots, 5.0]$ (top to bottom), both at regular intervals. Each line is the plot for each degree of freedom (DOF). For the data to be usable, some kind of filtering needs to be applied to the data. The sampled results show that the range of values are constrained to $[-1.0,1.0]$. This is due to the use of the tanh activation function at the output layer of the decoder. It appears that our trained VAE only learned the distributions of only a subset of the DOFs. As the latent variables changed, only a few of the DOFs changes shape while the rest remains relatively constant, despite changing the baseline values.

Unsatisfied with the above results, we found that the DCGAN generator model seem to be successful in producing noise-free data unlike the VAE decoder model. With our GAN model (explained in the next section), we trained another VAE model which decoder model is replaced with the same model/layers as the GAN generator (with layers and parameters in Table 8.4) with the same number of latent variables as our earlier VAE model. This second model was able to produce very smooth data which no longer need post-filtering to denoise (Fig. 8.12 - columns are the variations for the latent variable $z_{1} \in[-5.0,-3.333,-1.667,0.0,1.667,3.3335 .0]$ (left to right), and the rows are the variations for $z_{2} \in[-5.0,-3.333,-1.667,0.0,1.667,3.3335 .0]$ (top to bottom)).

Video demonstrations of the motions produced by the VAE model (Fig. 8.13, Fig. 8.14) can be found on the internet: https://youtu.be/PQ0d5k_9_dc. 
VAE output
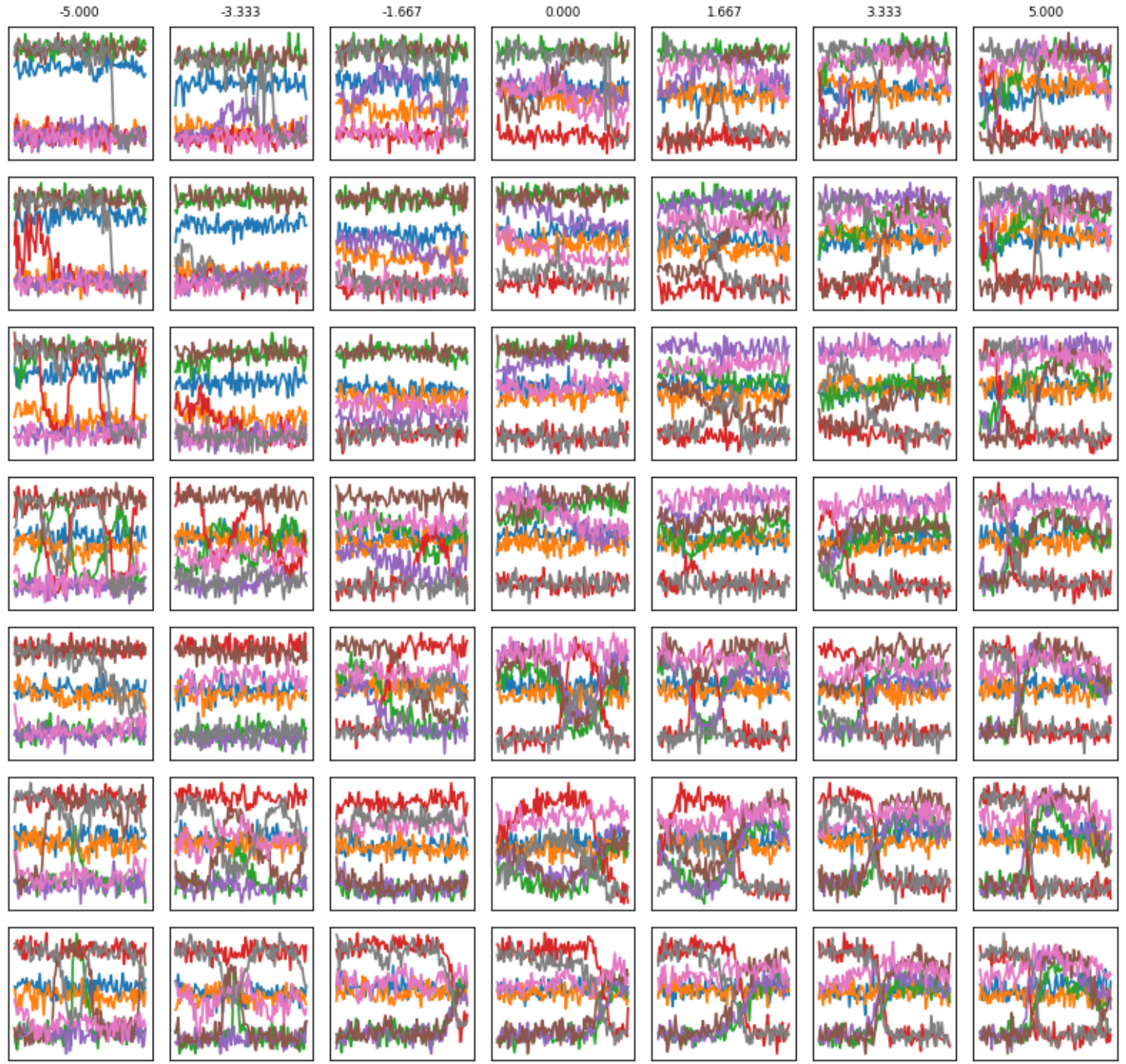

Figure 8.10: Unfiltered VAE output for various values of with latent variables $z$ of size 2. The columns are the variations for the latent variable $z_{1}$ (left to right), and the rows are the variations for $z_{2}$ (top to bottom). 
Filtered (DWT, sym4)
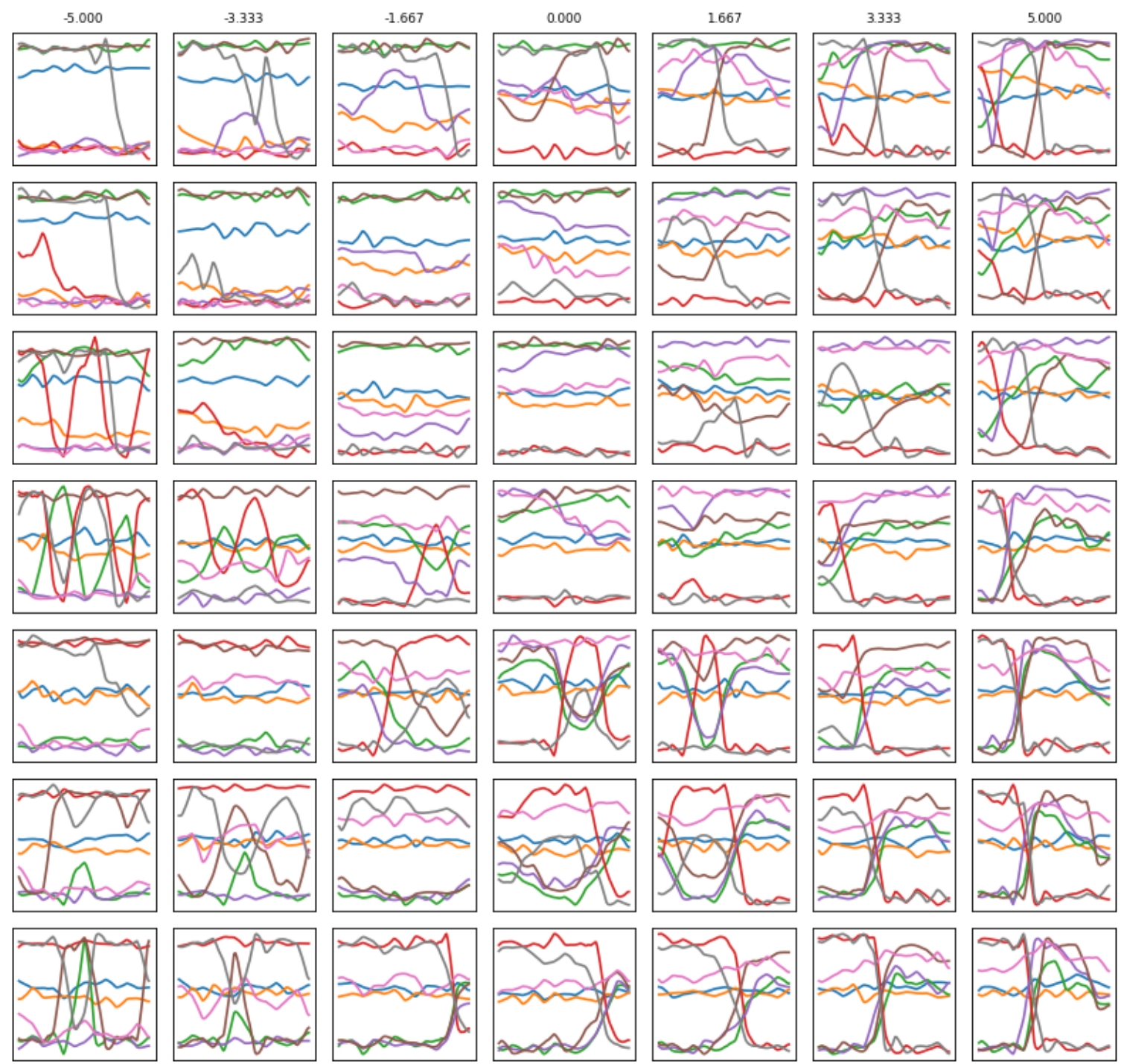

Figure 8.11: Output of VAE from Fig. 8.10 filtered using the symlet4 wavelet. 

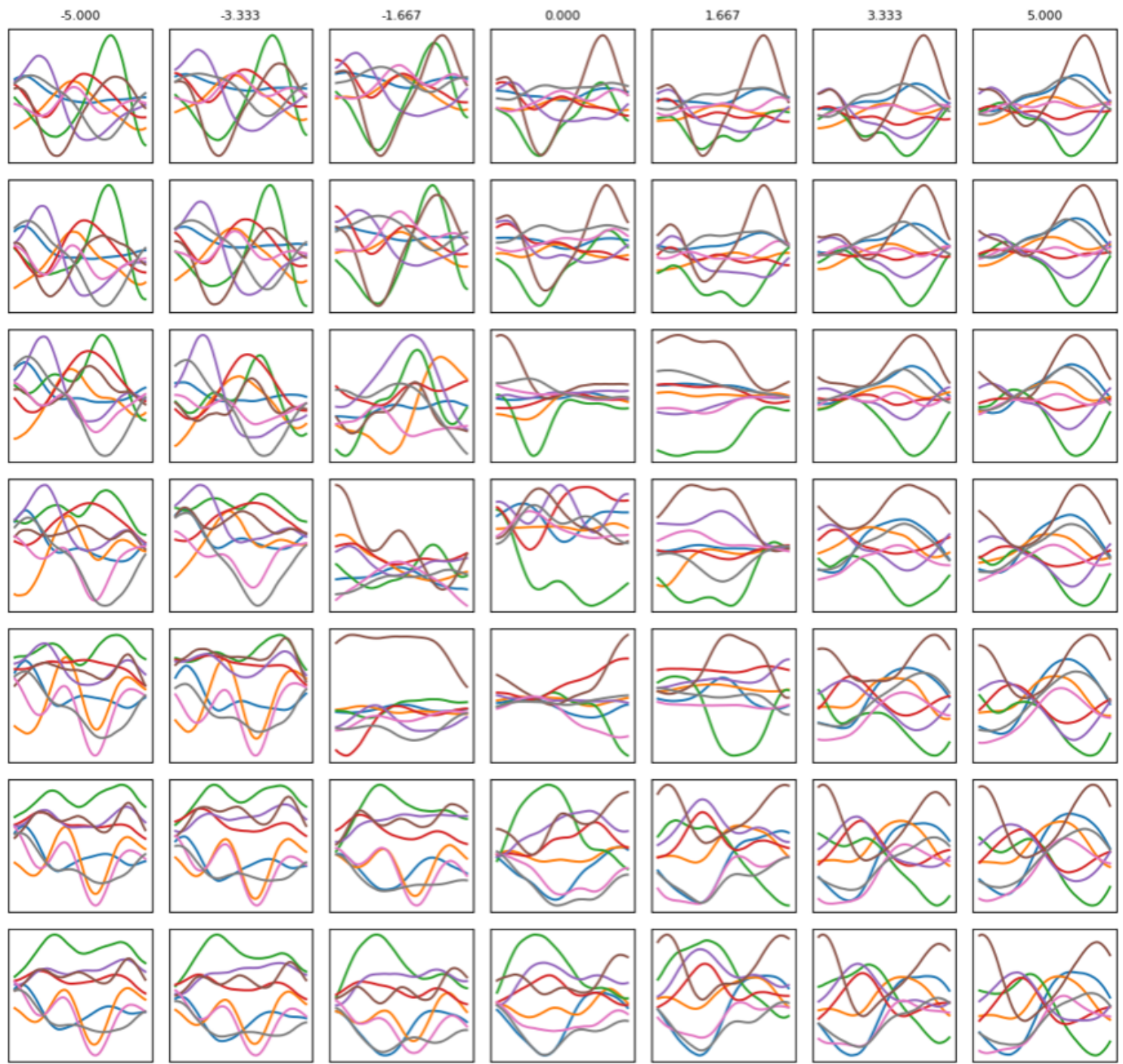

Figure 8.12: Output of VAE model with convolutional decoder model based on our GAN generator (Table 8.4) with latent variables $z$ of size 2 . The columns are the variations for the latent variable $z_{1}$ (left to right), and the rows are the variations for $z_{2}$ (top to bottom).
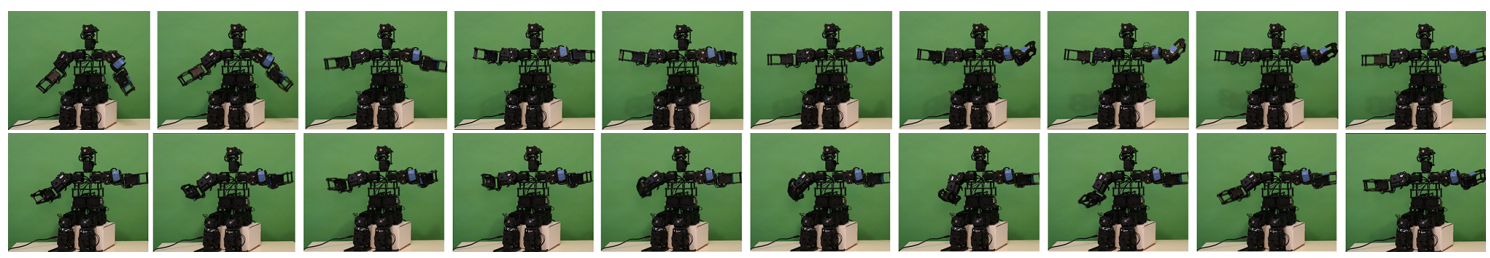

Figure 8.13: Two samples of gestural motions produced by the VAE motion generator model (Top and bottom). Each sample consists of 100 frames; each pose shown here are taken one for every 10 poses. 


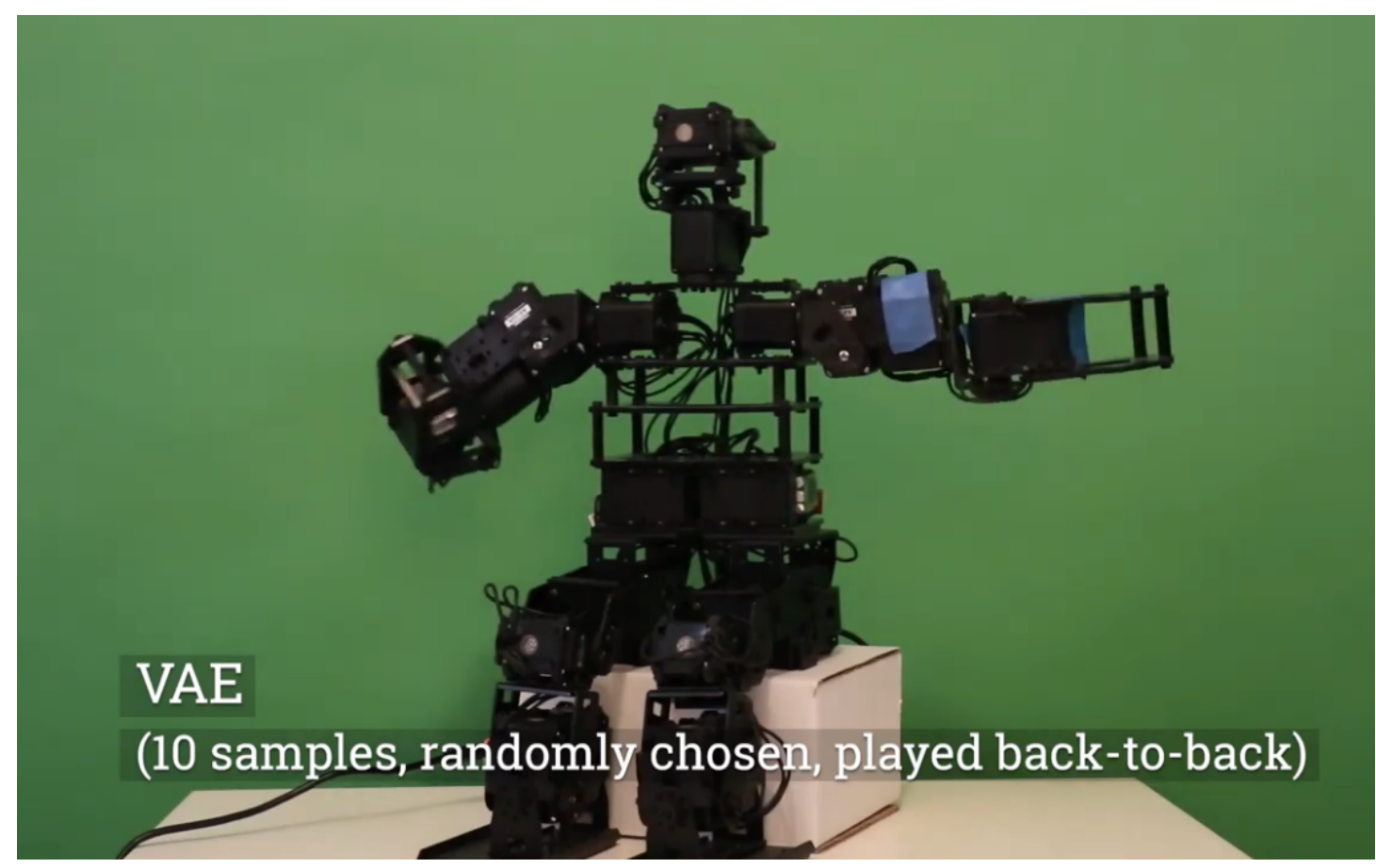

Figure 8.14: Video demonstrations of the motion samples produced by the VAE model (https://youtu.be/PQ0d5k_9_dc).

\subsection{Generative Adversarial Network}

The Generative Adversarial Network (GAN) model is able to produce new samples that do not exist in its training data with high quality, and these new samples can sometimes be difficult to distinguish from real data [99] [106]. GAN has been shown to produce samples that appear to be sharper than samples that were generated by VAE in the case of image data. GAN has found applications in information security (REFs), image and video manipulation (REFs), and image synthesis (REFs). In this work, we use GAN to develop a generative model that produces motion data for a humanoid robot with the 'natural' and 'expressive' qualities of motion like in motion capture data.

The main idea of the GAN framework is to train two networks simultaneously where they compete with each other as they obtain better performance. The first 


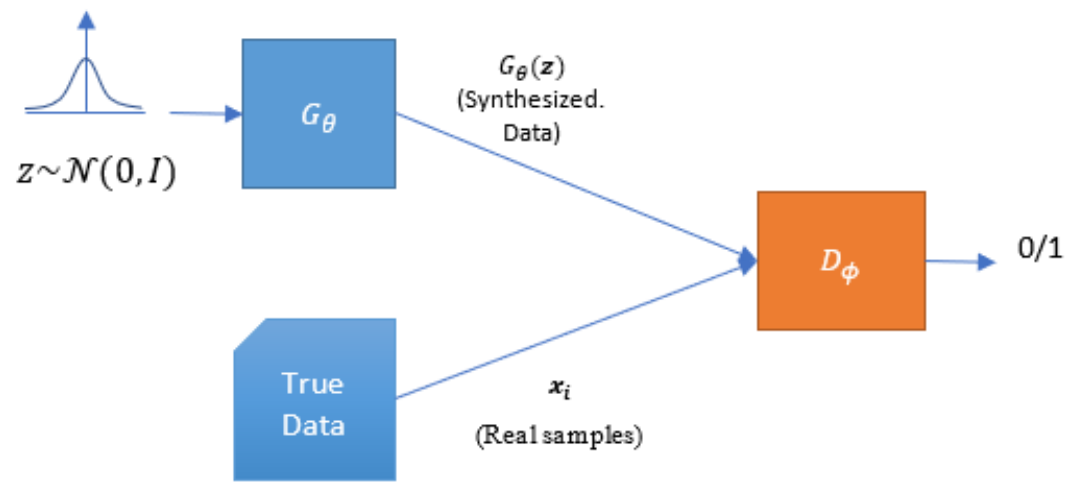

Figure 8.15: Basic architecture of GAN. $G_{\theta}$ is the generator network with parameters (weights) $\theta, D$ is the discriminator network with parameters (i.e. weights) $\psi$. "Synth. Data" $G_{\theta}(z)$ is synthetic data produced by the generator. $\mathbf{x}$ is feature vector of the "real data" which is sampled from the training data.

network is called the discriminator and it is a classifying network, which task is to correctly label its inputs as 'true data' or 'fake data'. The second network is called the generator and its task is to produce new objects or data points (e.g. MNIST image data). During training, the discriminator network $\left(D_{\phi}\right)$ receives 'true data' inputs from the training set $X$ and 'fake data' $\left(G_{\theta}(Z)\right)$ produced by the generator network $G$ (Fig. 8.16). The goal of the discriminator network is to get better at classifying the data from the training dataset versus data produced by the generator. Meanwhile, the goal of the generator network is to improve the quality of its output such that the generated data is classified by the discriminator as 'true data' i.e. fooling the discriminator network. At the same time, as the output of the generator networks improves, the discriminator network must also improve its ability to recognize these improved generator outputs and label them correctly. Both the discriminator network and the generator network modify their parameters using the error calculated from the classification errors of the discriminator. Figure 8.15 shows the basic architecture of a GAN.

In the GAN framework, typically the discriminator and generator are implemented 
using neural network architectures, but there are no restrictions of the learning models to use. One of the most successful models of GAN is called Deep Convolutional Generative Adversarial Network (DCGAN) [106]. In DCGAN, the generator and discriminator networks are made of convolutional layers. DCGAN was shown to be successful in three tasks. The first task of DCGAN is strictly generation of new samples based on the LSUN and CIFAR datasets that the authors claimed to have the most variety and sharpness compared to other generative models like VAE. The second contribution of DCGAN is showing that image manipulation can be done by using simple algebraic operations on the input feature vector $\mathrm{z}$ for the generator.

The input to the DCGAN generator network (and in most GAN models) is a vector of arbitrary size with uniform distribution, typically with zero mean and one standard deviation. To show their results, the authors of [106] collected the sets of $z$ vectors that produce one kind of data, such as: portrait of male faces, female faces, male faces with sunglasses, and so on. Each set of the $z$ vectors is averaged and considered as the representative latent variables for that class of data. Algebraic vector operations like addition and subtraction are applied to these $\mathrm{z}$ vector (i.e. latent) representations to produce new images. For example, the latent representation of a male person wearing sunglasses is subtracted with the latent representation of a male person without sunglasses, and then adding the latent representation for female face (without sunglasses), produces an image of a female wearing sunglasses. Other uses of the learned latent variables include changing the orientation of the object in the picture, e.g. one feature from the latent vector can linearly change the orientation of a picture of a person from facing left to facing to the right.

A GAN model consists of two networks: a generator and a discriminator. The generator takes an input vector of arbitrary size initialized with normal noise distribution $z N(0, I)$. The output of the generator is the data to be synthesized. For 
example, if the generator is to produce MNIST image data, then the output of the generator is a vector of size 784 (representing the $28 \times 28$ pixels image of MNIST data). The input to the discriminator is the data to be classified. The output of the discriminator is the predicted class. During training, the error of the discriminator is calculated as the sum of two terms: the classification error for the synthesized data and the classification error for the "true" data. This error is used in backpropagation to update the parameters (i.e. weights) of the discriminator. The discriminator error is also used to compute the backpropagation for the generator and update its parameters.

The loss function of the discriminator network $D$ :

$$
\begin{aligned}
& J^{(D)}=-\frac{1}{2} \mathbb{E}_{\mathbf{x} p_{\text {data }}} \log D(\mathbf{x})-\frac{1}{2} \mathbb{E}_{\mathbf{z}} \log (1-D(G(\mathbf{z}))) \\
& =-\frac{1}{2}\left(\mathbb{E}_{\mathbf{x} p_{\text {data }}} \log D(\mathbf{x})+\mathbb{E}_{\mathbf{z}} \log (1-D(G(\mathbf{z})))\right)
\end{aligned}
$$

Where $G(z)$ is the output of the generator, and $D(x)$ is the output of the discriminator given true data $x$, and $D(G(z))$ is the output of the discriminator given the synthesized data $G(z)$. In practice, we use the sigmoid cross-entropy loss to compute the loss for the discriminator:

$$
C E=-\sum_{i=1}^{C^{\prime}=2} t_{i} \log \left(f\left(s_{i}\right)\right)=-t_{1} \log \left(f\left(s_{1}\right)\right)-\left(1-t_{1}\right) \log \left(1-f\left(s_{1}\right)\right)
$$

Where $C E$ means 'Cross Entropy loss'. For binary classification (e.g. motion capture $=1$, generated (fake sample $=0$ ), there are only two classes. $t_{1}$ is the ground truth label, $s_{1}$ is the score for class $C_{1}$ where $C_{1} \in C^{\prime}$ The score and target class for 


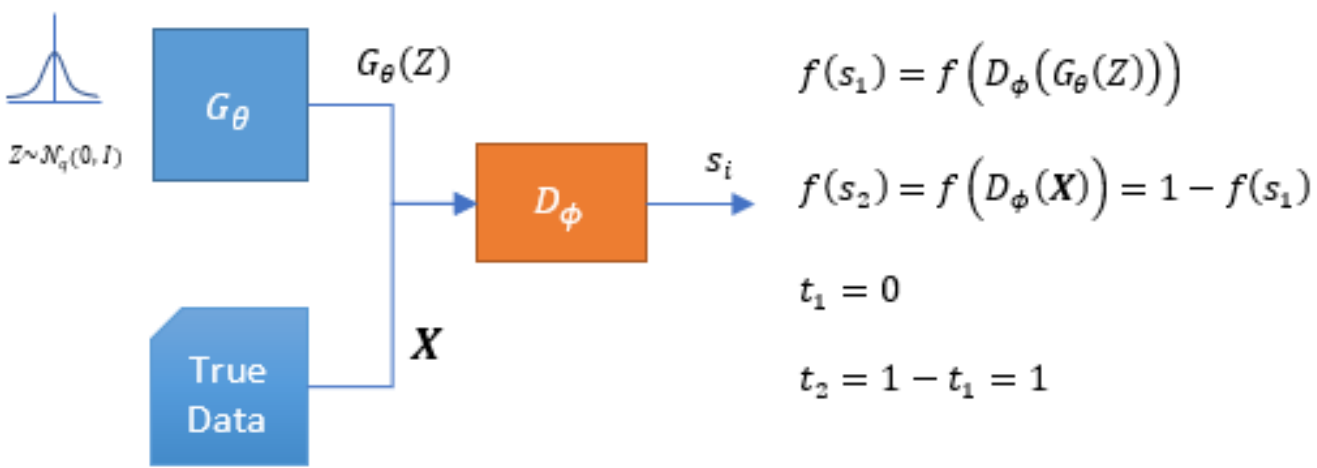

Figure 8.16: Discriminator loss

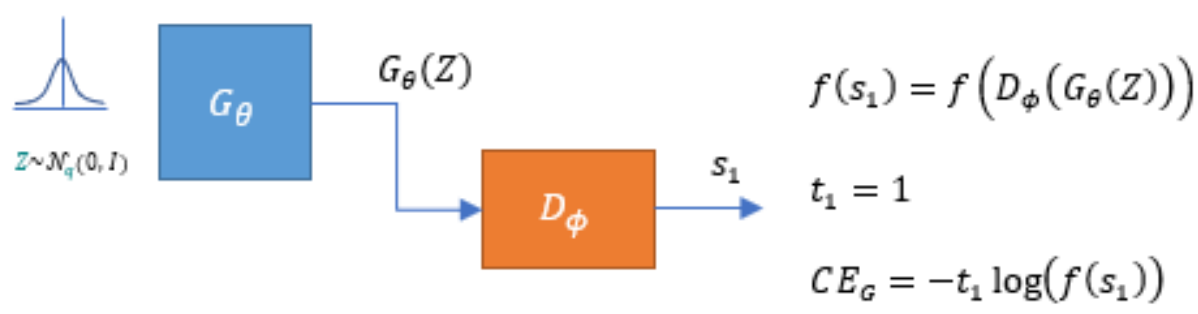

Figure 8.17: Generator loss

the class $C_{2}$ is $s_{2}=1-s_{1}$ and $t_{2}=1-t_{1} . f\left(s_{i}\right)$ is the sigmoid activation function:

$$
f\left(s_{i}\right)=\frac{1}{1+e^{-s_{i}}}
$$

The loss of the generator network is computed using sigmoid cross-entropy but only with respect of the expected 'true' label as 1 (i.e. we want the generator to produce samples that is classified by the discriminator as 1 . Figure 8.16 illustrates the computation of the cross-entropy loss for the discriminator network $C E_{D}$. Figure 8.17 illustrates the computation of the cross-entropy loss for the generator network $C E_{G}$ where the desired target value is $1\left(t_{1}=1\right)$. Notice that $C E_{G}$ does not depend on the true data $\mathbf{x}$. 


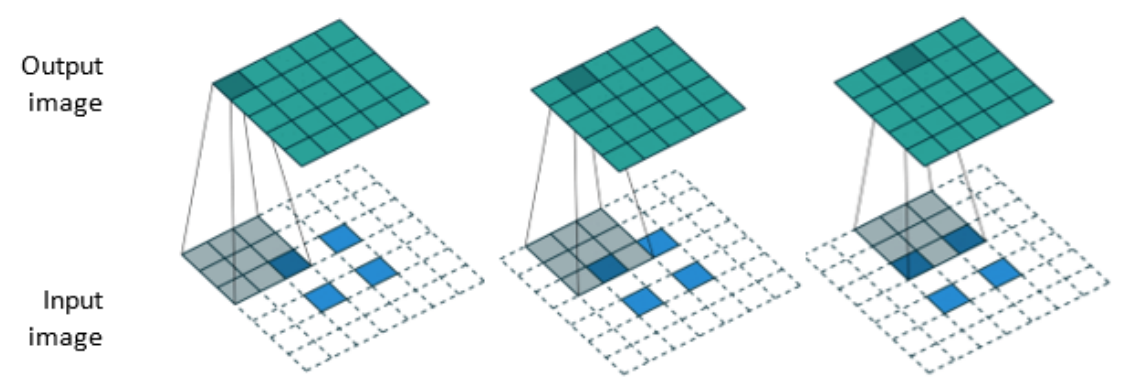

Figure 8.18: Three steps of the operation of the transposed convolution layer (images from $[215])$.

\subsubsection{The Transposed Convolution Layer}

A component of the generator network is the transposed convolution layer [215] (Fig. 8.18). The transposed convolution performs convolution on an input image of dimensions $n \times n$ into another image $m \times m$, where $m>n$, effectively upscaling the input image. It is not the same as a deconvolution or inverse convolution process. In deconvolution or inverse convolution, the input is the result image of convolution and the output is the original, pre-convoluted image. Transposed convolution performs normal convolution where the input image is padded with zeros between the values. As a network layer, the weights of a transposed convolution kernel are also learned during the training session. The amount of padding, stride, and kernel size are dependent upon the desired upscaling factor.

\subsubsection{The Motion Generator GAN Model}

Figure 8.19 shows the generator network of our GAN model which was modeled after the DCGAN architecture [106]. The input of the generator is a vector $\mathbf{z}$ of size 100 which values are sampled from a zero-mean, one standard deviation $Z=N_{1} 00(0, I)$. The input $\mathbf{Z}$ is first projected using a fully connected layer into a convolutional feature representation with many feature maps similar to DCGAN. The dimensions of the 
convolutional feature representation can be described by an $n \times n \times k$ where each feature is two dimensional with size $n \times n$ and $k$ is the number of features (depth). We used three transposed convolution layers [215] and mimic the DCGAN model where each successive transposed convolution layer has half the depth (number of features) of the previous transposed convolution layer, but the dimensions of the successive features are doubled from the previous. The increase of feature dimensions and reduction of number of features is done to upscale the features to the desired output dimensions. Our output needs to be $8 \times 100$ representing 8 DOFs and 100 timesteps of joint angle data. Since the shape of this data is non-square while it is easier to use the transposed convolution layers with square features, we used the generator model that would generate a $28 \times 28$ feature (suitable for generating MNIST data). We then reshaped the feature into a vector of size $784(28 \times 28=784)$ and added a fully connected network to output a vector of size 800 , then reshape the vector to $8 \times 100$ to obtain the motion data. Adding the fully connected layer was against the recommendation by Radford et. al. [106], but we found it to be convenient to map the output feature vector to the dimensions that we need $(8 \times 100)$, and our results show that the addition of this layer does not seem to negatively impact the generator's ability to produce interesting data. We explain our results below.

\subsubsection{The Discriminator Model}

Our discriminator network is based on the discriminator network used in the DCGAN model $[106]^{1}$. The discriminator network consists of two convolution layers and a fully connected, feed forward layer for the output layer with one value.

\footnotetext{
${ }^{1} \mathrm{My}$ implementation is based on the implementation described in Tensorflow website [216].
} 


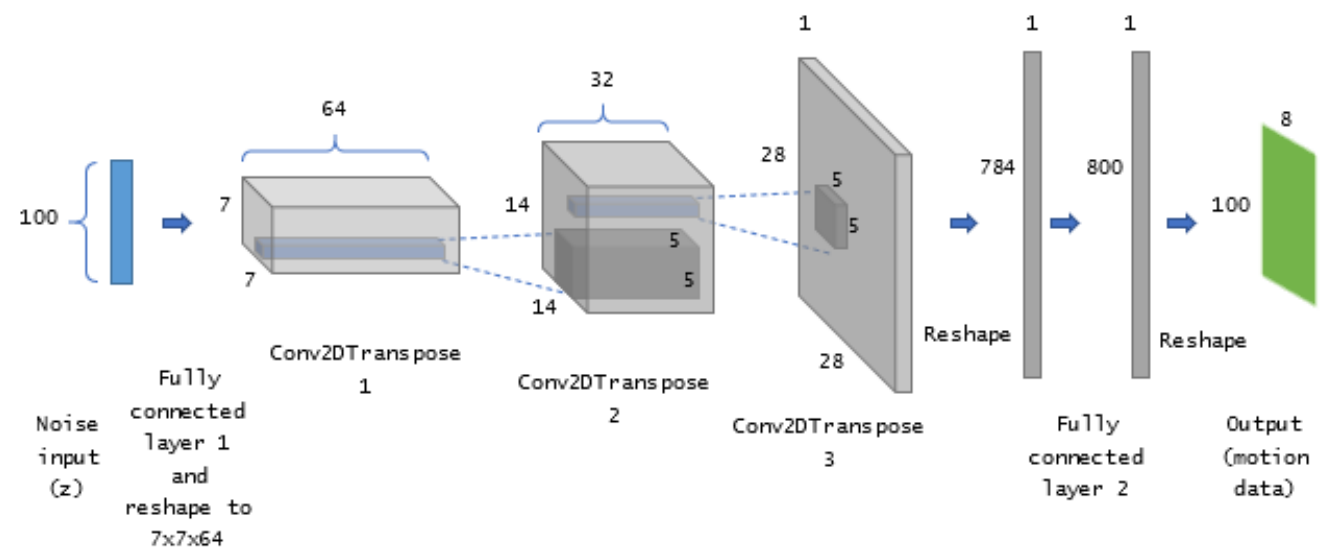

Figure 8.19: Our GAN generator model based on the generator of the DCGAN architecture [106].

Table 8.4: GAN generator parameters

\begin{tabular}{|c|c|c|}
\hline Layer & Parameters & \\
\hline Input $(X)$ & Dimension $/$ size $=800$ & \\
\hline Fully connected (FC1) & $\begin{array}{l}\text { units }=3136(7 \times 7 \times 64) \\
\text { none/linear }\end{array}$ & activation $=$ \\
\hline \multicolumn{3}{|l|}{ Batch normalization } \\
\hline Rectified Linear units (ReLu) & activation & \\
\hline Reshape & shape $=(-1,7,7,64)$ & \\
\hline Conv2DTranspose (conv1) & $\begin{array}{l}\text { filters }=64, \quad \text { kernel }=(5,5), \\
\text { padding='same' }\end{array}$ & strides $=(1,1)$, \\
\hline \multicolumn{3}{|l|}{ Batch normalization } \\
\hline ReLu & activation & \\
\hline Conv2DTranspose (conv2) & $\begin{array}{l}\text { filters }=32, \quad \text { kernel }=(5,5), \\
\text { padding='same' }\end{array}$ & strides $=(2,2)$, \\
\hline \multicolumn{3}{|l|}{ Batch normalization } \\
\hline ReLu & activation & \\
\hline Conv2DTranspose (conv3) & $\begin{array}{l}\text { filters }=1, \quad \text { kernel }=(5,5), \\
\text { padding='same' }\end{array}$ & strides $=(2,2)$, \\
\hline \multicolumn{3}{|l|}{ Batch normalization } \\
\hline Reshape & shape $=(-1,784)$ & \\
\hline Dropout & probability $=0.3$ & \\
\hline Fully connected (FC2) & units $=800$ (motion data shape) & \\
\hline
\end{tabular}


Table 8.5: GAN discriminator parameters

\begin{tabular}{ll}
\hline Layer & Parameters \\
\hline Reshape & shape= $=(-1$, input dimensions, 1$)$ \\
Conv2D (conv1) & filters=64, kernel=(5,5), strides=(2,2), padding='same' \\
Leaky ReLu & activation \\
Dropout & probability=0.3 \\
Conv2D (conv2) & filter=128, kernel=(5,5), strides=(2,2), padding='same' \\
Leaky ReLu & activation \\
Dropout & probability=0.3 \\
Fully connected (FC3) & units=1 (class) \\
\hline
\end{tabular}

\subsubsection{Generator Input and Output Modes}

GAN training is commonly done by feeding the generator network input vector of some random values $\mathbf{z}$ [99] [106]. This makes it difficult to determine a priori the mapping of each input feature to the properties of the synthesized output $G_{\theta}(\mathbf{z})$. The process has to be done in reverse; by observing the variations on $G_{\theta}(\mathbf{z})$ and the values of $\mathbf{z}$, then the user can try to find the relationship between each input feature $z_{i}$ to the properties of the output. Even when one relationship is found in one model, there is no guarantee that the next trained model will produce the same relationship. What we are able to see is that certain combinations of values of $z_{i}$ and their relative values with each other create certain patterns. In a way, this is similar to the issue with the latent variables $\mathbf{z}$ of the VAE.

Generative models such as GAN also prone to the generator only producing one 'best' pattern of output that always minimize the loss. In the case of GAN, the pattern that always 'fool' the discriminator. This behavior is known as mode collapse [99] where 'mode' refers to the variety of generated pattern, or type of output.

Our goal is to avoid mode collapse and have high variety of output pattern as possible. Additionally, we want that each $z_{i} \in \mathbf{z}$ has some meaningful interpretation 
to some properties of $G_{\theta}(\mathbf{z})$. We built several models with $\mathbf{z}$ of size two, four, and eight.

\subsubsection{Results}

Our GAN motion generator model was able to produce data that appear to have similar properties as the motion data in our dataset. In particular, the generated data have distinguishable low frequency components and not dominated by high frequency parts, compared to the raw results from the VAE model. The generated data do appear to have a small amount of noise. However, the data produced often do not immediately correspond to any meaningful behaviors or gestures.

The cost function used was the sigmoid cross entropy, which seems to help the generator not stuck on producing the same output (mode collapse).

We trained three GAN models with the input to the generator $\mathbf{z}$ with three sizes: $|\mathbf{z}| \in\{2,4,8\}$. By using $\mathbf{z}$ with smaller sizes, we aim to find correlation between each $z_{i}$ and how they affect the generated data. Fig. 8.20 shows 16 samples of the generated output at epoch 100 during training for $|\mathbf{z}|=2$; similarly for Fig.8.21 and 8.22. In these figures, the grayscale boxes are the representations of $\mathbf{z}$ where grey levels indicate the range of values, where 0 : black, 1 : white. The values of $\mathbf{z}$ are randomized for each of the 16 samples. The waveform plots next to the squares are the generated data which correspond to motion data.

We can see in some of the figures above that certain patterns of $\mathbf{z}$ will create output data that have similar shapes. For example, in the first column of Fig. 8.20 has $z_{1}$ (top square) with lower value than $z_{2}$ (bottom square) which generates data with large undulations. As the value of $z_{1}$ gets lower (darker), the amplitude of the left side of the signal increases while the right side decreases. This pattern is also visible in other similar values of z's in Fig. 8.20. Meanwhile, when $z_{2}$ is lower than 
Epoch: 100

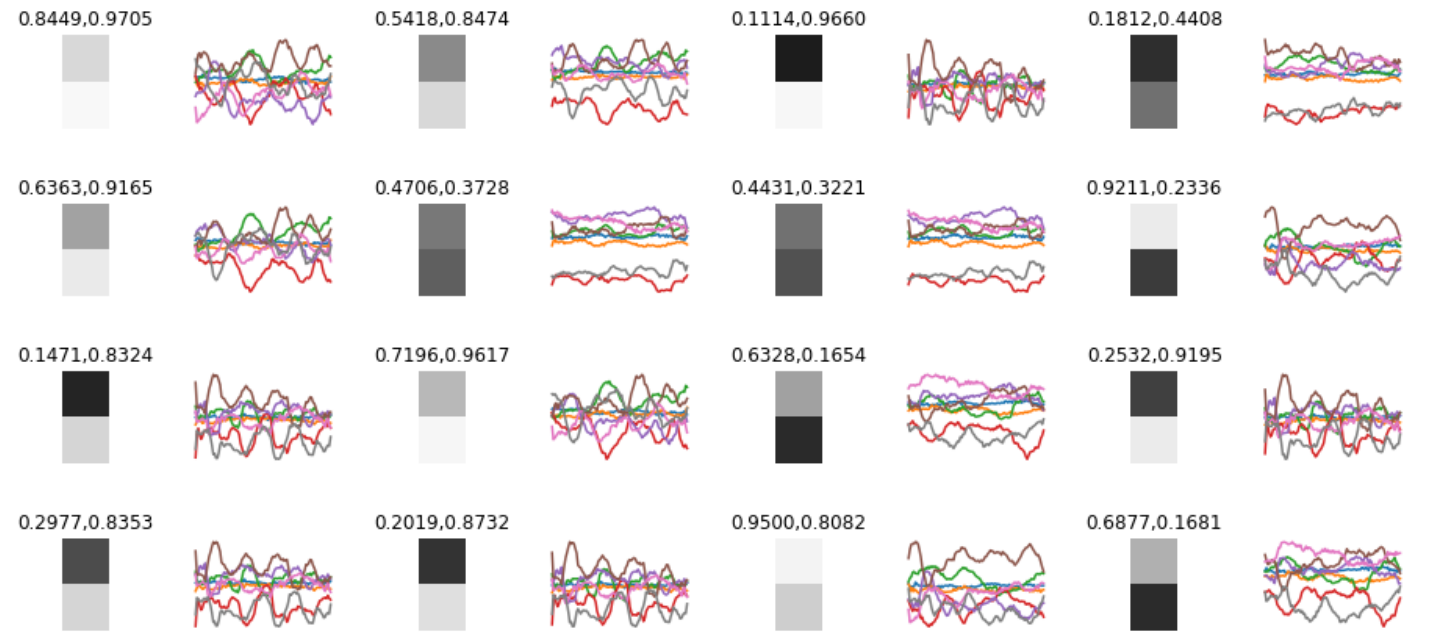

Figure 8.20: Samples of generator output with $|\mathbf{z}|=2$ and random values of $z_{i}$ during training at epoch 100.

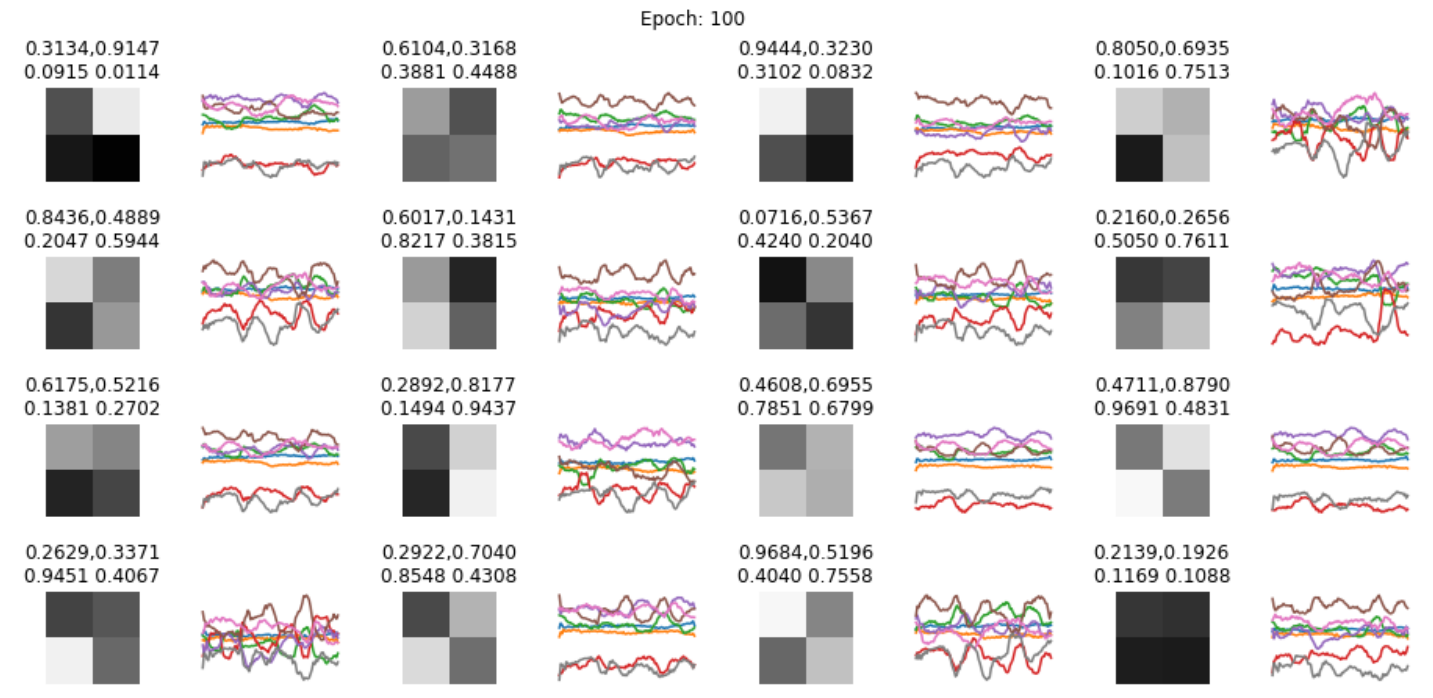

Figure 8.21: Samples of generator output with $|\mathbf{z}|=4$ and random values of $z_{i}$ during training at epoch 100 . 
Epoch: 100
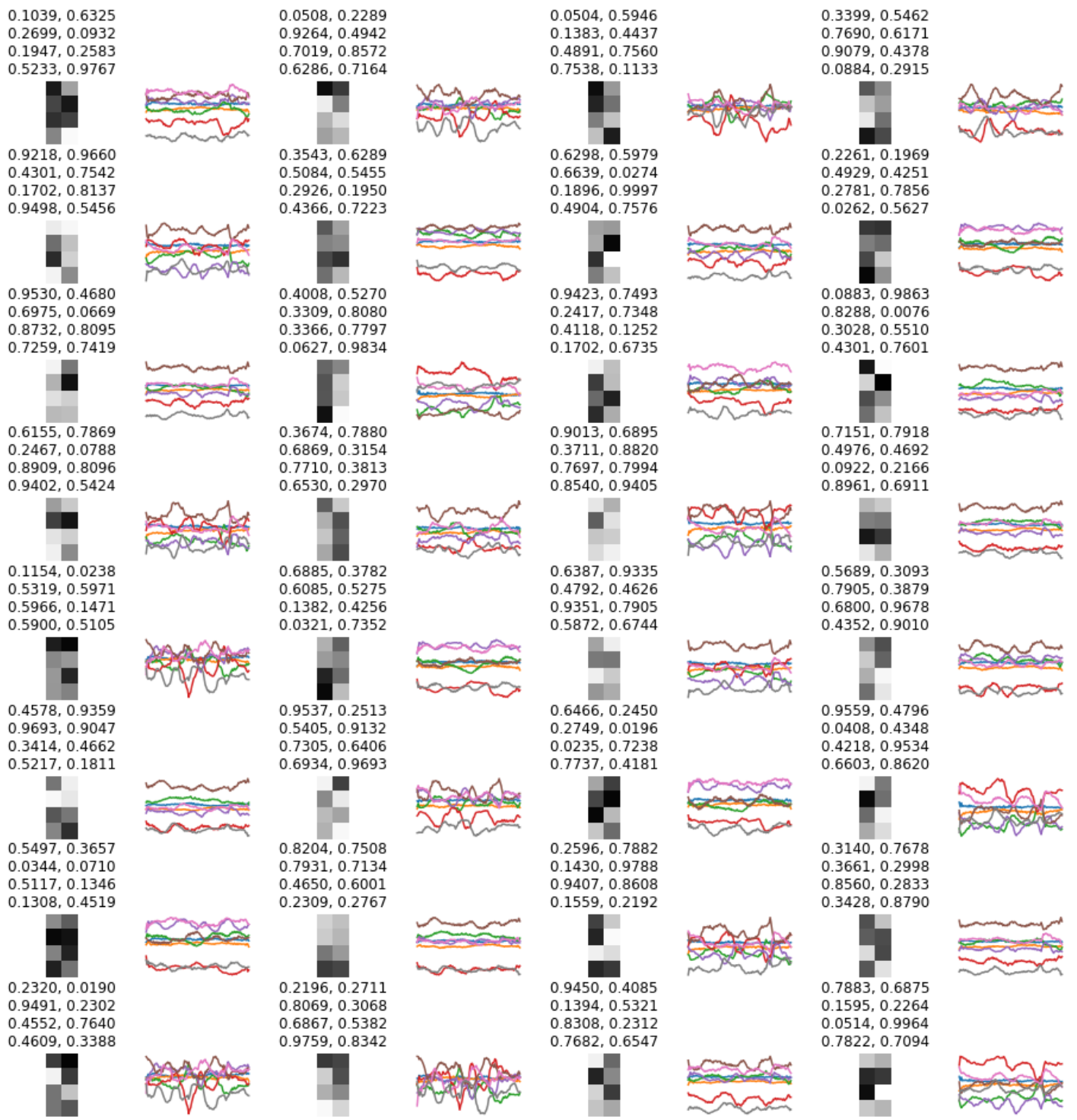

Figure 8.22: Samples of generator output with $|\mathbf{z}|=8$ and random values of $z_{i}$ during training at epoch 100. 
$z_{1}$, the output signal appear to have smaller and fewer large undulations. Fig. 8.23 shows the variations of the generated data when varying $z_{1}$ and $z_{2}$ gradually. The columns represent the range of $z_{1}$, and the rows for $z_{2}$. If the effects of each $z_{i}$ is well understood, then $\mathbf{z}$ can be used as parameters to generate the types of motion desired by the user.

The effects of $\mathbf{z}$ values become more difficult to analyze in the models with $\mathbf{z}=4$ and $\mathbf{z}=8$. However, with increasing number of $|\mathbf{z}|$ there are more variations of the generated data (motion).

In all the GAN models we have trained, the generated signals appear slightly noisy, but with much lower noise than the output of the VAE decoder. Fig. 8.24 shows the closeups of two examples of the generated output.

Video demonstrations of some of the motion samples produced by the GAN model (Fig. 8.25, Fig. 8.26) can be viewed on the internet: https : //youtu . be/Km0x3jNr45k

\subsection{Evaluation of Motion Quality}

To evaluate the quality of the motions produced by these three motion generators, motion data produced from the generators are collected and given to our trained motion evaluators presented in Chapter 7. The evaluation results are given in Table 8.6 for the REBeL motion data with motion texturing (including KB interpolation and wavelets), Table 8.7 for GAN-generated motion data, and Table 8.8 for VAEgenerated motion data. All evaluations are done as test data on fully trained models.

Note that this evaluation is still experimental particularly due to the relatively small number of generated samples and more rigorous tests should be done in the future. The evaluation of the REBeL data (Table 8.6) uses only REBeL data as the test data and the goal is to check if these motions possess qualities of human motions 


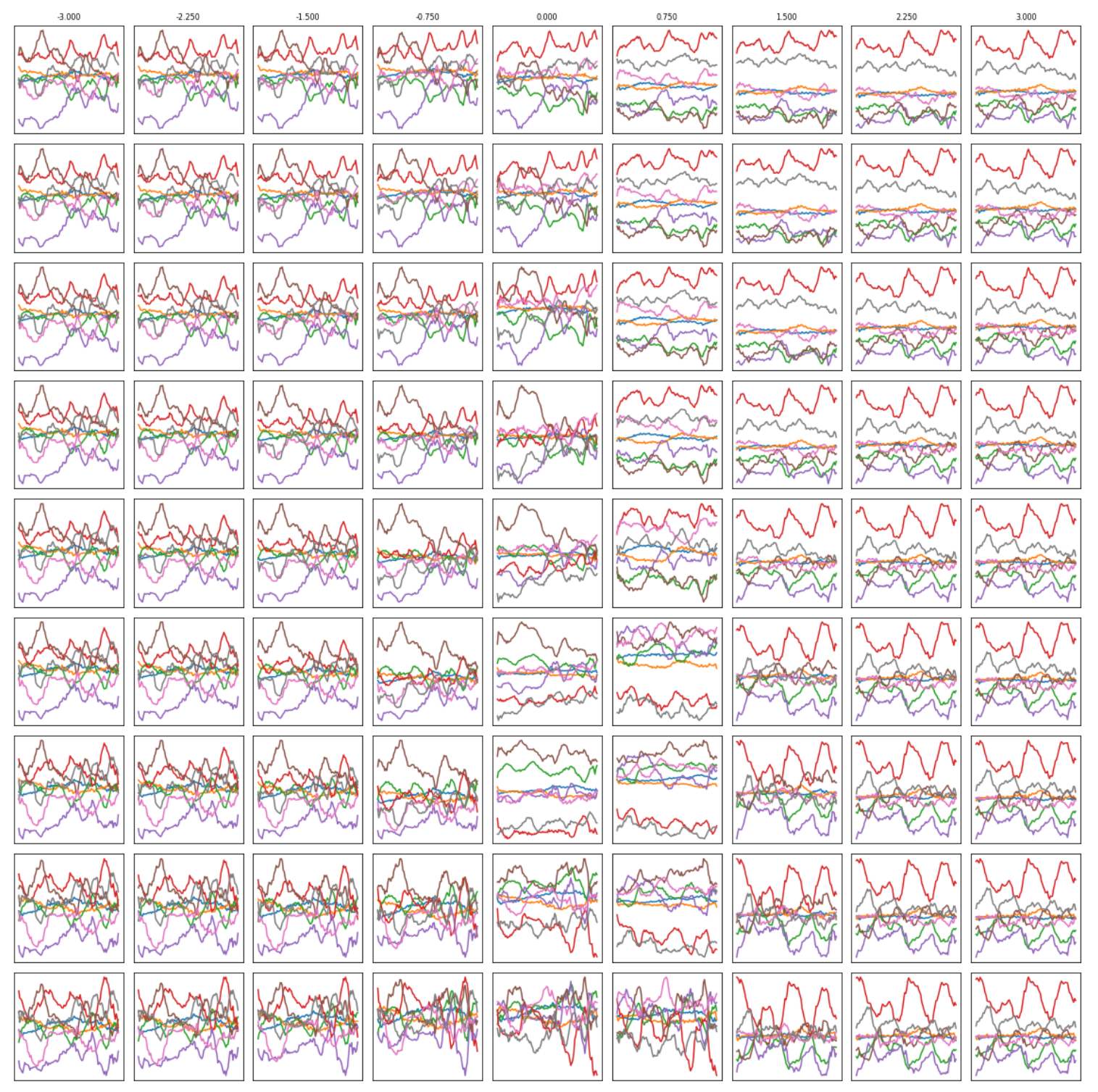

Figure 8.23: Variations of output of the GAN generator by varying the values of $\mathbf{z}$ where $|\mathbf{z}|=2$. The columns represent the range of $z_{1}$, and the rows for $z_{2}$. 

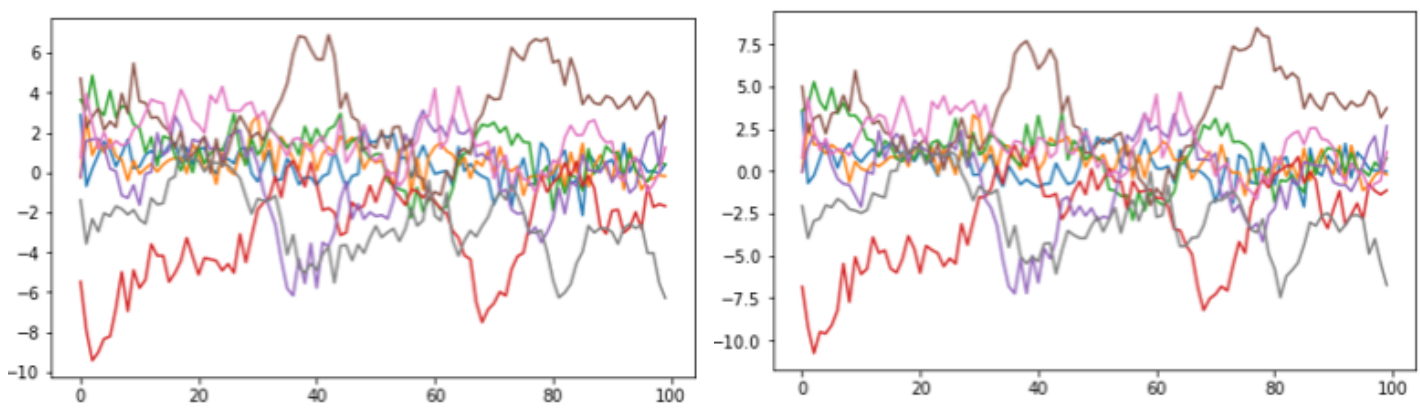

Figure 8.24: Close up of some samples generated by the GAN generator.
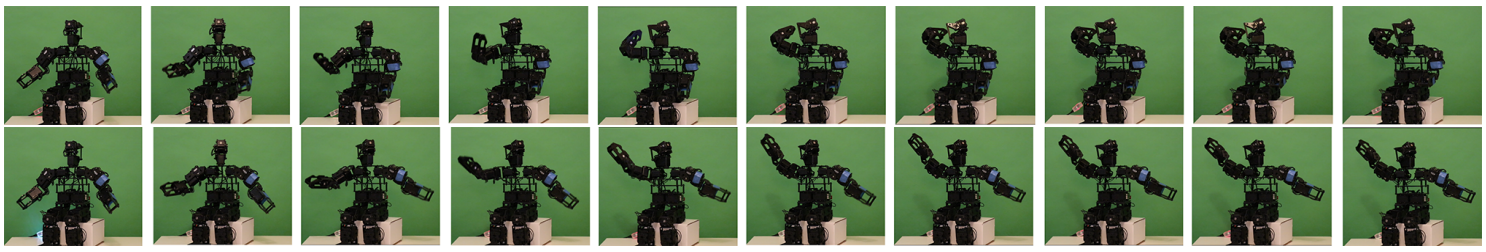

Figure 8.25: Two samples of gestural motions produced by the GAN motion generator model (Top and bottom). Each sample consists of 100 frames; each pose shown here are taken one for every 10 poses.

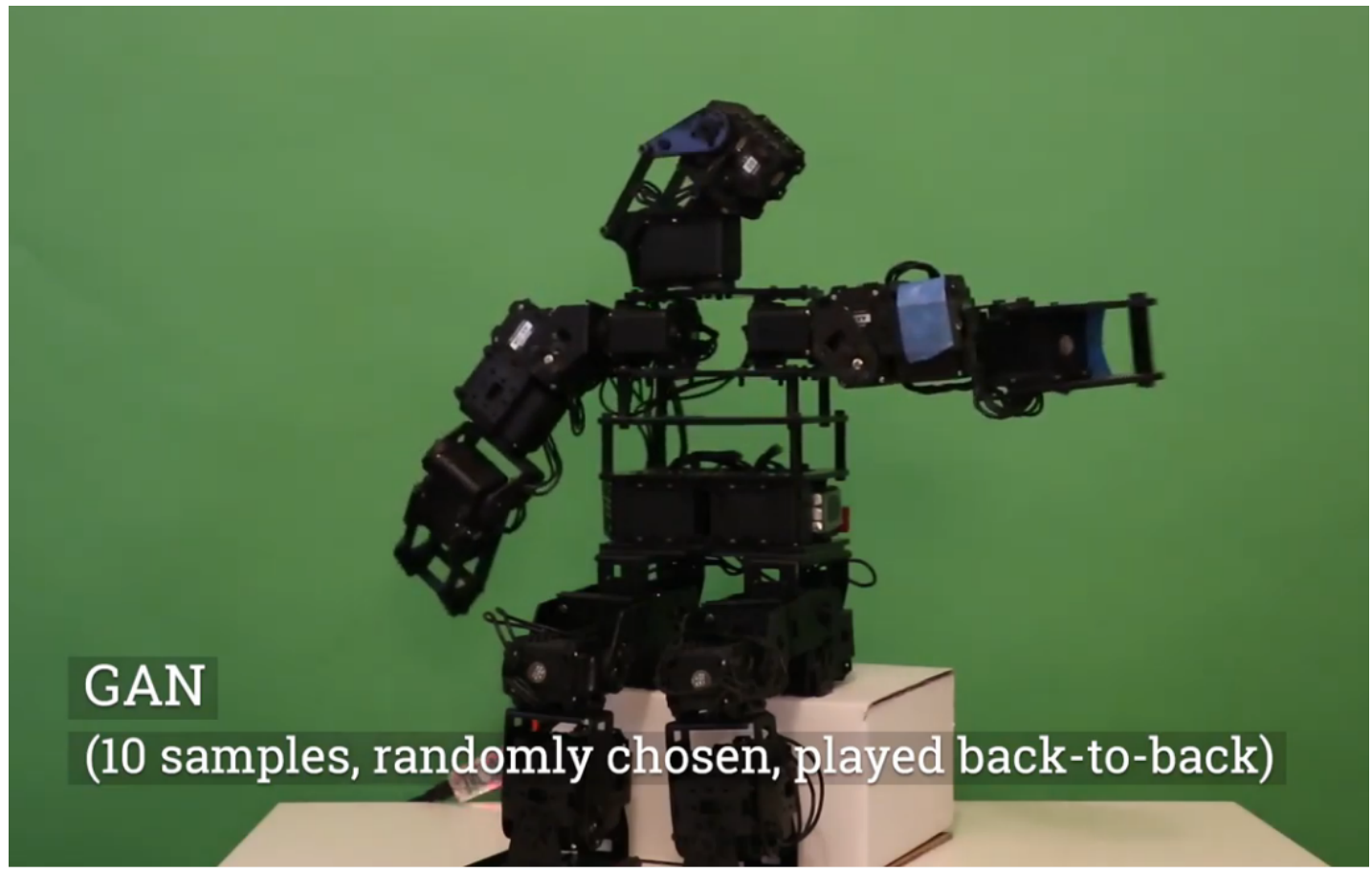

Figure 8.26: Video demonstrations of the motion samples produced by the GAN model (https://youtu.be/Km0x3jNr45k). 
as captured by a motion capture system. The results show that only about $78 \%$ are deemed 'good' (classified as the "motion capture"-class) by the decision tree model, almost $90 \%$ are deemed 'good' by the SVM model, and only about $61 \%$ are 'good' according to the Naïve Bayes model. This result is consistent with our expectations; some motion textures may make the motions appear more natural, but some textures are creating effects that never occur in human motion capture data.

The GAN and VAE results are evaluated using datasets that contain roughly about $50 \%$ human motion capture data [198] and 50\% of generated data of the GAN (50/48 split) and VAE model, respectively. Therefore, we expect that if the generated data have the qualities of motion capture data, the accuracy of the evaluation should be around $50 \%$ on average. For the GAN-generated dataset, the results in Table 8.7 show that the accuracy of the evaluation averaged at $53.94 \%$ with only $8.3 \%$ of the GAN samples considered having 'bad' quality by the Decision Tree model, the SVM model considers $100 \%$ of the GAN-generated data as 'good', while only 1 sample was considered 'bad' by the Naïve Bayes model. Meanwhile, the results for the VAEgenerated dataset as shown in Table 8.8 shows an average of $51.54 \%$ accuracy for all three evaluator models, All these results between the REBeL dataset, GAN dataset, and VAE dataset as evaluated by the motion evaluator models are encouraging and indicates that:

- Motion texturing can help robot motions to have similar qualities as human motion capture samples.

- The three motion generator models presented here can produce motion data that have similar qualities as human motion capture samples.

We do notice that some of the samples produced either by the VAE or GAN models tend to only consist of few types of gestures. For example, in every batch of 


\begin{tabular}{c|c|c|c|c|c}
\hline Model & \% Accuracy & TP & TN & FP & FN \\
\hline Dec. Tree & 22.14 & 0 & 401 & 1410 & 0 \\
\hline SVM & 9.55 & 0 & 173 & 1638 & 0 \\
\hline Naïve Bayes & 38.6 & 0 & 699 & 1112 & 0 \\
\hline
\end{tabular}

Table 8.6: Evaluation of motion data generated by REBeL with additional texturing (TP: True Positive, TN: True Negative, FP: False Positive, FN: False Negative). Dataset: 0 motion capture samples, 1811 REBeL samples.

\begin{tabular}{c|c|c|c|c|c}
\hline Model & \% Accuracy & TP & TN & FP & FN \\
\hline Dec. Tree & 57.88 & 48 & 4 & 44 & 2 \\
\hline SVM & 56.0 & 50 & 0 & 48 & 0 \\
\hline Naïve Bayes & 47.98 & 47 & 1 & 47 & 3 \\
\hline
\end{tabular}

Table 8.7: Evaluation of motion data generated by GAN motion generator (TP: True Positive, TN: True Negative, FP: False Positive, FN: False Negative). Dataset consists of 50 motion capture samples and 48 GAN-generated samples.

samples that was generated by the GAN motion generator ${ }^{2}$, about $50 \%$ of samples produced many samples where the head is turned to the left, the right arm of the robot is raised up and bent at the elbow as if scratching the head, and the left arm is lowered with a slight bend at the elbow (Fig. 8.25 top). In each of those samples, the position of the head and arms may deviate slightly, but the overall pose is similar. So, the GAN model was able to produce a gesture with some variations.

These results give us the confidence that the generator models work reasonably well in producing motion data that come from the same distribution as human motion

${ }^{2}$ We tested generating with batches of 8, 16, 32 samples per batch.

\begin{tabular}{c|c|c|c|c|c}
\hline Model & \% Accuracy & TP & TN & FP & FN \\
\hline Dec. Tree & 55.56 & 316 & 13 & 306 & 4 \\
\hline SVM & 56.18 & 320 & 0 & 319 & 0 \\
\hline Naïve Bayes & 48.87 & 304 & 6 & 313 & 16 \\
\hline
\end{tabular}

Table 8.8: Evaluation of motion data generated by VAE motion generator (TP: True Positive, TN: True Negative, FP: False Positive, FN: False Negative). Dataset consists of 320 motion capture samples and 319 VAE-generated samples. 
data.

In addition, we found that generator models can work cross-domain; models for generating one type of dataset (images) also worked well for generating motion data: our VAE and GAN models were based on architectures that were originally used and trained to produce samples resembling the MNIST [214] and CIFAR image data [106] and Belgian street sign datasets [217].

\subsection{Contributions}

My contributions in relation to the work presented in this chapter are as follows:

- The presented work is the first in literature to introduce using generative models to generate novel robot motion data that do not exist in the training data or from prior knowledge. Other works have demonstrated using generative models to produce new images, music segments, and texts, but generation of robot motion data is novel to the best of my knowledge.

- Demonstrated motion generation of gesture-like motions can be achieved using three different models: a LSTM network, a VAE network, and a GAN network.

- Introduced a new method to discretize continuous-valued data using wavelets which results in improved quality of the generated motion data with the LSTM network.

- Showed that using transposed convolution layers [215], the VAE and GAN motion generators produced more stable results than using only fully-connected, feedforward layers. The motions produced by the VAE and GAN models using the transposed convolution layers exhibit properties in human motion capture data: interesting variety of trajectories, low amount of high frequency noise, 
and relatively smooth progressions of motion trajectories. In contrast, using only fully-connected, feedforward network produces motion data that have large amount of high frequency noise. 


\section{CHAPTER 9 \\ CONCLUSIONS}

In this dissertation I presented several new ideas and contributions for expressive motion generation for humanoid robots.

- Robot Expressive Behavior Language (REBeL) - REBeL is a tool to compose behaviors for robots or other agents by defining the behaviors using algebraic expressions with probabilistic algebraic operators called behavior expressions. I extended operators from regular expressions such as concatenation, union, and repetition by adding probabilistic behaviors for the operators in behavior expressions. Additionally, I introduced new operators such as subtraction and concurrency. All these operators can be used to compose more complex robot behaviors as behavior expressions support hierarchical composition and reusing other behaviors. Behavior expressions provide composition features that are difficult or impossible to do in other robot motion editor tools. I demonstrated behavior expressions using several examples of composing complex robot behaviors. The main limitations of using behavior expressions in REBeL is its reliance of existing behaviors and inability to specify timing information. Currently, all timing information are hard-coded values. The former limitation is not severe as simple gestures can be done easily using common robot motion editors. To address the latter limitation, I introduced other methods using music information and interpolation parameters. This work was accepted at the International Symposium on Multi-Valued Logic $2020^{1}$.

\footnotetext{
${ }^{1}$ No citation entry yet.
} 
- Music to Motion - using music information to specify the timing for executing the behaviors composed using behavior expressions. Timing information affects pauses and speed, and ultimately influences the expressive quality of the executed motions. I demonstrated that using timing information from music as timing information for motion can make motions appear more dynamic and expressive without requiring manual work from the user. Data were obtained using standard methods of surveys and questionnaires [21][6], and the hypothesis tested using multiple methods of multiple-measure ANOVA and Friedman tests. Post-hoc analysis were also performed using Scheffe and Wilcoxon SignedRank tests to find the significantly different ranked categories. This work has been published in the Internatonal Journal of Social Robotics in 2018 [130].

- Motion Texturing - using wavelets as parameters to create expressive execution of gestures and robot motion. I extended the work of Burderlin and Williams [13] and showed that using the combination of Kochanek-Bartels interpolation and wavelet analysis various motion textures such as stuttering, shaking, laziness, followthrough, exaggeration, etc. can be realized by using a few set of parameters of the KB interpolation and levels of filtering using differnt wavelet kernels. This work is unique by experimenting with established methods of $\mathrm{KB}$ interpolation and wavelet analysis and found various motion textures that can be created, where in contrast, previous works develop custom equations of motions to produce a specific set of motion textures [14] [12].

- Evaluating quality of robot motion - Several classifier models were trained to discriminate betwen motion capture data and robot motion data, and act as motion evaluators: LSTM network, decision tree, naïve Bayes, and support vector machine $(\mathrm{SVM})$. Included in this work is the creation of a new motion 
dataset by combining motion capture data from [198] and existing robot motion data for the HROS-1 robot to train the motion evaluator. The trained motion evaluators are able to achieve over $98 \%$ accuracy on average, and also tested against motion data generated by REBeL's behavior expressions with motion textures added, and motion data generated by our motion generator models (Chapter 8). Our results show that using motion evaluators have the potential to be a viable alternative to subjective evaluation methods (e.g. surveys, interviews, questionnaires) when evaluating qualities of robot motions for social and entertainment robots.

- Automatic Robot Gesture Generation - three generative models were built as generator of motion data: a LSTM network, a variational auto encoder (VAE) model, and a generative adversarial network (GAN) that was based on the DCGAN architecture. All three models are able to produce novel motion data that have similar characteristics as the samples of motion capture data (continuous progressions, no sudden jerks, no jitter) while not merely reproducing the samples from the training dataset. Some notable gestures were produced: "shy" and a few deictic gestures. Currently in the three models that I have built, there is no human-readable way to condition the generators to produce a specific output. I plan to investigate this in future works.

The contributions that have been presented in this dissertation are focused on novel ways to produce expressive gestures and motions for humanoid robots in the social and entertainment robotics field (e.g. robot theater). The mission of this work is to reduce the amount of manual work a user have to do to create expressive humanoid robot motions when using the current tools and methods. The vision of my work is ultimately: an intelligent, autonomous system that can help future humanoid 
robots produce their own expressive communicative gestures in any social situation, with no or minimal involvement of human artists in the creation of their gestures. 


\section{REFERENCES}

[1] C. Breazeal, C. D. Kidd, A. L. Thomaz, G. Hoffman, and M. Berlin, "Effects of nonverbal communication on efficiency and robustness in human-robot teamwork," in 2005 IEEE/RSJ international conference on intelligent robots and systems, IEEE, 2005, pp. 708-713.

[2] R. Kirby, J. Forlizzi, and R. Simmons, "Affective social robots," Robotics and Autonomous Systems, vol. 58, no. 3, pp. 322-332, 2010.

[3] K. Dautenhahn, "Roles and functions of robots in human society: implications from research in autism therapy," Robotica, vol. 21, no. 4, S0263574703004922, 2003.

[4] C. L. Sidner, C. Lee, C. Kidd, N. Lesh, and C. Rich, "Explorations in engagement for humans and robots," arXiv preprint cs/0507056, 2005.

[5] W. A. Bainbridge, J. Hart, E. S. Kim, and B. Scassellati, "The effect of presence on human-robot interaction," in RO-MAN 2008-The 17th IEEE International Symposium on Robot and Human Interactive Communication, IEEE, 2008, pp. 701-706.

[6] L. Takayama, D. Dooley, and W. Ju, "Expressing thought: improving robot readability with animation principles," Proceedings of the 6th ACM/IEEE International Conference on Human-Robot Interaction (HRI), pp. 69-76, 2011.

[7] C. Breazeal, "Sociable machines: Expressive social exchange between humans and robots," PhD Thesis, p. 264, 2000.

[8] C. Breazeal, "Role of expressive behaviour for robots that learn from people," Philosophical Transactions of the Royal Society B: Biological Sciences, vol. 364, no. 1535, pp. 3527-3538, 2009.

[9] G. Hoffman and G. Weinberg, "Interactive improvisation with a robotic marimba player," Autonomous Robots, vol. 31, no. 2-3, pp. 133-153, 2011.

[10] T. Poanessa, S. Hakkoum, and B. Dunn, "Robot Theater Final Report," Portland State University, Department of Electrical and Computer Engineering, Tech. Rep., 2017. 
[11] K. Perlin and A. Goldberg, "Improv: A system for scripting interactive actors in virtual worlds," in Proceedings of the 23rd annual conference on Computer graphics and interactive techniques, 1996, pp. 205-216.

[12] H. Knight and R. Simmons, "Laban head-motions convey robot state: A call for robot body language," Proceedings - IEEE International Conference on Robotics and Automation, vol. 2016-June, pp. 2881-2888, 2016.

[13] A. Bruderlin and L. Williams, "Motion signal processing," in Proceedings of the 22nd annual conference on Computer graphics and interactive techniques, 1995, pp. 97-104.

[14] D. Chi, M. Costa, L. Zhao, and N. Badler, "The EMOTE model for effort and shape," in Proceedings of the 27th annual conference on Computer graphics and interactive techniques - SIGGRAPH '00, New York, New York, USA: ACM Press, 2000, pp. 173-182, ISBN: 1581132085.

[15] K. Granat, Rme, https ://github . com/Interbotix/HROS1-Framework / wiki/rme, [Online; accessed 21-May-2019], 2015.

[16] Aldebaran, Choreographe suite, http://doc.aldebaran.com/2-4/software/ choregraphe/index.html, 2020.

[17] A. J. van Breemen, "Animation engine for believable interactive user-interface robots," in 2004 IEEE/RSJ International Conference on Intelligent Robots and Systems (IROS)(IEEE Cat. No. 04CH37566), IEEE, vol. 3, 2004, pp. 28732878.

[18] T. Fong, I. Nourbakhsh, and K. Dautenhahn, "A Survey of Socially Interactive Robots: Concepts, Design, and Applications," Robotics and Autonomous Systems, vol. 42, no. 3, pp. 143-166, 2003. arXiv: arXiv:1011.1669v3.

[19] J. Cassell, "Embodied conversational interface agents," Communications of the $A C M$, vol. 43, no. 4, pp. 70-78, 2002.

[20] R. W. Picard, Affective computing. MIT press, 2000.

[21] C. Bartneck, J. Reichenbach, and A. V. Breemen, "In your face, robot! The influence of a character's embodiment on how users perceive its emotional expressions," In design and emotion 2004 conference, no. 2004, pp. 1-19, 2004. 
[22] a. Weiss, J. Igelsbock, M. Tscheligi, A. Bauer, K. Kuhnlenz, D. Wollherr, and M. Buss, "Robots asking for directions - The willingness of passers-by to support robots," Human-Robot Interaction (HRI), 2010 5th ACM/IEEE International Conference on, pp. 23-30, 2010.

[23] A. Bruce, I. Nourbakhsh, and R. Simmons, "The Role of Expressiveness and Attention in Human-Robot Interaction," Tech. Rep., 2001.

[24] G. Hoffman and C. Breazeal, "Effects of anticipatory action on human-robot teamwork efficiency, fluency, and perception of team," in Proceedings of the ACM/IEEE international conference on Human-robot interaction, 2007, pp. 18.

[25] A. v. d. Oord, S. Dieleman, H. Zen, K. Simonyan, O. Vinyals, A. Graves, N. Kalchbrenner, A. Senior, and K. Kavukcuoglu, "Wavenet: A generative model for raw audio," arXiv preprint arXiv:1609.03499, 2016.

[26] D. Adiwardana, M.-T. Luong, D. R. So, J. Hall, N. Fiedel, R. Thoppilan, Z. Yang, A. Kulshreshtha, G. Nemade, Y. Lu, et al., "Towards a human-like open-domain chatbot," arXiv preprint arXiv:2001.09977, 2020.

[27] B. Robins, K. Dautenhahn, and P. Dickerson, "From isolation to communication: A case study evaluation of robot assisted play for children with autism with a minimally expressive humanoid robot," in 2009 Second International Conferences on Advances in Computer-Human Interactions, IEEE, 2009, pp. 205-211.

[28] K. Strabala, M. K. Lee, A. Dragan, J. Forlizzi, S. S. Srinivasa, M. Cakmak, and V. Micelli, "Toward seamless human-robot handovers," Journal of HumanRobot Interaction, vol. 2, no. 1, pp. 112-132, 2013.

[29] T. Geijtenbeek, M. Van De Panne, and A. F. Van Der Stappen, "Flexible muscle-based locomotion for bipedal creatures," ACM Transactions on Graphics (TOG), vol. 32, no. 6, pp. 1-11, 2013.

[30] T. McGeer et al., "Passive dynamic walking," I. J. Robotic Res., vol. 9, no. 2, pp. 62-82, 1990.

[31] M. Raibert, K. Blankespoor, G. Nelson, R. Playter, and B. Team, "BigDog, the Rough-Terrain Quadruped Robot," 2008. 
[32] Z. Xie, G. Berseth, P. Clary, J. Hurst, and M. van de Panne, "Feedback control for cassie with deep reinforcement learning," in 2018 IEEE/RSJ International Conference on Intelligent Robots and Systems (IROS), IEEE, 2018, pp. 12411246 .

[33] U. Saranli, A. A. Rizzi, and D. E. Koditschek, "Model-based dynamic selfrighting maneuvers for a hexapedal robot," The International Journal of Robotics Research, vol. 23, no. 9, pp. 903-918, 2004.

[34] N. Morozovsky and T. Bewley, "Skysweeper: A low dof, dynamic high wire robot," in 2013 IEEE/RSJ International Conference on Intelligent Robots and Systems, IEEE, 2013, pp. 2339-2344.

[35] B. J. Stephens and C. G. Atkeson, "Dynamic balance force control for compliant humanoid robots," in 2010 IEEE/RSJ international conference on intelligent robots and systems, IEEE, 2010, pp. 1248-1255.

[36] J. J. Kuffner, S. Kagami, K. Nishiwaki, M. Inaba, and H. Inoue, "Dynamicallystable motion planning for humanoid robots," Autonomous robots, vol. 12, no. 1, pp. 105-118, 2002.

[37] T. Ribeiro and A. Paiva, "The illusion of robotic life," Proceedings of the seventh annual ACM/IEEE international conference on Human-Robot Interaction - HRI '12, no. 1937, p. 383, 2012.

[38] S. Kopp, P. A. Tepper, K. Ferriman, K. Striegnitz, and J. Cassell, "Trading Spaces: How Humans and Humanoids Use Speech and Gesture to Give Directions," Conversational Informatics: An Engineering Approach, pp. 133-160, 2007.

[39] A. D. Dragan, S. Bauman, J. Forlizzi, and S. S. Srinivasa, "Effects of robot motion on human-robot collaboration," in 2015 10th ACM/IEEE International Conference on Human-Robot Interaction (HRI), IEEE, 2015, pp. 51-58.

[40] M. Heerink, B. Kröse, V. Evers, and B. Wielinga, "Measuring acceptance of an assistive social robot: A suggested toolkit," Proceedings - IEEE International Workshop on Robot and Human Interactive Communication, pp. 528533, 2009.

[41] M. Saerbeck and A. J. N. Van Breemen, "Design guidelines and tools for creating believable motion for personal robots," in Proceedings - IEEE Inter- 
national Workshop on Robot and Human Interactive Communication, IEEE, 2007, pp. 386-391, ISBN: 1424416345.

[42] R. Laban and F. C. Lawrence, Effort: economy of human movement. Macdonald \& Evans, 1979.

[43] O. Johnston and F. Thomas, The illusion of life: Disney animation. Disney Editions New York, 1981.

[44] H. Knight, "Eight lessons learned about non-verbal interactions through robot theater," Lecture Notes in Computer Science (including subseries Lecture Notes in Artificial Intelligence and Lecture Notes in Bioinformatics), vol. 7072 LNAI, pp. $42-51,2011$.

[45] J. Lasseter, "Principles of traditional animation applied to 3d computer animation," in Proceedings of the 14th annual conference on Computer graphics and interactive techniques, 1987, pp. 35-44.

[46] M. Eadweard and M. A. Ventura, "Muybridge's complete human and animal locomotion: All 781 plates from the 1887 animal locomotion [m]," Ed. Anita Ventura Mozley. Mineola: Dover, 1979.

[47] R. Williams, The animator's survival kit: a manual of methods, principles and formulas for classical, computer, games, stop motion and internet animators. Macmillan, 2012.

[48] R. Parent, Computer animation: algorithms and techniques. Newnes, 2012.

[49] WIRED, How animators created the spider-verse, https : / / www . youtube . com/watch?v=1-wUKu_V2Lk, [Online; accessed 20-January-2020], 2019.

[50] N. Y. F. Academy. (2015). Stage vs. screen: What's the big difference? Accessed: 2020-04-23.

[51] 1. Kondo Kagaku co., Hearttoheart4, https://kondo-robot .com/product/ hearttoheart4, [Online; accessed 21-May-2019], 2020.

[52] Lynxmotion, Lynxmotion visual sequencer (seq-01), http: //www . lynxmotion . com/p-443-lynxmotion-visual-sequencer-seq-01.aspx, [Online; accessed 21-May-2019], 2020. 
[53] ROBOTIS, $R+$ motion 1.0, http : / / emanual . robotis . com / docs / en / software/rplus1/motion/, [Online; accessed 21-May-2019], 2020.

[54] ROBOTIS, Robotis op3, http://emanual.robotis.com/docs/en/platform/ op3/tutorials, [Online; accessed 21-May-2019], 2020.

[55] R3n33, Winrme, http://blog.trossenrobotics.com/2015/09/02/winrme/, [Online; accessed 21-February-2020], 2015.

[56] LEGO, Lego mindstorms ev3, https : / / www . lego . com / en-us / themes / mindstorms/about, [Online; accessed 21-April-2020], 2020.

[57] Pololu, Maestro usb servo controllers, https://www.pololu.com/category/ 102 / maestro-usb-servo-controllers, [Online; accessed 21-April-2020], 2020 .

[58] A. Nakazawa, S. Nakaoka, and K. Ikeuchi, "Synthesize stylistic human motion from examples," pp. 3899-3904, 2004.

[59] S. Nakaoka, S. Kajita, and K. Yokoi, "Intuitive and flexible user interface for creating whole body motions of biped humanoid robots," in 2010 IEEE/ RSJ International Conference on Intelligent Robots and Systems, IEEE, 2010, pp. $1675-1682$.

[60] Y. Kuroki, B. Blank, T. Mikami, P. Mayeux, A. Miyamoto, R. Playter, K. Nagasaka, M. Raibert, M. Nagano, and J. Yamaguchi, "Motion creating system for a small biped entertainment robot," in Proceedings 2003 IEEE/RSJ International Conference on Intelligent Robots and Systems (IROS 2003)(Cat. No. 03CH37453), IEEE, vol. 2, 2003, pp. 1394-1399.

[61] G. Pierris and M. G. Lagoudakis, "An interactive tool for designing complex robot motion patterns," in 2009 IEEE International Conference on Robotics and Automation, IEEE, 2009, pp. 4013-4018.

[62] A. Van Breemen and Y. Xue, "Advanced animation engine for user-interface robots," in 2006 IEEE/RSJ International Conference on Intelligent Robots and Systems, IEEE, 2006, pp. 1824-1830.

[63] M. Kamermans, A primer on bézier curves, https : //pomax . github.io/ bezierinfo/, [Online; accessed 23-June-2019], 2018. 
[64] K. Perlin, "An image synthesizer," ACM Siggraph Computer Graphics, vol. 19, no. 3, pp. 287-296, 1985.

[65] B. De Carolis, C. Pelachaud, I. Poggi, and M. Steedman, "Apml, a markup language for believable behavior generation," in Life-like characters, Springer, 2004, pp. 65-85.

[66] M. Giuliani, "MultiML - A General Purpose Representation Language for Multimodal Human Utterances," pp. 165-172, 2008.

[67] S. Kopp, B. Krenn, S. Marsella, A. N. Marshall, C. Pelachaud, H. Pirker, K. R. Thórisson, and H. Vilhjálmsson, "Towards a common framework for multimodal generation: The behavior markup language," in International workshop on intelligent virtual agents, Springer, 2006, pp. 205-217.

[68] A. Kranstedt and S. Kopp, "MURML : A Multimodal Utterance Representation Markup Language for Conversational Agents," AAMAS'02 Workshop Embodied conversational agents - let's specify and evaluate them!., pp. 5-10, 2002.

[69] J. Gratch, J. Rickel, E. Andre, N. Badler, J. Cassell, and E. Petajan, "Creating virtual humans: Some assembly required," IEEE Intelligent Systems, no. July, 2002 .

[70] D. Reidsma, H. Van Welbergen, and J. Zwiers, "LNAI 6895 - Multimodal Plan Representation for Adaptable BML Scheduling," Tech. Rep., 2011, pp. 296308.

[71] J. Cassell, H. H. Vilhjálmsson, and T. Bickmore, "Beat: The behavior expression animation toolkit," in Life-Like Characters, Springer, 2004, pp. 163-185.

[72] J. Lee and S. Marsella, "Nonverbal behavior generator for embodied conversational agents," in International Workshop on Intelligent Virtual Agents, Springer, 2006, pp. 243-255.

[73] A. Hartholt, D. Traum, S. C. Marsella, A. Shapiro, G. Stratou, A. Leuski, L.-P. Morency, and J. Gratch, "All Together Now: Introducing the Virtual Human Toolkit," in 13th International Conference on Intelligent Virtual Agents, Edinburgh, UK, Aug. 2013. 
[74] Y. Xu, C. Pelachaud, and S. Marsella, "Compound Gesture Generation: A Model Based on Ideational Units," in Intelligent Virtual Agents, Boston, MA: Springer, Aug. 2014, pp. 477-491.

[75] P. Aggarwal and D. Traum, "The BML Sequencer: A Tool for Authoring Multicharacter Animations," in 11th International Conference on Intelligent Virtual Agents (IVA 2011), ReykjavÃk, Iceland, Sep. 2011.

[76] M. Neff, M. Kipp, I. Albrecht, and H.-P. Seidel, "Gesture modeling and animation based on a probabilistic re-creation of speaker style," ACM Transactions on Graphics (TOG), vol. 27, no. 1, pp. 1-24, 2008.

[77] A. Barré, Mokka - motion kinematic and kinetic analyzer, https://biomechanical-toolkit.github.io/mokka/, [Online; accessed 21-April-2020], 2013.

[78] B. Foundation, Home of the blender project - free and open 3d creation software, http://www. blender.org/, [Online; accessed 21-May-2019], 2020.

[79] A. Menache, Understanding motion capture for computer animation. Elsevier, 2011.

[80] J. Min, Y.-L. Chen, and J. Chai, "Interactive generation of human animation with deformable motion models," ACM Transactions on Graphics (TOG), vol. 29, no. 1, pp. 1-12, 2009.

[81] K. Yamane, J. K. Hodgins, and H. B. Brown, "Controlling a motorized marionette with human motion capture data," International Journal of Humanoid Robotics, vol. 1, no. 04, pp. 651-669, 2004.

[82] K. Yamane and J. Hodgins, "Simultaneous tracking and balancing of humanoid robots for imitating human motion capture data," in 2009 IEEE/RSJ International Conference on Intelligent Robots and Systems, IEEE, 2009, pp. 25102517.

[83] L. Kovar, M. Gleicher, and F. Pighin, "Motion Graphs," Tech. Rep., 2002.

[84] R. Heck and M. Gleicher, "Parametric motion graphs," in Proceedings of the 2007 symposium on Interactive 3D graphics and games, 2007, pp. 129-136.

[85] M. Unuma, K. Anjyo, and R. Takeuchi, "Fourier principles for emotion-based human figure animation," in Proceedings of the 22nd annual conference on Computer graphics and interactive techniques, 1995, pp. 91-96. 
[86] K. Amaya, A. Bruderlin, and T. Calvert, "Emotion from motion," in Graphics interface, Toronto, Canada, vol. 96, 1996, pp. 222-229.

[87] A. Witkin and M. Kass, "Spacetime constraints," ACM Siggraph Computer Graphics, vol. 22, no. 4, pp. 159-168, 1988.

[88] M. Gleicher, "Motion editing with spacetime constraints," in Proceedings of the 1997 symposium on Interactive 3D graphics, 1997, 139-ff.

[89] J. T. Ngo and J. Marks, "Spacetime constraints revisited," in Proceedings of the 20th annual conference on Computer graphics and interactive techniques, 1993, pp. 343-350.

[90] S. Hoshyari, H. Xu, E. Knoop, S. Coros, and M. Bächer, "Vibration-minimizing motion retargeting for robotic characters," ACM Transactions on Graphics (TOG), vol. 38, no. 4, pp. 1-14, 2019.

[91] S. Schaal, "Dynamic movement primitives-a framework for motor control in humans and humanoid robotics," in Adaptive motion of animals and machines, Springer, 2006, pp. 261-280.

[92] B. Lim, S. Ra, and F. C. Park, "Movement primitives, principal component analysis, and the efficient generation of natural motions," in Proceedings of the 2005 IEEE international conference on robotics and automation, IEEE, 2005, pp. $4630-4635$.

[93] K. Sims, "Evolving virtual creatures," in Proceedings of the 21st annual conference on Computer graphics and interactive techniques, 1994, pp. 15-22.

[94] V. Braitenberg, Vehicles: Experiments in synthetic psychology. MIT press, 1986.

[95] L. Kovar, M. Gleicher, and F. Pighin, "Motion graphs," in ACM SIGGRAPH 2008 classes, 2008, pp. 1-10.

[96] O. Arikan and D. A. Forsyth, "Interactive motion generation from examples," ACM Transactions on Graphics (TOG), vol. 21, no. 3, pp. 483-490, 2002.

[97] J Wang, D. Fleet, A Hertzmann, R Urtasun, A Geiger, J Popovic, T Darrell, N Lawrence, and P Fua, "Gaussian process models for human pose and motion," IEEE transactions on pattern analysis and machine intelligence, vol. 30, no. 2, pp. 283-298, 2008. 
[98] N. Heess, D. TB, S. Sriram, J. Lemmon, J. Merel, G. Wayne, Y. Tassa, T. Erez, Z. Wang, S. Eslami, et al., "Emergence of locomotion behaviours in rich environments," arXiv preprint arXiv:1707.02286, 2017.

[99] I. Goodfellow, J. Pouget-Abadie, M. Mirza, B. Xu, D. Warde-Farley, S. Ozair, A. Courville, and Y. Bengio, "Generative adversarial nets," in Advances in neural information processing systems, 2014, pp. 2672-2680.

[100] A. Graves, "Generating sequences with recurrent neural networks," arXiv preprint arXiv:1308.0850, 2013.

[101] K. Gregor, I. Danihelka, A. Graves, D. J. Rezende, and D. Wierstra, "Draw: A recurrent neural network for image generation," arXiv preprint arXiv:1502. 04623, 2015.

[102] D. Hasegawa, N. Kaneko, S. Shirakawa, H. Sakuta, and K. Sumi, "Evaluation of speech-to-gesture generation using bi-directional lstm network," in Proceedings of the 18th International Conference on Intelligent Virtual Agents, 2018, pp. $79-86$.

[103] M. Liwicki and H. Bunke, "Iam-ondb-an on-line english sentence database acquired from handwritten text on a whiteboard," in Eighth International Conference on Document Analysis and Recognition (ICDAR'05), IEEE, 2005, pp. 956-961.

[104] D. P. Kingma and M. Welling, "Auto-encoding variational bayes," arXiv preprint arXiv:1312.6114, 2013.

[105] A. Roberts, J. Engel, and D. Eck, "Hierarchical variational autoencoders for music," in NIPS Workshop on Machine Learning for Creativity and Design, 2017.

[106] A. Radford, L. Metz, and S. Chintala, "Unsupervised representation learning with deep convolutional generative adversarial networks," arXiv preprint arXiv:1511.06434, 2015.

[107] A. Augello, E. Cipolla, I. Infantino, A. Manfre, G. Pilato, and F. Vella, "Creative robot dance with variational encoder," arXiv preprint arXiv:1707.01489, 2017.

[108] A. M. Turing, "Computing machinery and intelligence," in Parsing the Turing Test, Springer, 2009, pp. 23-65. 
[109] Y. LeCun, S. Chopra, R. Hadsell, M. Ranzato, and F. J. Huang, "A Tutorial on Energy-Based Learning Yann," Predicting Structured Data, vol. 28, no. 3, pp. 391-397, 2016. arXiv: arXiv:1011.1669v3.

[110] C. Cortes and V. Vapnik, "Support-vector networks," Machine learning, vol. 20, no. 3, pp. 273-297, 1995.

[111] M. A. Boden, "Creativity and artificial intelligence," Artificial Intelligence, vol. 103, no. 1-2, pp. 347-356, 1998.

[112] G. Fauconnier and M. Turner, "Conceptual integration networks," Cognitive science, vol. 22, no. 2, pp. 133-187, 1998.

[113] T. Veale, M. T. Keane, D. O’Donoghue, M. T. Keane, and M. T. Keane, "Belief modelling, intentionality and perlocution in metaphor comprehension," in Proceedings of the Sixteenth Annual Meeting of the Cognitive Science Society, Citeseer, 1994.

[114] S. Coulson and T. Oakley, "Metonymy and conceptual blending," PRAGMATICS AND BEYOND NEW SERIES, pp. 51-80, 2003.

[115] M. Eppe, E. Maclean, R. Confalonieri, O. Kutz, M. Schorlemmer, E. Plaza, and K.-U. Kühnberger, "A computational framework for conceptual blending," Artificial Intelligence, vol. 256, pp. 105 -129, 2018.

[116] J. F. Sowa, "Semantic networks," 1987.

[117] A. Mordvintsev, C. Olah, and M. Tyka, "Deepdream-a code example for visualizing neural networks," Google Research, vol. 2, no. 5, 2015.

[118] J. Engel, C. Resnick, A. Roberts, S. Dieleman, D. Eck, K. Simonyan, and M. Norouzi, "Neural audio synthesis of musical notes with wavenet autoencoders," 2017.

[119] F. C. Pereira and A. Cardoso, "Dr. divago: Searching for new ideas in a multidomain environment," Proceedings of the 8th Cognitive Science of Natural Language Processing (CSNLP), 1999.

[120] R. Tubb and S. Dixon, "A four strategy model of creative parameter space interaction.," in ICCC, 2014, pp. 16-22. 
[121] G. A. Wiggins, "A preliminary framework for description, analysis and comparison of creative systems," Knowledge-Based Systems, vol. 19, pp. 449-458, 2006.

[122] C. Bartl and D. Dörner, "Psi: A theory of the integration of cognition, emotion and motivation," in Proceedings of the 2nd European Conference on Cognitive Modelling, Nottingham University Press, 1998, pp. 66-73.

[123] A. Augello, I. Infantino, U. Maniscalco, G. Pilato, R. Rizzo, and F. Vella, "Robotic intelligence and computational creativity," Encyclopedia with Semantic Computing and Robotic Intelligence, vol. 2, no. 01, p. 1850 011, 2018.

[124] S. McGregor, M. M. McGinity, and S. Griffiths, "How many robots does it take? creativity, robots and multi-agent systems," in The AISB15's 2nd International Symposium on Computational Creativity (CC2015), 2015, p. 23.

[125] W. Garage, Ros, http://www . willowgarage . com/pages/software/rosplatform, [Online; accessed 14-January-2020], 2020.

[126] ROS.org, Nasa-gm robonaut2, http://wiki.ros.org/Robots/Robonaut2, [Online; accessed 14-January-2020], 2020.

[127] P. Burgess, Animating multiple led backpacks, 2012.

[128] ROBOTIS, Dynamixel ax-12a, http://robotis.us/ax-12a/, [Online; accessed 21-May-2019], 2020.

[129] Q. Williams, S. Bogner, M. Kelley, C. Castillo, M. Lukac, D. H. Kim, J. S. Allen, M. I. Sunardi, S. Hossain, and M. Perkowski, "An emotional mimicking humanoid biped robot and its quantum control based on the constraint satisfaction model," 2007.

[130] M. Sunardi and M. Perkowski, "Music to motion: Using music information to create expressive robot motion," International Journal of Social Robotics, vol. 10, no. 1, pp. 43-63, 2018.

[131] Lynxmotion, Lynxmotion - al5a arm hardware-only kit, 2020.

[132] D. McNeill, Hand and mind: What gestures reveal about thought. University of Chicago Press, 1992. 
[133] J. E. Hopcroft, R. Motwani, and J. D. Ullman, "Introduction to automata theory, languages, and computation," Acm Sigact News, vol. 32, no. 1, pp. 60$65,2001$.

[134] S. Zhao, "Humanoid social robots as a medium of communication," New Media ES Society, vol. 8, no. 3, pp. 401-419, 2006.

[135] A. Bruderlin and L. Williams, "Motion signal processing," in Proceedings of the 22nd annual conference on Computer graphics and interactive techniques - SIGGRAPH '95, 1995, pp. 97-104, ISBN: 0897917014.

[136] P. Norvig, (how to write a (lisp) interpreter (in python)), https://norvig. com/lispy.html, [Online; accessed 9-June-2020], 2020.

[137] E. R. Gansner, "Drawing graphs with graphviz," Technical report, ATEST Bell Laboratories, Murray, Tech. Rep, Tech. Rep., 2009.

[138] G. R. Andrews and F. B. Schneider, "Concepts and notations for concurrent programming," ACM Computing Surveys (CSUR), vol. 15, no. 1, pp. 3-43, 1983.

[139] C. A. R. Hoare, "Communicating sequential processes," Communications of the ACM, vol. 21, no. 8, pp. 666-677, 1978.

[140] K. Pullen and C. Bregler, "Motion capture assisted animation: Texturing and synthesis," in Proceedings of the 29th annual conference on Computer graphics and interactive techniques, 2002, pp. 501-508.

[141] D. H. Kochanek and R. H. Bartels, "Interpolating splines with local tension, continuity, and bias control," in Proceedings of the 11th annual conference on Computer graphics and interactive techniques, 1984, pp. 33-41.

[142] R. Polikar, The Wavelet Tutorial, http://web.iitd.ac.in/ sumeet/WaveletTutorial.pdf.

[143] A. Camurri, G. Volpe, G. D. Poli, and M. Leman, "Communicating expressiveness and affect in multimodal interactive systems," IEEE Multimedia, vol. 12, no. 1, pp. 43-53, 2005.

[144] E. Hein, Scales and emotions, http : / / www . ethanhein . com / wp / 2010 / scales-and-emotions, [Online; accessed 29-January-2017], 2010. 
[145] C. Stanislavski, Building a Character. A \& C Black, 2013.

[146] R. Von Laban and R. Lange, Laban's principles of dance and movement notation. Princeton Book Co Pub, 1975.

[147] M. Perkowski, T. Sasao, J.-H. Kim, M. Lukac, J. Allen, and S. Gebauer, "Hahoe kaist robot theatre: Learning rules of interactive robot behavior as a multiple-valued logic synthesis problem," in Multiple-Valued Logic, 2005. Proceedings. 35th International Symposium on, IEEE, 2005, pp. 236-248.

[148] D. Lee, S. Park, M. Hahn, and N. Lee, "Robot actors and authoring tools for live performance system," in Information Science and Applications (ICISA), 2014 International Conference on, IEEE, 2014, pp. 1-3.

[149] M. I. Sunardi, "Expressive motion synthesis for robot actors in robot theatre," Master's thesis, Portland State University, 1900 SW Fourth Ave., Suite 160, Portland, OR 97201, Jun. 2010.

[150] Merriam-Webster.com, Expressive, https : / / www . merriam-webster .com / dictionary/expressive, [Online; accessed 19-June-2017], 2017.

[151] Merriam-Webster.com, Dynamic, https : / / www . merriam-webster . com / dictionary/dynamic, [Online; accessed 19-June-2017], 2017.

[152] M. P. Michalowski, S. Sabanovic, and H. Kozima, "A dancing robot for rhythmic social interaction," in Human-Robot Interaction (HRI), 2007 2nd ACM/ IEEE International Conference on, IEEE, 2007, pp. 89-96.

[153] F. C. R. Mannell and J. Harrington, An introduction to phonetics and phonology, http: / / clas.mq.edu.au/speech/phonetics / index.html, [Online; accessed 29-January-2017], Macquarie University, 2014.

[154] C Breazeal, "A context-dependent attention system for a social robot," in 1999 International Joint Conference on Artificial Intelligence, 1999.

[155] S. Levine, V. Koltun, and C. Theobalt, "Body language animation synthesis from prosody," ACM Transactions on Graphics, vol. 28, no. 5, 2009.

[156] C. Busso, Z. Deng, U. Neumann, and S. Narayanan, "Natural head motion synthesis driven by acoustic prosodic features," Computer Animation and Virtual Worlds, vol. 16, no. 3-4, pp. 283-290, 2005. 
[157] A. Bhutada, "Universal event and motion editor for robots' theatre," Master's thesis, Portland State University, 1900 SW Fourth Ave., Suite 160, Portland, OR 97201, Jun. 2011.

[158] M. E. Sargin, E. Erzin, Y. Yemez, A. M. Tekalp, A. T. Erdem, C Erdem, and M Ozkan, "Prosody-driven head-gesture animation," in 2007 IEEE International Conference on Acoustics, Speech and Signal Processing-ICASSP'07, IEEE, vol. 2, 2007, pp. II-677.

[159] M. Scheeff, J. Pinto, K. Rahardja, S. Snibbe, and R. Tow, "Experiences with sparky, a social robot," in Socially intelligent agents, Springer, 2002, pp. 173180.

[160] R. Fernando, GPU Gems: Programming Techniques, Tips and Tricks for RealTime Graphics. Pearson Higher Education, 2004.

[161] Muse-sequencer.org, Main page, http://www .muse-sequencer .org/, [Online; accessed 19-June-2017], 2016.

[162] MIDI.org, Summary of midi messages, https://www .midi.org/specifications/item/table-1-summary-of-midi-message, [Online; accessed 3February-2017], 2016.

[163] Medonis.com, Asc16 - advanced servo controller, http://www.medonis.com/ asc16.html, [Online; accessed 29-January-2017], 2008.

[164] Robosavvy.com, Khr-1 humanoid robot kit + roboard embedded 1ghz linux board, https : / / robosavvy . com / store / khr-1-humanoid-robot-kitroboard-embedded-1ghz-linux-board .html, [Online; accessed 29-January2017], Jan. 2015.

[165] R. A. Fisher, Statistical methods for research workers. Genesis Publishing Pvt Ltd, 1925.

[166] H. Scheffé, "An analysis of variance for paired comparisons," Journal of the American Statistical Association, vol. 47, no. 259, pp. 381-400, 1952.

[167] M. Hollander, D. A. Wolfe, and E. Chicken, Nonparametric statistical methods. John Wiley \& Sons, 2013. 
[168] G. E. Meek, C. Ozgur, and K. Dunning, "Comparison of the t vs. wilcoxon signed-rank test for likert scale data and small samples," Journal of Modern Applied Statistical Methods, vol. 6, no. 1, p. 10, 2007.

[169] R. Johnson, "Choosing between parametric and non-parametric tests," Journal of Undergraduate Research at Minnesota State University, Mankato, vol. 9, no. 1, p. 6, 2014.

[170] M. Pilhofer and H. Day, Music theory for dummies. John Wiley \& Sons, 2015.

[171] MIDI.org, The complete midi 1.0 detailed specification, https://www .midi. org/specifications/item/the-midi-1-0-specification, [Online; accessed 3-February-2017], 2016.

[172] I. Daubechies, "Ten Lectures of Wavelets," p. 357, 1992.

[173] S. G. Mallat, "A theory for multiresolution signal decomposition: The wavelet representation," IEEE transactions on pattern analysis and machine intelligence, vol. 11, no. 7, pp. 674-693, 1989.

[174] Y. Li, T. Wang, and H.-Y. Shum, "Motion texture: A two-level statistical model for character motion synthesis," in Proceedings of the 29th annual conference on Computer graphics and interactive techniques, 2002, pp. 465-472.

[175] D. M. Chi, "A motion control scheme for animating expressive arm movements," no. January, 1999.

[176] Y.-F. Ma and H.-J. Zhang, "Motion texture: A new motion based video representation," in Object recognition supported by user interaction for service robots, IEEE, vol. 2, 2002, pp. 548-551.

[177] R. C. Nelson and R. Polana, "Qualitative recognition of motion using temporal texture," CVGIP: Image understanding, vol. 56, no. 1, pp. 78-89, 1992.

[178] K. Granat. (2015). Rme. [Online; accessed 21-February-2020].

[179] E. Catmull and R. Rom, "A class of local interpolating splines," in Computer aided geometric design, Elsevier, 1974, pp. 317-326.

[180] P. J. Burt and E. H. Adelson, "A multiresolution spline with application to image mosaics," ACM Transactions on Graphics (TOG), vol. 2, no. 4, pp. 217$236,1983$. 
[181] M. Antonini, M. Barlaud, P. Mathieu, and I. Daubechies, "Image coding using wavelet transform," IEEE Transactions on image processing, vol. 1, no. 2, pp. 205-220, 1992.

[182] A. Skodras, C. Christopoulos, and T. Ebrahimi, "The jpeg 2000 still image compression standard," IEEE Signal processing magazine, vol. 18, no. 5, pp. 36-58, 2001.

[183] A. Grossmann and J. Morlet, "Decomposition of hardy functions into square integrable wavelets of constant shape," SIAM journal on mathematical analysis, vol. 15, no. 4, pp. 723-736, 1984.

[184] A. Graps, "An introduction to wavelets," IEEE computational science and engineering, vol. 2, no. 2, pp. 50-61, 1995.

[185] G. Lee, R. Gommers, F. Waselewski, K. Wohlfahrt, and A. O'Leary, "Pywavelets: A python package for wavelet analysis," Journal of Open Source Software, vol. 4, no. 36, p. 1237, 2019.

[186] M. K. Pan, E. Knoop, M. Bächer, and G. Niemeyer, "Fast handovers with a robot character: Small sensorimotor delays improve perceived qualities," in IEEE/RSJ International Conference on Intelligent Robots and Systems (IROS), 2019.

[187] A. V. Breemen, "Animation engine for believable interactive user-interface robots," in IEEE/RSJ International Conference on Intelligent Robots and Systems - IROS '04, vol. 3, IEEE, 2004, pp. 2873-2878, ISBN: 0-7803-8463-6.

[188] C. Bartneck, D. Kulić, E. Croft, and S. Zoghbi, "Measurement instruments for the anthropomorphism, animacy, likeability, perceived intelligence, and perceived safety of robots," International Journal of Social Robotics, vol. 1, no. 1, pp. 71-81, 2009.

[189] M. Mori, K. F. MacDorman, and N. Kageki, "The uncanny valley [from the field]," IEEE Robotics $\& 3$ Automation Magazine, vol. 19, no. 2, pp. 98-100, 2012 .

[190] A. Weiss and C. Bartneck, "Meta analysis of the usage of the Godspeed Questionnaire Series," in Proceedings - IEEE International Workshop on Robot and Human Interactive Communication, vol. 2015-Novem, IEEE, 2015, pp. 381388, ISBN: 9781467367042. 
[191] N. S. Pollard, J. K. Hodgins, M. J. Riley, and C. G. Atkeson, "Adapting human motion for the control of a humanoid robot," in Proceedings 2002 IEEE international conference on robotics and automation (Cat. No. 02CH37292), IEEE, vol. 2, 2002, pp. 1390-1397.

[192] S. Kim, C. Kim, B. You, and S. Oh, "Stable whole-body motion generation for humanoid robots to imitate human motions," in 2009 IEEE/RSJ International Conference on Intelligent Robots and Systems, IEEE, 2009, pp. 2518-2524.

[193] L. Boutin, A. Eon, S. Zeghloul, and P. Lacouture, "An auto-adaptable algorithm to generate human-like locomotion for different humanoid robots based on motion capture data," in 2010 IEEE/RSJ International Conference on Intelligent Robots and Systems, IEEE, 2010, pp. 1256-1261.

[194] S. Gaertner, M. Do, T. Asfour, R. Dillmann, C. Simonidis, and W. Seemann, "Generation of human-like motion for humanoid robots based on markerbased motion capture data," in ISR 2010 (41st International Symposium on Robotics) and ROBOTIK 2010 (6th German Conference on Robotics), VDE, 2010, pp. 1-8.

[195] Z. Wang, J. Chai, and S. Xia, "Combining recurrent neural networks and adversarial training for human motion synthesis and control," IEEE transactions on visualization and computer graphics, 2019.

[196] J. Ho and S. Ermon, "Generative adversarial imitation learning," in Advances in neural information processing systems, 2016, pp. 4565-4573.

[197] J. Merel, Y. Tassa, D. Tb, S. Srinivasan, J. Lemmon, Z. Wang, G. Wayne, and N. H. Deepmind, "Learning human behaviors from motion capture by adversarial imitation," Tech. Rep. arXiv: 1707.02201v2.

[198] B. Hahne, The Motionbuilder-friendly BVH Conversion Release of CMU's Motion Capture Database, 2011.

[199] M. E. Maron, "Automatic indexing: An experimental inquiry," Journal of the $A C M$ (JACM), vol. 8, no. 3, pp. 404-417, 1961.

[200] S. Hochreiter and J. Schmidhuber, "Long short-term memory," Neural computation, vol. 9, no. 8, pp. 1735-1780, 1997.

[201] A. Graves, "Supervised sequence labelling with recurrent neural networks," PhD thesis, Technische Universit at München, 2008. 
[202] X. Glorot and Y. Bengio, "Understanding the difficulty of training deep feedforward neural networks," in Proceedings of the thirteenth international conference on artificial intelligence and statistics, 2010, pp. 249-256.

[203] S. Ioffe and C. Szegedy, "Batch normalization: Accelerating deep network training by reducing internal covariate shift," arXiv preprint arXiv:1502.03167, 2015 .

[204] K. He, X. Zhang, S. Ren, and J. Sun, "Deep residual learning for image recognition," in Proceedings of the IEEE conference on computer vision and pattern recognition, 2016, pp. 770-778.

[205] A. Graves, "Generating Sequences With Recurrent Neural Networks," pp. 143, 2013. arXiv: 1308.0850.

[206] O. M. Andrychowicz, B. Baker, M. Chociej, R. Jozefowicz, B. McGrew, J. Pachocki, A. Petron, M. Plappert, G. Powell, A. Ray, et al., "Learning dexterous in-hand manipulation," The International Journal of Robotics Research, vol. 39, no. 1, pp. 3-20, 2020.

[207] Z. Wang, J. Chai, and S. Xia, "Combining Recurrent Neural Networks and Adversarial Training for Human Motion Synthesis and Control," 2018. arXiv: 1806.08666

[208] D. Holden, "Edinburgh Research Explorer Fast Neural Style Transfer for Motion Data," 2017.

[209] C. M. Bishop, "Mixture Density Networks," 1994.

[210] D. Ha and D. Eck, "A neural representation of sketch drawings," arXiv preprint arXiv:1704.03477, 2017.

[211] T. Salimans, D. P. Kingma, and M. Welling, Markov chain monte carlo and variational inference: Bridging the gap, 2014. arXiv: 1410.6460 [stat.CO].

[212] C. Doersch, "Tutorial on variational autoencoders," arXiv preprint arXiv:1606. 05908, 2016.

[213] D. P. Kingma and J. Ba, "Adam: A method for stochastic optimization," arXiv preprint arXiv:1412.6980, 2014. 
[214] Keras. (2020). Example of vae on mnist dataset using mlp. Accessed: 2020-0526.

[215] P.-L. Pröve, An Introduction to different Types of Convolutions in Deep Learning.

[216] Tensorflow.org, Deep convolutional generative adversarial network, https:// www . tensorflow.org/tutorials/generative/dcgan, [Online; accessed 16June-2020], 2020.

[217] M. Mathias, R. Timofte, R. Benenson, and L. Van Gool, "Traffic sign recognition - how far are we from the solution?" In The 2013 international joint conference on Neural networks (IJCNN), IEEE, 2013, pp. 1-8. 


\section{APPENDIX A MOTION DATASET}

The works in Chapter 7 and 8 used two motion datasets: 1) a collection of sampled motion capture data from the CMU Motion Capture Database that have been converted into BVH format by Hahne [198], and 2) a collection of HROS-1 robot motion data generated using REBeL/behavior expressions, based on the pose data created by Mekana Burch. The attribution for the CMU Motion Capture Database is as follows:

The data used in this project was obtained from mocap.cs.cmu.edu. The database was created with funding from NSF EIA-0196217.

These datasets accompany this dissertation as two files: mocap_cleaned_dataset .csv and robot_cleaned_dataset.csv. More detailed descriptions about these two files are summarized in the table below. 
Details of motion dataset files.

\begin{tabular}{|c|c|c|}
\hline & Motion capture dataset & HROS-1 motion dataset \\
\hline File name & mocap_cleaned_dataset.csv & robot_cleaned_dataset.csv \\
\hline File type & $\begin{array}{l}\text { Comma-separated } \quad \text { values } \\
(\mathrm{CSV})\end{array}$ & CSV \\
\hline Encoding & UTF-8 & UTF-8 \\
\hline Size & $488 \mathrm{MB}(500,692 \mathrm{~KB})$ & $314 \mathrm{MB}(321,632 \mathrm{~KB})$ \\
\hline $\begin{array}{l}\text { Required applica- } \\
\text { tion software }\end{array}$ & $\begin{array}{l}\text { Microsoft Excel, any text ed- } \\
\text { itor, Python csv or Pandas } \\
\text { packages }\end{array}$ & Same \\
\hline $\begin{array}{l}\text { Hardware require- } \\
\text { ments }\end{array}$ & $\mathrm{N} / \mathrm{A}$ & $\mathrm{N} / \mathrm{A}$ \\
\hline Description & $\begin{array}{l}\text { Each data point is a motion } \\
\text { of } 100 \text { frames for } 8 \text { joints (see } \\
\text { Chapter } 7 \text { for more details). } \\
\text { Contains } 51918 \text { data points. }\end{array}$ & $\begin{array}{l}\text { Each data point is a motion } \\
\text { of } 100 \text { frames for } 8 \text { joints (see } \\
\text { Chapter } 7 \text { for more details). } \\
\text { Contains } 39470 \text { data points. }\end{array}$ \\
\hline
\end{tabular}

